Electronic Supplementary Information for

\title{
Phosphine-catalyzed Vinylation at Low Acetylene Pressure
}

\author{
Nikolai A. Sitte, ${ }^{\dagger, \#}$ Maximilian Menche, ${ }^{+, \neq, \# ~ P a v e l ~ T u z ̌ i n a, ~}{ }^{\S}$ Frank Bienewald,, Ansgar Schäfer, ${ }^{\ddagger}$ Peter \\ Comba,ll Frank Rominger, ${ }^{\perp}$ A. Stephen K. Hashmi, ${ }^{\perp}{ }^{\perp}$ and Thomas Schaub ${ }^{*}+, \S$ \\ ${ }^{\dagger}$ Catalysis Research Laboratory (CaRLa), Im Neuenheimer Feld 584, D-69120 Heidelberg, Germany \\ ‡BASF SE, Quantum Chemistry, Carl-Bosch-Straße 38, D-67056 Ludwigshafen, Germany \\ §BASF SE, Chemical Synthesis Research, Carl-Bosch-Straße 38, D-67056 Ludwigshafen, Germany \\ IInstitute of Inorganic Chemistry \& Interdisciplinary Center for Scientific Computing, Heidelberg University, Im \\ Neuenheimer Feld 270, D-69120 Heidelberg, Germany \\ ${ }^{\perp}$ Institute of Organic Chemistry, Heidelberg University, Im Neuenheimer Feld 270, D-69120 Heidelberg, Germany
}




\section{Table of Contents}

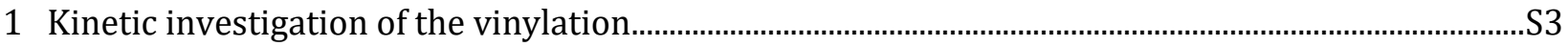

2 Additional Computational Information on the Reaction Mechanism.......................................................S6

2.1 Addition of Phosphine to Acetylene in trans-Configuration .................................................... S6

2.2 Alternative Addition Pathways from C............................................................................................. S8

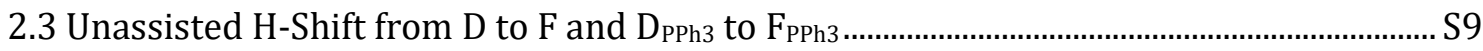

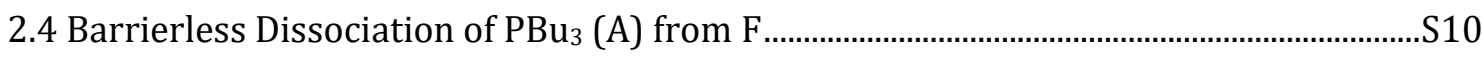

2.5 Potential Effects of Addition of Phenol to the Reaction System ..........................................S11

2.6 Calculations on the Experimentally Observed Dicationic Double Phosphine Addition

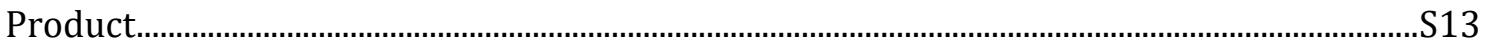

2.7 Activating Acetylene with the Pyrrolidone Anion as Alternative Pathway.......................S15

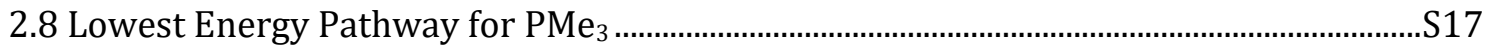

2.9 Additional Product Degradation Pathways ....................................................................................S18

2.10 Dimerization by Wittig-type Reaction Pathways ........................................................................ 20

2.11 Degradation by Wittig-type Reaction Pathways......................................................................S20

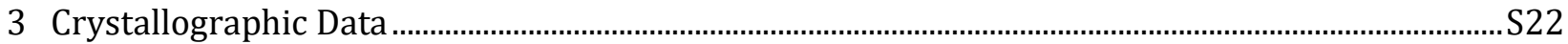

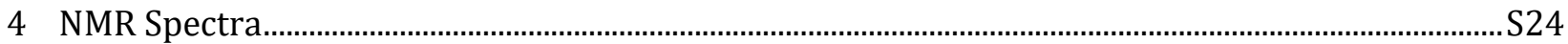

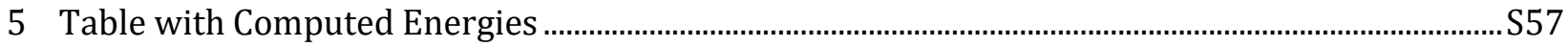

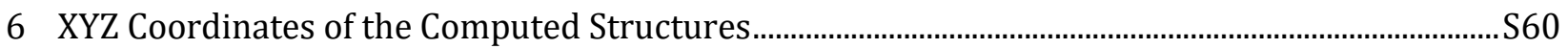




\title{
1 Kinetic investigation of the vinylation
}

\author{
$\mathrm{P}(n \mathrm{Bu})_{3}(10 \mathrm{~mol} \%)$ \\ $\mathrm{C}_{2} \mathrm{H}_{2}$ (atm.) \\ DMAA, $1-5 \mathrm{~h}, 130^{\circ} \mathrm{C}$
}

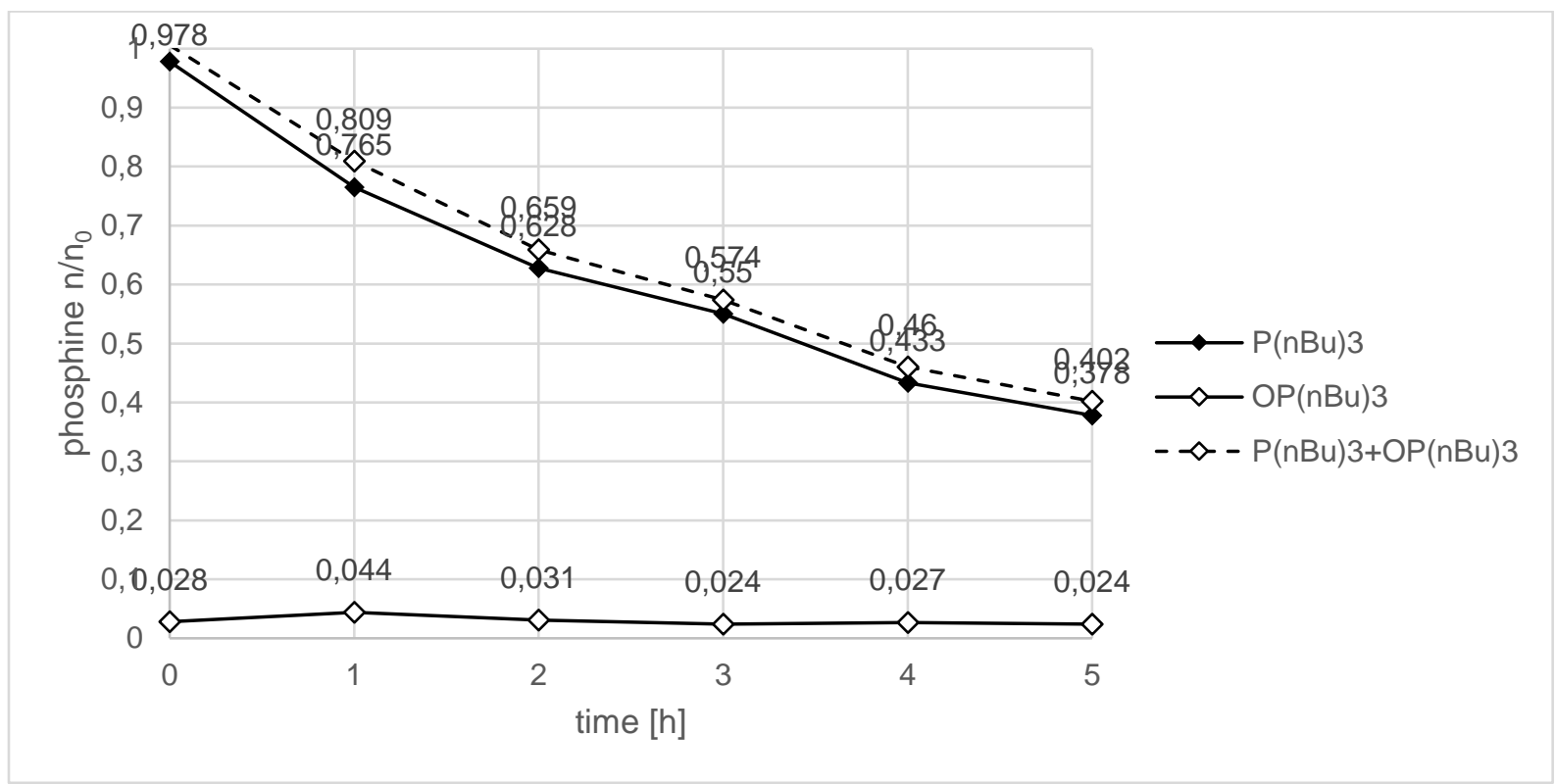

Scheme S-1. Kinetic investigation of the side reaction between tri- $n$-butylphosphine and acetylene. Amount versus time diagram of $\mathrm{P}(\mathrm{nBu})_{3}(\bullet), \mathrm{OP}(\mathrm{nBu})_{3}(\circ)$ and the sum $(\circ$, dashed). 

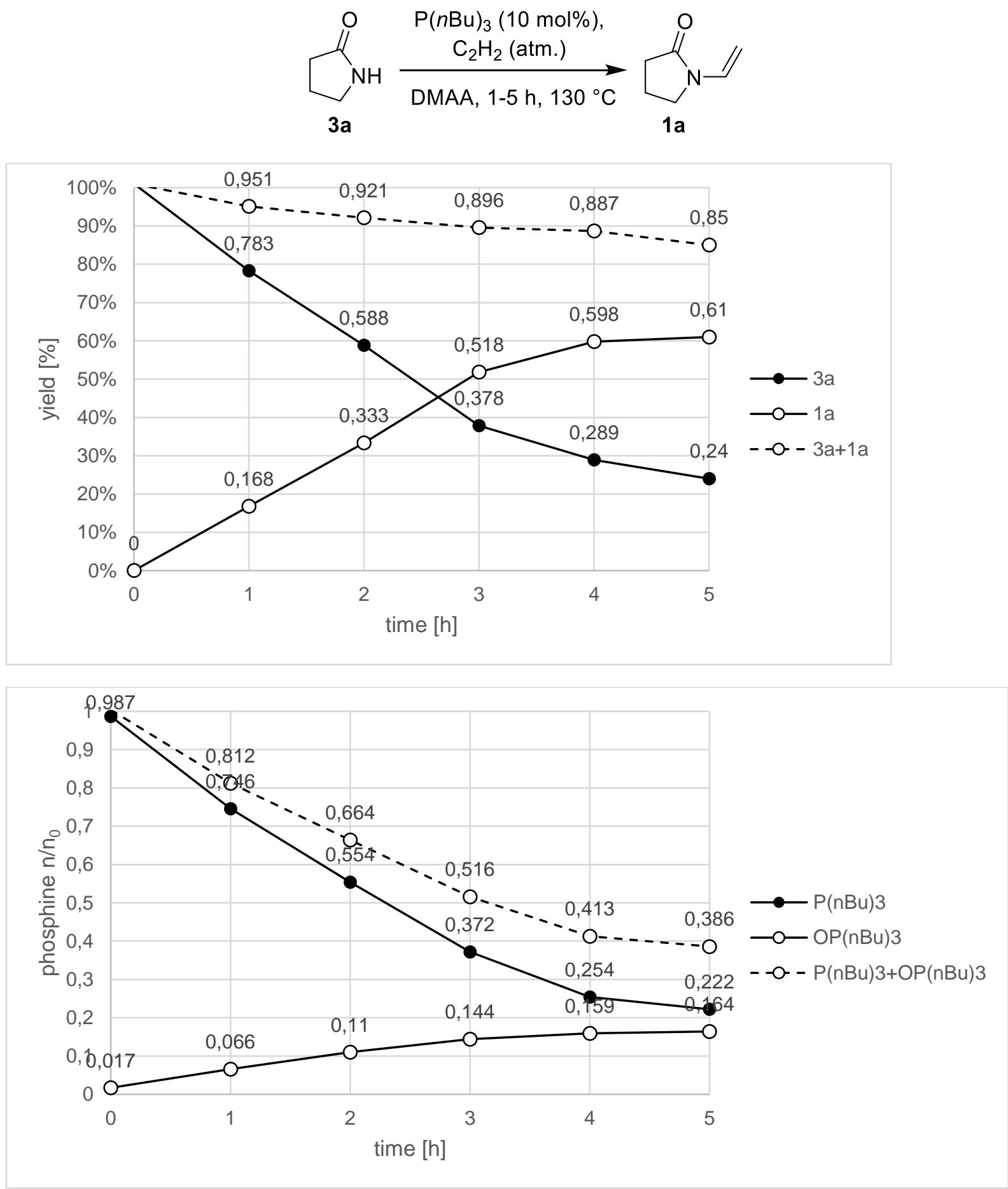

Scheme S-2. Kinetic investigation of the phosphine catalyzed vinylation of 3a. Top. Yield versus time diagram of 3a $(\bullet), \mathbf{1 a}(\circ)$ and the sum $\left(\circ\right.$, dashed). Bottom. Catalyst amount versus time diagram of $\mathrm{P}(n \mathrm{Bu})_{3}(\bullet), \mathrm{OP}(n \mathrm{Bu})_{3}(\circ)$ and the sum (o, dashed). 

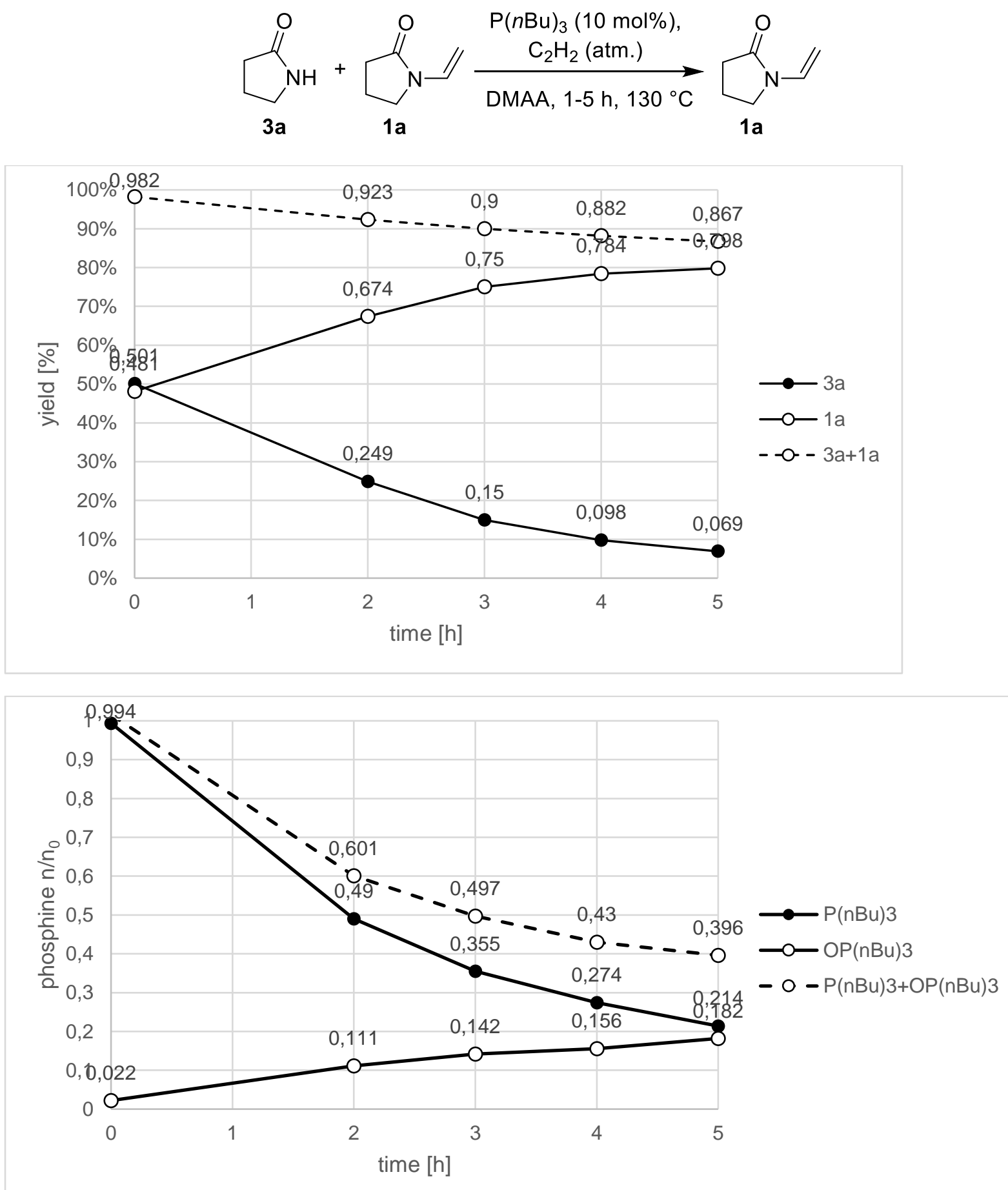

Scheme S-3. Kinetic investigation of the phosphine catalyzed vinylation of 3a with an equimolar amount of product 1a present. Top. Yield versus time diagram of $\mathbf{3 a}(\bullet), \mathbf{1 a}(\circ)$ and the sum (०, dashed). Bottom. Catalyst amount versus time diagram of $\mathrm{P}(n \mathrm{Bu})_{3}(\bullet), \mathrm{OP}(n \mathrm{Bu})_{3}(\circ)$ and the sum $(\circ$, dashed). 


\section{Additional Computational Information on the Reaction Mechanism}

In order to better understand the underlying reactivity, various additional mechanistic pathways were investigated. In the following, the information for these additional pathways and the lowest energy pathway employing $\mathrm{PMe}_{3}$, are provided.

\subsection{Addition of Phosphine to Acetylene in trans-Configuration}

The initial activation of acetylene by the phosphines can happen in cis- and trans-configuration in respect to the position of the hydrogen atom at $\beta$ position and the activating phosphine (scheme S-4). For both phosphines, $\mathrm{PBu}_{3}(\mathbf{A})$ and $\mathrm{PPh}_{3}\left(\mathbf{A}_{\mathrm{PPh}}\right)$, the initial addition of the catalyst is favored in the cisconfiguration: $\mathbf{T S}-\mathbf{A B}_{\text {cis }}\left(\Delta G^{+}=99.2 \mathrm{~kJ} \mathrm{~mol}^{-1}\right)$ is $8.2 \mathrm{~kJ} \mathrm{~mol}^{-1}$ more feasible than $\mathbf{T S}-\mathbf{A B}_{\text {trans }}$ $\left(\Delta G^{+}=107.4 \mathrm{~kJ} \mathrm{~mol}^{-1}\right)$ and TS-AB ${ }_{\text {cis/PPh }}\left(\Delta G^{+}=122.8 \mathrm{~kJ} \mathrm{~mol}^{-1}\right)$ is $27.6 \mathrm{~kJ} \mathrm{~mol}^{-1}$ more feasible than TS-AB trans/PPh3 $\left(\Delta G^{+}=150.4 \mathrm{~kJ} \mathrm{~mol}^{-1}\right)$. The resulting intermediates show a significantly more stable trans-configuration for phosphine $\mathbf{A}\left(\Delta G^{413}=62.5 \mathrm{~kJ} \mathrm{~mol}^{-1} \mathrm{vs} .36 .2 \mathrm{~kJ} \mathrm{~mol}^{-1}\right)$ and a slightly more stable trans-configuration for APPh3 $\left(\Delta G^{413}=105.6 \mathrm{~kJ} \mathrm{~mol}^{-1} \mathrm{vs}\right.$. $\left.95.1 \mathrm{~kJ} \mathrm{~mol}^{-1}\right)$. The subsequent proton transfer transition states, where the $\mathrm{NH}$ proton of the pyrrolidone substrate is transferred onto the $\beta$ position of the activated acetylene, is significantly lower in energy for the trans-configuration, both in the case of catalyst $A\left(\Delta G^{+}=84.2 \mathrm{~kJ} \mathrm{~mol}^{-1} \mathrm{vs} .50 .2 \mathrm{~kJ} \mathrm{~mol}^{-1}\right)$ and Apph $\left(\Delta G^{\ddagger}=119.8 \mathrm{~kJ} \mathrm{~mol}^{-1}\right.$ vs. $\left.105.9 \mathrm{~kJ} \mathrm{~mol}^{-1}\right)$. However, as the barriers of the proton transfer transition states $\mathbf{T S}-\mathbf{B C}_{\text {cis }}$ and TS$\mathbf{B C}_{\text {trans }}$ (as well as $\mathbf{T S}-\mathbf{B C}_{\text {cis/PPh3 }}$ and $\mathbf{T S}-\mathbf{B C}_{\text {trans/PPh3 }}$ ) remain below the energy barriers of phosphine addition, the latter transition state remains the rate-determining step of this reaction cascade and therefore, the cis-pathway remains preferred.
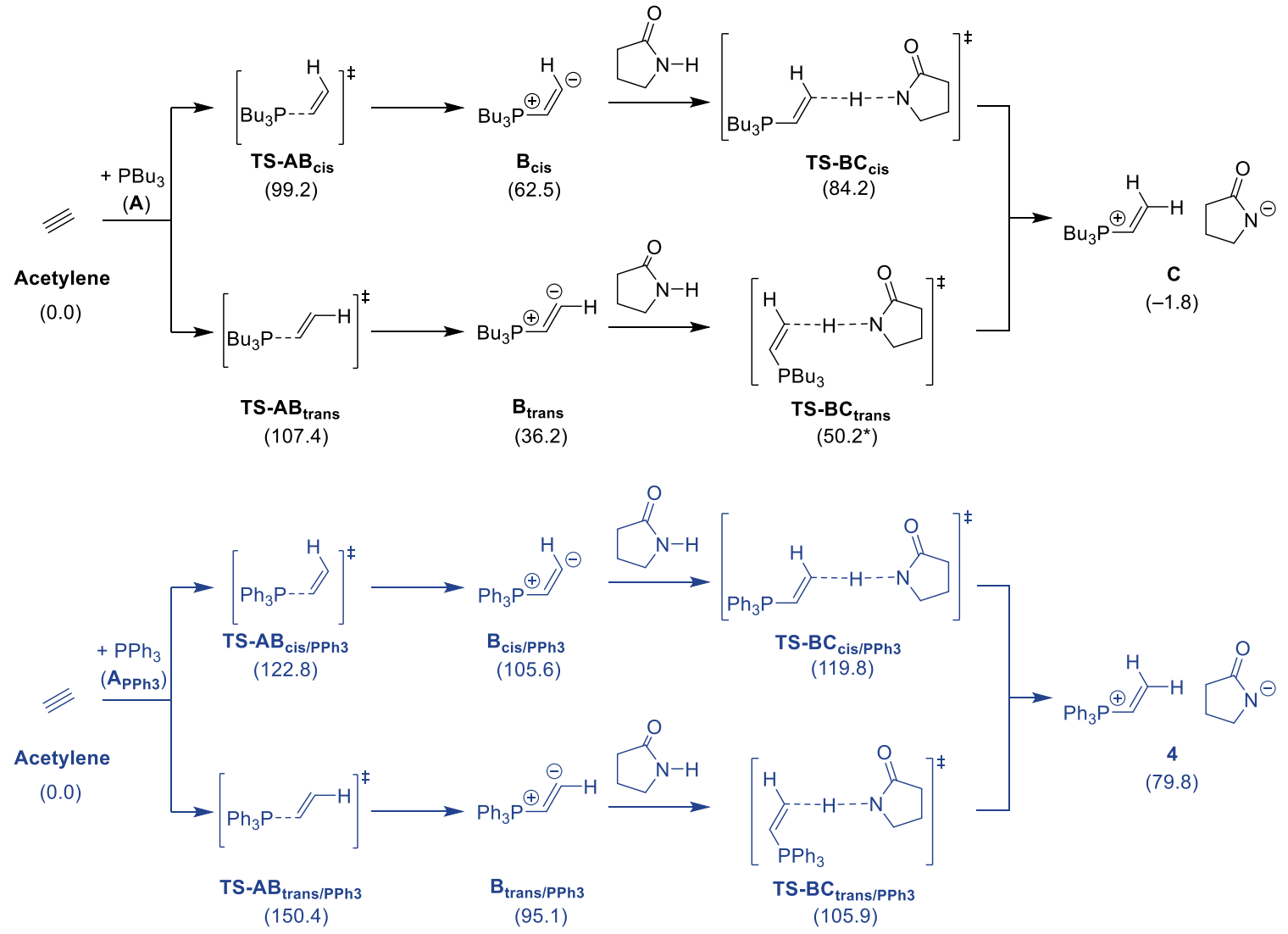

Scheme S-4: Catalytic pathways for the.cis- and trans-activation of acetylene with $\mathrm{PBu}_{3}\left(\mathbf{A}\right.$; black) and $\mathrm{PPh}$ ( $\mathbf{A P P h}_{3}$; red). $\Delta G^{413}$ in kJ mol-1; RI-PBE0-D3(BJ)/def2-QZVPP//BP86/def2-SV(P); COSMO-RS (DMF). *A converged structure 
of TS-BC trans could not be located. The reported energy is from a structure close to the minimum, for which some bond distances have been frozen.

Having established that the cis-pathway is preferred, a potential cis-trans isomerization of intermediated $\mathbf{B}_{\text {cis }}$ and $\mathbf{B}_{\text {trans }}$ was investigated (Scheme S-5) even though - due to the fact that the proton transfer transition state energies are lower than the acetylene activation - this will not lead to a reduction of the overall barrier of this two-step procedure. For this, two pathways were considered: (1) an inversion around the $\beta$ positioned carbon atom and (2) rotation around the $\mathrm{C}=\mathrm{C}$ bond. For neither of the two pathways a suitable transition state could be characterized. Structures that were not fully converged, but close to the expected minimum and exhibited the correct reaction mode were significantly higher in energy than the acetylene activation and proton transfer transition states. Based on this, any cis-trans isomerization was not further considered.

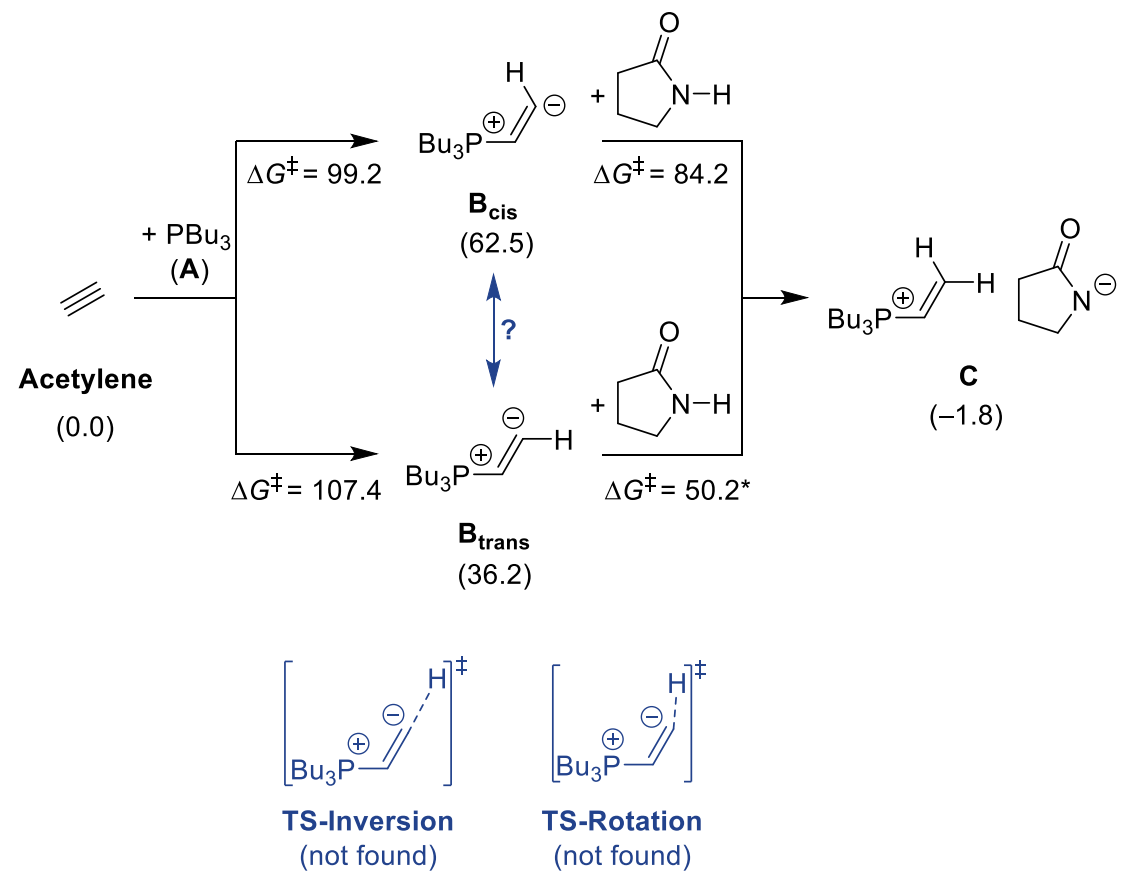

Scheme S-5: Potential isomerization pathways between $\mathbf{B}_{\text {cis }}$ and $\mathbf{B}_{\text {trans }}$ via inversion or rotation. Both transition states could not be located, however, energies from structures exhibiting the desired reaction mode were significantly higher

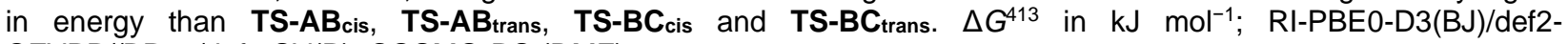
QZVPP//BP86/def2-SV(P); COSMO-RS (DMF). 


\subsection{Alternative Addition Pathways from C}

Apart from the addition of the pyrrolidone anion to the beta position of the cation (TS-CD in Scheme S-6; $\Delta G^{+}=23.4 \mathrm{~kJ} \mathrm{~mol}^{-1}$ ), which was proposed to be a key step in the main catalytic pathway that continues via a protonation-deprotonation cascade from $\mathbf{D}\left(\Delta G^{413}=-57.3 \mathrm{~kJ} \mathrm{~mol}^{-1}\right)$, further potential additions of the pyrrolidone anion to the cation were investigated (Scheme S-6). This investigation considered the addition of the oxygen atom of the pyrrolidone unit to the activated acetylene (TS-S1; $\Delta G^{\ddagger}=41.3 \mathrm{~kJ} \mathrm{~mol}^{-1}$ ) as well as the $\mathrm{N}$-addition to the a position of the cation in C (TS-S2; $\Delta G^{\ddagger}=72.2 \mathrm{~kJ} \mathrm{~mol}^{-1}$ ). Both alternative pathways exhibit a higher energy barrier then the previously investigated transition state TS-CD. Due to the fact that intermediate $\mathbf{D}\left(\Delta G^{413}=-57.3 \mathrm{~kJ} \mathrm{~mol}^{-1}\right)$, which follows TS-CD, is also the most stable intermediate of the three (D, S1, and $\mathbf{S 3}$ ) the reverse reaction from $\mathbf{D}$ to $\mathbf{C}$ will be significantly less prominent compared to the reverse reactions from $\mathbf{S 1}$ $\left(\Delta G^{413}=19.4 \mathrm{~kJ} \mathrm{~mol}^{-1}\right)$ and S3 $\left(\Delta G^{413}=50.5 \mathrm{~kJ} \mathrm{~mol}^{-1}\right)$. The combination of these factors leads us to the conclusion that neither of the two alternative pathways is relevant to the observed reactivity and its associated reaction mechanism. One should note that proceeding over TS-S2 and S3 would most likely lead to the same overall product as $\mathrm{P}(n \mathrm{Bu})_{3}(\mathbf{A})$ could be eliminated from $\mathbf{S} 3$ to yield the desired product NVP.
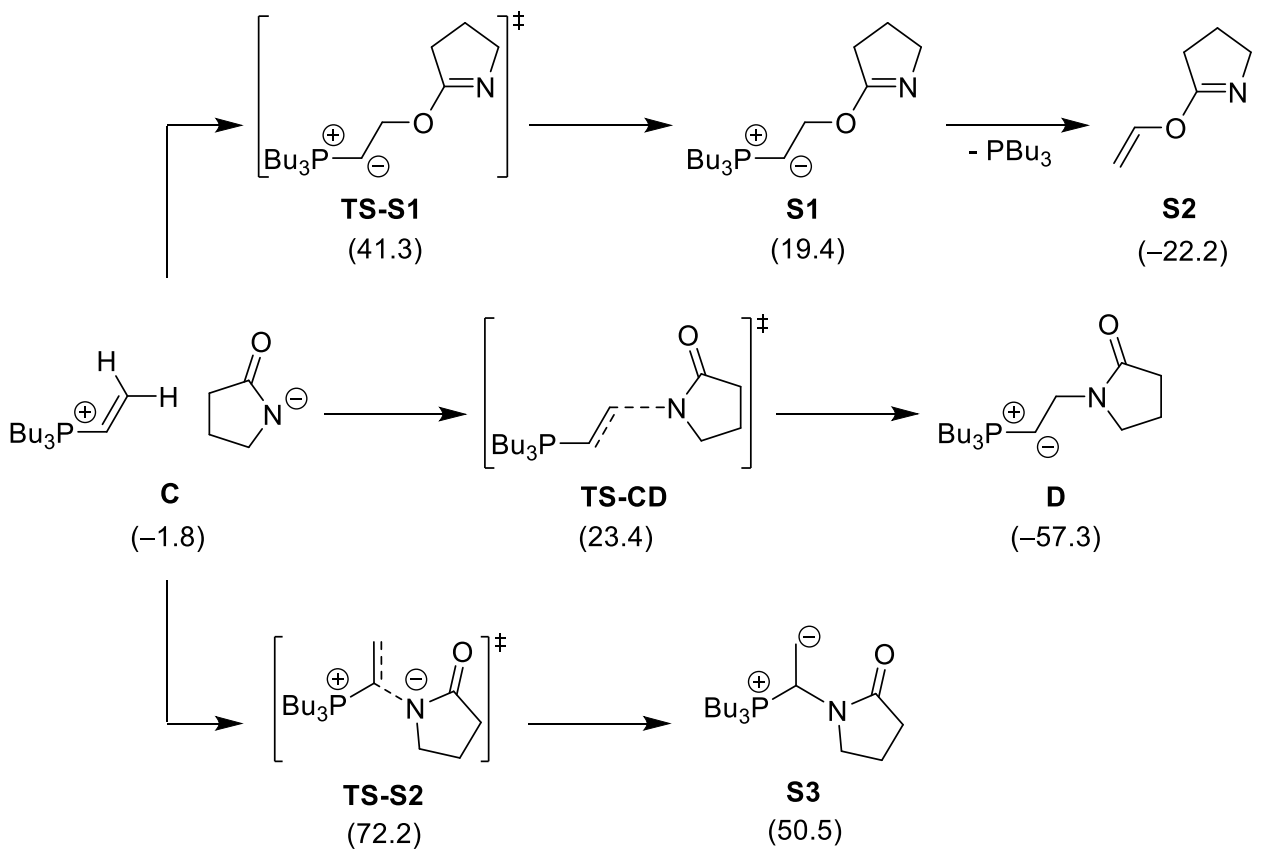

Scheme S-6: Alternative pathways for nucleophilic addition from C. Neither O-addition of the pyrrolidone anion (TS-S1) nor addition to the a carbon atom of the activated acetylene (TS-S2) are as feasible as the previously shown TS-CD. $\Delta G^{413}$ in $\mathrm{kJ} \mathrm{mol}^{-1}$; referenced to A, pyrrolidone and acetylene; RI-PBE0-D3(BJ)/def2-QZVPP//BP86/def2-SV(P); COSMO-RS (DMF). 


\subsection{Unassisted $\mathrm{H}$-Shift from $\mathrm{D}$ to $\mathrm{F}$ and $\mathrm{DPPh}_{\mathrm{PP}}$ to $\mathrm{F}_{\mathrm{PPh} 3}$}

In addition to the previously presented protonation-deprotonation cascade over TS-DE and TS-EF (see Figure 1), a direct unassisted $\mathrm{H}$-shift from $\mathbf{D}$ to $\mathbf{F}$ was also investigated (Scheme S-7). In both systems, employing $\mathrm{P}(n \mathrm{Bu})_{3}(\mathbf{A})$ and $\mathrm{PPh}_{3}\left(\mathbf{A P P h}_{3}\right)$, the direct $\mathrm{H}$-shift is significantly higher in energy than the pyrrolidone-assisted pathway (TS-S3: $\Delta G^{\ddagger}=212.2 \mathrm{~kJ} \mathrm{~mol}^{-1}$; TS-S3Pph3: $\Delta G^{\ddagger}=252.4 \mathrm{~kJ} \mathrm{~mol}^{-1}$ ) and will not take part in the reaction cascade.
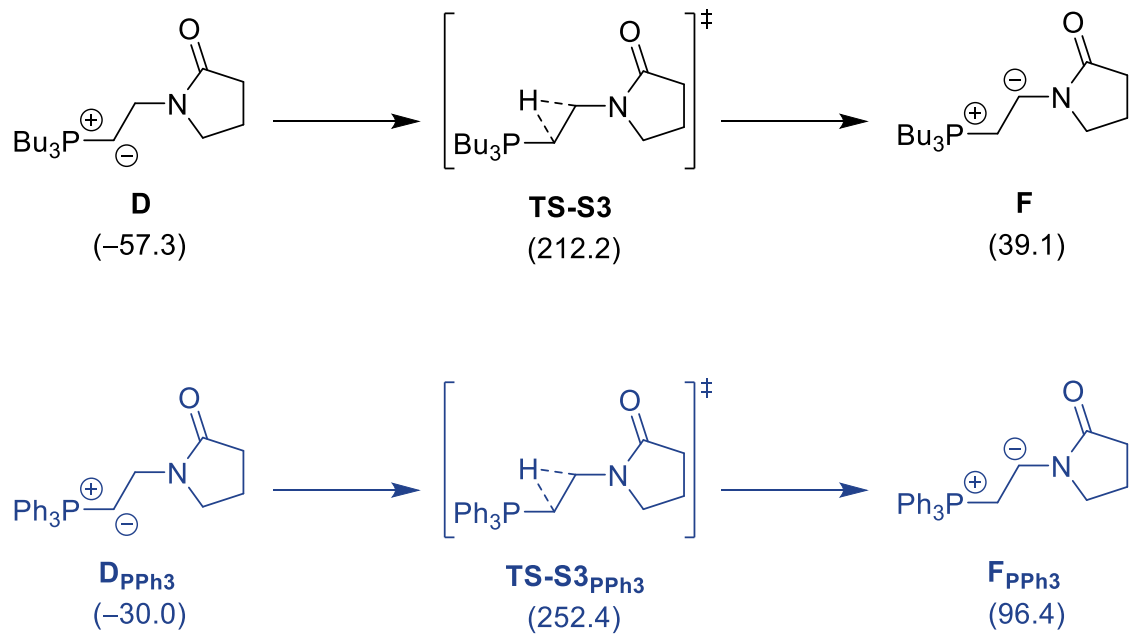

Scheme S-7: Alternative pathways for unassisted H-shift from $\mathbf{D}$ to $\mathbf{F}$ and $\mathbf{D p p h}$ to $\mathbf{F p p h}$. The unassisted H-shift is significantly less feasible than the protonation-deprotonation cascade. $\Delta G^{413}$ in $\mathrm{kJ} \mathrm{mol}^{-1}$; referenced to $\mathbf{A}$ (or $\mathbf{A P P h}$ ), pyrrolidone and acetylene; RI-PBE0-D3(BJ)/def2-QZVPP//BP86/def2-SV(P); COSMO-RS (DMF). 


\subsection{Barrierless Dissociation of $\mathrm{PBu}_{3}(\mathrm{~A})$ from $\mathrm{F}$}

After formation of intermediate $\mathbf{F}$, the dissociation of phosphine ligand - either $\mathrm{P}(n \mathrm{Bu})_{3}(\mathbf{A})$ or $\mathrm{PPh}_{3}$ (APPh3) - is required to form the desired product NVP and regenerate the phosphine catalyst. To investigate this dissociation step, the bond length of the C-P bond in intermediate $\mathbf{F}$ was scanned until a significant separation of the two fragments was observed. The energy plot $(\Delta E)$ does not show any significant barriers over the course of the reaction coordinate and indicates that this reaction takes place as barrierless dissociation of phosphine A. Due to the highly exergonic nature of this step, an extremely quick dissociation and therefore regeneration of catalyst is assumed.
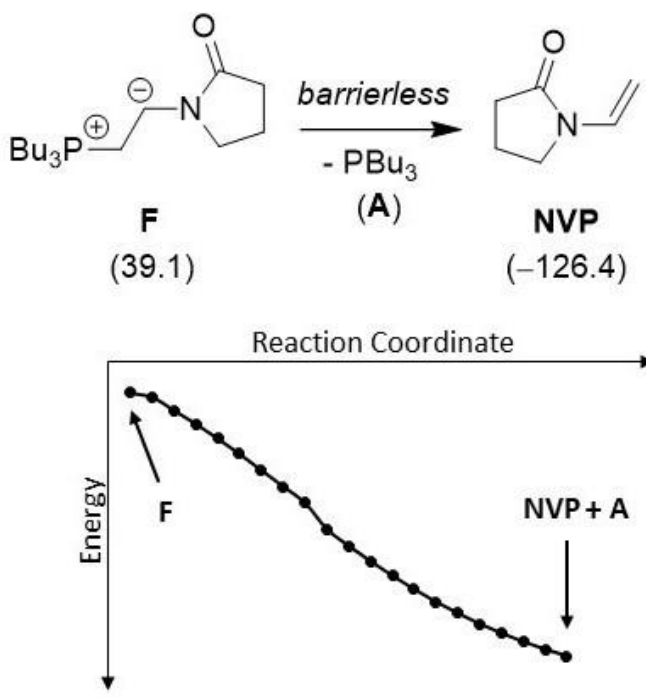

Figure S-1: Scan of the C-P bond length to investigate the dissociation of phosphine $\mathbf{A}$ from $\mathbf{F}$ leading to generation of desired NVP and regeneration of phosphine catalyst. $\Delta G\left(F\right.$ and NVP) and $\Delta E$ (bond length scan) in kJ mol${ }^{-1}$; referenced to A, pyrrolidone and acetylene; RI-PBE0-D3(BJ)/def2-QZVPP//BP86/def2-SV(P); COSMO-RS (DMF). 


\subsection{Potential Effects of Addition of Phenol to the Reaction System}

After the initial calculations on the minimum energy pathway for the transformation of acetylene and pyrrolidone to NVP were completed and the significantly more feasible protonation-deprotonation cascade (compared to the unassisted $\mathrm{H}$-shift) was observed, an investigation into the effect of phenol was conducted. The aim of this set of calculations was to probe if the addition of compounds facilitating such a cascade could lead to a reduction of the overall energy barrier and therefore increase activity of the reactive system. The calculations show a significant stabilization of TS-DE (to TS-DE Phenol) $_{\text {) }}$ (TS-DE: $\Delta G^{\ddagger}=-9.2 \mathrm{~kJ} \mathrm{~mol}^{-1}$; TS-DEPhenol: $\Delta G^{+}=-62.2 \mathrm{~kJ} \mathrm{~mol}^{-1}$ ) and of $\mathbf{E}$ (to Ehenol) (E: $\Delta G^{413}=-35.8 \mathrm{~kJ} \mathrm{~mol}^{-1} ; \mathbf{E}_{\text {Phenol }}: \Delta G^{413}=-86.8 \mathrm{~kJ} \mathrm{~mol}^{-1}$ ), which does fit the general intention of the idea of using phenol as an additional reagent. However, the calculations show that the stabilization of TSEF to TS-EF $F_{\text {Phenol }}$ is significantly less strong (TS-EF: $\Delta G^{+}=50.2 \mathrm{~kJ} \mathrm{~mol}^{-1}$; TS-EF Phenol: $\Delta G^{\ddagger}=41.3 \mathrm{~kJ} \mathrm{~mol}^{-1}$ ). Overall, this leads to an increase of the rate-determining energy barrier and therefore the direct opposite of the intended effect.
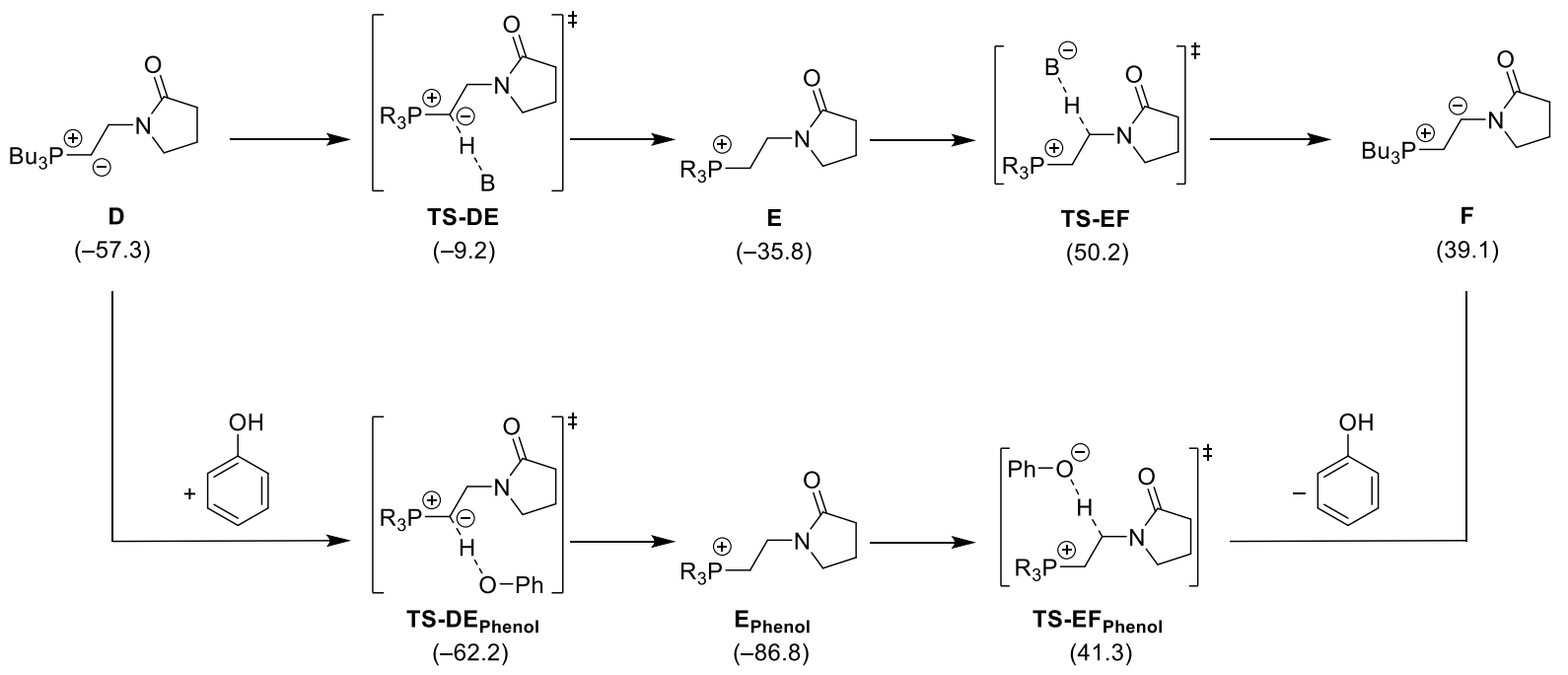

Scheme S-8: Investigation of the effect of phenol on the protonation-deprotonation cascade, which represents the ratedetermining step of the catalytic cycle. The phenolate anion does lower the energy of transition state TS-EF but leads to a significantly higher stabilization of intermediate $\mathbf{E}$ leading to an overall increase in reaction barrier. $\Delta G^{413}$ in $\mathrm{kJ} \mathrm{mol}^{-1}$; referenced to A, pyrrolidone and acetylene; RI-PBE0-D3(BJ)/def2-QZVPP//BP86/def2-SV(P); COSMO-RS (DMF).

To further understand what effects the addition of phenol might have on the whole reaction cascade, other potential phenolate ion pairs and phenol-containing intermediates were calculated. Neither the phenolate ion pair $\mathbf{C}_{\text {Phenol }}\left(\Delta G^{413}=-56.9 \mathrm{~kJ} \mathrm{~mol}^{-1}\right)$, which is significantly more stable than the previously shown pyrrolidone ion pair $\mathbf{C}\left(\Delta G^{413}=-1.8 \mathrm{~kJ} \mathrm{~mol}^{-1}\right)$, nor the phenolate addition product $\mathbf{S 4}$ $\left(\Delta G^{413}=-56.6 \mathrm{~kJ} \mathrm{~mol}^{-1}\right)$ are as low in energy as $E_{\text {Phenol }}\left(\Delta G^{413}=-86.8 \mathrm{~kJ} \mathrm{~mol}^{-1}\right)$, the intermediate of the protonation-deprotonation cascade presented in the previous scheme. 


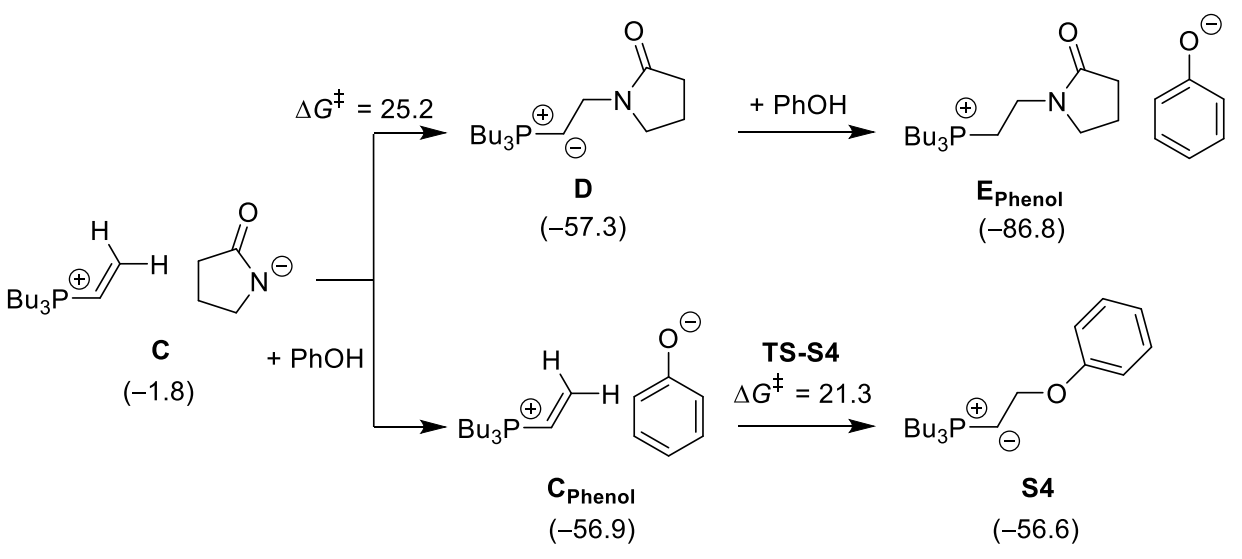

Scheme S-9: Overview of different intermediates and reaction pathways that were calculated to understand the effect of the addition of phenol to the catalytic system. The previously investigated intermediate EPhenol is the most stable species of the four intermediates. $\Delta G^{413}$ in $\mathrm{kJ} \mathrm{mol}^{-1}$; referenced to $\mathrm{A}$, pyrrolidone and acetylene; RI-PBE0-D3(BJ)/def2QZVPP//BP86/def2-SV(P); COSMO-RS (DMF).

All in all, the calculations have shown that the addition of phenol should not lead to an improvement in reaction performance, which was later confirmed by experiments. The calculations would also suggest that instead of the desired product the intermediate $\mathbf{E}_{\text {Phenol }}$ is formed. This was proven wrong in later experiments and could then also be rationalized by computing further reaction pathways (see the following section). 


\subsection{Calculations on the Experimentally Observed Dicationic Double Phosphine Addition Product}

After the results of the mechanistic experiments revealed that the addition of a second equivalent of phosphine to the activated acetylene species was preferred over the nucleophilic addition when employing $\mathrm{P}(n \mathrm{Bu})_{3} \cdot \mathrm{HBF}_{4}$ or phenol, further computational investigations were employed to understand this sharp difference in behavior compared to employing pyrrolidone (scheme S-10).

The quantum-chemical investigation of the $\mathrm{P}(n \mathrm{Bu})_{3} \cdot \mathrm{HBF}_{4}$ system is problematic as the generation of $\mathrm{BF}_{4}^{-}$from $\mathrm{P}(n \mathrm{Bu})_{3} \cdot \mathrm{HBF}_{4}$ will most likely be accompanied by a large error if not extensively investigated. Therefore, only the addition of the second phosphine molecule to intermediate $\mathbf{C}_{\mathrm{BF} 4}$ (TS-S5 $5_{\mathrm{BF} 4}$ : $\left.\Delta G^{\ddagger}=52.4 \mathrm{~kJ} \mathrm{~mol}^{-1}\right)$ and the subsequent intermediate $S 5_{\mathrm{BF} 4}\left(\Delta G^{413}=4.6 \mathrm{~kJ} \mathrm{~mol}^{-1}\right)$ were investigated. Afterwards, the same reaction steps were calculated for the phenol and pyrrolidone systems, both of which do not suffer from the same methodical problem as $\mathrm{P}(n \mathrm{Bu})_{3} \cdot \mathrm{HBF}_{4}$. The reaction barrier for phosphine addition is feasible for both pyrrolidone and phenol with barriers of 61.4 and $71.3 \mathrm{~kJ} \mathrm{~mol}^{-1}$, respectively (TS-S5: $\Delta G^{+}=69.5 \mathrm{~kJ} \mathrm{~mol}^{-1}$ and TS-S5 $5_{\text {Phenol }}: \Delta G^{+}=4.5 \mathrm{~kJ} \mathrm{~mol}^{-1}$ ). In both cases, the addition of the anion to the cation (representing the transition state towards the desired vinylated product) is more feasible than the addition of the second equivalent of phosphine (see schemes S-6 and S-9). However, this is where the differences between pyrrolidone and phenol become pronounced. Similar to all other intermediates that incorporate phenol, the subsequent phenol intermediates $\mathbf{S} 5_{\text {Phenol }}\left(\Delta G^{413}=-47.9 \mathrm{~kJ} \mathrm{~mol}^{-1}\right)$ and $\mathbf{S} \boldsymbol{6}_{\text {Phenol }}\left(\Delta G^{413}=-101.1 \mathrm{~kJ} \mathrm{~mol}^{-1}\right)$ are significantly more stable than their pyrrolidone counterparts S5 $\left(\Delta G^{413}=1.1 \mathrm{~kJ} \mathrm{~mol}^{-1}\right)$ and $\mathbf{S 6}$ $\left(\Delta G^{413}=37.6 \mathrm{~kJ} \mathrm{~mol}^{-1}\right)$. This leads to the observation that $\mathbf{S} 6$ phenol represents the most stable species of the catalytic system and once it has been formed it will not be active anymore towards the desired vinylation reaction. On the other hand, intermediate $\mathbf{S} 6$ is significantly less stable than $\mathbf{C}$, which shows that there is no thermodynamic driving force for the second addition of phosphine when employing pyrrolidone and the originally desired vinylation pathway remains intact. This also explains why no signs of double phosphine addition were observed when carrying out the catalysis with pyrrolidone as substrate, but only in the experiments with phenol as additive or in the mechanistic experiments with $\mathrm{P}(n \mathrm{Bu})_{3} \cdot \mathrm{HBF}_{4}$. 


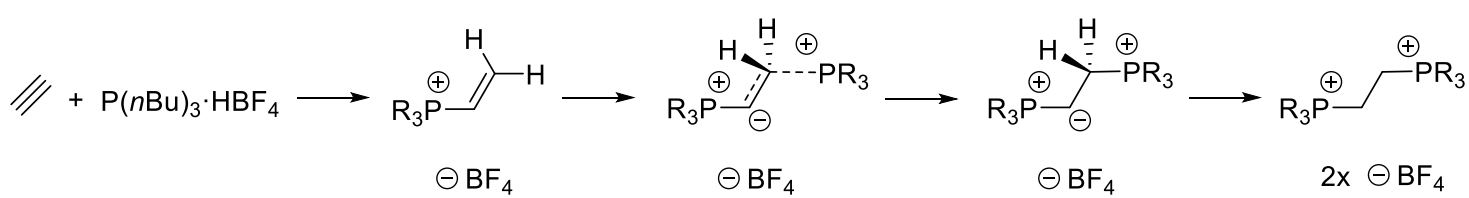

$\mathrm{C}_{\mathrm{BF} 4} \quad \mathrm{TS}-\mathrm{S} 5_{\mathrm{BF} 4} \quad \mathrm{~S} 5_{\mathrm{BF} 4}$

(0.0) (52.4) (4.6)
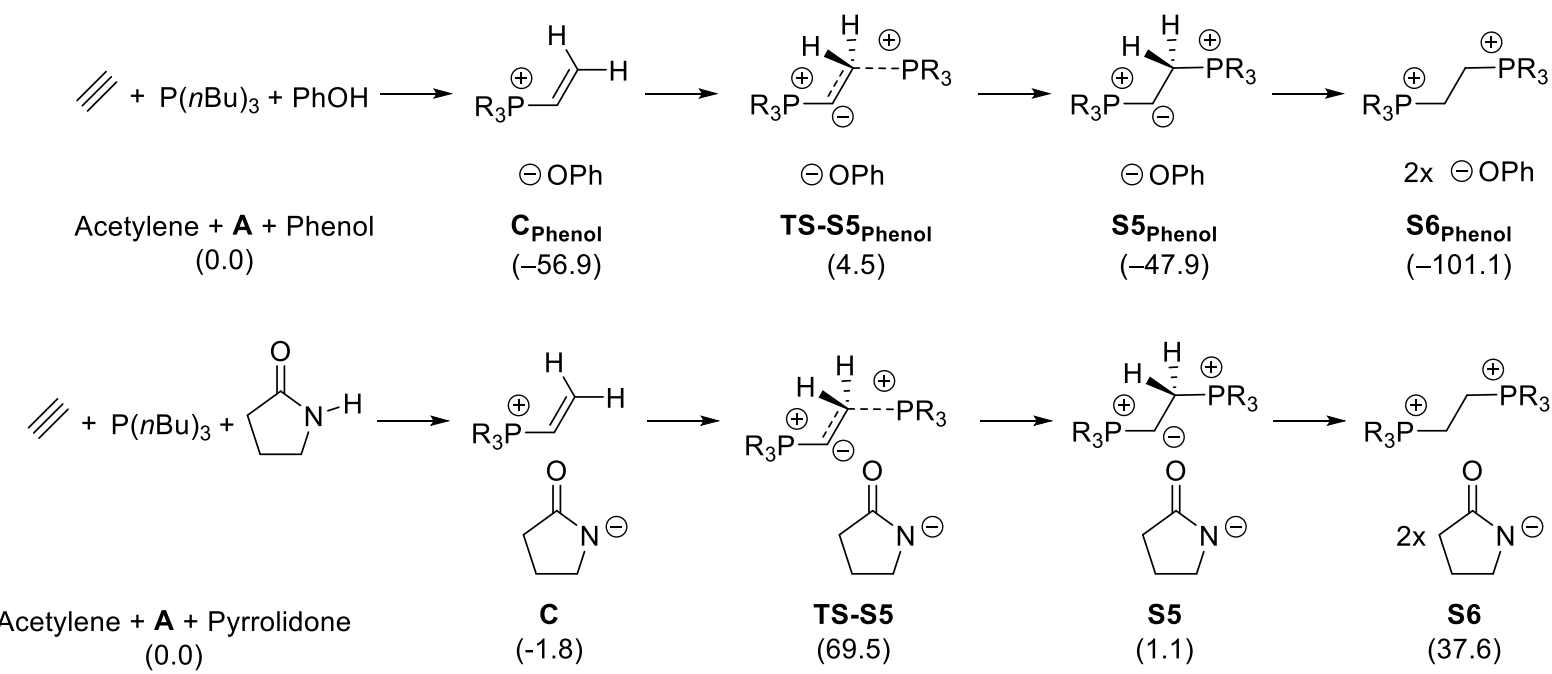

Scheme S-10: Investigation of the addition of a second equivalent of phosphine to the activated acetylene intermediate. The addition is significantly favored for the phenol system, but less feasible in the case of using pyrrolidone. $\Delta G^{413}$ in $\mathrm{kJ} \mathrm{mol}^{-1}$; RI-PBE0-D3(BJ)/def2-QZVPP//BP86/def2-SV(P); COSMO-RS (DMF). 


\subsection{Activating Acetylene with the Pyrrolidone Anion as Alternative Pathway}

As an alternative to the nucleophilic reaction cascade and the various investigated alternative pathways, a Reppe-type pathway was also investigated (see scheme S-11). The reaction pathway begins with the activation of acetylene by the employed phosphine $\mathrm{PR}_{3}$. The formed zwitter ionic intermediate will subsequently deprotonate the substrate forming the pyrrolidone anion. This anionic species continues to activate the next acetylene molecule to form the NVP anion, which only needs to be re-protonated to form the desired product. This re-protonation can occur via the substrate, which leads to the parallel formation of the next equivalent of pyrrolidone anion and therefore completing the catalytic cycle.

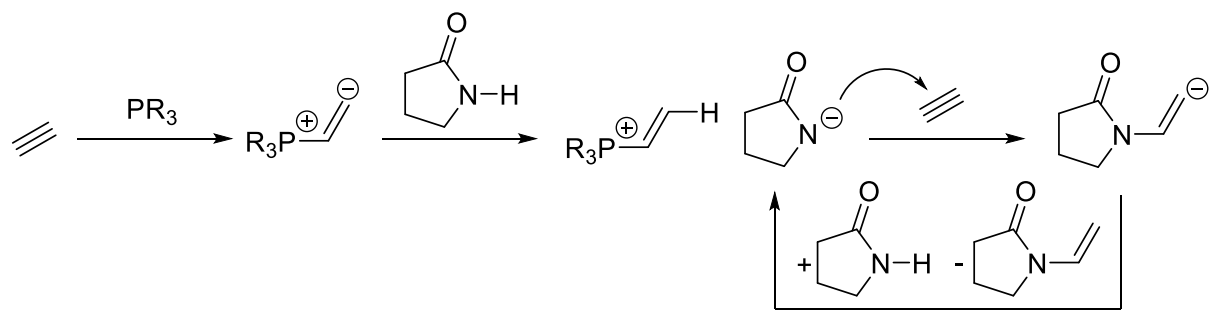

Scheme S-11: General schematic of the investigated Reppe-type reaction pathway. $\Delta G^{413}$ in kJ mol-1; RI-PBE0D3(BJ)/def2-QZVPP//BP86/def2-SV(P); COSMO-RS (DMF).

In this pathway, the initial steps $\mathbf{A} \rightarrow \mathbf{T S}-\mathbf{A B}_{\text {cis }} \rightarrow \mathbf{B}_{\text {cis }} \rightarrow \mathbf{T S}-\mathbf{B C}_{\text {cis }} \rightarrow \mathbf{C}$ are identical to the activation steps of the nucleophilic reaction mechanism. These reaction steps are resulting in the slightly exergonic formation of $\mathbf{C}\left(\Delta G^{413}=-1.8 \mathrm{~kJ} \mathrm{~mol}^{-1}\right)$ with a reaction barrier of $99.2 \mathrm{~kJ} \mathrm{~mol}^{-1}$ (TS-AB cis; $\Delta G^{+}=99.2 \mathrm{~kJ} \mathrm{~mol}^{-1}$; see figure 1 in the main paper). From $\mathbf{C}$, the activation of acetylene can be achieved with moderate barriers (TS-S6 cis: $\Delta G^{\ddagger}=67.2 \mathrm{~kJ} \mathrm{~mol}^{-1}$; TS-S6 $6_{\text {trans: }}: \Delta G^{\ddagger}=88.7 \mathrm{~kJ} \mathrm{~mol}^{-1}$ ) to form intermediate $\mathbf{S} 7_{\text {cis }}\left(\Delta G^{413}=-9.0 \mathrm{~kJ} \mathrm{~mol}^{-1}\right)$ and $\mathbf{S} 7_{\text {trans }}\left(\Delta G^{413}=-10.8 \mathrm{~kJ} \mathrm{~mol}^{-1}\right)$, respectively. This could then continue to form product NVP via a protonation step. However, one must note, that the acetylene activation transitions states TS-S6 $\boldsymbol{6}_{\text {cis }}$ and $\mathbf{T S}-\mathbf{S} \mathbf{6}_{\text {trans }}$ are in competition with the formation of ylide $\mathbf{D}\left(\Delta G^{413}=-57.3 \mathrm{~kJ} \mathrm{~mol}^{-1}\right)$, which has been previously described to form via a low barrier addition step (TS-CD; $\Delta G^{\ddagger}=23.4 \mathrm{~kJ} \mathrm{~mol}^{-1}$; see figure 1 in the main paper). Therefore, the formation of $\mathbf{D}$ and the subsequent steps of the nucleophilic vinylation pathways are much more feasible and in better agreement with the experimental results. To illustrate this energetic behavior, a comparison of the potential energy surfaces of the previously described nucleophilic pathway and the Reppe-type mechanism can be found below (see scheme S-13).
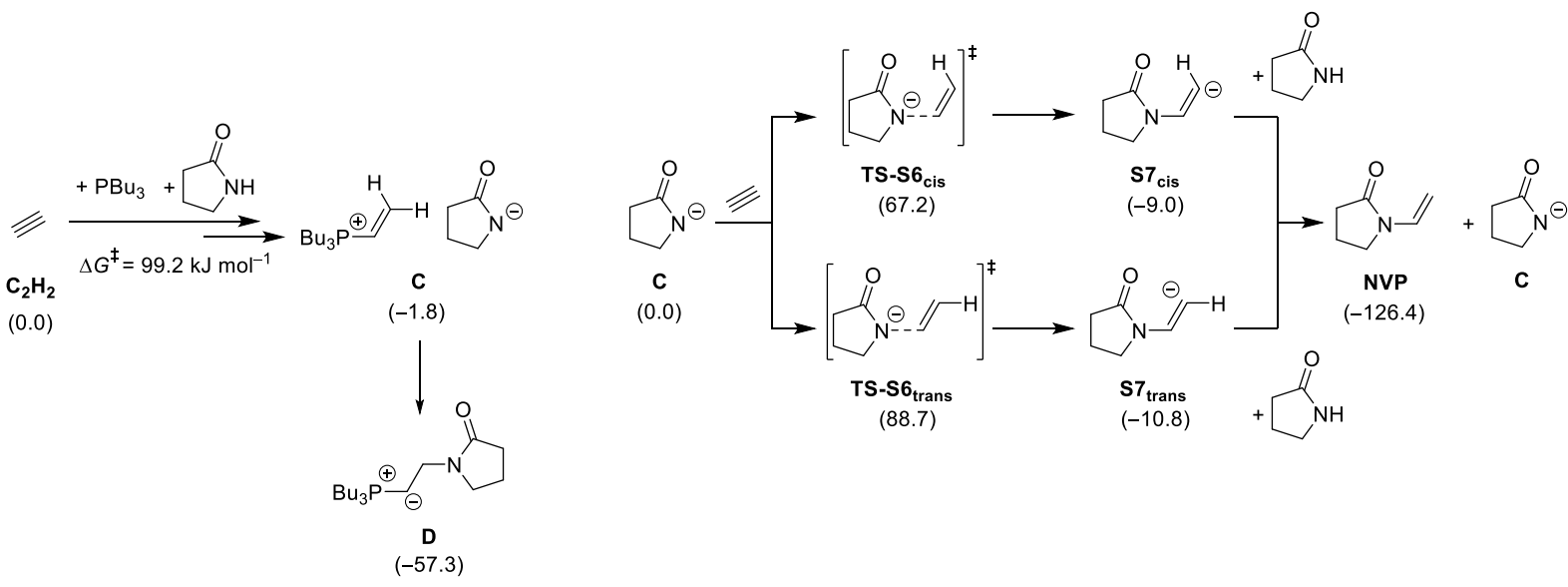

Scheme S-12: Investigation of the Reppe-type reaction pathways for the vinylation of pyrrolidone to NVP. $\triangle G^{413}$ in kJ $\mathrm{mol}^{-1}$; RI-PBE0-D3(BJ)/def2-QZVPP//BP86/def2-SV(P); COSMO-RS (DMF). 


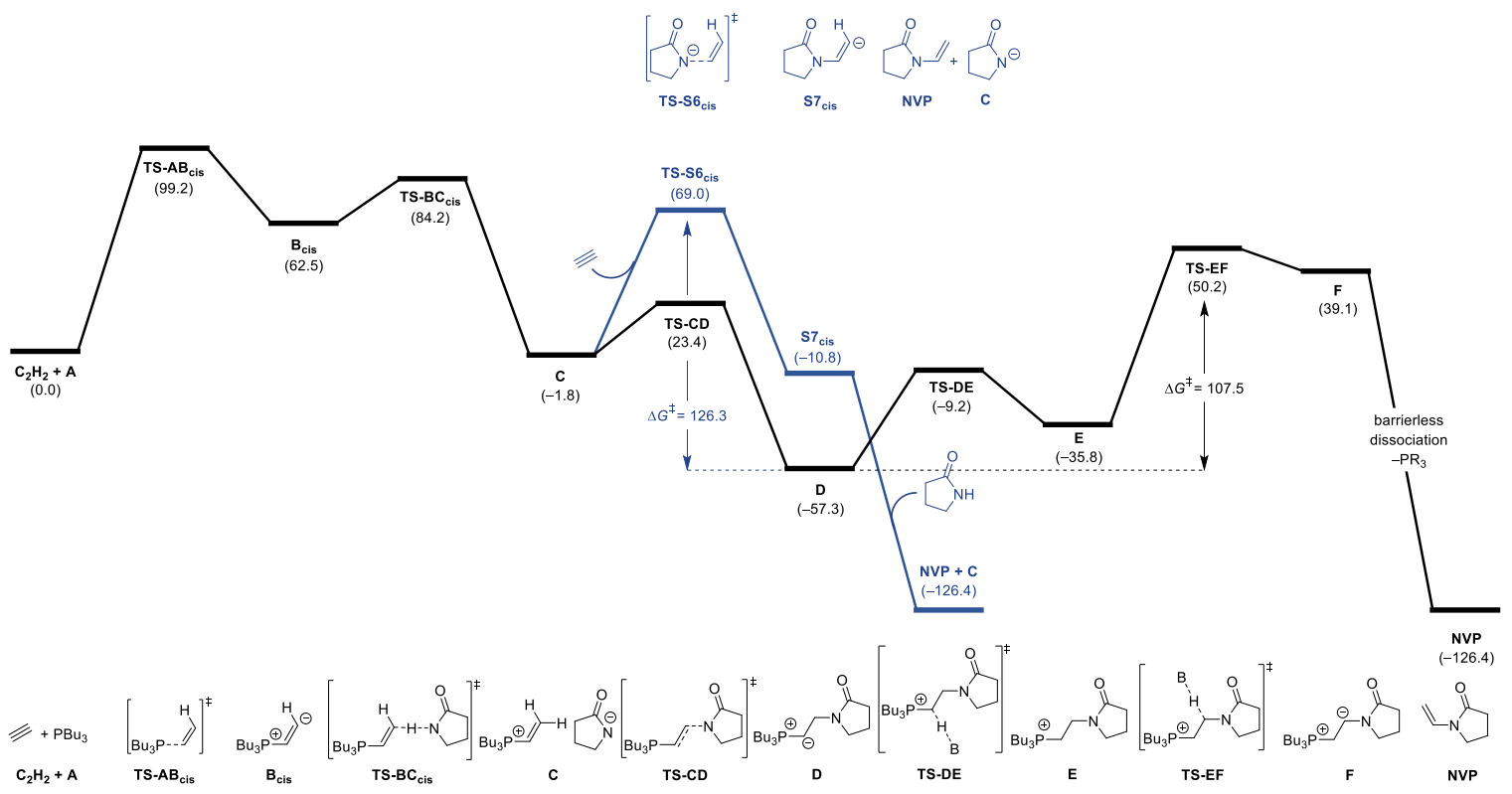

Scheme S-13: Comparison of the nucleophilic pathway (black) and the Reppe-type pathway (blue). $\Delta G^{413} \mathrm{in} \mathrm{kJ} \mathrm{mol}^{-1}$; RI-PBE0-D3(BJ)/def2-QZVPP//BP86/def2-SV(P); COSMO-RS (DMF). 


\subsection{Lowest Energy Pathway for $\mathrm{PMe}_{3}$}

In addition to the calculations employing the phosphine $\mathrm{P}(n \mathrm{Bu})_{3}(\mathbf{A})$ and $\mathrm{PPh}_{3}\left(\mathbf{A}_{\mathrm{PPh}}\right)$, the nucleophilic reaction pathway was also investigated for $\mathrm{PMe}_{3}\left(\mathrm{APMe}_{\mathrm{PM}}\right)$ and is shown in scheme $\mathrm{S}-14$. The reaction initially sets off via activation of acetylene by $A_{\text {PMe3 }}$ via TS-AB $B_{\text {cis/PMe3 }}\left(\Delta G^{\ddagger}=102.7 \mathrm{~kJ} \mathrm{~mol}^{-1}\right)$ or TS$\mathbf{A B}_{\text {trans/PMe3 }}\left(\Delta G^{\ddagger}=131.1 \mathrm{~kJ} \mathrm{~mol}^{-1}\right)$ to form $\mathbf{B}_{\text {cis/PMe3 }}\left(\Delta G^{413}=75.8 \mathrm{~kJ} \mathrm{~mol}^{-1}\right)$ or $\mathbf{B}_{\text {trans/PMe3 }}$ $\left(\Delta G^{413}=62.5 \mathrm{~kJ} \mathrm{~mol}^{-1}\right)$, respectively. Subsequent proton transfer via $\mathbf{T S}-\mathbf{B C}_{\text {cis/PMe3 }}$ $\left(\Delta G^{\ddagger}=103.9 \mathrm{~kJ} \mathrm{~mol}^{-1}\right)$ or $\mathbf{T S}-\mathbf{B C}_{\text {trans/PMe3 }}\left(\Delta G^{+}=68.1 \mathrm{~kJ} \mathrm{~mol}^{-1}\right)$ will recombine the cis- and trans pathways and lead to ion pair $\mathbf{C}_{\mathrm{PMe} 3}\left(\Delta G^{413}=20.9 \mathrm{~kJ} \mathrm{~mol}^{-1}\right)$. Similar to the previously investigated pathways with $\mathbf{A}$ and $\mathbf{A}_{\mathbf{P P h} 3}$, the cis pathway is significantly favored over the trans pathway. The reaction pathway continues with the addition of the pyrrolidone anion to the cation via TS-CDPMe3 $\left(\Delta G^{\ddagger}=49.9 \mathrm{~kJ} \mathrm{~mol}^{-1}\right)$ leading to the highly stable ylide $D_{\text {PMe3 }}\left(\Delta G^{413}=-51.1 \mathrm{~kJ} \mathrm{~mol}^{-1}\right)$. The ylide $\mathrm{D}$ can subsequently follow two reaction pathways: firstly, an unassisted $\mathrm{H}$-shift from $\beta$ position to $\alpha$ position (in respect to the phosphorus atom) or secondly, undergo a protonation/deprotonation cascade that will achieve the same $\mathrm{H}$-shift in two steps. The unassisted $\mathrm{H}$-shift TS-S3PMe3 $\left(\Delta G^{\ddagger}=226.9 \mathrm{~kJ} \mathrm{~mol}^{-1}\right)$ exhibits a very high activation energy that is not feasible at the employed reaction conditions. The second pathway starts with the protonation of $\mathbf{D}_{\mathrm{PMe} 3}$ via a second unit of pyrrolidone with a moderate barrier of $42.5 \mathrm{~kJ} \mathrm{~mol}^{-1}\left(\mathrm{TS}_{-}-\mathrm{DE}_{\mathrm{PMe}}\right.$ : $\Delta G^{\ddagger}=-8.6 \mathrm{~kJ} \mathrm{~mol}^{-1}$ ) forming $\mathrm{E}_{\mathrm{PMe} 3}$ $\left(\Delta G^{413}=-16.2 \mathrm{~kJ} \mathrm{~mol}^{-1}\right)$. This then undergoes re-protonation in a position via TS-EF PMe3 $\left(\Delta G^{\ddagger}=77.2 \mathrm{~kJ} \mathrm{~mol}^{-1}\right)$. The formed intermediate $\mathbf{F}\left(\Delta G^{413}=59.8 \mathrm{~kJ} \mathrm{~mol}^{-1}\right)$ can undergo barrierless dissociation of phosphine APMe3 to liberate the desired product NVP $\left(\Delta G^{413}=-126.4 \mathrm{~kJ} \mathrm{~mol}^{-1}\right)$. The overall reaction barrier of $128.3 \mathrm{~kJ} \mathrm{~mol}^{-1}$ falls in between the determined barriers for phosphines $\mathbf{A}$ $\left(107.5 \mathrm{~kJ} \mathrm{~mol}^{-1}\right)$ and Apph3 $\left(154.4 \mathrm{~kJ} \mathrm{~mol}^{-1}\right)$, which generally agrees with the significantly better performance than Apph, but worse performance than A (see table 3 in the main paper).

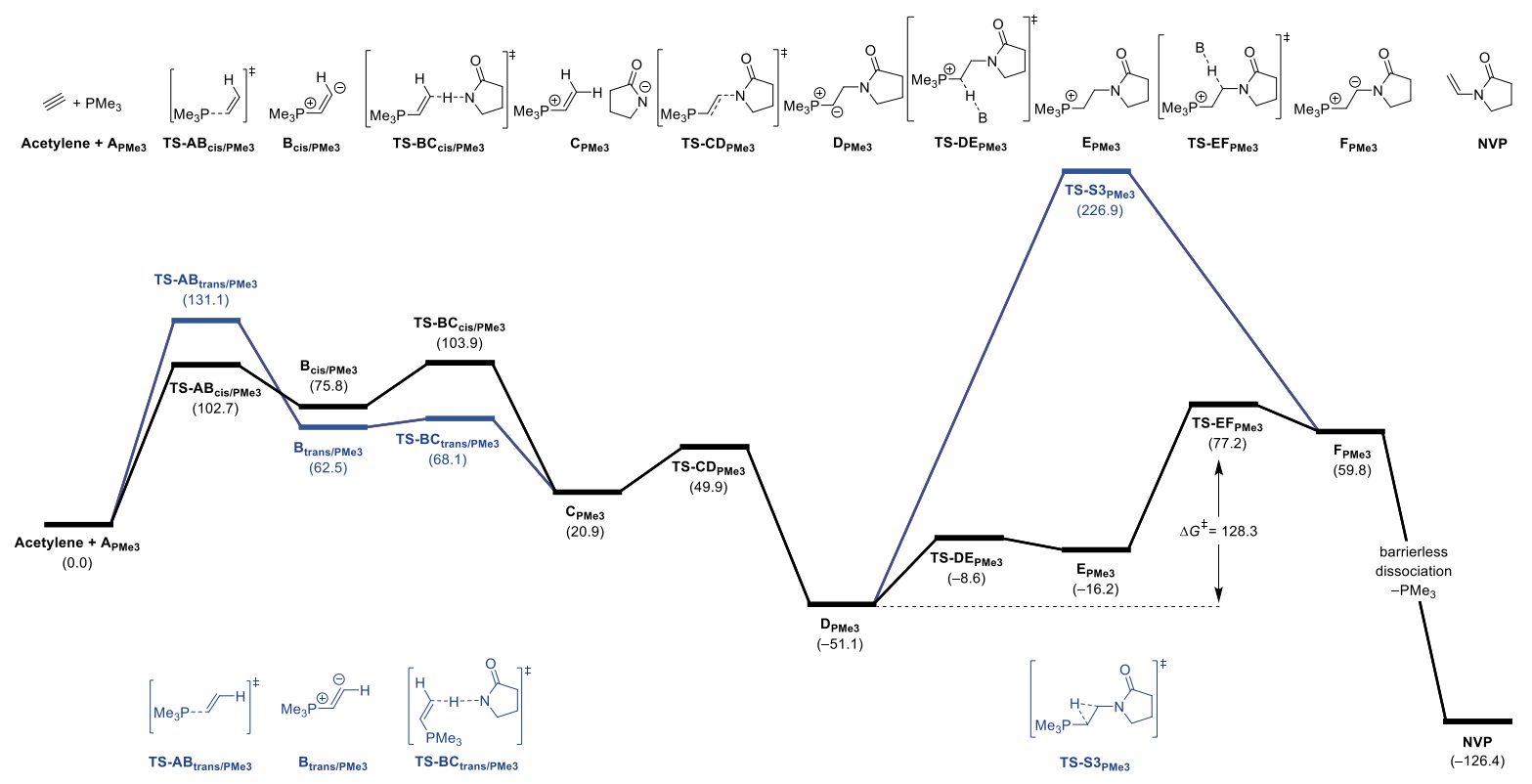

Scheme S-14: Calculated lowest energy pathway for the vinylation of pyrrolidone catalyzed by $\mathrm{PMe}_{3}$ ( $\mathrm{APMe}_{\mathrm{P}}$; black). Alternative pathways like the trans-configured activation pathway and the unassisted $\mathrm{H}$-shift are shown in blue. $\Delta G^{413}$ in $\mathrm{kJ} \mathrm{mol}^{-1}$; RI-PBE0-D3(BJ)/def2-QZVPP//BP86/def2-SV(P); COSMO-RS (DMF). 


\subsection{Additional Product Degradation Pathways}

In addition to the Wittig-type degradation pathway, which is presented in figure S-3, the quantumchemical investigation was extended to another potential pathway starting from $\mathbf{B}_{\text {cis }}$ to ensure that all possible options were studied (see scheme S-15). In this pathway, the $\beta$ carbon atom performs a nucleophilic attack towards the NVP carbonyl group (TS-S7; $\Delta G^{+}=129.8 \mathrm{~kJ} \mathrm{~mol}^{-1}$ ) leading to intermediate $\mathbf{S 8}\left(\Delta G^{413}=78.4 \mathrm{~kJ} \mathrm{~mol}^{-1}\right)$. Subsequently, a series of intermediates were investigated, where no connecting transitions states could be located. $\mathbf{S 9}\left(\Delta G^{413}=68.1 \mathrm{~kJ} \mathrm{~mol}^{-1}\right)$ including an epoxide unit can be formed by rearrangement of the oxygen atom. In the next step, the hydrogen atom shifts towards the pyrrolidone core forming the highly endergonic intermediate $\mathbf{S 1 0}$ $\left(\Delta G^{413}=181.9 \mathrm{~kJ} \mathrm{~mol}^{-1}\right)$. Now the oxygen atom can shift towards the non-cyclic carbon atom of the epoxide unit to form a stabilizing $\mathrm{P}-\mathrm{O}$ interaction similarly to the same interaction in the previously investigated Wittig-type reaction pathway $\left(\mathbf{S 1 1} ; \Delta G^{413}=-19.8 \mathrm{~kJ} \mathrm{~mol}^{-1}\right)$. These intermediates might be able to barrierlessly interconvert; however, this was not further investigated due to the high energies that these intermediates exhibit. Phosphine oxide elimination can then occur via TS-S8 $\left(\Delta G^{\ddagger}=100.3 \mathrm{~kJ} \mathrm{~mol}^{-1}\right)$ to form the strongly exergonic degradation product $\mathbf{M}_{\mathrm{NVP}}$ $\left(\Delta G^{413}=-108.9 \mathrm{~kJ} \mathrm{~mol}^{-1}\right)$.

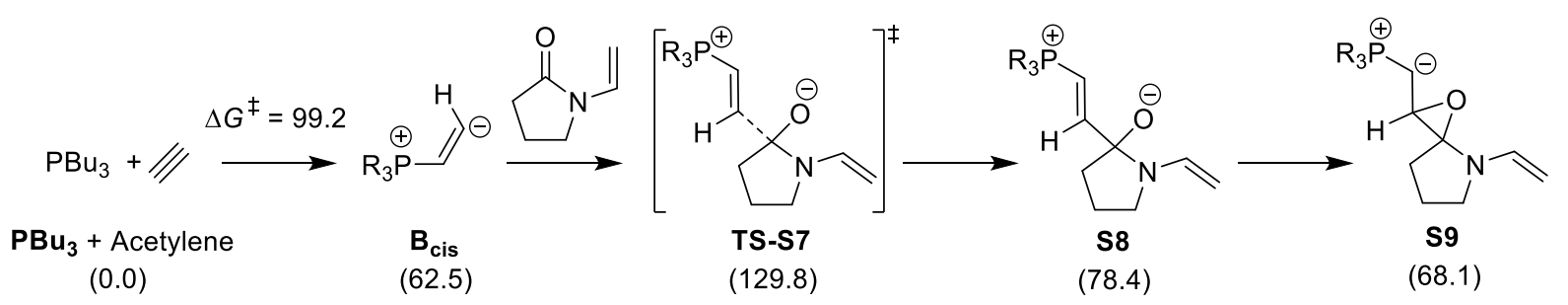

$(0.0)$

$(68.1)$
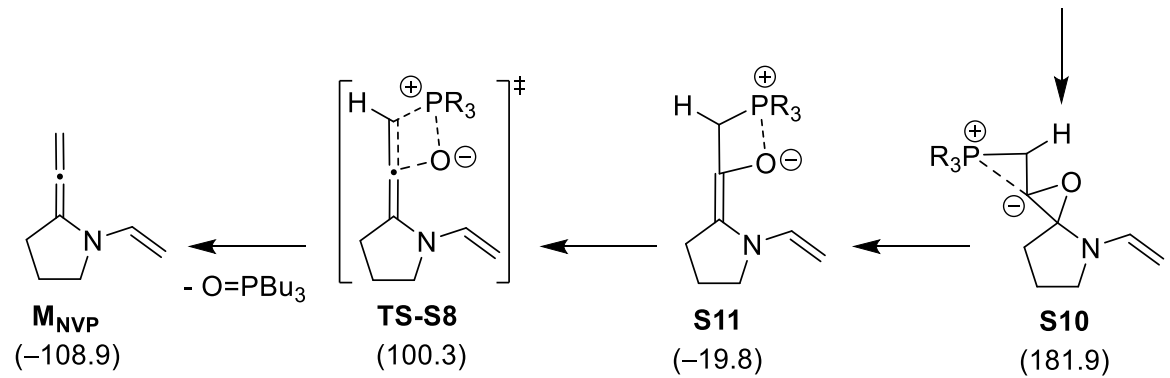

Scheme S-15: Investigation of an alternative product degradation pathway leading to allene $\mathbf{M}_{\mathrm{NvP}} . \Delta G^{413}$ in kJ mol${ }^{-1}$; RI-PBE0-D3(BJ)/def2-QZVPP//BP86/def2-SV(P); COSMO-RS (DMF).

After the kinetic studies (vide supra) showed that the phosphine concentration can lower over time at reaction conditions (even when acetylene is the only other system component), quantum-chemical calculations were employed to investigate a potential phosphine-induced acetylene polymerization as well as other cyclization compounds that could lead to this experimental observation (see scheme S16). As most catalytic pathways, this cascade also begins with the activation of acetylene by the employed phosphine (A). The formed intermediate $\boldsymbol{B}_{\text {trans }}\left(\Delta G^{413}=36.2 \mathrm{~kJ} \mathrm{~mol}^{-1}\right)$ can then continue to activate a second unit of acetylene via TS-S9 $\left(\Delta G^{+}=115.6 \mathrm{~kJ} \mathrm{~mol}^{-1}\right)$ leading to $\mathbf{S 1 2}$ $\left(\Delta G^{413}=-33.6 \mathrm{~kJ} \mathrm{~mol}^{-1}\right)$. In opposite to the previous activation step, the trans-configured transition state is given, because TS-S9 is favored as a follow-up of $\mathbf{B}_{\text {trans }}$ over $\mathbf{B}_{\text {cis }}$ reducing the overall barrier of the polymerization pathway. The second chain extension step proceeds via TS-S10 $\left(\Delta G^{\ddagger}=11.8 \mathrm{~kJ} \mathrm{~mol}^{-1}\right)$ to $\mathbf{S 1 3}\left(\Delta G^{413}=-183.3 \mathrm{~kJ} \mathrm{~mol}^{-1}\right)$. Further extension steps were not investigated but based on this trend are assumed to show lower barrier than TS-S9 and continue with the highly exergonic nature of the first steps. Moreover, two cyclization steps forming five-membered rings were envisioned that could compete with the chain extension transition states: Firstly, $\mathrm{P}$-containing heterocycle $\mathbf{S 1 4}\left(\Delta G^{413}=-134.8 \mathrm{~kJ} \mathrm{~mol}^{-1}\right)$ can be formed from $\mathbf{S 1 2}$ and secondly, $\mathbf{S 1 5}$ $\left(\Delta G^{413}=-362.5 \mathrm{~kJ} \mathrm{~mol}^{-1}\right)$, which contains a cyclopentadiene unit, can be formed from S13. Scans of the $\mathrm{P}-\mathrm{C}$ and $\mathrm{C}-\mathrm{C}$ bond lengths (for S14 and S15, respectively) were used to show that barrierless 
formation of these species is possible from S12 and S13 (see figure S-2). While it is hard to say without experimental evidence which of these products is formed (and to what amounts), the calculations help rationalize potentially relevant processes and can give guidance to the synthetic chemists trying to isolate the formed side products.

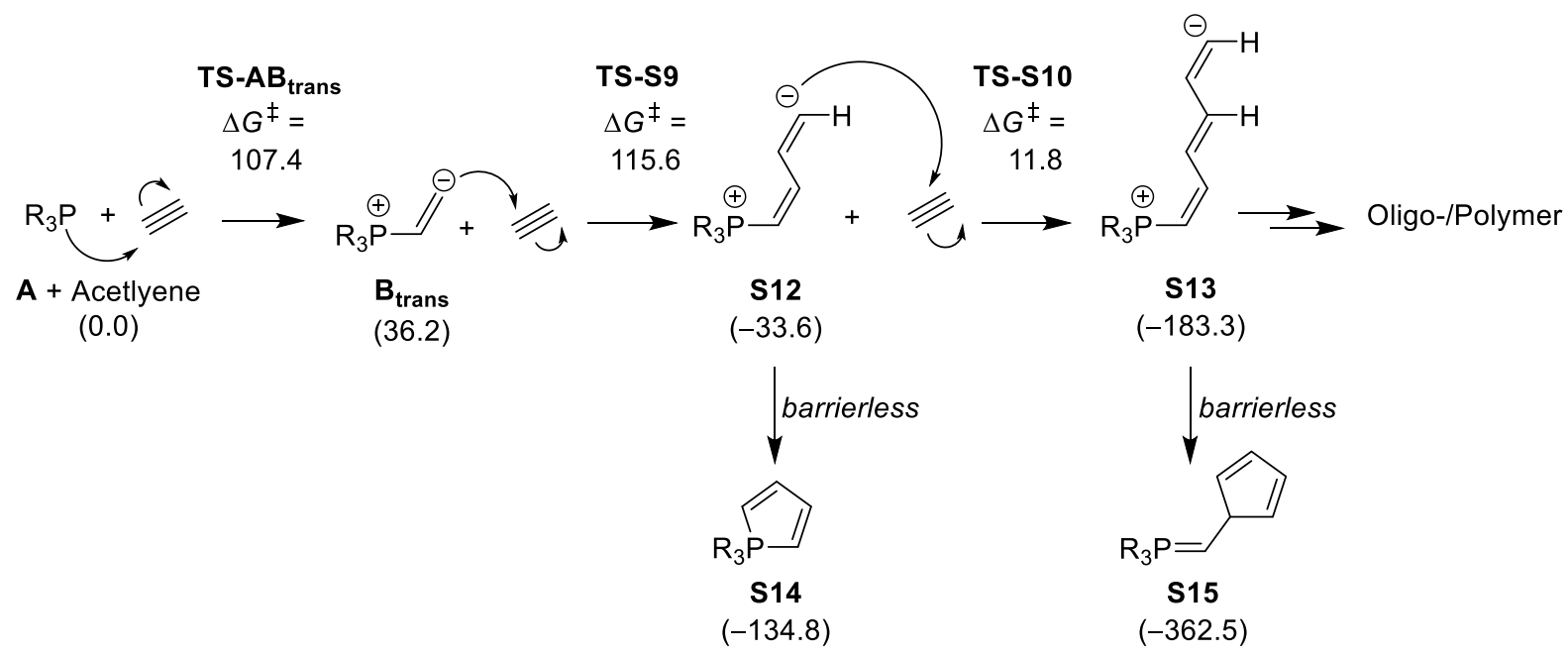

Scheme S-16: Investigation of phosphine-induced polymerization and cyclization pathways as a potential reason for diminishing phosphine concentrations. $\Delta G^{413}$ in $\mathrm{kJ} \mathrm{mol}^{-1}$; RI-PBE0-D3(BJ)/def2-QZVPP//BP86/def2-SV(P); COSMORS (DMF).

(a)

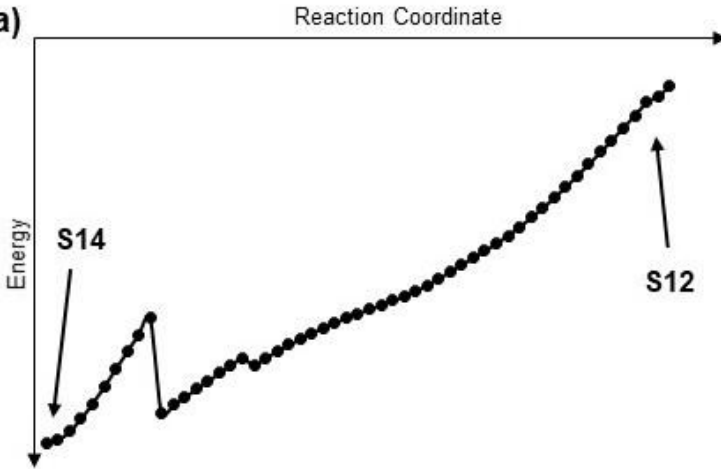

(b)

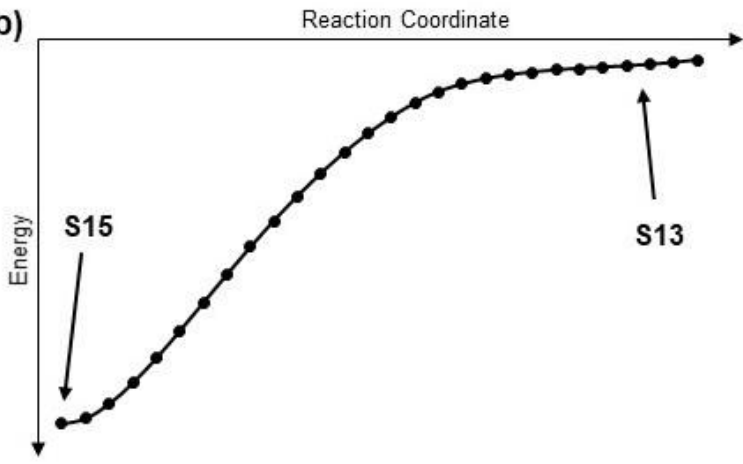

Figure S-2: Scans showing the barrierless formation of (a) S14 and (b) S15 from S12 and S13, respectively. The energy change at the beginning of the left plot is caused by a change of confirmation of the substituents of $\mathrm{PR}_{3}$ and does not represent a reaction barrier. $\Delta E^{413}$ in $\mathrm{kJ} \mathrm{mol}^{-1}$; RI-PBE0-D3(BJ)/def2-QZVPP//BP86/def2-SV(P); COSMO-RS (DMF). 


\subsection{Dimerization by Wittig-type Reaction Pathways}

Alternative to the Wittig-type reactivity of ylide $\mathbf{D}$ (see Figure 3 in the manuscript) and the possibility of ylide S19 to react with pyrrolidone or NVP (see Figure S-3), another pathway constituting of a Wittigtype dimerization reaction (meaning ylide $\mathbf{D}$ reacting with pyrrolidone or NVP) was investigated (see scheme S-17). Ylide $\mathbf{D}$ is formed according to the previously described pathway with a reaction barrier of $99.2 \mathrm{~kJ} \mathrm{~mol}^{-1}$ (see figure 1 in the main paper). From here, similar to the Wittig-type pathways described in figure 3 in the main paper, the reagent - either pyrrolidone or NVP - can add to ylide $\mathbf{D}$ either in cis- or trans configuration (TS-S11 cis/H: $\Delta G^{+}=73.9 \mathrm{~kJ} \mathrm{~mol}^{-1}$; TS-S11 trans $/ \mathrm{H}_{\text {: }}$

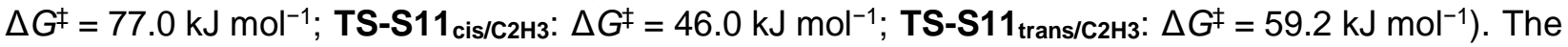
formed intermediates $\mathbf{S 1 6}$ exhibit the typical $\mathrm{P}-\mathrm{O}$ interaction $\left(\mathbf{S} 16_{\mathrm{cis} / \mathrm{H}}: \Delta G^{413}=34.4 \mathrm{~kJ} \mathrm{~mol}^{-1}\right.$;

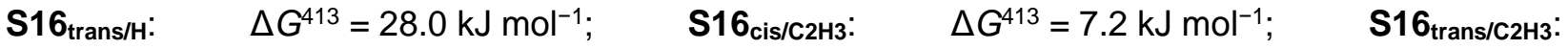

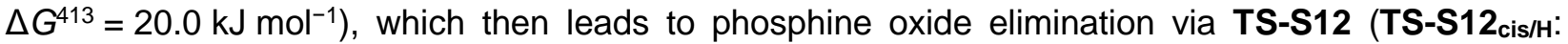

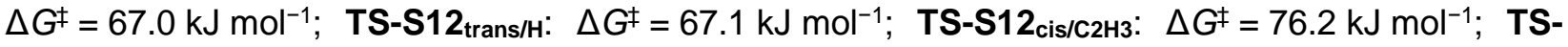
S12 trans/C2H3: $_{\text {(ch }} G^{+}=67.3 \mathrm{~kJ} \mathrm{~mol}^{-1}$ ). Interestingly, the final product $\mathbf{S 1 7}$ is shows a more stable cis configuration for pyrrolidone as educt, while exhibiting a more stable trans configuration for NVP as educt $\quad\left(\mathbf{S} 17_{\text {cis } / \mathrm{H}}: \quad \Delta G^{413}=-183.2 \mathrm{~kJ} \mathrm{~mol}^{-1} ; \quad \mathbf{S} 17_{\text {trans } / \mathrm{H}}: \quad \Delta G^{413}=-171.8 \mathrm{~kJ} \mathrm{~mol}^{-1} ; \quad \mathbf{S} 17_{\text {cis } / \mathbf{C}_{2 \mathrm{H} 3} \text { : }}\right.$ : $\Delta G^{413}=-168.6 \mathrm{~kJ} \mathrm{~mol}^{-1} ; \mathbf{S 1 7}$ trans $\left./ \mathrm{C}_{2 \mathrm{H} 3}: \Delta G^{413}=-179.9 \mathrm{~kJ} \mathrm{~mol}^{-1}\right)$. Looking at the lowest overall reaction barriers for these pathways shows that with $131.2 \mathrm{~kJ} \mathrm{~mol}^{-1}$ (for pyrrolidone as substrate) and $124.4 \mathrm{~kJ} \mathrm{~mol}^{-1}$ (for NVP as substrate) these pathways are less feasible than the other Wittig-type reaction pathways (or the desired vinylation pathway). This also explains why such dimer species were not observed, while the benzaldehyde Wittig reaction, which exhibits a significantly lower activation energy, was experimentally observed.

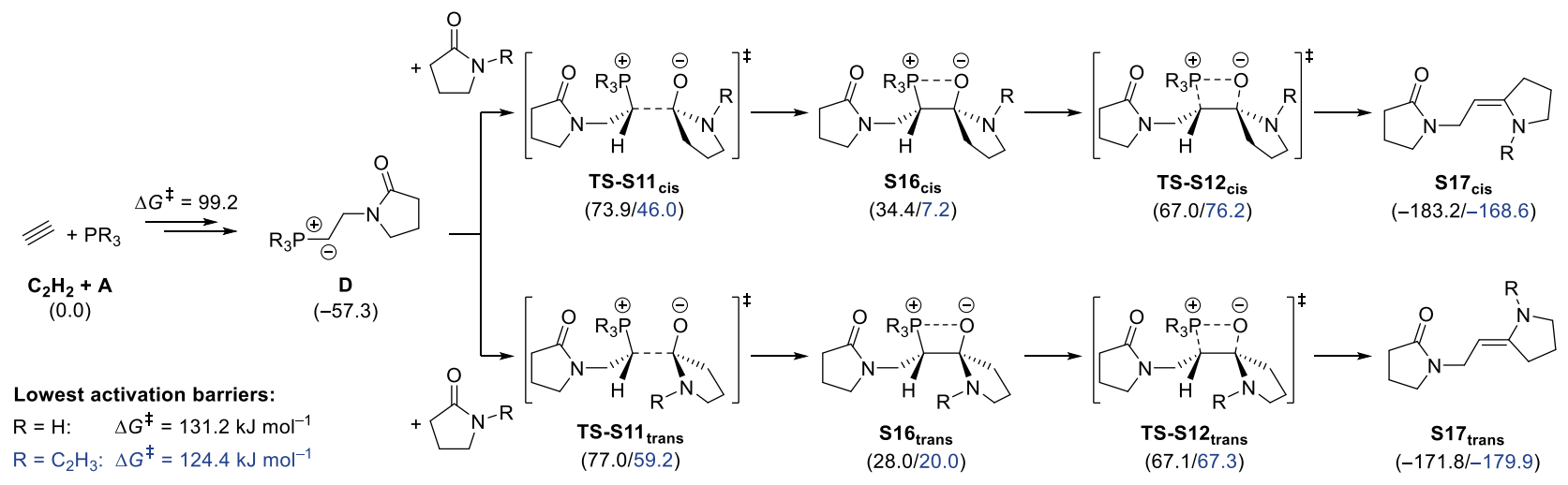

Scheme S-17: Investigation of Wittig-type dimerization pathway based on ylide $\mathbf{D}$ and pyrrolidone ( $\mathrm{R}=\mathrm{H}$; black) or NVP $\left(\mathrm{R}=\mathrm{C}_{2} \mathrm{H}_{3}\right.$; blue). $\Delta \mathrm{G}^{413}$ in $\mathrm{kJ} \mathrm{mol}^{-1}$; RI-PBE0-D3(BJ)/def2-QZVPP//BP86/def2-SV(P); COSMO-RS (DMF).

\subsection{Degradation by Wittig-type Reaction Pathways}

The feasibility of the Wittig-type pathway with benzaldehyde led to further investigations dealing with the reaction of ylides with other carbonyl compounds. First and foremost, the reactions of the substrate pyrrolidone and the product NVP were of importance as both are abundant during the vinylation reaction. Moreover, this was motivated by the observation of phosphine oxide when heating a mixture of pyrrolidone, NVP, acetylene and phosphine A (cf. Scheme S-3). The investigation revealed that a second equivalent of acetylene can support similar steps as pyrrolidone to form a reactive ylide (see Figure S-3 and cf. Figure 1). The initial activation step TS-A $\mathbf{B}_{\text {cis }}$ and the subsequent intermediate $\mathbf{B}_{\text {cis }}$ are identical to the activation shown for the vinylation (cf. Figure 1). Instead of protonation of $\mathbf{B}_{\text {cis }}$ by pyrrolidone as shown above, a proton will be transferred to $\mathbf{B}_{\text {cis }}$ from acetylene via TS-S13 $\left(\Delta G^{\ddagger}=73.7 \mathrm{~kJ} \mathrm{~mol}^{-1}\right)$ leading to $\mathbf{S} 18\left(\Delta G^{413}=16.3 \mathrm{~kJ} \mathrm{~mol}^{-1}\right)$. Subsequently, the acetylene anion can 
re-protonate by deprotonating intermediate $\mathbf{S 1 8}$ in the a position (TS-S14; $\Delta G^{\ddagger}=38.3 \mathrm{~kJ} \mathrm{~mol}{ }^{-1}$ ). The formed intermediate $\mathbf{S 1 9}$ can then undergo addition of pyrrolidone analogous to the previously mentioned Wittig-type pathway. This addition represents the rate-determining reaction barrier with an activation energy of $114.5 \mathrm{~kJ} \mathrm{~mol}^{-1}$ (TS-S15) and leads to intermediate $\mathbf{S 2 0}\left(\Delta G^{413}=29.0 \mathrm{~kJ} \mathrm{~mol}^{-1}\right)$, which - similarly to the previously discussed intermediate $\mathbf{H}_{\text {cis }} / \mathbf{H}_{\text {trans }}$ (see Figure 3 ) - exhibits a stabilizing $\mathrm{P}-\mathrm{O}$ interaction. In the final step of the reaction cascade, phosphine oxide is eliminated via TS-S16 $\left(\Delta G^{\ddagger}=101.9 \mathrm{~kJ} \mathrm{~mol}^{-1}\right)$ leading to the allene product S21 $\left(\Delta G^{413}=-93.4 \mathrm{~kJ} \mathrm{~mol}^{-1}\right)$. The same conceptual reactions steps can take place with the addition of NVP instead of pyrrolidone (see Scheme S-7; blue pathway). Differences between both pathways only occur from TS-S15NvP onwards, where the additions of NVP takes place $\left(\Delta G^{\ddagger}=-38.8 \mathrm{~kJ} \mathrm{~mol}^{-1}\right)$. In contrast to the pathway with pyrrolidone, the subsequent phosphine oxide elimination TS-S16 NVP $\left(\Delta G^{+}=-23.3 \mathrm{~kJ} \mathrm{~mol}^{-1}\right)$ starting from S20 NVP $\left(\Delta G^{413}=-112.3 \mathrm{~kJ} \mathrm{~mol}^{-1}\right)$ and leading to allene product $\mathbf{S 2 1} \mathbf{N V P}_{\mathrm{NV}}\left(\Delta G^{413}=-235.3 \mathrm{~kJ} \mathrm{~mol}^{-1}\right)$ constitutes the rate determining step with a reaction barrier of $103.1 \mathrm{~kJ} \mathrm{~mol}^{-1}$. With the barriers for both pathways, these should be accessible at the reaction conditions used. However, while the substrate pyrrolidone is present the formation of ylide $\mathbf{D}$ is far more favorable and will lead to only a small concentration of the active species for these Wittig-type pathways. This prediction is in agreement with the rather small amounts of phosphine oxides observed in our experiments. One should note that the compounds S21 and S21 Nvp could not be experimentally characterized, which is assumed to be due to its high reactivity to lead to various follow-up oligo- and/or polymeric products. In any case, the calculations show that such a degradation via Wittig-type reactions is plausible.

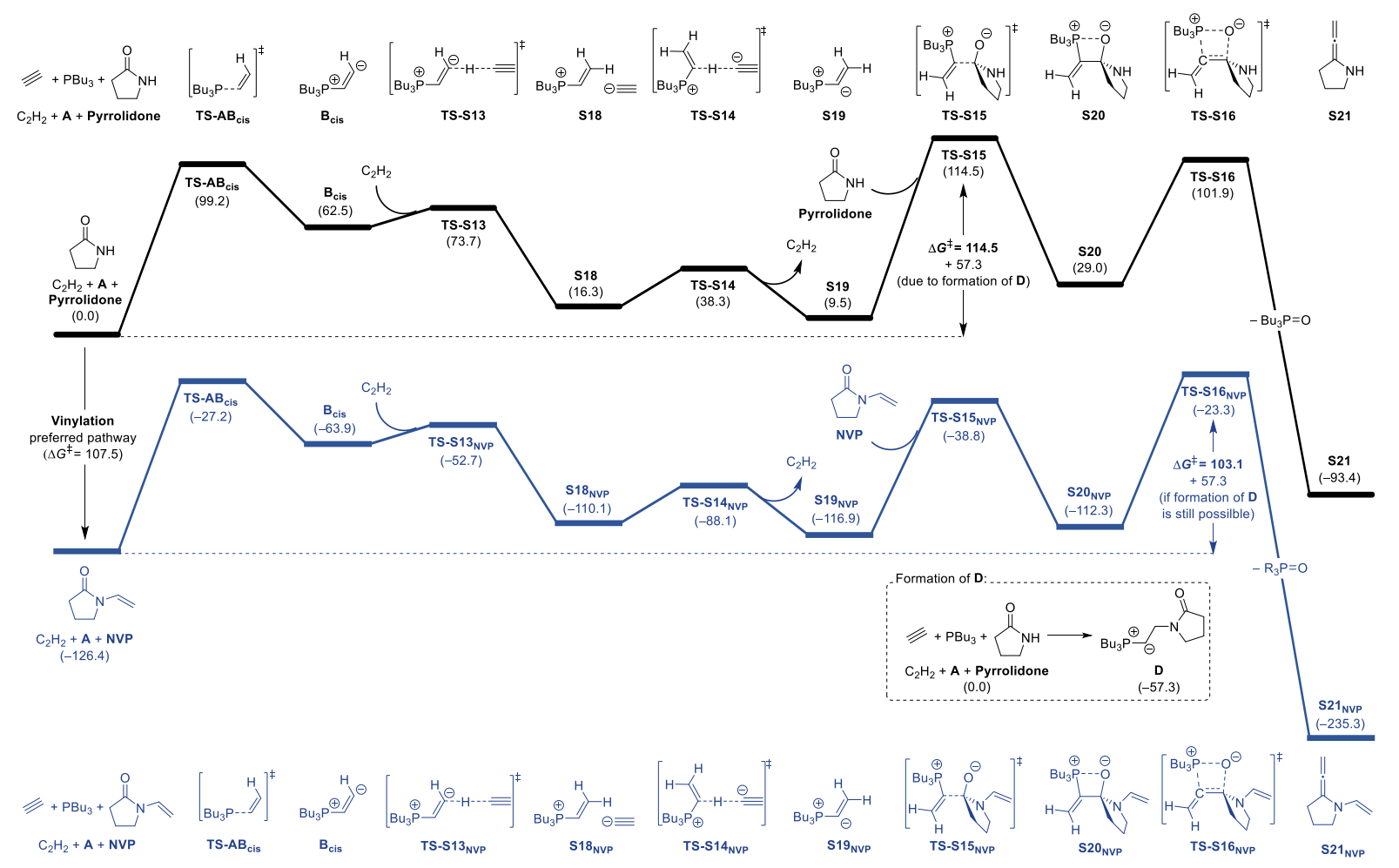

Figure S-3. Calculated lowest energy pathway for the 'degradation' reaction of pyrrolidone (black) and the vinylation product NVP (blue). $\Delta G^{413}$ in $\mathrm{kJ} \mathrm{mol}^{-1}$; RI-PBE0-D3(BJ)/def2-QZVPP//BP86/def2-SV(P); COSMO-RS (DMF). 


\section{Crystallographic Data}

\section{1,2-Bis(tri-n-butylphosphonium)ethane Tetrafluoroborate (5)}

Colourless crystal (column), dimensions $0.165 \times 0.110 \times 0.065 \mathrm{~mm}^{3}$, crystal system monoclinic, space group $\mathrm{C} 2 / \mathrm{c}, \quad \mathrm{Z}=16, \mathrm{a}=20.0988(10) \AA, \quad \mathrm{b}=23.8727(12) \AA, \quad \mathrm{c}=14.8488(7) \AA$, alpha=90 deg, beta=90.8465(15) deg, gamma $=90 \mathrm{deg}, \mathrm{V}=7123.9(6) \AA^{3}$, rho=1.145 $\mathrm{g} / \mathrm{cm}^{3}, T=200(2) \mathrm{K}$, Theta $\max =$ $22.722 \mathrm{deg}$, radiation MoKa, lambda=0.71073 $\AA, 0.5 \mathrm{deg}$ omega-scans with CCD area detector, covering the asymmetric unit in reciprocal space with a mean redundancy of 5.90 and a completeness of $99.9 \%$ to a resolution of $0.92 \AA$, 28611 reflections measured, 4791 unique $(R(\mathrm{int})=0.0445), 3114$ observed $(I>2 \sigma(I))$, intensities were corrected for Lorentz and polarization effects, an empirical scaling and absorption correction was applied using SADABS ${ }^{[1]}$ based on the Laue symmetry of the reciprocal space, $\mathrm{mu}=0.18 \mathrm{~mm}^{-1}, \mathrm{~T}_{\min }=0.93, \mathrm{~T}_{\max }=0.96$, structure solved with SHELXT-2018/2 (Sheldrick 2015) ${ }^{[2]}$ and refined against $F^{2}$ with a Full-matrix least-squares algorithm using the SHELXL-2018/3 (Sheldrick, 2018) software ${ }^{[3]}, 423$ parameters refined, hydrogen atoms were treated using appropriate riding models, goodness of fit 1.04 for observed reflections, final residual values $R 1(F)=0.068, w R\left(F^{2}\right)=0.168$ for observed reflections, residual electron density -0.37 to $0.28 \mathrm{e}^{-3}$. CCDC 2090396 contains the supplementary crystallographic data for this paper. The data can be obtained free of charge from The Cambridge Crystallographic Data Centre via www.ccdc.cam.ac.uk/structures.

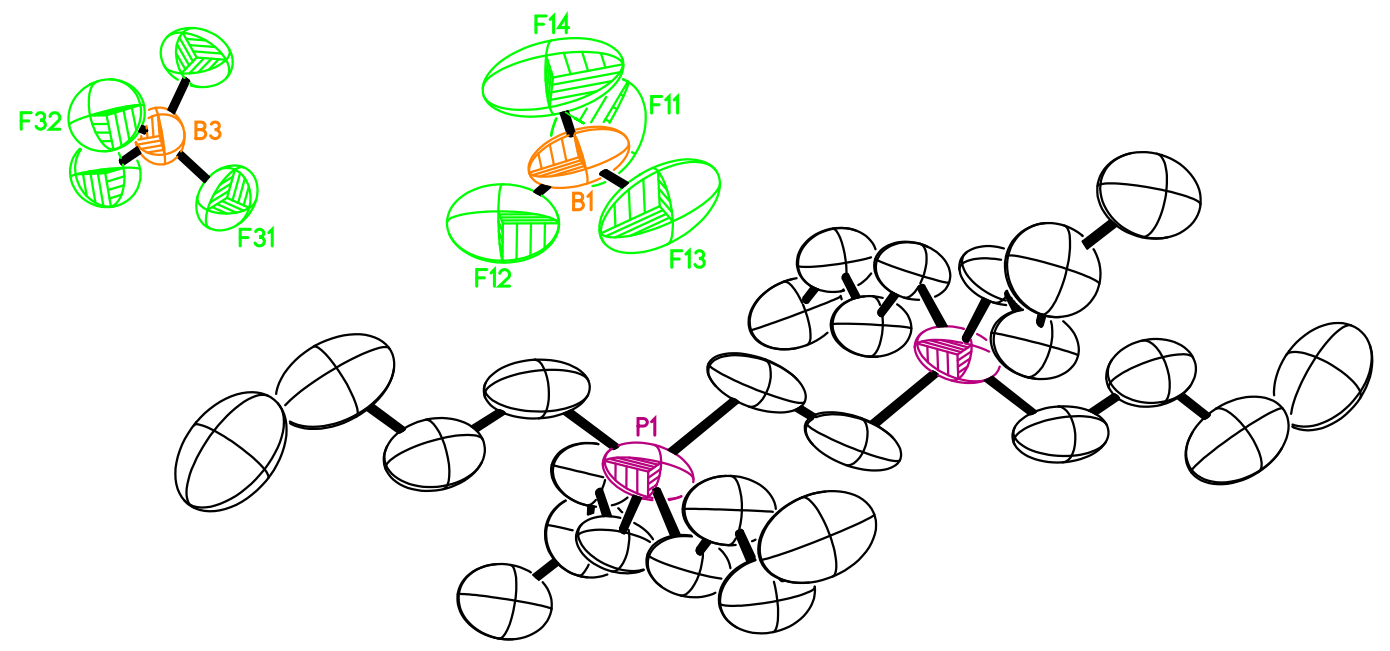

Figure S-4. Molecular structure of 5. Anisotropic displacement ellipsoids are drawn at a 50\% probability level.

[1] (SADABS-2016/2 - Bruker AXS area detector scaling and absorption correction)

Krause, L., Herbst-Irmer, R., Sheldrick G.M. \& Stalke D., J. Appl. Cryst. 48 (2015) 3-10.

[2] (SHELXT - Integrated space-group and crystal structure determination)

Sheldrick G. M., Acta Cryst. A71 (2015) 3-8.

[3] (program SHELXL-2018/3 (Sheldrick, 2018) for structure refinement)

Sheldrick G. M., Acta Cryst. (2015). C71, 3-8

Programs: APEX, APEX2, SMART, SAINT, SAINT-Plus:

Bruker (2007). "Program name(s)". Bruker AXS Inc., Madison, Wisconsin, USA. 


\section{NMR Spectra}

NS-184-A2-pure2, C6D6, 1H<smiles>C=CN1CCCC1=O</smiles>

1a, ${ }^{1} \mathrm{H}, \mathrm{C}_{6} \mathrm{D}_{6}, 300 \mathrm{MHz}$

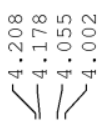

Vं
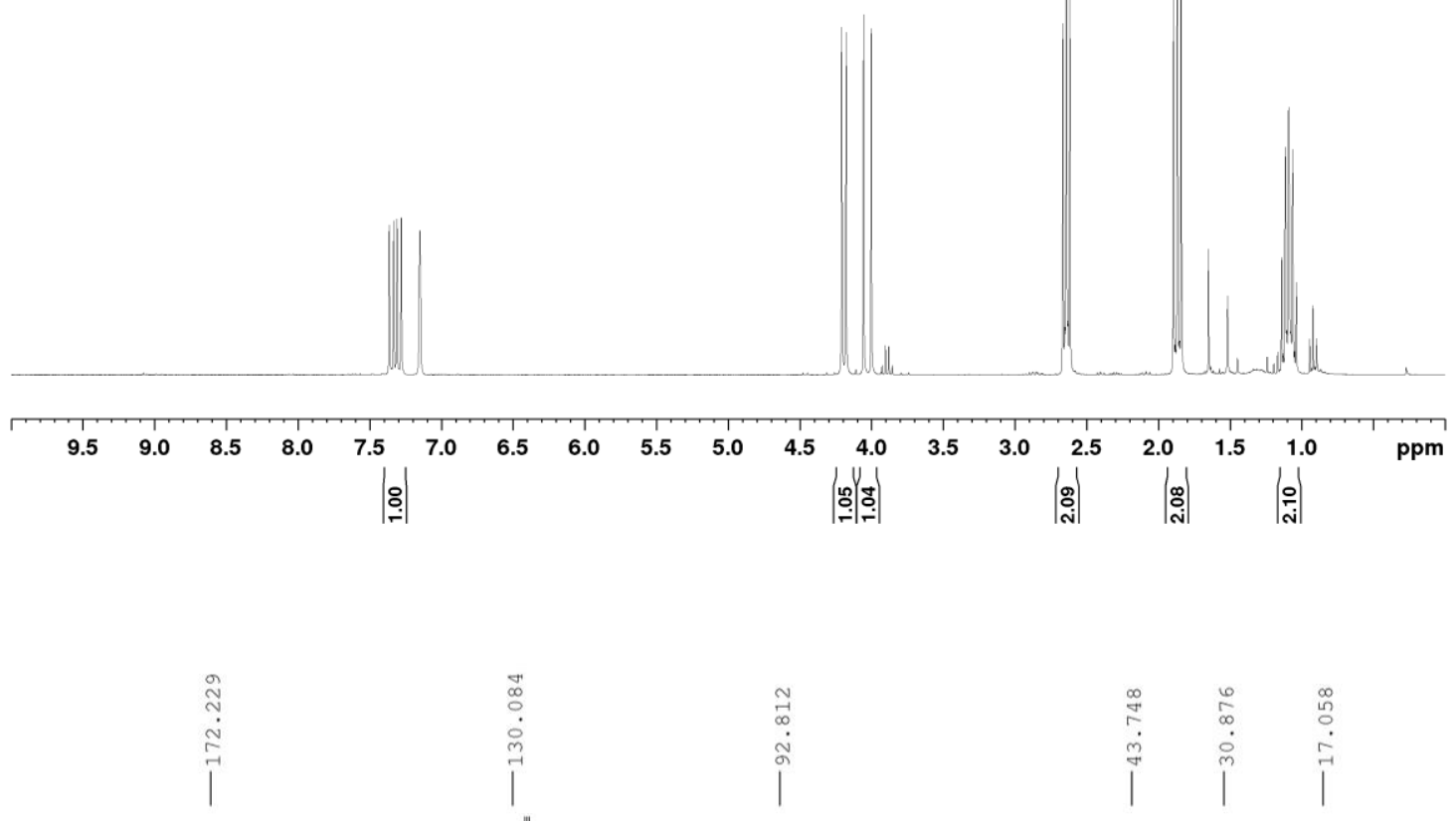

NS-184-A2-pure2, C6D6, 13C<smiles>C=CN1CCCC1=O</smiles>

1a, ${ }^{13} \mathrm{C}\left\{{ }^{1} \mathrm{H}\right\}, \mathrm{C}_{6} \mathrm{D}_{6}, 75.6 \mathrm{MHz}$

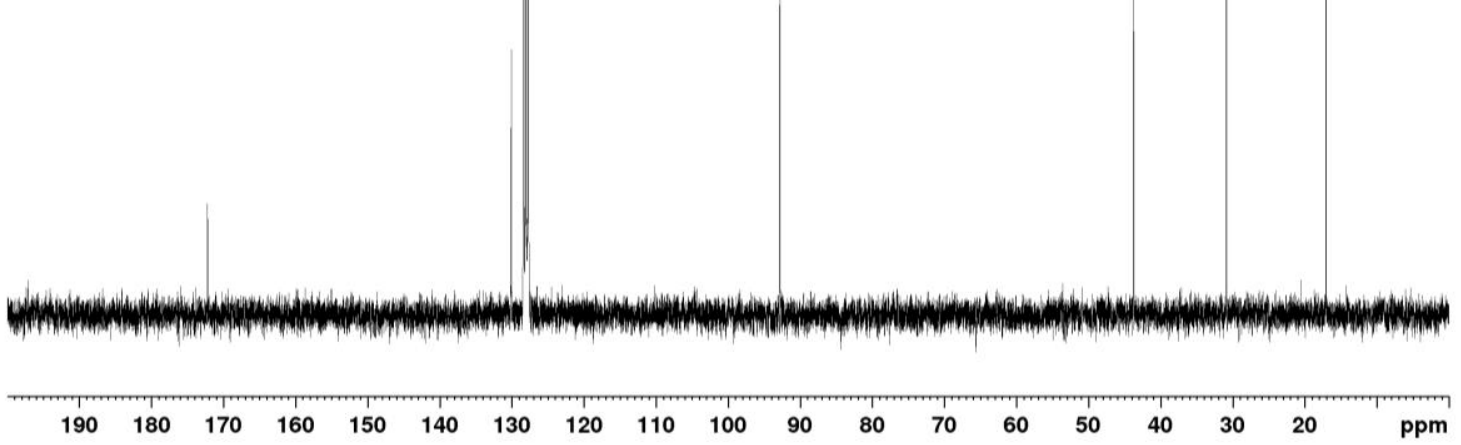



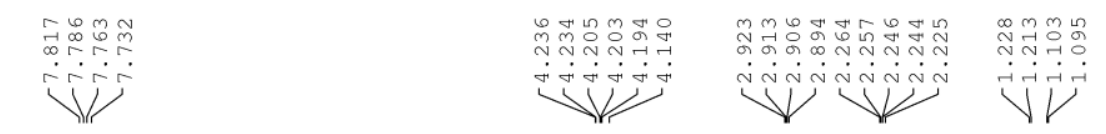

NS-184-B2-pure, C6D6, 1H

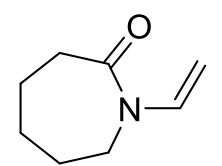

1b, ${ }^{1} \mathrm{H}, \mathrm{C}_{6} \mathrm{D}_{6}, 300 \mathrm{MHz}$
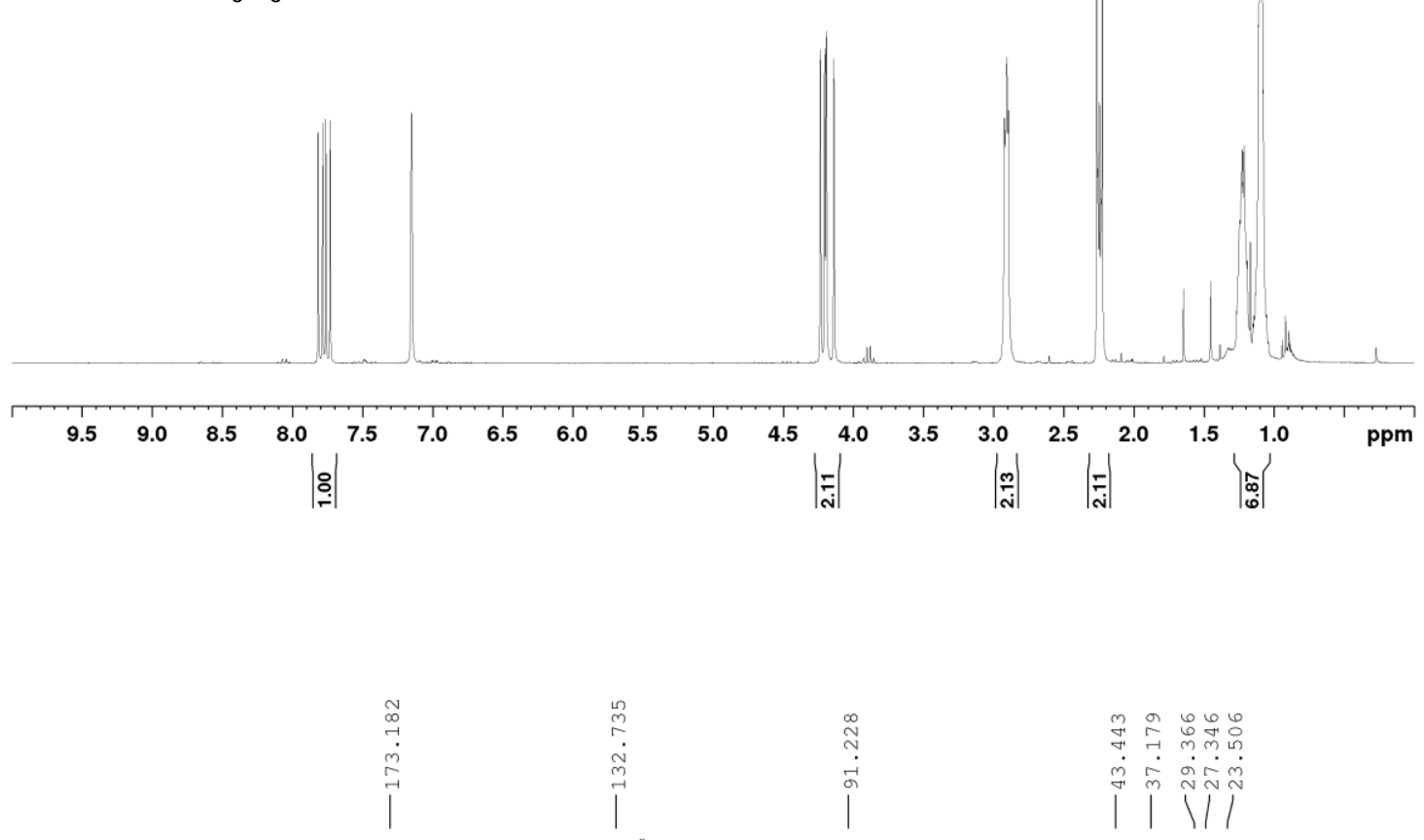

NS-184-B2-pure, C6D6, 13C

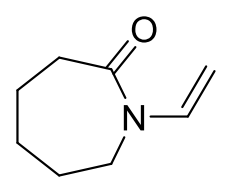

1b, ${ }^{13} \mathrm{C}\left\{{ }^{1} \mathrm{H}\right\}, \mathrm{C}_{6} \mathrm{D}_{6}, 75.6 \mathrm{MHz}$

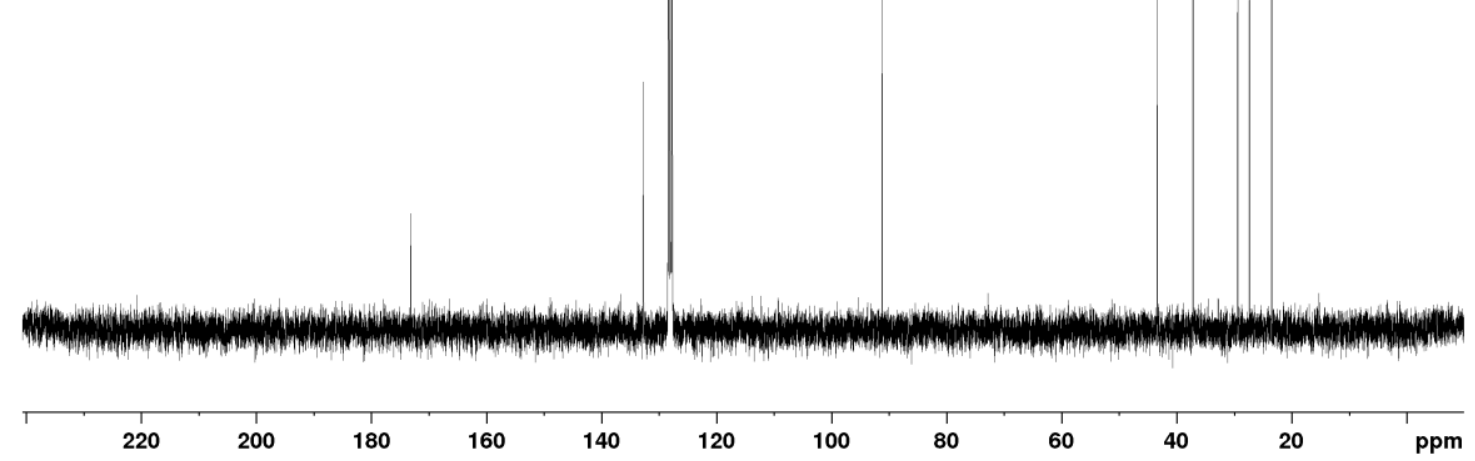




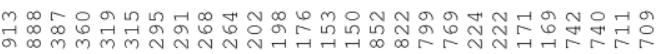

ising
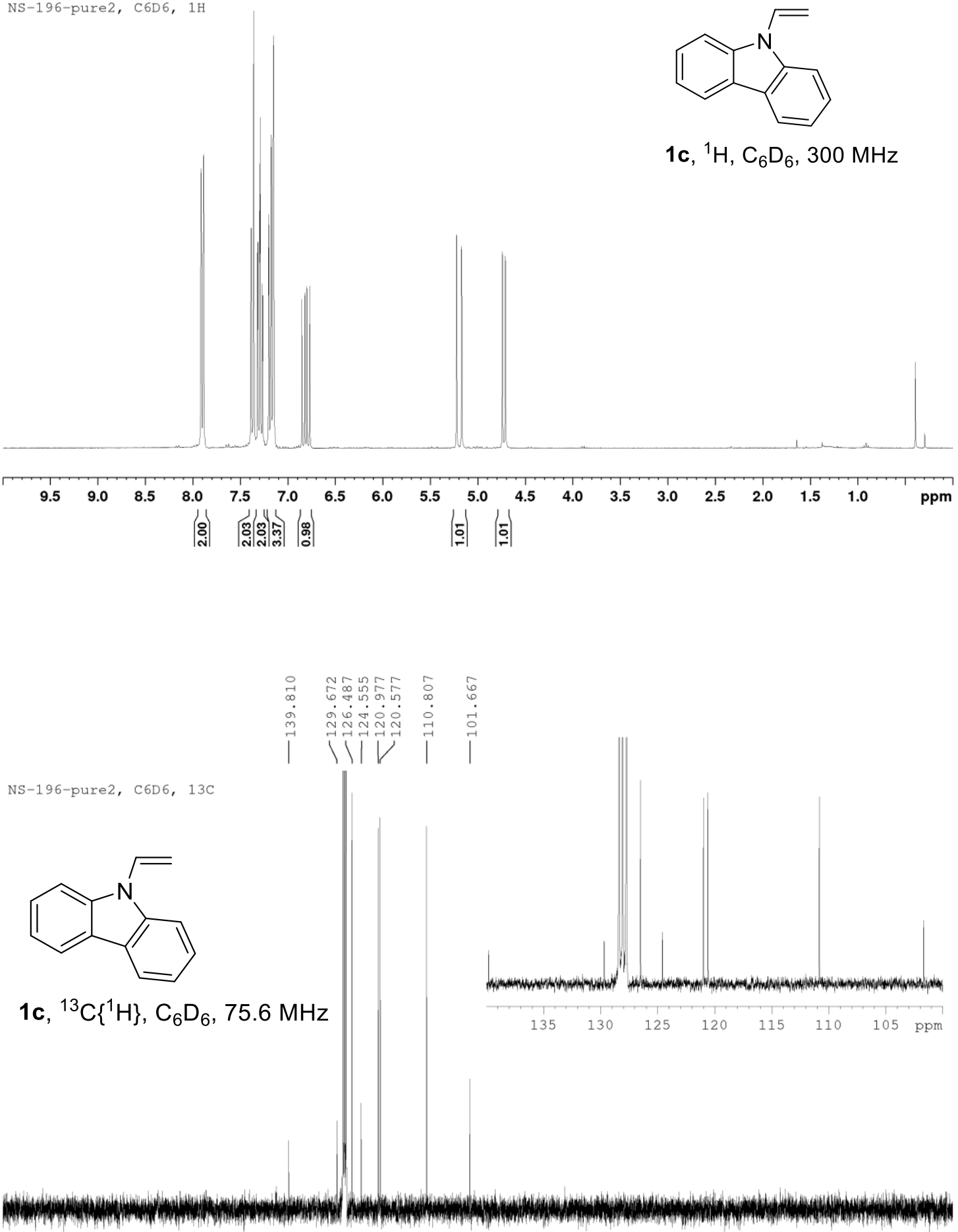

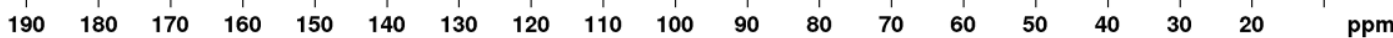



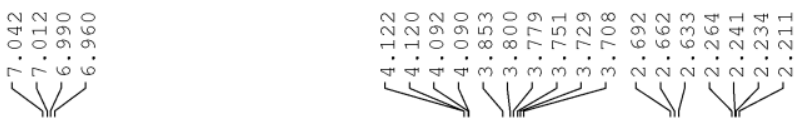

Von

NS-188-2-pure, C6D 6, 1H

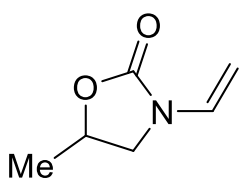

1d, ${ }^{1} \mathrm{H}, \mathrm{C}_{6} \mathrm{D}_{6}, 300 \mathrm{MHz}$
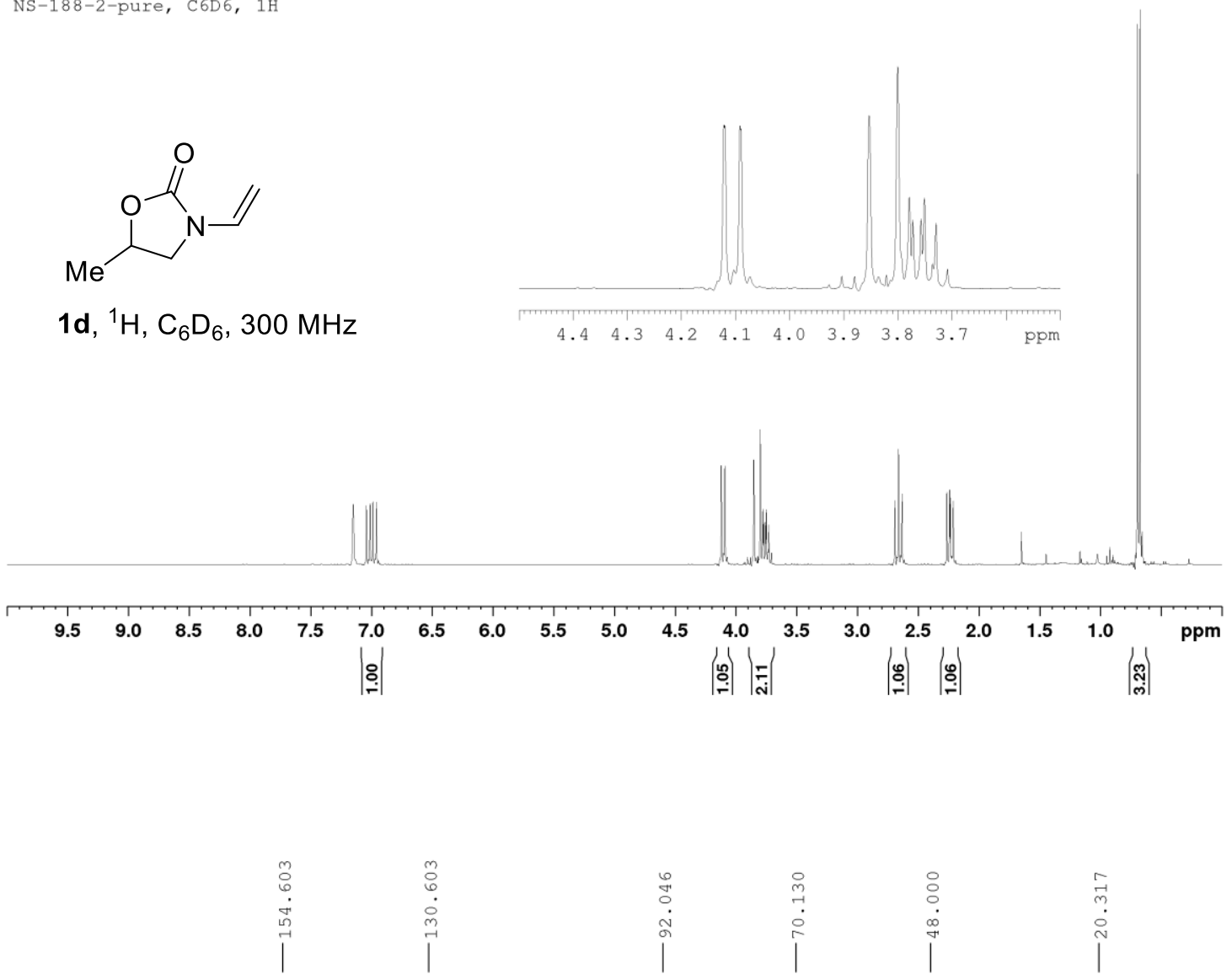

NS-188-2-pure, C6D6, 13C<smiles>C=CN1CC(C)OC1=O</smiles>

1d, ${ }^{13} \mathrm{C}\left\{{ }^{1} \mathrm{H}\right\}, \mathrm{C}_{6} \mathrm{D}_{6}, 76.5 \mathrm{MHz}$

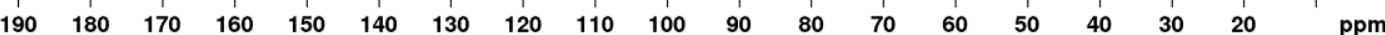



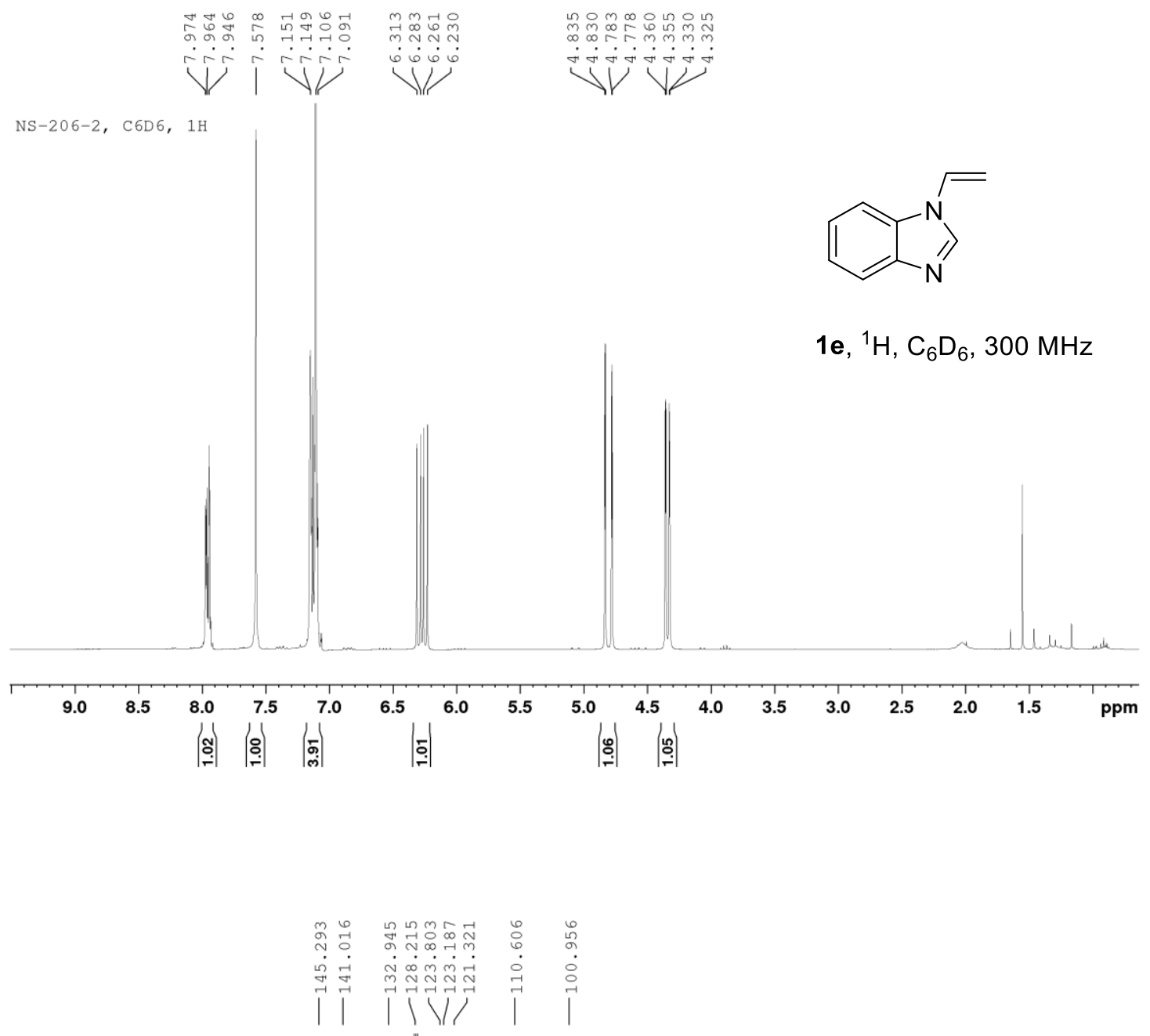

NS-206-2, C6D 6, $13 \mathrm{C}$<smiles>C=Cn1cnc2ccccc21</smiles>

1e, ${ }^{13} \mathrm{C}\left\{{ }^{1} \mathrm{H}\right\}, \mathrm{C}_{6} \mathrm{D}_{6}, 75.6 \mathrm{MHz}$
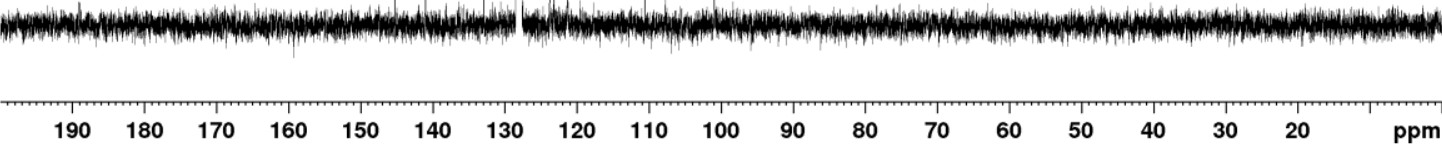


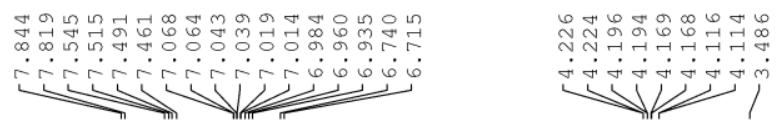

NS-217-pure, C6D 6, 1H<smiles>C=CN1Cc2ccccc2C1=O</smiles>

1g, ${ }^{1} \mathrm{H}, \mathrm{C}_{6} \mathrm{D}_{6}, 300 \mathrm{MHz}$
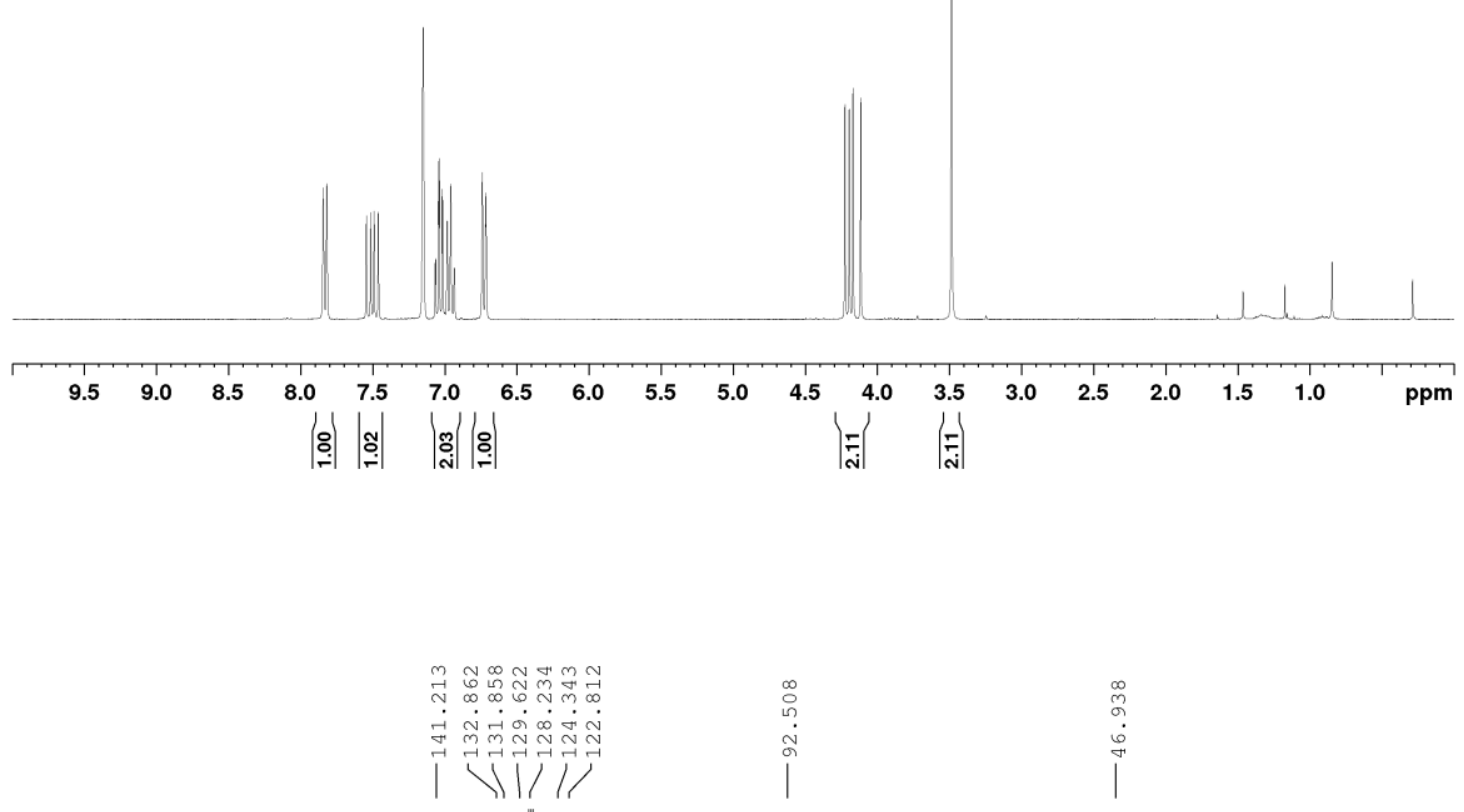

NS-217-pure, C6D6, $13 \mathrm{C}$
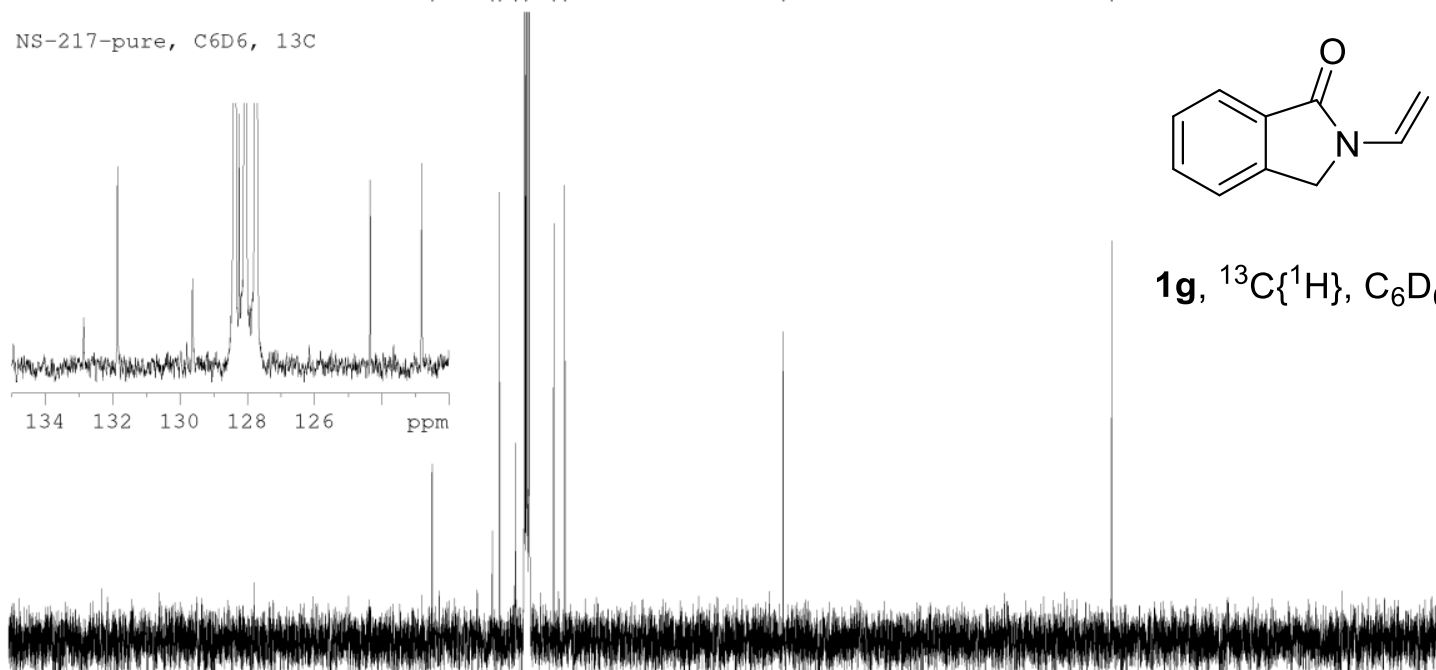

1g, ${ }^{13} \mathrm{C}\left\{{ }^{1} \mathrm{H}\right\}, \mathrm{C}_{6} \mathrm{D}_{6}, 75.6 \mathrm{MHz}$

$\begin{array}{lllllll}134 & 132 & 130 & 128 & 126\end{array}$

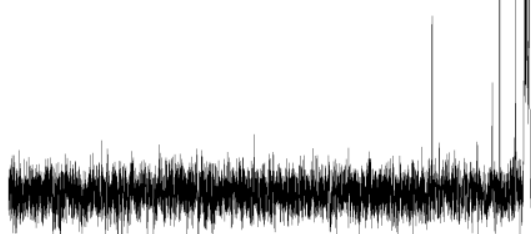
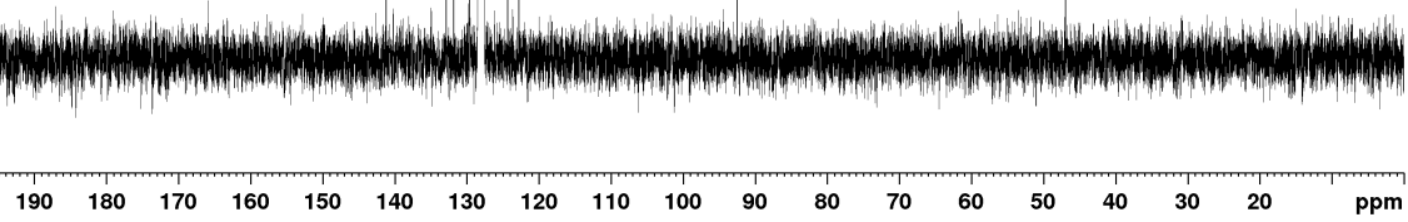
NS-202-3-pure, C6D6, 1H
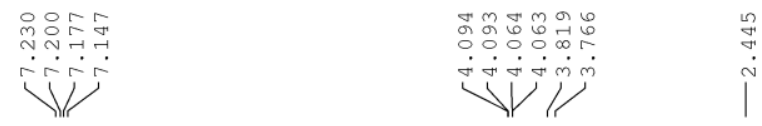

(1)

1i, ${ }^{1} \mathrm{H}, \mathrm{C}_{6} \mathrm{D}_{6}, 300 \mathrm{MHz}$
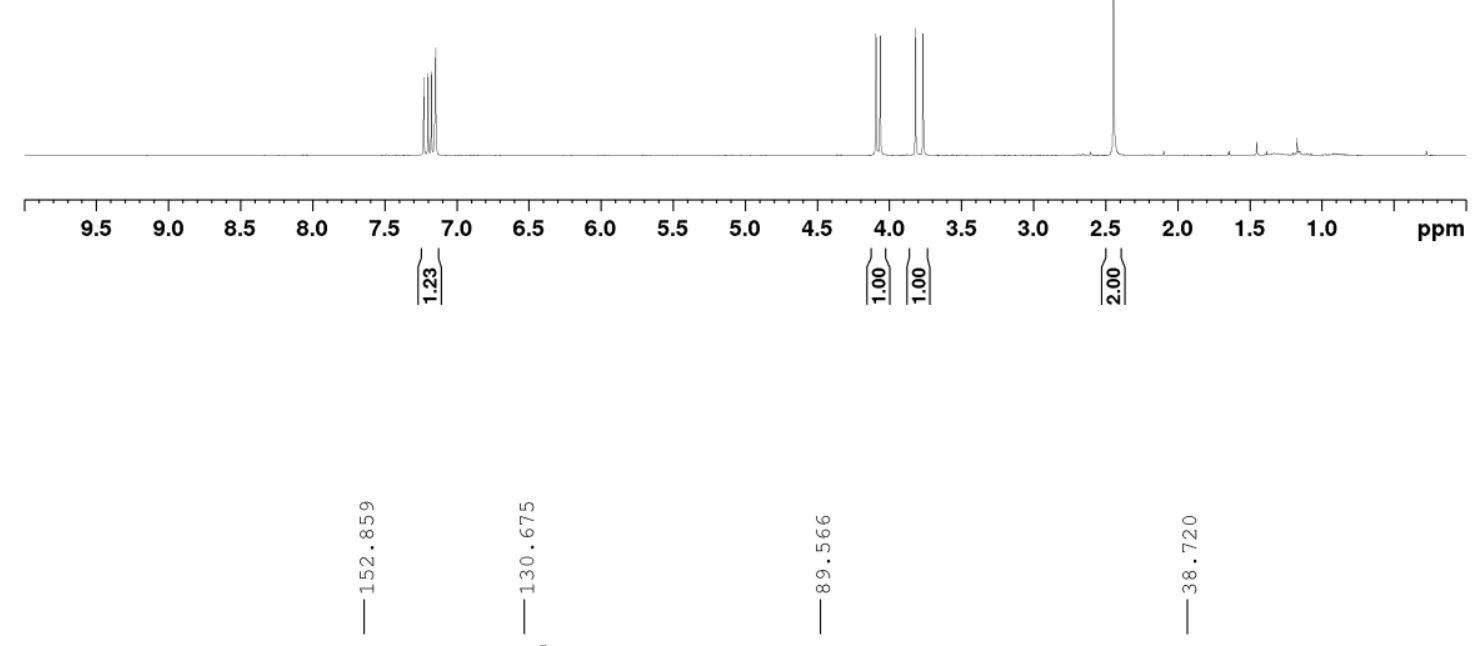

NS-202-3-pure, C6D6, 13C<smiles>C=CN1CCN(C=C)C1=O</smiles>

1i, ${ }^{13} \mathrm{C}\left\{{ }^{1} \mathrm{H}\right\}, \mathrm{C}_{6} \mathrm{D}_{6}, 75.6 \mathrm{MHz}$ 


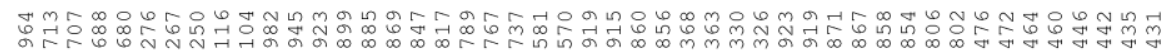

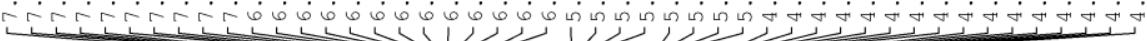
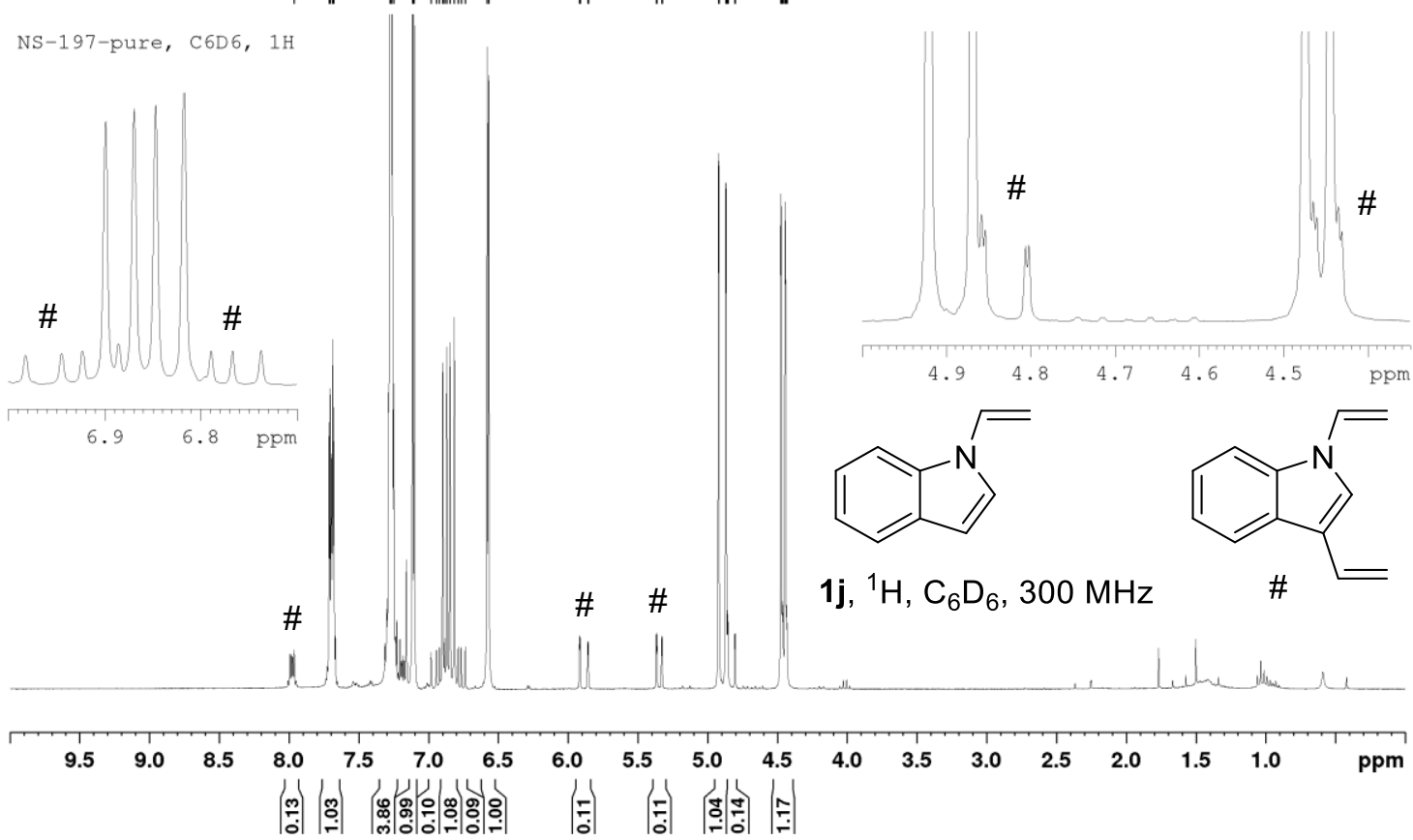

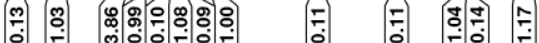

NS-197-pure, C6D 6, 13C<smiles>C=Cn1ccc2ccccc21</smiles>

1j, ${ }^{13} \mathrm{C}\left\{{ }^{1} \mathrm{H}\right\}, \mathrm{C}_{6} \mathrm{D}_{6}, 75.6 \mathrm{MHz}$
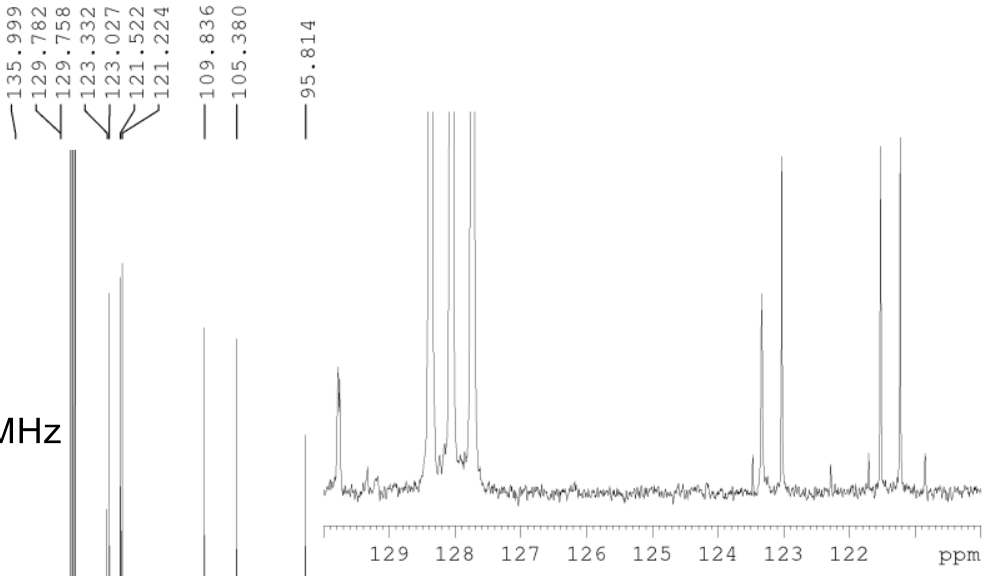

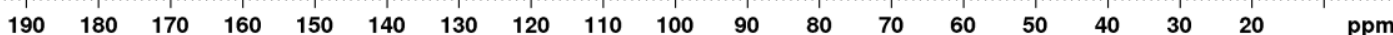


NS-215-2, C6D 6, 1H<smiles></smiles>

1I, ${ }^{1} \mathrm{H}, \mathrm{C}_{6} \mathrm{D}_{6}, 300 \mathrm{MHz}$

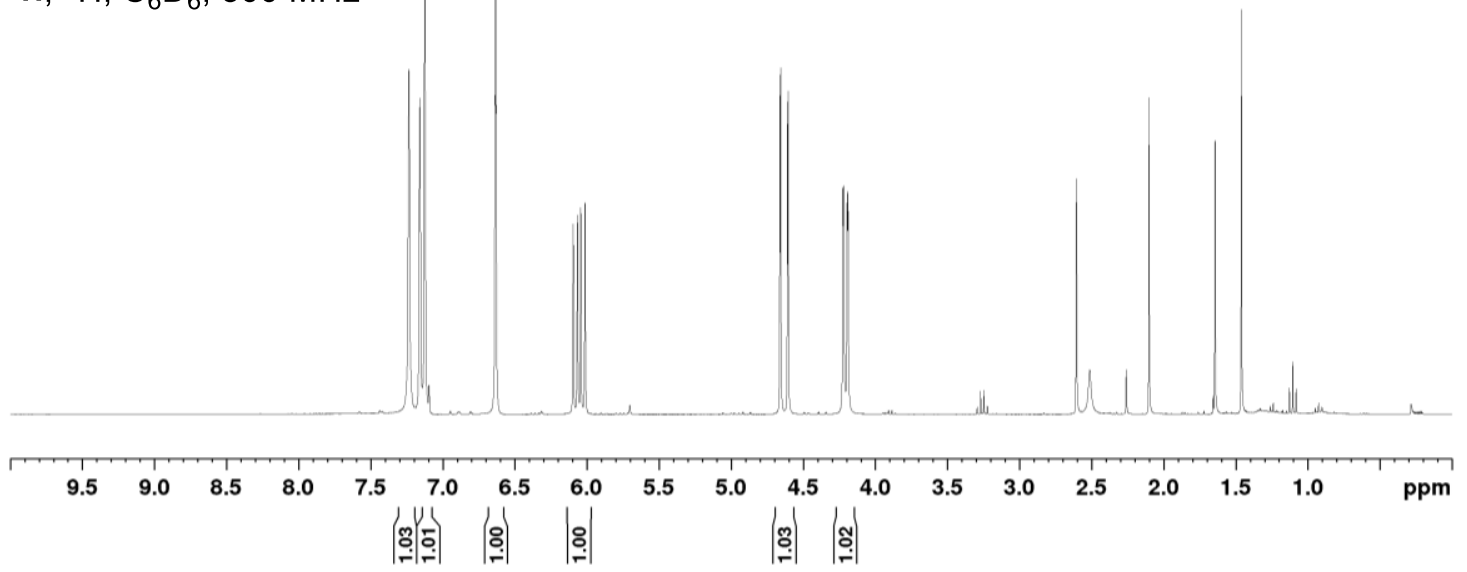

NS-215-2, C6D 6, $13 \mathrm{C}$

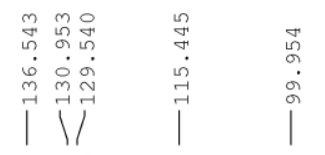<smiles>C=Cn1ccnc1</smiles>

1I, ${ }^{13} \mathrm{C}\left\{{ }^{1} \mathrm{H}\right\}, \mathrm{C}_{6} \mathrm{D}_{6}, 75.6 \mathrm{MHz}$

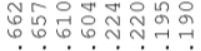

பேர்

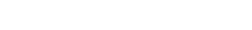


NS-224, C6D 6, $1 \mathrm{H}$

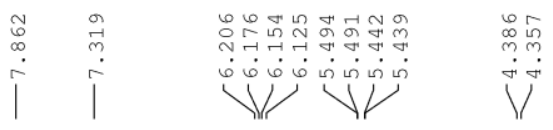

$\mathbb{N}_{N}^{\mathrm{N}}$

$1 \mathrm{~m},{ }^{1} \mathrm{H}, \mathrm{C}_{6} \mathrm{D}_{6}, 300 \mathrm{MHz}$
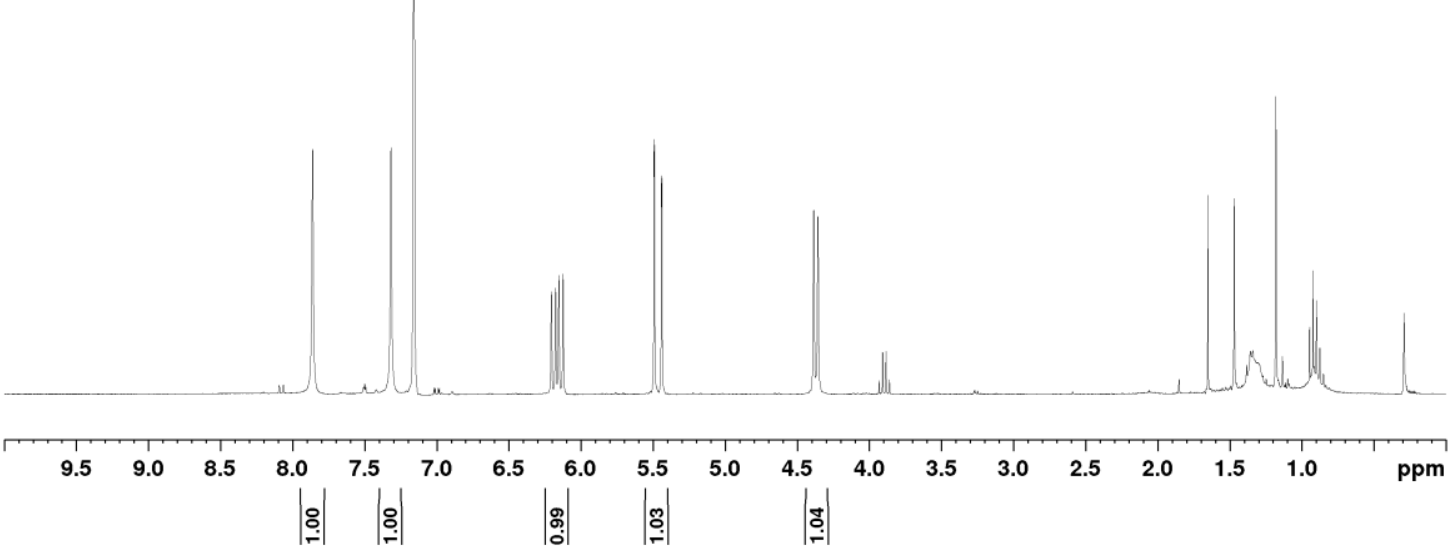

$$
\text { }
$$

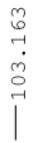

NS-224, C6D 6, 13C

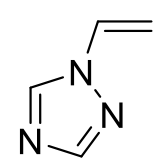

$1 \mathrm{~m},{ }^{13} \mathrm{C}\left\{{ }^{1} \mathrm{H}\right\}, \mathrm{C}_{6} \mathrm{D}_{6}, 75.6 \mathrm{MHz}$

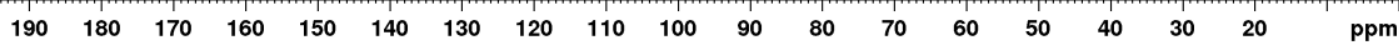



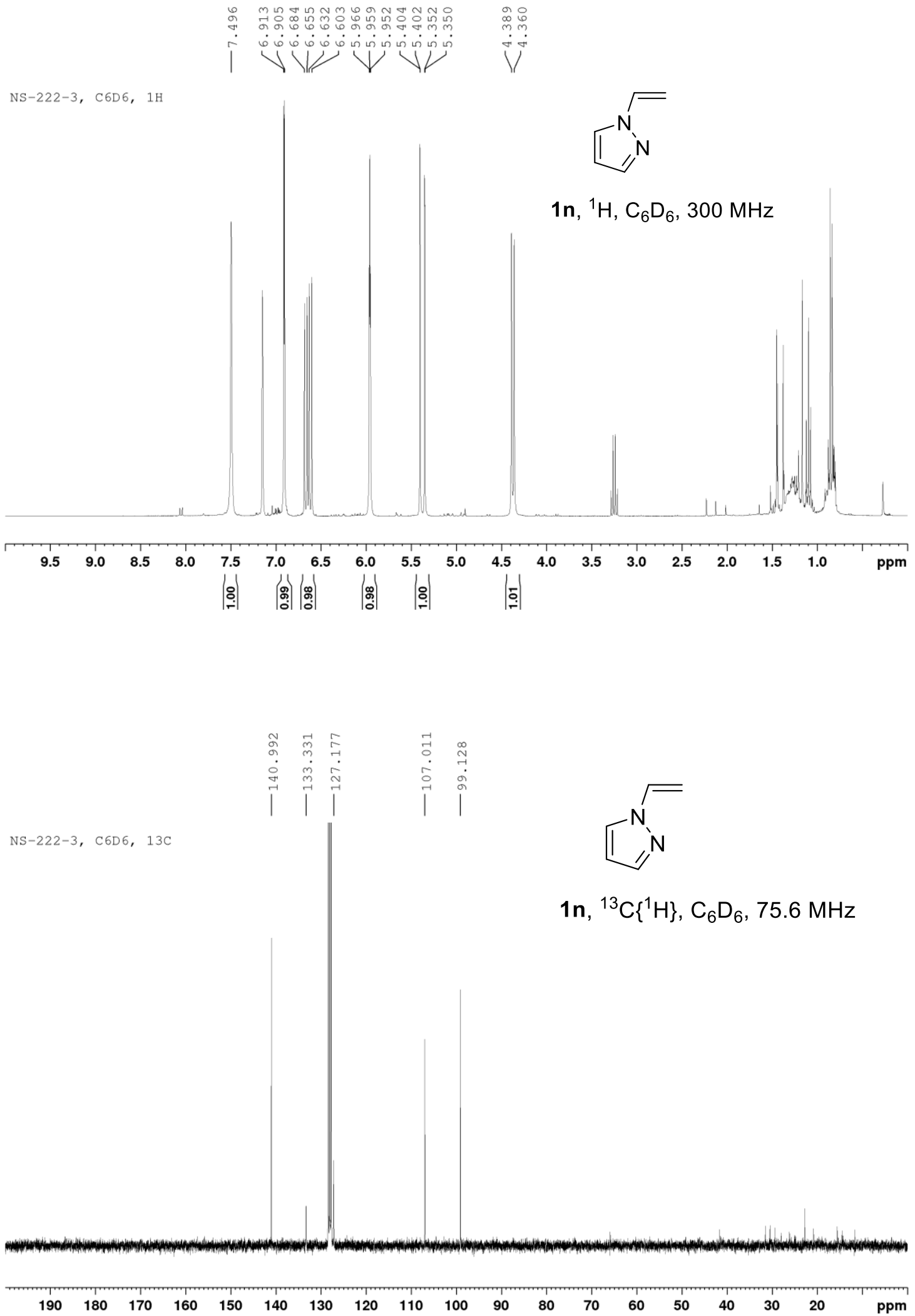
${ }^{1} \mathrm{H}$ NMR (300 MHz, $300 \mathrm{~K}$ ), before reaction (DMAA, $\mathrm{C}_{6} \mathrm{D}_{6}$, trimethoxybenzene as internal std.*)

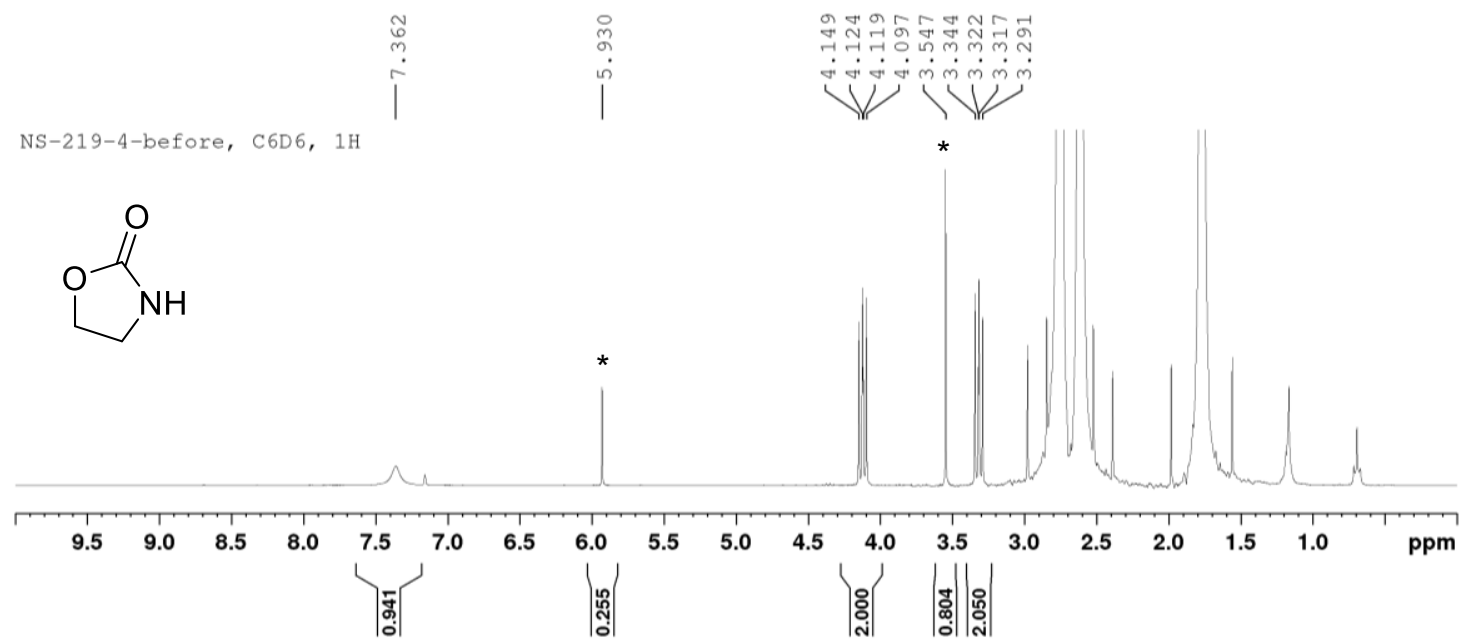

${ }^{1} \mathrm{H}$ NMR (300 MHz, $300 \mathrm{~K}$ ), after $160^{\circ} \mathrm{C}, 16 \mathrm{~h}$ (DMAA, $\mathrm{C}_{6} \mathrm{D}_{6}$, trimethoxybenzene as internal std. $\left.{ }^{*}\right)$

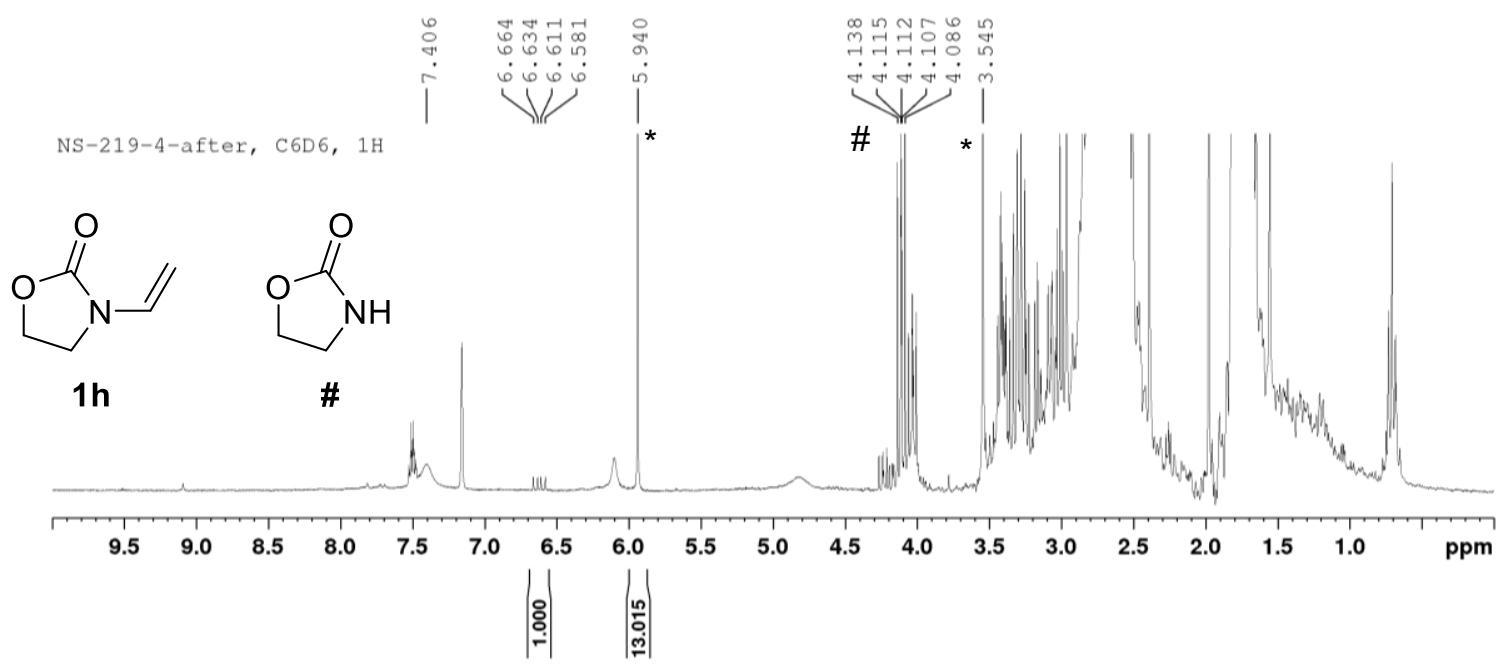

Yield: Integral STD before/Integral STD after $=0.255 / 13.02=2 \%$ 
${ }^{1} \mathrm{H}$ NMR (300 MHz, $300 \mathrm{~K}$ ), before reaction (DMAA, $\mathrm{C}_{6} \mathrm{D}_{6}$, trimethoxybenzene as internal std. ${ }^{*}$ )

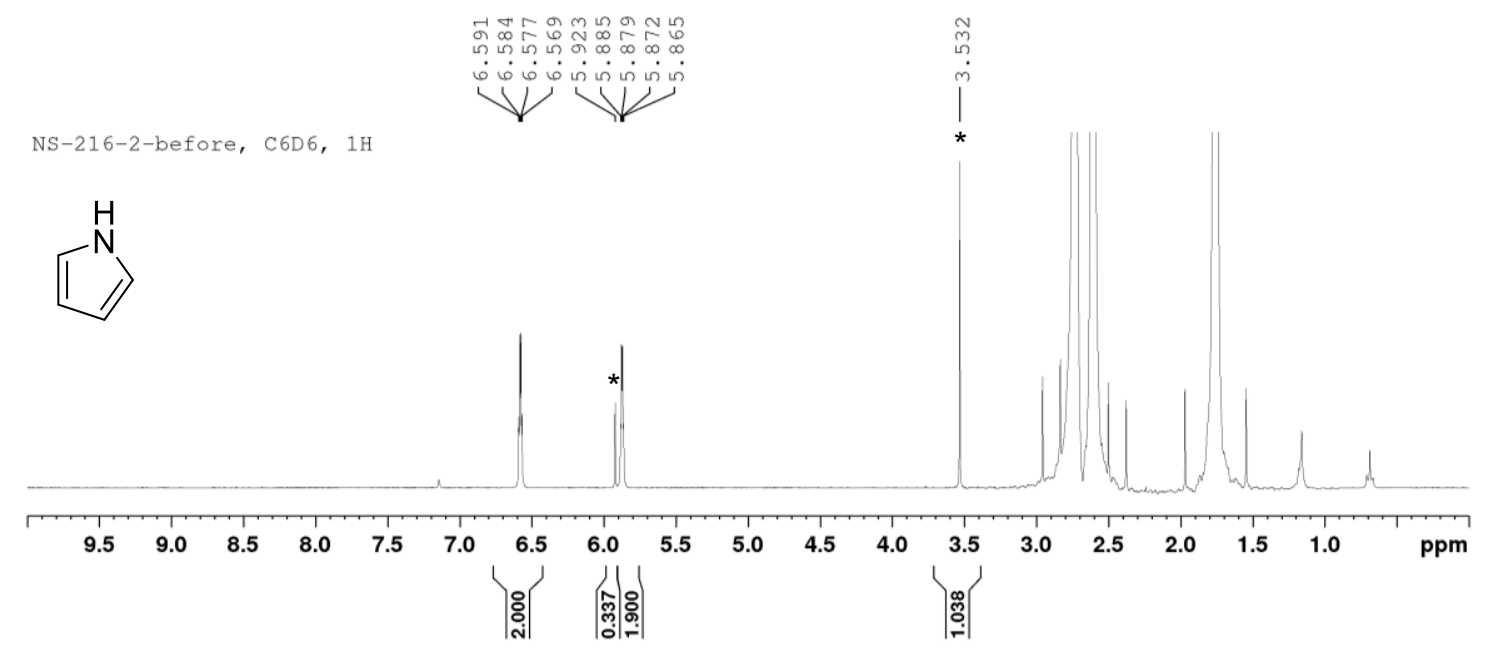

${ }^{1} \mathrm{H}$ NMR (300 MHz, $300 \mathrm{~K}$ ), after $140{ }^{\circ} \mathrm{C}, 16 \mathrm{~h}$ (DMAA, $\mathrm{C}_{6} \mathrm{D}_{6}$, trimethoxybenzene as internal std. $\left.{ }^{*}\right)$

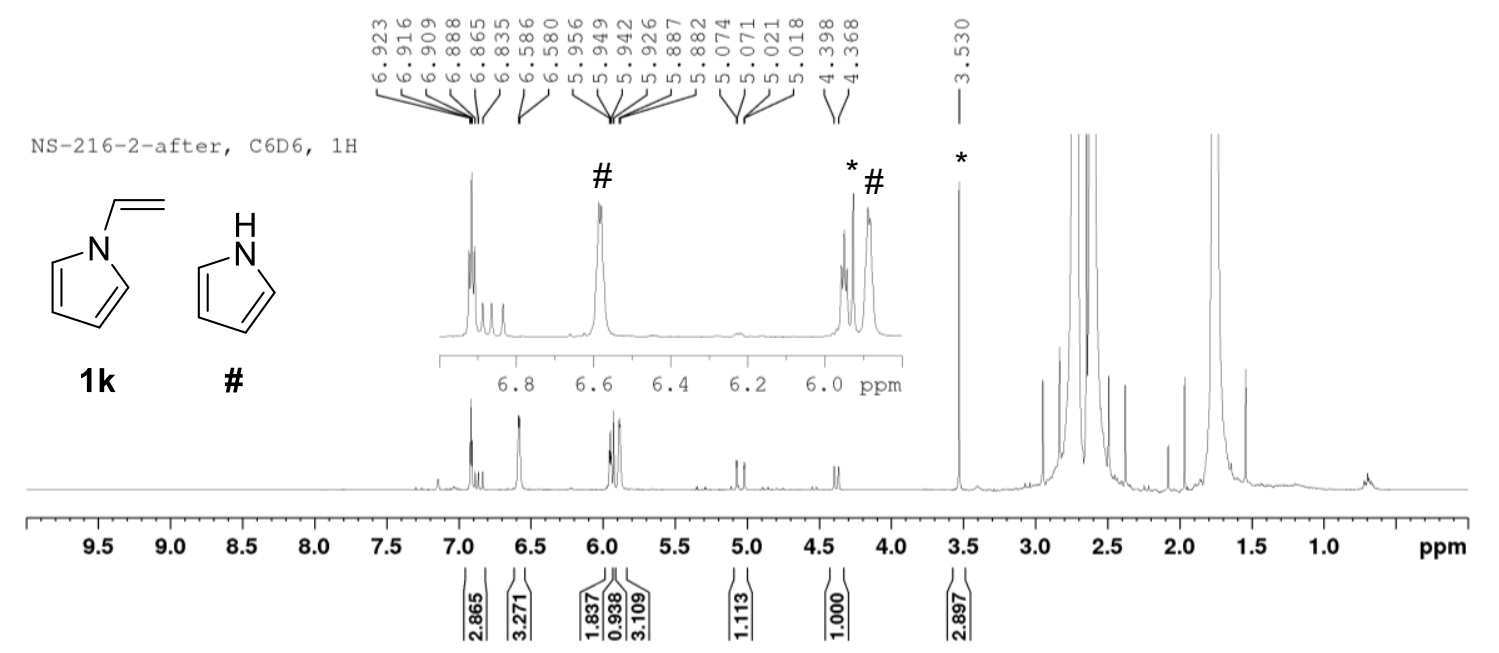




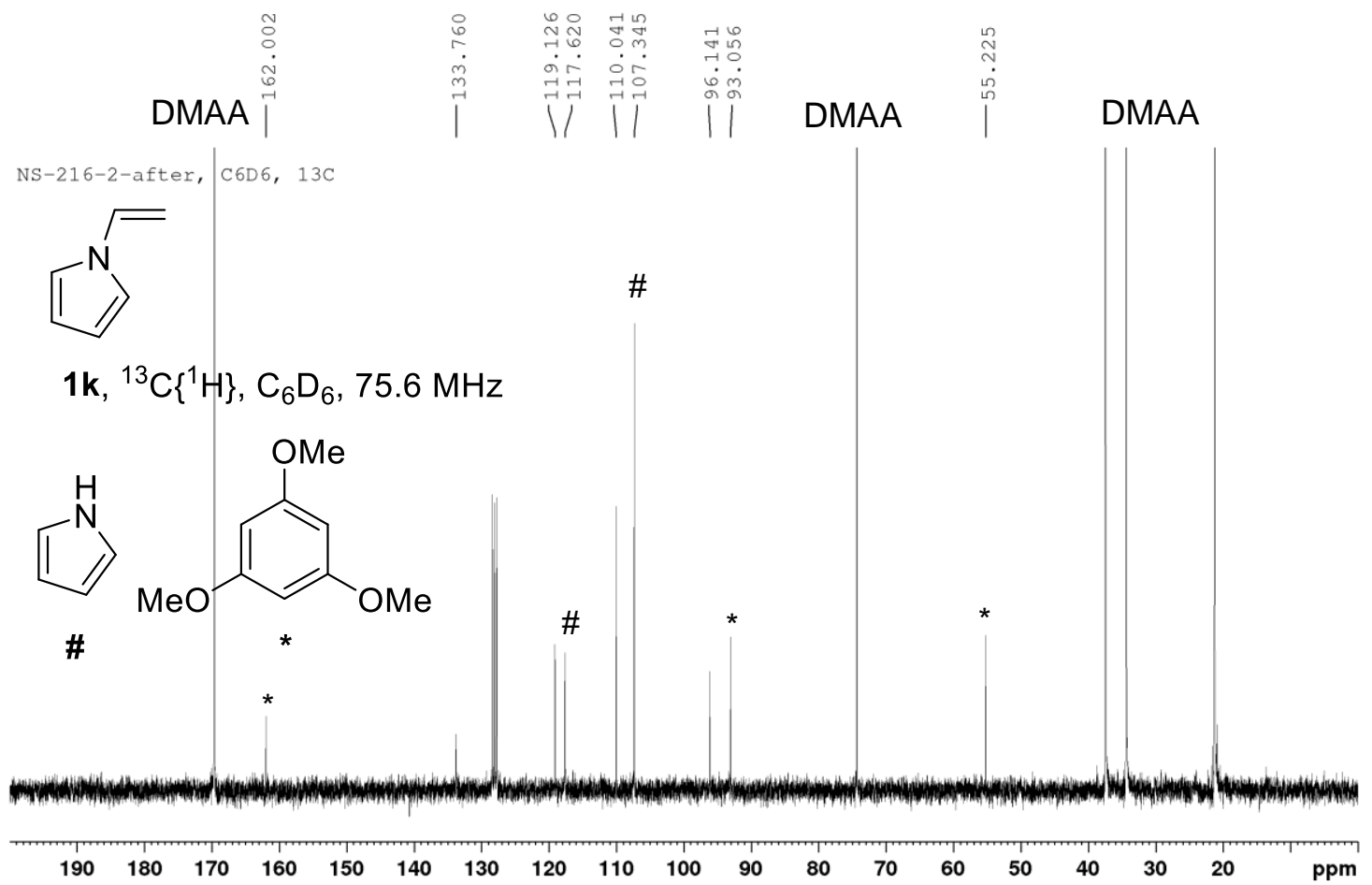


${ }^{1} \mathrm{H}$ NMR (300 MHz, $300 \mathrm{~K}$ ), before reaction (DMAA, $\mathrm{C}_{6} \mathrm{D}_{6}$ as internal std.*)

NS-230-B-C2H2， C6D 6, 1H
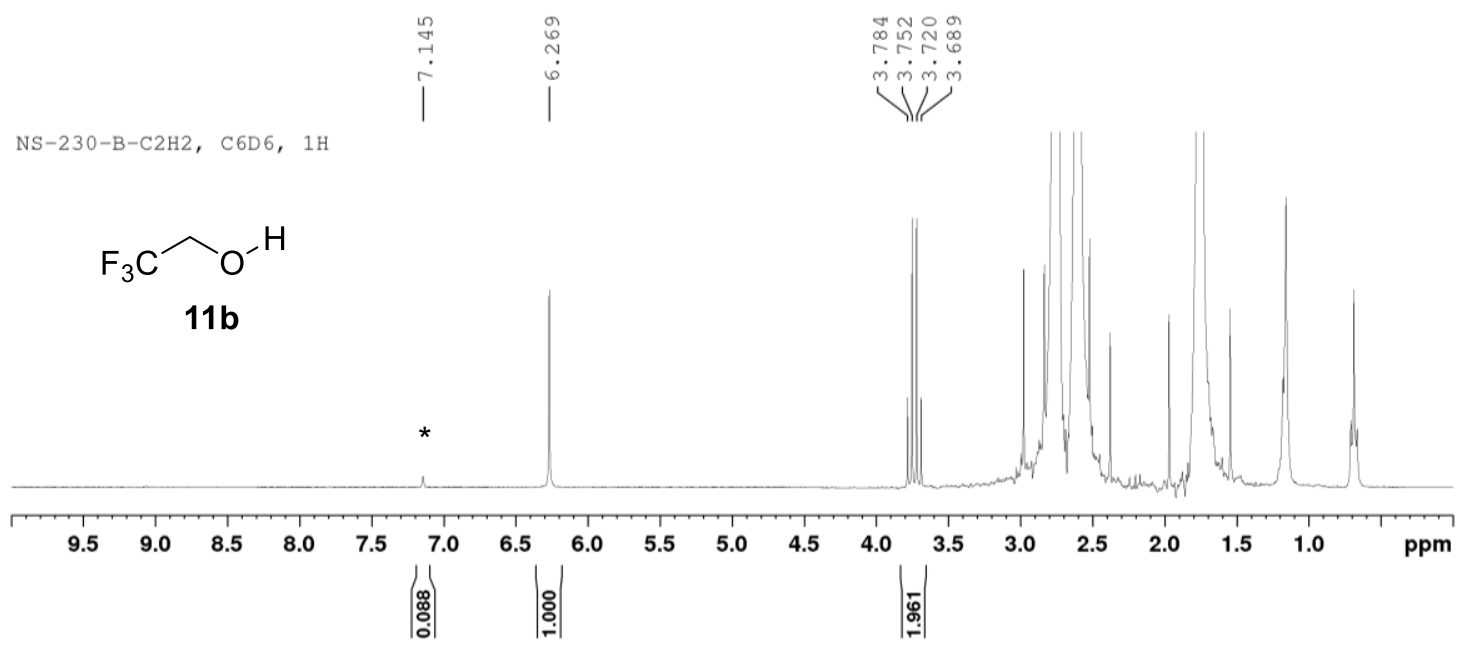

${ }^{13} \mathrm{C}\left\{{ }^{1} \mathrm{H}\right\}$ NMR (75.6 MHz, $300 \mathrm{~K}$ ), before reaction (DMAA, $\mathrm{C}_{6} \mathrm{D}_{6}$ as internal std.)

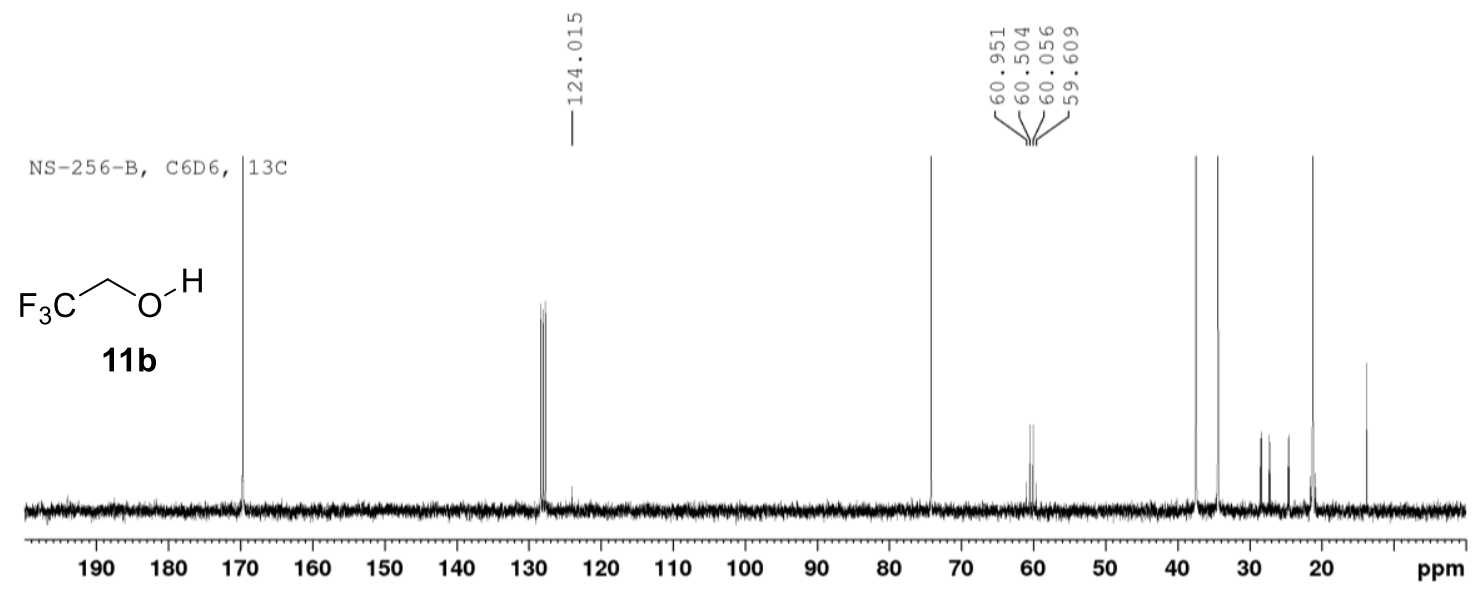

${ }^{19} \mathrm{~F}$ NMR (283 MHz, $300 \mathrm{~K}$ ), before reaction (DMAA, $\mathrm{C}_{6} \mathrm{D}_{6}$ as internal std.)

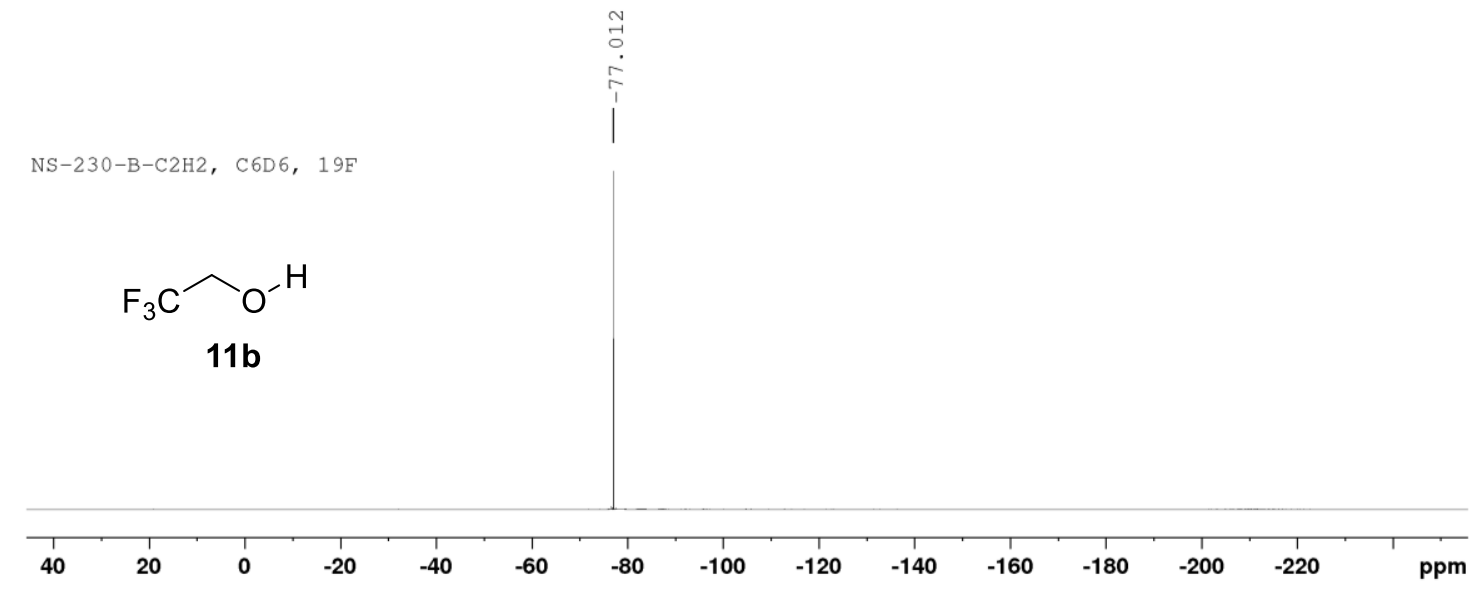


${ }^{1} \mathrm{H}$ NMR (300 MHz, $300 \mathrm{~K}$ ), after $140{ }^{\circ} \mathrm{C}$ for $5 \mathrm{~h}$ (DMAA, $\mathrm{C}_{6} \mathrm{D}_{6}$ as internal std.*)

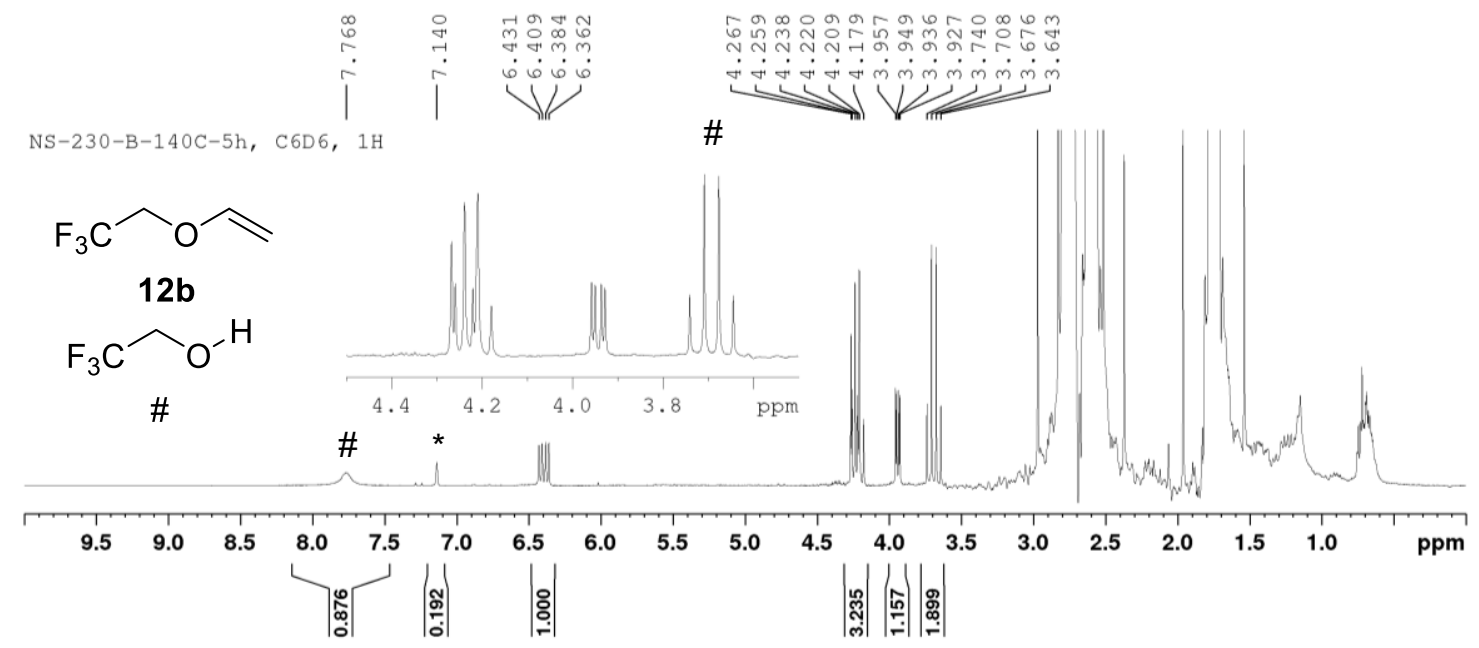

Yield: Integral STD before/Integral STD after $=0.088 / 0.192=45 \%$

${ }^{13} \mathrm{C}\left\{{ }^{1} \mathrm{H}\right\}$ NMR (75.6 MHz, $300 \mathrm{~K}$ ), after $140{ }^{\circ} \mathrm{C}$ for $5 \mathrm{~h}$ (DMAA, $\mathrm{C}_{6} \mathrm{D}_{6}$ as internal std.)

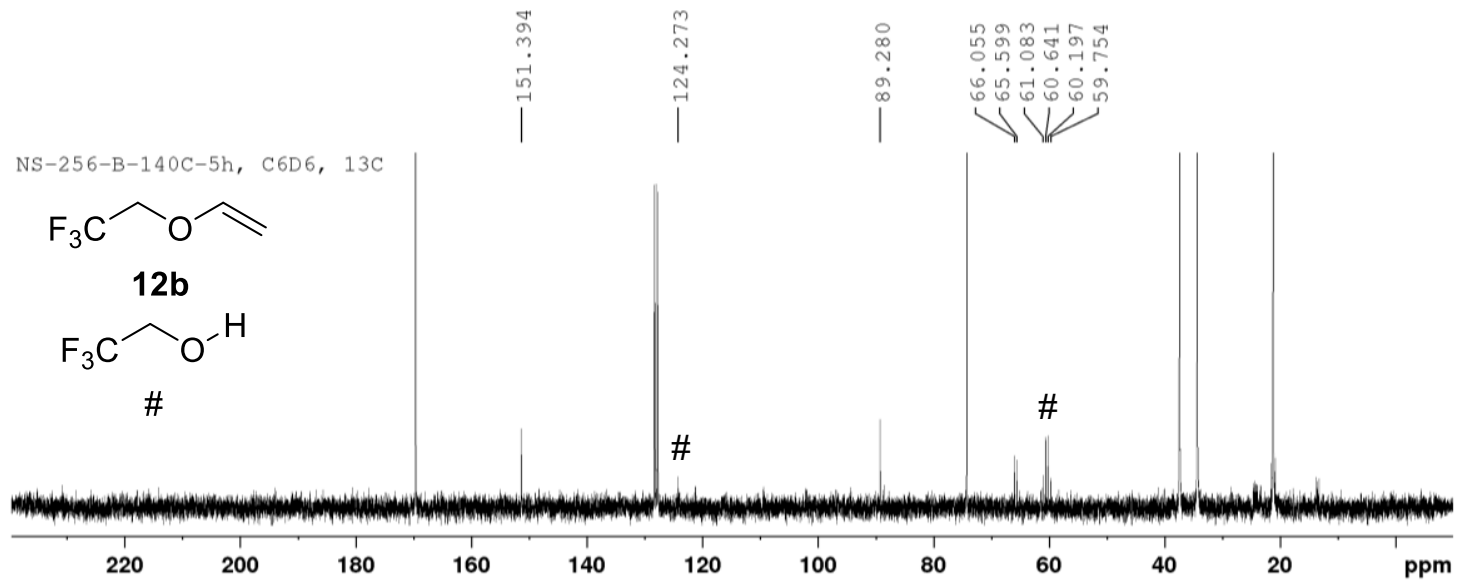

${ }^{19} \mathrm{~F}$ NMR (283 MHz, $300 \mathrm{~K}$ ), after $140{ }^{\circ} \mathrm{C}$ for $5 \mathrm{~h}$ (DMAA, $\mathrm{C}_{6} \mathrm{D}_{6}$ as internal std.)

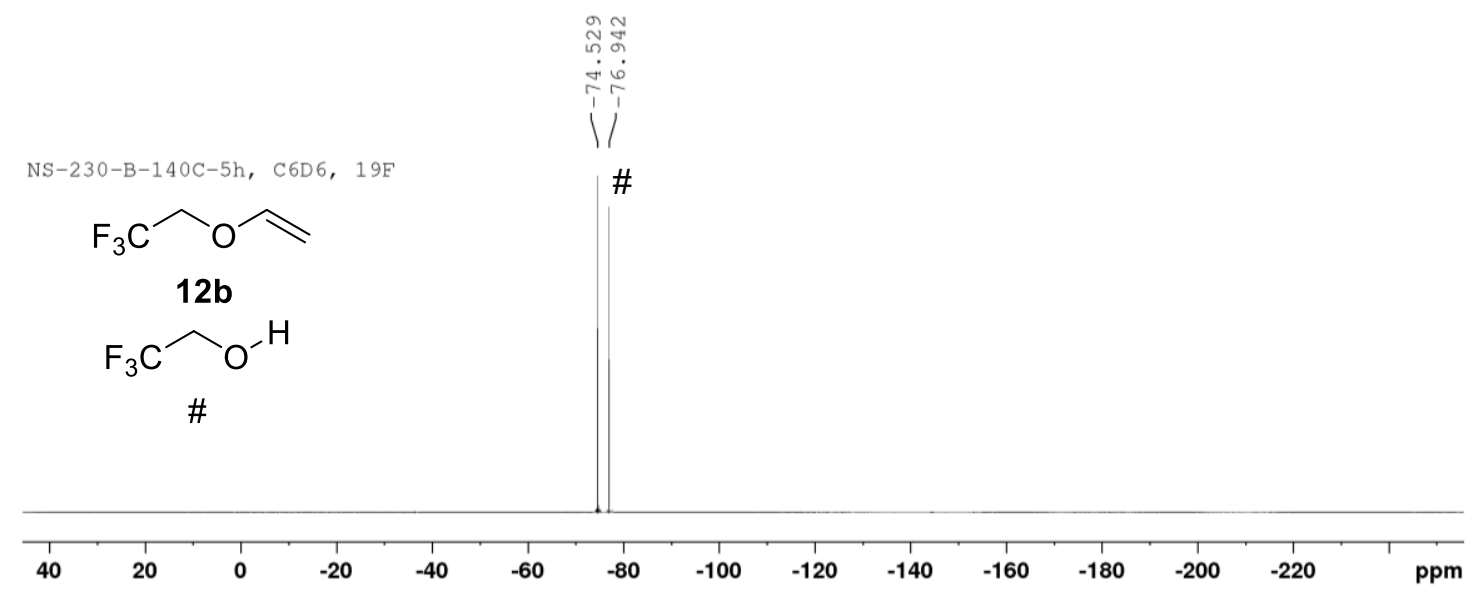


${ }^{1} \mathrm{H}$ NMR (300 MHz, $300 \mathrm{~K}$ ), before reaction (DMAA, $\mathrm{C}_{6} \mathrm{D}_{6}$ as internal std.*)

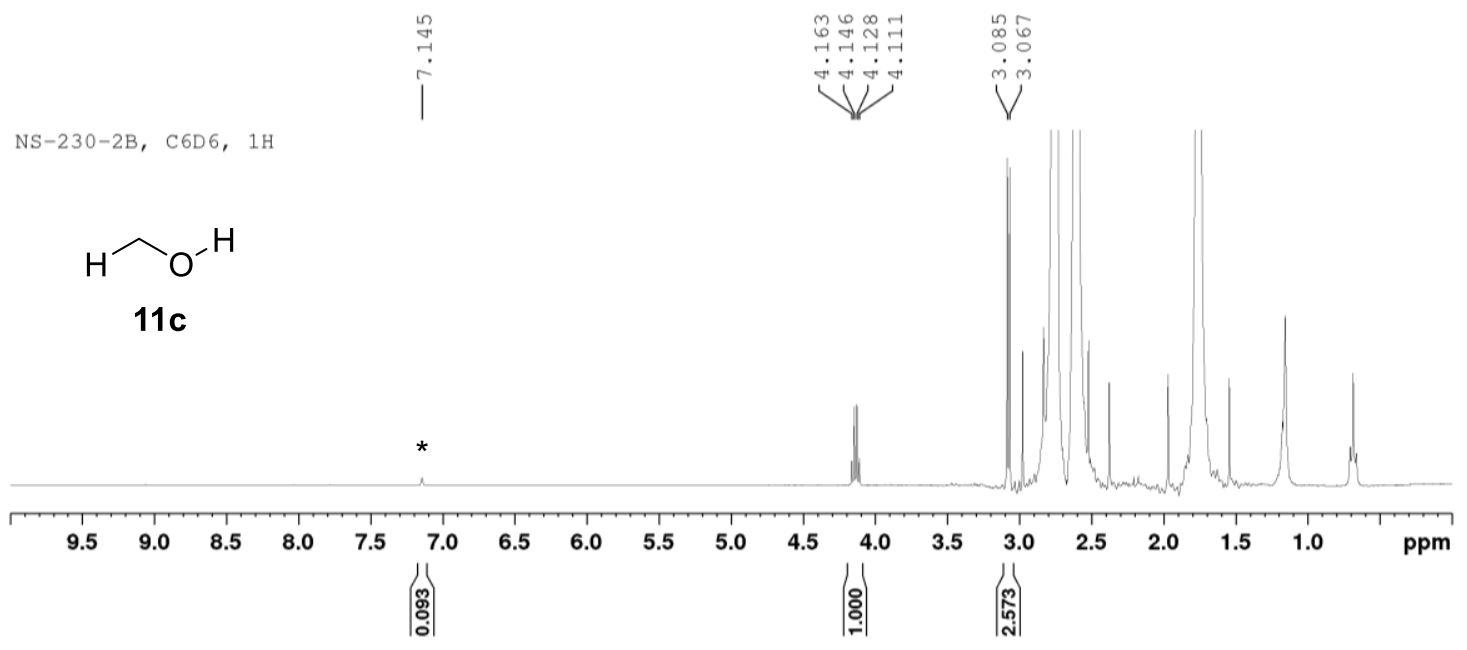

${ }^{13} \mathrm{C}\left\{{ }^{1} \mathrm{H}\right\}$ NMR (75.6 MHz, $300 \mathrm{~K}$ ), before reaction (DMAA, $\mathrm{C}_{6} \mathrm{D}_{6}$ as internal std.)

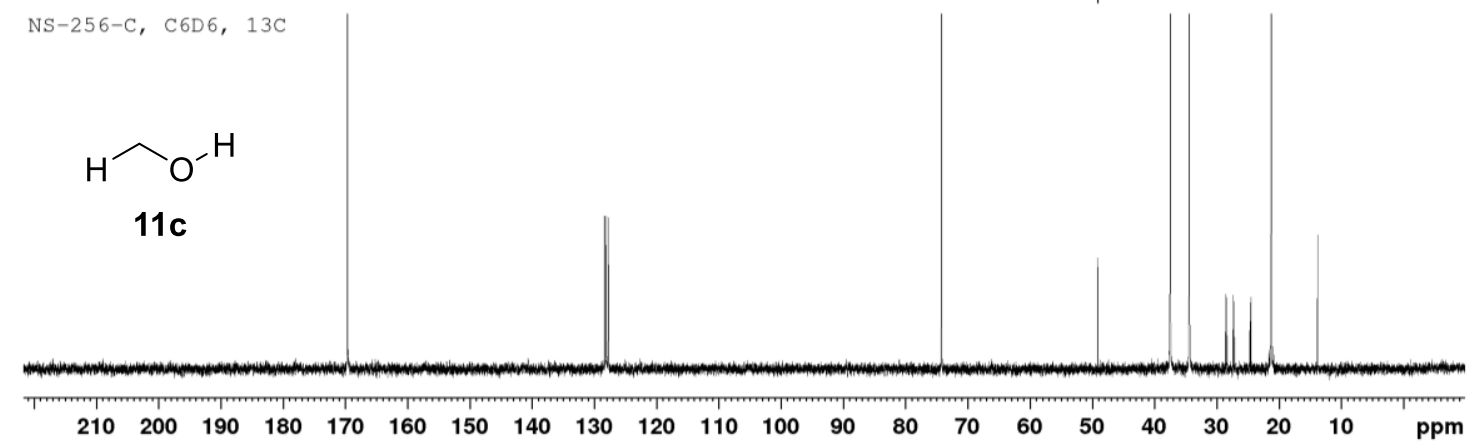


${ }^{1} \mathrm{H}$ NMR (300 MHz, $300 \mathrm{~K}$ ), after $140{ }^{\circ} \mathrm{C}$ for $5 \mathrm{~h}$ (DMAA, $\mathrm{C}_{6} \mathrm{D}_{6}$ as internal std.*)

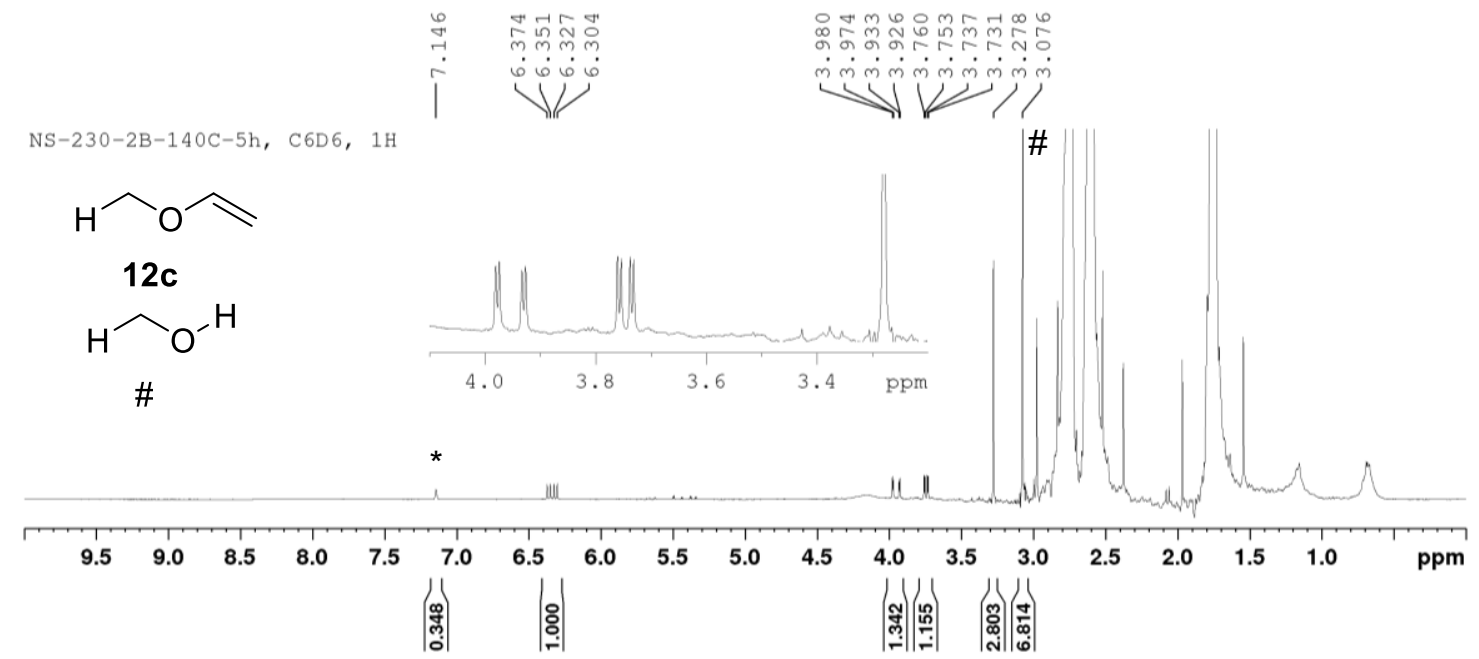

Yield: Integral STD before/Integral STD after $=0.093 / 0.348=27 \%$

${ }^{13} \mathrm{C}\left\{{ }^{1} \mathrm{H}\right\}$ NMR $\left(75.6 \mathrm{MHz}, 300 \mathrm{~K}\right.$ ), after $140{ }^{\circ} \mathrm{C}$ for $5 \mathrm{~h}$ (DMAA, $\mathrm{C}_{6} \mathrm{D}_{6}$ as internal std.)

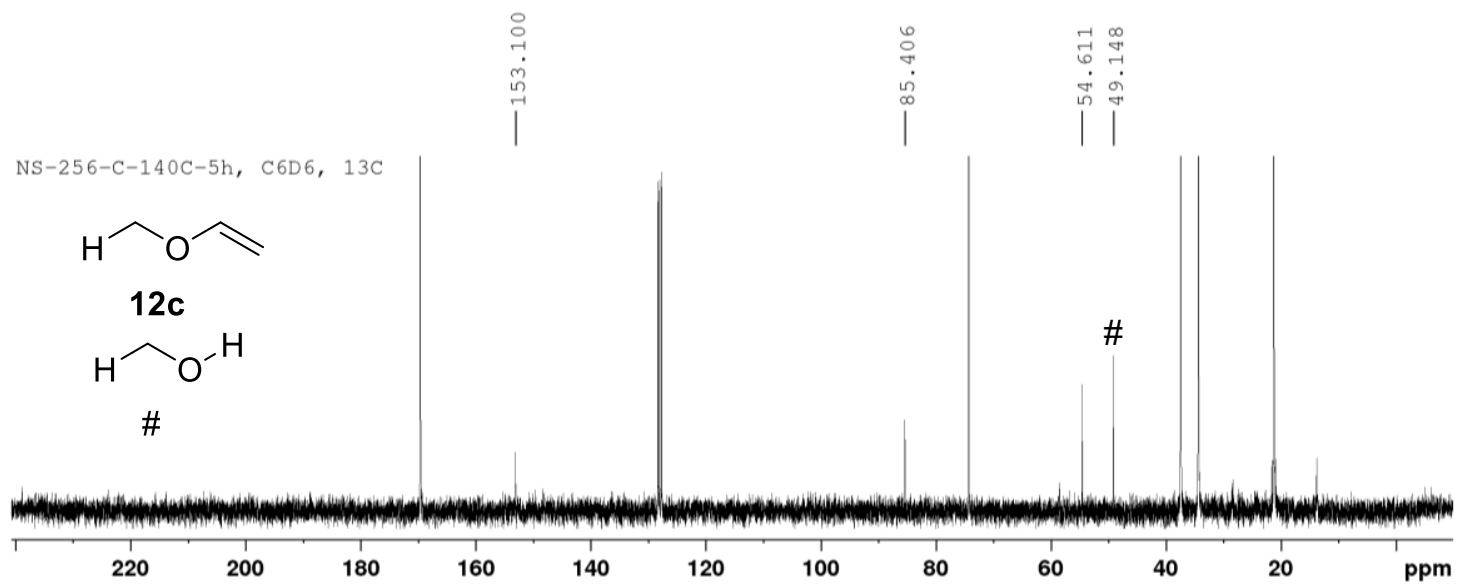


${ }^{1} \mathrm{H}$ NMR (300 MHz, $300 \mathrm{~K}$ ), before reaction (DMAA, $\mathrm{C}_{6} \mathrm{D}_{6}$ as internal std.*)

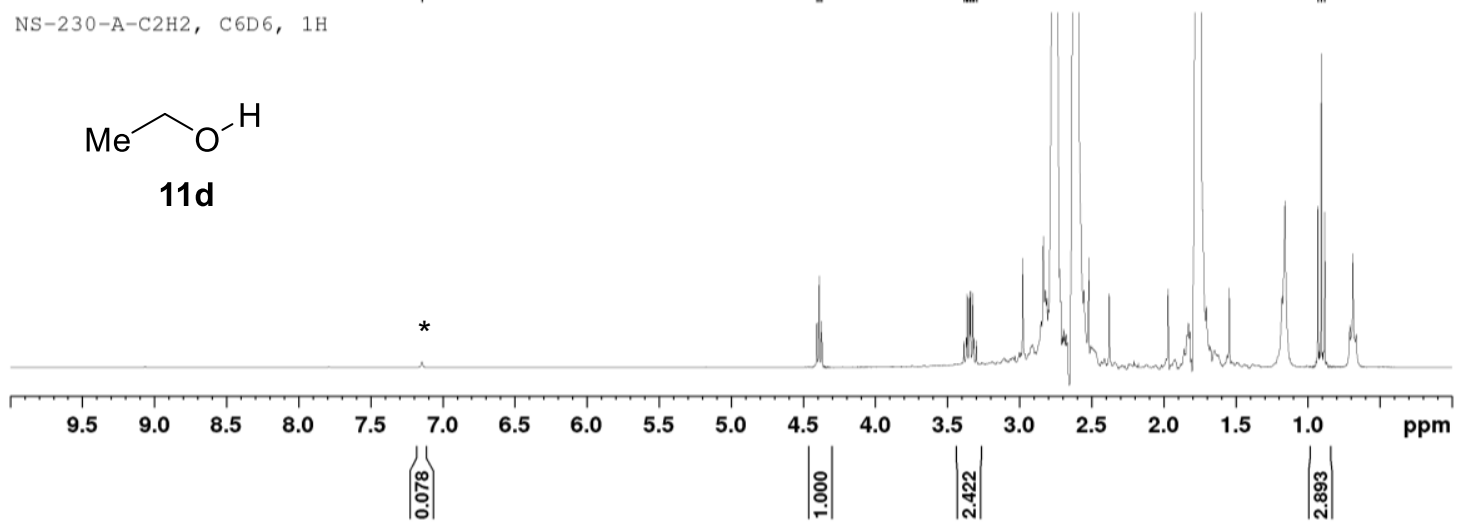

${ }^{13} \mathrm{C}\left\{{ }^{1} \mathrm{H}\right\}$ NMR (75.6 MHz, $300 \mathrm{~K}$ ), before reaction (DMAA, $\mathrm{C}_{6} \mathrm{D}_{6}$ as internal std.)

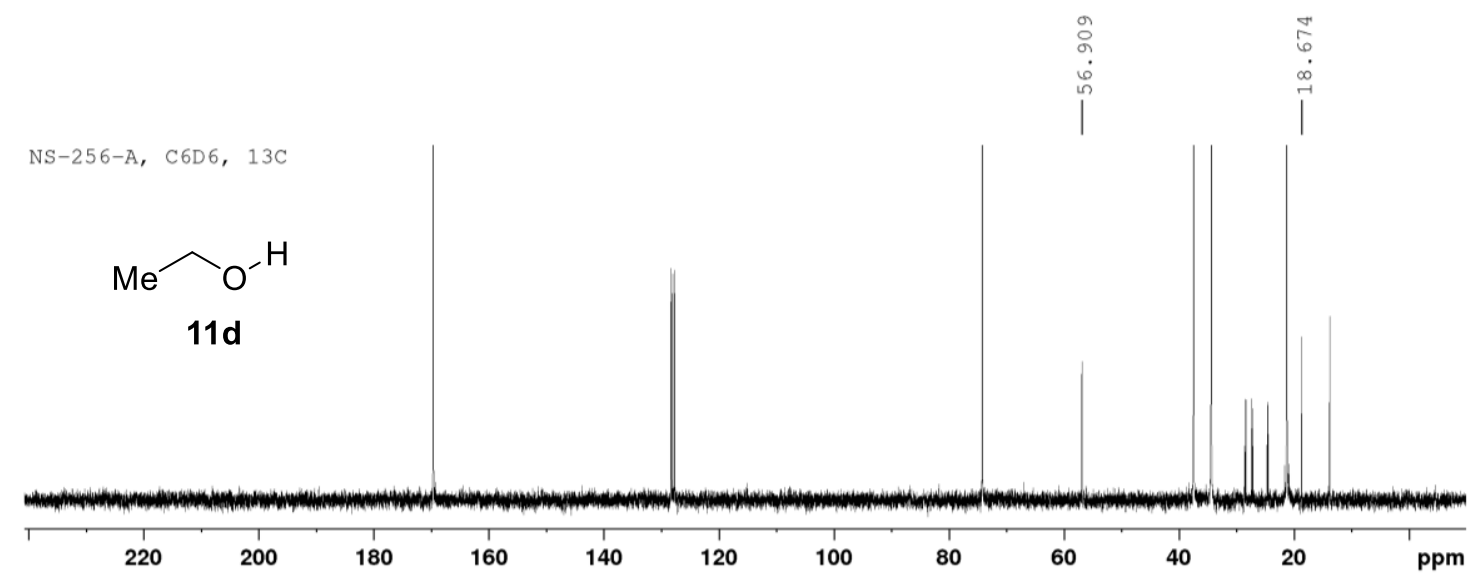


${ }^{1} \mathrm{H}$ NMR (300 MHz, $\left.300 \mathrm{~K}\right)$, after $140{ }^{\circ} \mathrm{C}$ for $5 \mathrm{~h}$ (DMAA, $\mathrm{C}_{6} \mathrm{D}_{6}$ as internal std. $\left.{ }^{*}\right)$

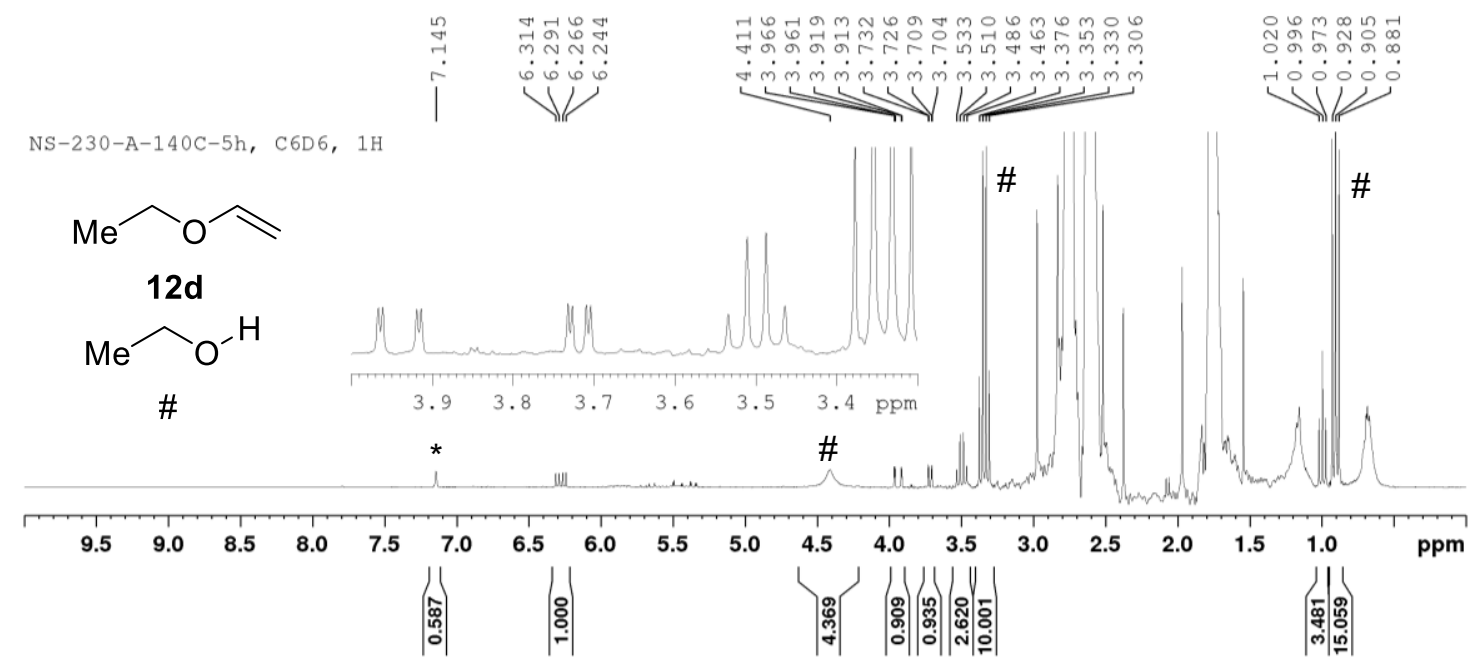

Yield: Integral STD before/Integral STD after $=0.078 / 0.587=13 \%$

${ }^{13} \mathrm{C}\left\{{ }^{1} \mathrm{H}\right\}$ NMR (75.6 MHz, $300 \mathrm{~K}$ ), after $140{ }^{\circ} \mathrm{C}$ for $5 \mathrm{~h}$ (DMAA, $\mathrm{C}_{6} \mathrm{D}_{6}$ as internal std.)

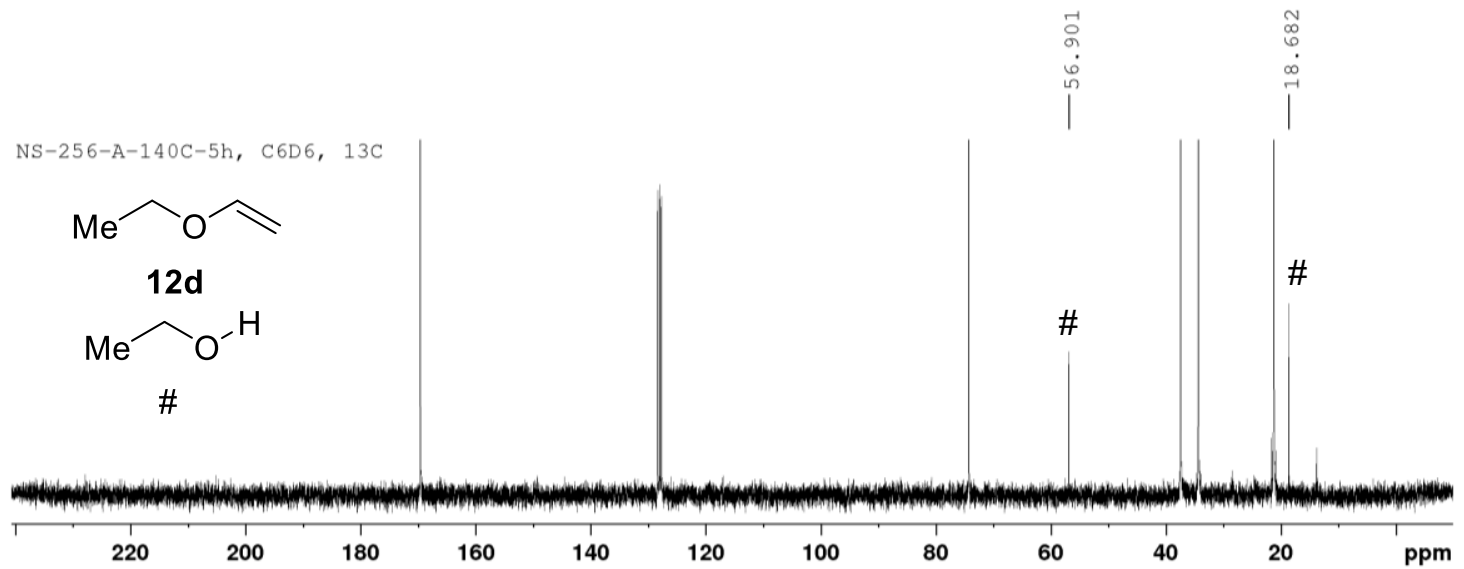

No ${ }^{13} \mathrm{C}$ NMR signals of $12 \mathrm{~d}$ could be observed (probably due to low concentration). 
${ }^{31} \mathrm{P}$ NMR (122 MHz, $300 \mathrm{~K}$ ), before reaction (DMAA, $\mathrm{C}_{6} \mathrm{D}_{6}$ as internal std.)

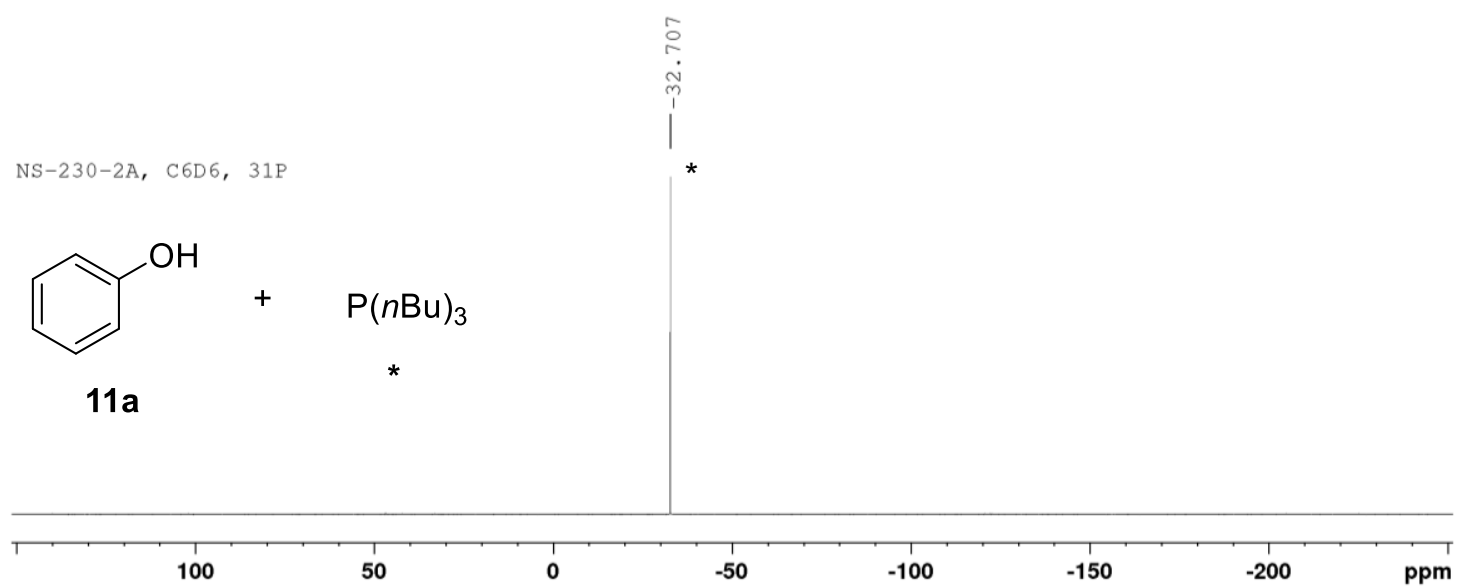

${ }^{31} \mathrm{P}$ NMR (122 MHz, $300 \mathrm{~K}$ ), after $120^{\circ} \mathrm{C}, 2 \mathrm{~h}$ (DMAA, $\mathrm{C}_{6} \mathrm{D}_{6}$ as internal std.)

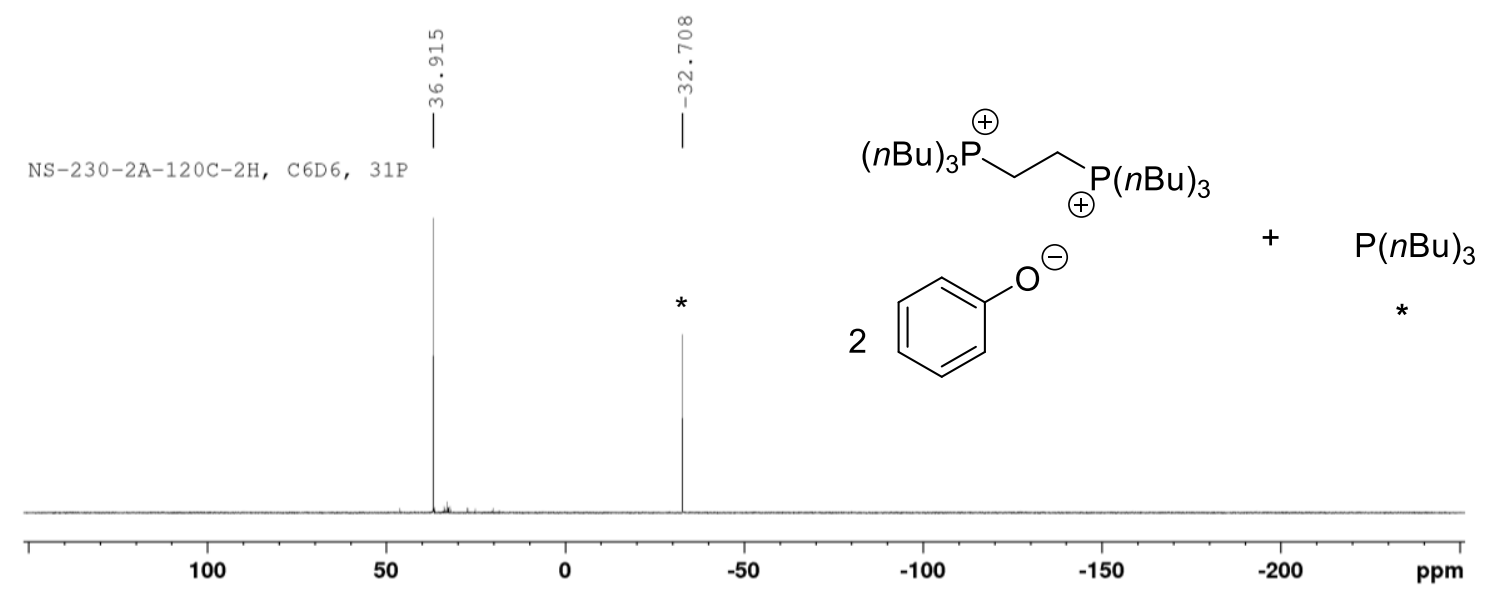




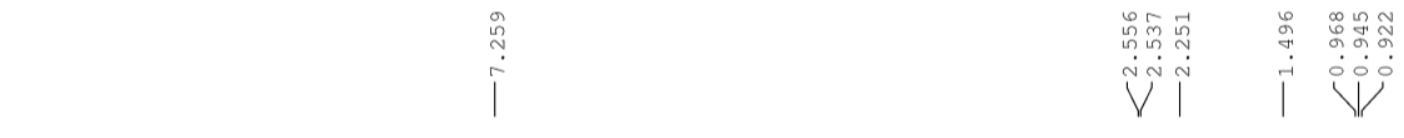

NS-095, $\mathrm{CDCl} 3,1 \mathrm{H}$

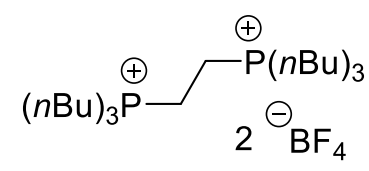

5, ${ }^{1} \mathrm{H}, \mathrm{CDCl}_{3}, 300 \mathrm{MHz}$

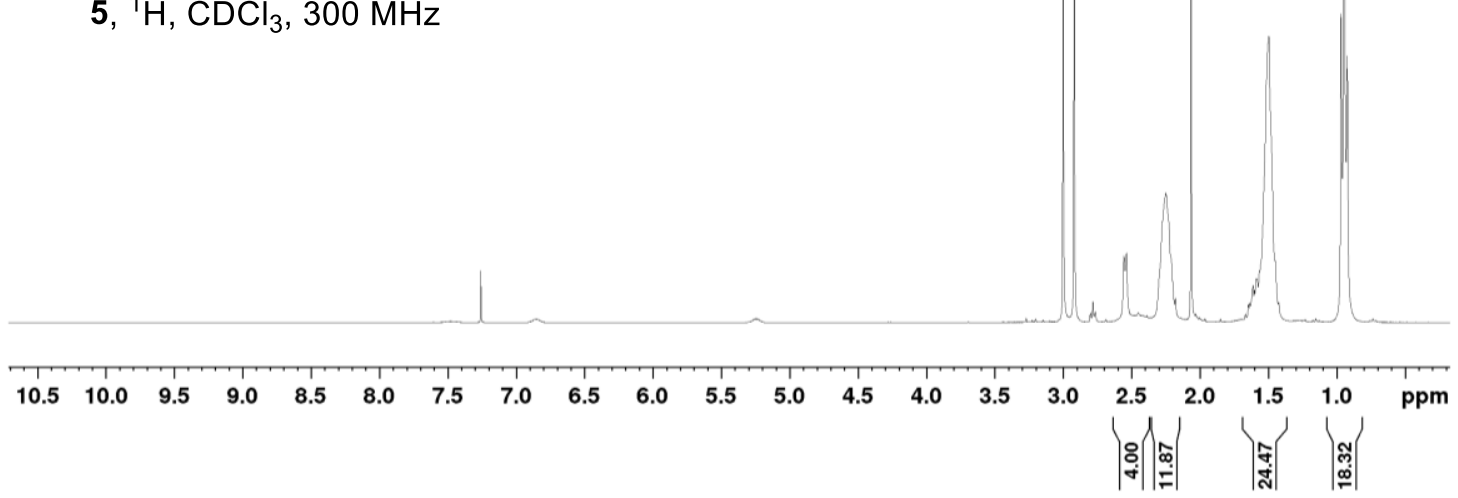

NS-095, CDC13, 31P

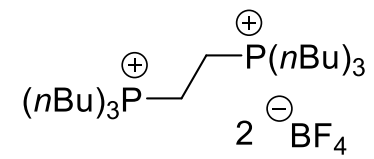

5, ${ }^{31} \mathrm{P}, \mathrm{CDCl}_{3}, 122 \mathrm{MHz}$

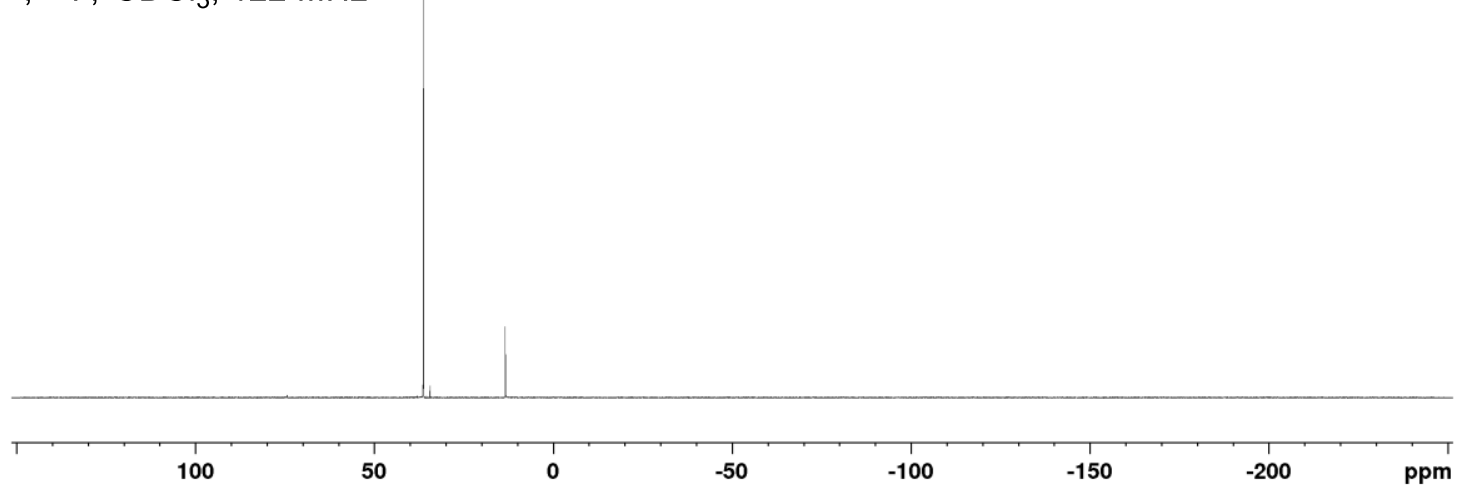




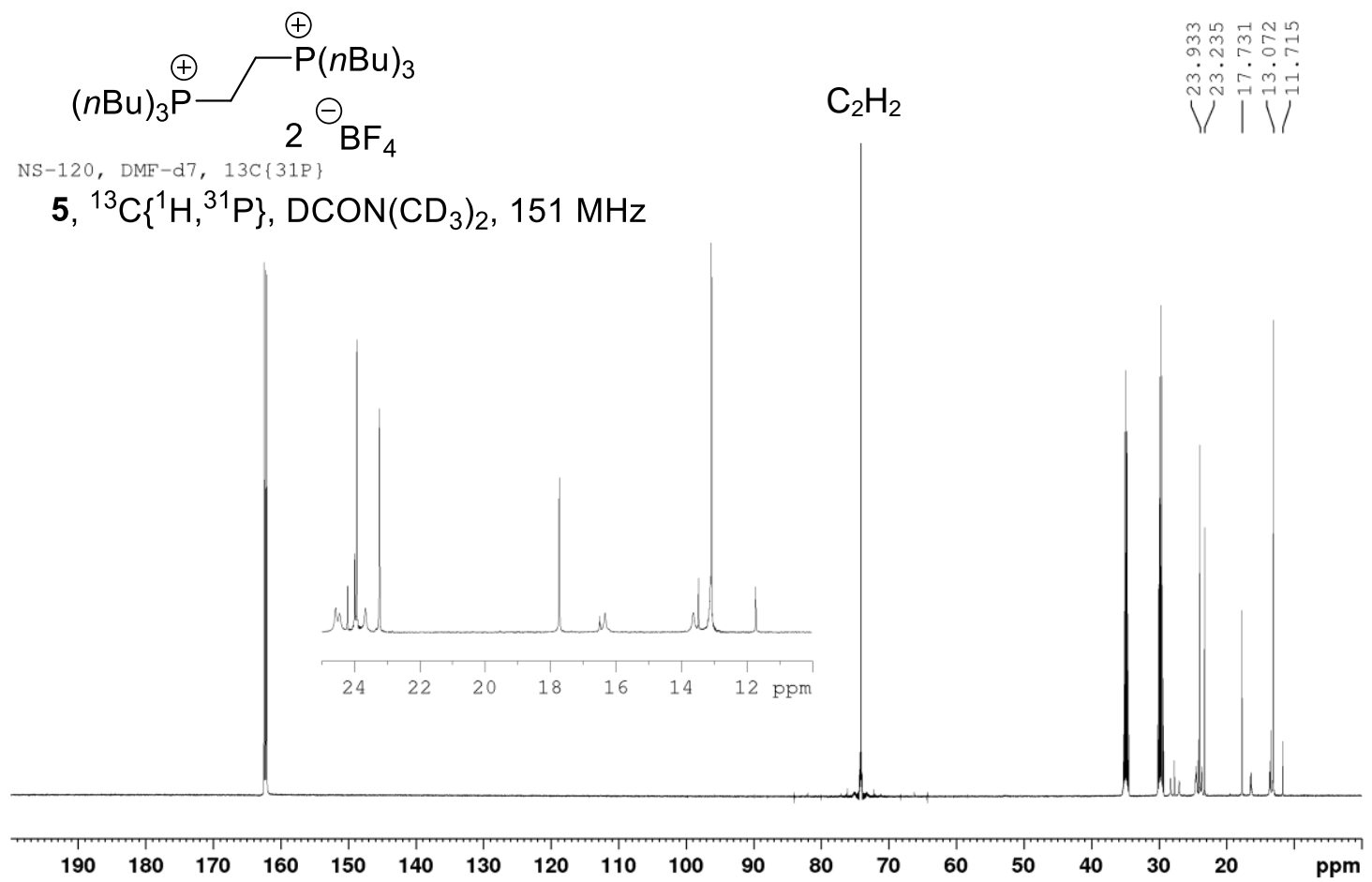

$\mathbf{5},{ }^{13} \mathrm{C}\left\{{ }^{1} \mathrm{H}\right\}, \operatorname{DCON}\left(\mathrm{CD}_{3}\right)_{2}, 151 \mathrm{MHz}$

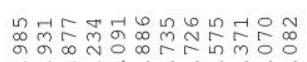

- 0.0.

ก้N N N

NS-120, DMF-d7, $13 \mathrm{C}$

$\mathrm{C}_{2} \mathrm{H}_{2}$

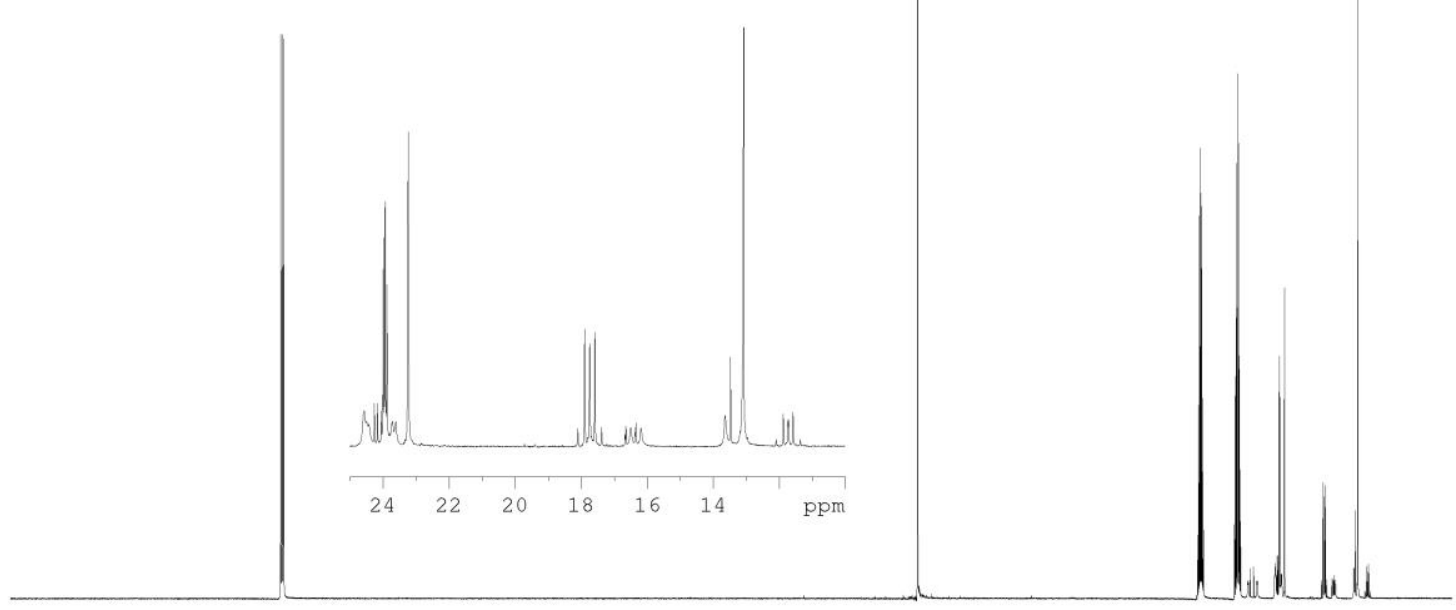

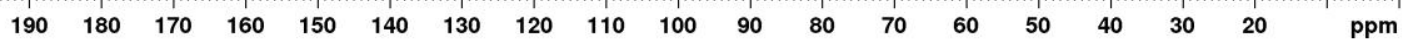




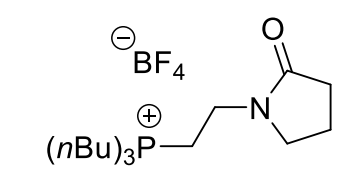

NS $110-B, \operatorname{CDCl} 3,1 \mathrm{H}$

7, ${ }^{1} \mathrm{H}, \mathrm{CDCl}_{3}, 600 \mathrm{MHz}$
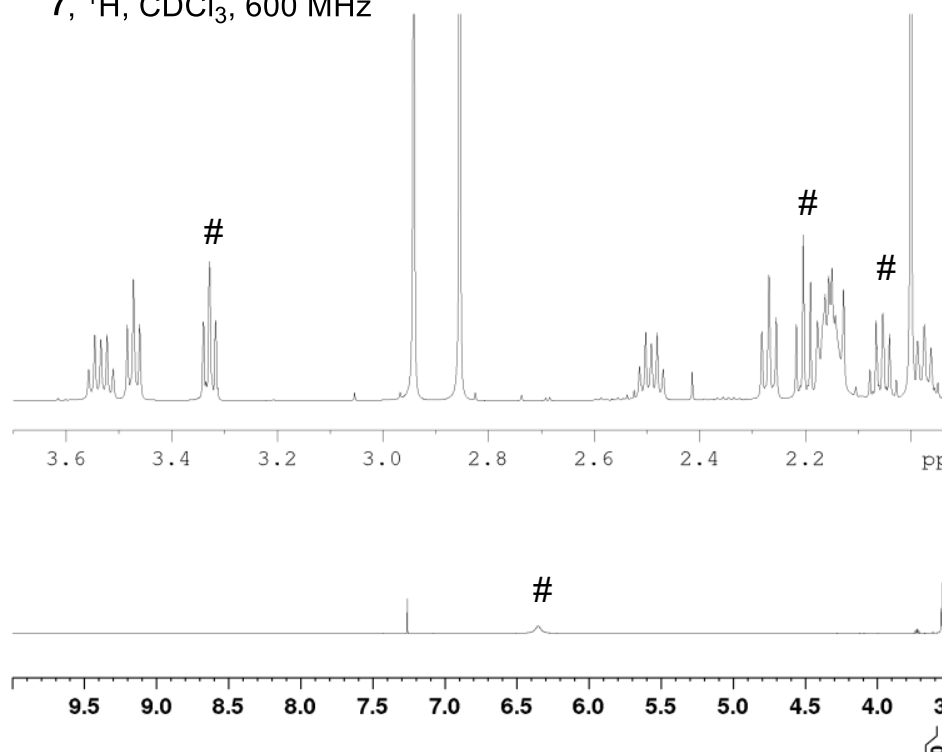

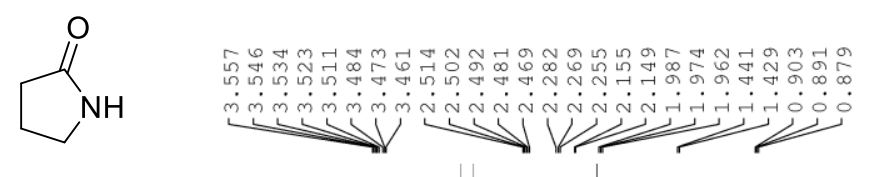

\#

DMAA

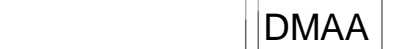

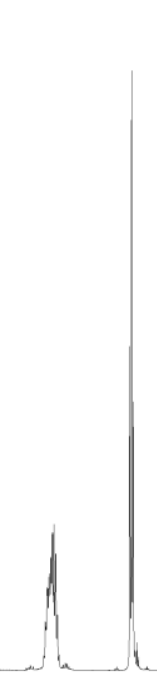
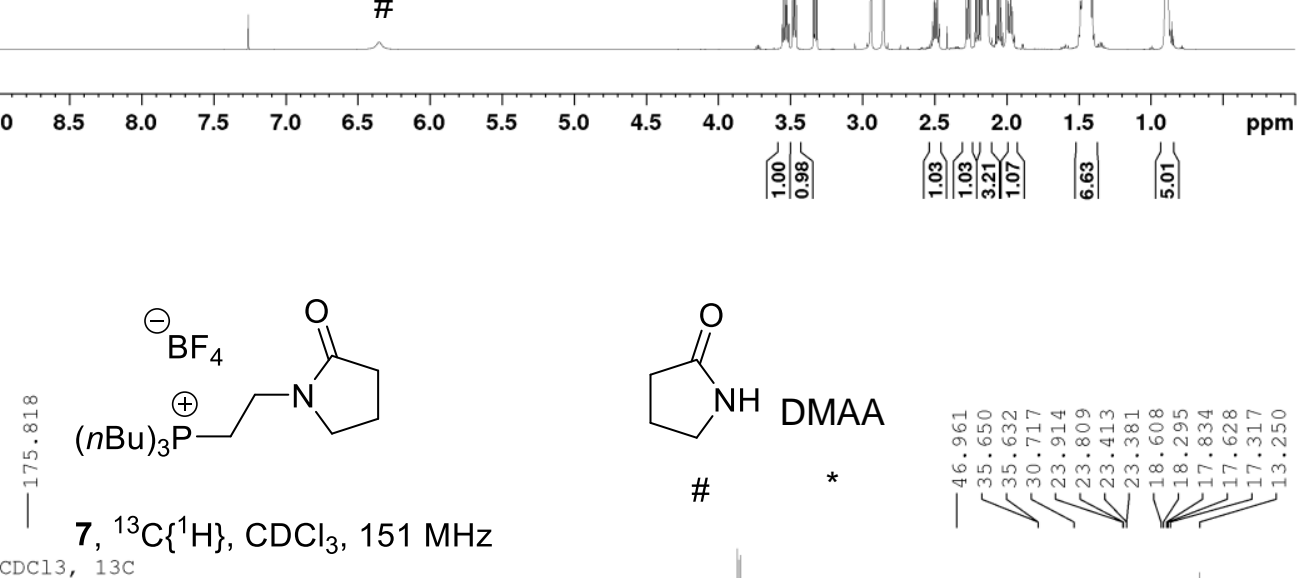
NS $110-B, \operatorname{CDCl} 3,13 \mathrm{C}$
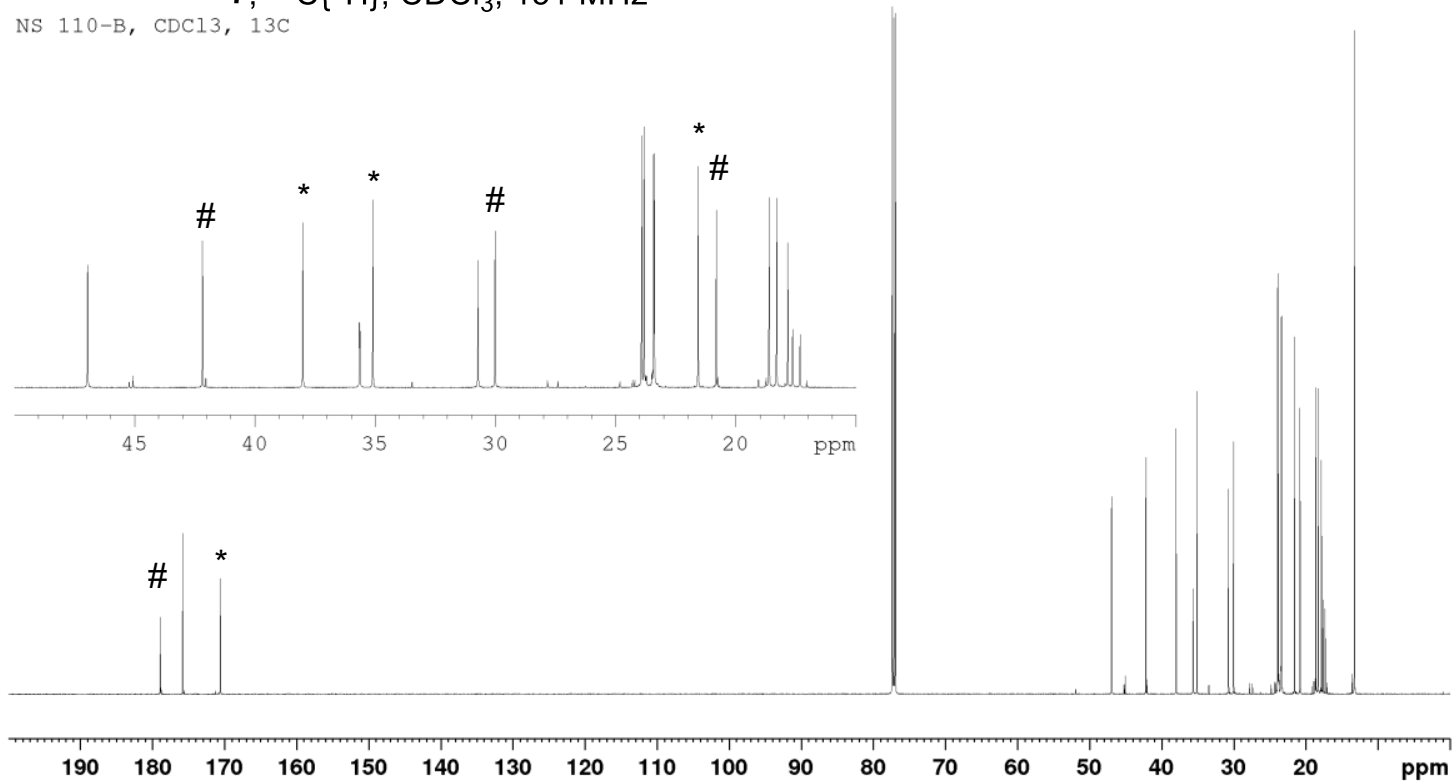
NS $110-\mathrm{B}, \mathrm{CDCl} 3,31 \mathrm{P}$

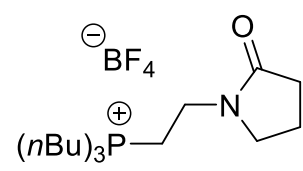

7, ${ }^{31} \mathrm{P}, \mathrm{CDCl}_{3}, 243 \mathrm{MHz}$

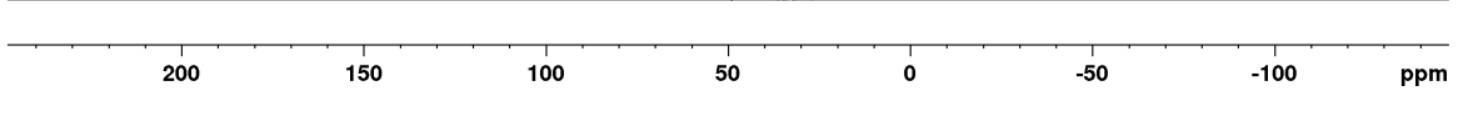


${ }^{1} \mathrm{H}$ NMR (300 MHz, $300 \mathrm{~K}$ ), before reaction (DMAA, $\mathrm{C}_{6} \mathrm{D}_{6}$ as internal std.*)

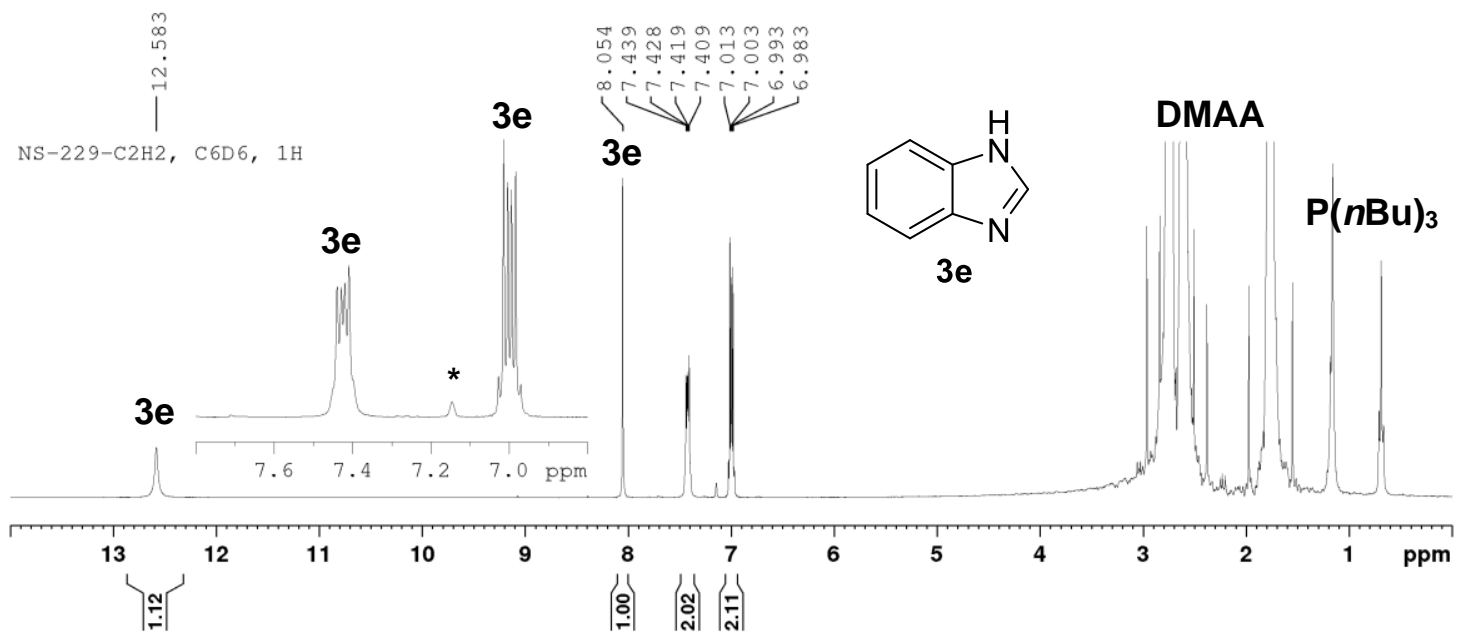

${ }^{1} \mathrm{H}$ NMR (300 MHz, $300 \mathrm{~K}$ ), after $5 \mathrm{~h}$ at $120{ }^{\circ} \mathrm{C}$ (DMAA, $\mathrm{C}_{6} \mathrm{D}_{6}$ as internal std.)

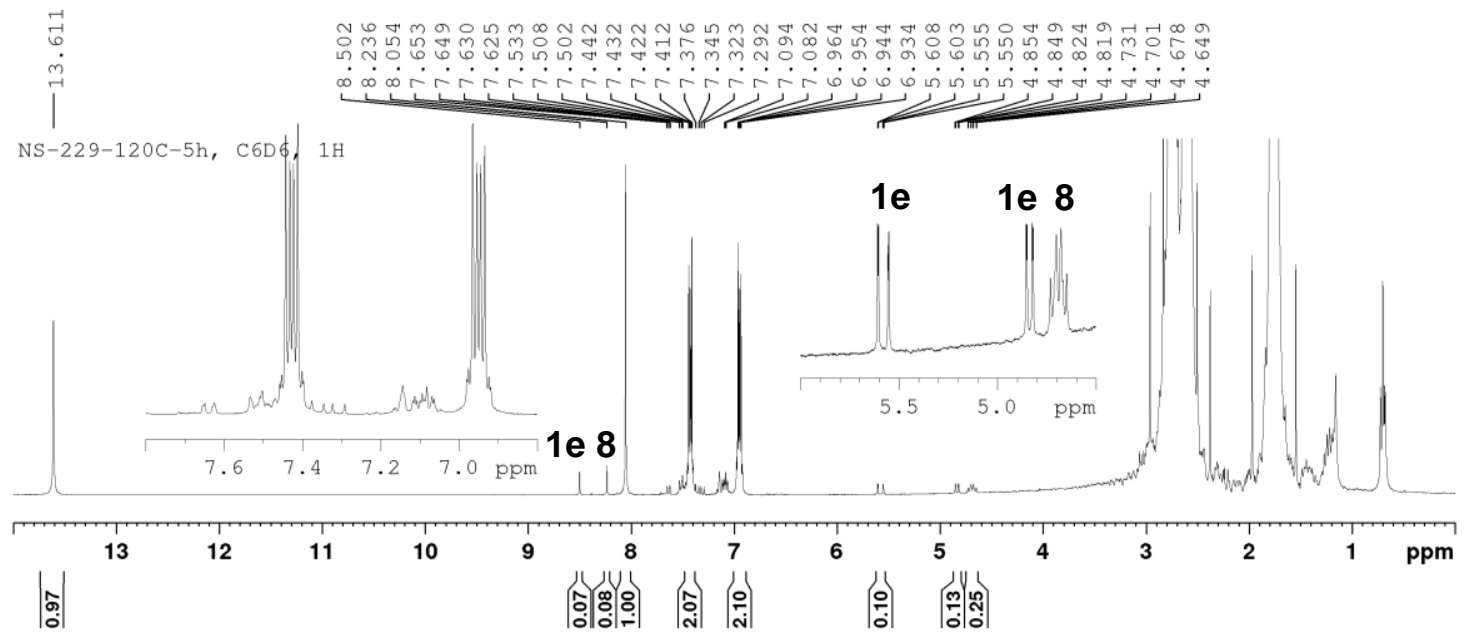

${ }^{1} \mathrm{H}$ NMR (300 MHz, $300 \mathrm{~K}$ ), after $1 \mathrm{~h}$ at $160{ }^{\circ} \mathrm{C}$ (DMAA, $\mathrm{C}_{6} \mathrm{D}_{6}$ as internal std.)

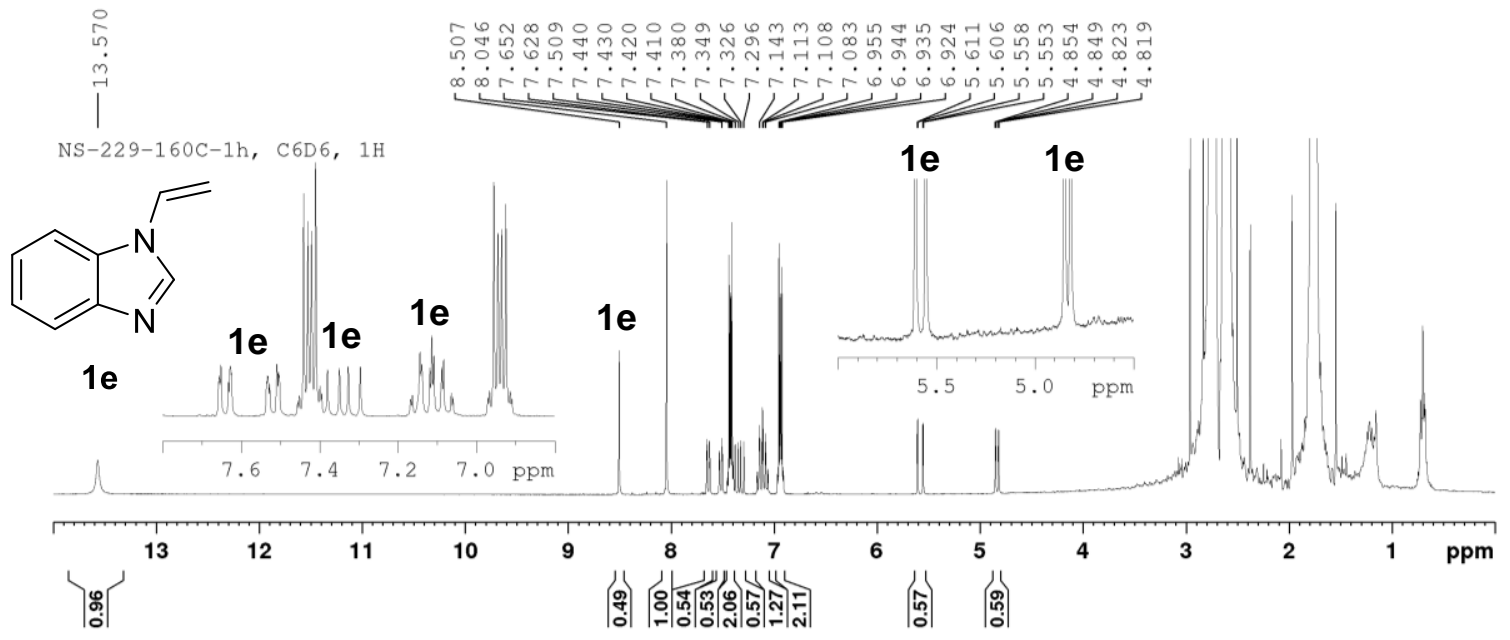


${ }^{31} \mathrm{P}$ NMR (122 MHz, $300 \mathrm{~K}$ ), before reaction (DMAA, $\mathrm{C}_{6} \mathrm{D}_{6}$ as internal std.)

NS-229-C2H2, C6D6, 31P

$\mathrm{P}(n \mathrm{Bu})_{3}$

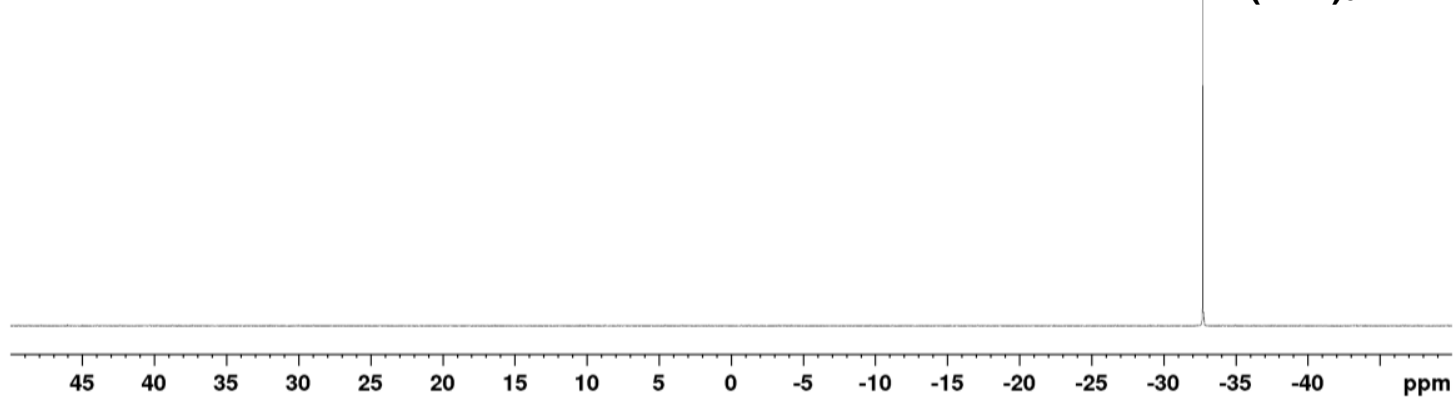

${ }^{31} \mathrm{P}$ NMR (122 MHz, $300 \mathrm{~K}$ ), after $5 \mathrm{~h}$ at $120^{\circ} \mathrm{C}$ (DMAA, $\mathrm{C}_{6} \mathrm{D}_{6}$ as internal std.)

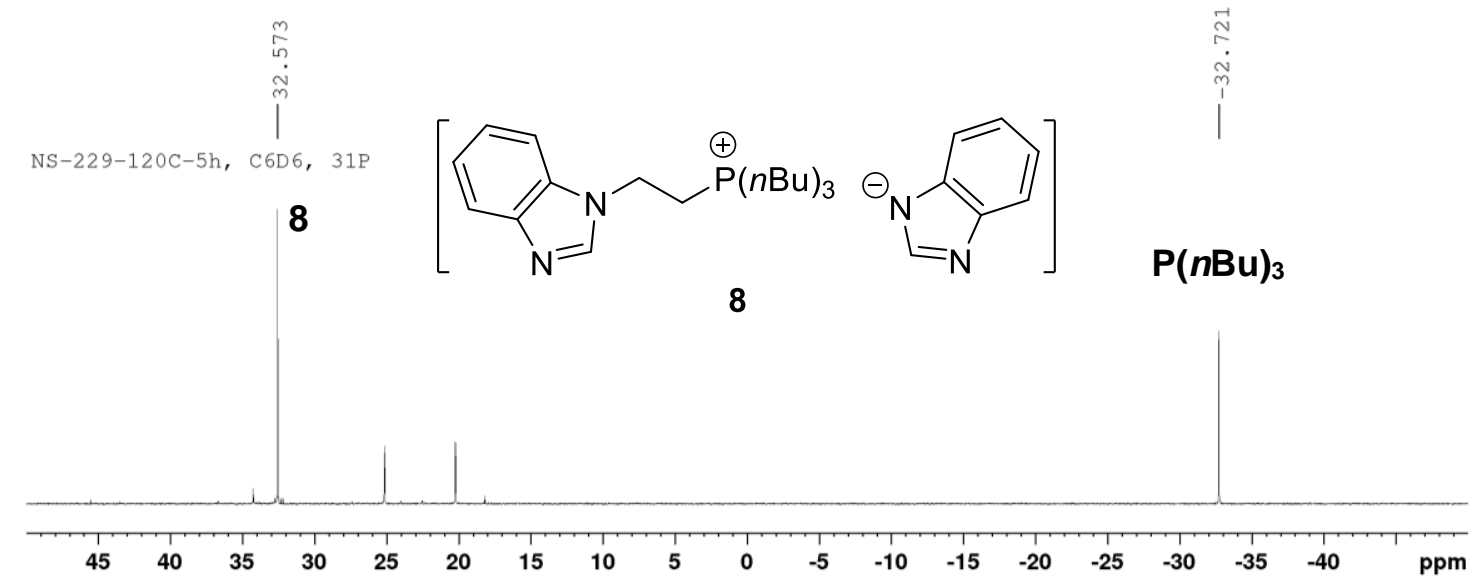

${ }^{31} \mathrm{P}$ NMR (122 MHz, $300 \mathrm{~K}$ ), after $1 \mathrm{~h}$ at $160{ }^{\circ} \mathrm{C}$ (DMAA, $\mathrm{C}_{6} \mathrm{D}_{6}$ as internal std.)
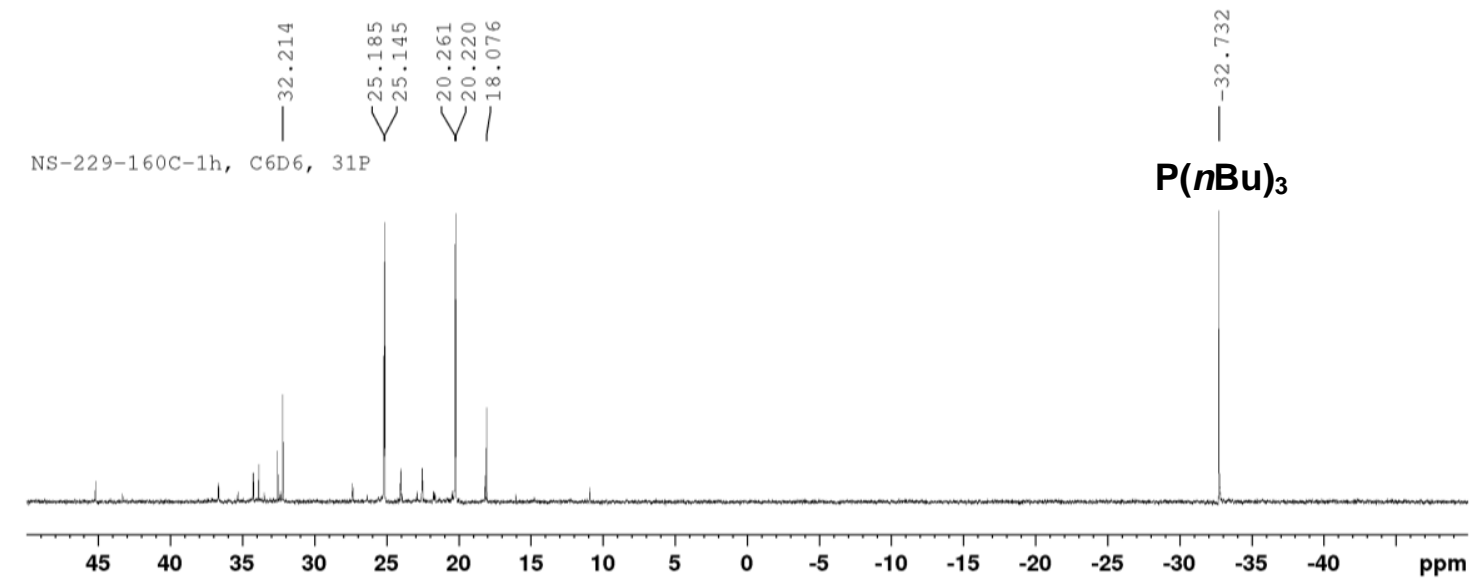


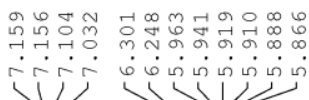

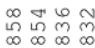

$\dot{m} \dot{m} \dot{m}$

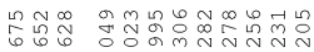

in

vin

ขึن

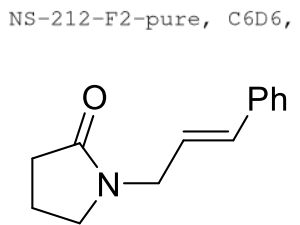

$10,{ }^{1} \mathrm{H}, \mathrm{C}_{6} \mathrm{D}_{6}, 300 \mathrm{MHz}$
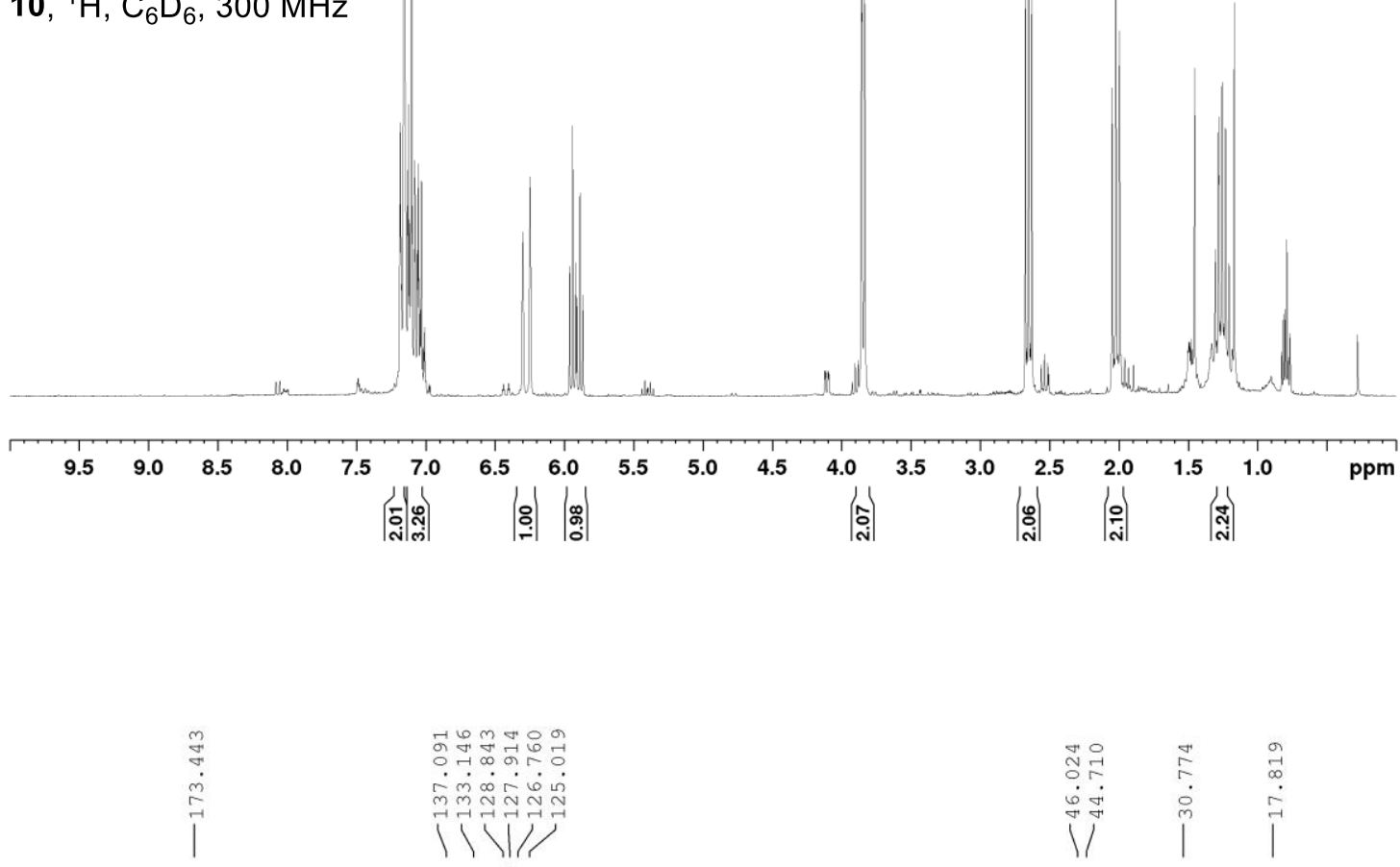

NS-212-F2-pure, C6D6, $13 C$<smiles>O=C1CCCN1C/C=C/c1ccccc1</smiles>

$10,{ }^{13} \mathrm{C}\left\{{ }^{1} \mathrm{H}\right\}, \mathrm{C}_{6} \mathrm{D}_{6}, 75.6 \mathrm{MHz}$
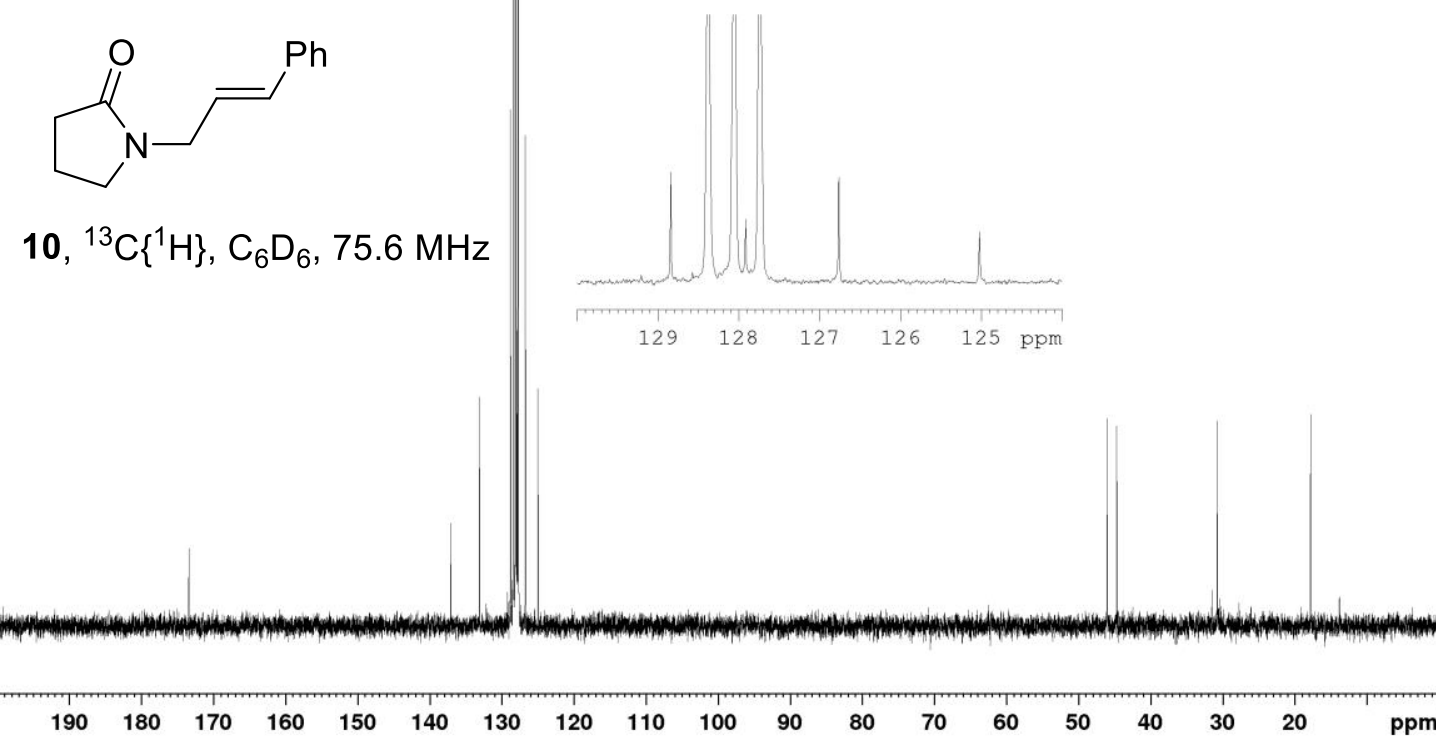

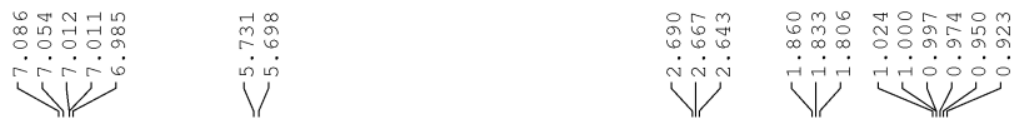

NS-213-F1-pure, C6D6, 1H<smiles>O=C1CCCN1/C=C\c1ccccc1</smiles>

14-cis, ${ }^{1} \mathrm{H}, \mathrm{C}_{6} \mathrm{D}_{6}, 300 \mathrm{MHz}$
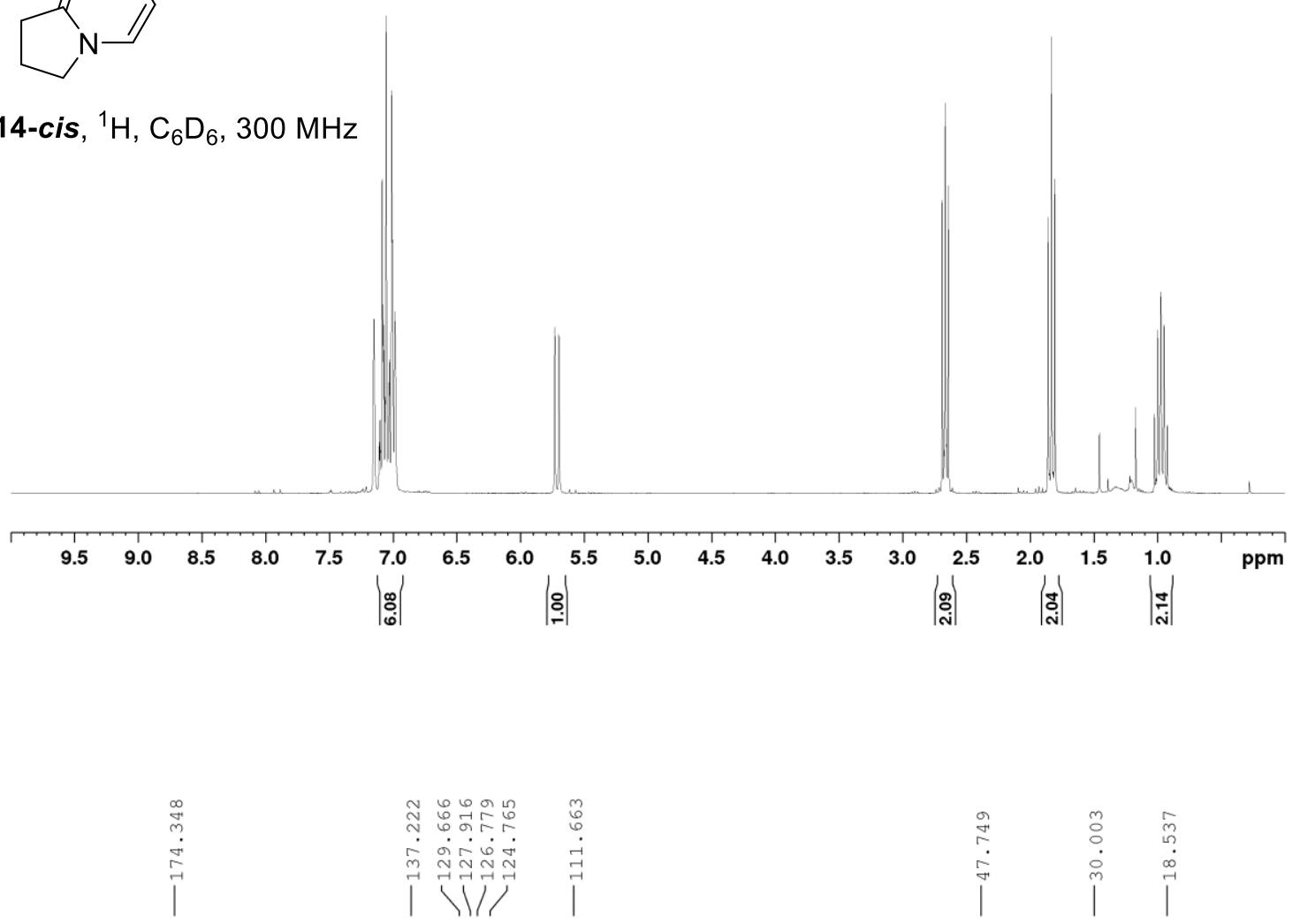

NS-213-F1-pure, C6D6, 13C<smiles>O=C1CCCN1/C=C\c1ccccc1</smiles>

14-cis, ${ }^{13} \mathrm{C}\left\{{ }^{1} \mathrm{H}\right\}, \mathrm{C}_{6} \mathrm{D}_{6}, 75.6 \mathrm{MHz}$
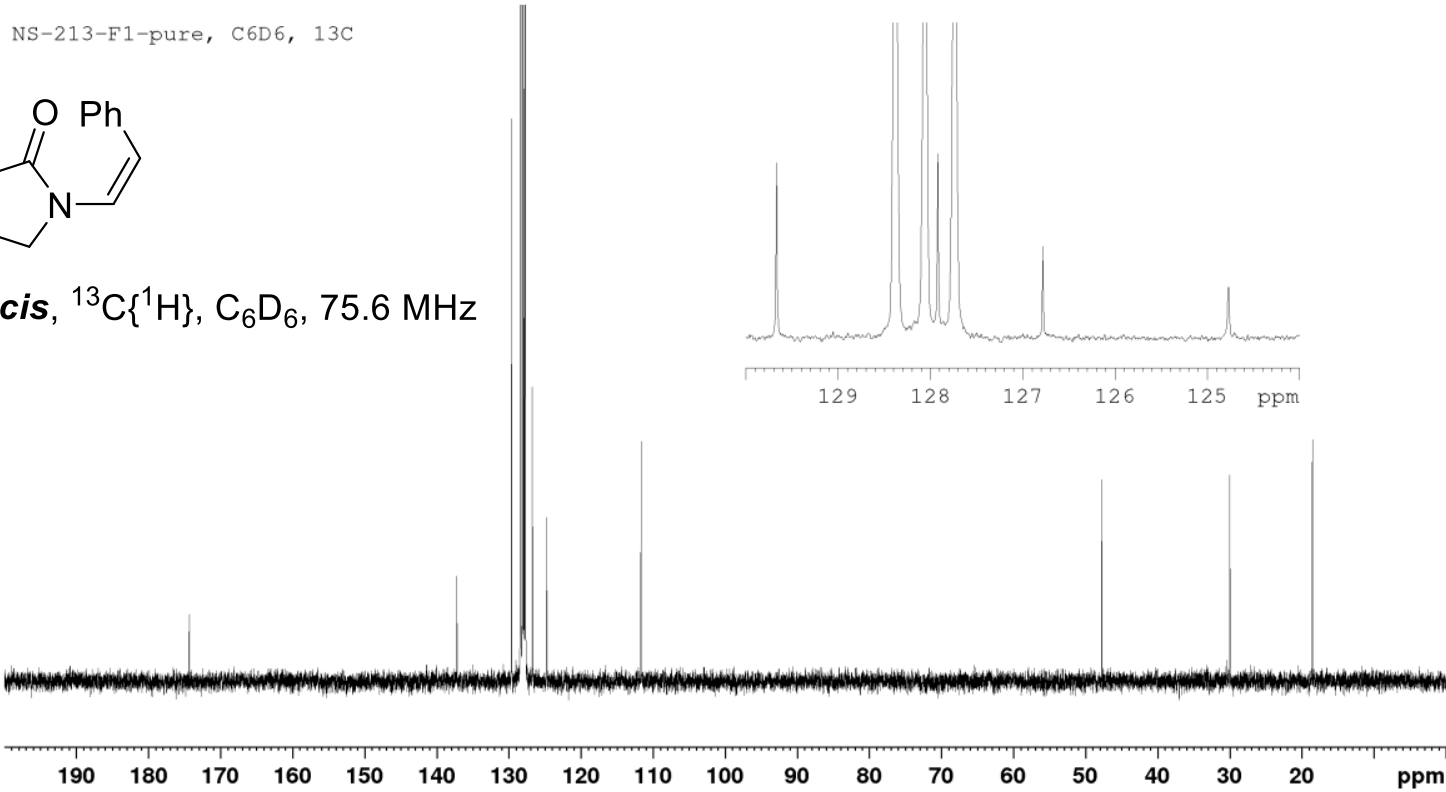

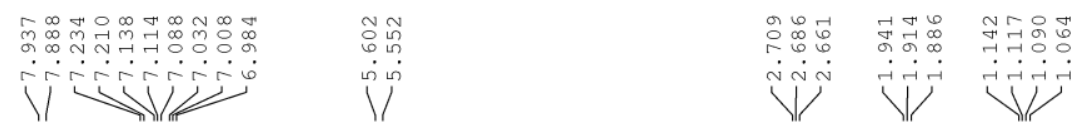

NS-213-F2-pure, C6D6, 1H<smiles>O=C1CCCN1/C=C/c1ccccc1</smiles>

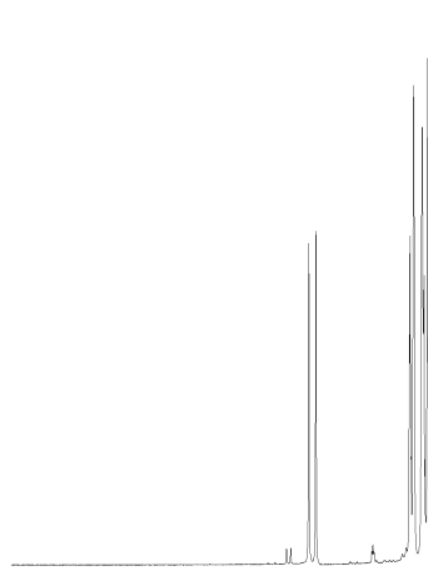

14-trans, ${ }^{1} \mathrm{H}, \mathrm{C}_{6} \mathrm{D}_{6}, 300 \mathrm{MHz}$
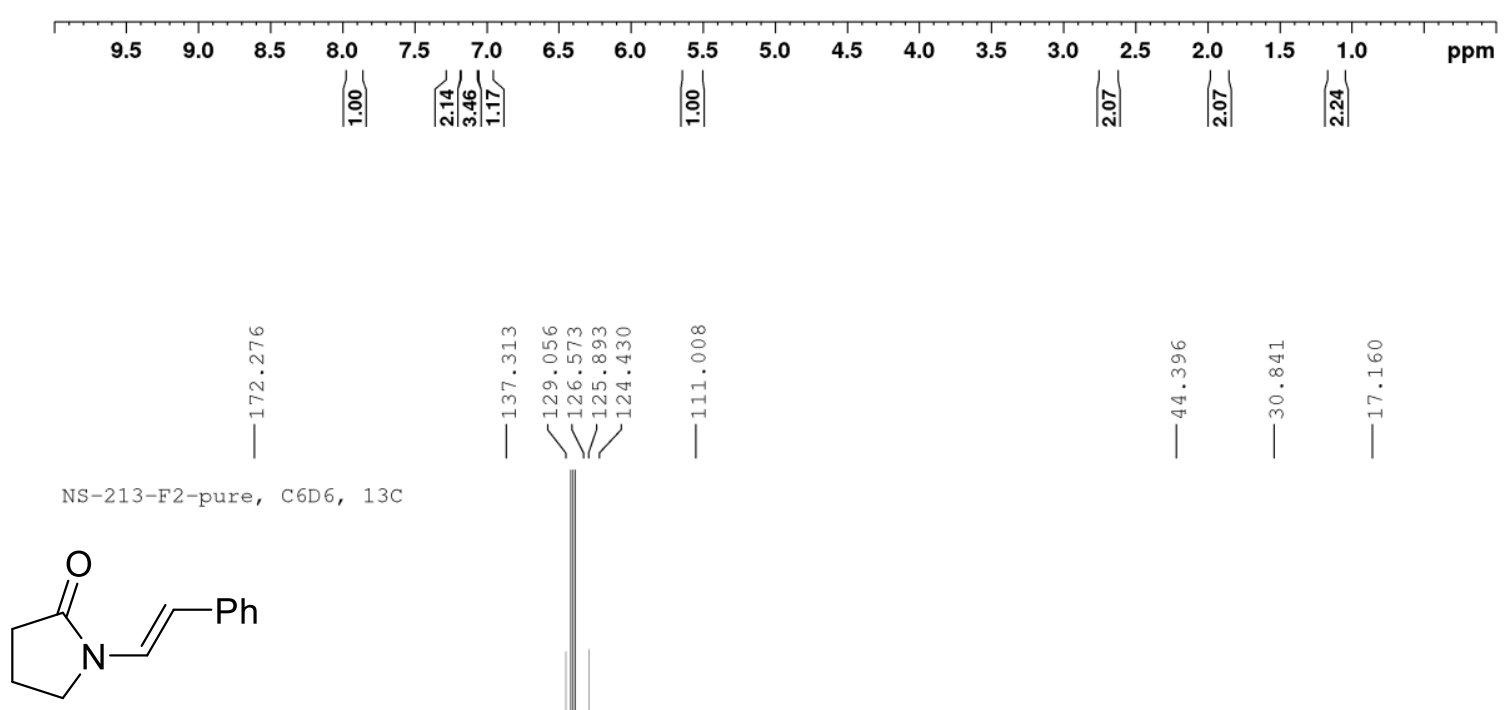

14-trans, ${ }^{13} \mathrm{C}\left\{{ }^{1} \mathrm{H}\right\}, \mathrm{C}_{6} \mathrm{D}_{6}, 75.6 \mathrm{MHz}$
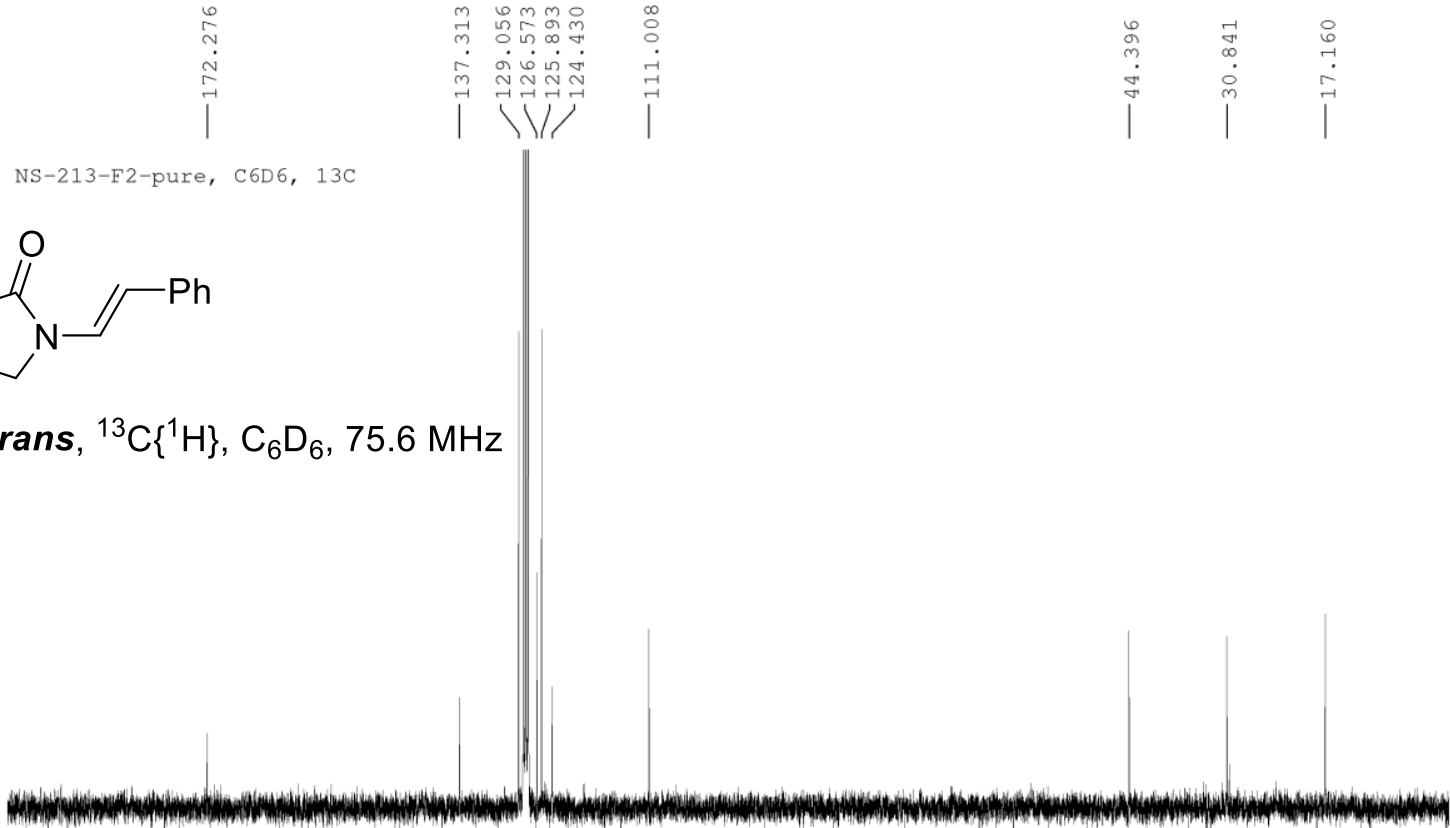

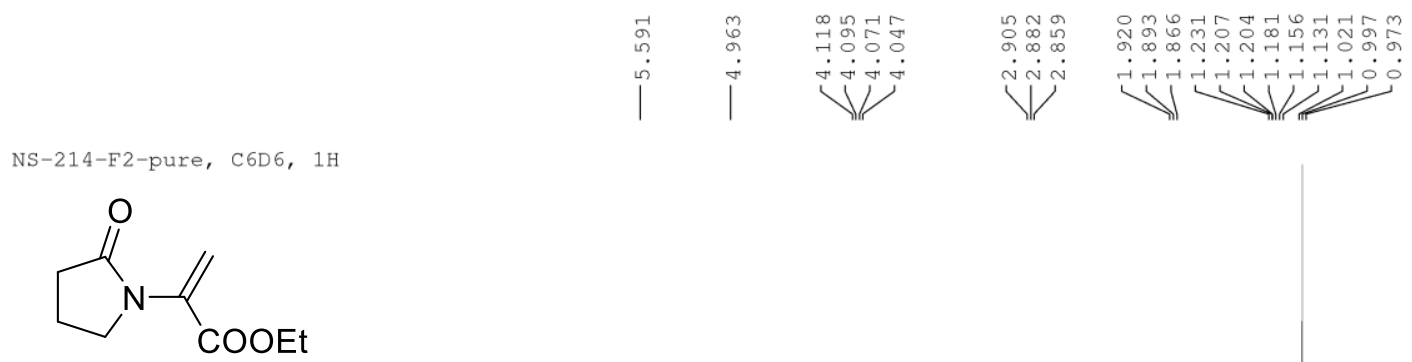

16a, ${ }^{1} \mathrm{H}, \mathrm{C}_{6} \mathrm{D}_{6}, 300 \mathrm{MHz}$
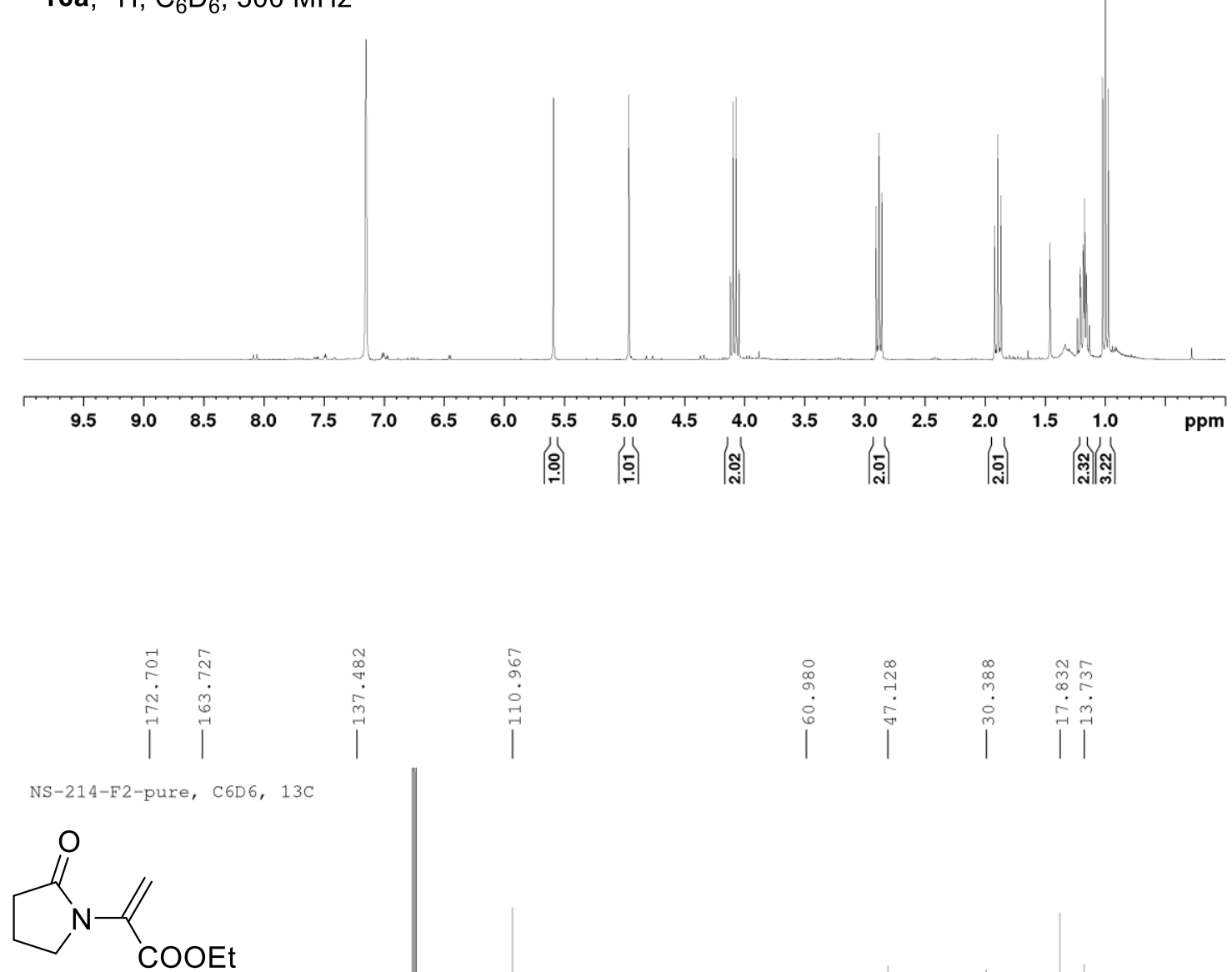

$16 a,{ }^{13} \mathrm{C}\left\{{ }^{1} \mathrm{H}\right\}, \mathrm{C}_{6} \mathrm{D}_{6}, 75.6 \mathrm{MHz}$

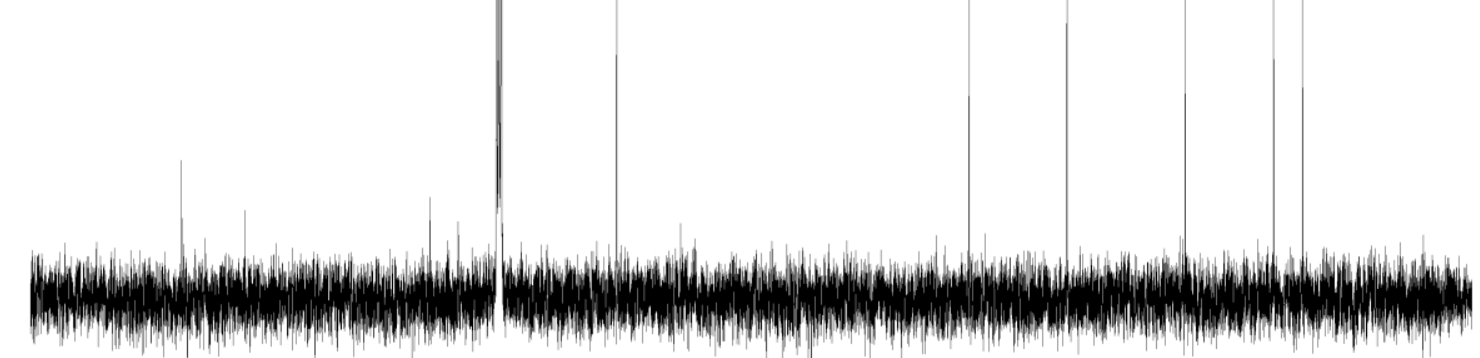

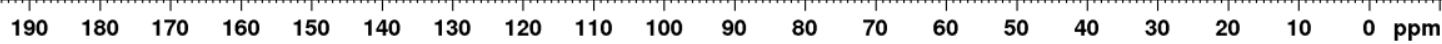




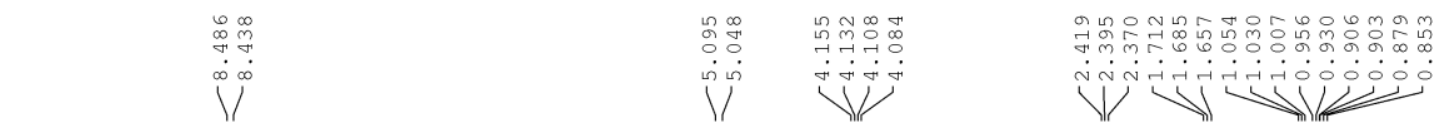

NS-214-F1-pure, C6D6, 1H<smiles>CCOC(=O)C=CN1CCCC1=O</smiles>

16b, ${ }^{1} \mathrm{H}, \mathrm{C}_{6} \mathrm{D}_{6}, 300 \mathrm{MHz}$

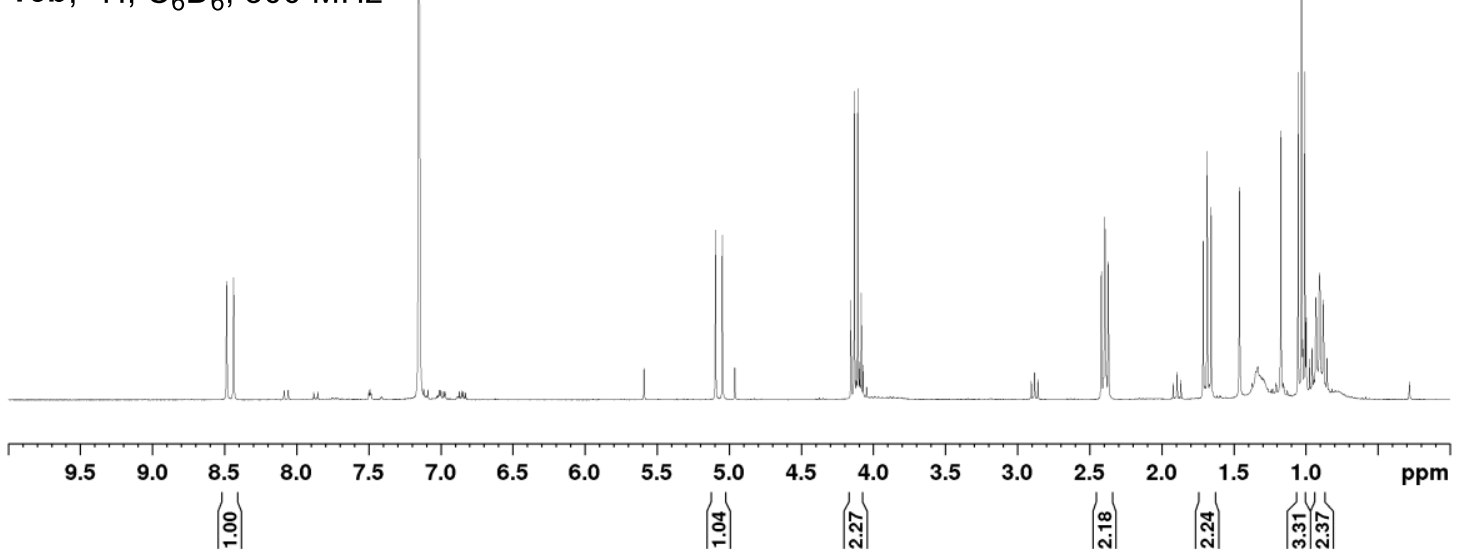

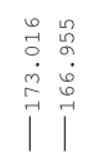
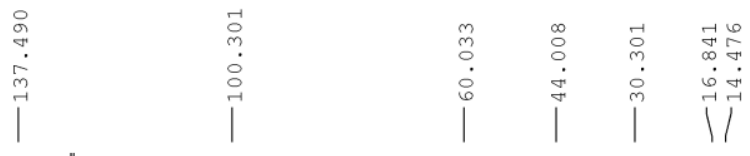

NS-214-F1-pure, C6D6, 13C<smiles>CCOC(=O)C=CN1CCCC1=O</smiles>

16b, ${ }^{13} \mathrm{C}\left\{{ }^{1} \mathrm{H}\right\}, \mathrm{C}_{6} \mathrm{D}_{6}, 75.6 \mathrm{MHz}$

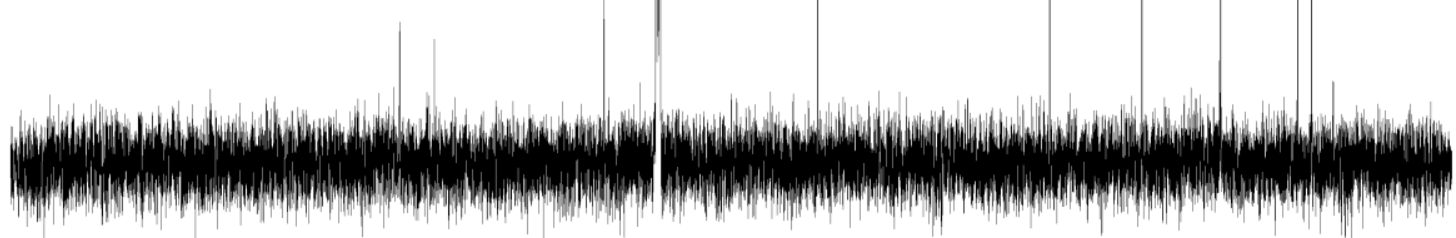

\begin{tabular}{|c|c|c|c|c|c|c|c|c|c|c|}
\hline $\begin{array}{c}1 \\
220\end{array}$ & 200 & $\begin{array}{c}1 \\
180\end{array}$ & $\begin{array}{c}1 \\
160\end{array}$ & $\begin{array}{c}1 \\
140\end{array}$ & $\begin{array}{c}1 \\
120\end{array}$ & $\begin{array}{c}1 \\
100\end{array}$ & 80 & 60 & 40 & ppm \\
\hline
\end{tabular}


<smiles>CCOC(=O)C=Cn1ccnc1</smiles>

$17,{ }^{1} \mathrm{H}, \mathrm{C}_{6} \mathrm{D}_{6}, 400 \mathrm{MHz}$
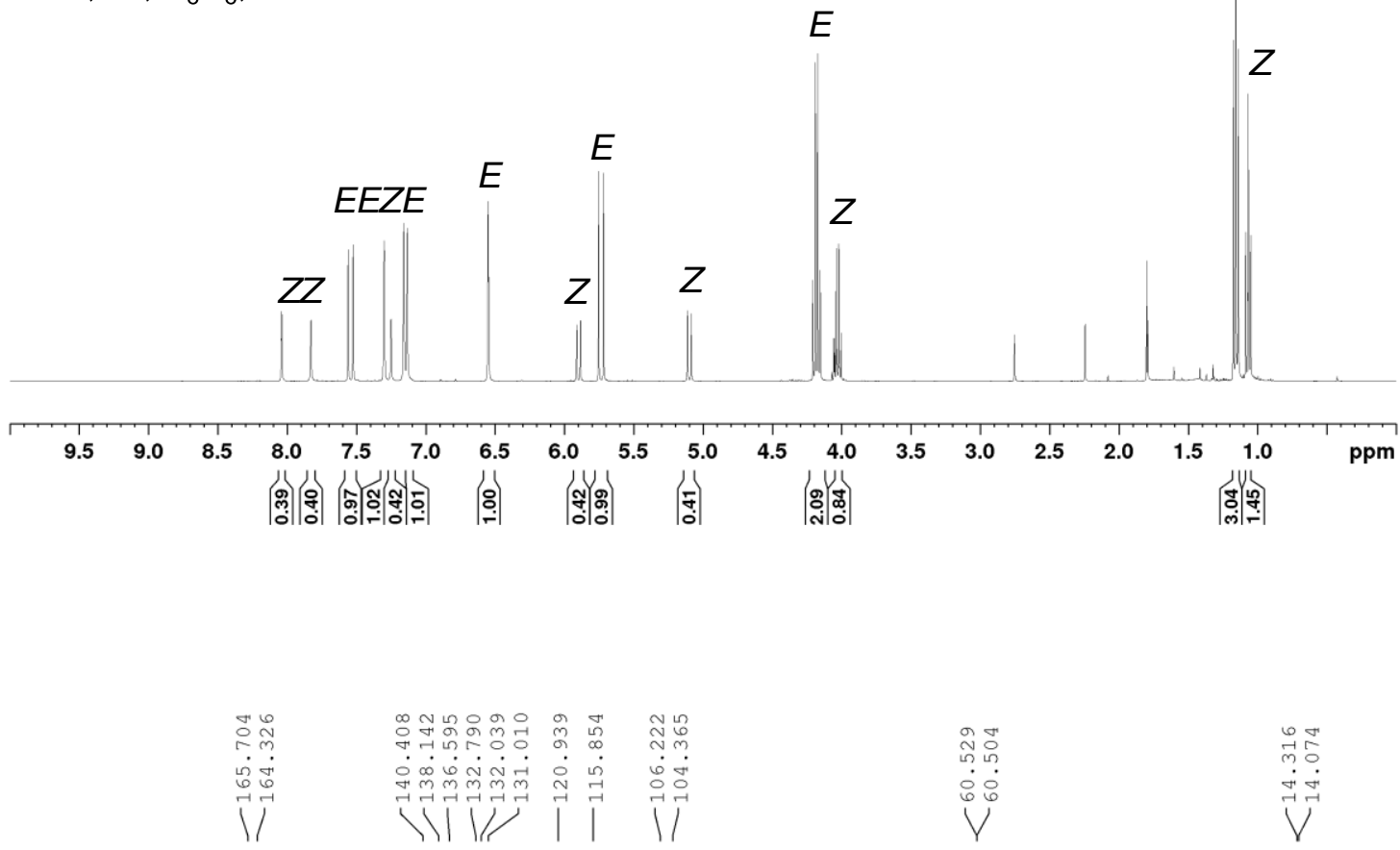

NS-195, C6D 6, 13C<smiles>CCOC(=O)C=Cn1ccnc1</smiles>

17, ${ }^{13} \mathrm{C}\left\{{ }^{1} \mathrm{H}\right\}, \mathrm{C}_{6} \mathrm{D}_{6}, 101 \mathrm{MHz}$

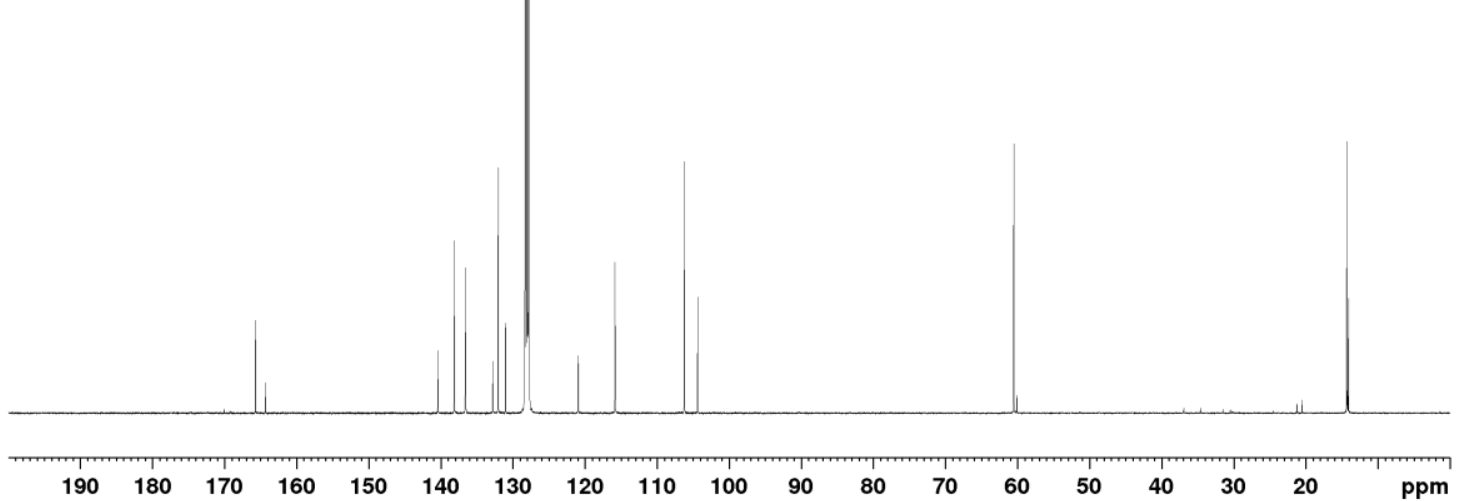




\section{Table with Computed Energies}

\begin{tabular}{|c|c|c|c|c|c|}
\hline Structure & $\begin{array}{l}\text { Number of } \\
\text { Imaginary } \\
\text { Modes }\end{array}$ & $\begin{array}{l}E(\text { Opt) } \\
\text { BP86/ } \\
\text { def2-SV(P) } \\
\text { [Hartree] }\end{array}$ & $\begin{array}{l}\text { E(SP) } \\
\text { PBE0-D3(BJ)/ } \\
\text { def2-QZVPP } \\
\text { [Hartree] }\end{array}$ & $\begin{array}{l}\Delta G(\text { therm }) \\
{\left[\mathrm{kJ} \mathrm{mol}^{-1}\right]}\end{array}$ & $\begin{array}{l}\Delta G(\text { solv }) \\
{\left[\mathrm{kJ} \mathrm{mol}^{-1}\right]}\end{array}$ \\
\hline A & 0 & -814.43463 & -814.43069 & 730.7 & $\begin{array}{c}-11.6 \\
\end{array}$ \\
\hline A_PMe3 & 0 & -460.92297 & -460.89770 & 155.2 & 8.5 \\
\hline A_PPh3 & 0 & -1035.73249 & -1035.67485 & 493.6 & -31.6 \\
\hline Acetylene & 0 & -77.26481 & -77.26103 & -22.9 & 18.2 \\
\hline B_cis & 0 & -891.67466 & -891.67927 & 783.6 & -39.3 \\
\hline B_cis-PMe3 & 0 & -538.15761 & -538.14020 & 211.9 & -25.7 \\
\hline B_cis-PPh3 & 0 & -1112.96751 & -1112.91482 & 559.2 & -51.6 \\
\hline B_trans & 0 & -891.68841 & -891.69307 & 785.6 & -31.4 \\
\hline B_trans-PMe3 & 0 & -538.16658 & -538.15036 & 215.5 & -16.0 \\
\hline B_trans-PPh3 & 0 & -1112.97003 & -1112.92017 & 558.5 & -47.4 \\
\hline Benzaldehyde & 0 & -345.32797 & -345.32200 & 145.6 & -4.0 \\
\hline C & 0 & -1178.16047 & -1178.18211 & 1036.3 & -43.5 \\
\hline C_BF4 & 0 & -1316.51073 & -1316.64475 & 830.7 & -48.9 \\
\hline C_Phenol & 0 & -1198.99776 & -1199.00427 & 1003.8 & -54.7 \\
\hline C_PMe3 & 0 & -824.63789 & -824.63568 & 458.0 & -33.3 \\
\hline C_PPh3 & 1 & -1399.42288 & -1399.39472 & 798.7 & -64.4 \\
\hline D & 0 & -1178.18626 & -1178.21127 & 1047.9 & -34.1 \\
\hline D_PMe3 & 0 & -824.67418 & -824.67726 & 477.7 & -15.9 \\
\hline D_PPh3 & 0 & -1399.47248 & -1399.44539 & 811.8 & -54.2 \\
\hline $\mathrm{E}$ & 0 & -1464.62955 & -1464.67065 & 1288.5 & -54.4 \\
\hline E_Phenol & 0 & -1485.46705 & -1485.49303 & 1259.7 & -64.6 \\
\hline E_PMe3 & 0 & -1111.11214 & -1111.12939 & 719.9 & -43.4 \\
\hline E_PPh3 & 0 & -1685.90285 & -1685.89385 & 1060.0 & -78.7 \\
\hline$F$ & 0 & -1178.14875 & -1178.16868 & 1038.1 & -39.7 \\
\hline F_PMe3 & 0 & -824.63084 & -824.62844 & 466.8 & -22.2 \\
\hline F_PPh3 & 0 & -1399.43682 & -1399.40008 & 816.5 & -51.5 \\
\hline G_cis & 0 & -1523.52392 & -1523.55742 & 1290.5 & -61.9 \\
\hline H_cis & 0 & -1523.53894 & -1523.57297 & 1301.3 & -51.1 \\
\hline H_trans & 0 & -1523.54078 & -1523.57277 & 1305.1 & -50.5 \\
\hline I_cis & 0 & -633.93302 & -633.93350 & 461.8 & -25.1 \\
\hline I_trans & 0 & -633.93957 & -633.93841 & 459.7 & -25.5 \\
\hline$\overline{N V P}$ & 0 & -363.77040 & -363.77830 & 226.4 & -6.8 \\
\hline OPBu3 & 0 & -889.63760 & -889.66908 & 737.1 & -20.7 \\
\hline Phenol & 0 & -307.24439 & -307.25379 & 138.7 & -1.5 \\
\hline Pyrrolidone & 0 & -286.42510 & -286.44014 & 155.4 & -7.2 \\
\hline S1 & 0 & -1178.15412 & -1178.17736 & 1042.3 & -40.8 \\
\hline S10 & 0 & -1255.42598 & -1255.44812 & 1111.0 & -52.6 \\
\hline S11 & 0 & -1255.51307 & -1255.53175 & 1119.6 & -43.2 \\
\hline S12 & 0 & -968.99936 & -969.00271 & 859.7 & -52.4 \\
\hline S13 & 0 & -1046.34297 & -1046.33947 & 928.5 & -76.7 \\
\hline S14 & 0 & -969.05494 & -969.06121 & 874.5 & -14.7 \\
\hline S15 & 0 & -1046.42341 & -1046.43013 & 943.4 & -32.8 \\
\hline S16_cis-C2H3 & 0 & -1541.95425 & -1542.00233 & 1384.2 & -52.7 \\
\hline S16_cis-H & 0 & -1464.60283 & -1464.65749 & 1315.4 & -45.6 \\
\hline
\end{tabular}




\begin{tabular}{|c|c|c|c|c|c|}
\hline S16_trans-C2H3 & 0 & -1541.95191 & -1542.00062 & 1388.0 & -48.2 \\
\hline S16_trans-H & 0 & -1464.60585 & -1464.66001 & 1315.6 & -45.6 \\
\hline S17_cis-C2H3 & 0 & -652.34538 & -652.35837 & 533.3 & -28.1 \\
\hline S17_cis-H & 0 & -575.01747 & -575.03568 & 477.6 & -17.8 \\
\hline S17_trans-C2H3 & 0 & -652.35485 & -652.36776 & 540.8 & -22.3 \\
\hline S17_trans-H & 0 & -575.00959 & -575.02847 & 472.8 & -20.6 \\
\hline S18 & 0 & -968.96828 & -968.97484 & 830.1 & -46.0 \\
\hline S18_NVP & 0 & -968.96828 & -968.97484 & 830.1 & -46.0 \\
\hline S19 & 0 & -968.98106 & -968.98236 & 830.1 & -33.1 \\
\hline S19_NVP & 0 & -968.98106 & -968.98236 & 830.1 & -33.1 \\
\hline S2 & 0 & -363.72933 & -363.73800 & 219.8 & -1.7 \\
\hline S20 & 0 & -1178.14953 & -1178.18146 & 1050.9 & -29.0 \\
\hline S20_NVP & 0 & -1255.49702 & -1255.52300 & 1121.5 & -34.3 \\
\hline S21 & 0 & -288.52431 & -288.52407 & 214.1 & -0.4 \\
\hline S21_NVP & 0 & -365.87200 & -365.86482 & 280.1 & -3.6 \\
\hline S3 & 0 & -1178.14409 & -1178.16636 & 1039.4 & -35.6 \\
\hline S4 & 0 & -1198.99368 & -1199.00985 & 1024.2 & -38.5 \\
\hline S5 & 0 & -1992.60189 & -1992.64115 & 1851.6 & -62.3 \\
\hline S5_BF4 & 0 & -2130.95927 & -2131.10934 & 1659.3 & -64.7 \\
\hline S5_Phenol & 0 & -2013.44051 & -2013.46804 & 1834.6 & -70.5 \\
\hline S6 & 0 & -2279.04392 & -2279.10075 & 2097.6 & -72.5 \\
\hline S6_Phenol & 0 & -2320.73048 & -2320.77158 & 2060.3 & -81.6 \\
\hline S7_cis & 0 & -363.11553 & -363.12783 & 185.9 & -122.4 \\
\hline S7_trans & 0 & -363.12301 & -363.13458 & 187.6 & -108.1 \\
\hline S8 & 0 & -1255.47131 & -1255.49012 & 1117.1 & -51.8 \\
\hline S9 & 0 & -1255.47354 & -1255.49645 & 1117.9 & -46.4 \\
\hline TS-AB_cis & 1 & -891.67273 & -891.66856 & 784.4 & -31.5 \\
\hline TS-AB_cis-PMe3 & 1 & -538.15679 & -538.13265 & 211.8 & -18.7 \\
\hline TS-AB_cis-PPh3 & 1 & -1112.96648 & -1112.90800 & 554.5 & -47.6 \\
\hline TS-AB_trans & 1 & -891.67449 & -891.66776 & 779.5 & -20.6 \\
\hline TS-AB_trans-PMe3 & 1 & -538.15374 & -538.12619 & 209.9 & -5.3 \\
\hline TS-AB_trans-PPh3 & 1 & -1112.95746 & -1112.89749 & 547.5 & -40.6 \\
\hline TS-BC_cis & 1 & -1178.12691 & -1178.14279 & 1022.1 & -46.5 \\
\hline TS-BC_cis-PMe3 & 1 & -824.60792 & -824.60254 & 450.3 & -29.7 \\
\hline TS-BC_cis-PPh3 & 1 & -1399.41350 & -1399.37549 & 789.9 & -66.1 \\
\hline TS-BC_trans & 1 & -1178.14258 & -1178.16225 & 1030.0 & -37.4 \\
\hline TS-BC_trans-PMe3 & 1 & -824.62176 & -824.61892 & 452.5 & -24.6 \\
\hline TS-BC_trans-PPh3 & 1 & -1399.41555 & -1399.38371 & 790.1 & -58.6 \\
\hline TS-CD & 1 & -1178.15775 & -1178.17908 & 1047.8 & -37.7 \\
\hline TS-CD_PMe3 & 1 & -824.63781 & -824.63575 & 474.3 & -20.4 \\
\hline TS-CD_PPh3 & 1 & -1399.43172 & -1399.40121 & 818.4 & -51.7 \\
\hline TS-DE & 1 & -1464.61788 & -1464.65679 & 1280.8 & -56.5 \\
\hline TS-DE_Phenol & 1 & -1485.45982 & -1485.48986 & 1266.5 & -55.2 \\
\hline TS-DE_PMe3 & 1 & -1111.11127 & -1111.12599 & 713.3 & -38.1 \\
\hline TS-DE_PPh3 & 1 & -1685.90197 & -1685.89014 & 1060.6 & -74.4 \\
\hline TS-DG_cis & 1 & -1523.52057 & -1523.54867 & 1291.0 & -57.9 \\
\hline TS-DH_trans & 1 & -1523.52190 & -1523.54877 & 1294.8 & -53.3 \\
\hline TS-EF & 1 & -1464.59563 & -1464.62831 & 1274.5 & -65.7 \\
\hline TS-EF_Phenol & 1 & -1485.42991 & -1485.44784 & 1259.4 & -54.9 \\
\hline TS-EF_PMe3 & 1 & -1111.07778 & -1111.08650 & 703.9 & -46.7 \\
\hline TS-EF PPh3 & 1 & -1685.87475 & -1685.85332 & 1049.4 & -73.8 \\
\hline
\end{tabular}




\begin{tabular}{llcccc}
\hline TS-HI_cis & 1 & -1523.50354 & -1523.53383 & 1293.7 & -47.9 \\
\hline TS-HI_trans & 1 & -1523.50401 & -1523.53408 & 1290.7 & -48.1 \\
\hline TS-S1 & 1 & -1178.14163 & -1178.16232 & 1036.3 & -52.3 \\
\hline TS-S10 & 1 & -1046.26461 & -1046.26452 & 902.6 & -52.5 \\
\hline TS-S11_cis-C2H3 & 1 & -1541.94126 & -1541.98285 & 1376.8 & -57.7 \\
\hline TS-S11_cis-H & 1 & -1464.59292 & -1464.63866 & 1307.4 & -47.6 \\
\hline TS-S11_trans-C2H3 & 1 & -1541.93570 & -1541.97942 & 1379.8 & -56.4 \\
\hline TS-S11_trans-H & 1 & -1464.58728 & -1464.63561 & 1306.4 & -51.5 \\
\hline TS-S12_cis-C2H3 & 1 & -1541.92884 & -1541.97639 & 1381.2 & -48.8 \\
\hline TS-S12_cis-H & 1 & -1464.58986 & -1464.64319 & 1308.7 & -43.9 \\
\hline TS-S12_trans-C2H3 & 1 & -1541.93074 & -1541.97999 & 1379.4 & -46.5 \\
\hline TS-S12_trans-H & 1 & -1464.58732 & -1464.64187 & 1306.5 & -45.0 \\
\hline TS-S13 & 1 & -968.93752 & -968.94160 & 828.2 & -74.0 \\
\hline TS-S13_NVP & 1 & -968.93752 & -968.94160 & 828.2 & -74.0 \\
\hline TS-S14 & 1 & -968.96522 & -968.96835 & 830.9 & -41.9 \\
\hline TS-S14_NVP & 1 & -968.96522 & -968.96835 & 830.9 & -41.9 \\
\hline TS-S15 & 1 & -1178.11787 & -1178.14319 & 1044.5 & -37.6 \\
\hline TS-S15_NVP & 1 & -1255.46739 & -1255.48671 & 1109.1 & -43.7 \\
\hline TS-S16 & 1 & -1178.11809 & -1178.15069 & 1045.1 & -31.1 \\
\hline TS-S16_NVP & 1 & -1255.46133 & -1255.48845 & 1119.0 & -33.5 \\
\hline TS-S2 & 1 & -1178.14144 & -1178.16026 & 1046.1 & -36.6 \\
\hline TS-S3 & 1 & -1178.08008 & -1178.09801 & 1030.8 & -44.7 \\
\hline TS-S3_PMe3 & 1 & -824.56305 & -824.55892 & 455.5 & -26.4 \\
\hline TS-S3_PPh3 & 1 & -1399.37211 & -1399.33203 & 799.7 & -57.3 \\
\hline TS-S4 & 1 & -1198.99163 & -1199.00707 & 1028.9 & -51.2 \\
\hline TS-S5 & 1 & -1992.58841 & -1992.61970 & 1863.9 & -62.6 \\
\hline TS-S5_BF4 & 1 & -2130.95076 & -2131.08932 & 1651.2 & -61.4 \\
\hline TS-S5_Phenol & 1 & -2013.43162 & -2013.45037 & 1835.2 & -65.2 \\
\hline TS-S6_cis & 1 & -363.09647 & -363.10364 & 171.2 & -95.0 \\
\hline TS-S6_trans & 1 & -363.08924 & -363.09685 & 173.1 & -93.3 \\
\hline TS-S7 & 1 & -1255.45257 & -1255.46841 & 1107.9 & -48.3 \\
\hline TS-S8 & 1 & -1255.45762 & -1255.47987 & 1104.2 & -43.9 \\
\hline TS-S9 & 1 & -968.94160 & -968.94250 & 833.6 & -35.2 \\
\hline & & & & & \\
\hline & & & & & \\
\hline
\end{tabular}




\section{$6 \mathrm{XYZ}$ Coordinates of the Computed Structures}

$X Y Z$ coordinates of all computed structures are given in the following format:

\section{Compound}

Total energy [Hartree] at the level of geometry optimization

$\mathrm{XYZ}$ coordinate

\begin{tabular}{cccc} 
A & \multicolumn{4}{c}{} \\
E(BP86/def2-SV(P) $=-814.4346321965$ \\
P & 0.0005532 & 2.4724129 & -0.0016149 \\
C & -1.5507755 & 1.7008133 & 0.7550368 \\
C & 0.1225437 & 1.7036881 & -1.7235466 \\
C & 1.4322581 & 1.7021762 & 0.9627602 \\
H & -1.4248785 & 1.8237457 & 1.8576141 \\
H & -2.3691974 & 2.4088018 & 0.4826451 \\
C & -2.0105034 & 0.2685611 & 0.4297895 \\
H & 0.7698576 & 2.4113592 & -2.2937845 \\
C & 0.6317954 & 0.2712579 & -1.9612581 \\
H & -0.8941539 & 1.8297525 & -2.1683306 \\
H & 2.3240294 & 1.8233089 & 0.3014128 \\
H & 1.6076377 & 2.4121112 & 1.8056588 \\
C & 1.3798217 & 0.2714509 & 1.5276528 \\
C & -3.3157964 & -0.1174766 & 1.1519151 \\
H & -2.1681187 & 0.1699012 & -0.6697509 \\
H & -1.2174119 & -0.4658216 & 0.6951224 \\
C & 0.6607269 & -0.1114498 & -3.4531793 \\
H & 1.6617345 & 0.1698165 & -1.5459162 \\
H & 0.0032247 & -0.4630475 & -1.4099406 \\
C & 2.6533927 & -0.1107954 & 2.3050578 \\
H & 0.5026007 & 0.1731111 & 2.2091627 \\
H & 1.2180478 & -0.4651385 & 0.7096273 \\
C & -3.8064543 & -1.5318597 & 0.8149850 \\
H & -3.1642102 & -0.0280897 & 2.2539645 \\
H & -4.1091714 & 0.6241266 & 0.8949862 \\
C & 1.1988407 & -1.5249272 & -3.7116819 \\
H & -0.3690355 & -0.0214380 & -3.8740228 \\
H & 1.2803441 & 0.6314511 & -4.0094291 \\
C & 2.6048194 & -1.5239161 & 2.9017575 \\
H & 3.5359731 & -0.0217906 & 1.6280193 \\
H & 2.8215738 & 0.6331023 & 3.1194842 \\
H & -4.7477597 & -1.7773241 & 1.3555302 \\
H & -4.0076596 & -1.6423076 & -0.2749802 \\
H & -3.0501495 & -2.3008219 & 1.0926648 \\
H & 1.2033179 & -1.7680754 & -4.7974630 \\
H & 2.2428392 & -1.6362459 & -3.3395011 \\
H & 0.5793615 & -2.2949325 & -3.1980631 \\
H & 3.5399025 & -1.7662931 & 3.4539358 \\
H & 1.7563663 & -1.6342952 & 3.6150439
\end{tabular}

H $\quad 2.4737134 \quad-2.2949021 \quad 2.1086363$

Acetylene

$\mathrm{E}(\mathrm{BP} 86 / \mathrm{def} 2-\mathrm{SV}(\mathrm{P}))=-77.26481098239$

C $\quad 0.0000000 \quad 0.0000000 \quad-0.6098462$

C $\quad 0.0000000 \quad 0.0000000 \quad 0.6098440$

$\mathrm{H} \quad 0.0000000 \quad 0.0000000 \quad 1.6928948$

H $\quad 0.0000000 \quad 0.0000000 \quad-1.6928926$

A_PMe3
$\begin{array}{lccc}\text { E(BP86/def2-SV(P) }=-460.9229674910 \\ \text { P } & -0.0001210 & 0.9396458 & 0.0003427 \\ \text { C } & -1.6439426 & 0.0481153 & -0.0896988 \\ \text { C } & 0.8994930 & 0.0480802 & -1.3784132 \\ \text { C } & 0.7443474 & 0.0477101 & 1.4683629 \\ \text { H } & -2.2774514 & 0.3510550 & 0.7732586 \\ \text { H } & -2.1780624 & 0.3498220 & -1.0179540 \\ \text { H } & -1.5375836 & -1.0615630 & -0.0833526 \\ \text { H } & 1.9701374 & 0.3504660 & -1.3780888 \\ \text { H } & 0.8413277 & -1.0615295 & -1.2887073 \\ \text { H } & 0.4683135 & 0.3502411 & -2.3585953 \\ \text { H } & 1.8087148 & 0.3500761 & 1.5848809 \\ \text { H } & 0.2087896 & 0.3495633 & 2.3958026 \\ \text { H } & 0.6960374 & -1.0616824 & 1.3721623\end{array}$

\section{A PPh3}

$\mathrm{E}(\mathrm{BP} 86 /$ def2-SV(P)) $=-1035.732491715$

$\begin{array}{llll}\mathrm{P} & 0.0696237 & 1.3840302 & 0.0691836\end{array}$

$\begin{array}{llll}\text { C } & -4.3008820 & -0.2557504 & -0.3680701\end{array}$

C $\quad-3.2732549 \quad-0.8672335-1.1064951$

C $-1.9477292 \quad-0.4126166-0.9884991$

C $\quad-1.6329354 \quad 0.6660586-0.1332780$

$\begin{array}{llll}\text { C } & -2.6776690 & 1.2839199 & 0.5934009\end{array}$

$\begin{array}{llll}\text { C } & -3.9988648 & 0.8206975 & 0.4854886\end{array}$

$\mathrm{H} \quad-5.3399040 \quad-0.6137002-0.4619767$

H $-3.5029477 \quad-1.7093861 \quad-1.7811399$

$\mathrm{H} \quad-1.1488785-0.9012724 \quad-1.5699621$

H $\quad-2.4506579 \quad 2.1421596 \quad 1.2497632$

H $\quad-4.8005743 \quad 1.3113622 \quad 1.0628294$

C $\quad 2.4068321 \quad-0.4479900 \quad-3.5583836$

$\begin{array}{llll}\text { C } & 1.6763164 & 0.7479277 & -3.6789556\end{array}$ 


$\begin{array}{cccc}\mathrm{C} & 0.9886321 & 1.2667165 & -2.5699655 \\ \mathrm{C} & 1.0021172 & 0.5890114 & -1.3283898 \\ \mathrm{C} & 1.7405534 & -0.6099053 & -1.2179333 \\ \mathrm{C} & 2.4395895 & -1.1222197 & -2.3255034 \\ \mathrm{H} & 2.9561696 & -0.8513427 & -4.4255694 \\ \mathrm{H} & 1.6518146 & 1.2875411 & -4.6407176 \\ \mathrm{H} & 0.4334139 & 2.2162780 & -2.6649185 \\ \mathrm{H} & 1.7713327 & -1.1475837 & -0.2559798 \\ \mathrm{H} & 3.0137087 & -2.0586541 & -2.2230755 \\ \mathrm{C} & 1.8184221 & -0.7635330 & 3.8525606 \\ \mathrm{C} & 2.4923640 & 0.3039442 & 3.2313888 \\ \mathrm{C} & 1.9404615 & 0.9193328 & 2.0966251 \\ \mathrm{C} & 0.7176624 & 0.4643511 & 1.5485651 \\ \mathrm{C} & 0.0493439 & -0.6072264 & 2.1802815 \\ \mathrm{C} & 0.5954043 & -1.2131902 & 3.3265600 \\ \mathrm{H} & 2.2451567 & -1.2404621 & 4.7507215 \\ \mathrm{H} & 3.4496216 & 0.6684311 & 3.6407247 \\ \mathrm{H} & 2.4644563 & 1.7699334 & 1.6266676 \\ \mathrm{H} & -0.9084929 & -0.9717013 & 1.7744436 \\ \mathrm{H} & 0.0597941 & -2.0479277 & 3.8096088\end{array}$

\begin{tabular}{lccc}
\multicolumn{4}{l}{ B_cis } \\
E(BP86/def2-SV(P)) $=-891.6746630762$ \\
C & 0.1506525 & 1.7600952 & -4.2051492 \\
C & 1.0672231 & 1.2302894 & -3.3540463 \\
P & 0.6426976 & 0.5948615 & -1.6860733 \\
H & -0.8908660 & 1.8157438 & -3.7647313 \\
H & 2.1455672 & 1.0849030 & -3.5949150 \\
C & -0.2784798 & 1.9232852 & -0.7907903 \\
C & -0.4532893 & -0.8871011 & -1.9034461 \\
C & 2.1239245 & 0.0771423 & -0.6799936 \\
H & -1.3949483 & -0.4793290 & -2.3392071 \\
C & -0.7392330 & -1.8192122 & -0.7137313 \\
H & 0.0294654 & -1.4557040 & -2.7316460 \\
H & 2.4320836 & -0.9143697 & -1.0859438 \\
C & 2.0319917 & 0.0320635 & 0.8567468 \\
H & 2.9250963 & 0.7909946 & -0.9871742 \\
H & -0.8073224 & 2.4458447 & -1.6231641 \\
C & -1.2707860 & 1.5767498 & 0.3328765 \\
H & 0.5022446 & 2.6334451 & -0.4306232 \\
C & -1.6804871 & -2.9782067 & -1.0926743 \\
H & 0.2182780 & -2.2447476 & -0.3332620 \\
H & -1.1831076 & -1.2507740 & 0.1339687 \\
C & 3.3598361 & -0.3849024 & 1.5184247 \\
H & 1.7364626 & 1.0352843 & 1.2412998 \\
H & 1.2285683 & -0.6701218 & 1.1748224 \\
C & -1.9289722 & 2.8330291 & 0.9339164 \\
H & -2.0680284 & 0.9087382 & -0.0671418 \\
H & -0.7642790 & 1.0072699 & 1.1452179 \\
C & -1.9580640 & -3.9395210 & 0.0706245 \\
H & -2.6420465 & -2.5575357 & -1.4693650
\end{tabular}

$\begin{array}{cccc}\mathrm{H} & -1.2400475 & -3.5416099 & -1.9486981 \\ \mathrm{C} & 3.2896081 & -0.4185099 & 3.0507861 \\ \mathrm{H} & 3.6594433 & -1.3885889 & 1.1350424 \\ \mathrm{H} & 4.1643884 & 0.3167523 & 1.1951140 \\ \mathrm{C} & -2.9394041 & 2.5167075 & 2.0445266 \\ \mathrm{H} & -1.1336304 & 3.5065441 & 1.3317295 \\ \mathrm{H} & -2.4317044 & 3.4049185 & 0.1193780 \\ \mathrm{H} & -2.6420832 & -4.7603599 & -0.2379952 \\ \mathrm{H} & -1.0193388 & -4.4083234 & 0.4442497 \\ \mathrm{H} & -2.4333897 & -3.4128568 & 0.9291524 \\ \mathrm{H} & 4.2631492 & -0.7233476 & 3.4939191 \\ \mathrm{H} & 3.0298174 & 0.5810319 & 3.4676236 \\ \mathrm{H} & 2.5189899 & -1.1393849 & 3.4064382 \\ \mathrm{H} & -3.3923226 & 3.4457640 & 2.4553000 \\ \mathrm{H} & -3.7697691 & 1.8773913 & 1.6680050 \\ \mathrm{H} & -2.4578885 & 1.9756574 & 2.8906090\end{array}$

\section{B_cis-PMe3}

E(BP86/def2-SV(P) $)=-538.1576140527$

$\begin{array}{lllll}\text { C } & -0.4606305 & 0.8596457 & -2.6012588\end{array}$

$\begin{array}{lllll}\text { C } & 0.5568223 & 0.6352785 & -1.7294147\end{array}$

$\begin{array}{lllll}\mathrm{P} & 0.2022498 & 0.0303317 & -0.0451523\end{array}$

$\begin{array}{llll}\mathrm{H} & -1.4834165 & 0.6368642 & -2.1718887\end{array}$

H $1.6368831 \quad 0.7878672 \quad-1.9447575$

$\begin{array}{llll}\text { C } & -0.9081062 & 1.2001575 & 0.8340467\end{array}$

$\begin{array}{llll}\text { C } & -0.7181198 & -1.5576325 & -0.1168395\end{array}$

$\begin{array}{llll}\text { C } & 1.5993225 & -0.2815071 & 1.1372124\end{array}$

H $\quad-1.5510297-1.4367958-0.8422712$

H $\quad-0.0405120 \quad-2.3557845 \quad-0.4895495$

$\begin{array}{llll}\mathrm{H} & 2.2923907 & -1.0276289 & 0.6911985\end{array}$

$\begin{array}{llll}\mathrm{H} & 2.1597982 & 0.6655436 & 1.2938477\end{array}$

$\begin{array}{llll}\mathrm{H} & -1.7540229 & 1.4410167 & 0.1550852\end{array}$

$\begin{array}{llll}\mathrm{H} & -0.3534770 & 2.1390472 & 1.0493917\end{array}$

$\begin{array}{llll}\mathrm{H} & -1.2981927 & 0.7688938 & 1.7813683\end{array}$

$\begin{array}{llll}\mathrm{H} & -1.1254658 & -1.8451403 & 0.8767069\end{array}$

$\begin{array}{llll}\mathrm{H} & 1.2455065 & -0.6601570 & 2.1222748\end{array}$

\section{B_cis-PPh3}

$\mathrm{E}(\mathrm{BP} 86 /$ def2-SV(P)) $=-1112.967508658$

$\begin{array}{llll}\text { C } & -0.0560789 & 0.7115946 & -3.4634469\end{array}$

$\begin{array}{lllll}\text { C } & 0.8206170 & 0.7907484 & -2.4478469\end{array}$

$\begin{array}{lllll}\mathrm{P} & 0.3296772 & 0.2613260 & -0.7328311\end{array}$

$\begin{array}{llll}\mathrm{H} & -1.0428715 & 0.2251653 & -3.2574040\end{array}$

$\begin{array}{llll}\mathrm{H} & 1.8406720 & 1.2265478 & -2.4792030\end{array}$

$\begin{array}{llll}\text { C } & -2.4499417 & 3.5143862 & 1.1165956\end{array}$

$\begin{array}{llll}\text { C } & -2.2617251 & 3.4476779 & -0.2754825\end{array}$

$\begin{array}{llll}\text { C } & -1.4160685 & 2.4745745 & -0.8316866\end{array}$

$\begin{array}{llll}\text { C } & -0.7538974 & 1.5496833 & 0.0059755\end{array}$

$\begin{array}{llll}\text { C } & -0.9429893 & 1.6224935 & 1.4064885\end{array}$

$\begin{array}{llll}\text { C } & -1.7858097 & 2.6031198 & 1.9557436\end{array}$

$\begin{array}{llll}\mathrm{H} & -3.1105413 & 4.2838665 & 1.5497588\end{array}$ 


$\begin{array}{cccc}\mathrm{H} & -2.7711291 & 4.1666505 & -0.9383602 \\ \mathrm{H} & -1.2478281 & 2.4378252 & -1.9212086 \\ \mathrm{H} & -0.4184587 & 0.9183859 & 2.0732064 \\ \mathrm{H} & -1.9224724 & 2.6540104 & 3.0488399 \\ \mathrm{C} & -2.0060002 & -3.7541321 & -0.9857173 \\ \mathrm{C} & -1.0016322 & -3.4549584 & -1.9244369 \\ \mathrm{C} & -0.3233957 & -2.2265066 & -1.8671290 \\ \mathrm{C} & -0.6469322 & -1.2872771 & -0.8615404 \\ \mathrm{C} & -1.6590709 & -1.5887006 & 0.0743390 \\ \mathrm{C} & -2.3347089 & -2.8199921 & 0.0110129 \\ \mathrm{H} & -2.5392304 & -4.7181107 & -1.0361731 \\ \mathrm{H} & -0.7463572 & -4.1814759 & -2.7133960 \\ \mathrm{H} & 0.4506738 & -1.9827414 & -2.6120202 \\ \mathrm{H} & -1.9336663 & -0.8553355 & 0.8499408 \\ \mathrm{H} & -3.1274627 & -3.0454122 & 0.7433753 \\ \mathrm{C} & 3.9714192 & -0.4378294 & 2.0688909 \\ \mathrm{C} & 3.5640245 & 0.8658159 & 1.7287295 \\ \mathrm{C} & 2.4178824 & 1.0688236 & 0.9465345 \\ \mathrm{C} & 1.6494397 & -0.0375296 & 0.4981443 \\ \mathrm{C} & 2.0742224 & -1.3469129 & 0.8341367 \\ \mathrm{C} & 3.2222149 & -1.5400363 & 1.6174587 \\ \mathrm{H} & 4.8730647 & -0.5941653 & 2.6836451 \\ \mathrm{H} & 4.1460510 & 1.7356470 & 2.0769159 \\ \mathrm{H} & 2.1097837 & 2.0964595 & 0.6883970 \\ \mathrm{H} & 1.4961103 & -2.2189460 & 0.4872247 \\ \mathrm{H} & 3.5324153 & -2.5647398 & 1.8825289\end{array}$

\section{B_trans}

\begin{tabular}{lccc}
\multicolumn{4}{c}{$\mathrm{E}(\mathrm{BP} 86 / \mathrm{def} 2-\mathrm{SV}(\mathrm{P}))=-891.6884062641$} \\
$\mathrm{C}$ & -1.1767359 & 2.2312173 & -2.8930843 \\
$\mathrm{C}$ & -1.7261438 & 1.0826222 & -2.4150356 \\
$\mathrm{P}$ & -0.8997267 & 0.6109695 & -0.8897287 \\
$\mathrm{C}$ & -1.6988127 & -0.8765670 & -0.1245351 \\
$\mathrm{C}$ & 0.8746815 & 0.2297626 & -1.2138460 \\
$\mathrm{C}$ & -0.9581492 & 2.0361994 & 0.2766543 \\
$\mathrm{C}$ & -1.0297542 & -1.5255879 & 1.0987659 \\
$\mathrm{H}$ & -2.7402757 & -0.5599662 & 0.1171553 \\
$\mathrm{H}$ & -1.7954360 & -1.6181652 & -0.9523369 \\
$\mathrm{C}$ & 1.0966072 & -0.9470661 & -2.1782676 \\
$\mathrm{H}$ & 1.2386060 & 1.1794455 & -1.6716891 \\
$\mathrm{H}$ & 1.4112120 & 0.0775817 & -0.2500578 \\
$\mathrm{C}$ & 0.0825193 & 2.1454135 & 1.4021487 \\
$\mathrm{H}$ & -0.8628145 & 2.8807229 & -0.4515236 \\
$\mathrm{H}$ & -1.9980689 & 2.0630912 & 0.6786793 \\
$\mathrm{H}$ & -1.6458737 & 2.5400454 & -3.8648398 \\
$\mathrm{H}$ & -2.5327241 & 0.3922285 & -2.7783271 \\
$\mathrm{C}$ & -1.7879249 & -2.7682570 & 1.6025253 \\
$\mathrm{H}$ & 0.0170812 & -1.8154194 & 0.8468772 \\
$\mathrm{H}$ & -0.9524803 & -0.7849852 & 1.9282185 \\
$\mathrm{C}$ & 2.5759267 & -1.1274637 & -2.5633949 \\
$\mathrm{H}$ & 0.7202723 & -1.8945826 & -1.7243789
\end{tabular}

$\begin{array}{crcc}\mathrm{H} & 0.4923479 & -0.7750018 & -3.0988202 \\ \mathrm{C} & -0.1022274 & 3.4270683 & 2.2367503 \\ \mathrm{H} & 0.0336150 & 1.2612836 & 2.0805153 \\ \mathrm{H} & 1.1075351 & 2.1471412 & 0.9654488 \\ \mathrm{C} & -1.1285451 & -3.4316680 & 2.8185300 \\ \mathrm{H} & -2.8351662 & -2.4788229 & 1.8552295 \\ \mathrm{H} & -1.8695178 & -3.5073279 & 0.7707299 \\ \mathrm{C} & 2.8104101 & -2.2931766 & -3.5330840 \\ \mathrm{H} & 2.9478444 & -0.1805229 & -3.0191596 \\ \mathrm{H} & 3.1825186 & -1.2808096 & -1.6392566 \\ \mathrm{C} & 0.9387381 & 3.5805142 & 3.3530107 \\ \mathrm{H} & -0.0589806 & 4.3097227 & 1.5570021 \\ \mathrm{H} & -1.1272962 & 3.4317976 & 2.6769376 \\ \mathrm{H} & -1.7014424 & -4.3242532 & 3.1536027 \\ \mathrm{H} & -0.0921448 & -3.7661419 & 2.5861916 \\ \mathrm{H} & -1.0673731 & -2.7296073 & 3.6807198 \\ \mathrm{H} & 3.8871580 & -2.3926413 & -3.7940606 \\ \mathrm{H} & 2.4805359 & -3.2616673 & -3.0928465 \\ \mathrm{H} & 2.2463437 & -2.1491698 & -4.4821360 \\ \mathrm{H} & 0.7740703 & 4.5139439 & 3.9351217 \\ \mathrm{H} & 0.8970167 & 2.7276672 & 4.0684492 \\ \mathrm{H} & 1.9725737 & 3.6204325 & 2.9411453\end{array}$

\section{B_trans-PMe3}

$\mathrm{E}(\mathrm{BP} 86 /$ def2-SV(P) $)=-538.1665761192$

$\begin{array}{llll}\text { C } & -1.3368454 & -0.0417921 & -2.0801373\end{array}$

$\begin{array}{llll}\text { C } & -1.0868188 & 0.8770004 & -1.1081753\end{array}$

$\begin{array}{llll}\mathrm{P} & 0.0055271 & 0.0938575 & 0.0670500\end{array}$

$\begin{array}{llll}\text { C } & -0.7560887 & -1.4039943 & 0.7930011\end{array}$

$\begin{array}{llll}\text { C } & 1.5630596 & -0.4486608 & -0.7264752\end{array}$

$\begin{array}{llll}\text { C } & 0.4919281 & 1.1696047 & 1.4875213\end{array}$

$\begin{array}{llll}\mathrm{H} & -0.0319885 & -1.9848182 & 1.4044857\end{array}$

$\begin{array}{llll}\mathrm{H} & -1.6276897 & -1.1077762 & 1.4157523\end{array}$

$\begin{array}{lllll}\mathrm{H} & -1.1159705 & -1.9987641 & -0.0744142\end{array}$

$\begin{array}{lllll}\mathrm{H} & 2.1704474 & -1.0927208 & -0.0544094\end{array}$

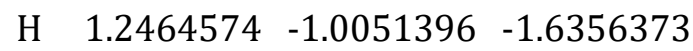

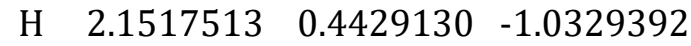

$\begin{array}{lllll}\mathrm{H} & 1.1711067 & 0.6434015 & 2.1921612\end{array}$

$\begin{array}{llll}\mathrm{H} & 1.0037100 & 2.0774002 & 1.1008874\end{array}$

$\begin{array}{llll}\mathrm{H} & -0.4203550 & 1.4894970 & 2.0363062\end{array}$

$\begin{array}{llll}\mathrm{H} & -2.0237742 & 0.3550796 & -2.8734667\end{array}$

$\begin{array}{llll}\mathrm{H} & -1.4044566 & 1.9349124 & -0.9115107\end{array}$

\section{B_trans-PPh3}

$\mathrm{E}(\mathrm{BP} 86 /$ def2-SV(P) $)=-1112.970029928$

C $\quad-1.77439590 .9207533 \quad-2.6684285$

$\begin{array}{llll}\text { C } & -1.4176454 & 1.5849994 & -1.5455886\end{array}$

$\begin{array}{lllll}\mathrm{P} & -0.4498885 & 0.4937923 & -0.4887986\end{array}$

$\begin{array}{llll}\mathrm{H} & -2.4033296 & 1.5233224 & -3.3727457\end{array}$

$\begin{array}{llll}\mathrm{H} & -1.5997584 & 2.6154486 & -1.1439330\end{array}$

$\begin{array}{llll}\text { C } & -2.9075185 & -3.1841155 & 0.9525422\end{array}$ 


$\begin{array}{cccc}\mathrm{C} & -2.0919954 & -2.4987337 & 1.8692347 \\ \mathrm{C} & -1.3469677 & -1.3814512 & 1.4533205 \\ \mathrm{C} & -1.4189052 & -0.9493940 & 0.1109968 \\ \mathrm{C} & -2.2311091 & -1.6428938 & -0.8131508 \\ \mathrm{C} & -2.9769718 & -2.7517170 & -0.3847185 \\ \mathrm{H} & -3.4902992 & -4.0610165 & 1.2818666 \\ \mathrm{H} & -2.0352164 & -2.8316472 & 2.9191245 \\ \mathrm{H} & -0.7151074 & -0.8441583 & 2.1792612 \\ \mathrm{H} & -2.2661396 & -1.2666896 & -1.8562549 \\ \mathrm{H} & -3.6172843 & -3.2875460 & -1.1055949 \\ \mathrm{C} & 3.3890491 & -1.1179248 & -2.5459876 \\ \mathrm{C} & 2.7214955 & 0.0012772 & -3.0785226 \\ \mathrm{C} & 1.5434752 & 0.4743036 & -2.4797199 \\ \mathrm{C} & 1.0308343 & -0.1702247 & -1.3343508 \\ \mathrm{C} & 1.6960706 & -1.2973772 & -0.8048885 \\ \mathrm{C} & 2.8743885 & -1.7673467 & -1.4114621 \\ \mathrm{H} & 4.3100470 & -1.4909527 & -3.0244445 \\ \mathrm{H} & 3.1176731 & 0.5060434 & -3.9751975 \\ \mathrm{H} & 0.9988503 & 1.3306669 & -2.9071383 \\ \mathrm{H} & 1.2875310 & -1.8197899 & 0.0761421 \\ \mathrm{H} & 3.3860999 & -2.6520535 & -0.9975196 \\ \mathrm{C} & 0.9042225 & 2.9907836 & 3.1985556 \\ \mathrm{C} & -0.4581442 & 2.8339504 & 2.8881533 \\ \mathrm{C} & -0.8461884 & 2.0383980 & 1.7980906 \\ \mathrm{C} & 0.1309008 & 1.3835030 & 1.0089031 \\ \mathrm{C} & 1.4975287 & 1.5562129 & 1.3188846 \\ \mathrm{C} & 1.8792489 & 2.3509893 & 2.4127928 \\ \mathrm{H} & 1.2072454 & 3.6162495 & 4.0547149 \\ \mathrm{H} & -1.2269896 & 3.3371151 & 3.4978462 \\ \mathrm{H} & -1.9178874 & 1.9187524 & 1.5644528 \\ \mathrm{H} & 2.2680511 & 1.0660942 & 0.7023212 \\ \mathrm{H} & 2.9490304 & 2.4723768 & 2.6512412\end{array}$

C

$\mathrm{E}(\mathrm{BP} 86 / \mathrm{def} 2-\mathrm{SV}(\mathrm{P}))=-1178.160468082$

$\begin{array}{llll}\text { C } & -3.1368671 & 0.3160158 & -0.9251400\end{array}$

$\begin{array}{llll}\text { C } & -4.6729336 & 0.0969014 & -0.9406622\end{array}$

$\begin{array}{llll}\text { C } & -5.1962082 & 1.3880979 & -0.2995569\end{array}$

$\begin{array}{lllll}\text { C } & -4.0518620 & 2.3918103 & -0.6230582\end{array}$

$\begin{array}{lllll}\mathrm{N} & -2.8270948 & 1.6052425 & -0.7340504\end{array}$

$\begin{array}{lllll}0 & -2.3265843 & -0.6456309 & -1.0927953\end{array}$

$\begin{array}{llll}\mathrm{H} & -4.9512816 & -0.8420658 & -0.4157414\end{array}$

$\begin{array}{llll}\mathrm{H} & -5.0044622 & -0.0141081 & -2.0014352\end{array}$

$\begin{array}{llll}\mathrm{H} & -6.1911177 & 1.7205857 & -0.6703556\end{array}$

$\begin{array}{llll}\mathrm{H} & -5.2768789 & 1.2573768 & 0.8040805\end{array}$

$\begin{array}{lllll}\mathrm{H} & -4.2746075 & 2.9332911 & -1.5829567\end{array}$

$\begin{array}{llll}\mathrm{H} & -3.9588812 & 3.1868142 & 0.1560540\end{array}$

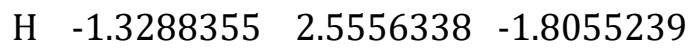

$\begin{array}{llll}\text { C } & -0.3849534 & 2.9517414 & -2.2734090\end{array}$

$\begin{array}{lllll}\text { C } & 0.8240711 & 2.4230913 & -1.9899704\end{array}$

$\begin{array}{llll}\mathrm{P} & 1.0247757 & 1.0167189 & -0.8584160\end{array}$ $\begin{array}{lrrc}\text { C } & -0.0249937 & 1.3007723 & 0.6089612 \\ \text { C } & 0.5032218 & -0.4805322 & -1.7610312 \\ \text { C } & 2.8330193 & 1.0385070 & -0.4548529 \\ \text { C } & -0.3125907 & 0.0923607 & 1.5217475 \\ \text { H } & 0.4303786 & 2.1564066 & 1.1614882 \\ \text { H } & -1.0176722 & 1.6352319 & 0.1661213 \\ \text { C } & 0.9672585 & -1.8592004 & -1.2543734 \\ \text { H } & -0.6359982 & -0.4227261 & -1.6691305 \\ \text { H } & 0.7928001 & -0.3218471 & -2.8244189 \\ \text { C } & 3.3039378 & 0.1779214 & 0.7309071 \\ \text { H } & 3.3588973 & 0.7406510 & -1.3926808 \\ \text { H } & 3.0892683 & 2.1087896 & -0.2756855 \\ \text { H } & -0.4557324 & 3.7909989 & -2.9918045 \\ \text { H } & 1.7540936 & 2.7954548 & -2.4628291 \\ \text { C } & -1.1794284 & 0.5078848 & 2.7242133 \\ \text { H } & -0.8745986 & -0.6552907 & 0.9183172 \\ \text { H } & 0.6224667 & -0.3897671 & 1.8913754 \\ \text { C } & 0.1925115 & -2.9825447 & -1.9704864 \\ \text { H } & 2.0646171 & -1.9974208 & -1.4081992 \\ \text { H } & 0.7850662 & -1.9469805 & -0.1595896 \\ \text { C } & 4.8285600 & 0.2453423 & 0.9330463 \\ \text { H } & 2.7963538 & 0.5199477 & 1.6612511 \\ \text { H } & 2.9985244 & -0.8818780 & 0.5813403 \\ \text { C } & -1.5547975 & -0.6741500 & 3.6270813 \\ \text { H } & -0.6409627 & 1.2814593 & 3.3230373 \\ \text { H } & -2.1022875 & 0.9962289 & 2.3374687 \\ \text { C } & 0.5724054 & -4.3859880 & -1.4824591 \\ \text { H } & -0.8950100 & -2.7973826 & -1.8187020 \\ \text { H } & 0.3723103 & -2.9051906 & -3.0691763 \\ \text { C } & 5.3151059 & -0.5856700 & 2.1272113 \\ \text { H } & 5.3364714 & -0.1026024 & 0.0027472 \\ \text { H } & 5.1334685 & 1.3100713 & 1.0678464 \\ \text { H } & -2.1855795 & -0.3449019 & 4.4823850 \\ \text { H } & -2.1280176 & -1.4436227 & 3.0630230 \\ \text { H } & -0.6506621 & -1.1706751 & 4.0488768 \\ \text { H } & -0.0093353 & -5.1681529 & -2.0182668 \\ \text { H } & 1.6535060 & -4.6015976 & -1.6454999 \\ \text { H } & 0.3667188 & -4.5059324 & -0.3943586 \\ \text { H } & 6.4190186 & -0.5177471 & 2.2428582 \\ \text { H } & 4.8558776 & -0.2361928 & 3.0794109 \\ \text { H } & 5.0555301 & -1.6615509 & 2.0057663\end{array}$

\section{C_BF4}

$\mathrm{E}(\mathrm{BP} 86 /$ def2-SV(P) $)=-1316.510725766$

$\begin{array}{lllll}\text { F } & -3.2792011 & 1.5908765 & -0.0913028\end{array}$

$\begin{array}{llll}\mathrm{H} & -2.3873324 & 3.3415598 & -0.2612744\end{array}$

$\begin{array}{lllll}\text { C } & -1.4735227 & 3.9338875 & -0.4594084\end{array}$

$\begin{array}{lllll}\text { C } & -0.2495376 & 3.3698042 & -0.5112187\end{array}$

$\begin{array}{lllll}\mathrm{P} & 0.0688003 & 1.5963536 & -0.2528265\end{array}$

$\begin{array}{llll}\text { C } & -0.5639452 & 1.1194849 & 1.4017510\end{array}$

$\begin{array}{llll}\text { C } & -0.7333208 & 0.6411560 & -1.6010545\end{array}$ 


$\begin{array}{cccc}\text { C } & 1.9144178 & 1.4987442 & -0.3441697 \\ \mathrm{C} & 0.0228476 & -0.1410158 & 2.0623983 \\ \mathrm{H} & -0.4180782 & 2.0142894 & 2.0476663 \\ \mathrm{H} & -1.6586065 & 0.9913713 & 1.2265665 \\ \mathrm{C} & -0.8186231 & -0.8884962 & -1.4266361 \\ \mathrm{H} & -1.7659579 & 1.0592417 & -1.6303835 \\ \mathrm{H} & -0.2073551 & 0.9349954 & -2.5399498 \\ \mathrm{C} & 2.5683512 & 0.1181746 & -0.5340080 \\ \mathrm{H} & 2.2045428 & 2.1718487 & -1.1843708 \\ \mathrm{H} & 2.2857065 & 1.9869612 & 0.5878680 \\ \mathrm{H} & -1.5818040 & 5.0220104 & -0.6250564 \\ \mathrm{H} & 0.6504710 & 3.9802400 & -0.7163844 \\ \mathrm{C} & -0.7498802 & -0.4936707 & 3.3472815 \\ \mathrm{H} & -0.0382619 & -1.0038241 & 1.3626990 \\ \mathrm{H} & 1.1023526 & 0.0097349 & 2.3015046 \\ \mathrm{C} & -1.4616283 & -1.5501511 & -2.6593457 \\ \mathrm{H} & 0.1872257 & -1.3329444 & -1.2458960 \\ \mathrm{H} & -1.4550000 & -1.0992914 & -0.5401226 \\ \mathrm{C} & 4.1060601 & 0.2025402 & -0.5256649 \\ \mathrm{H} & 2.2335765 & -0.5823993 & 0.2625017 \\ \mathrm{H} & 2.2319886 & -0.3228838 & -1.4993569 \\ \mathrm{C} & -0.1995165 & -1.7372247 & 4.0551009 \\ \mathrm{H} & -0.7223278 & 0.3787537 & 4.0424597 \\ \mathrm{H} & -1.8168347 & -0.6467187 & 3.0724934 \\ \mathrm{C} & -1.6417067 & -3.0628753 & -2.4833152 \\ \mathrm{H} & -2.4547489 & -1.0782189 & -2.8217097 \\ \mathrm{H} & -0.8398284 & -1.3425812 & -3.5633522 \\ \mathrm{C} & 4.7829731 & -1.1570527 & -0.7403273 \\ \mathrm{H} & 4.4377268 & 0.9162964 & -1.3162980 \\ \mathrm{H} & 4.4435618 & 0.6404517 & 0.4432505 \\ \mathrm{H} & -0.7870448 & -1.9681521 & 4.9707489 \\ \mathrm{H} & -0.2479179 & -2.6325795 & 3.3951326 \\ \mathrm{H} & 0.8627363 & -1.6015986 & 4.3635007 \\ \mathrm{H} & -2.1024839 & -3.5184582 & -3.3872783 \\ \mathrm{H} & -0.6697976 & -3.5788196 & -2.3043152 \\ \mathrm{H} & -2.3098252 & -3.2801351 & -1.6208580 \\ \mathrm{H} & 5.8905044 & -1.0606941 & -0.7268743 \\ \mathrm{H} & 4.4989218 & -1.8824048 & 0.0549309 \\ \mathrm{H} & 4.4967703 & -1.6038765 & -1.7191423 \\ \mathrm{~B} & -3.9693410 & 0.3087142 & 0.0489345 \\ \mathrm{~F} & -5.2076647 & 0.5073515 & 0.6207405 \\ \mathrm{~F} & -4.0422753 & -0.2711143 & -1.2263003 \\ \mathrm{~F} & -3.1361668 & -0.4976611 & 0.8906711\end{array}$

\section{C_Phenol}

E(BP86/def2-SV(P)) = -1198.997759972
O -2.2755403
$\begin{array}{ll}1.2471940 & 0.0056223\end{array}$
$\mathrm{H} \quad-1.6143058 \quad 3.0115262 \quad-0.3739430$
$\begin{array}{llll}\text { C } & -0.7659507 & 3.7306685 & -0.5203512\end{array}$
$\begin{array}{lllll}\text { C } & 0.5123647 & 3.2968934 & -0.4987819\end{array}$
P $\quad 0.9325418 \quad 1.5467621-0.2362070$

\begin{tabular}{cccc}
$\mathrm{C}$ & 0.3215120 & 1.0835271 & 1.4284086 \\
$\mathrm{C}$ & 0.0952724 & 0.5777704 & -1.5452061 \\
$\mathrm{C}$ & 2.7787742 & 1.5223472 & -0.3539422 \\
$\mathrm{C}$ & 0.8421797 & -0.2075391 & 2.0823225 \\
$\mathrm{H}$ & 0.5309956 & 1.9657179 & 2.0753481 \\
$\mathrm{H}$ & -0.7960613 & 1.0561191 & 1.2279492 \\
$\mathrm{C}$ & 0.1159626 & -0.9595325 & -1.4734312 \\
$\mathrm{H}$ & -0.9706918 & 0.9183196 & -1.3735164 \\
$\mathrm{H}$ & 0.4786517 & 0.9617036 & -2.5186010 \\
$\mathrm{C}$ & 3.4764553 & 0.1588448 & -0.5097748 \\
$\mathrm{H}$ & 3.0339230 & 2.1797752 & -1.2175316 \\
$\mathrm{H}$ & 3.1441699 & 2.0506876 & 0.5585555 \\
$\mathrm{H}$ & -0.9758144 & 4.8058466 & -0.6769703 \\
$\mathrm{H}$ & 1.3723146 & 3.9814393 & -0.6299108 \\
$\mathrm{C}$ & 0.1021170 & -0.5032040 & 3.4007479 \\
$\mathrm{H}$ & 0.7119984 & -1.0729569 & 1.3948734 \\
$\mathrm{H}$ & 1.9362024 & -0.1305832 & 2.2891358 \\
$\mathrm{C}$ & -0.6733724 & -1.5765319 & -2.6437713 \\
$\mathrm{H}$ & 1.1536440 & -1.3664729 & -1.4723680 \\
$\mathrm{H}$ & -0.3616400 & -1.2792737 & -0.5207679 \\
$\mathrm{C}$ & 5.0108603 & 0.2863912 & -0.4954653 \\
$\mathrm{H}$ & 3.1572690 & -0.5348593 & 0.3001674 \\
$\mathrm{H}$ & 3.1578228 & -0.3129571 & -1.4665841 \\
$\mathrm{C}$ & 0.5950955 & -1.7776243 & 4.0965233 \\
$\mathrm{H}$ & 0.2176760 & 0.3687855 & 4.0866515 \\
$\mathrm{H}$ & -0.9885730 & -0.5833977 & 3.1886256 \\
$\mathrm{C}$ & -0.7229741 & -3.1086482 & -2.5908561 \\
$\mathrm{H}$ & -1.7085545 & -1.1657880 & -2.6297755 \\
$\mathrm{H}$ & -5.1937587 & -0.7364434 & -0.0389133 \\
$\mathrm{H}$ & -3.8585684 & 0.1987971 & -2.1460104 \\
$\mathrm{H}$ & -3.5368359 & 0.2568573 & 2.1186989 \\
$\mathrm{C}$ & 5.7290404 & -1.0561382 & -0.6821146 \\
$\mathrm{H}$ & 5.3258036 & 0.9951317 & -1.2972807 \\
$\mathrm{H}$ & 5.3302662 & 0.7520467 & 0.4666919 \\
$\mathrm{H}$ & 0.0374860 & -1.9609978 & 5.0412213 \\
$\mathrm{H}$ & 0.4572719 & -2.6726789 & 3.4487111 \\
$\mathrm{H}$ & 1.6771808 & -1.7118803 & 4.3532454 \\
$\mathrm{H}$ & -1.2989690 & -3.5209832 & -3.4481726 \\
$\mathrm{H}$ & 0.2976144 & -3.5546637 & -2.6256098 \\
$\mathrm{H}$ & -1.2151945 & -3.4635977 & -1.6577154 \\
$\mathrm{H}$ & 6.8332829 & -0.9275394 & -0.6634900 \\
$\mathrm{H}$ & 5.4608205 & -1.7757555 & 0.1238503 \\
$\mathrm{H}$ & 5.4626737 & -1.5282928 & -1.6547805 \\
$\mathrm{C}$ & -6.1726820 & -0.3199964 & -0.0290586 \\
$\mathrm{C}$ & -5.5944971 & 0.2031055 & -1.2025391 \\
$\mathrm{C}$ & -3.4973057 & 0.7342297 & -1.1949316 \\
$\mathrm{C}$ & 0.7663302 & -0.0022670 \\
& -1169843 & 0.2348069 & 1.1785030 \\
\hline
\end{tabular}


H $\quad-5.8500247 \quad-0.6926810 \quad 2.0939379$

\section{C_PMe3}

E(BP86/def2-SV(P)) = -824.6378942916

C $\quad-1.8716631 \quad-0.7579554 \quad-0.4982542$

$\begin{array}{llll}\text { C } & -3.4140637 & -0.9220685 & -0.5621553\end{array}$

$\begin{array}{llll}\text { C } & -3.8887678 & -0.0459906 & 0.6039552\end{array}$

$\begin{array}{llll}\text { C } & -2.7355165 & 0.9913539 & 0.7142967\end{array}$

$\begin{array}{llll}\mathrm{N} & -1.5319335 & 0.3251351 & 0.2251961\end{array}$

$\begin{array}{lllll}0 & -1.0960922 & -1.5563854 & -1.0901889\end{array}$

$\mathrm{H} \quad-3.7045660 \quad-1.9924818 \quad-0.5023893$

H $\quad-3.7713141 \quad-0.5401494 \quad-1.5483737$

$\mathrm{H} \quad-4.8881678 \quad 0.4215350 \quad 0.4576227$

H $\quad-3.9359462-0.6518713 \quad 1.5377560$

H $\quad-2.9754235 \quad 1.9019345 \quad 0.0997182$

$\mathrm{H} \quad-2.6030173 \quad 1.3606050 \quad 1.7604014$

$\mathrm{H} \quad-0.1411512 \quad 1.4334040 \quad-0.7959414$

C $\quad 0.7775098 \quad 1.8154386-1.3246676$

C $\quad 1.9961832 \quad 1.2786404-1.1054120$

$\begin{array}{llll}\text { P } & 2.2284481 & -0.0935162 & 0.0514919\end{array}$

$\begin{array}{llll}\text { C } & 1.1697427 & 0.1205367 & 1.4938354\end{array}$

$\begin{array}{llll}\text { C } & 1.8094539 & -1.6554384 & -0.7524986\end{array}$

$\begin{array}{llll}\text { C } & 4.0031142 & -0.0734902 & 0.5194594\end{array}$

$\mathrm{H} \quad 1.2745066 \quad-0.7606641 \quad 2.1635385$

$\mathrm{H} \quad 1.4458263 \quad 1.0501342 \quad 2.0363172$

$\mathrm{H} \quad 0.0805660 \quad 0.1962930 \quad 1.0938186$

H $\quad 2.0530628 \quad-2.5051333 \quad-0.0773033$

H $\quad 0.6753131 \quad-1.6176485 \quad-0.9383941$

H $\quad 2.3659715 \quad-1.7573630 \quad-1.7085831$

H $\quad 4.2096033 \quad-0.9044493 \quad 1.2273691$

H $\quad 4.6393763 \quad-0.2044240 \quad-0.3828054$

$\begin{array}{llll}\mathrm{H} & 4.2579700 & 0.8913177 & 1.0089302\end{array}$

$\mathrm{H} \quad 0.6694470 \quad 2.6334098 \quad-2.0625745$

H $2.9015281 \quad 1.6192915-1.6441652$

\section{C_PPh3}

E(BP86/def2-SV(P) $)=-1399.422884966$

C $\quad-3.2369220 \quad-0.6122720 \quad-0.2860800$

$\begin{array}{llll}\text { C } & -4.7021980 & -0.7077830 & 0.2270390\end{array}$

$\begin{array}{llll}\text { C } & -5.2893470 & 0.6424950 & -0.2020890\end{array}$

C $\quad-4.3751660 \quad 1.0205200 \quad-1.4055020$

$\begin{array}{lllll}\mathrm{N} & -3.0959800 & 0.3661720 & -1.1788640\end{array}$

$\begin{array}{llll}0 & -2.3393510 & -1.4114370 & 0.1218340\end{array}$

$\mathrm{H} \quad-4.7360060-0.9141890 \quad 1.3188910$

$\mathrm{H} \quad-5.2024320 \quad-1.5676130 \quad-0.2808520$

H $\quad-6.3720890 \quad 0.6185290 \quad-0.4604270$

$\mathrm{H} \quad-5.1565980 \quad 1.3897040 \quad 0.6139520$

$\mathrm{H} \quad-4.8430130 \quad 0.6764440 \quad-2.3689070$

$\mathrm{H} \quad-4.2576960 \quad 2.1274090 \quad-1.5083450$

H $\quad-1.4759330 \quad 0.3948160 \quad-2.0517130$

C $\quad-0.5438150 \quad 0.2069570 \quad-2.6962240$

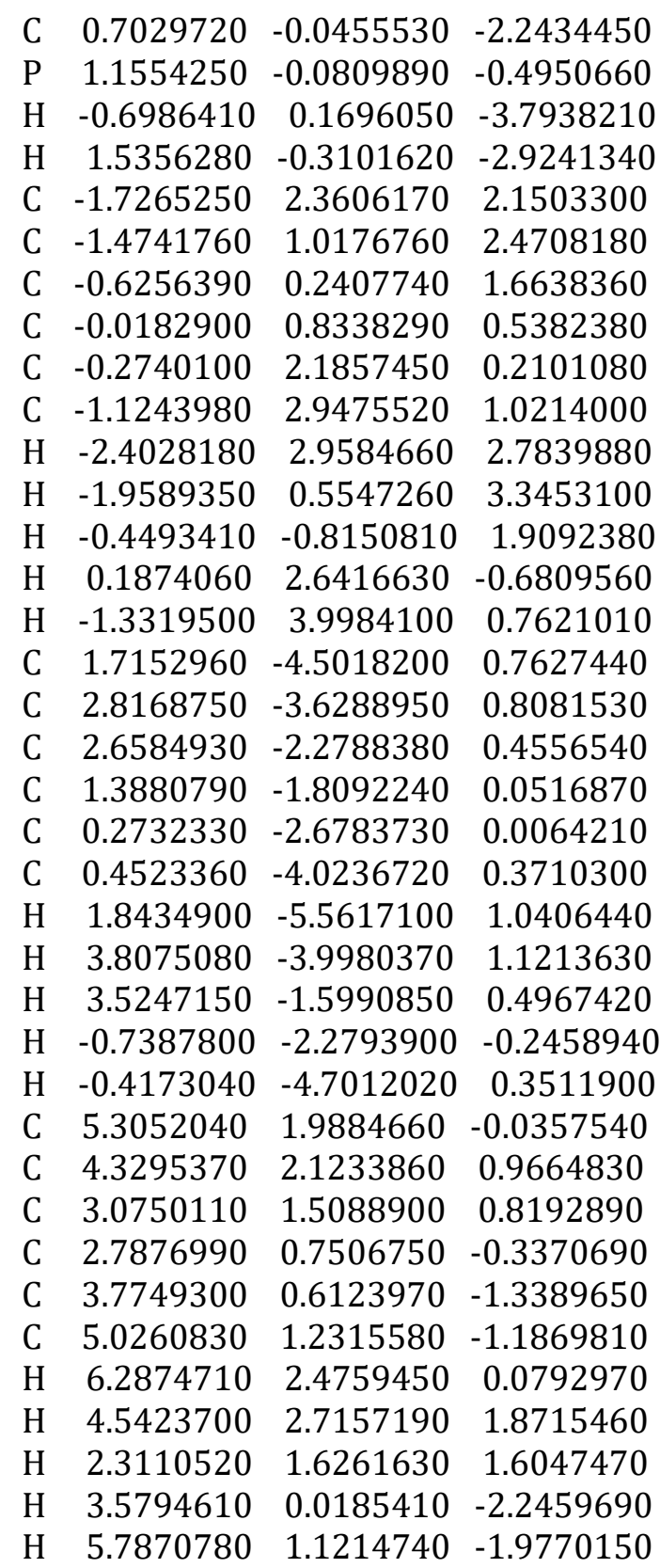

D

$\mathrm{E}(\mathrm{BP} 86 /$ def2-SV(P) $)=-1178.186259651$

$\begin{array}{llll}\text { C } & -0.7957313 & -3.5002187 & 2.2296318\end{array}$

$\begin{array}{llll}\text { C } & -0.2887226 & -4.9473877 & 2.1110203\end{array}$

$\begin{array}{llll}\text { C } & -1.3932429 & -5.6706813 & 1.3205000\end{array}$

$\begin{array}{llll}\text { C } & -2.0790881 & -4.5311464 & 0.5294228\end{array}$

$\begin{array}{llll}\mathrm{N} & -1.8449875 & -3.3571499 & 1.3577321\end{array}$

$\begin{array}{lllll}0 & -0.3191718 & -2.6117039 & 2.9435462\end{array}$

$\begin{array}{lllll}\mathrm{H} & -0.0744867 & -5.3749898 & 3.1127339\end{array}$

H $\quad 0.6777690 \quad-4.9185817 \quad 1.5557218$

$\begin{array}{llll}\mathrm{H} & -1.0153937 & -6.4761931 & 0.6560105\end{array}$

$\begin{array}{llll}\mathrm{H} & -2.1238893 & -6.1307475 & 2.0217430\end{array}$

$\begin{array}{llll}\mathrm{H} & -1.6301362 & -4.3847281 & -0.4845354\end{array}$ 


$\begin{array}{cccc}\text { H } & -3.1709913 & -4.7022131 & 0.3863868 \\ \mathrm{C} & -2.4433534 & -2.0386481 & 1.0405450 \\ \mathrm{C} & -1.9929906 & -1.4150037 & -0.2541397 \\ \mathrm{H} & -3.5497336 & -2.1908684 & 1.0505715 \\ \mathrm{H} & -2.2012488 & -1.4191800 & 1.9322611 \\ \mathrm{P} & -0.9163222 & -0.0952890 & -0.3389006 \\ \mathrm{H} & -2.6007991 & -1.5774097 & -1.1615734 \\ \mathrm{C} & -0.5706297 & 0.2171452 & -2.1389330 \\ \mathrm{C} & -1.5407029 & 1.5628368 & 0.3318146 \\ \mathrm{C} & 0.6369854 & -0.4709719 & 0.6105894 \\ \mathrm{C} & 0.8139313 & 0.7733112 & -2.5131285 \\ \mathrm{H} & -0.7274206 & -0.7712984 & -2.6257615 \\ \mathrm{H} & -1.3738298 & 0.8940239 & -2.5117061 \\ \mathrm{C} & -0.8817678 & 2.8942677 & -0.0703004 \\ \mathrm{H} & -2.6116555 & 1.5624963 & 0.0230126 \\ \mathrm{H} & -1.5482617 & 1.4477438 & 1.4416722 \\ \mathrm{C} & 1.5796355 & 0.6423833 & 1.0957073 \\ \mathrm{H} & 0.2691755 & -1.0547688 & 1.4904834 \\ \mathrm{H} & 1.1815385 & -1.2113120 & -0.0196790 \\ \mathrm{C} & 0.9864687 & 0.9546868 & -4.0323119 \\ \mathrm{H} & 0.9879170 & 1.7512610 & -2.0087133 \\ \mathrm{H} & 1.6077270 & 0.0863900 & -2.1383564 \\ \mathrm{C} & -1.5577275 & 4.1158138 & 0.5821391 \\ \mathrm{H} & 0.1984637 & 2.8951955 & 0.1966513 \\ \mathrm{H} & -0.9256322 & 3.0131896 & -1.1784859 \\ \mathrm{C} & 2.7745286 & 0.0744168 & 1.8864394 \\ \mathrm{H} & 1.9618226 & 1.2472360 & 0.2414453 \\ \mathrm{H} & 1.0210394 & 1.3468267 & 1.7541925 \\ \mathrm{C} & 2.3650110 & 1.5009889 & -4.4262919 \\ \mathrm{H} & 0.8115880 & -0.0240084 & -4.5387044 \\ \mathrm{H} & 0.1910893 & 1.6392061 & -4.4111623 \\ \mathrm{C} & -0.9303999 & 5.4538637 & 0.1692923 \\ \mathrm{H} & -2.6427351 & 4.1141978 & 0.3224363 \\ \mathrm{H} & -1.5117956 & 4.0075485 & 1.6914629 \\ \mathrm{C} & 3.7044392 & 1.1572191 & 2.4475723 \\ \mathrm{H} & 2.3874519 & -0.5619234 & 2.7153667 \\ \mathrm{H} & 3.1638509 & 1.8323025 & 3.1494658 \\ \mathrm{H} & 2.4550150 & 1.6173187 & -5.5288721 \\ \mathrm{H} & 2.5532433 & 2.4982143 & -3.9671439 \\ \mathrm{H} & 3.1816274 & 0.8212325 & -4.0928870 \\ \mathrm{H} & -1.4413762 & 6.3116933 & 0.6601569 \\ \mathrm{H} & 0.1466014 & 5.5010519 & 0.4493344 \\ & -0.9952653 & 5.6107895 & -0.9315333 \\ \mathrm{H} & 4.5576603 & 0.7109609 & 3.0050935 \\ & & & \end{array}$

\section{D_PMe3}

$\mathrm{E}(\mathrm{BP} 86 / \mathrm{def} 2-\mathrm{SV}(\mathrm{P}))=-824.6741783988$ $\begin{array}{llll}C & -0.6017083 & -0.8671556 & 1.6503154\end{array}$ $\begin{array}{llll}\text { C } & -0.5903855 & -2.3498288 & 2.0573876\end{array}$

$\begin{array}{cccc}\mathrm{C} & -1.8208386 & -2.9414285 & 1.3472246 \\ \mathrm{C} & -2.0327871 & -1.9912950 & 0.1449670 \\ \mathrm{~N} & -1.4897734 & -0.7245811 & 0.6183157 \\ \mathrm{O} & 0.1018264 & 0.0313438 & 2.1271421 \\ \mathrm{H} & -0.5904399 & -2.4595033 & 3.1618718 \\ \mathrm{H} & 0.3631508 & -2.7934529 & 1.6858064 \\ \mathrm{H} & -1.6931370 & -3.9988725 & 1.0331140 \\ \mathrm{H} & -2.7068882 & -2.8949128 & 2.0178239 \\ \mathrm{H} & -1.4831567 & -2.3422176 & -0.7651675 \\ \mathrm{H} & -3.1045695 & -1.8835952 & -0.1378806 \\ \mathrm{C} & -1.5670110 & 0.5313649 & -0.1781239 \\ \mathrm{C} & -0.5501759 & 0.7297978 & -1.2636692 \\ \mathrm{H} & -2.5961226 & 0.5486162 & -0.6053254 \\ \mathrm{H} & -1.5229200 & 1.3470165 & 0.5761879 \\ \mathrm{P} & 1.0091281 & 1.3546874 & -0.9737388 \\ \mathrm{C} & 1.6949928 & 2.0301668 & -2.5449982 \\ \mathrm{C} & 0.9790429 & 2.6991618 & 0.2770928 \\ \mathrm{H} & -0.6586036 & 0.1539803 & -2.2010330 \\ \mathrm{C} & 2.4195616 & 0.2910285 & -0.3503431 \\ \mathrm{H} & 2.7395991 & 2.3870680 & -2.4143660 \\ \mathrm{H} & 1.6863454 & 1.2260718 & -3.3132599 \\ \mathrm{H} & 1.0491315 & 2.8599391 & -2.9000126 \\ \mathrm{H} & 1.9895561 & 3.1467252 & 0.3925665 \\ \mathrm{H} & 0.2477085 & 3.4769533 & -0.0264046 \\ \mathrm{H} & 0.6688277 & 2.2400623 & 1.2415848 \\ \mathrm{H} & 3.3803526 & 0.8500655 & -0.2782040 \\ \mathrm{H} & 2.1294218 & -0.0815502 & 0.6559920 \\ \mathrm{H} & 2.5498720 & -0.5756558 & -1.0348656\end{array}$

\section{D_PPh3}

E(BP86/def2-SV(P)) $=-1399.472482614$

$\begin{array}{llll}C & -2.2172985 & -2.0620663 & 2.2316618\end{array}$

$\begin{array}{llll}\text { C } & -2.2725527 & -3.5962781 & 2.3612866\end{array}$

C $-3.0085273-4.0563810 \quad 1.0912292$

$\begin{array}{llll}\text { C } & -2.7364678 & -2.9212471 & 0.0725093\end{array}$

N $-2.4886610-1.7596234 \quad 0.9129496$

$\begin{array}{llll}0 & -1.9515531 & -1.2545948 & 3.1182878\end{array}$

H $\quad-2.7614231 \quad-3.8882525 \quad 3.3136828$

$\mathrm{H} \quad-1.2250353 \quad-3.9766149 \quad 2.4046404$

H $\quad-2.6785997 \quad-5.0475706 \quad 0.7150084$

H $\quad-4.1007177 \quad-4.1222273 \quad 1.2904010$

$\mathrm{H} \quad-1.8536432-3.1379549 \quad-0.5793042$

H $\quad-3.6020320-2.7390008 \quad-0.6062058$

$\begin{array}{llll}\text { C } & -2.4432525 & -0.3860681 & 0.3758977\end{array}$

$\begin{array}{llll}\text { C } & -1.4496236 & -0.1072650 & -0.7193837\end{array}$

H $-3.4732168-0.1519676 \quad 0.0074943$

$\mathrm{H} \quad-2.2650260 \quad 0.2497946 \quad 1.2718066$

P $\quad 0.09438290 .5738122 \quad-0.5076808$

H $\quad-1.6497214 \quad-0.4744154 \quad-1.7406388$

C $1.4217206 \quad 2.0670428-4.7189654$

$\begin{array}{llll}\text { C } & 1.1764363 & 3.0042773 & -3.7006335\end{array}$ 


$\begin{array}{cccc}\mathrm{C} & 0.7973344 & 2.5737177 & -2.4174414 \\ \mathrm{C} & 0.6673652 & 1.1967339 & -2.1411363 \\ \mathrm{C} & 0.9323809 & 0.2571099 & -3.1636813 \\ \mathrm{C} & 1.2961968 & 0.6931326 & -4.4482304 \\ \mathrm{H} & 1.7156011 & 2.4079797 & -5.7257046 \\ \mathrm{H} & 1.2788878 & 4.0832357 & -3.9049232 \\ \mathrm{H} & 0.6007887 & 3.3164282 & -1.6270348 \\ \mathrm{H} & 0.8567686 & -0.8229691 & -2.9542529 \\ \mathrm{H} & 1.4933398 & -0.0479395 & -5.2409398 \\ \mathrm{C} & -0.1729724 & 4.1604006 & 2.4663326 \\ \mathrm{C} & -1.1582456 & 3.9510430 & 1.4848367 \\ \mathrm{C} & -1.0530997 & 2.8626502 & 0.6039551 \\ \mathrm{C} & 0.0435792 & 1.9755397 & 0.6944906 \\ \mathrm{C} & 1.0238735 & 2.1857455 & 1.6866226 \\ \mathrm{C} & 0.9134922 & 3.2748913 & 2.5684383 \\ \mathrm{H} & -0.2593382 & 5.0113072 & 3.1626398 \\ \mathrm{H} & -2.0187236 & 4.6365522 & 1.4084128 \\ \mathrm{H} & -1.8281032 & 2.6736094 & -0.1578768 \\ \mathrm{H} & 1.8717350 & 1.4891873 & 1.7832221 \\ \mathrm{H} & 1.6795341 & 3.4266848 & 3.3468514 \\ \mathrm{C} & 3.6897088 & -2.0995710 & 0.9016200 \\ \mathrm{C} & 3.9573238 & -0.8970081 & 0.2221957 \\ \mathrm{C} & 2.8980289 & -0.0722671 & -0.1935490 \\ \mathrm{C} & 1.5607372 & -0.4476366 & 0.0635497 \\ \mathrm{C} & 1.3009762 & -1.6593273 & 0.7325555 \\ \mathrm{C} & 2.3604844 & -2.4794335 & 1.1571292 \\ \mathrm{H} & 4.5217314 & -2.7470885 & 1.2258130 \\ \mathrm{H} & 4.9989113 & -0.6008212 & 0.0112919 \\ \mathrm{H} & 3.1167447 & 0.8672911 & -0.7297756 \\ \mathrm{H} & 0.2527653 & -1.9593617 & 0.9040512 \\ \mathrm{H} & 2.1470053 & -3.4232152 & 1.6864947\end{array}$

E

$\mathrm{E}(\mathrm{BP} 86 /$ def2-SV $(\mathrm{P}))=-1464.629554339$

C $-2.0640051 \quad-4.5515667-1.1731302$

$\begin{array}{llll}\text { C } & -3.4698840 & -4.9683722 & -1.6265054\end{array}$

$\begin{array}{llll}\text { C } & -4.4163562 & -4.3130131 & -0.6030019\end{array}$

$\begin{array}{llll}\text { C } & -3.6097013 & -3.1126265 & -0.0480880\end{array}$

$\begin{array}{lllll}\mathrm{N} & -2.2199539 & -3.5379806 & -0.2486351\end{array}$

$\begin{array}{lllll}0 & -0.9829573 & -4.9852391 & -1.5669094\end{array}$

$\begin{array}{llll}\mathrm{H} & -3.5501510 & -6.0729595 & -1.6962595\end{array}$

$\begin{array}{llll}\mathrm{H} & -3.6186974 & -4.5662806 & -2.6555625\end{array}$

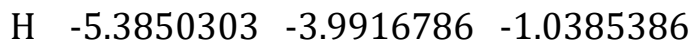

$\begin{array}{llll}\mathrm{H} & -4.6389201 & -5.0301648 & 0.2173814\end{array}$

$\begin{array}{llll}\mathrm{H} & -3.8102653 & -2.1491854 & -0.5760223\end{array}$

$\begin{array}{llll}\mathrm{H} & -3.8046042 & -2.9374514 & 1.0347698\end{array}$

$\begin{array}{llll}\text { C } & -1.0774173 & -2.7757303 & 0.2124406\end{array}$

$\begin{array}{llll}\text { C } & -0.7372205 & -1.6326581 & -0.7709828\end{array}$

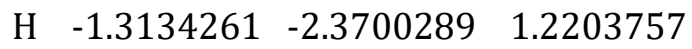

$\begin{array}{llll}\mathrm{H} & -0.2327390 & -3.4943988 & 0.3047491\end{array}$

$\begin{array}{llll}\mathrm{H} & -0.4584303 & -2.0668602 & -1.7583833\end{array}$
$\begin{array}{llll}\mathrm{P} & 0.6161801 & -0.5137493 & -0.2984004\end{array}$

$\begin{array}{lllll}\mathrm{H} & -1.6499500 & -0.9539504 & -0.9109365\end{array}$

$\begin{array}{llll}\text { C } & -3.1742832 & 1.1027649 & -0.3816638\end{array}$

$\begin{array}{llll}\text { C } & -4.4659306 & 1.9507494 & -0.2399271\end{array}$

$\begin{array}{llll}\text { C } & -4.0682029 & 2.9835645 & 0.8211724\end{array}$

$\begin{array}{llll}\text { C } & -2.5229044 & 3.0352022 & 0.6509356\end{array}$

$\begin{array}{llll}\mathrm{N} & -2.1106301 & 1.7301268 & 0.1405942\end{array}$

$\begin{array}{lllll}0 & -3.1823454 & -0.0236014 & -0.9626491\end{array}$

$\begin{array}{llll}\mathrm{H} & -5.3391060 & 1.3151984 & 0.0206733\end{array}$

$\begin{array}{llll}\mathrm{H} & -4.6895794 & 2.4221420 & -1.2270160\end{array}$

$\begin{array}{lllll}\mathrm{H} & -4.5576183 & 3.9770258 & 0.7100859\end{array}$

$\begin{array}{llll}\mathrm{H} & -4.3202674 & 2.5998217 & 1.8364170\end{array}$

$\begin{array}{llll}\mathrm{H} & -2.2427783 & 3.8566221 & -0.0635237\end{array}$

$\begin{array}{llll}\mathrm{H} & -2.0072080 & 3.2844429 & 1.6112067\end{array}$

$\begin{array}{llll}\text { C } & 0.5161968 & 0.8817610 & -1.4805012\end{array}$

$\begin{array}{llll}\text { C } & 0.2699951 & 0.0936383 & 1.3945578\end{array}$

$\begin{array}{llll}\text { C } & 2.2024838 & -1.4592149 & -0.4442098\end{array}$

C $\quad 1.1098896 \quad 1.2581896 \quad 1.9496296$

$\begin{array}{llll}\mathrm{H} & 0.2909343 & -0.7866530 & 2.0775003\end{array}$

$\begin{array}{llll}\mathrm{H} & -0.7889294 & 0.4639750 & 1.2593480\end{array}$

C $\quad \begin{array}{llll}1.7447116 & 1.7883301 & -1.6634777\end{array}$

$\begin{array}{llll}\mathrm{H} & -0.3897088 & 1.4308506 & -1.0763900\end{array}$

$\begin{array}{lllll}\mathrm{H} & 0.2145507 & 0.4287845 & -2.4529847\end{array}$

H $\quad 1.9810104 \quad-2.4805836-0.0566342$

$\begin{array}{llll}\text { C } & 3.4679111 & -0.9024661 & 0.2346653\end{array}$

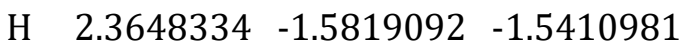

C $\quad 0.6813049 \quad 1.6256161 \quad 3.3821583$

$\begin{array}{llll}\mathrm{H} & 2.1987884 & 1.0250741 & 1.9421788\end{array}$

$\begin{array}{llll}\mathrm{H} & 0.9640851 & 2.1469883 & 1.2960361\end{array}$

C $\quad 1.4532261 \quad 2.9522333 \quad-2.6286604$

$\begin{array}{llll}\mathrm{H} & 2.0700712 & 2.2051215 & -0.6833237\end{array}$

$\begin{array}{llll}\mathrm{H} & 2.6095017 & 1.2011300 & -2.0548594\end{array}$

$\begin{array}{llll}\text { C } & 4.7134153 & -1.7542380 & -0.0724993\end{array}$

$\begin{array}{lllll}\mathrm{H} & 3.6516856 & 0.1480921 & -0.0868789\end{array}$

$\begin{array}{llll}\mathrm{H} & 3.3128001 & -0.8683579 & 1.3370505\end{array}$

$\begin{array}{llll}\text { C } & 2.6570486 & 3.8791600 & -2.8393739\end{array}$

$\begin{array}{lllll}\mathrm{H} & 1.1220707 & 2.5373716 & -3.6094663\end{array}$

$\begin{array}{lllll}\mathrm{H} & 0.5875744 & 3.5358352 & -2.2381774\end{array}$

$\begin{array}{llll}\text { C } & 5.9818935 & -1.2434647 & 0.6231011\end{array}$

H $44.5179735 \quad-2.8095772 \quad 0.2309080$

H $\quad 4.8751400 \quad-1.7798397 \quad-1.1758826$

$\begin{array}{llll}\text { C } & 1.4573649 & 2.8174453 & 3.9567785\end{array}$

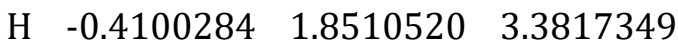

$\begin{array}{llll}\mathrm{H} & 0.8140279 & 0.7373432 & 4.0443309\end{array}$

$\begin{array}{llll}\mathrm{H} & 2.4103771 & 4.7065251 & -3.5402990\end{array}$

$\begin{array}{lllll}\mathrm{H} & 2.9884873 & 4.3401549 & -1.8810695\end{array}$

$\begin{array}{llll}\mathrm{H} & 3.5272373 & 3.3289701 & -3.2647942\end{array}$

H $\quad 6.8601816 \quad-1.87875820 .3751828$

$\begin{array}{llll}\mathrm{H} & 6.2233574 & -0.2013121 & 0.3142787\end{array}$

H $\quad 5.8677535 \quad-1.2465234 \quad 1.7308410$

H $\quad 1.1267915 \quad 3.0528277 \quad 4.9923832$ 
$\begin{array}{llll}\mathrm{H} & 2.5520716 & 2.6137978 & 3.9921311 \\ \mathrm{H} & 1.3063038 & 3.7324653 & 3.3411195\end{array}$

\section{E_Phenol}

$\mathrm{E}(\mathrm{BP} 86 /$ def2-SV(P)) $=-1485.467046297$

$\begin{array}{llll}\text { C } & -2.6990823 & -3.0669847 & 0.7068213\end{array}$

C $-3.3503896-4.4031891 \quad 0.3272763$

$\begin{array}{llll}\text { C } & -4.5979371 & -3.9981699 & -0.4778447\end{array}$

$\begin{array}{llll}\text { C } & -4.2338213 & -2.6055433 & -1.0482235\end{array}$

N $\quad-3.2581114 \quad-2.0931578-0.0869387$

$\begin{array}{llll}0 & -1.8035280 & -2.8805241 & 1.5339841\end{array}$

H $\quad-3.5574087 \quad-5.0060686 \quad 1.2354019$

H $\quad-2.6197081 \quad-4.9814137 \quad-0.2852709$

H $\quad-4.8709511-4.7202869-1.2747393$

H $\quad-5.4726541 \quad-3.9012158 \quad 0.2014489$

H $\quad-3.7901645 \quad-2.6783011-2.0721248$

H $\quad-5.1109262-1.9244162-1.1169085$

C $\quad-2.8872656-0.6881283 \quad 0.0107946$

C $-1.7655451-0.1846868-0.9162246$

H $\quad-3.7955458 \quad-0.0876715 \quad-0.2170453$

H $\quad-2.6318655-0.4825851 \quad 1.0710506$

$\mathrm{H} \quad-1.8950104 \quad-0.5358964 \quad-1.9645871$

P $\quad-0.0106583 \quad-0.4181070 \quad-0.4240053$

H $\quad-1.7877415 \quad 0.9582183 \quad-0.8779866$

$\begin{array}{lllll}0 & -1.1178057 & 2.4702191 & -0.1115538\end{array}$

C $\quad 0.8631824 \quad 0.7348469 \quad-1.5565447$

$\begin{array}{llll}\text { C } & 0.1017435 & 0.1232993 & 1.3215773\end{array}$

C $\quad 0.5298167 \quad-2.1661036-0.6623543$

C $\quad 1.4788530 \quad 0.5077322 \quad 1.8924030$

H $\quad-0.3854298 \quad-0.6841865 \quad 1.9152372$

$\mathrm{H} \quad-0.5461255 \quad 1.0400157 \quad 1.2889257$

C $\quad 2.3978370 \quad 0.7120052 \quad-1.6472379$

$\mathrm{H} \quad 0.4516764 \quad 1.7183399 \quad-1.1965686$

$\mathrm{H} \quad 0.4143927 \quad 0.5548370 \quad-2.5616839$

H $\quad-0.2525459-2.7754648 \quad-0.1591365$

C $\quad 1.9086144 \quad-2.5710435-0.1045202$

H $\quad 0.4682930 \quad-2.3616456 \quad-1.7586789$

C $\quad 1.3767709 \quad 0.8830999 \quad 3.3826962$

H $\quad 2.2206547 \quad-0.3147107 \quad 1.7717893$

H $\quad 1.8715197 \quad 1.3839619 \quad 1.3291904$

C $\quad 2.9269883 \quad 1.8713034-2.5132063$

$\mathrm{H} \quad 2.8499437 \quad 0.7832541 \quad-0.6328629$

H $\quad 2.7497357 \quad-0.2538906 \quad-2.0795687$

C $\quad 2.2512620-4.0380550-0.4233422$

H $\quad 2.7096903 \quad-1.9106512 \quad-0.5058472$

$\mathrm{H} \quad 1.9090035 \quad-2.4331706 \quad 1.0001136$

C $4.4557106 \quad 1.8837056-2.6383174$

$\mathrm{H} \quad 2.4672586 \quad 1.8083328 \quad-3.5275773$

$\mathrm{H} \quad 2.5732803 \quad 2.8346471 \quad-2.0797865$

$\begin{array}{llll}\text { C } & 3.6022019 & -4.4803521 & 0.1537215\end{array}$

H $\quad 1.4411827 \quad-4.6943655 \quad-0.0273292$
H $\quad 2.2510395 \quad-4.1830713 \quad-1.5297133$

C $\quad 2.7176646 \quad 1.3170216 \quad 3.9883073$

$\mathrm{H} \quad 0.6293274 \quad 1.7019399 \quad 3.4954546$

$\mathrm{H} \quad 0.9703272 \quad 0.0136349 \quad 3.9511042$

$\mathrm{H} \quad 4.8005494 \quad 2.7333095 \quad-3.2676238$

$\mathrm{H} \quad 4.9440027 \quad 1.9853486-1.6426923$

$\mathrm{H} \quad 4.8359663 \quad 0.9455836 \quad-3.1032121$

H $\quad 3.8198567 \quad-5.5417653-0.0968534$

H $\quad 4.4374551 \quad-3.8626251 \quad-0.2471353$

$\mathrm{H} \quad 3.6196374 \quad-4.3872624 \quad 1.2631193$

$\mathrm{H} \quad 2.6078034 \quad 1.5789964 \quad 5.0635370$

$\mathrm{H} \quad 3.4793954 \quad 0.5071929 \quad 3.9162507$

$\mathrm{H} \quad 3.1274954 \quad 2.2108317 \quad 3.4664114$

C $\quad-2.5853834 \quad 6.3990081 \quad 0.1190153$

$\begin{array}{llll}\text { C } & -3.1368038 & 5.4760552 & -0.7911948\end{array}$

$\begin{array}{llll}\text { C } & -2.6505602 & 4.1637682 & -0.8753637\end{array}$

$\begin{array}{llll}\text { C } & -1.5768846 & 3.6966242 & -0.0438186\end{array}$

$\begin{array}{llll}\text { C } & -1.0338327 & 4.6617597 & 0.8703746\end{array}$

C $\quad-1.5274536 \quad 5.9711351 \quad 0.9459546$

$\mathrm{H} \quad-2.9711075 \quad 7.4303188 \quad 0.1822045$

$\mathrm{H} \quad-3.9667418 \quad 5.7877452 \quad-1.4522077$

H $\quad-3.0904653 \quad 3.4518229-1.5975943$

$\mathrm{H} \quad-0.2031967 \quad 4.3375505 \quad 1.5231070$

H $\quad-1.0774816 \quad 6.6772448 \quad 1.6681523$

\section{E_PMe3}

$\mathrm{E}(\mathrm{BP} 86 /$ def2-SV(P)) $=-1111.112137321$

$\begin{array}{llll}C & -0.7637957 & -2.3766014 & 2.1604363\end{array}$

C $\quad-1.4308974 \quad-3.7243168 \quad 2.4656944$

C $\quad-2.7926030 \quad-3.6353119 \quad 1.7538419$

C $-2.5435906-2.6203394 \quad 0.6127081$

N $-1.4536396-1.7923094 \quad 1.1292575$

$\begin{array}{llll}0 & 0.2410850 & -1.9049529 & 2.7016781\end{array}$

H $\quad-1.4876784-3.8920395 \quad 3.5609765$

H $\quad-0.7849971 \quad-4.5315707 \quad 2.0471552$

H $-3.1671138-4.6091768 \quad 1.3762993$

$\mathrm{H} \quad-3.5580204 \quad-3.2283155 \quad 2.4501378$

$\mathrm{H} \quad-2.2453975-3.1286298 \quad-0.3383243$

H $-3.4370806-1.9972036 \quad 0.3871927$

C $-1.0684976-0.5165256 \quad 0.5318043$

$\begin{array}{llll}\text { C } & 0.1106190 & -0.5447718 & -0.4585058\end{array}$

$\mathrm{H} \quad-1.9532779-0.1389151 \quad-0.0228790$

$\mathrm{H} \quad-0.8760458 \quad 0.2327229 \quad 1.3265693$

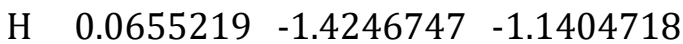

P $\quad 1.8194095 \quad-0.4003856 \quad 0.1816366$

$\mathrm{H} \quad 0.0545059 \quad 0.4079475 \quad-1.0976960$

$\begin{array}{llll}\text { C } & -0.1958143 & 2.9215735 & -0.7283525\end{array}$

C $-0.8503432 \quad 4.2452645 \quad-1.2110481$

C $\quad-0.3374813 \quad 4.3614330-2.6518888$

$\begin{array}{llll}\text { C } & -0.0670855 & 2.8733981 & -3.0169420\end{array}$

$\begin{array}{llll}\mathrm{N} & 0.2500008 & 2.1928467 & -1.7656715\end{array}$ 


$\begin{array}{crrr}\mathrm{O} & -0.1484212 & 2.6044516 & 0.4946158 \\ \mathrm{H} & -0.5835298 & 5.0902539 & -0.5408127 \\ \mathrm{H} & -1.9604179 & 4.1324598 & -1.1651407 \\ \mathrm{H} & -1.0369360 & 4.8667539 & -3.3549786 \\ \mathrm{H} & 0.6210524 & 4.9286331 & -2.6679808 \\ \mathrm{H} & -0.9755376 & 2.4253255 & -3.5046727 \\ \mathrm{H} & 0.7579090 & 2.7684758 & -3.7628009 \\ \mathrm{C} & 2.7535378 & 0.3377284 & -1.1962198 \\ \mathrm{C} & 1.8771663 & 0.7276470 & 1.5913770 \\ \mathrm{C} & 2.5914712 & -2.0031793 & 0.6127823 \\ \mathrm{H} & 1.4281990 & 0.2095974 & 2.4647614 \\ \mathrm{H} & 1.2383703 & 1.6121087 & 1.2864706 \\ \mathrm{H} & 2.1266323 & 1.2174846 & -1.5261156 \\ \mathrm{H} & 2.8378698 & -0.3875464 & -2.0341791 \\ \mathrm{H} & 2.0724368 & -2.4130135 & 1.5056487 \\ \mathrm{H} & 2.5095379 & -2.7089815 & -0.2418822 \\ \mathrm{H} & 3.7672584 & 0.6454764 & -0.8623665 \\ \mathrm{H} & 2.9290736 & 1.0178206 & 1.8018350 \\ \mathrm{H} & 3.6665454 & -1.8406416 & 0.8460505\end{array}$

\section{E_PPh3}

E(BP86/def2-SV(P)) $=-1685.902849529$

$\begin{array}{llll}\text { C } & -2.3462282 & -2.7608241 & 0.6671333\end{array}$

$\begin{array}{llll}\text { C } & -2.9612413 & -3.9343866 & -0.1092194\end{array}$

$\begin{array}{llll}\text { C } & -3.7629177 & -3.2601883 & -1.2359404\end{array}$

$\begin{array}{llll}\text { C } & -3.0363749 & -1.9094923 & -1.4460124\end{array}$

$\begin{array}{llll}\mathrm{N} & -2.4203949 & -1.6495587 & -0.1488202\end{array}$

$\begin{array}{llll}0 & -1.8483942 & -2.7918623 & 1.7904342\end{array}$

$\mathrm{H} \quad-3.5606113 \quad-4.5758826 \quad 0.5687199$

$\begin{array}{llll}\mathrm{H} & -2.1250681 & -4.5596409 & -0.5006777\end{array}$

$\mathrm{H} \quad-3.8160740 \quad-3.8575703 \quad-2.1697234$

H $\quad-4.8054773 \quad-3.0711890-0.8983066$

$\mathrm{H} \quad-2.2653763 \quad-1.9797906-2.2539501$

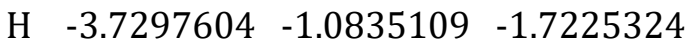

$\begin{array}{llll}\text { C } & -1.9494244 & -0.3362771 & 0.2591249\end{array}$

$\begin{array}{lllll}\text { C } & -0.7147760 & 0.2013825 & -0.4976795\end{array}$

$\mathrm{H} \quad-2.7633056 \quad 0.4080426 \quad 0.1116222$

$\begin{array}{llll}\mathrm{H} & -1.7692947 & -0.3909298 & 1.3519871\end{array}$

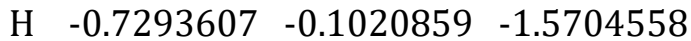

$\begin{array}{llll}\text { P } & 0.9221746 & -0.3152009 & 0.1059614\end{array}$

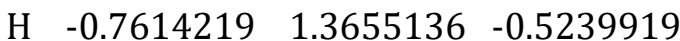

$\begin{array}{llll}\text { C } & -1.8199506 & 3.3967934 & 0.4485490\end{array}$

$\begin{array}{lllll}\text { C } & -2.6313079 & 4.7057935 & 0.2485801\end{array}$

$\begin{array}{llll}\text { C } & -1.9404360 & 5.3433222 & -0.9639003\end{array}$

$\begin{array}{lllll}\text { C } & -1.3469816 & 4.0998706 & -1.6852862\end{array}$

$\begin{array}{lllll}\mathrm{N} & -1.0831225 & 3.1162353 & -0.6413153\end{array}$

$\begin{array}{llll}0 & -1.9046799 & 2.7034056 & 1.4967873\end{array}$

$\begin{array}{llll}\mathrm{H} & -2.6310131 & 5.3204071 & 1.1738980\end{array}$

$\mathrm{H} \quad-3.6942771 \quad 4.4378751 \quad 0.0363311$

$\mathrm{H} \quad-2.6086804 \quad 5.9510919-1.6142485$

H $\quad-1.1115970 \quad 6.0051232-0.6231451$
$\begin{array}{lrrr}\mathrm{H} & -2.0790545 & 3.7091182 & -2.4447463 \\ \mathrm{H} & -0.4228344 & 4.3513861 & -2.2616986 \\ \mathrm{C} & 4.1863922 & 2.2259396 & -1.9925878 \\ \mathrm{C} & 2.9117803 & 2.7634914 & -1.7466280 \\ \mathrm{C} & 1.9059814 & 1.9857227 & -1.1457710 \\ \mathrm{C} & 2.1918416 & 0.6445927 & -0.7987533 \\ \mathrm{C} & 3.4786624 & 0.1058183 & -1.0293113 \\ \mathrm{C} & 4.4702704 & 0.8973696 & -1.6295026 \\ \mathrm{H} & 4.9667243 & 2.8463974 & -2.4644219 \\ \mathrm{H} & 2.6891082 & 3.8080920 & -2.0200983 \\ \mathrm{H} & 0.9077203 & 2.4376179 & -0.9329316 \\ \mathrm{H} & 3.7122976 & -0.9332568 & -0.7449018 \\ \mathrm{H} & 5.4711816 & 0.4724768 & -1.8123852 \\ \mathrm{C} & 1.6299083 & 0.7052734 & 4.5603037 \\ \mathrm{C} & 0.5864476 & 1.3236154 & 3.8537163 \\ \mathrm{C} & 0.3327643 & 1.0010118 & 2.5073936 \\ \mathrm{C} & 1.1449160 & 0.0315235 & 1.8791765 \\ \mathrm{C} & 2.2121287 & -0.5814022 & 2.5793383 \\ \mathrm{C} & 2.4466668 & -0.2462731 & 3.9202195 \\ \mathrm{H} & 1.8162291 & 0.9659931 & 5.6158641 \\ \mathrm{H} & -0.0528361 & 2.0779098 & 4.3411054 \\ \mathrm{H} & -0.4988571 & 1.5416505 & 1.9858419 \\ \mathrm{H} & 2.8639801 & -1.3189341 & 2.0823316 \\ \mathrm{H} & 3.2738750 & -0.7284851 & 4.4668794 \\ \mathrm{C} & 1.4985227 & -4.8310491 & -0.8346722 \\ \mathrm{C} & 1.1537072 & -4.4297723 & 0.4666835 \\ \mathrm{C} & 1.0090125 & -3.0663190 & 0.7761781 \\ \mathrm{C} & 1.2193526 & -2.0925169 & -0.2247622 \\ \mathrm{C} & 1.5471328 & -2.5012892 & -1.5403861 \\ \mathrm{C} & 1.6886826 & -3.8647083 & -1.8397179 \\ \mathrm{H} & 1.6152610 & -5.9020297 & -1.0704760 \\ \mathrm{H} & 0.9842063 & -5.1810975 & 1.2550057 \\ \mathrm{H} & 0.7009906 & -2.7685686 & 1.7890795 \\ \mathrm{H} & 1.7010016 & -1.7519941 & -2.3345469 \\ \mathrm{H} & 1.9522092 & -4.1737705 & -2.8647411 \\ & & & \end{array}$

$\mathbf{F}$

$\mathrm{E}(\mathrm{BP} 86 /$ def2-SV(P)) $=-1178.148749271$

C $\quad 0.7027076 \quad-3.4902182 \quad-0.1933169$

$\begin{array}{llll}\text { C } & 0.9712987 & -4.9117605 & 0.3123194\end{array}$

C $\quad-0.2832873-5.2482833 \quad 1.1348408$

$\begin{array}{llll}\text { C } & -1.3701185 & -4.3484629 & 0.5113969\end{array}$

$\begin{array}{llll}\mathrm{N} & -0.6321691 & -3.2312780 & -0.0706519\end{array}$

$\begin{array}{lllll}0 & 1.5725653 & -2.7108243 & -0.6631727\end{array}$

$\mathrm{H} \quad 1.9191265 \quad-4.9428750 \quad 0.8931031$

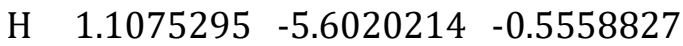

H $\quad-0.5661592 \quad-6.3231868 \quad 1.1142244$

$\begin{array}{llll}\mathrm{H} & -0.1269030 & -4.9656303 & 2.1995387\end{array}$

H $\quad-1.9606741 \quad-4.8774116 \quad-0.2800360$

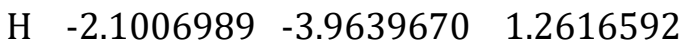

$\begin{array}{llll}\text { C } & -1.3580194 & -2.0392031 & -0.4027978\end{array}$ 


$\begin{array}{cccc}\mathrm{C} & -0.8130026 & -1.1875086 & -1.5119501 \\ \mathrm{H} & -1.4978897 & -0.9682331 & -2.3725574 \\ \mathrm{H} & 0.1423340 & -1.5905982 & -1.9165737 \\ \mathrm{P} & -0.3803255 & 0.3805689 & -0.7327677 \\ \mathrm{C} & 0.3259334 & 1.5839704 & -1.9749848 \\ \mathrm{C} & -1.9333461 & 1.1007006 & -0.0601672 \\ \mathrm{C} & 0.8758388 & 0.0307424 & 0.5608666 \\ \mathrm{C} & 0.3101937 & 3.0945536 & -1.6817575 \\ \mathrm{H} & 1.3640961 & 1.2313988 & -2.1780427 \\ \mathrm{H} & -0.2494558 & 1.3788543 & -2.9102491 \\ \mathrm{C} & -1.8251191 & 2.0587082 & 1.1385079 \\ \mathrm{H} & -2.4682737 & 1.5849749 & -0.9128088 \\ \mathrm{H} & -2.4940895 & 0.1829281 & 0.2434058 \\ \mathrm{C} & 1.8246644 & 1.1655454 & 0.9810772 \\ \mathrm{H} & 0.3079693 & -0.3689276 & 1.4278680 \\ \mathrm{H} & 1.4267053 & -0.8583269 & 0.1452719 \\ \mathrm{H} & -2.4400950 & -2.2454113 & -0.4658349 \\ \mathrm{C} & 0.9378020 & 3.9271574 & -2.8160186 \\ \mathrm{H} & -0.7399830 & 3.4327987 & -1.5230778 \\ \mathrm{H} & 0.8511210 & 3.3082875 & -0.7320754 \\ \mathrm{C} & -3.2039471 & 2.5370295 & 1.6299606 \\ \mathrm{H} & -1.3018395 & 1.5406012 & 1.9748236 \\ \mathrm{H} & -1.2012269 & 2.9455952 & 0.8816039 \\ \mathrm{C} & 2.8368055 & 0.6898593 & 2.0408533 \\ \mathrm{H} & 2.3880465 & 1.5445609 & 0.0967294 \\ \mathrm{H} & 1.2552944 & 2.0352330 & 1.3858455 \\ \mathrm{C} & 0.9090241 & 5.4374443 & -2.5479710 \\ \mathrm{H} & 1.9908334 & 3.5944072 & -2.9725070 \\ \mathrm{H} & 0.4041119 & 3.7052562 & -3.7704447 \\ \mathrm{C} & -3.1236298 & 3.4722759 & 2.8432342 \\ \mathrm{H} & -3.7317816 & 3.0531524 & 0.7934079 \\ \mathrm{H} & -3.8283176 & 1.6476229 & 1.8812435 \\ \mathrm{C} & 3.8202997 & 1.7835257 & 2.4760488 \\ \mathrm{H} & 2.2804757 & 0.3106020 & 2.9299441 \\ \mathrm{H} & 3.3956637 & -0.1858217 & 1.6370476 \\ \mathrm{H} & 1.3734734 & 6.0063978 & -3.3833543 \\ \mathrm{H} & -0.1338716 & 5.8094766 & -2.4278955 \\ \mathrm{H} & 1.4645827 & 5.6949956 & -1.6177262 \\ \mathrm{H} & -4.1349612 & 3.8013597 & 3.1689661 \\ \mathrm{H} & -2.6385690 & 2.9706528 & 3.7109798 \\ \mathrm{H} & -2.5314107 & 4.3871894 & 2.6130645 \\ \mathrm{H} & 4.5346073 & 1.4048845 & 3.2402158 \\ & 3.2896383 & 2.6577158 & 2.9179682\end{array}$

\section{F_PMe3}

$\mathrm{E}(\mathrm{BP} 86 / \mathrm{def} 2-\mathrm{SV}(\mathrm{P}))=-824.6308356669$

$\begin{array}{llll}\text { C } & 0.8124592 & -1.8620923 & -0.1558582\end{array}$

$\begin{array}{llll}\text { C } & 0.8505585 & -3.3951416 & -0.1359829\end{array}$

$\begin{array}{llll}\text { C } & -0.5415819 & -3.7815037 & 0.3921661\end{array}$

$\begin{array}{llll}\text { C } & -1.4257417 & -2.5872150 & -0.0283655\end{array}$

$\begin{array}{crrr}\mathrm{N} & -0.4945205 & -1.4648124 & -0.1138584 \\ \mathrm{O} & 1.8058024 & -1.1056504 & -0.2393709 \\ \mathrm{H} & 1.6949677 & -3.7543901 & 0.4901028 \\ \mathrm{H} & 1.0313382 & -3.7773812 & -1.1698646 \\ \mathrm{H} & -0.9247007 & -4.7465304 & -0.0024060 \\ \mathrm{H} & -0.5177051 & -3.8588730 & 1.5017022 \\ \mathrm{H} & -1.9290220 & -2.7571845 & -1.0146384 \\ \mathrm{H} & -2.2283434 & -2.3633652 & 0.7123616 \\ \mathrm{C} & -0.9729823 & -0.1090084 & -0.1684743 \\ \mathrm{C} & -0.3390257 & 0.7902227 & -1.2086266 \\ \mathrm{H} & -0.8771167 & 1.1064097 & -2.1436867 \\ \mathrm{H} & 0.6684661 & 0.4070835 & -1.4856362 \\ \mathrm{P} & -0.0320184 & 2.1217575 & -0.0313652 \\ \mathrm{C} & 0.7146687 & 3.6657837 & -0.8027442 \\ \mathrm{C} & -1.5888608 & 2.7924315 & 0.6680049 \\ \mathrm{C} & 1.1887361 & 1.6144134 & 1.2240315 \\ \mathrm{H} & 0.8337627 & 4.4899629 & -0.0654354 \\ \mathrm{H} & 1.7145707 & 3.4139864 & -1.2191247 \\ \mathrm{H} & 0.0676814 & 4.0216371 & -1.6344996 \\ \mathrm{H} & -1.3383325 & 3.5420941 & 1.4495405 \\ \mathrm{H} & -2.1772123 & 3.2959030 & -0.1318111 \\ \mathrm{H} & -2.1708288 & 1.9655298 & 1.1189969 \\ \mathrm{H} & 1.9396417 & 2.4240443 & 1.3809525 \\ \mathrm{H} & 0.6842915 & 1.3726820 & 2.1800088 \\ \mathrm{H} & 1.6599400 & 0.6770555 & 0.8318623 \\ \mathrm{H} & -2.0775597 & -0.0938516 & -0.1644240\end{array}$

\section{F_PPh3}

E(BP86/def2-SV(P)) = -1399.436817059

C $0.1486915-3.5954942-1.4019703$

$\begin{array}{llll}\text { C } & -0.2624812 & -5.0711545 & -1.3727701\end{array}$

$\begin{array}{llll}\text { C } & -1.5356138 & -5.0780975 & -0.5111827\end{array}$

$\begin{array}{llll}\text { C } & -2.1093373 & -3.6605994 & -0.7099715\end{array}$

$\begin{array}{llll}\mathrm{N} & -0.9427724 & -2.8391848 & -1.0423135\end{array}$

$\begin{array}{lllll}0 & 1.2765824 & -3.1565811 & -1.7070744\end{array}$

H $\quad 0.5646449 \quad-5.6967626-0.9748132$

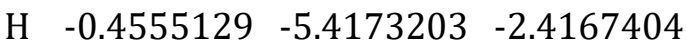

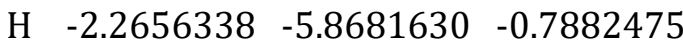

$\mathrm{H} \quad-1.2697061 \quad-5.2282161 \quad 0.5584873$

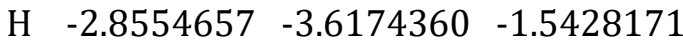

$\mathrm{H} \quad-2.6064975 \quad-3.2583941 \quad 0.2011518$

$\begin{array}{llll}\text { C } & -1.0686425 & -1.4293190 & -0.9648059\end{array}$

$\begin{array}{llll}\text { C } & -0.0788687 & -0.5347667 & -1.6515450\end{array}$

$\mathrm{H} \quad-0.3374070 \quad-0.0068021 \quad-2.6088611$

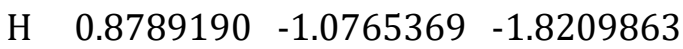

$\begin{array}{lllll}\mathrm{P} & 0.1161482 & 0.5844197 & -0.2506196\end{array}$

$\begin{array}{llll}\mathrm{H} & -2.1231505 & -1.1109162 & -0.9835030\end{array}$

$\begin{array}{llll}\text { C } & 2.4644996 & 4.4298596 & -1.5834317\end{array}$

$\begin{array}{llll}\text { C } & 1.1813847 & 4.5796874 & -1.0311280\end{array}$

C $\quad 0.4663138 \quad 3.4528353 \quad-0.5864134$

$\begin{array}{llll}\text { C } & 1.0343513 & 2.1642318 & -0.6839013\end{array}$ 


\begin{tabular}{|c|c|c|c|}
\hline & 2.3249515 & 0407 & \\
\hline & & & \\
\hline & & & \\
\hline & 0.731 & & \\
\hline & -0.5386270 & 3.5808801 & -0.15 \\
\hline & $2.7 \varepsilon$ & & \\
\hline & 40 & & \\
\hline & $-4.1<$ & 1.9 & \\
\hline & -3.7355701 & 2.010 & -0.3 \\
\hline & -2.4 & 1.61 & -0.7 \\
\hline & -1.5 & 1.1 & 0.2 \\
\hline & -1.9 & & \\
\hline & $-3.2<$ & 1.52 & \\
\hline & -5.16 & 2.29 & 1.2 \\
\hline & -4.4 & 2.36 & -1 \\
\hline & -2.1 & 1.6 & -1. \\
\hline & -1.2 & 0.71 & \\
\hline & -3.56 & 1.48 & 3.0 \\
\hline & 2.747 & -1.108 & 3.1 \\
\hline & 2.187 & 0.177 & 3.2 \\
\hline & 1.39 & 0.6 & 2.2 \\
\hline & 1.1255216 & -0.1429819 & 1.0684747 \\
\hline & 1.7111243 & -1.4276843 & 0.9773672 \\
\hline & 2.520 & -1.8953837 & 2.02270 \\
\hline & 3.371 & -1.4936 & 3.9935977 \\
\hline & 2.3779467 & 0.8123681 & 4.1324598 \\
\hline & 0.9998469 & 1.6975186 & $2.256261<$ \\
\hline & & -2.0582 & $0.082 \mathrm{~S}$ \\
\hline & & & \\
\hline
\end{tabular}

\section{G_cis}

$\mathrm{E}(\mathrm{BP} 86 / \mathrm{def} 2-\mathrm{SV}(\mathrm{P}))=-1523.523916437$

$\begin{array}{llll}\text { C } & -1.4390238 & -3.1330559 & -0.9937508\end{array}$

C $-2.4072288-3.9122171-1.8925694$

$\begin{array}{llll}\text { C } & -3.7968860 & -3.4027776 & -1.4699321\end{array}$

$\begin{array}{llll}\text { C } & -3.5193299 & -1.9823101 & -0.9249726\end{array}$

$\begin{array}{lllll}\mathrm{N} & -2.1289897 & -2.0657912 & -0.4695037\end{array}$

$\begin{array}{lllll}0 & -0.2495815 & -3.3926385 & -0.7858525\end{array}$

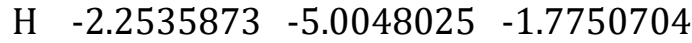

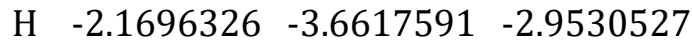

H $\quad-4.5415295 \quad-3.3977646-2.2930432$

H $\quad-4.2014181 \quad-4.0421118-0.6549140$

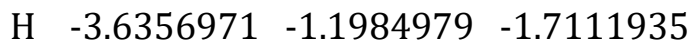

$\begin{array}{llll}\mathrm{H} & -4.1867289 & -1.6906473 & -0.0858901\end{array}$

$\begin{array}{llll}\text { C } & -1.5134862 & -1.1095426 & 0.4318381\end{array}$

$\begin{array}{lllll}\text { C } & -1.0879825 & 0.2422706 & -0.2168891\end{array}$

$\begin{array}{cccc}\mathrm{H} & -2.2122669 & -0.8529181 & 1.2602841\end{array}$

$\begin{array}{llll}\mathrm{H} & -0.6615092 & -1.6509879 & 0.8941163\end{array}$

$\begin{array}{lllll}\mathrm{P} & 0.7178877 & 0.5463371 & -0.0926563\end{array}$

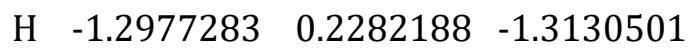

$\begin{array}{llll}\text { C } & -1.8488623 & 1.4853078 & 0.5671885\end{array}$

\begin{tabular}{cccc}
$\mathrm{O}$ & -1.6085053 & 1.4372559 & 1.8569970 \\
$\mathrm{C}$ & 1.0676281 & 2.0647459 & -1.0856268 \\
$\mathrm{C}$ & 1.2301471 & 0.8961035 & 1.6342587 \\
$\mathrm{C}$ & 1.6820767 & -0.8504085 & -0.8218665 \\
$\mathrm{H}$ & 0.5673667 & 1.7519362 & 1.9104427 \\
$\mathrm{H}$ & 2.3026456 & 1.2007294 & 1.5961617 \\
$\mathrm{C}$ & 0.9902891 & -0.2167862 & 2.6710790 \\
$\mathrm{H}$ & 1.6583141 & -0.6849504 & -1.9245922 \\
$\mathrm{C}$ & 3.1303550 & -1.0386316 & -0.3298952 \\
$\mathrm{H}$ & 1.0867732 & -1.7806525 & -0.6464535 \\
$\mathrm{H}$ & 0.5912541 & 1.8936946 & -2.0786872 \\
$\mathrm{C}$ & 2.5400191 & 2.4766078 & -1.2578186 \\
$\mathrm{H}$ & 0.4826774 & 2.8786006 & -0.6002799 \\
$\mathrm{C}$ & 1.6189107 & 0.1303544 & 4.0318572 \\
$\mathrm{H}$ & -0.1121903 & -0.3013720 & 2.7862272 \\
$\mathrm{H}$ & 1.3845392 & -1.2007941 & 2.3243570 \\
$\mathrm{C}$ & 3.8277181 & -2.2118777 & -1.0427955 \\
$\mathrm{H}$ & 3.7286634 & -0.1106942 & -0.4788946 \\
$\mathrm{H}$ & 3.1276506 & -1.2321846 & 0.7670240 \\
$\mathrm{C}$ & 2.6865010 & 3.8030504 & -2.0270630 \\
$\mathrm{H}$ & 3.0285831 & 2.5791673 & -0.2615427 \\
$\mathrm{H}$ & 3.0985481 & 1.6789948 & -1.7994766 \\
$\mathrm{C}$ & 1.3353042 & -0.9260156 & 5.1075246 \\
$\mathrm{H}$ & 2.7219283 & 0.2582368 & 3.9138979 \\
$\mathrm{H}$ & 1.2252005 & 1.1179868 & 4.3655138 \\
$\mathrm{C}$ & 5.2557937 & -2.4599242 & -0.5406697 \\
$\mathrm{H}$ & 3.2129051 & -3.1313613 & -0.9107586 \\
$\mathrm{H}$ & -3.5 .0946702 & 1.8288771 & -1.9665223 \\
$\mathrm{H}$ & -3.8763459 & 1.0918982 & 2.2041285 \\
$\mathrm{H}$ & -6.3919499 & 0.9722496 & 1.7142528 \\
$\mathrm{H}$ & 3.8458473 & -2.0132559 & -2.1405509 \\
$\mathrm{C}$ & 4.1467087 & 4.2296691 & -2.2241373 \\
$\mathrm{H}$ & 2.1863309 & 3.7065809 & -3.0191780 \\
$\mathrm{H}$ & 2.1314539 & 4.6022576 & -1.4825947 \\
$\mathrm{H}$ & 1.7931942 & -0.6452523 & 6.0818444 \\
$\mathrm{H}$ & 0.2410919 & -1.0471531 & 5.2716121 \\
$\mathrm{H}$ & 1.7426130 & -1.9217664 & 4.8183410 \\
$\mathrm{H}$ & 5.7300475 & -3.3106598 & -1.0775735 \\
$\mathrm{H}$ & 5.9029065 & -1.5658338 & -0.6904065 \\
$\mathrm{H}$ & 5.2673504 & -2.7024120 & 0.5461513 \\
$\mathrm{H}$ & 4.2138496 & 5.1903443 & -2.7800829 \\
$\mathrm{H}$ & 4.6639107 & 4.3696276 & -1.2480234 \\
$\mathrm{H}$ & 4.7195300 & 3.4679535 & -2.8001641 \\
$\mathrm{C}$ & -6.1209758 & 1.3282182 & -0.4172375 \\
$\mathrm{C}$ & -5.1911776 & 1.5730407 & -1.4465705 \\
$\mathrm{C}$ & -3.8163861 & 1.6148286 & -1.1551469 \\
$\mathrm{H}$ & -3.2881543 & 1.1916730 & 1.1848041 \\
$\mathrm{H}$ & 1.1424054 & 0.9006231 \\
\hline
\end{tabular}




\section{H $\quad-1.4731883 \quad 2.4193931 \quad 0.0083085$}

\section{H_cis}

$\mathrm{E}(\mathrm{BP} 86 / \mathrm{def} 2-\mathrm{SV}(\mathrm{P}))=-1523.538937017$

C $\quad-1.4269417 \quad-2.0522317 \quad 2.2833415$

$\begin{array}{llll}\text { C } & -1.5963232 & -3.5637919 & 2.4916377\end{array}$

C $-2.8418246 \quad-3.9212610 \quad 1.6620910$

$\begin{array}{llll}\text { C } & -2.8745101 & -2.8336389 & 0.5607305\end{array}$

$\begin{array}{lllll}\mathrm{N} & -2.1725804 & -1.7076804 & 1.1718825\end{array}$

$\begin{array}{lllll}0 & -0.7369339 & -1.2844238 & 2.9499852\end{array}$

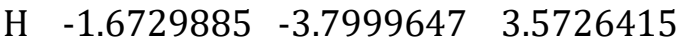

$\mathrm{H} \quad-0.6768362 \quad-4.0666232 \quad 2.1107449$

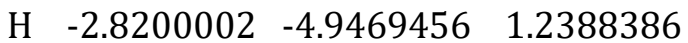

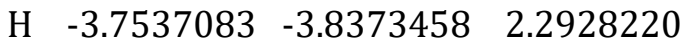

H $\quad-2.3572058 \quad-3.1739369-0.3703145$

$\begin{array}{llll}\mathrm{H} & -3.9097860 & -2.5386999 & 0.2751320\end{array}$

$\begin{array}{llll}\text { C } & -2.2884502 & -0.3454060 & 0.6801469\end{array}$

$\begin{array}{llll}\text { C } & -1.5692287 & -0.0153262 & -0.6499752\end{array}$

$\begin{array}{llll}\mathrm{H} & -3.3725589 & -0.1303253 & 0.5322870\end{array}$

$\begin{array}{llll}\mathrm{H} & -1.9377302 & 0.3235088 & 1.4941399\end{array}$

$\begin{array}{lllll}\mathrm{P} & 0.3066217 & 0.3293181 & -0.5009715\end{array}$

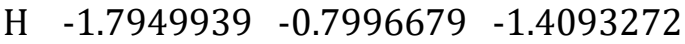

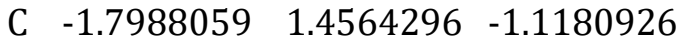

$\begin{array}{lllll}0 & -0.6361278 & 2.0401373 & -0.6098718\end{array}$

$\begin{array}{lllll}\text { C } & -5.4700554 & 3.4463800 & 0.1218438\end{array}$

$\begin{array}{llll}\text { C } & -4.2309988 & 3.8444818 & 0.6519954\end{array}$

$\begin{array}{lllll}\text { C } & -3.0478724 & 3.1966140 & 0.2545605\end{array}$

$\begin{array}{llll}\text { C } & -3.0870874 & 2.1414066 & -0.6761850\end{array}$

$\begin{array}{llll}\text { C } & -4.3341111 & 1.7584839 & -1.2167972\end{array}$

$\begin{array}{llll}\text { C } & -5.5181555 & 2.3998433 & -0.8176218\end{array}$

$\begin{array}{llll}\mathrm{H} & -6.3980325 & 3.9555394 & 0.4323398\end{array}$

$\begin{array}{llll}\mathrm{H} & -4.1836927 & 4.6730861 & 1.3792307\end{array}$

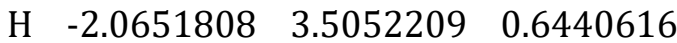

$\begin{array}{llll}\mathrm{H} & -4.3784606 & 0.9509811 & -1.9704147\end{array}$

$\begin{array}{llll}\mathrm{H} & -6.4848073 & 2.0874899 & -1.2483794\end{array}$

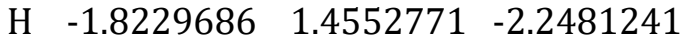

$\begin{array}{llll}\text { C } & 1.1496626 & 0.7992052 & 1.0857629\end{array}$

$\begin{array}{llll}\text { C } & 0.8018098 & -1.5446370 & -0.5087024\end{array}$

$\begin{array}{llll}\text { C } & 1.2062121 & 0.9117932 & -2.0382540\end{array}$

$\begin{array}{llll}\text { C } & 1.1961644 & 2.2769002 & 1.4947870\end{array}$

$\begin{array}{llll}\mathrm{H} & 0.6475323 & 0.1844038 & 1.8706192\end{array}$

$\begin{array}{llll}\mathrm{H} & 2.1825882 & 0.3943191 & 0.9924926\end{array}$

$\begin{array}{lllll}\text { C } & 2.1488724 & -2.0440201 & 0.0418873\end{array}$

$\begin{array}{llll}\mathrm{H} & -0.0127456 & -2.0584606 & 0.0487248\end{array}$

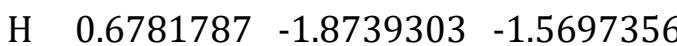

$\begin{array}{llll}\text { C } & 2.5011219 & 0.2404507 & -2.5184126\end{array}$

H $\quad 0.43562880 .8675866 \quad-2.8439067$

H $\quad 1.3629850 \quad 1.9954820 \quad-1.8547632$

C $\quad 2.0262964 \quad 2.4934655 \quad 2.7735946$

$\begin{array}{llll}\mathrm{H} & 1.6157833 & 2.8943750 & 0.6679745\end{array}$

$\begin{array}{llll}\mathrm{H} & 0.1612218 & 2.6490962 & 1.6449273\end{array}$

$\begin{array}{cccc}\text { C } & 2.3850633 & -3.5456757 & -0.2112648 \\ \text { H } & 2.9992459 & -1.4732696 & -0.3961806 \\ \text { H } & 2.1881706 & -1.8608448 & 1.1403856 \\ \text { C } & 3.0377080 & 0.8882258 & -3.8101307 \\ \text { H } & 3.2855552 & 0.3149272 & -1.7294903 \\ \text { H } & 2.3431617 & -0.8461377 & -2.7018035 \\ \text { C } & 2.0701686 & 3.9602277 & 3.2229252 \\ \text { H } & 1.6071797 & 1.8633833 & 3.5932909 \\ \text { H } & 3.0669891 & 2.1240106 & 2.6095472 \\ \text { C } & 3.7030413 & -4.0670818 & 0.3771832 \\ \text { H } & 1.5314138 & -4.1251787 & 0.2155733 \\ \text { H } & 2.3630200 & -3.7403205 & -1.3100788 \\ \text { C } & 4.3464247 & 0.2609648 & -4.3087451 \\ \text { H } & 2.2592802 & 0.8128014 & -4.6062457 \\ \text { H } & 3.1876086 & 1.9797402 & -3.6370500 \\ \text { H } & 2.6746910 & 4.0856342 & 4.1486795 \\ \text { H } & 2.5172035 & 4.6117778 & 2.4377535 \\ \text { H } & 1.0477440 & 4.3483810 & 3.4330941 \\ \text { H } & 3.8410864 & -5.1525427 & 0.1744209 \\ \text { H } & 4.5788255 & -3.5316106 & -0.0551717 \\ \text { H } & 3.7384318 & -3.9237859 & 1.4811930 \\ \text { H } & 4.7021496 & 0.7510702 & -5.2419256 \\ \text { H } & 5.1568295 & 0.3562589 & -3.5506619 \\ \text { H } & 4.2200318 & -0.8239133 & -4.5266711\end{array}$

\section{H_trans}

$\mathrm{E}(\mathrm{BP} 86 /$ def2-SV(P) $)=-1523.540781051$

$\begin{array}{llll}\text { C } & -0.3663190 & -3.9305177 & 0.8764895\end{array}$

$\begin{array}{llll}\text { C } & -0.4267553 & -5.1154228 & -0.0970349\end{array}$

$\begin{array}{llll}\text { C } & -1.8136136 & -4.9929590 & -0.7512364\end{array}$

$\begin{array}{llll}\text { C } & -2.1331809 & -3.4808791 & -0.6585137\end{array}$

$\begin{array}{llll}\mathrm{N} & -1.3499365 & -3.0382037 & 0.4918623\end{array}$

$\begin{array}{lllll}0 & 0.4209144 & -3.7681717 & 1.8061686\end{array}$

H $-0.2481922 \quad-6.0686851 \quad 0.4411247$

H $0.4009536 \quad-4.9948669-0.8340046$

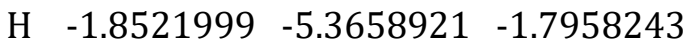

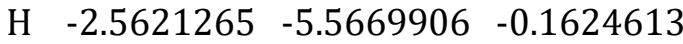

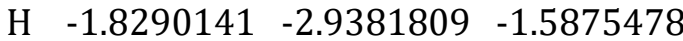

$\begin{array}{lllll}\mathrm{H} & -3.2160364 & -3.2755973 & -0.4995101\end{array}$

C $\quad-1.6114473-1.7957166 \quad 1.1974551$

$\begin{array}{llll}\text { C } & -1.4615830 & -0.4976074 & 0.3842595\end{array}$

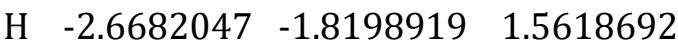

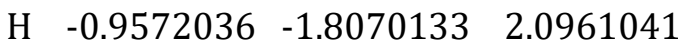

$\begin{array}{lllll}\mathrm{P} & 0.1588829 & 0.5075906 & 0.2532772\end{array}$

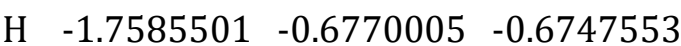

$\begin{array}{llll}\text { C } & -2.2379272 & 0.7499957 & 0.8933325\end{array}$

$\begin{array}{llll}0 & -1.3705804 & 1.7523827 & 0.4650965\end{array}$

$\begin{array}{llll}\text { C } & 0.4698995 & 1.4880009 & -1.3131179\end{array}$

$\begin{array}{llll}\text { C } & 0.8462032 & 1.2038493 & 1.8413042\end{array}$

$\begin{array}{llll}\text { C } & 1.3398907 & -0.9833822 & 0.0191291\end{array}$

$\begin{array}{llll}\text { C } & -6.2745152 & 1.0402950 & -0.7212868\end{array}$ 


$\begin{array}{cccc}\mathrm{C} & -5.1993340 & 1.6779691 & -1.3666675 \\ \mathrm{C} & -3.9029804 & 1.5955257 & -0.8324753 \\ \mathrm{C} & -3.6585444 & 0.8717663 & 0.3522348 \\ \mathrm{C} & -4.7464056 & 0.2551225 & 1.0051658 \\ \mathrm{C} & -6.0444573 & 0.3310433 & 0.4706020 \\ \mathrm{H} & -7.2933844 & 1.1085337 & -1.1385907 \\ \mathrm{H} & -5.3760250 & 2.2542013 & -2.2911119 \\ \mathrm{H} & -3.0536842 & 2.1115693 & -1.3082973 \\ \mathrm{H} & -4.5800596 & -0.2761741 & 1.9596316 \\ \mathrm{H} & -6.8842704 & -0.1549783 & 0.9959243 \\ \mathrm{H} & -2.3184658 & 0.6835746 & 2.0190459 \\ \mathrm{H} & 1.7835023 & 0.6679215 & 2.1038013 \\ \mathrm{H} & 0.0941739 & 0.9362703 & 2.6187339 \\ \mathrm{C} & 1.0653451 & 2.7253379 & 1.8419311 \\ \mathrm{H} & 1.0930944 & -1.4171590 & -0.9816590 \\ \mathrm{C} & 2.8683173 & -0.8601129 & 0.1702715 \\ \mathrm{H} & 1.0066263 & -1.7407054 & 0.7636594 \\ \mathrm{H} & -0.3697191 & 1.1734738 & -1.9751899 \\ \mathrm{H} & 0.2250486 & 2.5334234 & -1.0311468 \\ \mathrm{C} & 1.7953860 & 1.4211629 & -2.0822076 \\ \mathrm{C} & 1.4951667 & 3.2641381 & 3.2186322 \\ \mathrm{H} & 1.8425603 & 2.9963574 & 1.0881703 \\ \mathrm{H} & 0.1160220 & 3.2083669 & 1.5236743 \\ \mathrm{C} & 3.5892597 & -2.1972148 & -0.0913172 \\ \mathrm{H} & 3.2918565 & -0.0834018 & -0.5033206 \\ \mathrm{H} & 3.1170307 & -0.5314256 & 1.2061730 \\ \mathrm{C} & 1.7563711 & 2.2821270 & -3.3605503 \\ \mathrm{H} & 2.6328237 & 1.7748070 & -1.4381685 \\ \mathrm{H} & 3.9201320 & 2.6347619 & -3.5433736 \\ \mathrm{H} & 2.0368193 & 0.3713954 & -2.3640599 \\ \mathrm{C} & 1.7233306 & 4.7816095 & 3.2249124 \\ \mathrm{H} & 0.7142362 & 3.0021290 & 3.9709966 \\ \mathrm{H} & 2.4256385 & 2.7457155 & 3.5519195 \\ \mathrm{C} & 5.1065790 & -2.1262143 & 0.1257755 \\ \mathrm{H} & 3.1477765 & -2.9790945 & 0.5695396 \\ \mathrm{H} & 3.3787309 & -2.5253435 & -1.1374730 \\ \mathrm{C} & 3.0693622 & 2.2552404 & -4.1537269 \\ \mathrm{H} & 0.9190592 & 1.9331001 & -4.0101713 \\ \mathrm{H} & 1.5065809 & 3.3340607 & -3.0857682 \\ \mathrm{H} & 2.0185481 & 5.1456256 & 4.2340145 \\ \mathrm{H} & 2.5293874 & 5.0733264 & 2.5137119 \\ & 0.8008415 & 5.3285869 & 2.9249079 \\ \mathrm{H} & 5.5935724 & -3.1063518 & -0.0753567 \\ \mathrm{H} & -1.2212257 & -4.4770396\end{array}$

\section{I_cis}

$\mathrm{E}(\mathrm{BP} 86 / \mathrm{def} 2-\mathrm{SV}(\mathrm{P}))=-633.9330174075$ C $\quad-0.8607188 \quad-3.23753110 .6098058$ $\begin{array}{cccc}\text { C } & -2.1818685 & -3.7023738 & -0.0216077 \\ \text { C } & -2.9397845 & -2.3982211 & -0.3249489 \\ \text { C } & -1.8181325 & -1.3410325 & -0.4759822 \\ \text { N } & -0.7346726 & -1.8852565 & 0.3342296 \\ \text { O } & -0.0439602 & -3.9176918 & 1.2200741 \\ \text { H } & -2.7131887 & -4.4010948 & 0.6568178 \\ \text { H } & -1.9332384 & -4.2731073 & -0.9465689 \\ \text { H } & -3.5842353 & -2.4538231 & -1.2267732 \\ \text { H } & -3.5902808 & -2.1291797 & 0.5356273 \\ \text { H } & -1.5004953 & -1.2120167 & -1.5402536 \\ \text { H } & -2.1289971 & -0.3382268 & -0.1034829 \\ \text { C } & 0.4767935 & -1.1471050 & 0.6672631 \\ \text { C } & 1.3173488 & -0.8029628 & -0.5400755 \\ \text { H } & 1.0535012 & -1.8453816 & 1.3180541 \\ \text { H } & 0.2267463 & -0.2476280 & 1.2710273 \\ \text { C } & 1.7869339 & 0.4057011 & -0.9418437 \\ \text { H } & 2.4911935 & 0.3904920 & -1.7964344 \\ \text { H } & 1.6393901 & -1.6848617 & -1.1266081 \\ \text { C } & 1.2379497 & 4.3802507 & 0.6723396 \\ \text { C } & 2.4791083 & 3.9716865 & 0.1533107 \\ \text { C } & 2.6325316 & 2.6803311 & -0.3724121 \\ \text { C } & 1.5600337 & 1.7531932 & -0.3731709 \\ \text { C } & 0.3107465 & 2.1916307 & 0.1316723 \\ \text { C } & 0.1535281 & 3.4857890 & 0.6518134 \\ \text { H } & 1.1126608 & 5.3968324 & 1.0808101 \\ \text { H } & 3.3343965 & 4.6680704 & 0.1513486 \\ \text { H } & 3.6092227 & 2.3697497 & -0.7814423 \\ \text { H } & -0.5610437 & 1.5199507 & 0.0845355 \\ \text { H } & -0.8314690 & 3.8038171 & 1.0328751\end{array}$

\section{I_trans}

$\mathrm{E}(\mathrm{BP} 86 / \mathrm{def} 2-\mathrm{SV}(\mathrm{P}))=-633.9395740834$

C $\quad-0.6907871 \quad-2.4543329 \quad 2.5043179$

$\begin{array}{llll}\text { C } & -1.9948188 & -3.2266433 & 2.2501782\end{array}$

$\begin{array}{llll}\text { C } & -3.0194834 & -2.1351784 & 1.8913569\end{array}$

$\begin{array}{llll}\text { C } & -2.1542157 & -0.9839475 & 1.3224442\end{array}$

$\begin{array}{llll}\mathrm{N} & -0.8630338 & -1.1877243 & 1.9696419\end{array}$

$\begin{array}{llll}0 & 0.3278728 & -2.8639845 & 3.0489334\end{array}$

H $\quad-2.2612280 \quad-3.8413347 \quad 3.1344623$

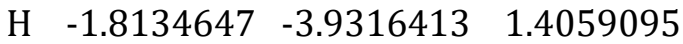

$\begin{array}{llll}\mathrm{H} & -3.7965247 & -2.4688360 & 1.1724178\end{array}$

H $\quad-3.5405225 \quad-1.7891901 \quad 2.8109210$

H $\quad-2.0613008$ - $1.0375844 \quad 0.2099603$

$\begin{array}{llll}\mathrm{H} & -2.5640779 & 0.0226247 & 1.5671775\end{array}$

$\begin{array}{llll}\text { C } & 0.2141579 & -0.2093027 & 1.9375772\end{array}$

$\begin{array}{llll}\text { C } & 0.7383283 & 0.0598180 & 0.5492579\end{array}$

$\begin{array}{llll}\mathrm{H} & 1.0140022 & -0.6510422 & 2.5767875\end{array}$

$\begin{array}{llll}\mathrm{H} & -0.1247351 & 0.7425338 & 2.4117438\end{array}$

$\begin{array}{llll}\text { C } & 0.7664708 & 1.2859186 & -0.0274797\end{array}$

H $\quad 0.3635355 \quad 2.13517690 .5597336$

$\begin{array}{llll}\mathrm{H} & 1.1213023 & -0.8269334 & 0.0092132\end{array}$ 


$\begin{array}{lrrr}\mathrm{C} & 2.2490535 & 2.4629024 & -3.9071229 \\ \mathrm{C} & 1.6763465 & 3.4045430 & -3.0359577 \\ \mathrm{C} & 1.2005299 & 3.0010805 & -1.7786802 \\ \mathrm{C} & 1.2833823 & 1.6506478 & -1.3574351 \\ \mathrm{C} & 1.8661452 & 0.7153045 & -2.2504526 \\ \mathrm{C} & 2.3406059 & 1.1160347 & -3.5061026 \\ \mathrm{H} & 2.6256778 & 2.7753547 & -4.8952755 \\ \mathrm{H} & 1.5994699 & 4.4628158 & -3.3368239 \\ \mathrm{H} & 0.7519211 & 3.7464806 & -1.0995311 \\ \mathrm{H} & 1.9541213 & -0.3430742 & -1.9556560 \\ \mathrm{H} & 2.7912695 & 0.3695139 & -4.1815172\end{array}$

\section{NVP}

$\mathrm{E}(\mathrm{BP} 86 / \mathrm{def} 2-\mathrm{SV}(\mathrm{P}))=-363.7703950595$
C $\quad 0.2935019-0.2073216 \quad 1.4102444$
$\begin{array}{llll}\text { C } & -0.9805786 & -1.0587290 & 1.4371857\end{array}$
$\begin{array}{llll}\text { C } & -1.7257383 & -0.6748540 & 0.1472921\end{array}$
$\begin{array}{llll}\text { C } & -0.6053453 & -0.2028178 & -0.8102658\end{array}$
$\begin{array}{lllll}\mathrm{N} & 0.4510675 & 0.2479457 & 0.0913879\end{array}$
$\begin{array}{lllll}0 & 1.0646680 & 0.0251484 & 2.3276817\end{array}$
$\begin{array}{llll}\mathrm{H} & -1.5490291 & -0.8788260 & 2.3725289\end{array}$

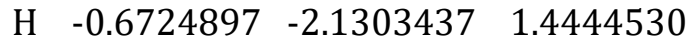
$\begin{array}{llll}\mathrm{H} & -2.3216768 & -1.5036200 & -0.2876976\end{array}$
$\begin{array}{lllll}\mathrm{H} & -2.4224475 & 0.1675356 & 0.3488857\end{array}$
$\mathrm{H} \quad-0.2303874 \quad-1.0261359-1.4658998$
$\begin{array}{llll}\mathrm{H} & -0.9296759 & 0.6287301 & -1.4744169\end{array}$
$\begin{array}{llll}\text { C } & 1.5704176 & 0.9710342 & -0.3098548\end{array}$
$\begin{array}{lllll}\text { C } & 1.8512553 & 1.3670097 & -1.5726189\end{array}$
$\begin{array}{lllll}\mathrm{H} & 2.7701750 & 1.9423198 & -1.7677007\end{array}$
H $\quad 1.2021498 \quad 1.1366491-2.4344172$
$\begin{array}{llll}\text { H } & 2.2341335 & 1.1962756 & 0.5432123\end{array}$

\section{OPBu3}

$\mathrm{E}(\mathrm{BP} 86 /$ def2-SV $(\mathrm{P}))=-889.6376035286$

$\begin{array}{lrrr}\mathrm{O} & 3.3927873 & 1.0468437 & -1.2449394 \\ \mathrm{P} & 2.0241408 & 0.6234895 & -0.7404892 \\ \mathrm{C} & 1.7713188 & -1.2097024 & -0.9480108 \\ \mathrm{C} & 1.8266205 & 1.0281582 & 1.0651238 \\ \mathrm{C} & 0.6718719 & 1.4962019 & -1.6756450 \\ \mathrm{C} & 0.7363098 & -1.9673470 & -0.0990085 \\ \mathrm{H} & 1.5856602 & -1.3673519 & -2.0365487 \\ \mathrm{H} & 2.7944207 & -1.6111861 & -0.7603471 \\ \mathrm{C} & 0.4321836 & 1.1821944 & 1.6942702 \\ \mathrm{H} & 2.4284847 & 0.2653297 & 1.6128928 \\ \mathrm{H} & 2.4006881 & 1.9792161 & 1.1573961 \\ \mathrm{C} & -0.7513357 & 0.9164156 & -1.7386950 \\ \mathrm{H} & 0.6598519 & 2.5403926 & -1.2838640 \\ \mathrm{H} & 1.1055677 & 1.5676592 & -2.7002873 \\ \mathrm{C} & 0.6941948 & -3.4743861 & -0.4160873 \\ \mathrm{H} & 0.9711627 & -1.8355501 & 0.9830551 \\ \mathrm{H} & -0.2812075 & -1.5386594 & -0.2482684\end{array}$

$\begin{array}{lrrr}\mathrm{C} & 0.4904685 & 1.5378503 & 3.1917307 \\ \mathrm{H} & -0.1335198 & 1.9790656 & 1.1579276 \\ \mathrm{H} & -0.1592726 & 0.2470267 & 1.5683014 \\ \mathrm{C} & -1.7045821 & 1.7705113 & -2.5955737 \\ \mathrm{H} & -0.7169398 & -0.1142556 & -2.1621350 \\ \mathrm{H} & -1.1780360 & 0.8155970 & -0.7145914 \\ \mathrm{C} & -0.3132302 & -4.2505640 & 0.4421831 \\ \mathrm{H} & 0.4511209 & -3.6130204 & -1.4960667 \\ \mathrm{H} & 1.7144486 & -3.9039279 & -0.2778663 \\ \mathrm{C} & -0.8924990 & 1.7255185 & 3.8283669 \\ \mathrm{H} & 1.0478323 & 0.7385685 & 3.7348567 \\ \mathrm{H} & 1.0926731 & 2.4675973 & 3.3207688 \\ \mathrm{C} & -3.1236937 & 1.1945225 & -2.6871054 \\ \mathrm{H} & -1.7456238 & 2.8031267 & -2.1757526 \\ \mathrm{H} & -1.2759847 & 1.8769723 & -3.6198117 \\ \mathrm{H} & -0.3200014 & -5.3320330 & 0.1819752 \\ \mathrm{H} & -0.0717372 & -4.1680799 & 1.5262464 \\ \mathrm{H} & -1.3489199 & -3.8657683 & 0.3012188 \\ \mathrm{H} & -0.8115878 & 1.9806864 & 4.9081220 \\ \mathrm{H} & -1.4597837 & 2.5453744 & 3.3320204 \\ \mathrm{H} & -1.5059225 & 0.7996378 & 3.7476604 \\ \mathrm{H} & -3.7830999 & 1.8367118 & -3.3118057 \\ \mathrm{H} & -3.1204716 & 0.1771027 & -3.1401489 \\ \mathrm{H} & -3.5943584 & 1.1100613 & -1.6810681\end{array}$

\section{Pyrrolidone}

$\mathrm{E}(\mathrm{BP} 86 /$ def2-SV(P) $)=-286.4250976048$

C $1.0009746 \quad-0.0250510 \quad 0.9728398$

$\begin{array}{llll}\text { C } & -0.4778469 & -0.4563234 & 1.0453929\end{array}$

$\begin{array}{llll}\text { C } & -1.1065180 & 0.1018536 & -0.2444577\end{array}$

$\begin{array}{lllll}\text { C } & 0.0863268 & 0.1994666 & -1.2310348\end{array}$

$\begin{array}{lllll}\mathrm{N} & 1.2185006 & 0.3722739 & -0.3331365\end{array}$

$\begin{array}{lllll}0 & 1.8339801 & -0.0469736 & 1.8669257\end{array}$

$\begin{array}{llll}\mathrm{H} & -0.9447394 & -0.0907787 & 1.9827773\end{array}$

$\begin{array}{llll}\mathrm{H} & -0.5021038 & -1.5701984 & 1.0812294\end{array}$

$\begin{array}{llll}\mathrm{H} & -1.9328841 & -0.5191894 & -0.6490585\end{array}$

$\mathrm{H} \quad-1.5129115 \quad 1.1198104-0.0569092$

$\mathrm{H} \quad 0.1861409 \quad-0.7292194 \quad-1.8470642$

$\mathrm{H} \quad-0.0148224 \quad 1.0559021 \quad-1.9343450$

H $\quad 2.1659030 \quad 0.5884273-0.6531592$

\section{S1}

$\mathrm{E}(\mathrm{BP} 86 / \mathrm{def} 2-\mathrm{SV}(\mathrm{P}))=-1178.154123694$

C $1.2759035 \quad-1.2882135 \quad-4.0876699$

$\begin{array}{llll}\text { C } & 0.2197516 & -1.5676390 & -5.1541432\end{array}$

$\begin{array}{llll}\text { C } & 0.6149864 & -0.5324359 & -6.2260082\end{array}$

$\begin{array}{llll}\text { C } & 2.1025049 & -0.2273347 & -5.8683069\end{array}$

$\begin{array}{llll}\mathrm{N} & 2.2819159 & -0.5658560 & -4.4556283\end{array}$

$\begin{array}{lllll}0 & 1.1025241 & -1.8417458 & -2.8876756\end{array}$

$\begin{array}{llll}\mathrm{H} & -0.8154143 & -1.4625909 & -4.7632894\end{array}$

H $\quad 0.3241882-2.6201745 \quad-5.5097626$ 


\begin{tabular}{|c|c|c|c|}
\hline & & & \\
\hline & 0.0022418 & 0.3888597 & -6.1 \\
\hline & 199 & & \\
\hline & 488 & 43 & \\
\hline & 3.1444721 & -1.6175079 & -2.427 \\
\hline & 2.1968708 & -1.5651756 & -1.85337 \\
\hline & 2.0877838 & -0.2939008 & \\
\hline & 0342 & -0.0 & \\
\hline & 7339 & -2.4 & -1.2 \\
\hline & 2.4015277 & 0.6205339 & -1.6 \\
\hline & -0.8971603 & 0.1 & -0.5 \\
\hline & 26 & -1.5 & \\
\hline & 1.37 & 1.5 & \\
\hline & 1.86 & -1.9 & \\
\hline & 0.15 & -2.2 & 0.7 \\
\hline & 0.45 & 2270 & 2.7 \\
\hline & -2.05 & 0.0 & \\
\hline & -0.9 & -0.5 & \\
\hline & -0.92 & 234 & \\
\hline & 2.216 & 934 & 1.6 \\
\hline & 0.31 & 2.3 & 1.7 \\
\hline & 1.82 & 2.1 & 0.1 \\
\hline & 0.47 & -2.5 & \\
\hline & 1.15 & -0.5 & \\
\hline & -0.56 & -0.7 & 2.7 \\
\hline & -3.4 & & -0.2 \\
\hline & $-2.0^{\prime}$ & -1. & \\
\hline & -2.02 & 537 & \\
\hline & $0.89^{\prime}$ & $3.5^{\prime}$ & 2.4 \\
\hline & -0.51 & 12 & \\
\hline & -0.1 & 1.6 & 2.5 \\
\hline & 0.10 & -2.2 & 5.0 \\
\hline & -0.21 & -3.2 & 3.10 \\
\hline & 1.49 & -2.9 & 3.49 \\
\hline & -4.65 & 0.0 & 0.67 \\
\hline & $-3.4 \varepsilon$ & 94 & -0.7 \\
\hline & -3.5186359 & 4263 & -1.1 \\
\hline & -0.1427496 & 7808 & 3.1689393 \\
\hline & 1.727 & 429 & 3.0 \\
\hline & 1.36 & 4.2 & 1.6 \\
\hline & 0.135 & -3.2 & 5.60 \\
\hline & 0.8044977 & 9766 & 5.5109109 \\
\hline & -0.9224059 & -1.8 & 5.11884 \\
\hline & -5.61 & 5653 & 0.13 \\
\hline & -4.6755514 & 53776 & 1.11532 \\
\hline & -4.6326764 & 0.7314674 & 1.5231752 \\
\hline & 0.3145035 & 5.3148276 & 3.6202777 \\
\hline & -0.96 & 4.7 & 2.4 \\
\hline & & & \\
\hline
\end{tabular}

S10

\begin{tabular}{|c|c|c|c|}
\hline \multicolumn{4}{|c|}{$(\mathrm{BP} 86 /$ def2-SV(P)) = -1255.425979119 } \\
\hline & 0365 & 783 & \\
\hline & & & \\
\hline & & & \\
\hline & & & \\
\hline & -1.9 & & \\
\hline & & & \\
\hline & & & \\
\hline & -4.8 & & -2 \\
\hline & -3.4 & & \\
\hline & & & \\
\hline & & & \\
\hline & -0.8 & & \\
\hline & -0.5 & & \\
\hline & & & \\
\hline & & & \\
\hline & -0.2 & & \\
\hline & -1.4 & & \\
\hline ) & -1.2 & & \\
\hline & & & \\
\hline & & & \\
\hline & -2.6 & & \\
\hline & & & \\
\hline & & & -0 \\
\hline & & & \\
\hline & & & \\
\hline & & & \\
\hline & & & 453 \\
\hline & 0.95 & -2.6 & -0 . \\
\hline & & & \\
\hline $\mathrm{I}$ & & & \\
\hline & & & \\
\hline & & & \\
\hline & & & \\
\hline & & & \\
\hline & & & \\
\hline & 0.60 & -0.6 & \\
\hline & & & \\
\hline & & & \\
\hline & & & \\
\hline & 2.94 & & \\
\hline & 19 & -0. & \\
\hline & & & \\
\hline 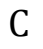 & & & \\
\hline $\mathrm{H}$ & -1.17 & -1.69 & 676 \\
\hline & -0.84 & -3.260 & 4.1 \\
\hline & & -4.7 & \\
\hline & & & \\
\hline $\mathrm{H}$ & 1.92 & -3.0 & 485 \\
\hline 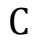 & 4.4474072 & 1.5849331 & 1.1484762 \\
\hline t & 2.6812945 & 2.7189047 & 0.5579863 \\
\hline
\end{tabular}




$\begin{array}{cccc}\mathrm{H} & 2.6986867 & 2.2390598 & 2.2720980 \\ \mathrm{H} & 0.3578694 & -3.1202385 & 6.3219176 \\ \mathrm{H} & 1.5514986 & -3.2152548 & 4.9837923 \\ \mathrm{H} & 1.2233740 & -1.6420353 & 5.7853767 \\ \mathrm{H} & 2.6224426 & -5.4026734 & -1.6848302 \\ \mathrm{H} & 3.2479033 & -4.3661570 & -0.3593583 \\ \mathrm{H} & 1.8116297 & -5.4092526 & -0.0835187 \\ \mathrm{H} & 5.0529191 & 2.4837884 & 1.3984842 \\ \mathrm{H} & 4.7489936 & 0.7723614 & 1.8483377 \\ \mathrm{H} & 4.7327611 & 1.2654507 & 0.1207602 \\ \mathrm{H} & -2.7935697 & -0.4258592 & -0.4441492\end{array}$

\section{S11}

$\mathrm{E}(\mathrm{BP} 86 / \mathrm{def} 2-\mathrm{SV}(\mathrm{P}))=-1255.513068957$

C $\quad-2.2434588 \quad 1.9315020-2.0965592$

$\begin{array}{lllll}\text { C } & -3.7158361 & 2.2636362 & -1.9724413\end{array}$

$\begin{array}{llll}\text { C } & -3.7907489 & 3.6901896 & -2.5606890\end{array}$

C $\quad-2.7048534 \quad 3.6799303 \quad-3.6532950$

$\begin{array}{llll}\mathrm{N} & -1.7318092 & 2.6987274 & -3.1804043\end{array}$

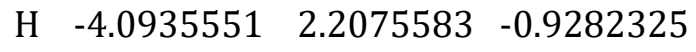

$\mathrm{H} \quad-4.3471633 \quad 1.5700688-2.5833389$

H $\quad-4.7936916 \quad 3.9538872-2.9585206$

$\begin{array}{lllll}\mathrm{H} & -3.5275261 & 4.4347743 & -1.7772545\end{array}$

$\mathrm{H} \quad-3.1113798 \quad 3.3786486-4.6526130$

$\begin{array}{lllll}\mathrm{H} & -2.2226794 & 4.6764024 & -3.7888066\end{array}$

$\begin{array}{llll}\text { C } & -0.5208789 & 2.5136890 & -3.8134652\end{array}$

$\begin{array}{llll}\text { C } & -0.0681827 & 3.1961471 & -4.9044156\end{array}$

$\begin{array}{llll}\mathrm{H} & 0.9256861 & 2.9540851 & -5.3130215\end{array}$

$\begin{array}{lllll}\mathrm{H} & -0.6473127 & 3.9866844 & -5.4107766\end{array}$

$\mathrm{H} \quad 0.0873150 \quad 1.7276002-3.3350966$

C $\quad-1.5504618 \quad 1.0287331-1.3400174$

$\begin{array}{lllll}0 & -0.2518341 & 0.6756670 & -1.4279057\end{array}$

$\begin{array}{lllll}\text { C } & -2.1144446 & 0.2271466 & -0.1907007\end{array}$

$\begin{array}{llll}\mathrm{P} & -0.4355779 & -0.3386542 & 0.4109001\end{array}$

$\begin{array}{llll}\mathrm{H} & -2.6933751 & 0.7665729 & 0.5942099\end{array}$

C $\quad-1.0266573 \quad-1.1977663 \quad 2.0104045$

$\begin{array}{llll}\text { C } & 0.3779572 & -1.7121292 & -0.5508290\end{array}$

$\begin{array}{llll}\text { C } & 0.6422162 & 1.0109328 & 1.0925346\end{array}$

$\begin{array}{llll}\text { C } & -0.0351453 & -1.5955842 & 3.1180920\end{array}$

$\begin{array}{llll}\mathrm{H} & -1.7785260 & -0.4969312 & 2.4453724\end{array}$

$\begin{array}{llll}\mathrm{H} & -1.6124336 & -2.0913762 & 1.6868258\end{array}$

$\begin{array}{llll}\text { C } & 0.9212232 & -2.9721921 & 0.1373371\end{array}$

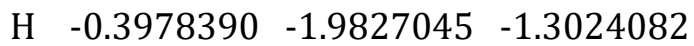

H $\quad 1.1674662 \quad-1.2044742 \quad-1.1425148$

$\begin{array}{llll}\text { C } & 2.0553015 & 1.0812833 & 0.4915935\end{array}$

$\begin{array}{llll}\mathrm{H} & 0.0902709 & 1.9491283 & 0.8614226\end{array}$

$\begin{array}{lllll}\mathrm{H} & 0.6900608 & 0.9130984 & 2.1996157\end{array}$

$\begin{array}{lrrr}\text { C } & -0.7239486 & -2.2636710 & 4.3239926\end{array}$

$\begin{array}{lllll}\mathrm{H} & 0.7450038 & -2.2841166 & 2.7255045\end{array}$

$\mathrm{H} \quad 0.5115094 \quad-0.6938588 \quad 3.4804674$

$\begin{array}{llll}\text { C } & 1.4569759 & -3.9908440 & -0.8868708\end{array}$

$\begin{array}{cccc}\mathrm{H} & 1.7440294 & -2.7029859 & 0.8383679 \\ \mathrm{H} & 0.1311764 & -3.4626184 & 0.7508211 \\ \mathrm{C} & 2.8556914 & 2.2886944 & 1.0116577 \\ \mathrm{H} & 2.6164067 & 0.1449245 & 0.7233383 \\ \mathrm{H} & 1.9576186 & 1.1450827 & -0.6134186 \\ \mathrm{C} & 0.2486963 & -2.6439828 & 5.4483514 \\ \mathrm{H} & -1.5090969 & -1.5784593 & 4.7229507 \\ \mathrm{H} & -1.2662834 & -3.1743992 & 3.9758983 \\ \mathrm{C} & 2.0330998 & -5.2572883 & -0.2400747 \\ \mathrm{H} & 0.6341279 & -4.2691621 & -1.5861455 \\ \mathrm{H} & 2.2367816 & -3.5021138 & -1.5171484 \\ \mathrm{C} & 4.2702702 & 2.3697897 & 0.4234856 \\ \mathrm{H} & 2.2974358 & 3.2233297 & 0.7694728 \\ \mathrm{H} & 2.9155650 & 2.2466597 & 2.1252250 \\ \mathrm{H} & -0.2817519 & -3.1238649 & 6.3002598 \\ \mathrm{H} & 1.0233025 & -3.3596731 & 5.0903066 \\ \mathrm{H} & 0.7798422 & -1.7492367 & 5.8453906 \\ \mathrm{H} & 2.4064844 & -5.9698588 & -1.0081629 \\ \mathrm{H} & 2.8840075 & -5.0164097 & 0.4365847 \\ \mathrm{H} & 1.2654316 & -5.7901873 & 0.3660976 \\ \mathrm{H} & 4.8187320 & 3.2586523 & 0.8066140 \\ \mathrm{H} & 4.8706520 & 1.4677021 & 0.6815709 \\ \mathrm{H} & 4.2404821 & 2.4448585 & -0.6867754 \\ \mathrm{H} & -2.7043684 & -0.6812442 & -0.4727633\end{array}$

\section{S12}

$\mathrm{E}(\mathrm{BP} 86 /$ def2-SV(P) $)=-968.9993635498$

C $0.3605312 \quad 1.5132715 \quad-3.8374558$

$\begin{array}{llll}\text { C } & 1.2049692 & 0.8994393 & -2.9070271\end{array}$

$\begin{array}{lllll}\mathrm{P} & 0.6509498 & 0.3264954 & -1.3401782\end{array}$

$\begin{array}{lllll}\mathrm{H} & 0.8580568 & 2.0030019 & -4.6988882\end{array}$

$\begin{array}{llll}\mathrm{H} & 2.2937123 & 0.8429685 & -3.0771195\end{array}$

$\begin{array}{lllll}\text { C } & -0.2268218 & 1.7010734 & -0.4573366\end{array}$

$\begin{array}{llll}\text { C } & -0.4609771 & -1.1679664 & -1.4435138\end{array}$

$\begin{array}{llll}\text { C } & 2.1747250 & -0.1709836 & -0.4078506\end{array}$

H $\quad-1.4490718-0.7772057-1.7797070$

$\begin{array}{llll}\text { C } & -0.6153840 & -2.0878160 & -0.2193279\end{array}$

$\begin{array}{lllll}\mathrm{H} & -0.0531885 & -1.7379848 & -2.3094473\end{array}$

$\begin{array}{llll}\mathrm{H} & 2.4642087 & -1.1786915 & -0.7854481\end{array}$

$\begin{array}{llll}\text { C } & 2.1101721 & -0.1585129 & 1.1298707\end{array}$

$\begin{array}{llll}\mathrm{H} & 2.9634570 & 0.5334156 & -0.7564821\end{array}$

H $\quad-0.7254855 \quad 2.2690217 \quad-1.2800186$

$\begin{array}{lllll}\text { C } & -1.2459059 & 1.3657924 & 0.6448952\end{array}$

$\begin{array}{llll}\mathrm{H} & 0.5835980 & 2.3608199 & -0.0703807\end{array}$

$\begin{array}{llll}\text { C } & -1.5806552 & -3.2575349 & -0.4894113\end{array}$

$\begin{array}{llll}\mathrm{H} & 0.3784177 & -2.5017127 & 0.0696974\end{array}$

H $\quad-0.9816262 \quad-1.5100332 \quad 0.6593323$

$\begin{array}{llll}\text { C } & 3.4477352 & -0.5681944 & 1.7744859\end{array}$

$\begin{array}{llll}\mathrm{H} & 1.8348916 & 0.8611220 & 1.4839955\end{array}$

H $\quad 1.3071136 \quad-0.8388238 \quad 1.4915119$

$\begin{array}{llll}\text { C } & -1.9135102 & 2.6352198 & 1.2073749\end{array}$ 


$\begin{array}{cccc}\mathrm{H} & -2.0383808 & 0.7003416 & 0.2309695 \\ \mathrm{H} & -0.7631931 & 0.8033332 & 1.4777514 \\ \mathrm{C} & -1.7402702 & -4.1989374 & 0.7112816 \\ \mathrm{H} & -2.5759587 & -2.8479620 & -0.7803267 \\ \mathrm{H} & -1.2188686 & -3.8332875 & -1.3735998 \\ \mathrm{C} & 3.4107055 & -0.5451970 & 3.3079086 \\ \mathrm{H} & 3.7265317 & -1.5888832 & 1.4214113 \\ \mathrm{H} & 4.2530107 & 0.1112622 & 1.4081535 \\ \mathrm{C} & -2.9572366 & 2.3403713 & 2.2925741 \\ \mathrm{H} & -1.1260116 & 3.3102512 & 1.6181815 \\ \mathrm{H} & -2.3870651 & 3.1928451 & 0.3669738 \\ \mathrm{H} & -2.4419907 & -5.0302067 & 0.4809049 \\ \mathrm{H} & -0.7669010 & -4.6535755 & 1.0052213 \\ \mathrm{H} & -2.1403020 & -3.6602894 & 1.6001085 \\ \mathrm{H} & 4.3903402 & -0.8463253 & 3.7395996 \\ \mathrm{H} & 3.1723189 & 0.4719298 & 3.6933972 \\ \mathrm{H} & 2.6398390 & -1.2430214 & 3.7059929 \\ \mathrm{H} & -3.4189464 & 3.2784938 & 2.6715575 \\ \mathrm{H} & -3.7787459 & 1.6987074 & 1.9009077 \\ \mathrm{H} & -2.5047550 & 1.8131330 & 3.1633493 \\ \mathrm{C} & -1.0573812 & 1.7480940 & -3.6916496 \\ \mathrm{C} & -1.5545112 & 3.0164487 & -3.8886664 \\ \mathrm{H} & -1.6525968 & 0.9792715 & -3.1348989 \\ \mathrm{H} & -0.8495431 & 3.6270212 & -4.5286739\end{array}$

\section{S13}

\begin{tabular}{lccc}
\multicolumn{4}{c}{ E(BP86/def2-SV(P) $=-1046.342971750$} \\
C & -3.5555284 & 1.0952951 & 0.1346156 \\
C & -2.6245241 & 0.6727094 & 1.1046223 \\
P & -0.9731492 & 0.2082122 & 0.7800967 \\
H & -4.5688159 & 1.3131875 & 0.5274067 \\
H & -2.9315299 & 0.6004202 & 2.1608302 \\
C & -0.9060582 & -1.2775719 & -0.3388855 \\
C & 0.0162317 & 1.5766608 & -0.0144302 \\
C & -0.2258552 & -0.2009446 & 2.4233026 \\
H & -0.2618192 & 1.5804693 & -1.0929094 \\
C & 1.5464911 & 1.5830963 & 0.1515671 \\
H & -0.4242152 & 2.5050945 & 0.4142783 \\
H & 0.0232135 & 0.7727902 & 2.9050689 \\
C & 0.9813518 & -1.1546361 & 2.4568749 \\
H & -1.0654464 & -0.6346266 & 3.0123786 \\
H & -1.7618290 & -1.1169914 & -1.0342414 \\
C & 0.3734004 & -1.5879600 & -1.1342511 \\
H & -1.2015609 & -2.1376009 & 0.3053412 \\
C & 2.2007530 & 2.7836298 & -0.5590355 \\
H & 1.8084452 & 1.6199867 & 1.2345200 \\
H & 1.9876441 & 0.6412387 & -0.2460002 \\
C & 1.4750586 & -1.4175488 & 3.8916886 \\
H & 0.7053042 & -2.1260715 & 1.9859382 \\
H & 1.8200609 & -0.7419089 & 1.8526393 \\
C & 0.2200999 & -2.8411996 & -2.0168445
\end{tabular}

$\begin{array}{cccc}\mathrm{H} & 0.6301971 & -0.7203421 & -1.7845114 \\ \mathrm{H} & 1.2373573 & -1.7311928 & -0.4460406 \\ \mathrm{C} & 3.7251205 & 2.8265243 & -0.3940485 \\ \mathrm{H} & 1.9411927 & 2.7511229 & -1.6429295 \\ \mathrm{H} & 1.7530184 & 3.7268863 & -0.1678336 \\ \mathrm{C} & 2.6632213 & -2.3858916 & 3.9530430 \\ \mathrm{H} & 1.7569820 & -0.4473521 & 4.3647024 \\ \mathrm{H} & 0.6313463 & -1.8199809 & 4.5003257 \\ \mathrm{C} & 1.4773305 & -3.1623819 & -2.8350733 \\ \mathrm{H} & -0.0400337 & -3.7127490 & -1.3711450 \\ \mathrm{H} & -0.6479226 & -2.6990229 & -2.7016888 \\ \mathrm{H} & 4.1619424 & 3.7038768 & -0.9193722 \\ \mathrm{H} & 4.0169175 & 2.8993622 & 0.6782602 \\ \mathrm{H} & 4.2058174 & 1.9128265 & -0.8115136 \\ \mathrm{H} & 2.9944842 & -2.5508459 & 5.0015303 \\ \mathrm{H} & 2.4004841 & -3.3796489 & 3.5246041 \\ \mathrm{H} & 3.5364315 & -1.9950603 & 3.3832265 \\ \mathrm{H} & 1.3300575 & -4.0710415 & -3.4590314 \\ \mathrm{H} & 1.7400609 & -2.3255786 & -3.5208718 \\ \mathrm{H} & 2.3564971 & -3.3462382 & -2.1765407 \\ \mathrm{C} & -3.3964900 & 1.2613435 & -1.2540939 \\ \mathrm{C} & -4.4449612 & 1.5720860 & -2.1393211 \\ \mathrm{H} & -2.3937810 & 1.1260135 & -1.7075338 \\ \mathrm{H} & -5.4500877 & 1.7329852 & -1.6989808 \\ \mathrm{C} & -4.3021245 & 1.8542270 & -3.5361779 \\ \mathrm{C} & -5.1005995 & 2.8145686 & -4.1321454 \\ \mathrm{H} & -6.0827664 & 2.9352983 & -3.5881176 \\ \mathrm{H} & -3.3574151 & 1.5144753 & -4.0232927\end{array}$

\section{S15}

$\mathrm{E}(\mathrm{BP} 86 /$ def2-SV(P) $)=-1046.423414271$

$\begin{array}{llll}\text { C } & -2.3866823 & -3.4015784 & 1.8964960\end{array}$

$\begin{array}{llll}\text { C } & -3.0593498 & -4.5854402 & 1.9324027\end{array}$

C $\quad-2.7052066 \quad-5.3763460 \quad 0.7391126$

$\begin{array}{llll}\text { C } & -1.8224767 & -4.6701032 & -0.0202340\end{array}$

$\begin{array}{llll}\text { C } & -1.5213999 & -3.3357263 & 0.6438586\end{array}$

$\begin{array}{llll}\mathrm{H} & -2.4373948 & -2.5862141 & 2.6357403\end{array}$

$\begin{array}{llll}\mathrm{H} & -3.7634296 & -4.9164528 & 2.7136035\end{array}$

$\mathrm{H} \quad-3.1053700 \quad-6.3784646 \quad 0.5136716$

$\begin{array}{llll}\mathrm{H} & -1.3749707 & -4.9686533 & -0.9805677\end{array}$

$\begin{array}{llll}\text { C } & -1.6787274 & -2.1275399 & -0.2705247\end{array}$

$\begin{array}{llll}\mathrm{P} & -0.7370389 & -0.7114613 & -0.1665843\end{array}$

H $\quad-2.7009049-1.9509037 \quad-0.6553215$

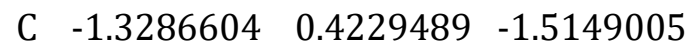

$\begin{array}{llll}\text { C } & -0.7835535 & 0.3676963 & 1.4046636\end{array}$

$\begin{array}{llll}\text { C } & 1.0579191 & -1.1722745 & -0.3950939\end{array}$

$\begin{array}{llll}\text { C } & 2.1918872 & -0.2424017 & 0.0656379\end{array}$

H $1.1398366-2.1537377 \quad 0.1282503$

H $\quad 1.1530364 \quad-1.4154824 \quad-1.4774532$

C $\quad-0.2851738 \quad 1.3139453 \quad-2.2104428$

$\begin{array}{llll}\mathrm{H} & -1.8042496 & -0.2569822 & -2.2560112\end{array}$ 


$\begin{array}{cccc}\mathrm{H} & -2.1395217 & 1.0509054 & -1.0780257 \\ \mathrm{C} & -0.3364195 & 1.8407121 & 1.3964740 \\ \mathrm{H} & -1.8560221 & 0.3034101 & 1.7036539 \\ \mathrm{H} & -0.2237555 & -0.2092619 & 2.1792430 \\ \mathrm{C} & 3.5862498 & -0.8436347 & -0.1925966 \\ \mathrm{H} & 2.1215345 & 0.7401695 & -0.4542324 \\ \mathrm{H} & 2.0890515 & -0.0278491 & 1.1543822 \\ \mathrm{C} & -0.9000111 & 2.1821748 & -3.3230955 \\ \mathrm{H} & 0.2155425 & 1.9771628 & -1.4685651 \\ \mathrm{H} & 0.5184804 & 0.6789993 & -2.6507339 \\ \mathrm{C} & -0.4847003 & 2.5208466 & 2.7714520 \\ \mathrm{H} & 0.7225517 & 1.9292018 & 1.0643803 \\ \mathrm{H} & -0.9411430 & 2.4088429 & 0.6515487 \\ \mathrm{C} & 4.7358237 & 0.0627718 & 0.2665169 \\ \mathrm{H} & 3.6586473 & -1.8302894 & 0.3228461 \\ \mathrm{H} & 3.6939445 & -1.0622860 & -1.2813349 \\ \mathrm{C} & 0.1250016 & 3.0753308 & -4.0339198 \\ \mathrm{H} & -1.4015288 & 1.5195559 & -4.0671650 \\ \mathrm{H} & -1.7088127 & 2.8155889 & -2.8873440 \\ \mathrm{C} & -0.0720653 & 3.9987576 & 2.7694686 \\ \mathrm{H} & -1.5430893 & 2.4279201 & 3.1120887 \\ \mathrm{H} & 0.1249429 & 1.9634137 & 3.5212909 \\ \mathrm{H} & 5.7257438 & -0.4018923 & 0.0620659 \\ \mathrm{H} & 4.7121082 & 1.0452064 & -0.2572918 \\ \mathrm{H} & 4.6806325 & 0.2661537 & 1.3600928 \\ \mathrm{H} & -0.3538755 & 3.6869324 & -4.8300014 \\ \mathrm{H} & 0.6167081 & 3.7766632 & -3.3218441 \\ \mathrm{H} & 0.9276188 & 2.4703995 & -4.5134181 \\ \mathrm{H} & -0.1891117 & 4.4558055 & 3.7771029 \\ \mathrm{H} & 0.9927640 & 4.1229064 & 2.4671845 \\ \mathrm{H} & -0.6911002 & 4.5922791 & 2.0588282 \\ \mathrm{H} & -0.4542798 & -3.3917250 & 0.9946453\end{array}$

\section{S16_cis-C2H3}

$\begin{array}{lccc}\text { E(BP86/def2-SV }(\mathrm{P}))=-1541.954248183 \\ \mathrm{C} & -0.8650598 & -3.2990383 & 0.8207146 \\ \mathrm{C} & -0.5059652 & -4.7201443 & 0.3646131 \\ \mathrm{C} & -1.4278828 & -4.9745869 & -0.8405971 \\ \mathrm{C} & -1.7210484 & -3.5562311 & -1.3882445 \\ \mathrm{~N} & -1.5514278 & -2.6989087 & -0.2187843 \\ \mathrm{O} & -0.5780215 & -2.7646758 & 1.8891894 \\ \mathrm{H} & -0.6263390 & -5.4377833 & 1.2018890 \\ \mathrm{H} & 0.5722135 & -4.7268586 & 0.0807223 \\ \mathrm{H} & -0.9846118 & -5.6382788 & -1.6118484 \\ \mathrm{H} & -2.3764641 & -5.4430683 & -0.4989450 \\ \mathrm{H} & -1.0099863 & -3.2726166 & -2.2036942 \\ \mathrm{H} & -2.7515131 & -3.4569132 & -1.7991658 \\ \mathrm{C} & -2.0859814 & -1.3521459 & -0.1302058 \\ \mathrm{C} & -1.3752587 & -0.2427875 & -0.9400068 \\ \mathrm{H} & -3.1446239 & -1.3701014 & -0.4782869 \\ \mathrm{H} & -2.1188515 & -1.0978069 & 0.9496902\end{array}$

P $\quad 0.2100651 \quad 0.4875929 \quad-0.1825306$

$\begin{array}{llll}\mathrm{H} & -1.1843591 & -0.6070293 & -1.9770108\end{array}$

$\begin{array}{llll}0 & -1.2706900 & 1.8210351 & -0.0170504\end{array}$

$\begin{array}{llll}\text { C } & 0.4979160 & 0.4852411 & 1.6517312\end{array}$

$\begin{array}{llll}\text { C } & 1.3456820 & -0.9950045 & -0.6965631\end{array}$

$\begin{array}{llll}\text { C } & 1.1224640 & 1.8696150 & -1.0563500\end{array}$

C $\quad 0.2015642 \quad 1.7757723 \quad 2.4287344$

H $\quad-0.0909666 \quad-0.3788429 \quad 2.0412193$

$\begin{array}{llll}\mathrm{H} & 1.5624080 & 0.1978992 & 1.7905712\end{array}$

C $\quad 2.6095832 \quad-1.3730251 \quad 0.0943284$

H $\quad 0.6791512 \quad-1.8841288-0.7274727$

H $\quad 1.6103133 \quad-0.7950518$ - 1.7643862

C $\quad 2.6579899 \quad 1.8740897 \quad-1.0933803$

H $\quad 0.7142090 \quad 1.8764456 \quad-2.0939826$

$\begin{array}{llll}\mathrm{H} & 0.7311610 & 2.7934525 & -0.5835520\end{array}$

C $\quad 0.4472048 \quad 1.6100712 \quad 3.9395062$

$\begin{array}{llll}\mathrm{H} & 0.8429212 & 2.6033184 & 2.0425428\end{array}$

$\begin{array}{lllll}\mathrm{H} & -0.8438534 & 2.0948040 & 2.2373973\end{array}$

$\begin{array}{llll}\text { C } & 3.4548939 & -2.4562267 & -0.6028378\end{array}$

H $3.2553619 \quad-0.4831550 \quad 0.2745742$

H $\quad 2.3100795 \quad-1.7489066 \quad 1.0993682$

C $\quad 3.2088629 \quad 3.1282227 \quad-1.7998226$

H $3.0654392 \quad 1.8398719-0.0558530$

H $\quad 3.0481339 \quad 0.9704614 \quad-1.6131942$

C $\quad 0.1675984 \quad 2.8902024 \quad 4.7380181$

$\begin{array}{llll}\mathrm{H} & -0.1941836 & 0.7821528 & 4.3237391\end{array}$

$\mathrm{H} \quad 1.4998122 \quad 1.2831727 \quad 4.1158347$

$\begin{array}{llll}\text { C } & 4.6917209 & -2.8796567 & 0.2005575\end{array}$

H $\quad 2.8130772 \quad-3.3487768-0.7981458$

$\mathrm{H} \quad 3.7702833 \quad-2.0859511-1.6075175$

C $\quad 4.7417228 \quad 3.1785026-1.8423345$

H $\quad 2.8033668 \quad 3.1692436-2.8386299$

H $\quad 2.8181903 \quad 4.0379787 \quad-1.2867768$

$\mathrm{H} \quad 0.3402823 \quad 2.7374285 \quad 5.8266066$

H $\quad 0.8245667 \quad 3.7267005 \quad 4.4072591$

$\mathrm{H} \quad-0.8863356 \quad 3.2248148 \quad 4.6071118$

$\mathrm{H} \quad 5.2767706 \quad-3.6622764 \quad-0.3317203$

H $\quad 5.3728161 \quad-2.0168728 \quad 0.3806316$

H $\quad 4.4066382 \quad-3.2904361 \quad 1.1956394$

H $\quad 5.1035832 \quad 4.0955408 \quad-2.3579193$

H $\quad 5.1766127 \quad 3.1774657 \quad-0.8169971$

$\mathrm{H} \quad 5.1627108 \quad 2.3007950 \quad-2.3834526$

C $\quad-2.1276201 \quad 1.1430339-0.8611798$

N $\quad-3.5275516 \quad 1.0695421-0.3500063$

C $\quad-4.5358331 \quad 1.2503188-1.3824181$

C $\quad-3.7327630 \quad 1.2701848 \quad-2.6991186$

$\begin{array}{llll}\text { C } & -2.3639328 & 1.7972648 & -2.2530996\end{array}$

$\mathrm{H} \quad-5.3037082 \quad 0.4386273-1.3614020$

$\mathrm{H} \quad-5.0863640 \quad 2.2121908-1.2176181$

H $\quad-3.6373931 \quad 0.2395839 \quad-3.1117393$

$\begin{array}{lllll}\mathrm{H} & -4.2192607 & 1.8933553 & -3.4795305\end{array}$ 


$\begin{array}{llll}\mathrm{H} & -2.3934474 & 2.8967212 & -2.0843091 \\ \mathrm{H} & -1.5457210 & 1.5809826 & -2.9735939 \\ \mathrm{C} & -3.8377644 & 1.1772123 & 0.9868052 \\ \mathrm{C} & -5.0811853 & 1.2563292 & 1.5348258 \\ \mathrm{H} & -5.1861119 & 1.3517063 & 2.6271484 \\ \mathrm{H} & -6.0056408 & 1.2301451 & 0.9333557 \\ \mathrm{H} & -2.9396493 & 1.2181965 & 1.6269495\end{array}$

\section{S16_cis-H}

$\mathrm{E}(\mathrm{BP} 86 / \mathrm{def} 2-\mathrm{SV}(\mathrm{P}))=-1464.602833344$

C $-1.6408766 \quad-2.3179600 \quad 2.2904806$

$\begin{array}{llll}\text { C } & -1.8036881 & -3.8431195 & 2.3509638\end{array}$

C $-3.0555738-4.1253956 \quad 1.5017736$

$\begin{array}{llll}\text { C } & -3.1127294 & -2.9315450 & 0.5165321\end{array}$

$\begin{array}{llll}\mathrm{N} & -2.4082512 & -1.8704796 & 1.2282854\end{array}$

$\begin{array}{llll}0 & -0.9430582 & -1.6118908 & 3.0123402\end{array}$

H $-1.8695186 \quad-4.1843614 \quad 3.4043065$

$\mathrm{H} \quad-0.8857640 \quad-4.3017568 \quad 1.9150946$

$\begin{array}{llll}\mathrm{H} & -3.0289828 & -5.1020354 & 0.9753830\end{array}$

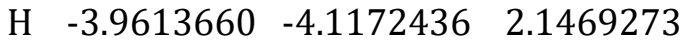

H $\quad-2.6095426 \quad-3.1709453 \quad-0.4532190$

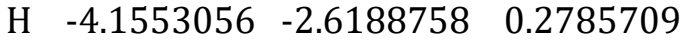

$\begin{array}{llll}\text { C } & -2.5173991 & -0.4651738 & 0.8900695\end{array}$

$\begin{array}{llll}\text { C } & -1.8662592 & 0.0309527 & -0.4191375\end{array}$

$\begin{array}{llll}\mathrm{H} & -3.6096047 & -0.2274479 & 0.8183814\end{array}$

$\begin{array}{llll}\mathrm{H} & -2.1157829 & 0.0991778 & 1.7571052\end{array}$

$\begin{array}{lllll}\mathrm{P} & 0.0343787 & 0.3572665 & -0.5659580\end{array}$

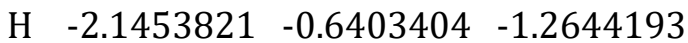

$\begin{array}{llll}0 & -0.8860433 & 1.9123783 & -1.1697643\end{array}$

$\begin{array}{lllll}\text { C } & 0.7784202 & 1.0423027 & 0.9904768\end{array}$

$\begin{array}{llll}\text { C } & 0.5468170 & -1.5066184 & -0.3623451\end{array}$

$\begin{array}{lllll}\text { C } & 1.1151887 & 0.7134194 & -2.0759708\end{array}$

$\begin{array}{llll}\text { C } & 0.6642760 & 2.5527137 & 1.2325408\end{array}$

$\begin{array}{lllll}\mathrm{H} & 0.3141594 & 0.4661455 & 1.8250874\end{array}$

$\begin{array}{llll}\mathrm{H} & 1.8475521 & 0.7317254 & 0.9696888\end{array}$

C $\quad 1.7985478-1.9016393 \quad 0.4427490$

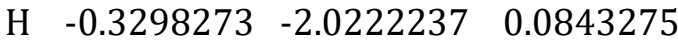

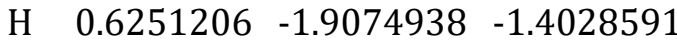

$\begin{array}{llll}\text { C } & 2.5380385 & 0.1522291 & -2.1864819\end{array}$

$\begin{array}{lllll}\mathrm{H} & 0.4992234 & 0.3762327 & -2.9428521\end{array}$

$\begin{array}{llll}\mathrm{H} & 1.1211777 & 1.8215818 & -2.1433844\end{array}$

$\begin{array}{llll}\text { C } & 1.3839089 & 2.9887338 & 2.5212943\end{array}$

$\begin{array}{llll}\mathrm{H} & 1.3839089 & 3.1080718 & 0.3633289\end{array}$

$\begin{array}{llll}\mathrm{H} & -0.4124811 & 2.8265943 & 1.2787171\end{array}$

$\begin{array}{llll}\text { C } & 2.1547646 & -3.3951545 & 0.3140762\end{array}$

$\begin{array}{llll}\mathrm{H} & 2.6850854 & -1.3038299 & 0.1315313\end{array}$

H $\quad 1.6272136 \quad-1.6664731 \quad 1.5174665$

$\begin{array}{lllll}\text { C } & 3.2466138 & 0.6277238 & -3.4704292\end{array}$

$\begin{array}{lllll}\mathrm{H} & 3.1462168 & 0.4685652 & -1.3065440\end{array}$

H $\quad 2.5313686 \quad-0.9609513 \quad-2.1782772$

C $\quad 1.2773770 \quad 4.4960568 \quad 2.7889430$

$\begin{array}{crrr}\mathrm{H} & 0.9603154 & 2.4265695 & 3.3872565 \\ \mathrm{H} & 2.4605291 & 2.6967776 & 2.4690509 \\ \mathrm{C} & 3.3701193 & -3.8084958 & 1.1548311 \\ \mathrm{H} & 1.2706755 & -4.0093537 & 0.6112159 \\ \mathrm{H} & 2.3426223 & -3.6368392 & -0.7595303 \\ \mathrm{C} & 4.6778244 & 0.0931916 & -3.6104507 \\ \mathrm{H} & 2.6425757 & 0.3173004 & -4.3558994 \\ \mathrm{H} & 3.2595415 & 1.7426730 & -3.4897335 \\ \mathrm{H} & 1.7998293 & 4.7833217 & 3.7288664 \\ \mathrm{H} & 1.7277100 & 5.0876431 & 1.9593168 \\ \mathrm{H} & 0.2143526 & 4.8139138 & 2.8829271 \\ \mathrm{H} & 3.6024014 & -4.8904409 & 1.0362599 \\ \mathrm{H} & 4.2789957 & -3.2364553 & 0.8590166 \\ \mathrm{H} & 3.1959700 & -3.6174234 & 2.2379518 \\ \mathrm{H} & 5.1572754 & 0.4542620 & -4.5473743 \\ \mathrm{H} & 5.3178753 & 0.4193940 & -2.7592530 \\ \mathrm{H} & 4.6957641 & -1.0204407 & -3.6319218 \\ \mathrm{C} & -2.1680869 & 1.5013415 & -0.7925643 \\ \mathrm{~N} & -2.6611407 & 2.3332695 & 0.3431561 \\ \mathrm{C} & -3.0221859 & 3.6090756 & -0.3051335 \\ \mathrm{C} & -3.5799286 & 3.2678645 & -1.7295379 \\ \mathrm{C} & -3.1992162 & 1.7781648 & -1.9268166 \\ \mathrm{H} & -3.7370206 & 4.1724325 & 0.3316283 \\ \mathrm{H} & -2.0984336 & 4.2173103 & -0.3985156 \\ \mathrm{H} & -4.6796321 & 3.4212381 & -1.7898633 \\ \mathrm{H} & -3.1169686 & 3.9152057 & -2.5041502 \\ \mathrm{H} & -2.7587701 & 1.5619079 & -2.9227986 \\ \mathrm{H} & -4.0886517 & 1.1192926 & -1.7951086 \\ \mathrm{H} & -3.5277938 & 1.9083831 & 0.7123745\end{array}$

S16_trans-C2H3

$\mathrm{E}(\mathrm{BP} 86 /$ def2-SV(P)) $=-1541.951913559$

$\begin{array}{llll}\text { C } & -1.5694741 & -2.2275244 & 2.4729122\end{array}$

$\begin{array}{llll}\text { C } & -1.7793729 & -3.7456236 & 2.5450315\end{array}$

$\begin{array}{llll}\text { C } & -3.0451955 & -3.9930134 & 1.7058612\end{array}$

$\begin{array}{llll}\text { C } & -3.0681024 & -2.8059849 & 0.7120458\end{array}$

$\begin{array}{llll}\mathrm{N} & -2.3257734 & -1.7616119 & 1.4136475\end{array}$

$\begin{array}{lllll}0 & -0.8466693 & -1.5409071 & 3.1914005\end{array}$

H $-1.8477856 \quad-4.0785518 \quad 3.6009098$

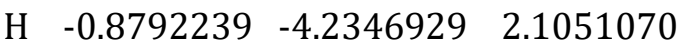

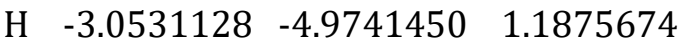

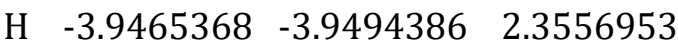

$\begin{array}{llll}\mathrm{H} & -2.5772327 & -3.0685745 & -0.2575254\end{array}$

H $\quad-4.0996577 \quad-2.4592402 \quad 0.4759073$

$\begin{array}{llll}\text { C } & -2.4001644 & -0.3572136 & 1.0551279\end{array}$

$\begin{array}{llll}\text { C } & -1.7085315 & 0.0814408 & -0.2586028\end{array}$

$\begin{array}{llll}\mathrm{H} & -3.4814070 & -0.1025368 & 0.9526127\end{array}$

$\begin{array}{llll}\mathrm{H} & -2.0093377 & 0.2098843 & 1.9262009\end{array}$

P $\quad 0.2019046 \quad 0.3476023 \quad-0.2293196$

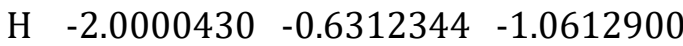

$\begin{array}{lllll}0 & -0.6352130 & 2.0442955 & -0.5531902\end{array}$ 


\begin{tabular}{|c|c|c|c|}
\hline & 56580 & 7887944 & \\
\hline & & -1.5480790 & \\
\hline & & & \\
\hline & & & \\
\hline & 145102 & 0.1345692 & 2. \\
\hline & 336 & & 128 \\
\hline & & & \\
\hline & 7876 & -2.0 & \\
\hline & 0.6711982 & -1.83 & -1.3 \\
\hline & 5991 & 0.21 & -1.8 \\
\hline & 49 & & \\
\hline & 1.4 & 1.9 & \\
\hline & 1.65 & 2.47 & \\
\hline & 1.32 & 2.90 & \\
\hline & 85 & & 1. \\
\hline & & -3.5 & \\
\hline & $2.7^{\prime}$ & -1.5 & \\
\hline & 1.71 & -1.9 & \\
\hline & 3.45 & 0.84 & -3.0 \\
\hline & 3.3 & 0.3 & \\
\hline & 2.5 & -0.8 & \\
\hline & 1.61 & 3.93 & \\
\hline & & & \\
\hline & 2.71 & 2.1 & $3 .($ \\
\hline & 3.2 & -4.7 & \\
\hline & 1.1 & & \\
\hline & 2.27 & -3.7 & -0 \\
\hline & 4.84 & 0.2 & -3.2 \\
\hline & 2.8 & 0.7 & -3 \\
\hline & 3.5 & 1.9 & \\
\hline & & & \\
\hline & 2.05 & 4.62 & \\
\hline & 632 & 4.26 & 3.8 \\
\hline & 3.4 & -5.2 & \\
\hline & 4.2 & -3.6 & \\
\hline & & -4.0 & \\
\hline & 5.3528372 & 0.69 & -4.1 \\
\hline & 5.50 & & -2.3 \\
\hline & 476 & -0.8 & -3 \\
\hline & -1.95 & & -0.6 \\
\hline & -2.9208237 & 2.42 & 8608 \\
\hline & -3.1 & 3.6 & -0.6 \\
\hline & & 3.0 & $-2 .($ \\
\hline & -2.56 & & -1.98 \\
\hline $\mathrm{H}$ & -4.0673503 & 4.234 & -0.3877840 \\
\hline & -2.2873204 & 4.3004013 & -0.6662017 \\
\hline & -4.39 & 2.78 & -2.3 \\
\hline & -2.9300177 & 3.6535 & -2.8916284 \\
\hline H & -3.8776395 & 1.8846794 & 0.4030900 \\
\hline & -2.4667309 & 2.6855769 & 1.1952922 \\
\hline & -2.21087 & 1.0645629 & -3.1177324 \\
\hline
\end{tabular}

$\begin{array}{llll}\mathrm{C} & -2.6090920 & 1.3019302 & -4.3989936 \\ \mathrm{H} & -2.2515555 & 0.6440666 & -5.2066911 \\ \mathrm{H} & -3.2884918 & 2.1256303 & -4.6731408 \\ \mathrm{H} & -1.5357541 & 0.2111036 & -2.9266986\end{array}$

\section{S16_trans-H}

$\mathrm{E}(\mathrm{BP} 86 /$ def2-SV(P)) $=-1464.605849803$

C $\quad-1.6308423 \quad-2.0014801 \quad 2.5009146$

C $\quad-1.7923008-3.5190799 \quad 2.6656816$

$\begin{array}{llll}\text { C } & -3.0435190 & -3.8577388 & 1.8367700\end{array}$

$\begin{array}{llll}\text { C } & -3.0929328 & -2.7374322 & 0.7691455\end{array}$

$\begin{array}{llll}\mathrm{N} & -2.3876458 & -1.6283098 & 1.4062229\end{array}$

$\begin{array}{llll}0 & -0.9384400 & -1.2500248 & 3.1833929\end{array}$

H $-1.8580827 \quad-3.7878647 \quad 3.7397268$

H $-0.8741245-4.0062134 \quad 2.2615478$

H $-3.0197043 \quad-4.8698860 \quad 1.3820155$

H $-3.9500522-3.7992395 \quad 2.4780823$

$\mathrm{H} \quad-2.5866275-3.0455088-0.1786665$

H $\quad-4.1328163 \quad-2.4381047 \quad 0.5049506$

$\begin{array}{llll}\text { C } & -2.5090197 & -0.2520988 & 0.9545298\end{array}$

$\begin{array}{llll}\text { C } & -1.8482893 & 0.1120797 & -0.3969530\end{array}$

$\mathrm{H} \quad-3.5991207 \quad-0.0365746 \quad 0.8607873$

$\mathrm{H} \quad-2.1207514 \quad 0.3878890 \quad 1.7756514$

P $\quad 0.0356385 \quad 0.4122426 \quad-0.3043213$

$\mathrm{H} \quad-2.1156904-0.6401081 \quad-1.1721273$

$\begin{array}{llll}0 & -0.9056398 & 2.1599144 & -0.4215199\end{array}$

C $\quad 0.8742565 \quad 0.8926581 \quad 1.2811730$

C $0.5081476-1.4667739-0.3173856$

$\begin{array}{llll}\text { C } & 0.9680739 & 0.9613921 & -1.8334428\end{array}$

$\begin{array}{llll}\text { C } & 0.9951739 & 2.3826543 & 1.6287098\end{array}$

$\begin{array}{llll}\mathrm{H} & 0.3331973 & 0.3342241 & 2.0821335\end{array}$

H $1.8856846 \quad 0.4318586 \quad 1.2255984$

C $\quad 1.8261015 \quad-1.9842218 \quad 0.2861429$

H $\quad-0.3317866 \quad-1.9848676 \quad 0.1946515$

$\mathrm{H} \quad 0.4303393 \quad-1.7765866-1.3884887$

$\begin{array}{lllll}\text { C } & 2.3205056 & 0.3336903 & -2.2024601\end{array}$

$\mathrm{H} \quad 0.2331428 \quad 0.8122115 \quad-2.6583739$

$\mathrm{H} \quad 1.0644971 \quad 2.0601144 \quad-1.7090726$

C $\quad 1.7673351 \quad 2.6077127 \quad 2.9411570$

$\mathrm{H} \quad 1.5044968 \quad 2.9274822 \quad 0.8003026$

$\mathrm{H} \quad-0.0181306 \quad 2.8304393 \quad 1.6921813$

$\begin{array}{llll}\text { C } & 2.0714285 & -3.4802043 & 0.0088687\end{array}$

H $\quad 2.6990761-1.4074981-0.0963616$

H $\quad 1.8119651 \quad-1.8261445 \quad 1.3891860$

$\begin{array}{llll}\text { C } & 2.9082750 & 0.9569585 & -3.4838037\end{array}$

H $\quad 3.0502972 \quad 0.4726801 \quad-1.3707947$

H $\quad 2.2211066-0.7645196 \quad-2.3548768$

C $\quad 1.8935659 \quad 4.0896201 \quad 3.3198156$

H $\quad 1.2585913 \quad 2.0535758 \quad 3.7650786$

$\begin{array}{llll}\mathrm{H} & 2.7854328 & 2.1573904 & 2.8585258\end{array}$

$\begin{array}{llll}\text { C } & 3.3592528 & -4.0186884 & 0.6468009\end{array}$ 


$\begin{array}{cccc}\mathrm{H} & 1.1975561 & -4.0680937 & 0.3803521 \\ \mathrm{H} & 2.1011352 & -3.6489340 & -1.0940522 \\ \mathrm{C} & 4.2669636 & 0.3645587 & -3.8811151 \\ \mathrm{H} & 2.1815793 & 0.8225453 & -4.3194220 \\ \mathrm{H} & 3.0076814 & 2.0590415 & -3.3428029 \\ \mathrm{H} & 2.4513949 & 4.2213305 & 4.2738048 \\ \mathrm{H} & 2.4320665 & 4.6650371 & 2.5326645 \\ \mathrm{H} & 0.8922386 & 4.5602047 & 3.4461799 \\ \mathrm{H} & 3.5041693 & -5.0998928 & 0.4269851 \\ \mathrm{H} & 4.2554987 & -3.4766834 & 0.2674961 \\ \mathrm{H} & 3.3433330 & -3.8993214 & 1.7540179 \\ \mathrm{H} & 4.6610941 & 0.8371927 & -4.8078266 \\ \mathrm{H} & 5.0256170 & 0.5151650 & -3.0795612 \\ \mathrm{H} & 4.1935079 & -0.7308664 & -4.0683722 \\ \mathrm{C} & -2.0985779 & 1.5852530 & -0.8485099 \\ \mathrm{C} & -3.3778634 & 2.3124511 & -0.3089212 \\ \mathrm{C} & -3.8080482 & 3.2378829 & -1.4565407 \\ \mathrm{C} & -3.4960643 & 2.3441868 & -2.6743032 \\ \mathrm{~N} & -2.2230111 & 1.6760313 & -2.3539614 \\ \mathrm{H} & -4.8707029 & 3.5617463 & -1.3920901 \\ \mathrm{H} & -3.1670697 & 4.1477835 & -1.4805402 \\ \mathrm{H} & -4.3054792 & 1.5845819 & -2.8054652 \\ \mathrm{H} & -3.4143122 & 2.8970742 & -3.6355913 \\ \mathrm{H} & -4.2011628 & 1.5882549 & -0.1121220 \\ \mathrm{H} & -3.1455475 & 2.8324182 & 0.6430419 \\ \mathrm{H} & -1.4560619 & 2.3134329 & -2.6144411\end{array}$

\section{S17_cis-C2H3}

\begin{tabular}{lccc}
\multicolumn{4}{c}{$\mathrm{E}(\mathrm{BP} 86 / \mathrm{def} 2-\mathrm{SV}(\mathrm{P}))=-652.3453807428$} \\
$\mathrm{C}$ & -0.1850600 & -2.2065928 & 2.0885133 \\
$\mathrm{C}$ & -0.2940849 & -3.7385240 & 2.1643433 \\
$\mathrm{C}$ & -1.4818203 & -4.0777444 & 1.2472058 \\
$\mathrm{C}$ & -1.5100580 & -2.9072563 & 0.2336594 \\
$\mathrm{~N}$ & -0.9054822 & -1.8084689 & 0.9741434 \\
$\mathrm{O}$ & 0.4279940 & -1.4634356 & 2.8449817 \\
$\mathrm{H}$ & -0.4103228 & -4.0690712 & 3.2168217 \\
$\mathrm{H}$ & 0.6636882 & -4.1679475 & 1.7889829 \\
$\mathrm{H}$ & -1.3965315 & -5.0647077 & 0.7462143 \\
$\mathrm{H}$ & -2.4257632 & -4.0804323 & 1.8352977 \\
$\mathrm{H}$ & -0.9270200 & -3.1504788 & -0.6903995 \\
$\mathrm{H}$ & -2.5429009 & -2.6438866 & -0.0916503 \\
$\mathrm{C}$ & -0.9411729 & -0.4222770 & 0.5308094 \\
$\mathrm{C}$ & -0.0895420 & -0.1815291 & -0.6998727 \\
$\mathrm{H}$ & -2.0141056 & -0.1432254 & 0.3669234 \\
$\mathrm{H}$ & -0.5694662 & 0.1675591 & 1.3984245 \\
$\mathrm{H}$ & 0.0855106 & -1.0670944 & -1.3354930 \\
$\mathrm{C}$ & 0.5465783 & 0.9476542 & -1.1188734 \\
$\mathrm{~N}$ & 0.5078760 & 2.2543034 & -0.5995997 \\
$\mathrm{C}$ & 1.4957171 & 3.1281487 & -1.2427536 \\
$\mathrm{C}$ & 1.7345405 & 2.4513431 & -2.6023140 \\
$\mathrm{C}$ & 1.5427438 & 0.9593468 & -2.2771973
\end{tabular}

$\begin{array}{crrr}\mathrm{H} & 1.0829370 & 4.1569389 & -1.3288123 \\ \mathrm{H} & 2.4317591 & 3.1891678 & -0.6346293 \\ \mathrm{H} & 0.9630090 & 2.7918454 & -3.3276244 \\ \mathrm{H} & 2.7320020 & 2.6867179 & -3.0288592 \\ \mathrm{H} & 1.1878329 & 0.3588711 & -3.1412959 \\ \mathrm{H} & 2.5041273 & 0.5076687 & -1.9381908 \\ \mathrm{C} & -0.3463651 & 2.7387476 & 0.3818485 \\ \mathrm{C} & -0.2438781 & 3.9312392 & 1.0228005 \\ \mathrm{H} & -1.0241762 & 4.2218652 & 1.7433700 \\ \mathrm{H} & 0.5986610 & 4.6265189 & 0.8752945 \\ \mathrm{H} & -1.1972269 & 2.0747359 & 0.5979314\end{array}$

\section{S17_cis-H}

$\mathrm{E}(\mathrm{BP} 86 /$ def2-SV(P) $)=-575.0174701648$

C $\quad-0.3471698 \quad-0.9059481 \quad 1.8251420$

$\begin{array}{llll}\text { C } & 0.0486810 & -2.3001616 & 2.3298799\end{array}$

$\begin{array}{llll}\text { C } & -0.9757213 & -3.2441480 & 1.6750390\end{array}$

$\begin{array}{llll}\text { C } & -1.4372654 & -2.4653231 & 0.4202060\end{array}$

$\begin{array}{llll}\mathrm{N} & -1.2404460 & -1.0709226 & 0.8003832\end{array}$

$\begin{array}{llll}0 & 0.0886531 & 0.1741894 & 2.2429585\end{array}$

$\mathrm{H} \quad 0.0649161 \quad-2.3261446 \quad 3.4391510$

$\mathrm{H} \quad 1.0899146 \quad-2.4996551 \quad 1.9854506$

$\begin{array}{llll}\mathrm{H} & -0.5656730 & -4.2443062 & 1.4227059\end{array}$

$\begin{array}{llll}\mathrm{H} & -1.8409378 & -3.3979327 & 2.3563171\end{array}$

$\mathrm{H} \quad-0.8248072-2.7182072 \quad-0.4811702$

$\begin{array}{llll}\mathrm{H} & -2.5032083 & -2.6550564 & 0.1600218\end{array}$

$\begin{array}{lllll}\text { C } & -1.7382984 & 0.0557930 & -0.0125657\end{array}$

$\begin{array}{llll}\text { C } & -0.9370816 & 0.3982344 & -1.2421414\end{array}$

$\begin{array}{llll}\mathrm{H} & -2.7897139 & -0.1883244 & -0.2839001\end{array}$

$\begin{array}{llll}\mathrm{H} & -1.7677337 & 0.9237823 & 0.6852991\end{array}$

$\begin{array}{llll}\mathrm{H} & -1.2545185 & -0.0017585 & -2.2181218\end{array}$

$\begin{array}{llll}\text { C } & 0.1553095 & 1.2214452 & -1.1982481\end{array}$

$\begin{array}{lllll}\mathrm{N} & 0.6826929 & 1.8258569 & -0.0723480\end{array}$

$\begin{array}{llll}\text { C } & 1.9128985 & 2.5581355 & -0.3437832\end{array}$

$\begin{array}{lllll}\text { C } & 1.7850894 & 2.8707414 & -1.8486927\end{array}$

$\begin{array}{llll}\text { C } & 1.0116799 & 1.6465641 & -2.3856787\end{array}$

$\begin{array}{lllll}\mathrm{H} & 1.9866813 & 3.4744742 & 0.2838450\end{array}$

$\begin{array}{llll}\mathrm{H} & 2.8263847 & 1.9407327 & -0.1372806\end{array}$

$\mathrm{H} \quad 1.1802162 \quad 3.7941120 \quad-1.9869214$

$\begin{array}{lllll}\mathrm{H} & 2.7669767 & 3.0273363 & -2.3430924\end{array}$

$\begin{array}{llll}\mathrm{H} & 0.4105314 & 1.8592891 & -3.2946615\end{array}$

$\mathrm{H} \quad 1.7188164 \quad 0.8231930 \quad-2.6430410$

H $\quad 0.4931332 \quad 1.4240091 \quad 0.8652479$

\section{S17_trans-C2H3}

$\mathrm{E}(\mathrm{BP} 86 /$ def2-SV(P) $)=-652.3548463144$

C $\quad-0.9369893 \quad-1.0565595 \quad 2.3680515$

$\begin{array}{llll}\text { C } & -0.5493488 & -2.4078031 & 2.9887771\end{array}$

$\begin{array}{llll}\text { C } & -1.2960386 & -3.4475839 & 2.1349584\end{array}$

$\begin{array}{llll}\text { C } & -1.4903862 & -2.7313440 & 0.7768606\end{array}$

$\begin{array}{llll}\mathrm{N} & -1.5123105 & -1.3206537 & 1.1434243\end{array}$ 


$\begin{array}{cccc}\mathrm{O} & -0.7485004 & 0.0587442 & 2.8511174 \\ \mathrm{H} & -0.7971662 & -2.4277469 & 4.0700487 \\ \mathrm{H} & 0.5577834 & -2.5108649 & 2.9060079 \\ \mathrm{H} & -0.7558613 & -4.4116379 & 2.0289273 \\ \mathrm{H} & -2.2895233 & -3.6675240 & 2.5838916 \\ \mathrm{H} & -0.6525132 & -2.9496494 & 0.0682402 \\ \mathrm{H} & -2.4378788 & -3.0209890 & 0.2670485 \\ \mathrm{C} & -1.8635399 & -0.2539599 & 0.1979158 \\ \mathrm{C} & -0.8190673 & 0.0359866 & -0.8549351 \\ \mathrm{H} & -2.8243747 & -0.5483356 & -0.2854111 \\ \mathrm{H} & -2.0651430 & 0.6403187 & 0.8229171 \\ \mathrm{H} & -0.7802998 & -0.6516216 & -1.7168222 \\ \mathrm{C} & 0.0587012 & 1.0751380 & -0.7728936 \\ \mathrm{C} & 0.1104324 & 2.1281741 & 0.3249850 \\ \mathrm{C} & 1.5223031 & 2.7299183 & 0.1898075 \\ \mathrm{C} & 1.8316376 & 2.5843450 & -1.3086890 \\ \mathrm{~N} & 1.0655186 & 1.3982878 & -1.7032574 \\ \mathrm{H} & 1.5867654 & 3.7819292 & 0.5374855 \\ \mathrm{H} & 2.2478118 & 2.1314878 & 0.7834273 \\ \mathrm{H} & 1.5038480 & 3.4751688 & -1.9006425 \\ \mathrm{H} & 2.9129002 & 2.4256570 & -1.5194032 \\ \mathrm{H} & -0.6654010 & 2.9051525 & 0.1246650 \\ \mathrm{H} & -0.0960236 & 1.6987153 & 1.3280154 \\ \mathrm{C} & 1.2906115 & 0.7606012 & -2.9105062 \\ \mathrm{C} & 2.1647815 & 1.1424786 & -3.8773357 \\ \mathrm{H} & 2.2589387 & 0.5314933 & -4.7886485 \\ \mathrm{H} & 2.7893001 & 2.0477568 & -3.8029757 \\ \mathrm{H} & 0.6790324 & -0.1450797 & -3.0550515\end{array}$

\section{S17_trans-H}

E(BP86/def2-SV(P)) = -575.0095882183
$\begin{array}{llll}C & -0.2956053 & -1.0959977 & 1.8803292\end{array}$
$\begin{array}{llll}\text { C } & 0.0772808 & -2.5465746 & 2.2303673\end{array}$
$\begin{array}{llll}\text { C } & -0.9676918 & -3.3970387 & 1.4879619\end{array}$
$\begin{array}{llll}\text { C } & -1.4018461 & -2.4907071 & 0.3112919\end{array}$
$\begin{array}{llll}\mathrm{N} & -1.1807153 & -1.1460787 & 0.8255514\end{array}$
$\begin{array}{llll}0 & 0.1339642 & -0.0752837 & 2.4162863\end{array}$
$\mathrm{H} \quad 0.1002844 \quad-2.6911793 \quad 3.3302284$
$\mathrm{H} \quad 1.1118327-2.7279579 \quad 1.8560773$
H $\quad-0.5844962 \quad-4.3797945 \quad 1.1418588$
H $\quad-1.8402973-3.5903547 \quad 2.1498096$
$\mathrm{H} \quad-0.7860300 \quad-2.6753850 \quad-0.6049780$
H $\quad-2.4693745 \quad-2.6348953 \quad 0.0259648$
C $-1.6233582 \quad 0.06185250 .1128855$
$\begin{array}{llll}\text { C } & -0.8692538 & 0.3731202 & -1.1551777\end{array}$
$\mathrm{H} \quad-2.7111892-0.0665825 \quad-0.0991952$
$\mathrm{H} \quad-1.5221166 \quad 0.8851710 \quad 0.8504925$
H $-1.1470679-0.1839874 \quad-2.0693213$
C $\quad 0.1523806 \quad 1.2760114-1.2198370$
C $\quad 0.6634958 \quad 2.1759906-0.0981515$
$\begin{array}{llll}\text { C } & 2.0059534 & 2.7149279 & -0.6408888\end{array}$

$\begin{array}{crrr}\mathrm{C} & 1.7914090 & 2.7173771 & -2.1676758 \\ \mathrm{~N} & 0.9192341 & 1.5626595 & -2.3420173 \\ \mathrm{H} & 2.2723801 & 3.7161957 & -0.2427119 \\ \mathrm{H} & 2.8294751 & 2.0133522 & -0.3829147 \\ \mathrm{H} & 1.3116031 & 3.6741551 & -2.5052044 \\ \mathrm{H} & 2.7381349 & 2.5994060 & -2.7414028 \\ \mathrm{H} & -0.0600497 & 3.0121787 & 0.0506985 \\ \mathrm{H} & 0.7481021 & 1.6407007 & 0.8711064 \\ \mathrm{H} & 0.6035617 & 1.2787185 & -3.2714332\end{array}$

\section{S18}

$\mathrm{E}(\mathrm{BP} 86 / \mathrm{def} 2-\mathrm{SV}(\mathrm{P}))=-968.9682752283$

$\begin{array}{llll}\text { C } & -4.2388747 & 0.4352072 & -0.4527814\end{array}$

$\begin{array}{llll}\text { C } & -3.0585659 & 1.0607808 & -0.2908765\end{array}$

$\begin{array}{llll}\mathrm{P} & -1.4357137 & 0.2719779 & -0.1301914\end{array}$

$\begin{array}{llll}\mathrm{H} & -2.9203717 & 2.1710437 & -0.2561015\end{array}$

$\begin{array}{llll}\text { C } & -0.7548526 & 0.9774681 & 1.4061820\end{array}$

$\begin{array}{llll}\text { C } & -1.6746408 & -1.5590170 & -0.1048568\end{array}$

$\begin{array}{llll}\text { C } & -0.5040531 & 0.8551079 & -1.5981380\end{array}$

$\begin{array}{llll}\text { C } & -1.1403216 & 3.6505012 & -0.1087327\end{array}$

$\begin{array}{lllll}\text { C } & -1.1266575 & 4.8795099 & -0.3368390\end{array}$

$\begin{array}{llll}\mathrm{H} & -4.3473582 & -0.6650061 & -0.5016504\end{array}$

$\mathrm{H} \quad-1.1169178 \quad 5.9486885-0.5339074$

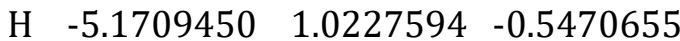

$\begin{array}{llll}\text { C } & 0.7416252 & 0.8349273 & 1.7254093\end{array}$

$\begin{array}{llll}\mathrm{H} & -0.9684948 & 2.0824840 & 1.1582914\end{array}$

$\begin{array}{llll}\mathrm{H} & -1.4080104 & 0.6252514 & 2.2379880\end{array}$

$\begin{array}{llll}\text { C } & 0.8809026 & 0.2630693 & -1.9049077\end{array}$

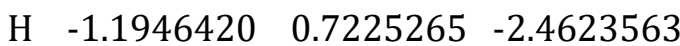

H $\quad-0.4778871 \quad 1.9648101-1.3608804$

$\begin{array}{llll}\text { C } & -0.4668938 & -2.4095362 & 0.3324952\end{array}$

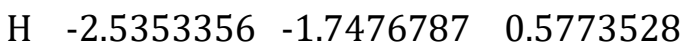

H $\quad-2.0100145 \quad-1.8465042 \quad-1.1286513$

C $\quad 1.0773677 \quad 1.4960783 \quad 3.0752340$

H $1.0785674 \quad-0.2281150 \quad 1.7408445$

H $1.32401851 .3502025 \quad 0.9282502$

$\begin{array}{llll}\text { C } & 1.5621291 & 1.0118797 & -3.0659870\end{array}$

H $\quad 1.5345860 \quad 0.3179440 \quad-1.0062753$

H $\quad 0.7978478 \quad-0.8182337 \quad-2.1678990$

$\begin{array}{lrrr}\text { C } & -0.7574077 & -3.9192508 & 0.2541053\end{array}$

$\mathrm{H} \quad 0.4199248 \quad-2.1721917 \quad-0.2975066$

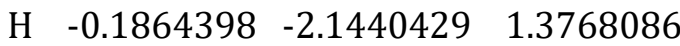

$\begin{array}{llll}\text { C } & 2.5719927 & 1.4458497 & 3.4156158\end{array}$

$\begin{array}{llll}\mathrm{H} & 0.7306842 & 2.5541094 & 3.0460628\end{array}$

$\begin{array}{llll}\mathrm{H} & 0.4913515 & 0.9976138 & 3.8837861\end{array}$

$\begin{array}{llll}\text { C } & 2.9471722 & 0.4525197 & -3.4143680\end{array}$

$\begin{array}{llll}\mathrm{H} & 0.9025133 & 0.9690981 & -3.9641856\end{array}$

$\begin{array}{llll}\mathrm{H} & 1.6424398 & 2.0899549 & -2.7976461\end{array}$

$\begin{array}{llll}\text { C } & 0.4247657 & -4.7848219 & 0.7080934\end{array}$

$\begin{array}{llll}\mathrm{H} & -1.6535235 & -4.1517745 & 0.8767504\end{array}$

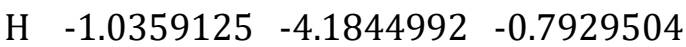




$\begin{array}{crrc}\mathrm{H} & 2.7780159 & 1.9278922 & 4.3967204 \\ \mathrm{H} & 2.9450929 & 0.3974251 & 3.4718167 \\ \mathrm{H} & 3.1790101 & 1.9775221 & 2.6484847 \\ \mathrm{H} & 3.4101777 & 1.0180796 & -4.2525581 \\ \mathrm{H} & 3.6409338 & 0.5158974 & -2.5454488 \\ \mathrm{H} & 2.8916718 & -0.6168649 & -3.7215786 \\ \mathrm{H} & 0.1818851 & -5.8677063 & 0.6379461 \\ \mathrm{H} & 1.3265401 & -4.6019109 & 0.0812122 \\ \mathrm{H} & 0.7026185 & -4.5710257 & 1.7648902\end{array}$

\section{S18_NVP}

\begin{tabular}{lccc}
\multicolumn{4}{c}{ E(BP86/def2-SV(P)) $=-968.9682752283$} \\
C & -4.2388747 & 0.4352072 & -0.4527814 \\
C & -3.0585659 & 1.0607808 & -0.2908765 \\
P & -1.4357137 & 0.2719779 & -0.1301914 \\
H & -2.9203717 & 2.1710437 & -0.2561015 \\
C & -0.7548526 & 0.9774681 & 1.4061820 \\
C & -1.6746408 & -1.5590170 & -0.1048568 \\
C & -0.5040531 & 0.8551079 & -1.5981380 \\
C & -1.1403216 & 3.6505012 & -0.1087327 \\
C & -1.1266575 & 4.8795099 & -0.3368390 \\
H & -4.3473582 & -0.6650061 & -0.5016504 \\
H & -1.1169178 & 5.9486885 & -0.5339074 \\
H & -5.1709450 & 1.0227594 & -0.5470655 \\
C & 0.7416252 & 0.8349273 & 1.7254093 \\
H & -0.9684948 & 2.0824840 & 1.1582914 \\
H & -1.4080104 & 0.6252514 & 2.2379880 \\
C & 0.8809026 & 0.2630693 & -1.9049077 \\
H & -1.1946420 & 0.7225265 & -2.4623563 \\
H & -0.4778871 & 1.9648101 & -1.3608804 \\
C & -0.4668938 & -2.4095362 & 0.3324952 \\
H & -2.5353356 & -1.7476787 & 0.5773528 \\
H & -2.0100145 & -1.8465042 & -1.1286513 \\
C & 1.0773677 & 1.4960783 & 3.0752340 \\
H & 1.0785674 & -0.2281150 & 1.7408445 \\
H & 1.3240185 & 1.3502025 & 0.9282502 \\
C & 1.5621291 & 1.0118797 & -3.0659870 \\
H & 1.5345860 & 0.3179440 & -1.0062753 \\
H & 0.7978478 & -0.8182337 & -2.1678990 \\
C & -0.7574077 & -3.9192508 & 0.2541053 \\
H & 0.4199248 & -2.1721917 & -0.2975066 \\
H & -0.1864398 & -2.1440429 & 1.3768086 \\
C & 2.5719927 & 1.4458497 & 3.4156158 \\
H & 0.7306842 & 2.5541094 & 3.0460628 \\
H & 0.4913515 & 0.9976138 & 3.8837861 \\
C & 2.9471722 & 0.4525197 & -3.4143680 \\
H & 0.9025133 & 0.9690981 & -3.9641856 \\
H & 1.6424398 & 2.0899549 & -2.7976461 \\
C & 0.4247657 & -4.7848219 & 0.7080934 \\
H & -1.6535235 & -4.1517745 & 0.8767504 \\
H & -1.0359125 & -4.1844992 & -0.7929504 \\
& & &
\end{tabular}

$\begin{array}{cccc}\mathrm{H} & 2.7780159 & 1.9278922 & 4.3967204 \\ \mathrm{H} & 2.9450929 & 0.3974251 & 3.4718167 \\ \mathrm{H} & 3.1790101 & 1.9775221 & 2.6484847 \\ \mathrm{H} & 3.4101777 & 1.0180796 & -4.2525581 \\ \mathrm{H} & 3.6409338 & 0.5158974 & -2.5454488 \\ \mathrm{H} & 2.8916718 & -0.6168649 & -3.7215786 \\ \mathrm{H} & 0.1818851 & -5.8677063 & 0.6379461 \\ \mathrm{H} & 1.3265401 & -4.6019109 & 0.0812122 \\ \mathrm{H} & 0.7026185 & -4.5710257 & 1.7648902\end{array}$

\section{S19}

$\mathrm{E}(\mathrm{BP} 86 /$ def2-SV(P) $)=-968.9810597551$

C $\quad-2.3748164 \quad 1.1993990-3.3240975$

$\begin{array}{llll}\text { C } & -1.9735871 & 1.5403940 & -2.0766239\end{array}$

$\begin{array}{llll}\mathrm{P} & -0.9116665 & 0.4892585 & -1.1529499\end{array}$

$\begin{array}{llll}\mathrm{H} & -2.8310831 & 3.2917041 & -1.0708305\end{array}$

$\begin{array}{llll}\text { C } & -1.7467102 & 0.2861332 & 0.4897732\end{array}$

$\begin{array}{llll}\text { C } & -0.4851308 & -1.2231939 & -1.7986127\end{array}$

$\begin{array}{llll}\text { C } & 0.6772132 & 1.4242895 & -0.9427705\end{array}$

$\begin{array}{lllll}\text { C } & -3.3145577 & 4.1403083 & -0.5404694\end{array}$

$\begin{array}{llll}\text { C } & -3.8484086 & 5.0621379 & 0.0614887\end{array}$

$\begin{array}{llll}\mathrm{H} & -2.1075014 & 0.2689878 & -3.8855099\end{array}$

$\begin{array}{llll}\mathrm{H} & -4.3246933 & 5.8814037 & 0.5854348\end{array}$

$\begin{array}{llll}\mathrm{H} & -3.0431622 & 1.8786135 & -3.8989843\end{array}$

$\begin{array}{llll}\text { C } & -1.2809035 & -0.8189085 & 1.4518934\end{array}$

$\begin{array}{cccc}\mathrm{H} & -1.6987236 & 1.2897221 & 0.9701838\end{array}$

$\begin{array}{llll}\mathrm{H} & -2.8195724 & 0.1443257 & 0.2211391\end{array}$

$\begin{array}{llll}\text { C } & 1.5380961 & 1.1890188 & 0.3105121\end{array}$

$\begin{array}{llll}\mathrm{H} & 1.2642861 & 1.2316159 & -1.8708205\end{array}$

$\begin{array}{lllll}\mathrm{H} & 0.3541389 & 2.4885832 & -0.9959991\end{array}$

$\begin{array}{llll}\text { C } & 0.7749511 & -1.9577997 & -1.3081456\end{array}$

$\mathrm{H} \quad-1.3963260-1.8414812 \quad-1.6232071$

$\begin{array}{lllll}\mathrm{H} & -0.4283690 & -1.0849328 & -2.9047482\end{array}$

$\begin{array}{llll}\text { C } & -2.0948350 & -0.8335126 & 2.7600768\end{array}$

$\mathrm{H}-1.3745301 \quad-1.8145674 \quad 0.9592620$

H $\quad-0.2018727 \quad-0.6940043 \quad 1.6969886$

$\begin{array}{llll}\text { C } & 2.8025015 & 2.0692314 & 0.3244712\end{array}$

$\begin{array}{llll}\mathrm{H} & 0.9377373 & 1.4082722 & 1.2233524\end{array}$

$\begin{array}{llll}\mathrm{H} & 1.8391437 & 0.1195673 & 0.3844944\end{array}$

$\begin{array}{llll}\text { C } & 0.9485852 & -3.3426242 & -1.9604768\end{array}$

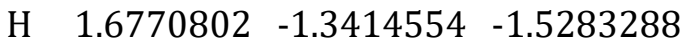

H $\quad 0.7460625 \quad-2.0801310 \quad-0.2019021$

$\begin{array}{llll}\text { C } & -1.6567608 & -1.9338066 & 3.7354473\end{array}$

H $-2.0080593 \quad 0.1623631 \quad 3.2545229$

H $\quad-3.1759254 \quad-0.95640412 .5151162$

C $\quad 3.6863623 \quad 1.8404909 \quad 1.5577723$

$\begin{array}{llll}\mathrm{H} & 3.3939139 & 1.8728064 & -0.6006217\end{array}$

$\begin{array}{llll}\mathrm{H} & 2.5007644 & 3.1414134 & 0.2707969\end{array}$

$\begin{array}{llll}\text { C } & 2.1985794 & -4.0894496 & -1.4774484\end{array}$

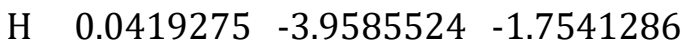

H $\quad 0.9876104 \quad-3.2221062 \quad-3.0687182$ 


$\begin{array}{cccc}\mathrm{H} & -2.2644031 & -1.9140259 & 4.6667401 \\ \mathrm{H} & -1.7680293 & -2.9457282 & 3.2837390 \\ \mathrm{H} & -0.5888897 & -1.8153626 & 4.0295335 \\ \mathrm{H} & 4.5865409 & 2.4934573 & 1.5374381 \\ \mathrm{H} & 3.1347070 & 2.0594795 & 2.5000689 \\ \mathrm{H} & 4.0385020 & 0.7852992 & 1.6141896 \\ \mathrm{H} & 2.2918545 & -5.0834411 & -1.9680633 \\ \mathrm{H} & 3.1270961 & -3.5166139 & -1.7012068 \\ \mathrm{H} & 2.1690152 & -4.2598872 & -0.3771988\end{array}$

\section{S19_NVP}

\begin{tabular}{lccc}
\multicolumn{4}{c}{ E(BP86/def2-SV(P) $=-968.9810597551$} \\
$\mathrm{C}$ & -2.3748164 & 1.1993990 & -3.3240975 \\
$\mathrm{C}$ & -1.9735871 & 1.5403940 & -2.0766239 \\
$\mathrm{P}$ & -0.9116665 & 0.4892585 & -1.1529499 \\
$\mathrm{H}$ & -2.8310831 & 3.2917041 & -1.0708305 \\
$\mathrm{C}$ & -1.7467102 & 0.2861332 & 0.4897732 \\
$\mathrm{C}$ & -0.4851308 & -1.2231939 & -1.7986127 \\
$\mathrm{C}$ & 0.6772132 & 1.4242895 & -0.9427705 \\
$\mathrm{C}$ & -3.3145577 & 4.1403083 & -0.5404694 \\
$\mathrm{C}$ & -3.8484086 & 5.0621379 & 0.0614887 \\
$\mathrm{H}$ & -2.1075014 & 0.2689878 & -3.8855099 \\
$\mathrm{H}$ & -4.3246933 & 5.8814037 & 0.5854348 \\
$\mathrm{H}$ & -3.0431622 & 1.8786135 & -3.8989843 \\
$\mathrm{C}$ & -1.2809035 & -0.8189085 & 1.4518934 \\
$\mathrm{H}$ & -1.6987236 & 1.2897221 & 0.9701838 \\
$\mathrm{H}$ & -2.8195724 & 0.1443257 & 0.2211391 \\
$\mathrm{C}$ & 1.5380961 & 1.1890188 & 0.3105121 \\
$\mathrm{H}$ & 1.2642861 & 1.2316159 & -1.8708205 \\
$\mathrm{H}$ & 0.3541389 & 2.4885832 & -0.9959991 \\
$\mathrm{C}$ & 0.7749511 & -1.9577997 & -1.3081456 \\
$\mathrm{H}$ & -1.3963260 & -1.8414812 & -1.6232071 \\
$\mathrm{H}$ & -0.4283690 & -1.0849328 & -2.9047482 \\
$\mathrm{C}$ & -2.0948350 & -0.8335126 & 2.7600768 \\
$\mathrm{H}$ & -1.3745301 & -1.8145674 & 0.9592620 \\
$\mathrm{H}$ & -0.2018727 & -0.6940043 & 1.6969886 \\
$\mathrm{C}$ & 2.8025015 & 2.0692314 & 0.3244712 \\
$\mathrm{H}$ & 0.9377373 & 1.4082722 & 1.2233524 \\
$\mathrm{H}$ & 1.8391437 & 0.1195673 & 0.3844944 \\
$\mathrm{C}$ & 0.9485852 & -3.3426242 & -1.9604768 \\
$\mathrm{H}$ & 1.6770802 & -1.3414554 & -1.5283288 \\
$\mathrm{H}$ & 0.7460625 & -2.0801310 & -0.2019021 \\
$\mathrm{C}$ & -1.6567608 & -1.9338066 & 3.7354473 \\
$\mathrm{H}$ & -2.0080593 & 0.1623631 & 3.2545229 \\
$\mathrm{H}$ & -3.1759254 & -0.9564041 & 2.5151162 \\
$\mathrm{C}$ & 3.6863623 & 1.8404909 & 1.5577723 \\
$\mathrm{H}$ & 3.3939139 & 1.8728064 & -0.6006217 \\
$\mathrm{H}$ & 2.5007644 & 3.1414134 & 0.2707969 \\
$\mathrm{C}$ & 2.1985794 & -4.0894496 & -1.4774484 \\
$\mathrm{H}$ & 0.0419275 & -3.9585524 & -1.7541286 \\
$\mathrm{H}$ & 0.9876104 & -3.2221062 & -3.0687182
\end{tabular}

$\begin{array}{crcc}\mathrm{H} & -2.2644031 & -1.9140259 & 4.6667401 \\ \mathrm{H} & -1.7680293 & -2.9457282 & 3.2837390 \\ \mathrm{H} & -0.5888897 & -1.8153626 & 4.0295335 \\ \mathrm{H} & 4.5865409 & 2.4934573 & 1.5374381 \\ \mathrm{H} & 3.1347070 & 2.0594795 & 2.5000689 \\ \mathrm{H} & 4.0385020 & 0.7852992 & 1.6141896 \\ \mathrm{H} & 2.2918545 & -5.0834411 & -1.9680633 \\ \mathrm{H} & 3.1270961 & -3.5166139 & -1.7012068 \\ \mathrm{H} & 2.1690152 & -4.2598872 & -0.3771988\end{array}$

\section{S2}

$\mathrm{E}(\mathrm{BP} 86 /$ def2-SV(P) $)=-363.7293289259$

C $\quad-0.1390239 \quad 0.0154634 \quad 0.0206082$

$\begin{array}{llll}\text { C } & -1.0598355 & 0.7396674 & -0.9419778\end{array}$

$\begin{array}{llll}\text { C } & -0.0343252 & 1.2129764 & -1.9959094\end{array}$

$\begin{array}{lllll}\text { C } & 1.1839927 & 0.2699264 & -1.7439945\end{array}$

$\begin{array}{llll}\mathrm{N} & 1.0512037 & -0.2454241 & -0.3775099\end{array}$

$\begin{array}{llll}0 & -0.6585496 & -0.3385050 & 1.2253722\end{array}$

$\begin{array}{llll}\mathrm{H} & -1.6329080 & 1.5543286 & -0.4500638\end{array}$

$\begin{array}{llll}\mathrm{H} & -1.8079319 & 0.0204949 & -1.3523075\end{array}$

$\begin{array}{llll}\mathrm{H} & -0.4161748 & 1.1673403 & -3.0373341\end{array}$

$\mathrm{H} \quad 0.2561907 \quad 2.2673677 \quad-1.7940893$

$\mathrm{H} \quad 1.1952905 \quad-0.5930978-2.4530510$

$\begin{array}{lllll}\mathrm{H} & 2.1609955 & 0.7877381 & -1.8670121\end{array}$

$\begin{array}{llll}\text { C } & 0.1330504 & -1.0561372 & 2.1028874\end{array}$

$\begin{array}{llll}\text { C } & -0.3317734 & -1.4029519 & 3.3141961\end{array}$

$\begin{array}{llll}\mathrm{H} & 0.3125354 & -1.9773134 & 3.9981742\end{array}$

$\begin{array}{llll}\mathrm{H} & -1.3477703 & -1.1262804 & 3.6433926\end{array}$

H $\quad 1.1350336 \quad-1.29559331 .7086188$

\section{S20}

$\mathrm{E}(\mathrm{BP} 86 / \mathrm{def} 2-\mathrm{SV}(\mathrm{P}))=-1178.149533857$

C $\quad-1.7163767 \quad 0.4649157 \quad-3.1440839$

$\begin{array}{llll}\text { C } & -1.3745566 & 0.8475551 & -1.9023544\end{array}$

$\begin{array}{lllll}\mathrm{P} & -0.2082066 & 0.1639220 & -0.6121997\end{array}$

$\begin{array}{llll}\text { C } & -1.0015295 & -0.4413943 & 0.9724497\end{array}$

$\begin{array}{llll}\text { C } & 0.1542445 & -1.5055002 & -1.5060379\end{array}$

$\begin{array}{lllll}\text { C } & 1.5555703 & 0.7681000 & -0.4388151\end{array}$

$\begin{array}{llll}\mathrm{H} & -1.3701011 & -0.4740252 & -3.6087747\end{array}$

$\begin{array}{llll}\mathrm{H} & -2.3681679 & 1.1237483 & -3.7500231\end{array}$

C $\quad-0.6748601 \quad-1.8106140 \quad 1.5826201$

$\begin{array}{cccc}\mathrm{H} & -0.8097532 & 0.3778155 & 1.6960529\end{array}$

$\begin{array}{llll}\mathrm{H} & -2.0914433 & -0.3781458 & 0.7487072\end{array}$

$\begin{array}{llll}\text { C } & 1.8905750 & 1.5001390 & 0.8696171\end{array}$

$\begin{array}{llll}\mathrm{H} & 2.2476581 & -0.0872199 & -0.5915909\end{array}$

$\mathrm{H} \quad 1.6961040 \quad 1.4556047 \quad-1.3042816$

$\begin{array}{llll}\text { C } & 1.3174370 & -2.4280485 & -1.0933743\end{array}$

$\begin{array}{lllll}\mathrm{H} & -0.8093396 & -2.0689243 & -1.4722317\end{array}$

$\begin{array}{llll}\mathrm{H} & 0.2988643 & -1.2430476 & -2.5795985\end{array}$

$\begin{array}{llll}\text { C } & -1.5155316 & -2.0896806 & 2.8435696\end{array}$

$\begin{array}{llll}\mathrm{H} & -0.8547497 & -2.6257083 & 0.8459581\end{array}$ 


$\begin{array}{cccc}\mathrm{H} & 0.4050498 & -1.8627733 & 1.8539554 \\ \mathrm{C} & 3.3274292 & 2.0533886 & 0.8846710 \\ \mathrm{H} & 1.1591424 & 2.3240326 & 1.0089035 \\ \mathrm{H} & 1.7619659 & 0.8054188 & 1.7336657 \\ \mathrm{C} & 1.2350655 & -3.8237645 & -1.7411546 \\ \mathrm{H} & 2.2866476 & -1.9633225 & -1.3903583 \\ \mathrm{H} & 1.3709983 & -2.5514048 & 0.0112909 \\ \mathrm{C} & -1.2054013 & -3.4435658 & 3.4949569 \\ \mathrm{H} & -1.3489653 & -1.2713163 & 3.5830391 \\ \mathrm{H} & -2.5981337 & -2.0415993 & 2.5777386 \\ \mathrm{C} & 3.6805464 & 2.7767685 & 2.1909453 \\ \mathrm{H} & 4.0525744 & 1.2228053 & 0.7109135 \\ \mathrm{H} & 3.4570007 & 2.7533792 & 0.0251693 \\ \mathrm{C} & 2.4254152 & -4.7300776 & -1.3995092 \\ \mathrm{H} & 0.2862600 & -4.3179790 & -1.4228955 \\ \mathrm{H} & 1.1581315 & -3.7078053 & -2.8481152 \\ \mathrm{H} & -1.8315530 & -3.6122026 & 4.3989708 \\ \mathrm{H} & -1.3970939 & -4.2858707 & 2.7920309 \\ \mathrm{H} & -0.1389098 & -3.5084111 & 3.8095064 \\ \mathrm{H} & 4.7187894 & 3.1764990 & 2.1687999 \\ \mathrm{H} & 2.9923186 & 3.6321779 & 2.3758942 \\ \mathrm{H} & 3.6028784 & 2.0929444 & 3.0668075 \\ \mathrm{H} & 2.3269606 & -5.7305422 & -1.8762743 \\ \mathrm{H} & 3.3859167 & -4.2863724 & -1.7474657 \\ \mathrm{H} & 2.5118211 & -4.8894638 & -0.3004385 \\ \mathrm{O} & -0.7922217 & 1.9548110 & -0.0797224 \\ \mathrm{C} & -1.7052443 & 2.1300034 & -1.1491821 \\ \mathrm{C} & -3.1702064 & 2.3134731 & -0.6685622 \\ \mathrm{C} & -3.1791990 & 3.7999975 & -0.2773019 \\ \mathrm{C} & -2.3122013 & 4.4544969 & -1.3808209 \\ \mathrm{~N} & -1.5169250 & 3.3457081 & -1.9431903 \\ \mathrm{H} & -3.4087429 & 1.6293597 & 0.1715088 \\ \mathrm{H} & -3.8785457 & 2.1120238 & -1.5042594 \\ \mathrm{H} & -4.1980283 & 4.2387571 & -0.2068418 \\ \mathrm{H} & -2.6819852 & 3.9107015 & 0.7100246 \\ \mathrm{H} & -2.9515933 & 4.9154088 & -2.1738562 \\ \mathrm{H} & -1.6767529 & 5.2731756 & -0.9679983 \\ \mathrm{H} & -0.5190460 & 3.5616486 & -2.0464542\end{array}$

\section{S20_NVP}

$\mathrm{E}(\mathrm{BP} 86 / \mathrm{def} 2-\mathrm{SV}(\mathrm{P}))=-1255.497022508$
C -1.9984693
$0.0376777-2.7301250$
$\begin{array}{llll}\text { C } & -1.4569432 & 0.5467074 & -1.6102745\end{array}$
$\begin{array}{llll}\mathrm{P} & -0.2522758 & -0.1357870 & -0.3468048\end{array}$
$\begin{array}{llll}\text { C } & -1.0315347 & -0.7652846 & 1.2334921\end{array}$
$\begin{array}{llll}\text { C } & 0.1139197 & -1.7857168 & -1.2707177\end{array}$
$\begin{array}{llll}\text { C } & 1.5034226 & 0.4824613 & -0.1592552\end{array}$

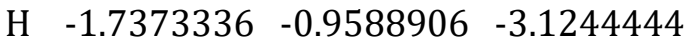
$\begin{array}{llll}\mathrm{H} & -2.7363115 & 0.6271015 & -3.3080948\end{array}$
$\begin{array}{lrrr}\text { C } & -0.6604150 & -2.1267847 & 1.8350002\end{array}$
$\begin{array}{llll}\mathrm{H} & -0.8593781 & 0.0551146 & 1.9607409\end{array}$

\begin{tabular}{|c|c|c|c|}
\hline & -2.1230305 & & \\
\hline & 593 & 423 & \\
\hline & 850 & & \\
\hline & 486839 & & \\
\hline & 683 & -2.6 & -0 \\
\hline & .8361864 & 704679 & -1.2 \\
\hline & .2316614 & 28874 & -2.3 \\
\hline & & & \\
\hline & 93 & & \\
\hline & 0.424 & 227 & 2.0 \\
\hline & 3.25 & 45 & \\
\hline & & & \\
\hline & & & \\
\hline & 1.22 & -4.0 & -1.5 \\
\hline & 2.25 & -2.2 & -1.1 \\
\hline & 1.37 & -2.8 & \\
\hline & -1.1 & -3. & \\
\hline & -1.3 & & \\
\hline & -2.5 & 669 & 2.8 \\
\hline & 3.5 & 2.4 & 2.5 \\
\hline & & & \\
\hline & $3.3^{\prime}$ & & \\
\hline & 2.44 & -4.9 & -1. \\
\hline & 0.2 & -4.5 & -1.2 \\
\hline & 1.13 & -3. & -2.6 \\
\hline & -1.7 & & \\
\hline & -1.3 & -4 . & \\
\hline & -0.0 & -3. & \\
\hline & 4.62 & & \\
\hline & 2.89 & 3.3 & \\
\hline & 3.5 & & \\
\hline & 2.3 & -5. & -1. \\
\hline & 3.3 & -4 & -1.6 \\
\hline & 2.548 & & -0.1 \\
\hline & -0.86 & 27 & 0.2 \\
\hline & 4 & & -0.8 \\
\hline & $-3.1^{\prime}$ & 2.1 & -0.4 \\
\hline & -3.136 & 4052 & -0.2 \\
\hline & -2.2244304 & 0834 & -1.3 \\
\hline & -1.3 & $3 .($ & -1.6 \\
\hline & -3.4 & 58 & 0.3 \\
\hline & -3.8593361 & 95928 & -1.3 \\
\hline & -4.1397902 & 6767 & -0.2 \\
\hline & -2.66 & & 0.7 \\
\hline & -2.80 & & -2.2 \\
\hline H & -1.60 & 555 & -1.0 \\
\hline & -0.2796116 & 3.2012311 & $-2.4 \mathrm{C}$ \\
\hline & 0.1 & & -3.1 \\
\hline & & 4.2651997 & -3.775 \\
\hline & -0.3701057 & 5.2889335 & -3.067537 \\
\hline & 0.2755958 & 2.2543168 & -2.618705 \\
\hline
\end{tabular}




\section{S21}

$\mathrm{E}(\mathrm{BP} 86 /$ def2-SV(P)) $=-288.5243065598$

$\begin{array}{cccc}\mathrm{C} & 0.3707837 & 0.1748704 & 0.3725904 \\ \mathrm{C} & -0.8788595 & -0.7138530 & 0.4037785 \\ \mathrm{C} & -1.6119838 & -0.3507501 & -0.9077399 \\ \mathrm{C} & -0.4610775 & 0.0721805 & -1.8400807 \\ \mathrm{~N} & 0.4352598 & 0.7534785 & -0.9118827 \\ \mathrm{C} & 1.2298294 & 0.3479517 & 1.3731811 \\ \mathrm{H} & -1.4923971 & -0.5513547 & 1.3141928 \\ \mathrm{H} & -0.5695427 & -1.7839691 & 0.4006298 \\ \mathrm{H} & -2.2136184 & -1.1885498 & -1.3187449 \\ \mathrm{H} & -2.2905547 & 0.5141230 & -0.7409089 \\ \mathrm{H} & 0.0049325 & -0.8281556 & -2.3230670 \\ \mathrm{H} & -0.7906704 & 0.7576503 & -2.6523608 \\ \mathrm{C} & 2.0580342 & 0.5215610 & 2.3873605 \\ \mathrm{H} & 2.9173090 & -0.1578922 & 2.5788971 \\ \mathrm{H} & 1.9454350 & 1.3586906 & 3.1109335 \\ \mathrm{H} & 1.3471205 & 1.0740185 & -1.2467789\end{array}$

\section{S21_NVP}

$\mathrm{E}(\mathrm{BP} 86 /$ def2-SV(P)) $=-365.8720016413$

$\begin{array}{llll}C & -0.0151352 & -0.2363963 & 0.8560848\end{array}$

$\begin{array}{llll}\text { C } & -1.2736819 & -1.0977472 & 0.8976570\end{array}$

$\begin{array}{llll}\text { C } & -2.0504238 & -0.6370842 & -0.3531869\end{array}$

$\begin{array}{llll}\text { C } & -0.9362061 & -0.2579412 & -1.3461926\end{array}$

$\begin{array}{llll}\mathrm{N} & 0.1579131 & 0.1854413 & -0.4839366\end{array}$

$\begin{array}{llll}\text { C } & 0.7768708 & 0.0425471 & 1.8874091\end{array}$

$\mathrm{H} \quad-1.8379600 \quad-0.97660351 .8446910$

H $\quad-0.9905379-2.1729450 \quad 0.8115228$

H $\quad-2.7332081 \quad-1.4149674 \quad-0.7546326$

$\mathrm{H} \quad-2.6603844 \quad 0.2611931-0.1126184$

$\mathrm{H} \quad-0.6168982 \quad-1.1256256 \quad-1.9765388$

H $\quad-1.2341718 \quad 0.5612929 \quad-2.0387192$

C $\quad 1.2673531 \quad 0.8584593 \quad-0.9600181$

$\begin{array}{llll}\text { C } & 1.5226697 & 1.1836042 & -2.2538178\end{array}$

$\mathrm{H} \quad 2.4402724 \quad 1.7412256-2.4978590$

H $\quad 0.8533197 \quad 0.9117848 \quad-3.0869208$

H $\quad 1.9755539 \quad 1.1371752-0.1596470$

C $\quad 1.5520003 \quad 0.2756243 \quad 2.9301115$

H $\quad 2.3879882 \quad-0.4014101 \quad 3.2107954$

H $1.4146660 \quad 1.1623728 \quad 3.5858163$

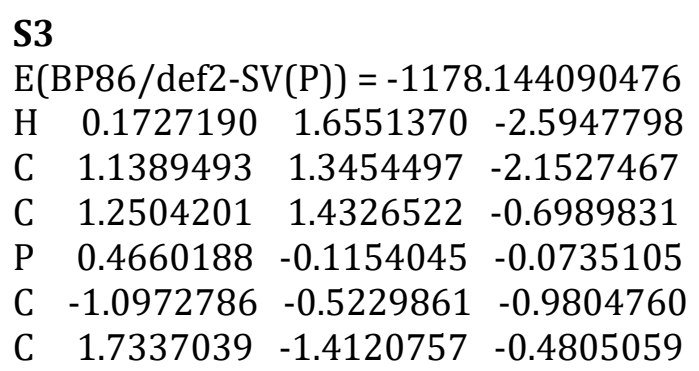

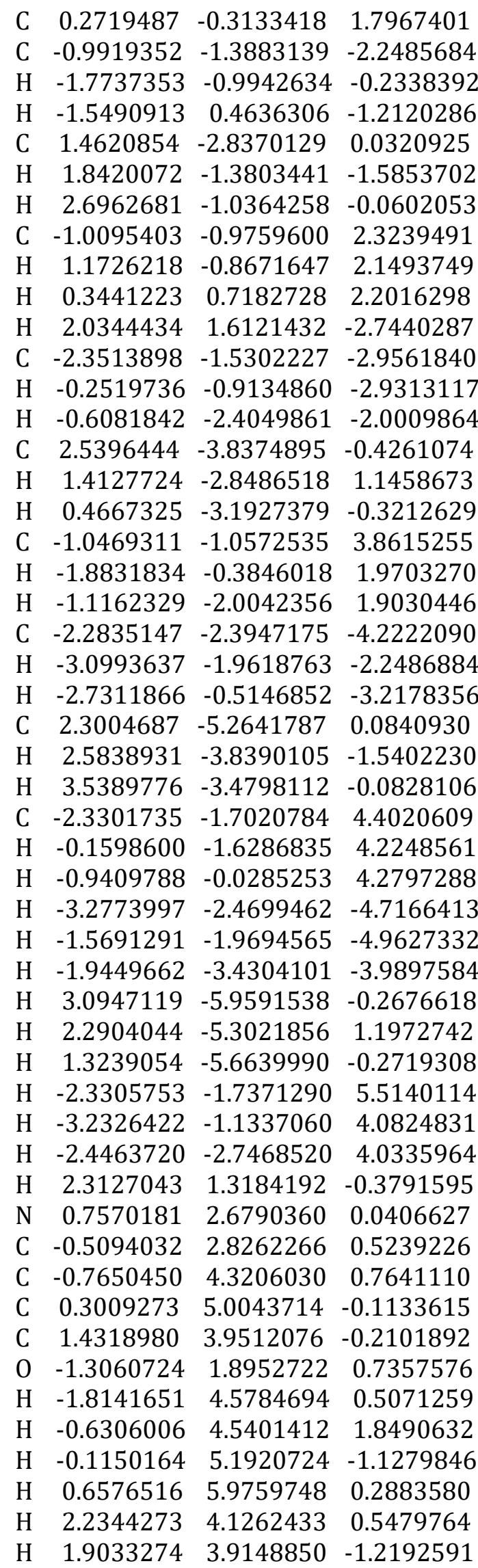




\section{S4}

$\mathrm{E}(\mathrm{BP} 86 /$ def2-SV(P) $)=-1198.993683152$

$0-2.4578766-3.6360626 \quad 1.1606967$

$\begin{array}{llll}\text { C } & -2.8296730 & -2.1800237 & 1.1195323\end{array}$

$\begin{array}{llll}\text { C } & -2.4539927 & -1.4604899 & -0.1146465\end{array}$

$\mathrm{H} \quad-3.9293977 \quad-2.2560647 \quad 1.2641735$

$\mathrm{H} \quad-2.4041593 \quad-1.7122525 \quad 2.0408670$

P $-1.3177692 \quad-0.1942382-0.2385838$

H $\quad-3.0679285-1.6177747 \quad-1.0198503$

$\begin{array}{llll}\text { C } & -0.9392441 & 0.0497449 & -2.0448200\end{array}$

$\begin{array}{llll}\text { C } & -1.9103356 & 1.4992813 & 0.3620029\end{array}$

$\begin{array}{llll}\text { C } & 0.2228169 & -0.5757753 & 0.7291724\end{array}$

$\begin{array}{llll}\text { C } & 0.4489514 & 0.5932888 & -2.4238005\end{array}$

$\mathrm{H} \quad-1.0878274 \quad-0.9580232-2.4924796$

$\begin{array}{lllll}\mathrm{H} & -1.7403223 & 0.7065470 & -2.4564778\end{array}$

C $\quad-1.2439696 \quad 2.8065937 \quad-0.1017302$

$\begin{array}{llll}\mathrm{H} & -2.9835717 & 1.4938971 & 0.0607370\end{array}$

H $\quad-1.9163272 \quad 1.4307962 \quad 1.4759995$

C $\quad 1.1746100 \quad 0.5497073 \quad 1.1674426$

H $\quad-0.1523759 \quad-1.1251296 \quad 1.6232788$

$\mathrm{H} \quad 0.7561799 \quad-1.3513648 \quad 0.1343713$

C $\quad 0.6407540 \quad 0.7063517 \quad-3.9474649$

$\mathrm{H} \quad 0.6156785 \quad 1.5932161 \quad-1.9620791$

H $\quad 1.2385328 \quad-0.0759188-2.0100834$

$\begin{array}{llll}\text { C } & -1.9137139 & 4.0604521 & 0.4929345\end{array}$

$\mathrm{H} \quad-0.1632245 \quad 2.8148384 \quad 0.1643539$

H $\quad-1.2886553 \quad 2.8737722 \quad-1.2140428$

$\begin{array}{llll}\text { C } & 2.3743121 & 0.0181318 & 1.9745317\end{array}$

$\mathrm{H} \quad 1.5543150 \quad 1.1077412 \quad 0.2814560$

$\mathrm{H} \quad 0.6241106 \quad 1.2919414 \quad 1.7903315$

C $\quad 2.0252298 \quad 1.2330102 \quad-4.3474580$

H $\quad 0.4712336 \quad-0.2939429-4.4114415$

$\begin{array}{lllll}\mathrm{H} & -0.1488075 & 1.3737340 & -4.3667094\end{array}$

C $\quad-1.2767112 \quad 5.3741360 \quad 0.0207486$

$\mathrm{H} \quad-2.9979356 \quad 4.0536017 \quad 0.2308139$

H $\quad-1.8710415 \quad 4.0031095 \quad 1.6063080$

C $\quad 3.3344826 \quad 1.1229028 \quad 2.4338130$

$\mathrm{H} \quad 1.9964757 \quad-0.5450744 \quad 2.8593110$

H $2.9264993 \quad-0.7289139 \quad 1.3581023$

H $\quad 2.1291526 \quad 1.3005542 \quad-5.4528023$

H $\quad 2.2099132 \quad 2.2493146 \quad-3.9306569$

$\mathrm{H} \quad 2.8361416 \quad 0.5671376 \quad-3.9745268$

$\mathrm{H} \quad-1.7851262 \quad 6.2564894 \quad 0.4685567$

$\mathrm{H} \quad-0.2006840 \quad 5.4283830 \quad 0.3030535$

H $\quad-1.3363368 \quad 5.4797086 \quad-1.0865081$

H $\quad 4.1883291 \quad 0.7039276 \quad 3.0109745$

$\begin{array}{llll}\mathrm{H} & 3.7576433 & 1.6801037 & 1.5671970\end{array}$

$\begin{array}{llll}\mathrm{H} & 2.8199623 & 1.8639571 & 3.0873695\end{array}$

C $1.6135213 \quad-4.6049237 \quad 1.4177032$

C $\quad 0.9008202 \quad-4.2310492 \quad 2.5725130$
C $\quad-0.4595833 \quad-3.8903563 \quad 2.4877732$

C $-1.1314725 \quad-3.9246245 \quad 1.2425070$

$\begin{array}{llll}\text { C } & -0.4090697 & -4.2967831 & 0.0850485\end{array}$

C $\quad 0.9505680 \quad-4.6405843 \quad 0.1771579$

H $\quad 2.6789160 \quad-4.8814251 \quad 1.4874774$

H $\quad 1.4077869-4.2179166 \quad 3.5529717$

H $\quad-1.0318609-3.6178880 \quad 3.3905409$

H $-0.9481095 \quad-4.3270586-0.8743196$

H $1.5001667 \quad-4.9427126-0.7313411$

\section{S5}

$\mathrm{E}(\mathrm{BP} 86 /$ def2-SV(P)) $=-1992.601888385$

C $\quad 0.4209068 \quad 0.4567428 \quad 1.6885952$

$\begin{array}{llll}\text { C } & 1.3953683 & -0.6006337 & 1.2572959\end{array}$

$\begin{array}{llll}\mathrm{P} & 0.8300292 & -2.1538637 & 0.7486773\end{array}$

$\begin{array}{llll}\text { C } & -0.4288707 & -2.9638672 & 1.8750793\end{array}$

$\begin{array}{llll}\text { C } & 0.0310327 & -2.0631764 & -0.9228834\end{array}$

C $\quad 2.3430176-3.2292272 \quad 0.7101523$

$\begin{array}{llll}\text { C } & -0.6450616 & -4.4870780 & 1.8393193\end{array}$

$\mathrm{H} \quad-0.1033150 \quad-2.65481792 .8953683$

H $-1.3708009-2.4023426 \quad 1.6581106$

C $-0.8407709-3.2145412 \quad-1.4488487$

$\mathrm{H} \quad-0.6205437 \quad-1.1657168 \quad-0.7969854$

H $\quad 0.8514222 \quad-1.8052562-1.6314866$

$\begin{array}{llll}\text { C } & 2.3732419 & -4.4091054 & -0.2756921\end{array}$

$\mathrm{H} \quad 3.1759547 \quad-2.5264367 \quad 0.4830633$

H $\quad 2.5014446 \quad-3.5891169 \quad 1.7532224$

P $\quad 0.6620380 \quad 2.0891160 \quad 0.8305444$

H $\quad 2.3565637 \quad-0.6607711 \quad 1.8013173$

C $\quad-1.7316641 \quad-4.9470981 \quad 2.8299130$

H $\quad-0.9299717 \quad-4.8194823 \quad 0.8163771$

H $\quad 0.3079876 \quad-5.0147069 \quad 2.0831314$

C $-1.3846842 \quad-2.9247737 \quad-2.8605730$

H $\quad-0.2810349 \quad-4.1777746 \quad-1.4674187$

H $\quad-1.7034352 \quad-3.3504776 \quad-0.7599252$

C $3.6973154-5.1925554-0.2171482$

$\mathrm{H} \quad 1.5293268 \quad-5.1058177-0.0707176$

H $\quad 2.2204728 \quad-4.0338011-1.3140938$

C $-1.9622435-6.4639851 \quad 2.8226882$

H $\quad-1.4521959-4.6151654 \quad 3.8577225$

H $-2.6842728 \quad-4.4199231 \quad 2.5899449$

C $\quad-2.2861187 \quad-4.0404729-3.4050611$

$\mathrm{H} \quad-1.9489908-1.9640621 \quad-2.8360806$

H $\quad-0.5295543 \quad-2.7620467 \quad-3.5594242$

C $3.7571843-6.3605425-1.2102678$

H $\quad 4.5441982-4.4927261-0.4104462$

H $\quad 3.8503019-5.5750067 \quad 0.8198105$

H $\quad-2.7526283-6.7589214 \quad 3.5481953$

$\mathrm{H} \quad-2.2810738-6.8210417 \quad 1.8169676$

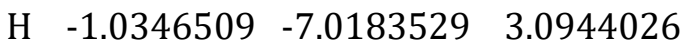

H $\quad-2.6658905 \quad-3.7972493 \quad-4.4223363$ 


\begin{tabular}{|c|c|c|c|}
\hline & 40 & 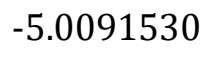 & \\
\hline & -3.1700408 & -4.2018731 & -2.7 \\
\hline & 865 & -6.9045974 & 1 \\
\hline & 14 & -7.0966490 & 93 \\
\hline & 3.6467832 & -6.0066110 & -2.2 \\
\hline & 2.4143549 & 2.5658312 & 1.1524744 \\
\hline & 0.2516388 & 1.8010364 & -0.9 \\
\hline & 0.4326660 & 0.7 & \\
\hline & -0.64 & 061 & \\
\hline & -0.5548816 & 3.2456571 & 1.57 \\
\hline & 3.0472999 & 3.77 & 0.43 \\
\hline & 2.47 & & \\
\hline & $2.9^{\prime}$ & & \\
\hline & 0.527 & 963 & -1.9 \\
\hline & 0.76 & 3953 & -1.16 \\
\hline & -0.85 & 377 & -0.84 \\
\hline & -0.4 & 4.7 & 1.2 \\
\hline & -1.5 & 2.7 & \\
\hline & -0.5 & 1629 & 2.6 \\
\hline & 4.48 & 4.03 & 0.91 \\
\hline & 3.0 & & -0.6 \\
\hline & 2.4 & 4.6 & 0.5 \\
\hline & -0.0 & 2.4 & \\
\hline & 7736 & 3.8 & -1.6 \\
\hline & 1.62 & 3.0 & -2.1 \\
\hline & -1.6 & 5.5 & 1.8 \\
\hline & 0.4 & & 1.6 \\
\hline & -0.4 & 4.9 & 216 \\
\hline & 5.1572242 & 5.21 & 0.18 \\
\hline & 4.48 & 4.2 & 2.00 \\
\hline & 5.0 & 3.1 & 0.7 \\
\hline & 0.17 & 3.5 & -4.45 \\
\hline & & 025 & -3.66 \\
\hline & -1.1639200 & 0217 & -3.23 \\
\hline & -1.6228266 & 3825 & 8537 \\
\hline & -2.6 & 5.0 & 1.4 \\
\hline & -1.6 & 5.3 & 2.97 \\
\hline & 6.1936463 & 9034 & 0.5572632 \\
\hline & 5.2161454 & 9851 & 2542 \\
\hline & $4.5 \mathrm{c}$ & 6.1 & 538 \\
\hline $\mathrm{I}$ & -0.2734065 & 3.1 & -5.42 \\
\hline & -0.2787875 & 8365 & -4.1944861 \\
\hline & 1.2601115 & 6389 & -4.6306430 \\
\hline & -2.49 & 7.55 & 288 \\
\hline & -0.6975244 & 6222 & 6322 \\
\hline $\mathrm{I}$ & -1.6461991 & 7.2352751 & 0.4998719 \\
\hline & -3.0980178 & 0.2810890 & 0.2959127 \\
\hline & -4.60 & 7809 & 0.1 \\
\hline & -5.2660052 & 0418 & 0.1619637 \\
\hline & -4.1122630 & 2.2194767 & -0.3508990 \\
\hline & -2.8624326 & 1.5670842 & 0.0262475 \\
\hline
\end{tabular}

$\begin{array}{rrrr}\mathrm{O} & -2.2331862 & -0.6001704 & 0.6054578 \\ \mathrm{H} & -4.9339117 & -0.7652879 & 0.9518282 \\ \mathrm{H} & -4.7522480 & -0.6047182 & -0.8221468 \\ \mathrm{H} & -6.1934320 & 1.3862225 & -0.4486387 \\ \mathrm{H} & -5.5285455 & 1.5934881 & 1.2068753 \\ \mathrm{H} & -4.1834457 & 2.3427458 & -1.4660347 \\ \mathrm{H} & -4.1786219 & 3.2538215 & 0.0688529\end{array}$

\section{S5_BF4}

$\mathrm{E}(\mathrm{BP} 86 / \mathrm{def} 2-\mathrm{SV}(\mathrm{P}))=-2130.959273033$

F $\quad-2.8649285 \quad 1.4996401 \quad 0.9079492$

$\begin{array}{llll}\text { C } & 0.2366006 & 0.5433755 & 1.6510367\end{array}$

$\begin{array}{llll}\text { C } & 1.1551983 & -0.4726227 & 1.0383453\end{array}$

$\begin{array}{lllll}\mathrm{P} & 0.5944230 & -2.0539416 & 0.6168031\end{array}$

$\begin{array}{llll}\text { C } & -0.3462114 & -2.9473270 & 1.9719536\end{array}$

$\begin{array}{llll}\text { C } & -0.5015639 & -2.0158870 & -0.8736203\end{array}$

$\begin{array}{llll}\text { C } & 2.1316939 & -3.0327609 & 0.2681277\end{array}$

$\begin{array}{lrrr}\text { C } & -0.4733109 & -4.4813890 & 1.9680601\end{array}$

H $\quad 0.1710376 \quad-2.6170881 \quad 2.9028277$

H $\quad-1.3476289-2.4582032 \quad 1.9802590$

C $-1.4320448 \quad-3.1998716-1.1887415$

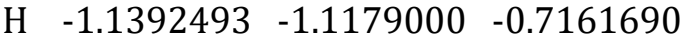

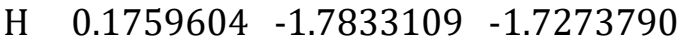

$\begin{array}{llll}\text { C } & 2.0221751 & -4.2315465 & -0.6890146\end{array}$

$\mathrm{H} \quad 2.8506474 \quad-2.2858350 \quad-0.1383815$

$\begin{array}{llll}\mathrm{H} & 2.5311211 & -3.3576415 & 1.2566989\end{array}$

$\begin{array}{llll}\mathrm{P} & 0.2963813 & 2.1957914 & 0.7794194\end{array}$

H $\quad 2.1905565 \quad-0.4953555 \quad 1.4266583$

C $\quad-1.2870323 \quad-5.0013144 \quad 3.1688111$

$\begin{array}{llll}\mathrm{H} & -0.9536095 & -4.8326148 & 1.0280261\end{array}$

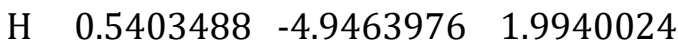

$\begin{array}{llll}\text { C } & -2.2647269 & -2.9428549 & -2.4591395\end{array}$

H $-0.8604080-4.1496978-1.3044287$

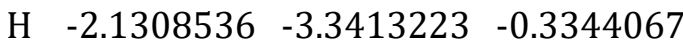

$\begin{array}{llll}\text { C } & 3.3722652 & -4.9395418 & -0.9030997\end{array}$

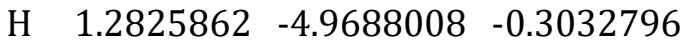

H $\quad 1.6317316 \quad-3.8889541 \quad-1.6744851$

$\begin{array}{lrrr}\text { C } & -1.4097670 & -6.5303236 & 3.2056836\end{array}$

$\mathrm{H} \quad-0.8152890 \quad-4.6403781 \quad 4.1133190$

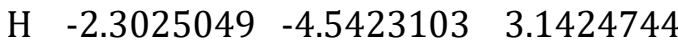

$\begin{array}{llll}\text { C } & -3.2569689 & -4.0717020 & -2.7678563\end{array}$

H $-2.8095170 \quad-1.9809954-2.3300225$

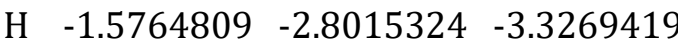

$\begin{array}{llll}\text { C } & 3.2852512 & -6.1298651 & -1.8670840\end{array}$

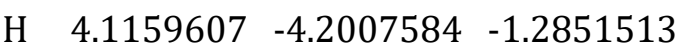

H $\quad 3.7654531 \quad-5.2843906 \quad 0.0824916$

H $\quad-2.0064330 \quad-6.8680769 \quad 4.0818498$

H $-1.9103375 \quad-6.9191758 \quad 2.2900622$

H $-0.4104989-7.0182355 \quad 3.2721988$

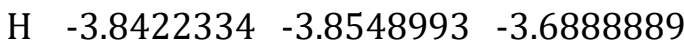

H $\quad-2.7376856 \quad-5.0461075 \quad-2.9205481$ 


$\begin{array}{cccc}\mathrm{H} & -3.9844854 & -4.2059201 & -1.9357236 \\ \mathrm{H} & 4.2759734 & -6.6180147 & -1.9995129 \\ \mathrm{H} & 2.5766798 & -6.9034417 & -1.4935498 \\ \mathrm{H} & 2.9303189 & -5.8115323 & -2.8734572 \\ \mathrm{~B} & -3.3488146 & 0.2506429 & 0.4020755 \\ \mathrm{~F} & -4.7171102 & 0.1540261 & 0.5309316 \\ \mathrm{~F} & -2.9463126 & 0.1633794 & -0.9723130 \\ \mathrm{~F} & -2.6832149 & -0.7943109 & 1.1176592 \\ \mathrm{C} & 2.0752017 & 2.6690700 & 0.7075540 \\ \mathrm{C} & -0.3641415 & 1.9986900 & -0.9212800 \\ \mathrm{H} & 0.4428761 & 0.7938414 & 2.7246659 \\ \mathrm{H} & -0.8420417 & 0.2675616 & 1.5933820 \\ \mathrm{C} & -0.6506720 & 3.4120138 & 1.7904888 \\ \mathrm{C} & 2.5062753 & 3.7310282 & -0.3194999 \\ \mathrm{H} & 2.3707103 & 2.9682872 & 1.7405740 \\ \mathrm{H} & 2.5829225 & 1.6979204 & 0.4913294 \\ \mathrm{C} & -0.9578025 & 3.2432914 & -1.6061160 \\ \mathrm{H} & 0.4579843 & 1.5434635 & -1.5175364 \\ \mathrm{H} & -1.1707793 & 1.2365780 & -0.8295367 \\ \mathrm{C} & -0.4222854 & 4.9184213 & 1.5760306 \\ \mathrm{H} & -1.7142893 & 3.1215556 & 1.6376728 \\ \mathrm{H} & -0.4056484 & 3.1387725 & 2.8446271 \\ \mathrm{C} & 4.0242667 & 3.9882489 & -0.2923507 \\ \mathrm{H} & 2.2129461 & 3.3989320 & -1.3415301 \\ \mathrm{H} & 1.9693980 & 4.6901250 & -0.1404847 \\ \mathrm{C} & -1.5489332 & 2.8908266 & -2.9836382 \\ \mathrm{H} & -1.7739334 & 3.6477327 & -0.9661756 \\ \mathrm{H} & -0.1968374 & 4.0509718 & -1.7173301 \\ \mathrm{H} & -1.0 .0694072 & 7.5806448 & 2.5270259 \\ \mathrm{C} & -1.3211480 & 5.7703712 & 2.4918327 \\ \mathrm{H} & 0.6444999 & 5.1757272 & 1.7728949 \\ \mathrm{H} & -0.6205223 & 5.1915333 & 0.5152180 \\ \mathrm{C} & 4.4808252 & 5.0260254 & -1.3256544 \\ \mathrm{H} & 4.3208323 & 4.3218770 & 0.7298900 \\ \mathrm{H} & 4.5594863 & 3.0250136 & -0.4649423 \\ \mathrm{C} & -2.2104785 & 4.0873153 & -3.6790038 \\ \mathrm{H} & -0.7431242 & 2.4755299 & -3.6345827 \\ \mathrm{H} & -2.2906016 & 2.0727203 & -2.8434195 \\ \mathrm{C} & -1.1186085 & 7.2792505 & 2.3054313 \\ \mathrm{H} & -2.3866990 & 5.5059885 & 2.2996102 \\ \mathrm{H} & -1.1259018 & 5.4938149 & 3.5548092 \\ \mathrm{H} & 5.5801208 & 5.1875122 & -1.2778156 \\ \mathrm{H} & 4.2336737 & 4.7026544 & -2.3619884 \\ \mathrm{H} & -2.6331928 & 3.7957412 & -4.6656271 \\ \mathrm{H} & -1.4844230 & 4.9139335 & -3.8554018 \\ & & & \\ \mathrm{H} & & \end{array}$

\section{S5_Phenol}

\begin{tabular}{|c|c|c|c|}
\hline \multicolumn{4}{|c|}{$\mathrm{E}(\mathrm{BP} 86 /$ def2-SV(P)) $=-2013.4405$} \\
\hline & & & \\
\hline & & & \\
\hline & & & \\
\hline & 7012082 & -2.9774007 & $2.07 / 6168$ \\
\hline & 64 & 620 & \\
\hline & & & \\
\hline & & & \\
\hline & 2682 & -2.6 & \\
\hline & 87096 & -2.7 & \\
\hline & & & \\
\hline & 46 & & \\
\hline & 9674 & & \\
\hline & 3.26 & & \\
\hline & & & \\
\hline & & & \\
\hline & & & \\
\hline & 2.5 & & \\
\hline & 0.2 & -5 . & \\
\hline & & & \\
\hline & & & \\
\hline & 82 & -2 & \\
\hline & & & \\
\hline & 91 & -3 & -0 \\
\hline & & & \\
\hline & & & \\
\hline & & & 60 \\
\hline & $0.4 \varepsilon$ & & \\
\hline & & -4 & \\
\hline & 5 & & \\
\hline & & & \\
\hline & -2.0 & & \\
\hline & -0.6 & -2 . & 84 \\
\hline & & & \\
\hline & & & \\
\hline & & & \\
\hline & -0.011 & -7.3694359 & 564 \\
\hline & & & \\
\hline & & & \\
\hline & -2.56 & 01 & 226 \\
\hline & -1.2133325 & 9344 & 813 \\
\hline & -2.62 & 762 & 027 \\
\hline & & & \\
\hline & & & \\
\hline $\mathrm{H}$ & 4.500 & 93975 & 094 \\
\hline & 1.5953756 & 3.3497140 & \\
\hline & 0.26 & & \\
\hline $\mathrm{H}$ & & 0.8958475 & \\
\hline$x^{2}+2$ & -0.5997374 & 0.1778333 & 310 \\
\hline $\mathrm{C}$ & -1.4430682 & 2.9726521 & 502 \\
\hline & 1.5691192 & 4.8610021 & 1.22 \\
\hline
\end{tabular}




\begin{tabular}{|c|c|c|c|}
\hline & 1.6576989 & 785406 & 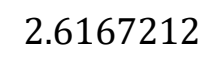 \\
\hline & & & \\
\hline & 68 & 46 & \\
\hline & 0521128 & 2603 & -0 . \\
\hline & -0.7178803 & 1.4543754 & -1.0223342 \\
\hline & -2.0393468 & 463 & 6862 \\
\hline & -2.0848037 & 2.0658063 & \\
\hline & -1.3796323 & 3.1947247 & 2. \\
\hline & 2.8472853 & 5.5724600 & 1.70 \\
\hline & 1.4362475 & 695 & 0.13 \\
\hline & 0.68 & 5.3 & \\
\hline & 0.47 & 2.6 & -3.2 \\
\hline & -0.181 & 623 & -1.6 \\
\hline & 55245 & 6801 & -1.6 \\
\hline & -3.4 & 4.5 & 1.1 \\
\hline & -1.3 & 5.0 & 0.6 \\
\hline $\mathrm{H}$ & -2.13 & 3.8 & \\
\hline & 2.83 & 7.08 & \\
\hline & 2.9861 & 5.3765366 & 2734 \\
\hline & 3.73 & 5.1 & 1.1 \\
\hline & 0.76 & 3.7 & \\
\hline & 1.20 & 85 & -3 \\
\hline 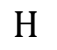 & -0.5278702 & 2.1 & -3.4 \\
\hline & -4.08 & 5.6 & 0.4 \\
\hline & -4.0 & 3.6 & \\
\hline 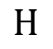 & -3.3 & 4.8 & \\
\hline$\pi$ & 3.76 & 7.5 & 1.8 \\
\hline & 2.7289448 & 7.3 & 0.36 \\
\hline & 1.98 & 7.5 & 1.9 \\
\hline $\mathrm{H}$ & 0.70 & 3.3 & -5. \\
\hline $\mathrm{H}$ & 0.03 & & -4.218 \\
\hline 2 & 1.7833676 & 4.1798604 & -4.1725674 \\
\hline $\mathrm{H}$ & -5.08 & 5.9 & 0.8060064 \\
\hline & -3.46 & 6.6 & 0.4 \\
\hline $\mathrm{H}$ & -4.2128164 & 5.4 & -0.672 \\
\hline & -2.0743145 & 0.2496277 & 0.1216455 \\
\hline & -5.7018246 & -1.7351565 & -0.6248512 \\
\hline & -5.20 & -0.7 & -1.5253815 \\
\hline & -3.99 & $-0.1 c$ & -1.28 \\
\hline $\mathrm{C}$ & -3.2068110 & -0.3659456 & -0.1085934 \\
\hline & -3.7404813 & -1.3542329 & 0.7900391 \\
\hline & -4.9498619 & -2.0155475 & 0.5335433 \\
\hline $\mathrm{H}$ & -6.6535901 & -2.2564959 & -0.8211087 \\
\hline $\mathrm{H}$ & -5.7796681 & -0.5334667 & -2.4408445 \\
\hline & -3.6212317 & 0.6516415 & -1.9938183 \\
\hline & -3.16 & -1.57795 & 1.70 \\
\hline & -5.3187 & $-2.7664 !$ & \\
\hline
\end{tabular}

\section{S6}

$\mathrm{E}(\mathrm{BP} 86 / \mathrm{def} 2-\mathrm{SV}(\mathrm{P}))=-2279.043919274$ $\begin{array}{llll}C & -0.7832651 & 0.6010075 & 0.3718523\end{array}$

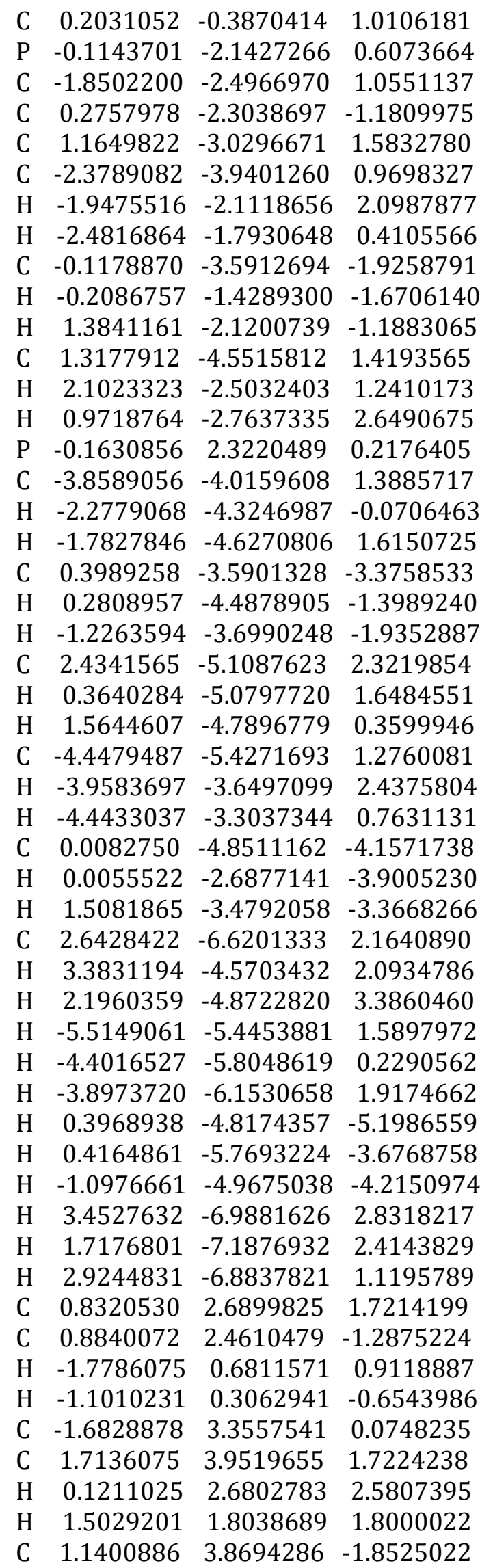




\begin{tabular}{|c|c|c|c|}
\hline & 1.8282602 & & \\
\hline & 0.3680470 & 8335748 & \\
\hline & 49847 & 26130 & 922 \\
\hline & 32743 & & 2861 \\
\hline & -2.4828958 & 92 & 768 \\
\hline & 2.4389785 & 1348271 & 3.0 \\
\hline & 2.4761564 & 3.8463403 & 0.9187748 \\
\hline & 1.1184950 & 4.8656524 & 1744 \\
\hline & 944 & 44 & -3.1 \\
\hline & 0.1737603 & 4.36 & -2 . \\
\hline & 1.6324019 & 987 & 480 \\
\hline & -2.9758877 & 5.5217687 & 383 \\
\hline & -1.3 & 4.8 & 361 \\
\hline & -0.82 & 5.38 & \\
\hline & 3.3766474 & 5.34 & \\
\hline & 1.6837552 & 4.23 & 927 \\
\hline & 3.0193423 & 3.2097682 & 005 \\
\hline & 2.30 & 5.21 & 608 \\
\hline & 2.98 & & 148 \\
\hline & 1.53 & 3.18 & -3 . \\
\hline & -2.96 & 6.95 & \\
\hline & -3.2492132 & 5.52 & -0 . \\
\hline & -3.76 & 4.92 & 305 \\
\hline & 3.88 & 5.4 & 49 \\
\hline & 4.1674380 & 5.25 & 572 \\
\hline & 2.82 & $6.2 \mathrm{C}$ & 373 \\
\hline & 2.94 & 5.1 & -4 \\
\hline & 1.36 & 5.7 & 370 \\
\hline & 2.82 & 5.8 & 526 \\
\hline & -3.9586879 & 7.4 & 364 \\
\hline & -2.7402768 & 6.98 & 909 \\
\hline & -2.20 & 7.5 & 92 \\
\hline & 0.16 & -0.3 & 18 \\
\hline & 1.2714121 & $-0.17 \mathrm{C}$ & 63 \\
\hline & -4.1524728 & 0.5052386 & 443 \\
\hline & -5.56 & 0.9 & -0 . \\
\hline & -5.68 & 0.2 & -1 . \\
\hline & -4.7083353 & -0.9459104 & -1. \\
\hline & -3.7003243 & -0.5392866 & 6362 \\
\hline & -3.5564038 & 1.1032792 & 1972 \\
\hline & -5.67 & 2.0 & -0 \\
\hline 1 & -6.3170747 & 0.5581137 & 875 \\
\hline & -6.7222869 & -0.0574492 & 0655 \\
\hline & -5.3213398 & 0.9322692 & 32636 \\
\hline & -5.2679281 & -1.8568193 & 320 \\
\hline & -4.2442973 & -1.2364321 & 0303 \\
\hline & 3.7061988 & -0.1742311 & -0.0189790 \\
\hline & 5.1960221 & 0.1356224 & 1529 \\
\hline & 5.84 & -1.2507222 & -0.2 \\
\hline & 4.644 & -2.18649 & -0.52 \\
\hline & 34303839 & -1.4708872 & -0.1386771 \\
\hline
\end{tabular}

$\begin{array}{cccc}\mathrm{O} & 2.8780297 & 0.7559843 & 0.2703604 \\ \mathrm{H} & 5.5947003 & 0.9016134 & 0.3884947 \\ \mathrm{H} & 5.2790326 & 0.5613157 & -1.3407558 \\ \mathrm{H} & 6.7195544 & -1.4069341 & -0.8699330 \\ \mathrm{H} & 6.1834745 & -1.4282062 & 0.8437047 \\ \mathrm{H} & 4.6286496 & -2.4313553 & -1.6239570 \\ \mathrm{H} & 4.7262052 & -3.1686532 & -0.0014086\end{array}$

\section{S6_Phenol}

$\mathrm{E}(\mathrm{BP} 86 /$ def2-SV(P)) $=-2320.730477246$

$\begin{array}{llll}\text { C } & -0.4874732 & 0.6408457 & 1.5872278\end{array}$

$\begin{array}{llll}\text { C } & 0.3430075 & -0.6616059 & 1.6139393\end{array}$

$\begin{array}{llll}\mathrm{P} & -0.4734848 & -2.1241936 & 0.8563023\end{array}$

$\begin{array}{llll}\text { C } & -2.1786414 & -2.2584417 & 1.5287753\end{array}$

$\begin{array}{llll}\text { C } & -0.5022592 & -1.8568484 & -0.9530947\end{array}$

C $\quad 0.5997245 \quad-3.5365691 \quad 1.3553433$

C $\quad-2.9034620-3.6113356 \quad 1.4043965$

H $\quad-2.1030533 \quad-1.9637721 \quad 2.6017402$

H $\quad-2.7195383-1.4324396 \quad 0.9913768$

C $-1.3457564-2.8126798-1.8174095$

$\mathrm{H} \quad-0.9546297 \quad-0.8361529 \quad-1.0402188$

H $\quad 0.5676808 \quad-1.8295324 \quad-1.2661188$

C $\quad 0.5030569-4.8415316 \quad 0.5430056$

$\mathrm{H} \quad 1.6430396 \quad-3.1385760 \quad 1.2981505$

H $\quad 0.3798003 \quad-3.7211386 \quad 2.4335920$

$\begin{array}{llll}\mathrm{P} & 0.3909801 & 2.1131349 & 0.9228553\end{array}$

C $-4.3422616-3.5382987 \quad 1.9480064$

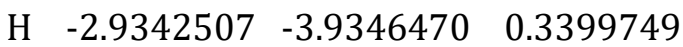

H $\quad-2.3441617 \quad-4.4022228 \quad 1.9570705$

C $-1.2041086-2.4780956-3.3136716$

$\mathrm{H} \quad-1.0633452 \quad-3.8767468-1.6510569$

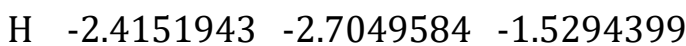

C $\quad 1.4287120-5.9337585 \quad 1.1088405$

$\mathrm{H} \quad-0.5454861 \quad-5.2173853 \quad 0.5191949$

H $\quad 0.7967863 \quad-4.6346523 \quad-0.5102964$

C $\quad-5.0975163 \quad-4.8692617 \quad 1.8389760$

$\mathrm{H} \quad-4.3130547 \quad-3.2095229 \quad 3.0137445$

H $\quad-4.8955339-2.7431395 \quad 1.3974793$

C $-2.0580112-3.3788964 \quad-4.2150029$

$\mathrm{H} \quad-1.4892171-1.4121405-3.4689555$

$\mathrm{H} \quad-0.1311816-2.5604009-3.6066350$

$\begin{array}{llll}\text { C } & 1.3708266 & -7.2412229 & 0.3084367\end{array}$

H $2.4727839 \quad-5.5443922 \quad 1.1177997$

H $1.1559475 \quad-6.1330258 \quad 2.1727489$

H $\quad-6.1312190 \quad-4.7793499 \quad 2.2390786$

H $\quad-5.1764882-5.2051619 \quad 0.7803325$

$\mathrm{H} \quad-4.5856940 \quad-5.6775622 \quad 2.4094778$

H $-1.9374593 \quad-3.1069663 \quad-5.2868106$

H $-1.7742686-4.4506846 \quad-4.1071627$

H $\quad-3.1398971-3.2908546 \quad-3.9666327$

H $\quad 2.0469081 \quad-8.0094011 \quad 0.7442797$ 


\begin{tabular}{|c|c|c|c|}
\hline & 0.3 & -7.6685226 & \\
\hline & 42456 & -7.0806354 & -0.7 \\
\hline & 2.0235483 & 2.2507016 & \\
\hline & 31521 & 91 & -0.8 \\
\hline & -0.8517768 & 0.9202637 & 2.6004236 \\
\hline & -1.4002202 & 0.5398691 & $0.913144 €$ \\
\hline & -0.7325989 & 3.5171624 & 1.3254600 \\
\hline & 2.7461925 & 3.60 & 1.7 \\
\hline & 1.84 & 1.9 & \\
\hline & 2.6182741 & 1.4328604 & 1.26 \\
\hline & 1.5213443 & 2.8214742 & -1.64 \\
\hline & 1.047 & 0.8 & $-0 . c$ \\
\hline & -0.44 & 1.8438597 & \\
\hline & -0.5 & 4.82 & \\
\hline & -1.76 & 3.1151235 & \\
\hline & -0.6203828 & 3.6958377 & 2.4 \\
\hline & 4.12 & 3.5 & 2.3 \\
\hline & 2.87 & & \\
\hline & 2.13 & 4.3 & 2.2 \\
\hline & 1.5252762 & 2.499 & -3.1 \\
\hline & 1.22 & 3.8 & -1. \\
\hline & 2.55 & 2.7 & -1.2 \\
\hline & -1.54 & 5.9 & \\
\hline & 0.4793453 & 5.21 & 43 \\
\hline & -0.7480820 & 4.625 & -0.5 \\
\hline & 4.87 & 4.88 & 2.3 \\
\hline & 4.00 & 3.20 & 31 \\
\hline & 4.73 & 2.75 & 391 \\
\hline & 2.4671379 & 3.403 & -3.95 \\
\hline & 1.8201057 & 1.4336963 & $-3.2 \varepsilon$ \\
\hline & 0.48 & 2.5 & -3.5 \\
\hline & -1.41 & 7.22 & 49 \\
\hline & -2.5860776 & 5.51 & 0.9 \\
\hline & -1.3872520 & 6.1039544 & $2.0^{\circ}$ \\
\hline & 5.8697336 & 4.7968738 & 943 \\
\hline & 5.055 & 5.22 & 325 \\
\hline & 4.307 & 5.6807372 & 084 \\
\hline & 2.4506227 & 3.1414442 & -5.0392851 \\
\hline & 2.1789668 & 4.4757598 & -3.86 \\
\hline & 3.51 & 3.30 & -3.6 \\
\hline 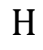 & -2.1408243 & 7.9845 & 3165 \\
\hline $\mathrm{I}$ & -0.3961361 & 7.6574734 & 0.3209807 \\
\hline & -1.6200866 & 7.0676781 & -0.8563290 \\
\hline & 0.618 & -0.9548268 & 221 \\
\hline & 1.3117789 & -0.5499767 & 1.02 \\
\hline 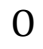 & -2.6349529 & 0.1889453 & -0.3510512 \\
\hline & -4.6171533 & 3.8513179 & -0.8969154 \\
\hline & -3.8900470 & 3.2580713 & -1.950004 \\
\hline & -3.2388911 & 2.0281326 & -1.7793410 \\
\hline & -3.2768542 & 1.3129045 & -0.5303468 \\
\hline & -4.0356216 & 1.9404230 & 0.5206042 \\
\hline
\end{tabular}

$\begin{array}{cccc}\mathrm{C} & -4.6857257 & 3.1704362 & 0.3353334 \\ \mathrm{H} & -5.1373178 & 4.8126424 & -1.0409441 \\ \mathrm{H} & -3.8380736 & 3.7651807 & -2.9308201 \\ \mathrm{H} & -2.6934619 & 1.5614735 & -2.6189602 \\ \mathrm{H} & -4.1155273 & 1.4070418 & 1.4849546 \\ \mathrm{H} & -5.2669868 & 3.6052630 & 1.1690875 \\ \mathrm{O} & 2.6710380 & -0.1785486 & -0.0979450 \\ \mathrm{C} & 4.6874090 & -3.8479605 & -0.4308061 \\ \mathrm{C} & 4.6294893 & -3.1658561 & 0.8012668 \\ \mathrm{C} & 3.9681069 & -1.9333162 & 0.9170350 \\ \mathrm{C} & 3.3248119 & -1.3042506 & -0.2078200 \\ \mathrm{C} & 3.4149926 & -2.0211441 & -1.4534732 \\ \mathrm{C} & 4.0762564 & -3.2533953 & -1.5544552 \\ \mathrm{H} & 5.2172622 & -4.8107526 & -0.5189386 \\ \mathrm{H} & 5.1194666 & -3.6015794 & 1.6914093 \\ \mathrm{H} & 3.9501018 & -1.3995400 & 1.8844040 \\ \mathrm{H} & 2.9619408 & -1.5528780 & -2.3454848 \\ \mathrm{H} & 4.1261082 & -3.7606128 & -2.5352769\end{array}$

S7_cis

$\mathrm{E}(\mathrm{BP} 86 / \mathrm{def} 2-\mathrm{SV}(\mathrm{P}))=-363.1155250755$

C $\quad-2.7224453 \quad 1.4471852-0.5024065$

C $-1.4097199 \quad 1.3999829-0.8660838$

$\mathrm{H} \quad-2.9306019 \quad 0.6484059 \quad 0.2842531$

$\mathrm{H} \quad-0.9466593 \quad 2.0797740 \quad-1.6146218$

$\begin{array}{llll}\mathrm{N} & -0.3392896 & 0.5179394 & -0.3861800\end{array}$

C $\quad 0.9868157 \quad 0.6112667 \quad-0.7381806$

$\begin{array}{llll}\text { C } & 1.7695692 & -0.4201306 & 0.1101475\end{array}$

C $\quad 0.6745335 \quad-1.3713720 \quad 0.6117859$

$\begin{array}{llll}\text { C } & -0.5827327 & -0.4763639 & 0.6441621\end{array}$

$\mathrm{H} \quad 0.5276123 \quad-2.1977297 \quad-0.1210643$

$\mathrm{H} \quad 0.8898069-1.8375187 \quad 1.6007089$

$\mathrm{H} \quad-0.7275369 \quad 0.0132407 \quad 1.6425640$

H $-1.5307796-1.0215723 \quad 0.4309581$

H $2.27554390 .1159966 \quad 0.9508530$

$\begin{array}{llll}0 & 1.4960369 & 1.3929115 & -1.5519512\end{array}$

H $\quad 2.5698469 \quad-0.9020157 \quad-0.4949444$

\section{S7_trans}

$\mathrm{E}(\mathrm{BP} 86 / \mathrm{def} 2-\mathrm{SV}(\mathrm{P}))=-363.1230077416$

$\begin{array}{llll}C & -2.6775012 & 0.9858191 & -0.4879109\end{array}$

C $-1.3865122 \quad 1.2710312-0.8158431$

$\mathrm{H} \quad-3.3160018 \quad 1.7459154 \quad-1.0346403$

H $\quad-0.9435181 \quad 2.0608583 \quad-1.4701345$

$\mathrm{N} \quad-0.3003856 \quad 0.4749780 \quad-0.2893455$

C $\quad 1.0347113 \quad 0.6457989 \quad-0.5446609$

$\begin{array}{llll}\text { C } & 1.7977272 & -0.4202662 & 0.2797292\end{array}$

C $\quad 0.7080652 \quad-1.4457315 \quad 0.6342740$

$\begin{array}{llll}\text { C } & -0.5775806 & -0.5943083 & 0.6612710\end{array}$

$\mathrm{H} \quad 0.6355729 \quad-2.2124218-0.1719240$

H $\quad 0.8845028 \quad-1.9852100 \quad 1.5932550$ 


$\begin{array}{cccc}\mathrm{H} & -0.7823122 & -0.1675322 & 1.6765611 \\ \mathrm{H} & -1.5219163 & -1.0989048 & 0.3554857 \\ \mathrm{H} & 2.2263511 & 0.0651245 & 1.1913252 \\ \mathrm{O} & 1.5645138 & 1.4990123 & -1.2722800 \\ \mathrm{H} & 2.6542837 & -0.8241628 & -0.3051620\end{array}$

\section{S8}

$\mathrm{E}(\mathrm{BP} 86 / \mathrm{def} 2-\mathrm{SV}(\mathrm{P}))=-1255.471310180$

$\begin{array}{llll}\text { C } & 0.4597111 & 0.2401367 & 3.1244063\end{array}$

$\begin{array}{llll}\text { C } & 1.9381561 & 0.2138734 & 3.6290035\end{array}$

$\begin{array}{llll}\text { C } & 1.8441813 & 0.3095013 & 5.1601592\end{array}$

$\begin{array}{llll}\text { C } & 0.5552442 & -0.4728516 & 5.4785840\end{array}$

$\begin{array}{llll}\mathrm{N} & -0.2921498 & -0.1797723 & 4.3410884\end{array}$

$\begin{array}{llll}\mathrm{H} & 2.4992481 & 1.0488934 & 3.1622844\end{array}$

$\mathrm{H} \quad 2.4248219-0.7450200 \quad 3.3354914$

$\mathrm{H} \quad 2.7361963 \quad-0.0959944 \quad 5.6849848$

$\begin{array}{llll}\mathrm{H} & 1.7152178 & 1.3702602 & 5.4691407\end{array}$

$\mathrm{H} \quad 0.7609931 \quad-1.5704243 \quad 5.5926921$

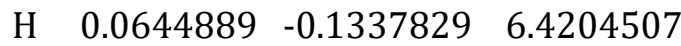

C $-1.6624627 \quad-0.1342286 \quad 4.3873160$

$\begin{array}{llll}\text { C } & -2.4843549 & -0.5820526 & 5.3774963\end{array}$

$\begin{array}{llll}\mathrm{H} & -3.5714623 & -0.4218718 & 5.2958378\end{array}$

$\mathrm{H} \quad-2.1090843 \quad-1.0942057 \quad 6.2803139$

$\begin{array}{llll}\mathrm{H} & -2.0800359 & 0.3773268 & 3.4990840\end{array}$

$\begin{array}{llll}\text { C } & 0.1492234 & -0.5159713 & 1.8541398\end{array}$

$\begin{array}{llll}0 & -0.0218668 & 1.3397483 & 2.4963620\end{array}$

$\begin{array}{llll}\text { C } & 1.0397259 & -0.8074689 & 0.8164675\end{array}$

$\begin{array}{llll}\mathrm{P} & 0.4835621 & -0.4701704 & -0.8075436\end{array}$

H $\quad 2.1269037 \quad-0.6696996 \quad 0.9685132$

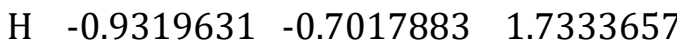

$\begin{array}{llll}\text { C } & 1.6726264 & -1.2019039 & -2.0201057\end{array}$

$\begin{array}{llll}\text { C } & -1.2101395 & -1.1832969 & -1.0408952\end{array}$

$\begin{array}{llll}\text { C } & 0.3820850 & 1.3708552 & -1.0374237\end{array}$

$\begin{array}{llll}\text { C } & 1.7142642 & -0.6275821 & -3.4470159\end{array}$

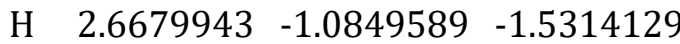

H $1.4698182-2.2974648-2.0295138$

$\begin{array}{llll}\text { C } & -1.6954475 & -1.4480448 & -2.4774917\end{array}$

H $-1.1968683 \quad-2.1294182-0.4549566$

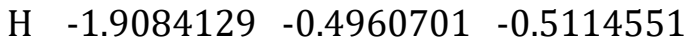

$\begin{array}{lrrr}\text { C } & -0.5503326 & 1.9844915 & -2.0948694\end{array}$

$\begin{array}{llll}\mathrm{H} & 0.1037511 & 1.7047858 & -0.0061009\end{array}$

H $\quad 1.4347276 \quad 1.7091389-1.1867256$

$\begin{array}{llll}\text { C } & 2.7758705 & -1.3145014 & -4.3259456\end{array}$

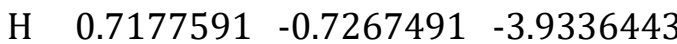

$\begin{array}{llll}\mathrm{H} & 1.9302145 & 0.4645777 & -3.4041271\end{array}$

$\begin{array}{llll}\text { C } & -3.1281770 & -2.0118470 & -2.5114960\end{array}$

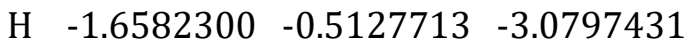

H $\quad-1.0111199 \quad-2.1713592-2.9789036$

$\begin{array}{llll}\text { C } & -0.5159693 & 3.5244247 & -2.0733713\end{array}$

$\mathrm{H} \quad-0.2818570 \quad 1.6272490 \quad-3.1161758$

$\begin{array}{llll}\mathrm{H} & -1.5969127 & 1.6481489 & -1.9101679\end{array}$ $\begin{array}{llll}\text { C } & 2.8390934 & -0.7492455 & -5.7507494\end{array}$

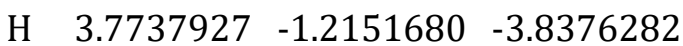

H $\quad 2.5648769-2.4090119-4.3660285$

$\begin{array}{llll}\text { C } & -3.6291022 & -2.3055309 & -3.9314098\end{array}$

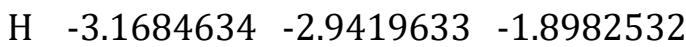

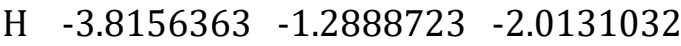

$\begin{array}{lllll}\text { C } & -1.4632666 & 4.1663584 & -3.0948884\end{array}$

$\begin{array}{lllll}\mathrm{H} & -0.7716390 & 3.8763107 & -1.0472572\end{array}$

H $\quad 0.5283092 \quad 3.8682891-2.2624828$

H $\quad 3.6159514 \quad-1.2661053 \quad-6.3556305$

H $\quad 1.8667878 \quad-0.8700226-6.2799560$

$\mathrm{H} \quad 3.0849552 \quad 0.3368070 \quad-5.7465715$

H $\quad-4.6659911 \quad-2.7070562 \quad-3.9174939$

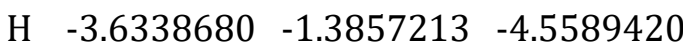

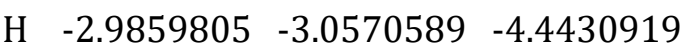

$\mathrm{H} \quad-1.4111247 \quad 5.2765297 \quad-3.0517970$

H $\quad-1.21046793 .8593141 \quad-4.1353952$

$\begin{array}{llll}\mathrm{H} & -2.5215572 & 3.8745488 & -2.9070533\end{array}$

\section{S9}

$\mathrm{E}(\mathrm{BP} 86 /$ def2-SV(P)) $=-1255.473543378$

$\begin{array}{llll}\text { C } & -2.2209018 & 2.3879683 & -2.1685493\end{array}$

$\begin{array}{llll}\text { C } & -3.4153161 & 2.9903729 & -1.4081703\end{array}$

$\begin{array}{llll}\text { C } & -4.1421581 & 3.8580113 & -2.4522318\end{array}$

$\begin{array}{llll}\text { C } & -3.8898828 & 3.1054518 & -3.7729753\end{array}$

$\begin{array}{lllll}\mathrm{N} & -2.5791892 & 2.5088111 & -3.5723548\end{array}$

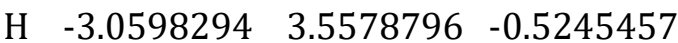

H $\quad-4.0754287 \quad 2.1681828$ - 1.0480114

H $\quad-5.2232132 \quad 3.9946322 \quad-2.2365960$

$\mathrm{H} \quad-3.6741718 \quad 4.8655061 \quad-2.5052844$

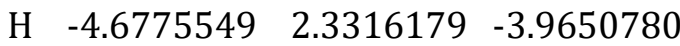

H $\quad-3.8707369 \quad 3.7800843 \quad-4.6596482$

$\begin{array}{llll}\text { C } & -1.7405545 & 2.1409470 & -4.5993054\end{array}$

$\begin{array}{llll}\text { C } & -2.0338880 & 2.0886124 & -5.9267452\end{array}$

$\mathrm{H} \quad-1.2429404 \quad 1.8251937-6.6468156$

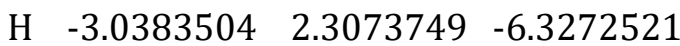

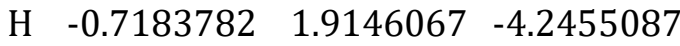

C $\quad-1.4189720 \quad 1.2676480-1.6226051$

$\begin{array}{lllll}0 & -0.8949937 & 2.7289451 & -1.8172232\end{array}$

$\begin{array}{llll}\text { C } & -1.5908350 & 0.6912367 & -0.2965360\end{array}$

$\begin{array}{llll}\mathrm{P} & -0.2747601 & -0.1102064 & 0.4646896\end{array}$

$\begin{array}{llll}\mathrm{H} & -2.2660091 & 1.2071024 & 0.4111677\end{array}$

H $-0.9384011 \quad 0.6294876 \quad-2.3913218$

$\begin{array}{lrrr}\text { C } & -0.9686538 & -1.0441174 & 1.9097329\end{array}$

$\begin{array}{llll}\text { C } & 0.5295222 & -1.2700225 & -0.7468117\end{array}$

$\begin{array}{llll}\text { C } & 1.1154317 & 0.9917164 & 1.1026038\end{array}$

$\begin{array}{llll}\text { C } & -0.0497324 & -1.4534914 & 3.0723434\end{array}$

H $-1.7837765 \quad-0.3810336 \quad 2.2824491$

H $\quad-1.4846432 \quad-1.9294812 \quad 1.4747400$

$\begin{array}{llll}\text { C } & 1.2178337 & -2.5311907 & -0.1939677\end{array}$

H $\quad-0.2947409-1.5592145 \quad-1.4359125$ 


$\begin{array}{cccc}\mathrm{H} & 1.2486412 & -0.6639692 & -1.3444650 \\ \mathrm{C} & 2.5212103 & 0.4417398 & 1.4005300 \\ \mathrm{H} & 1.1673986 & 1.7790483 & 0.3144458 \\ \mathrm{H} & 0.6900092 & 1.5045173 & 1.9978584 \\ \mathrm{C} & -0.8132098 & -2.1847153 & 4.1925717 \\ \mathrm{H} & 0.7725727 & -2.1090504 & 2.7051520 \\ \mathrm{H} & 0.4415073 & -0.5502154 & 3.5011279 \\ \mathrm{C} & 1.8093613 & -3.4127765 & -1.3094475 \\ \mathrm{H} & 2.0256938 & -2.2554232 & 0.5202292 \\ \mathrm{H} & 0.4823555 & -3.1350402 & 0.3865126 \\ \mathrm{C} & 3.4925214 & 1.5319348 & 1.8931196 \\ \mathrm{H} & 2.4739663 & -0.3705157 & 2.1619584 \\ \mathrm{H} & 2.9428875 & -0.0218525 & 0.4786180 \\ \mathrm{C} & 0.0802073 & -2.5918823 & 5.3713132 \\ \mathrm{H} & -1.6402525 & -1.5308070 & 4.5574725 \\ \mathrm{H} & -1.3076298 & -3.0896910 & 3.7674774 \\ \mathrm{C} & 2.4790403 & -4.6891158 & -0.7835294 \\ \mathrm{H} & 1.0003183 & -3.6815099 & -2.0279976 \\ \mathrm{H} & 2.5481518 & -2.8153275 & -1.8936771 \\ \mathrm{C} & 4.9083871 & 1.0081098 & 2.1666849 \\ \mathrm{H} & 3.5343840 & 2.3505981 & 1.1366652 \\ \mathrm{H} & 3.0793215 & 1.9956159 & 2.8196587 \\ \mathrm{H} & -0.5032266 & -3.1159410 & 6.1600580 \\ \mathrm{H} & 0.8947754 & -3.2783060 & 5.0461059 \\ \mathrm{H} & 0.5595759 & -1.7042838 & 5.8429721 \\ \mathrm{H} & 2.8931516 & -5.3007142 & -1.6151732 \\ \mathrm{H} & 3.3181772 & -4.4520878 & -0.0909463 \\ \mathrm{H} & 1.7561044 & -5.3272209 & -0.2261791 \\ \mathrm{H} & 5.5799720 & 1.8202126 & 2.5230827 \\ \mathrm{H} & 4.9041504 & 0.2111991 & 2.9452147 \\ \mathrm{H} & 5.3657009 & 0.5748389 & 1.2483102\end{array}$

\section{TS-AB_cis}

\begin{tabular}{lccc}
\multicolumn{4}{c}{ E(BP86/def2-SV(P) $=-891.6727261113$} \\
C & -0.7313497 & -0.4080898 & -2.1778712 \\
C & -0.8418825 & 0.3141307 & -1.0863300 \\
P & 0.3559401 & -0.0620147 & 0.5371778 \\
H & 0.0350406 & -1.1940692 & -2.3552333 \\
H & -1.5549085 & 1.1132352 & -0.8210177 \\
C & -0.3359925 & -1.6870006 & 1.1318150 \\
C & 2.0350417 & -0.3482477 & -0.1932064 \\
C & 0.5948318 & 1.0192304 & 2.0649855 \\
C & 1.8302680 & 0.8387683 & 2.9687350 \\
H & -0.3440482 & 0.9053468 & 2.6563415 \\
H & 0.5754627 & 2.0641136 & 1.6742065 \\
H & 2.5557848 & 0.6368657 & -0.1427794 \\
C & 2.9472362 & -1.4698766 & 0.3337032 \\
H & 1.8110205 & -0.5245844 & -1.2720330 \\
C & 0.1646173 & -2.3288025 & 2.4360427 \\
H & -1.4348145 & -1.5074682 & 1.1957465 \\
H & -0.2115435 & -2.3795694 & 0.2678159
\end{tabular}

$\begin{array}{cccc}\mathrm{C} & 1.8508632 & 1.8271889 & 4.1508679 \\ \mathrm{H} & 1.8731601 & -0.2018842 & 3.3614479 \\ \mathrm{H} & 2.7557738 & 0.9804908 & 2.3640502 \\ \mathrm{C} & 4.2710383 & -1.5615576 & -0.4485101 \\ \mathrm{H} & 3.1723730 & -1.3182994 & 1.4139563 \\ \mathrm{H} & 2.4167368 & -2.4472021 & 0.2589143 \\ \mathrm{C} & -0.5316316 & -3.6705026 & 2.7340793 \\ \mathrm{H} & 1.2651184 & -2.4902969 & 2.3951707 \\ \mathrm{H} & -0.0155786 & -1.6346855 & 3.2896514 \\ \mathrm{C} & 3.0875715 & 1.6772481 & 5.0467645 \\ \mathrm{H} & 1.7980156 & 2.8694641 & 3.7565702 \\ \mathrm{H} & 0.9276963 & 1.6860294 & 4.7612559 \\ \mathrm{C} & 5.1887349 & -2.6899426 & 0.0397658 \\ \mathrm{H} & 4.0450255 & -1.7032538 & -1.5313884 \\ \mathrm{H} & 4.8068384 & -0.5856251 & -0.3756404 \\ \mathrm{C} & -0.0681646 & -4.3173831 & 4.0456900 \\ \mathrm{H} & -1.6352290 & -3.5108215 & 2.7640461 \\ \mathrm{H} & -0.3503457 & -4.3718209 & 1.8858621 \\ \mathrm{H} & 3.0688622 & 2.4035749 & 5.8891276 \\ \mathrm{H} & 3.1495417 & 0.6557517 & 5.4861510 \\ \mathrm{H} & 4.0269577 & 1.8517916 & 4.4746344 \\ \mathrm{H} & 6.1343222 & -2.7252714 & -0.5450260 \\ \mathrm{H} & 5.4620372 & -2.5573522 & 1.1113581 \\ \mathrm{H} & 4.6969469 & -3.6842846 & -0.0586517 \\ \mathrm{H} & -0.5887797 & -5.2834256 & 4.2275003 \\ \mathrm{H} & 1.0262268 & -4.5237753 & 4.0326153 \\ \mathrm{H} & -0.2727157 & -3.6572228 & 4.9191389\end{array}$

\section{TS-AB_cis-PMe3}

$\mathrm{E}(\mathrm{BP} 86 /$ def2-SV(P) $)=-538.1567913114$

C $\quad-1.2709379-0.2190463 \quad-2.0931104$

$\begin{array}{llll}\text { C } & -1.0027921 & 0.6496737 & -1.1309972\end{array}$

$\begin{array}{lllll}\mathrm{P} & 0.1862833 & 0.0810049 & 0.3336326\end{array}$

$\mathrm{H} \quad-0.8507695 \quad-1.2524733 \quad-2.1013921$

$\mathrm{H} \quad-1.3479796 \quad 1.6904790 \quad-1.0016096$

$\begin{array}{llll}\text { C } & -0.5624689 & -1.4453115 & 1.0429227\end{array}$

$\begin{array}{llll}\text { C } & 1.7459516 & -0.4924464 & -0.4616355\end{array}$

$\begin{array}{llll}\text { C } & 0.8060219 & 0.9853428 & 1.8569525\end{array}$

$\begin{array}{cccc}\mathrm{H} & -0.0643608 & 1.3048893 & 2.4701869\end{array}$

$\begin{array}{llll}\mathrm{H} & 1.3610702 & 1.8949280 & 1.5402586\end{array}$

$\begin{array}{lllll}\mathrm{H} & 2.3472218 & 0.3904984 & -0.7694696\end{array}$

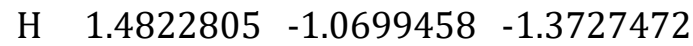

$\mathrm{H} \quad-1.4248003 \quad-1.1664557 \quad 1.6866600$

$\begin{array}{llll}\mathrm{H} & -0.9394004 & -2.0709178 & 0.2064757\end{array}$

$\mathrm{H} \quad 0.1724980 \quad-2.0261665 \quad 1.6419982$

H $\quad 2.3487892 \quad-1.1287720 \quad 0.2225927$

H $\quad 1.4766930 \quad 0.3530193 \quad 2.4839816$

\section{TS-AB_cis-PPh3}

$\mathrm{E}(\mathrm{BP} 86 / \mathrm{def} 2-\mathrm{SV}(\mathrm{P}))=-1112.966477380$ $\begin{array}{llll}\text { C } & -0.6520871 & 0.4533758 & -2.3467385\end{array}$ 


\begin{tabular}{|c|c|c|c|}
\hline & & & \\
\hline & & & \\
\hline & & 366 & \\
\hline & 15 & 03 & \\
\hline & -2.1 & -3.628 & \\
\hline & 0260 & -3.0 & \\
\hline & & & \\
\hline & -0.6 & & \\
\hline & -1.6 & -2.0 & \\
\hline & -2.3 & -3.1 & \\
\hline & -2.7 & -4 & \\
\hline & $-1 .($ & -3 & \\
\hline & 0.3 & -1.5 & \\
\hline & -1.8 & -1.5 & -0 \\
\hline & -3.16 & -3.5 & -0 \\
\hline & & -1. & \\
\hline & 3.6 & -0.4 & \\
\hline & & & \\
\hline & 1.81 & -0.7 & -0 \\
\hline & 2.2 & -2.0 & -0 . \\
\hline & 3.4 & -2.5 & \\
\hline & & -2.0 & \\
\hline & 4.2 & 0.2 & -2 \\
\hline & 2.1 & & \\
\hline & 17 & -2.6 & \\
\hline & & -3.5 & \\
\hline & & 2.5 & \\
\hline & 0.33 & 2.18 & 3.8 \\
\hline & -0.0 & 1.3 & \\
\hline & & 0.87 & \\
\hline & 2.2728105 & 1.2641312 & 7469 \\
\hline & 2.6464465 & 2.1006634 & 4080 \\
\hline & & 3.22 & \\
\hline & & & \\
\hline & -1.10 & 1.07 & 2.7 \\
\hline & 3.03 & 0.9012492 & 1.3604601 \\
\hline & 3.7055734 & 2.385195 & 3.246397 \\
\hline
\end{tabular}

\section{TS-AB_trans}

\begin{tabular}{lccc}
\multicolumn{4}{c}{ E(BP86/def2-SV $(\mathrm{P}))=-891.6744949712$} \\
$\mathrm{C}$ & 0.7818187 & 1.6918174 & -1.1169401 \\
$\mathrm{C}$ & 0.1499176 & 0.5820549 & -1.1856542 \\
$\mathrm{P}$ & 0.5381712 & -0.4692240 & 0.6963436 \\
$\mathrm{H}$ & 0.9388116 & 2.5356644 & -1.8030798 \\
$\mathrm{H}$ & -0.4927989 & -0.0847290 & -1.7760423 \\
$\mathrm{C}$ & -0.1712650 & -2.0206258 & 1.5037121 \\
$\mathrm{C}$ & 2.3596201 & -0.7893847 & 0.5386881 \\
$\mathrm{C}$ & 0.3550434 & 0.8848041 & 1.9497995 \\
$\mathrm{C}$ & 1.4630593 & 1.1138193 & 2.9926162 \\
$\mathrm{H}$ & -0.6278743 & 0.7181081 & 2.4502714 \\
$\mathrm{H}$ & 0.2678728 & 1.7923108 & 1.3021366
\end{tabular}

$\begin{array}{cccc}\mathrm{H} & 2.8111337 & -0.9730104 & 1.5395869 \\ \mathrm{C} & 2.7030362 & -1.9247100 & -0.4399658 \\ \mathrm{H} & 2.7627328 & 0.1788942 & 0.1614986 \\ \mathrm{C} & 0.4332462 & -2.5300428 & 2.8249960 \\ \mathrm{H} & -1.2580845 & -1.8064089 & 1.6355718 \\ \mathrm{H} & -0.1170742 & -2.8183765 & 0.7262087 \\ \mathrm{C} & 1.1364950 & 2.2779171 & 3.9462932 \\ \mathrm{H} & 1.6432860 & 0.1918127 & 3.5934299 \\ \mathrm{H} & 2.4201284 & 1.3361817 & 2.4680843 \\ \mathrm{C} & 4.2180344 & -2.0950680 & -0.6528251 \\ \mathrm{H} & 2.2782924 & -2.8903746 & -0.0754253 \\ \mathrm{H} & 2.2155429 & -1.7189569 & -1.4216921 \\ \mathrm{C} & -0.2331614 & -3.8235472 & 3.3323756 \\ \mathrm{H} & 1.5260839 & -2.7107853 & 2.6967563 \\ \mathrm{H} & 0.3406968 & -1.7422220 & 3.6078597 \\ \mathrm{C} & 2.2416207 & 2.5505921 & 4.9751634 \\ \mathrm{H} & 0.9490531 & 3.1976224 & 3.3441727 \\ \mathrm{H} & 0.1784633 & 2.0606555 & 4.4758105 \\ \mathrm{C} & 4.5722691 & -3.2234628 & -1.6301742 \\ \mathrm{H} & 4.6415883 & -1.1321851 & -1.0229201 \\ \mathrm{H} & 4.7081221 & -2.2853153 & 0.3315195 \\ \mathrm{C} & 0.3600463 & -4.3377995 & 4.6509201 \\ \mathrm{H} & -1.3272599 & -3.6452941 & 3.4587935 \\ \mathrm{H} & -0.1425568 & -4.6124293 & 2.5486304 \\ \mathrm{H} & 1.9725811 & 3.3957132 & 5.6469399 \\ \mathrm{H} & 2.4296242 & 1.6593043 & 5.6164714 \\ \mathrm{H} & 3.2031899 & 2.8111620 & 4.4773501 \\ \mathrm{H} & 5.6730010 & -3.3158803 & -1.7629128 \\ \mathrm{H} & 4.1941464 & -4.2074157 & -1.2699691 \\ \mathrm{H} & 4.1271004 & -3.0431639 & -2.6349070 \\ \mathrm{H} & -0.1431954 & -5.2716250 & 4.9862921 \\ \mathrm{H} & 1.4463488 & -4.5606698 & 4.5476198 \\ \mathrm{H} & 0.2502926 & -3.5865271 & 5.4656960\end{array}$

\section{TS-AB_trans-PMe3}

$\mathrm{E}(\mathrm{BP} 86 /$ def2-SV(P) $)=-538.1537438833$

$\begin{array}{llll}C & -1.3048907 & -0.3239480 & -1.9358416\end{array}$

$\begin{array}{llll}\text { C } & -1.2082303 & 0.7130701 & -1.1882788\end{array}$

$\begin{array}{llll}\mathrm{P} & 0.1837839 & 0.1826498 & 0.3694784\end{array}$

H $\quad-1.8864219 \quad-0.5339542 \quad-2.8459946$

H $-1.5233421 \quad 1.7485864-0.9962551$

C $\quad-0.5316931 \quad-1.3680974 \quad 1.0572858$

$\begin{array}{llll}\text { C } & 1.6648892 & -0.3794035 & -0.5694041\end{array}$

$\begin{array}{llll}\text { C } & 0.9299897 & 1.0106974 & 1.8799944\end{array}$

$\begin{array}{llll}\mathrm{H} & 1.6389107 & 0.3457287 & 2.4247366\end{array}$

$\mathrm{H} \quad 0.1156885 \quad 1.3102593 \quad 2.5753127$

H $1.4721041 \quad 1.9292885 \quad 1.5659392$

$\mathrm{H} \quad 2.2942250 \quad 0.4964179-0.8402730$

$\mathrm{H} \quad 2.2746614 \quad-1.1062786 \quad 0.0103004$

$\mathrm{H} \quad 1.2829974 \quad-0.8585597 \quad-1.4979690$

H $\quad 0.2462477 \quad-2.0224930 \quad 1.5074602$ 
$\begin{array}{llll}\mathrm{H} & -1.2962692 & -1.1214701 & 1.8261231 \\ \mathrm{H} & -1.0214502 & -1.8897935 & 0.2053852\end{array}$

\section{TS-AB_trans-PPh3}

$\mathrm{E}(\mathrm{BP} 86 /$ def2-SV(P)) $=-1112.957464200$

$\begin{array}{llll}C & -2.0767200 & 0.3357057 & -1.1185597\end{array}$

$\begin{array}{llll}\text { C } & -0.9588710 & 0.9216073 & -0.9103317\end{array}$

$\mathrm{P} \quad 0.4364590 \quad-0.1416136 \quad 0.3779250$

H $\quad-3.0031534 \quad 0.5570845 \quad-1.6633364$

$\mathrm{H} \quad-0.3718082 \quad 1.8262315-1.1392657$

C $-2.0941606-3.6917762 \quad 1.9160218$

C $-1.4022690 \quad-2.8941180 \quad 2.8488949$

C $-0.6054919-1.8270909 \quad 2.4077992$

C $-0.4839415-1.5568494 \quad 1.0259994$

C $-1.2016889-2.3439270 \quad 0.0899295$

C $-1.9919301-3.4168566 \quad 0.5436746$

$\mathrm{H} \quad-2.7172977 \quad-4.5322425 \quad 2.2654730$

H $-1.4799582-3.1088916 \quad 3.9277476$

H $\quad-0.0672357 \quad-1.2029028 \quad 3.1394734$

H $\quad-1.1276540 \quad-2.1297051-0.9860002$

H $\quad-2.5385420 \quad-4.0359428 \quad-0.1871960$

C $\quad 4.2547329 \quad-1.5675371-1.8664745$

$\begin{array}{llll}\text { C } & 3.9836338 & -0.1976785 & -1.6941141\end{array}$

C $\quad 2.8450033 \quad 0.2149176-0.9862509$

$\begin{array}{llll}\text { C } & 1.9639440 & -0.7416454 & -0.4179750\end{array}$

C $\quad 2.2427370 \quad-2.1173507 \quad-0.6004804$

C $3.3782615-2.5221212-1.3198920$

H $\quad 5.1471491 \quad-1.8899319 \quad-2.4279279$

$\mathrm{H} \quad 4.6619556 \quad 0.5592816 \quad-2.1224279$

$\mathrm{H} \quad 2.6414877 \quad 1.2932478 \quad-0.8696914$

$\mathrm{H} \quad 1.5685686-2.8744187 \quad-0.1684080$

H $3.5844526 \quad-3.5982447 \quad-1.4465267$

C $\quad 1.5787984 \quad 2.6326256 \quad 3.9336002$

C $\quad 0.2634702 \quad 2.5790691 \quad 3.4386816$

$\begin{array}{llll}\text { C } & -0.0542163 & 1.7292687 & 2.3673027\end{array}$

$\begin{array}{llll}\text { C } & 0.9427311 & 0.9047687 & 1.7887397\end{array}$

$\begin{array}{llll}\text { C } & 2.2636841 & 0.9653763 & 2.2907286\end{array}$

C $\quad 2.5762922 \quad 1.8265993 \quad 3.3548862$

H $\quad 1.8284224 \quad 3.3060890 \quad 4.7703619$

$\mathrm{H} \quad-0.5222062 \quad 3.2121171 \quad 3.8839190$

H $\quad-1.0825688 \quad 1.7026738 \quad 1.9683460$

$\begin{array}{llll}\mathrm{H} & 3.0491417 & 0.3303337 & 1.8500302\end{array}$

H $3.60868821 .8614475 \quad 3.7416238$

\section{TS-BC_cis}

$\mathrm{E}(\mathrm{BP} 86 / \mathrm{def} 2-\mathrm{SV}(\mathrm{P}))=-1178.126908421$

$\begin{array}{llll}C & 0.1119496 & 0.2902171 & 3.8975876\end{array}$

C $\quad 0.3248343 \quad 0.9017895 \quad 5.3048833$

$\begin{array}{llll}\text { C } & -0.5287847 & 0.0040838 & 6.2145299\end{array}$

C $-0.5717968-1.3363911 \quad 5.4318094$

N $\quad-0.4256963 \quad-0.9426013 \quad 4.0393844$

\begin{tabular}{|c|c|c|c|}
\hline & 89233 & 846 & \\
\hline & & 069 & \\
\hline & & & \\
\hline & 5 & 53 & \\
\hline & 7703 & & \\
\hline & 04861 & -2.0147958 & \\
\hline & 208 & & \\
\hline & & -17 r & \\
\hline & -0.3310573 & -2.14 & \\
\hline & 195 & -1.65 & \\
\hline & 124 & -0.7 & \\
\hline & & 27 & \\
\hline & -2.2 & -1. & \\
\hline & -0.4 & 0.9 & \\
\hline & 1.13 & -1.4 & \\
\hline & -1.6 & -0.9 & \\
\hline & 1.8 & -1.0 & \\
\hline & 1.65 & -1.1 & -2 \\
\hline & $1.0^{\prime}$ & -2.5 & \\
\hline & -1.5 & -1.9 & -2. \\
\hline & -1.6 & 0.1 & -3 . \\
\hline & -2.7 & -0.9 & \\
\hline & -0.1 & & \\
\hline & 0.5 & $1.9 !$ & -0.8 \\
\hline & -1. & & \\
\hline & 3.0 & -1.6 & \\
\hline & 0.9 & -1.5 & \\
\hline & & -0.0 & \\
\hline & -2.74 & $-0.0^{\prime}$ & -4 \\
\hline & $-1 . \varepsilon$ & & \\
\hline & -0.6 & & \\
\hline & & 3.31 & -0.1 \\
\hline & & 1.51 & -0.8 \\
\hline & 0.3 & 2.1 & -1. \\
\hline & 3.6 & -1.4 & \\
\hline & & -1.2 & \\
\hline & & -2.78 & -2.6 \\
\hline & $-2.7 !$ & 1.00 & -5.4 \\
\hline & -2.5 & -1.0 & $-4 . \varepsilon$ \\
\hline & -3.7 & -0.1 & -3. \\
\hline & 1.66 & 4.28 & -0.7 \\
\hline & -0.38 & 3.78 & 732 \\
\hline & & 3.12 & \\
\hline & & -1.8 & \\
\hline & 2.9 & -1.8936922 & -4.9 \\
\hline & 3.6666118 & -0.3413764 & 0007 \\
\hline & -3.5 & 0.79 & -6.1 \\
\hline & $-2.977 €$ & 2.00 & -4.9 \\
\hline & -1.7735301 & 1.0757697 & -5.939470 \\
\hline & 1.6672477 & 5.2488907 & -0.1731687 \\
\hline & 2.6908336 & 3.8618983 & -0.669356 \\
\hline
\end{tabular}




\section{H $\quad 1.4498690 \quad 4.5102532 \quad-1.7954355$}

\begin{tabular}{lccc}
\multicolumn{4}{l}{ TS-BC_cis-PMe3 } \\
E(BP86/def2-SV(P)) =-824.6079188561 \\
C & -0.5332425 & -0.2237893 & -1.9102863 \\
C & -1.1974840 & -0.0793326 & -3.3023193 \\
C & -0.0922774 & 0.5585312 & -4.1595897 \\
C & 1.2088791 & 0.1114984 & -3.4369200 \\
N & 0.8021563 & -0.0837549 & -2.0544609 \\
O & -1.1623299 & -0.4608751 & -0.8547336 \\
H & -2.1366488 & 0.5094785 & -3.2342498 \\
H & -1.4770188 & -1.0983416 & -3.6604317 \\
H & -0.1142161 & 0.2602919 & -5.2302969 \\
H & -0.1756242 & 1.6678426 & -4.1193141 \\
H & 1.6091662 & -0.8363724 & -3.8815148 \\
H & 2.0227326 & 0.8684086 & -3.5177008 \\
H & 1.5523838 & -0.1986386 & -1.0525826 \\
C & 1.7428328 & -0.4720784 & 0.3393652 \\
C & 1.3361221 & 0.4392869 & 1.2667626 \\
P & -0.0084908 & 0.1095642 & 2.4022311 \\
H & 1.4070194 & -1.5195121 & 0.5354440 \\
H & 1.5462562 & 1.5273280 & 1.1611673 \\
C & -1.4481123 & 1.1229580 & 1.9065023 \\
C & -0.5452947 & -1.6239934 & 2.2672958 \\
C & 0.3371086 & 0.4763192 & 4.1738770 \\
H & -0.8678459 & -1.7604293 & 1.2101144 \\
H & -1.3928844 & -1.8104256 & 2.9611130 \\
H & 0.2925989 & -2.3135095 & 2.5019728 \\
H & 1.1703209 & -0.1695320 & 4.5252647 \\
H & -0.5585727 & 0.3053941 & 4.8105050 \\
H & 0.6527005 & 1.5378494 & 4.2709538 \\
H & -1.6739734 & 0.8132514 & 0.8570089 \\
H & -2.3221972 & 0.9448753 & 2.5699273 \\
H & -1.1748980 & 2.2000351 & 1.9316744
\end{tabular}

\section{TS-BC_cis-PPh3}

$\mathrm{E}(\mathrm{BP} 86 /$ def2-SV(P)) $=-1399.413502592$

$\begin{array}{llll}\text { C } & 0.1081605 & 0.0346733 & -4.3946093\end{array}$

$\begin{array}{llll}\text { C } & 0.1277367 & 0.2682068 & -5.9287348\end{array}$

$\begin{array}{llll}\text { C } & 1.5553822 & -0.1373680 & -6.3266947\end{array}$

$\begin{array}{llll}\text { C } & 1.9480744 & -1.1462585 & -5.2108738\end{array}$

N $\quad \begin{array}{llll}1.1651335 & -0.7360183 & -4.0579336\end{array}$

$\begin{array}{lllll}0 & -0.7742581 & 0.4830709 & -3.6347843\end{array}$

$\mathrm{H} \quad-0.1494727 \quad 1.3150418 \quad-6.1749648$

H $\quad-0.6435106 \quad-0.3924700 \quad-6.3914352$

H $\quad 1.6433457 \quad-0.5671719 \quad-7.3487972$

H $\quad 2.2275855 \quad 0.7488241 \quad-6.2766109$

H $\quad 1.7049843 \quad-2.1966884 \quad-5.5235996$

H $\quad 3.0418379-1.1291111-4.9925637$

H $\quad 1.2470243 \quad-1.1580706 \quad-2.8278818$

C $\quad 0.9303122-1.3384366-1.4833564$

$\begin{array}{cccc}\mathrm{C} & 1.2376707 & -0.3881039 & -0.5666244 \\ \mathrm{P} & 0.2213020 & -0.0639217 & 0.8923457 \\ \mathrm{H} & 0.0312185 & -1.9658986 & -1.2782870 \\ \mathrm{H} & 2.0700888 & 0.3350436 & -0.6911328 \\ \mathrm{C} & -4.2809472 & -0.4333823 & -0.1404401 \\ \mathrm{C} & -3.8418684 & -0.7269864 & 1.1639769 \\ \mathrm{C} & -2.4765321 & -0.6464273 & 1.4799300 \\ \mathrm{C} & -1.5472261 & -0.2658106 & 0.4851431 \\ \mathrm{C} & -1.9813724 & 0.0254536 & -0.8292558 \\ \mathrm{C} & -3.3530434 & -0.0629640 & -1.1281833 \\ \mathrm{H} & -5.3538544 & -0.5037523 & -0.3870782 \\ \mathrm{H} & -4.5648605 & -1.0294004 & 1.9396697 \\ \mathrm{H} & -2.1371551 & -0.8932150 & 2.4993229 \\ \mathrm{H} & -1.2830784 & 0.2854858 & -1.6535729 \\ \mathrm{H} & -3.6773090 & 0.1545410 & -2.1589009 \\ \mathrm{C} & 1.2455513 & -2.9833979 & 4.3665580 \\ \mathrm{C} & 0.6788311 & -1.7301826 & 4.6590913 \\ \mathrm{C} & 0.3757541 & -0.8336091 & 3.6210183 \\ \mathrm{C} & 0.6410124 & -1.1912269 & 2.2794895 \\ \mathrm{C} & 1.2184404 & -2.4462500 & 1.9901720 \\ \mathrm{C} & 1.5156165 & -3.3385183 & 3.0333209 \\ \mathrm{H} & 1.4845911 & -3.6841169 & 5.1837189 \\ \mathrm{H} & 0.4739314 & -1.4448357 & 5.7042634 \\ \mathrm{H} & -0.0566625 & 0.1525649 & 3.8583171 \\ \mathrm{H} & 1.4484550 & -2.7128379 & 0.9449863 \\ \mathrm{H} & 1.9692159 & -4.3161064 & 2.8010104 \\ \mathrm{C} & 1.0582928 & 4.2945678 & 2.2502978 \\ \mathrm{C} & -0.1785603 & 3.9751155 & 1.6634131 \\ \mathrm{C} & -0.4639118 & 2.6523053 & 1.2876831 \\ \mathrm{C} & 0.4916865 & 1.6354860 & 1.5064174 \\ \mathrm{C} & 1.7392834 & 1.9613488 & 2.0906817 \\ \mathrm{C} & 2.0160497 & 3.2861564 & 2.4616855 \\ \mathrm{H} & 1.2790334 & 5.3346491 & 2.5420294 \\ \mathrm{H} & -0.9309367 & 4.7627173 & 1.4930852 \\ \mathrm{H} & -1.4318401 & 2.4086653 & 0.8207857 \\ \mathrm{H} & 2.4955890 & 1.1769456 & 2.2628448 \\ \mathrm{H} & 2.9887277 & 3.5325425 & 2.9188028\end{array}$

\section{TS-BC_trans}

$\mathrm{E}(\mathrm{BP} 86 /$ def2-SV(P) $)=-1178.142581073$

$\begin{array}{llll}\text { C } & -2.0027347 & -0.8220623 & 0.1313344\end{array}$

$\begin{array}{llll}\text { C } & -3.5347816 & -0.9809611 & 0.2603964\end{array}$

$\begin{array}{llll}\text { C } & -4.0182554 & 0.4534296 & 0.5279633\end{array}$

$\begin{array}{llll}\text { C } & -2.9111217 & 1.3187744 & -0.1286799\end{array}$

$\begin{array}{llll}\mathrm{N} & -1.7173944 & 0.4867522 & -0.0587279\end{array}$

$\begin{array}{lllll}0 & -1.1914944 & -1.7699492 & 0.1775095\end{array}$

H $\quad-3.7937570 \quad-1.7198024 \quad 1.0475923$

$\mathrm{H} \quad-3.9217702-1.3841391 \quad-0.7052084$

$\begin{array}{llll}\mathrm{H} & -5.0312002 & 0.6757592 & 0.1284821\end{array}$

$\begin{array}{llll}\mathrm{H} & -4.0406221 & 0.6465623 & 1.6239995\end{array}$

$\begin{array}{lllll}\mathrm{H} & -3.1709966 & 1.5645210 & -1.1904467\end{array}$ 


$\begin{array}{cccc}\mathrm{H} & -2.7574010 & 2.2896884 & 0.3950845 \\ \mathrm{H} & -0.6551856 & 0.9173806 & -0.3196634 \\ \mathrm{C} & 0.7555898 & 1.6456097 & -0.7458040 \\ \mathrm{C} & 2.0541484 & 1.2697954 & -0.6185972 \\ \mathrm{P} & 2.3819561 & -0.2833804 & 0.2423507 \\ \mathrm{C} & 1.5186601 & -0.2270783 & 1.8702129 \\ \mathrm{C} & 1.7511024 & -1.6822618 & -0.7679082 \\ \mathrm{C} & 4.2138836 & -0.4050862 & 0.4837222 \\ \mathrm{C} & 1.1762419 & -1.5481534 & 2.5791557 \\ \mathrm{H} & 2.1306867 & 0.4475820 & 2.5142950 \\ \mathrm{H} & 0.5856033 & 0.3308416 & 1.6239988 \\ \mathrm{C} & 2.2597046 & -3.1076255 & -0.4913667 \\ \mathrm{H} & 0.6392796 & -1.6296500 & -0.6070366 \\ \mathrm{H} & 1.9525230 & -1.3847665 & -1.8220999 \\ \mathrm{C} & 4.7453454 & -1.3712293 & 1.5569833 \\ \mathrm{H} & 4.6383549 & -0.6536780 & -0.5172143 \\ \mathrm{H} & 4.5451698 & 0.6357309 & 0.7084219 \\ \mathrm{H} & 0.6575318 & 2.6040155 & -1.3167085 \\ \mathrm{H} & 2.9694722 & 1.7802994 & -1.0058294 \\ \mathrm{C} & 0.4715685 & -1.3060286 & 3.9267381 \\ \mathrm{H} & 0.4969856 & -2.1320143 & 1.9194189 \\ \mathrm{H} & 2.0926491 & -2.1585541 & 2.7531434 \\ \mathrm{C} & 1.4931749 & -4.1449190 & -1.3340160 \\ \mathrm{H} & 3.3511756 & -3.1864721 & -0.7112445 \\ \mathrm{H} & 2.1345985 & -3.3609045 & 0.5853039 \\ \mathrm{C} & 6.2829646 & -1.3720068 & 1.6382066 \\ \mathrm{H} & 4.3292168 & -1.0885891 & 2.5513754 \\ \mathrm{H} & 4.3884241 & -2.4059958 & 1.3543471 \\ \mathrm{C} & 0.0788671 & -2.6061269 & 4.6402529 \\ \mathrm{H} & 1.1324439 & -0.6982251 & 4.5901450 \\ \mathrm{H} & -0.4389014 & -0.6878294 & 3.7496593 \\ \mathrm{C} & 1.9561856 & -5.5866528 & -1.0904342 \\ \mathrm{H} & 0.4071341 & -4.0468388 & -1.1072897 \\ \mathrm{H} & 1.6065874 & -3.8919211 & -2.4146932 \\ \mathrm{C} & 6.8313554 & -2.3139993 & 2.7177192 \\ \mathrm{H} & 6.7009559 & -1.6578423 & 0.6442185 \\ \mathrm{H} & 6.5213318 & -3.3673943 & 2.5329276 \\ \mathrm{H} & 6.6394386 & -0.3325949 & 1.8310063 \\ \mathrm{H} & -0.4325812 & -2.3997553 & 5.6064730 \\ \mathrm{H} & -0.6127857 & -3.2139185 & 4.0148602 \\ \mathrm{H} & 0.9714880 & -3.2350934 & 4.8609831 \\ \mathrm{H} & 1.3787007 & -6.3066092 & -1.7114816 \\ & 3.0341503 & -5.7192739 & -1.3393430 \\ \mathrm{H} & 1.8202980 & -5.8816637 & -0.0249991 \\ & & & \end{array}$

\section{TS-BC_trans-PMe3}

$\mathrm{E}(\mathrm{BP} 86 /$ def2-SV(P) $)=-824.6217574963$

$\begin{array}{llll}C & -1.9902539 & -0.7358382 & -0.1859827\end{array}$

$\begin{array}{llll}\text { C } & -3.5134400 & -0.9397471 & -0.0202971\end{array}$

$\begin{array}{cccc}\mathrm{C} & -3.9855878 & 0.3870864 & 0.5948064 \\ \mathrm{C} & -2.9201126 & 1.3968712 & 0.0952448 \\ \mathrm{~N} & -1.7186724 & 0.5909825 & -0.0803006 \\ \mathrm{O} & -1.1778734 & -1.6540267 & -0.3957448 \\ \mathrm{H} & -3.7300576 & -1.8443445 & 0.5855670 \\ \mathrm{H} & -3.9482924 & -1.1128369 & -1.0330059 \\ \mathrm{H} & -5.0197630 & 0.6792897 & 0.3118906 \\ \mathrm{H} & -3.9471533 & 0.3239323 & 1.7056093 \\ \mathrm{H} & -3.2366743 & 1.8672692 & -0.8712209 \\ \mathrm{H} & -2.7479356 & 2.2298293 & 0.8143943 \\ \mathrm{H} & -0.7001152 & 1.0824542 & -0.3775957 \\ \mathrm{C} & 0.6742093 & 1.8533789 & -0.8458360 \\ \mathrm{C} & 1.9767638 & 1.4764030 & -0.7502918 \\ \mathrm{P} & 2.3007247 & -0.0611715 & 0.1182326 \\ \mathrm{C} & 1.3412224 & -0.1407541 & 1.6672498 \\ \mathrm{C} & 1.8651116 & -1.5365925 & -0.8569494 \\ \mathrm{C} & 4.0828123 & -0.1705429 & 0.5483580 \\ \mathrm{H} & 1.4616781 & -1.1322788 & 2.1536888 \\ \mathrm{H} & 1.6760677 & 0.6689794 & 2.3495292 \\ \mathrm{H} & 0.2724794 & 0.0205880 & 1.4050555 \\ \mathrm{H} & 2.2807852 & -2.4556335 & -0.3883703 \\ \mathrm{H} & 0.7419758 & -1.5942455 & -0.8657675 \\ \mathrm{H} & 2.2570256 & -1.4253324 & -1.8901175 \\ \mathrm{H} & 4.2984024 & -1.1095769 & 1.1012787 \\ \mathrm{H} & 4.6890564 & -0.1540423 & -0.3832411 \\ \mathrm{H} & 4.3717272 & 0.7002597 & 1.1753906 \\ \mathrm{H} & 0.5622741 & 2.8046832 & -1.4256010 \\ \mathrm{H} & 2.8869230 & 1.9730737 & -1.1668299\end{array}$

\section{TS-BC_trans-PPh3}

$\mathrm{E}(\mathrm{BP} 86 / \mathrm{def} 2-\mathrm{SV}(\mathrm{P}))=-1399.415549858$

$\begin{array}{llll}\text { C } & -3.5688505 & -0.7106063 & -0.2960582\end{array}$

$\begin{array}{llll}\text { C } & -5.0877627 & -0.6266082 & 0.0012126\end{array}$

$\begin{array}{llll}\text { C } & -5.4495689 & 0.8024431 & -0.4303647\end{array}$

$\begin{array}{lllll}\text { C } & -4.3679713 & 1.1184968 & -1.4984525\end{array}$

$\begin{array}{llll}\mathrm{N} & -3.2237739 & 0.3075737 & -1.1136671\end{array}$

$\begin{array}{lllll}0 & -2.8240482 & -1.6065388 & 0.1506331\end{array}$

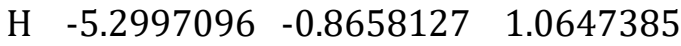

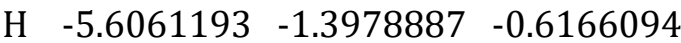

$\mathrm{H} \quad-6.4857484 \quad 0.9178516 \quad-0.8172913$

$\begin{array}{llll}\mathrm{H} & -5.3332786 & 1.4979516 & 0.4312239\end{array}$

$\begin{array}{llll}\mathrm{H} & -4.7351837 & 0.8514618 & -2.5247745\end{array}$

$\mathrm{H} \quad-4.1080412 \quad 2.2027176-1.5305856$

H $\quad-2.1214843 \quad 0.3877990 \quad-1.6910986$

$\begin{array}{llll}\text { C } & -0.8862447 & 0.3726588 & -2.5288699\end{array}$

$\begin{array}{llll}\text { C } & 0.4107854 & 0.1209081 & -2.2290088\end{array}$

$\begin{array}{llll}\mathrm{P} & 0.9635041 & 0.0261430 & -0.5100977\end{array}$

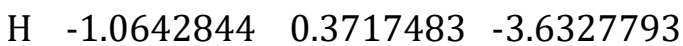

H $\quad 1.2388191 \quad-0.0850301 \quad-2.9462638$

C $\begin{array}{llll}-1.8855605 & 2.3519023 & 2.2953510\end{array}$

$\begin{array}{llll}\text { C } & -1.5092820 & 1.0421114 & 2.6338020\end{array}$ 


$\begin{array}{cccc}\mathrm{C} & -0.6604914 & 0.3090487 & 1.7873385 \\ \mathrm{C} & -0.1880150 & 0.8963515 & 0.5971854 \\ \mathrm{C} & -0.5748984 & 2.2110239 & 0.2496824 \\ \mathrm{C} & -1.4176593 & 2.9360108 & 1.1039562 \\ \mathrm{H} & -2.5550182 & 2.9216908 & 2.9613789 \\ \mathrm{H} & -1.8872766 & 0.5763985 & 3.5583913 \\ \mathrm{H} & -0.3834795 & -0.7232919 & 2.0486521 \\ \mathrm{H} & -0.2251615 & 2.6595751 & -0.6934043 \\ \mathrm{H} & -1.7217838 & 3.9598881 & 0.8309423 \\ \mathrm{C} & 1.4774855 & -4.3990386 & 0.7794049 \\ \mathrm{C} & 2.5981371 & -3.5501836 & 0.7822020 \\ \mathrm{C} & 2.4586736 & -2.1999264 & 0.4206868 \\ \mathrm{C} & 1.1904733 & -1.7019596 & 0.0469078 \\ \mathrm{C} & 0.0583378 & -2.5505253 & 0.0439265 \\ \mathrm{C} & 0.2147912 & -3.8954118 & 0.4196226 \\ \mathrm{H} & 1.5897365 & -5.4586700 & 1.0648980 \\ \mathrm{H} & 3.5893019 & -3.9378939 & 1.0708053 \\ \mathrm{H} & 3.3396264 & -1.5382974 & 0.4316059 \\ \mathrm{H} & -0.9575562 & -2.1616415 & -0.1923488 \\ \mathrm{H} & -0.6714650 & -4.5511990 & 0.4313112 \\ \mathrm{C} & 5.1212743 & 2.1003492 & -0.1721865 \\ \mathrm{C} & 4.1485465 & 2.3172955 & 0.8186291 \\ \mathrm{C} & 2.8930309 & 1.6936521 & 0.7259155 \\ \mathrm{C} & 2.6011855 & 0.8446610 & -0.3637042 \\ \mathrm{C} & 3.5850871 & 0.6262374 & -1.3560810 \\ \mathrm{C} & 4.8376037 & 1.2536066 & -1.2580507 \\ \mathrm{H} & 6.1049527 & 2.5930725 & -0.0986606 \\ \mathrm{H} & 4.3654598 & 2.9798434 & 1.6727951 \\ \mathrm{H} & 2.1328036 & 1.8727829 & 1.5032194 \\ \mathrm{H} & 3.3809747 & -0.0386093 & -2.2111010 \\ \mathrm{H} & 5.5967333 & 1.0790513 & -2.0383436\end{array}$

\section{TS-CD}

\begin{tabular}{lccc}
\multicolumn{4}{c}{ E(BP86/def2-SV(P) $=-1178.157748892$} \\
C & 0.0894519 & -0.7115398 & 1.1689475 \\
C & -0.3872421 & -2.0759042 & 1.7248185 \\
C & -1.6755782 & -1.6917053 & 2.4653082 \\
C & -2.1127879 & -0.4003020 & 1.7137919 \\
N & -0.8902028 & 0.2037979 & 1.1967569 \\
O & 1.2528973 & -0.5287813 & 0.7079149 \\
H & 0.3926187 & -2.5593611 & 2.3512303 \\
H & -0.5839672 & -2.7579235 & 0.8621389 \\
H & -2.4587375 & -2.4814158 & 2.4672156 \\
H & -1.4446305 & -1.4455929 & 3.5263097 \\
H & -2.8201794 & -0.6589570 & 0.8795765 \\
H & -2.6672449 & 0.3047409 & 2.3774651 \\
C & -0.9795661 & 1.0940673 & -0.9027450 \\
C & 0.1159559 & 0.6980343 & -1.6319867 \\
H & -1.9617519 & 0.6310392 & -1.1034516 \\
H & -1.0146144 & 2.0519827 & -0.3669267 \\
P & 1.7335802 & 1.3371077 & -1.2867340
\end{tabular}

$\begin{array}{cccc}\mathrm{C} & 2.2146160 & 2.4706710 & -2.6874231 \\ \mathrm{C} & 1.7265208 & 2.3606654 & 0.2517148 \\ \mathrm{H} & 0.0955730 & -0.2276889 & -2.2339700 \\ \mathrm{C} & 3.0227094 & 0.0048310 & -1.3458769 \\ \mathrm{C} & 3.6475478 & 3.0310086 & -2.6854810 \\ \mathrm{H} & 2.0285709 & 1.8812317 & -3.6154974 \\ \mathrm{H} & 1.4649308 & 3.2938229 & -2.6981029 \\ \mathrm{C} & 1.3149062 & 3.8364502 & 0.0949957 \\ \mathrm{H} & 1.0338975 & 1.8095127 & 0.9262897 \\ \mathrm{H} & 2.7401315 & 2.2819407 & 0.7023265 \\ \mathrm{C} & 4.1642804 & 0.1250353 & -0.3235895 \\ \mathrm{H} & 2.4591612 & -0.9283809 & -1.1440001 \\ \mathrm{H} & 3.4155022 & -0.0146537 & -2.3894252 \\ \mathrm{C} & 3.9203309 & 3.9777312 & -3.8691492 \\ \mathrm{H} & 3.8458580 & 3.5749084 & -1.7323556 \\ \mathrm{H} & 4.3818309 & 2.1928284 & -2.7170206 \\ \mathrm{C} & 1.1946435 & 4.5368662 & 1.4612338 \\ \mathrm{H} & 2.0504156 & 4.3901127 & -0.5333667 \\ \mathrm{H} & 0.3369563 & 3.9117892 & -0.4345602 \\ \mathrm{C} & 5.1994811 & -1.0039426 & -0.4746326 \\ \mathrm{H} & 4.6826042 & 1.1090922 & -0.4162959 \\ \mathrm{H} & 3.7127468 & 0.0764537 & 0.6912545 \\ \mathrm{C} & 5.3500502 & 4.5338176 & -3.8863113 \\ \mathrm{H} & 3.7172905 & 3.4358882 & -4.8228328 \\ \mathrm{H} & 3.1905958 & 4.8208031 & -3.8374703 \\ \mathrm{C} & 0.7840632 & 6.0109455 & 1.3526349 \\ \mathrm{H} & 0.4558810 & 3.9832476 & 2.0854502 \\ \mathrm{H} & 2.1689011 & 4.4567696 & 1.9987095 \\ \mathrm{C} & 6.3278929 & -0.9267378 & 0.5620453 \\ \mathrm{H} & 4.6763154 & -1.9841995 & -0.3856975 \\ \mathrm{H} & 5.6335941 & -0.9777619 & -1.5027405 \\ \mathrm{H} & 5.5104648 & 5.2119664 & -4.7532504 \\ \mathrm{H} & 5.5717439 & 5.1135513 & -2.9616576 \\ \mathrm{H} & 6.1032333 & 3.7164695 & -3.9561025 \\ \mathrm{H} & 0.7052887 & 6.4832834 & 2.3566367 \\ \mathrm{H} & 1.5237353 & 6.5988490 & 0.7625612 \\ \mathrm{H} & -0.2050552 & 6.1209298 & 0.8527806 \\ \mathrm{H} & 7.0539260 & -1.7599483 & 0.4344874 \\ \mathrm{H} & 6.8948858 & 0.0283854 & 0.4768158 \\ \mathrm{H} & 5.9264765 & -0.9856331 & 1.5987400\end{array}$

\section{TS-CD_PMe3}

$\begin{array}{cccc}\text { E(BP86/def2-SV }(P))=-824.6378146890 \\ \text { C } & 0.5924176 & -1.4854882 & -0.0602754 \\ \text { C } & 1.0257348 & -2.8742076 & -0.5848233 \\ \text { C } & 0.0478581 & -3.8112756 & 0.1382190 \\ \text { C } & -1.1673081 & -2.8767887 & 0.4073584 \\ \text { N } & -0.6256928 & -1.5250927 & 0.5040650 \\ \text { O } & 1.2963715 & -0.4445939 & -0.2007410 \\ \text { H } & 2.1014034 & -3.0698686 & -0.3883309 \\ \text { H } & 0.8862861 & -2.8919706 & -1.6930145\end{array}$ 


$\begin{array}{cccc}\mathrm{H} & -0.2237078 & -4.7237026 & -0.4364732 \\ \mathrm{H} & 0.4854157 & -4.1425846 & 1.1067626 \\ \mathrm{H} & -1.9093471 & -2.9545135 & -0.4322283 \\ \mathrm{H} & -1.7198165 & -3.1604524 & 1.3336416 \\ \mathrm{C} & -1.8105535 & 0.1164981 & -0.4675606 \\ \mathrm{C} & -0.9410656 & 0.9589450 & -1.1224169 \\ \mathrm{H} & -2.4283452 & -0.5886093 & -1.0512906 \\ \mathrm{H} & -2.1861728 & 0.3381619 & 0.5424941 \\ \mathrm{P} & 0.1475454 & 1.9859071 & -0.1780871 \\ \mathrm{C} & -0.5571435 & 3.6979860 & -0.1038621 \\ \mathrm{C} & 0.2846390 & 1.4914527 & 1.5837532 \\ \mathrm{H} & -0.6240511 & 0.7606054 & -2.1605444 \\ \mathrm{C} & 1.7631035 & 2.2394958 & -0.9960959 \\ \mathrm{H} & 0.0924380 & 4.3801634 & 0.4892329 \\ \mathrm{H} & -0.6697048 & 4.1019066 & -1.1330537 \\ \mathrm{H} & -1.5642695 & 3.6576186 & 0.3641383 \\ \mathrm{H} & 1.3555011 & 1.4465630 & 1.8672949 \\ \mathrm{H} & -0.2498828 & 2.2191590 & 2.2328160 \\ \mathrm{H} & -0.1359092 & 0.4639618 & 1.6804072 \\ \mathrm{H} & 2.3592914 & 2.9871417 & -0.4301341 \\ \mathrm{H} & 2.2747927 & 1.2567989 & -1.0292518 \\ \mathrm{H} & 1.5853437 & 2.6229422 & -2.0243944\end{array}$

\section{TS-CD_PPh3}

\begin{tabular}{lccc}
\multicolumn{4}{c}{ E(BP86/def2-SV(P) $=-1399.431724409$} \\
C & -0.4237500 & -0.7206657 & 1.1926767 \\
C & -0.5966688 & -1.3420843 & 2.5981595 \\
C & -1.7849442 & -2.2920226 & 2.3830691 \\
C & -1.7145419 & -2.5691717 & 0.8506079 \\
N & -1.0279651 & -1.4320587 & 0.2470233 \\
O & 0.2515525 & 0.3521752 & 1.0234648 \\
H & -0.7513473 & -0.5589691 & 3.3708788 \\
H & 0.3408410 & -1.8850797 & 2.8664945 \\
H & -1.7483437 & -3.2178260 & 2.9985630 \\
H & -2.7371992 & -1.7683382 & 2.6267839 \\
H & -1.1547003 & -3.5189073 & 0.6438699 \\
H & -2.7267545 & -2.7129296 & 0.4027918 \\
C & -1.5392269 & 0.5554434 & -1.2549392 \\
C & -0.3932146 & 0.8871071 & -1.9032183 \\
H & -2.3885214 & 0.1296119 & -1.8174860 \\
H & -1.7105044 & 0.8463291 & -0.2108197 \\
P & 1.0614664 & 1.5340792 & -1.0835144 \\
H & -0.2494101 & 0.6733827 & -2.9767724 \\
C & 3.1025665 & 3.7914360 & -4.6672226 \\
C & 2.7326667 & 4.5162309 & -3.5234395 \\
C & 2.1218487 & 3.8646537 & -2.4359750 \\
C & 1.8744207 & 2.4769716 & -2.4805373 \\
C & 2.2664667 & 1.7527274 & -3.6311590 \\
C & 2.8689087 & 2.4052549 & -4.7175364 \\
H & 3.5797341 & 4.3040300 & -5.5190893 \\
H & 2.9196373 & 5.6018530 & -3.4708020
\end{tabular}

$\begin{array}{crrr}\mathrm{H} & 1.8398168 & 4.4485049 & -1.5458958 \\ \mathrm{H} & 2.1153881 & 0.6606478 & -3.6777445 \\ \mathrm{H} & 3.1654741 & 1.8246143 & -5.6069612 \\ \mathrm{C} & 0.3196240 & 4.8646504 & 2.0581533 \\ \mathrm{C} & -0.4530172 & 4.8025669 & 0.8859573 \\ \mathrm{C} & -0.2273381 & 3.7825262 & -0.0540154 \\ \mathrm{C} & 0.7726208 & 2.8164817 & 0.1841178 \\ \mathrm{C} & 1.5365412 & 2.8671713 & 1.3676748 \\ \mathrm{C} & 1.3114366 & 3.8962107 & 2.2953032 \\ \mathrm{H} & 0.1421179 & 5.6658293 & 2.7948535 \\ \mathrm{H} & -1.2407588 & 5.5513047 & 0.6991035 \\ \mathrm{H} & -0.8387547 & 3.7337649 & -0.9705136 \\ \mathrm{H} & 2.2817833 & 2.0851389 & 1.5754741 \\ \mathrm{H} & 1.9074894 & 3.9310828 & 3.2223223 \\ \mathrm{C} & 4.4037887 & -1.6185445 & -0.4513581 \\ \mathrm{C} & 4.7118206 & -0.2492177 & -0.3895431 \\ \mathrm{C} & 3.6960373 & 0.7088421 & -0.5527995 \\ \mathrm{C} & 2.3618733 & 0.2899856 & -0.7418142 \\ \mathrm{C} & 2.0463686 & -1.0843337 & -0.8003886 \\ \mathrm{C} & 3.0742718 & -2.0302620 & -0.6623103 \\ \mathrm{H} & 5.2038087 & -2.3691360 & -0.3368109 \\ \mathrm{H} & 5.7514465 & 0.0804088 & -0.2261403 \\ \mathrm{H} & 3.9520506 & 1.7814877 & -0.5399770 \\ \mathrm{H} & 0.9916852 & -1.4028987 & -0.9035369 \\ \mathrm{H} & 2.8291129 & -3.1046569 & -0.7057248\end{array}$

\section{TS-DE}

\begin{tabular}{cccc}
\multicolumn{4}{c}{$\mathrm{E}(\mathrm{BP} 86 / \mathrm{def} 2-\mathrm{SV}(\mathrm{P}))=-1464.617883902$} \\
$\mathrm{C}$ & -1.3861146 & -1.3350054 & 1.6204492 \\
$\mathrm{C}$ & -2.2965374 & -2.5635617 & 1.7611920 \\
$\mathrm{C}$ & -3.5581036 & -2.1810401 & 0.9651002 \\
$\mathrm{C}$ & -3.0441466 & -1.1446701 & -0.0627665 \\
$\mathrm{~N}$ & -1.8724416 & -0.5698663 & 0.5987161 \\
$\mathrm{O}$ & -0.3647047 & -1.0873348 & 2.2773428 \\
$\mathrm{H}$ & -2.4739624 & -2.7993198 & 2.8309875 \\
$\mathrm{H}$ & -1.7667609 & -3.4401826 & 1.3192168 \\
$\mathrm{H}$ & -4.0608353 & -3.0438270 & 0.4803900 \\
$\mathrm{H}$ & -4.2980712 & -1.6972243 & 1.6396537 \\
$\mathrm{H}$ & -2.7567264 & -1.6262423 & -1.0305443 \\
$\mathrm{H}$ & -3.7853026 & -0.3513816 & -0.2994893 \\
$\mathrm{C}$ & -1.2509762 & 0.6821994 & 0.1735924 \\
$\mathrm{C}$ & -0.0681621 & 0.6049692 & -0.8015839 \\
$\mathrm{H}$ & -2.0217596 & 1.3103157 & -0.3455802 \\
$\mathrm{H}$ & -0.9605643 & 1.2334974 & 1.0933284 \\
$\mathrm{H}$ & -0.2648894 & -0.0665737 & -1.6674280 \\
$\mathrm{P}$ & 1.5855494 & 0.3260189 & -0.1888998 \\
$\mathrm{H}$ & -0.0498480 & 1.8094536 & -1.2899443 \\
$\mathrm{C}$ & -1.6491168 & 3.4701007 & -1.9575213 \\
$\mathrm{C}$ & -1.7738393 & 4.7890713 & -2.7687341 \\
$\mathrm{C}$ & -0.3727311 & 5.4017772 & -2.6498416 \\
$\mathrm{C}$ & 0.5150428 & 4.1450338 & -2.4251083
\end{tabular}




\begin{tabular}{|c|c|c|c|}
\hline & -0.3419632 & 396 & 27 \\
\hline & -2.6379892 & 8019522 & -1.5867796 \\
\hline & -2.5999227 & 5.4199548 & 355 \\
\hline & -2.0318572 & 4.5292073 & \\
\hline & -0.0546042 & 6.0091322 & -3.52 \\
\hline & -0.3207140 & 6.0567170 & -1.750571 \\
\hline & 31470 & 27 & \\
\hline & 1.4230952 & & $-1 . \varepsilon$ \\
\hline & 2.6718958 & 0.8873149 & -1.5797561 \\
\hline & 1.7925470 & 1.420 & 1.2788779 \\
\hline & 2.0300013 & -1.4255402 & 0.23 \\
\hline & 3.2074601 & 84 & 759 \\
\hline & 1.27 & & \\
\hline & 1.1683888 & 2.31 & 1.03 \\
\hline & 4.1584626 & 0.493 & -1.5920498 \\
\hline & 2.5447601 & 1.9 & -1.6209718 \\
\hline & 2.167 & & 949 \\
\hline & 1.125 & -1.8 & 700 \\
\hline & 3.2595646 & -1.6 & 1.1213230 \\
\hline & 2.1076582 & -1.9 & -0.74 \\
\hline & $3.18^{\prime}$ & 2.7 & \\
\hline & 3.8 & & \\
\hline & 3.67 & 2.4 & 569 \\
\hline & 4.9026055 & 1.0 & -2.8 \\
\hline & 4.66 & 0.8 & -0. \\
\hline & 4.2 & -0.6 & -1 \\
\hline & 3.48 & -3.2 & 1.3448442 \\
\hline & 4.18 & -1.2 & 0.6834262 \\
\hline & 3.1067423 & -1.20 & 2.1102073 \\
\hline & 6.3842759 & 719 & 335 \\
\hline & 4.39 & 69 & -3.74 \\
\hline & 4.8099032 & 2.1 & -2.8037822 \\
\hline & 4.6777546 & -3.50 & 2.2599789 \\
\hline & 2.55 & -3.6 & 1.7771815 \\
\hline & 3.63 & -3.7 & 0.3578811 \\
\hline & 4.5807354 & 179 & 3.3665219 \\
\hline & 2.5245751 & 429 & 2.7613858 \\
\hline & 2.7163811 & 82 & 3.7795036 \\
\hline & 6.889 & 1.10 & -3.7494153 \\
\hline & 6.9297258 & 1.03 & -1.9564120 \\
\hline & 6.5099610 & -0.4306199 & -2.9073201 \\
\hline & 4.8121467 & -4.6053618 & 2.3980612 \\
\hline & 5.6269174 & -3.1059213 & 1.8400481 \\
\hline & 4.5395177 & -3.0597001 & 3.2689285 \\
\hline & 4.5283477 & 3.8711136 & 4.2791448 \\
\hline & 5.2575166 & 2.3820645 & 3.5908647 \\
\hline & 5.0615653 & 3.8440162 & 2.5655821 \\
\hline
\end{tabular}

TS-DE_Phenol

$\mathrm{E}(\mathrm{BP} 86 / \mathrm{def} 2-\mathrm{SV}(\mathrm{P}))=-1485.459816009$ C $-1.3900629 \quad-1.7002019 \quad 1.8223181$
$\begin{array}{cccc}\mathrm{C} & -2.0625083 & -3.0809846 & 1.8704109 \\ \mathrm{C} & -3.3177048 & -2.9156320 & 0.9953350 \\ \mathrm{C} & -2.9421845 & -1.7630976 & 0.0329319 \\ \mathrm{~N} & -1.9617311 & -0.9960806 & 0.7946710 \\ \mathrm{O} & -0.4666015 & -1.3005167 & 2.5392452 \\ \mathrm{H} & -2.2616163 & -3.3838802 & 2.9191554 \\ \mathrm{H} & -1.3481137 & -3.8257853 & 1.4473950 \\ \mathrm{H} & -3.6122909 & -3.8385727 & 0.4535261 \\ \mathrm{H} & -4.1804006 & -2.6056506 & 1.6245965 \\ \mathrm{H} & -2.4992207 & -2.1453199 & -0.9210266 \\ \mathrm{H} & -3.8134567 & -1.1268754 & -0.2411436 \\ \mathrm{C} & -1.5070817 & 0.3486005 & 0.4057737 \\ \mathrm{C} & -0.3650467 & 0.4759689 & -0.5926906 \\ \mathrm{H} & -2.4004585 & 0.8676531 & -0.0109557 \\ \mathrm{H} & -1.2552595 & 0.8728672 & 1.3522328 \\ \mathrm{H} & -0.5462984 & -0.0293705 & -1.5660318 \\ \mathrm{P} & 1.3102554 & 0.2632011 & -0.0730483 \\ \mathrm{H} & -0.3084363 & 1.9929405 & -0.8343858 \\ \mathrm{O} & -0.1403737 & 3.1321571 & -0.8037584 \\ \mathrm{C} & 2.2968252 & 0.9634492 & -1.4746995 \\ \mathrm{C} & 1.5270513 & 1.2780632 & 1.4536055 \\ \mathrm{C} & 1.9080081 & -1.4751044 & 0.2496468 \\ \mathrm{C} & 2.9291702 & 1.8077057 & 1.8023676 \\ \mathrm{H} & 1.0969814 & 0.6594274 & 2.2749477 \\ \mathrm{H} & 0.8421750 & 2.1401531 & 1.2832971 \\ \mathrm{C} & 3.8052138 & 0.6828095 & -1.5688705 \\ \mathrm{H} & 2.0734890 & 2.0547360 & -1.4466274 \\ \mathrm{H} & 1.7822077 & 0.5823571 & -2.3879115 \\ \mathrm{H} & 1.0614572 & -1.9483392 & 0.7924073 \\ \mathrm{C} & 3.1983608 & -1.7017460 & 1.0587598 \\ \mathrm{H} & 2.1818313 & 3.4507330 & 3.0157570 \\ \mathrm{H} & 1.9710998 & -1.9598349 & -0.7534784 \\ \mathrm{H} & 2.5505413 & 1.9741930 & 3.9386435 \\ \mathrm{H} & 6.5051708 & 1.4767272 & -2.0161323 \\ \mathrm{H} & 3.6600644 & 0.9736057 & 1.8990184 \\ \mathrm{H} & 3.2980417 & 2.4583762 & 0.9764698 \\ \mathrm{C} & 4.4466918 & 1.3757977 & -2.7857937 \\ \mathrm{H} & 4.3229415 & 1.0220546 & -0.6432805 \\ \mathrm{H} & 3.9852624 & -0.4147655 & -1.6423728 \\ \mathrm{C} & 3.5448178 & -3.1963562 & 1.1956975 \\ \mathrm{H} & 4.0597998 & -1.1689422 & 0.5968332 \\ \mathrm{H} & 3.0668444 & -1.2708136 & 2.0771136 \\ \mathrm{C} & 5.9524348 & 1.1108738 & -2.9110661 \\ \mathrm{H} & 3.9274905 & 1.0381222 & -3.7135856 \\ \mathrm{C} & 4.2618781 & 2.4732015 & -2.7180786 \\ \mathrm{H} & 4.6748418 & -3.4518322 & 2.0321352 \\ & -3.7281620 & 1.6529204 \\ \mathrm{H} & & & \\ \mathrm{H} & & \end{array}$ 


$\begin{array}{rrrr}\mathrm{H} & 6.1674894 & 0.0229710 & -3.0147526 \\ \mathrm{H} & 5.0258608 & -4.5392844 & 2.1110668 \\ \mathrm{H} & 5.6982538 & -2.9603802 & 1.5829338 \\ \mathrm{H} & 4.6916416 & -3.0568801 & 3.0669871 \\ \mathrm{H} & 4.2543446 & 3.7726154 & 4.4243005 \\ \mathrm{H} & 5.0519396 & 2.3863331 & 3.6085210 \\ \mathrm{H} & 4.6753080 & 3.8787566 & 2.6826050 \\ \mathrm{C} & -2.8710300 & 5.5316848 & -2.8968620 \\ \mathrm{C} & -2.9561900 & 4.1307263 & -2.9829435 \\ \mathrm{C} & -2.0510776 & 3.3080982 & -2.2930610 \\ \mathrm{C} & -1.0239932 & 3.8712330 & -1.4836449 \\ \mathrm{C} & -0.9472421 & 5.2896955 & -1.4085262 \\ \mathrm{C} & -1.8554931 & 6.0997846 & -2.1030907 \\ \mathrm{H} & -3.5841111 & 6.1726031 & -3.4410634 \\ \mathrm{H} & -3.7425835 & 3.6643154 & -3.6024757 \\ \mathrm{H} & -2.1241001 & 2.2107899 & -2.3815619 \\ \mathrm{H} & -0.1532436 & 5.7307186 & -0.7832200 \\ \mathrm{H} & -1.7706130 & 7.1978691 & -2.0223933\end{array}$

\section{TS-DE_PMe3}

$\begin{array}{lccc}\text { E(BP86/def2-SV }(\mathrm{P}))=-1111.111267488 \\ \mathrm{C} & -0.3194284 & -3.1649040 & 0.6909414 \\ \mathrm{C} & -0.7389312 & -4.5591394 & 0.2035448 \\ \mathrm{C} & -2.1200889 & -4.3323274 & -0.4370430 \\ \mathrm{C} & -2.0912850 & -2.8416118 & -0.8530089 \\ \mathrm{~N} & -1.1452310 & -2.2488035 & 0.0882098 \\ \mathrm{O} & 0.6164539 & -2.8914009 & 1.4478762 \\ \mathrm{H} & -0.7277832 & -5.2863571 & 1.0414218 \\ \mathrm{H} & 0.0190831 & -4.9062888 & -0.5371517 \\ \mathrm{H} & -2.3329885 & -5.0043526 & -1.2942266 \\ \mathrm{H} & -2.9194288 & -4.4898409 & 0.3196132 \\ \mathrm{H} & -1.7435263 & -2.7110898 & -1.9085362 \\ \mathrm{H} & -3.0869024 & -2.3506243 & -0.7723250 \\ \mathrm{C} & -0.9851428 & -0.8043243 & 0.2669295 \\ \mathrm{C} & -0.0562860 & -0.0542468 & -0.6898024 \\ \mathrm{H} & -2.0006642 & -0.3548050 & 0.1782715 \\ \mathrm{H} & -0.6643480 & -0.6449870 & 1.3183958 \\ \mathrm{H} & -0.2663239 & -0.2766379 & -1.7610484 \\ \mathrm{P} & 1.7097478 & -0.1209803 & -0.4271829 \\ \mathrm{H} & -0.4049109 & 1.2175323 & -0.5322777 \\ \mathrm{C} & -0.0172284 & 3.3920503 & 0.3315386 \\ \mathrm{C} & -0.7290792 & 4.7373167 & 0.6214318 \\ \mathrm{C} & -1.9516169 & 4.6826540 & -0.3063924 \\ \mathrm{C} & -2.1704591 & 3.1527288 & -0.4845539 \\ \mathrm{~N} & -0.8586160 & 2.5503908 & -0.2927205 \\ \mathrm{O} & 1.1730015 & 3.1522877 & 0.6626046 \\ \mathrm{H} & -0.0448075 & 5.5950923 & 0.4499366 \\ \mathrm{H} & -1.0157749 & 4.7591257 & 1.6997519 \\ \mathrm{H} & -2.8511838 & 5.2069469 & 0.0846866 \\ \mathrm{H} & -1.7002022 & 5.1388581 & -1.2905688 \\ \mathrm{H} & -2.9109724 & 2.7720030 & 0.2692284\end{array}$

$\begin{array}{cccc}\mathrm{H} & -2.5943929 & 2.9043823 & -1.4871531 \\ \mathrm{C} & 2.4497518 & 1.2190829 & -1.4181945 \\ \mathrm{C} & 2.1127314 & 0.2140309 & 1.3135849 \\ \mathrm{C} & 2.5604909 & -1.6856897 & -0.8942909 \\ \mathrm{H} & 3.2155638 & 0.1897163 & 1.4490514 \\ \mathrm{H} & 1.6443083 & -0.5669742 & 1.9492114 \\ \mathrm{H} & 1.7298530 & 1.2424662 & 1.5238037 \\ \mathrm{H} & 3.5579862 & 1.1374985 & -1.4186027 \\ \mathrm{H} & 2.1275925 & 2.1640898 & -0.9091103 \\ \mathrm{H} & 2.0709573 & 1.1841501 & -2.4619415 \\ \mathrm{H} & 2.2343486 & -2.4829860 & -0.1920453 \\ \mathrm{H} & 3.6632170 & -1.5567778 & -0.8220102 \\ \mathrm{H} & 2.2960889 & -1.9649272 & -1.9371240\end{array}$

\section{TS-DE_PPh3}

$\mathrm{E}(\mathrm{BP} 86 /$ def2-SV(P) $)=-1685.901971991$

C $-1.5575865-1.02467691 .8635078$

$\begin{array}{llll}\text { C } & -2.2777242 & -2.3810355 & 1.8260585\end{array}$

$\begin{array}{llll}\text { C } & -3.2088469 & -2.2792704 & 0.6056897\end{array}$

$\begin{array}{llll}\text { C } & -2.5040223 & -1.2473670 & -0.3083472\end{array}$

$\begin{array}{lllll}\mathrm{N} & -1.7308891 & -0.4379990 & 0.6264621\end{array}$

$\begin{array}{llll}0 & -0.9152343 & -0.5496820 & 2.7978150\end{array}$

$\begin{array}{llll}\mathrm{H} & -2.7929057 & -2.5739117 & 2.7894774\end{array}$

$\begin{array}{llll}\mathrm{H} & -1.5030877 & -3.1732104 & 1.7015015\end{array}$

$\begin{array}{llll}\mathrm{H} & -3.3821419 & -3.2471277 & 0.0905431\end{array}$

H $\quad-4.1998128 \quad-1.88237130 .9170351$

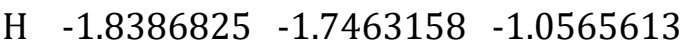

H

$\begin{array}{llll}\text { C } & -1.1749458 & 0.8636041 & 0.2810085\end{array}$

$\begin{array}{llll}\text { C } & -0.0716075 & 0.8914333 & -0.7900716\end{array}$

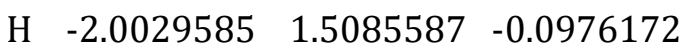

$\mathrm{H} \quad-0.8276642 \quad 1.3137759 \quad 1.2345045$

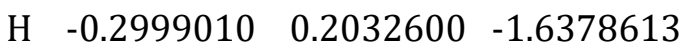

$\begin{array}{lllll}\text { P } & 1.5960788 & 0.4758126 & -0.2803620\end{array}$

H $\quad-0.1687454 \quad 2.0433275-1.3640333$

$\begin{array}{lllll}\text { C } & -0.7984368 & 4.3633543 & -1.2743438\end{array}$

$\begin{array}{llll}\text { C } & -1.6079506 & 5.4099753 & -2.0838020\end{array}$

$\begin{array}{lllll}\text { C } & -1.4353675 & 4.9330596 & -3.5326062\end{array}$

$\begin{array}{lllll}C & -1.1860566 & 3.4105701 & -3.3512904\end{array}$

$\begin{array}{lllll}\mathrm{N} & -0.5367369 & 3.2834643 & -2.0515170\end{array}$

$\begin{array}{lllll}0 & -0.4798636 & 4.5121695 & -0.0771494\end{array}$

$\mathrm{H} \quad-1.2463744 \quad 6.4394822 \quad-1.8774382$

H $\quad-2.67155895 .3649200 \quad-1.7490546$

H $\quad-2.2976353 \quad 5.1563560 \quad-4.1990633$

H $-0.5323622 \quad 5.4050223 \quad-3.9818778$

H $-2.1606521 \quad 2.8521057 \quad-3.3846052$

H $\quad-0.5591678 \quad 2.9855998 \quad-4.1716216$

$\begin{array}{llll}\text { C } & 4.5341370 & 1.4496474 & -3.7495281\end{array}$

$\begin{array}{llll}\text { C } & 3.3178092 & 2.1424508 & -3.6304343\end{array}$

$\begin{array}{llll}\text { C } & 2.4109561 & 1.8262542 & -2.6034695\end{array}$

$\begin{array}{llll}\text { C } & 2.7268080 & 0.7935857 & -1.6912908\end{array}$ 


$\begin{array}{lrrr}\mathrm{C} & 3.9594596 & 0.1099855 & -1.7998886 \\ \mathrm{C} & 4.8560897 & 0.4360464 & -2.8294825 \\ \mathrm{H} & 5.2397545 & 1.7046962 & -4.5578646 \\ \mathrm{H} & 3.0675537 & 2.9480286 & -4.3403792 \\ \mathrm{H} & 1.4716951 & 2.4066149 & -2.5012536 \\ \mathrm{H} & 4.2240188 & -0.6831147 & -1.0816637 \\ \mathrm{H} & 5.8136355 & -0.1046332 & -2.9107159 \\ \mathrm{C} & 3.2023077 & 3.1991240 & 3.1060156 \\ \mathrm{C} & 2.1121589 & 3.6114267 & 2.3238478 \\ \mathrm{C} & 1.5924631 & 2.7796239 & 1.3151528 \\ \mathrm{C} & 2.1782430 & 1.5123867 & 1.1032678 \\ \mathrm{C} & 3.2919175 & 1.1020024 & 1.8744790 \\ \mathrm{C} & 3.7960700 & 1.9440073 & 2.8761843 \\ \mathrm{H} & 3.5996810 & 3.8584812 & 3.8960363 \\ \mathrm{H} & 1.6454327 & 4.5975116 & 2.4809704 \\ \mathrm{H} & 0.7467382 & 3.1777729 & 0.7077674 \\ \mathrm{H} & 3.7702244 & 0.1246944 & 1.6977332 \\ \mathrm{H} & 4.6592796 & 1.6176648 & 3.4797456 \\ \mathrm{C} & 1.8716940 & -4.0456171 & 0.8146664 \\ \mathrm{C} & 1.7176057 & -3.0879941 & 1.8316543 \\ \mathrm{C} & 1.6783138 & -1.7178484 & 1.5203919 \\ \mathrm{C} & 1.8025457 & -1.2948404 & 0.1776599 \\ \mathrm{C} & 1.9337533 & -2.2645985 & -0.8454740 \\ \mathrm{C} & 1.9722898 & -3.6308295 & -0.5258600 \\ \mathrm{H} & 1.9072150 & -5.1190160 & 1.0649470 \\ \mathrm{H} & 1.6197128 & -3.4037121 & 2.8833590 \\ \mathrm{H} & 1.5143686 & -0.9844085 & 2.3241704 \\ \mathrm{H} & 2.0130272 & -1.9523497 & -1.9000290 \\ \mathrm{H} & 2.0844640 & -4.3761517 & -1.3308251\end{array}$

\section{TS-DG_cis}

$\begin{array}{lccc}\mathrm{E}(\mathrm{BP} 86 / \mathrm{def} 2-\mathrm{SV}(\mathrm{P}))=-1523.520567935 \\ \mathrm{C} & -0.4654116 & -3.0923098 & 0.3256913 \\ \mathrm{C} & -1.4445272 & -4.0957716 & -0.3013567 \\ \mathrm{C} & -2.8305358 & -3.4834431 & -0.0319119 \\ \mathrm{C} & -2.5447183 & -1.9676804 & 0.0891415 \\ \mathrm{~N} & -1.1558995 & -1.9243786 & 0.5408948 \\ \mathrm{O} & 0.7302818 & -3.2788594 & 0.5690445 \\ \mathrm{H} & -1.2910892 & -5.1085153 & 0.1252722 \\ \mathrm{H} & -1.2160575 & -4.1637937 & -1.3909413 \\ \mathrm{H} & -3.5815175 & -3.7090009 & -0.8176613 \\ \mathrm{H} & -3.2307091 & -3.8635243 & 0.9335392 \\ \mathrm{H} & -2.6636404 & -1.4428051 & -0.8911908 \\ \mathrm{H} & -3.2086747 & -1.4496779 & 0.8154420 \\ \mathrm{C} & -0.5402807 & -0.7222926 & 1.0961211 \\ \mathrm{C} & -0.2231173 & 0.3862733 & 0.0899566 \\ \mathrm{H} & -1.2308006 & -0.3185672 & 1.8756430 \\ \mathrm{H} & 0.3655844 & -1.0723073 & 1.6332933 \\ \mathrm{P} & 1.4680097 & 0.5978465 & -0.3997646 \\ \mathrm{H} & -0.8704665 & 0.3734155 & -0.8093740 \\ \mathrm{C} & -0.7575889 & 2.1428088 & 1.0928395\end{array}$

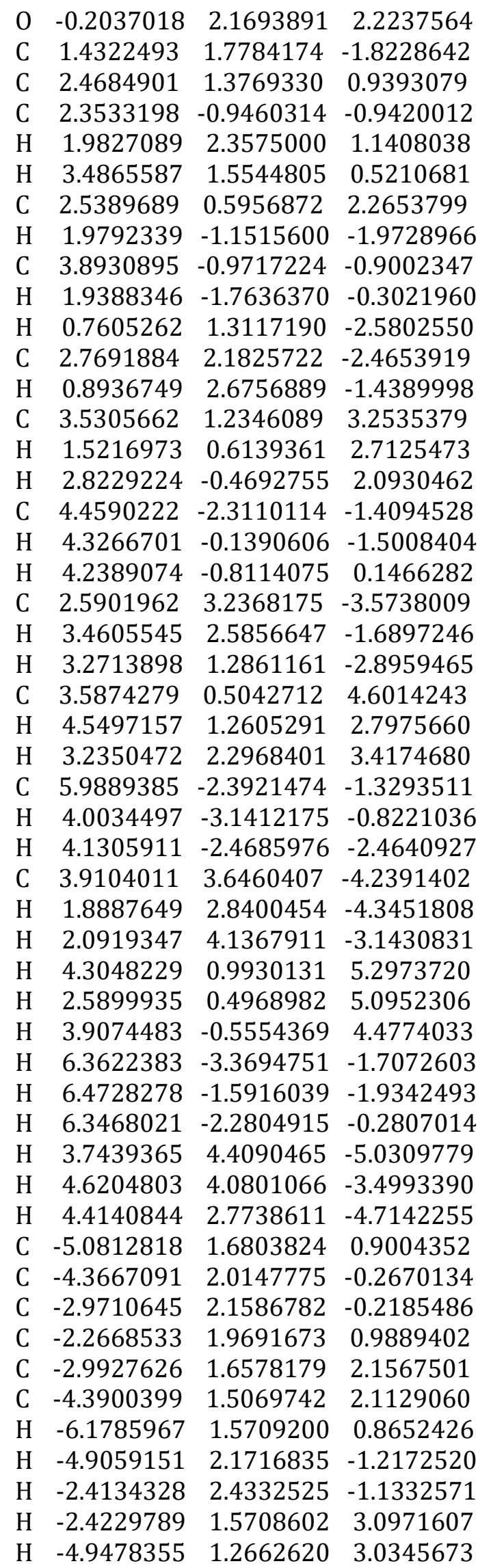




\section{H $\quad-0.3890433 \quad 2.8308093 \quad 0.2631609$}

\section{TS-DH_trans}

$\mathrm{E}(\mathrm{BP} 86 / \mathrm{def2}-\mathrm{SV}(\mathrm{P}))=-1523.521902537$

C $\quad 0.3714038-2.41562990 .9571133$

$\begin{array}{llll}\text { C } & -0.0709990 & -3.8793749 & 0.8084190\end{array}$

$\begin{array}{llll}\text { C } & -1.2967517 & -3.8096846 & -0.1197750\end{array}$

$\begin{array}{llll}\text { C } & -1.0737239 & -2.5080873 & -0.9281634\end{array}$

$\begin{array}{llll}\mathrm{N} & -0.2451789 & -1.6981269 & -0.0413468\end{array}$

$\begin{array}{llll}\mathrm{O} & 1.1657424 & -1.9577440 & 1.7815495\end{array}$

$\begin{array}{llll}\mathrm{H} & -0.2652444 & -4.3306044 & 1.8032634\end{array}$

$\begin{array}{llll}\mathrm{H} & 0.7733275 & -4.4484136 & 0.3531892\end{array}$

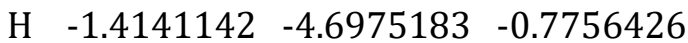

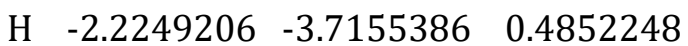

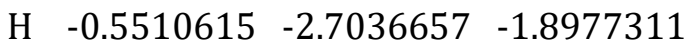

H $\quad-2.0225528 \quad-1.9789973-1.1709838$

$\begin{array}{llll}\text { C } & -0.0398567 & -0.2578755 & -0.2296625\end{array}$

$\begin{array}{llll}\text { C } & 0.8525258 & 0.1956046 & -1.3719731\end{array}$

$\begin{array}{llll}\mathrm{H} & -1.0485132 & 0.1913052 & -0.3858886\end{array}$

$\begin{array}{llll}\mathrm{H} & 0.3381069 & 0.1142448 & 0.7473959\end{array}$

$\begin{array}{lllll}\mathrm{P} & 2.6242652 & 0.0431893 & -1.2022879\end{array}$

$\begin{array}{llll}\mathrm{H} & 0.5469159 & -0.1985846 & -2.3631633\end{array}$

$\begin{array}{lllll}\text { C } & 0.5715453 & 2.2316338 & -1.5995738\end{array}$

$\begin{array}{lllll}\text { O } & 1.5995425 & 2.8038058 & -2.0831964\end{array}$

$\begin{array}{lllll}\text { C } & 3.2969512 & 0.5652062 & -2.8336874\end{array}$

$\begin{array}{llll}\text { C } & 3.1436326 & 1.2096495 & 0.1261681\end{array}$

$\begin{array}{llll}\text { C } & 3.2042016 & -1.6758790 & -0.7852326\end{array}$

$\begin{array}{llll}\text { C } & -3.0845928 & 2.1730079 & -3.9702315\end{array}$

$\begin{array}{llll}\text { C } & -3.1614665 & 2.1750197 & -2.5671776\end{array}$

$\begin{array}{llll}\text { C } & -1.9835634 & 2.1884625 & -1.7975761\end{array}$

$\begin{array}{llll}\text { C } & -0.7141489 & 2.1897681 & -2.4137200\end{array}$

$\begin{array}{llll}\text { C } & -0.6517876 & 2.2219321 & -3.8236011\end{array}$

$\begin{array}{lllll}\text { C } & -1.8226336 & 2.2036328 & -4.5956410\end{array}$

H $-4.0058383 \quad 2.1667239-4.5771678$

$\mathrm{H} \quad-4.1454726 \quad 2.1819238-2.0674180$

$\begin{array}{lllll}\mathrm{H} & -2.0503293 & 2.2337623 & -0.6956034\end{array}$

$\mathrm{H} \quad 0.3422573 \quad 2.2814681 \quad-4.2976460$

$\begin{array}{llll}\mathrm{H} & -1.7562177 & 2.2262131 & -5.6972577\end{array}$

$\begin{array}{llll}\mathrm{H} & 0.3385218 & 2.3698001 & -0.4983245\end{array}$

$\begin{array}{lllll}\text { C } & 4.5471517 & 1.0778868 & 0.7387364\end{array}$

$\begin{array}{lllll}\mathrm{H} & 2.9640037 & 2.2048610 & -0.3496883\end{array}$

$\begin{array}{llll}\mathrm{H} & 2.3771135 & 1.0882443 & 0.9252725\end{array}$

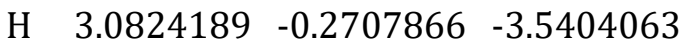

$\begin{array}{llll}\mathrm{H} & 2.6303725 & 1.4331334 & -3.0748810\end{array}$

$\begin{array}{llll}\text { C } & 4.7640881 & 1.0190043 & -2.9222386\end{array}$

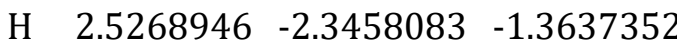

H $\quad 2.9349971 \quad-1.8216656 \quad 0.2869714$

C $4.6692259-2.0629636-1.0589052$

$\begin{array}{llll}\text { C } & 4.8162408 & 2.1705940 & 1.7902365\end{array}$

$\begin{array}{lllll}\mathrm{H} & 4.6595008 & 0.0789921 & 1.2202662\end{array}$

$\begin{array}{llll}\mathrm{H} & 5.3281290 & 1.1372113 & -0.0530395\end{array}$

$\begin{array}{cccc}\mathrm{C} & 5.1494788 & 1.4200934 & -4.3584145 \\ \mathrm{H} & 4.9097277 & 1.8990586 & -2.2557220 \\ \mathrm{H} & 5.4627149 & 0.2288266 & -2.5643695 \\ \mathrm{C} & 4.9810483 & -3.5027470 & -0.6082621 \\ \mathrm{H} & 4.8888627 & -1.9688684 & -2.1474241 \\ \mathrm{H} & 5.3611364 & -1.3625032 & -0.5398504 \\ \mathrm{C} & 6.2052963 & 2.0651552 & 2.4324549 \\ \mathrm{H} & 4.6959055 & 3.1707600 & 1.3125475 \\ \mathrm{H} & 4.0321148 & 2.1141998 & 2.5812612 \\ \mathrm{C} & 6.5924132 & 1.9267422 & -4.4789202 \\ \mathrm{H} & 5.0023908 & 0.5450273 & -5.0351759 \\ \mathrm{H} & 4.4435760 & 2.2056106 & -4.7144181 \\ \mathrm{C} & 6.4304369 & -3.9240963 & -0.8827778 \\ \mathrm{H} & 4.7606167 & -3.5984974 & 0.4805300 \\ \mathrm{H} & 4.2831723 & -4.2050447 & -1.1228874 \\ \mathrm{H} & 6.3648718 & 2.8688590 & 3.1846908 \\ \mathrm{H} & 6.3404205 & 1.0889505 & 2.9512940 \\ \mathrm{H} & 7.0132536 & 2.1540449 & 1.6709395 \\ \mathrm{H} & 6.8372446 & 2.2054858 & -5.5276142 \\ \mathrm{H} & 6.7592047 & 2.8272663 & -3.8456073 \\ \mathrm{H} & 7.3252773 & 1.1516203 & -4.1578949 \\ \mathrm{H} & 6.6200127 & -4.9665032 & -0.5440906 \\ \mathrm{H} & 6.6697364 & -3.8754936 & -1.9693516 \\ \mathrm{H} & 7.1524783 & -3.2629791 & -0.3520764\end{array}$

\section{TS-EF}

$\mathrm{E}(\mathrm{BP} 86 /$ def2-SV(P) $)=-1464.595627785$

C $\quad-0.5443731-1.7682235 \quad 2.4106428$

$\begin{array}{llll}\text { C } & -0.9991389 & -3.1203129 & 2.9760062\end{array}$

$\begin{array}{llll}\text { C } & -2.1687420 & -3.5268700 & 2.0617199\end{array}$

$\begin{array}{llll}\text { C } & -1.8711008 & -2.7925855 & 0.7372568\end{array}$

$\begin{array}{llll}\mathrm{N} & -1.1049421 & -1.6098408 & 1.1738094\end{array}$

\begin{tabular}{lllll}
\hline & 0.2347753 & -0.9686294 & 2.9642707
\end{tabular}

H $\quad-1.2542916 \quad-3.0270361 \quad 4.0530113$

H $\quad-0.1411782-3.8319775 \quad 2.9100137$

$\mathrm{H} \quad-2.2724500 \quad-4.6244758 \quad 1.9277044$

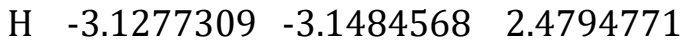

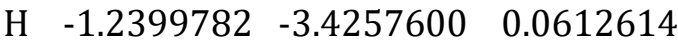

H $\quad-2.7855138 \quad-2.4938701 \quad 0.1709553$

$\begin{array}{llll}\text { C } & -1.0133555 & -0.3925294 & 0.3925387\end{array}$

$\begin{array}{llll}\text { C } & 0.1087996 & -0.3369658 & -0.6097792\end{array}$

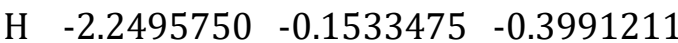

$\begin{array}{llll}\mathrm{H} & -0.9816033 & 0.4722628 & 1.0899738\end{array}$

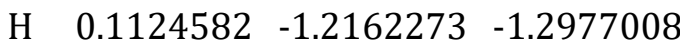

$\begin{array}{llll}\mathrm{P} & 1.9119234 & -0.2090935 & -0.0056157\end{array}$

$\mathrm{H} \quad 0.0029557 \quad 0.5707192 \quad-1.2521701$

$\begin{array}{llll}\text { C } & -4.3394966 & -0.6713228 & -1.2531258\end{array}$

$\begin{array}{lllll}\text { C } & -5.4477172 & 0.1111687 & -2.0085604\end{array}$

$\begin{array}{llll}\text { C } & -5.0536079 & 1.5781728 & -1.7835509\end{array}$

$\begin{array}{llll}\text { C } & -3.5149912 & 1.4882809 & -1.5851495\end{array}$

$\begin{array}{llll}\text { N } & -3.2828217 & 0.1556939 & -1.0551281\end{array}$ 


\begin{tabular}{cccc}
$\mathrm{O}$ & -4.4345522 & -1.8647637 & -0.9193261 \\
$\mathrm{H}$ & -6.4537099 & -0.1687272 & -1.6311931 \\
$\mathrm{H}$ & -5.4097000 & -0.1750336 & -3.0865044 \\
$\mathrm{H}$ & -5.3436833 & 2.2662065 & -2.6080964 \\
$\mathrm{H}$ & -5.5257614 & 1.9565644 & -0.8488830 \\
$\mathrm{H}$ & -2.9849955 & 1.6467310 & -2.5638644 \\
$\mathrm{H}$ & -3.1334614 & 2.2768663 & -0.8922552 \\
$\mathrm{C}$ & 2.9751183 & 0.2232736 & -1.4634036 \\
$\mathrm{C}$ & 1.9382974 & 1.1026148 & 1.2750630 \\
$\mathrm{C}$ & 2.4419741 & -1.8204088 & 0.7156394 \\
$\mathrm{H}$ & 1.0376719 & 0.8342800 & 1.8815546 \\
$\mathrm{H}$ & 1.7392094 & 2.0662235 & 0.7497632 \\
$\mathrm{H}$ & 2.3383398 & 0.8864075 & -2.0956024 \\
$\mathrm{H}$ & 3.0933382 & -0.7227784 & -2.0421297 \\
$\mathrm{H}$ & 1.9590773 & -1.8308333 & 1.7209721 \\
$\mathrm{H}$ & 1.9326774 & -2.6027648 & 0.1063498 \\
$\mathrm{C}$ & 4.3427215 & 0.8936456 & -1.2301973 \\
$\mathrm{C}$ & 3.1537438 & 1.2059812 & 2.2134257 \\
$\mathrm{C}$ & 3.9492020 & -2.1197754 & 0.8097660 \\
$\mathrm{C}$ & 5.0911684 & 1.1683295 & -2.5481169 \\
$\mathrm{H}$ & 4.1956787 & 1.8555238 & -0.6877330 \\
$\mathrm{H}$ & 4.9773632 & 0.2588278 & -0.5720194 \\
$\mathrm{C}$ & 2.9755440 & 2.3323366 & 3.2486006 \\
$\mathrm{H}$ & 3.2732912 & 0.2409831 & 2.7556318 \\
$\mathrm{H}$ & 4.0954921 & 1.3750083 & 1.6429614 \\
$\mathrm{C}$ & 4.2237916 & -3.4836821 & 1.4707402 \\
$\mathrm{H}$ & 4.4059288 & -2.1120695 & -0.2072746 \\
$\mathrm{H}$ & 4.4649968 & -1.3224888 & 1.3912559 \\
$\mathrm{C}$ & 6.4457594 & 1.8579859 & -2.3409528 \\
$\mathrm{H}$ & 5.2399868 & 0.2054892 & -3.0914173 \\
$\mathrm{H}$ & 4.4481868 & 1.7952914 & -3.2097570 \\
$\mathrm{C}$ & 4.1506172 & 2.4416677 & 4.2284099 \\
$\mathrm{H}$ & 2.8403717 & 3.3030833 & 2.7153917 \\
$\mathrm{H}$ & 6.1960836 & -3.8533522 & 0.5662017 \\
$\mathrm{H}$ & 2.0306096 & 2.1544954 & 3.8114319 \\
$\mathrm{C}$ & 5.7177044 & -3.8168321 & 1.5713010 \\
$\mathrm{H}$ & 3.7663163 & -3.4921015 & 2.4871535 \\
$\mathrm{H}$ & 3.6992377 & -4.2809681 & 0.8933739 \\
$\mathrm{H}$ & 6.9586340 & 2.0381148 & -3.3110125 \\
$\mathrm{H}$ & 6.3273505 & 2.8434497 & -1.8363888 \\
$\mathrm{H}$ & 7.1251500 & 1.2392112 & -1.7121349 \\
$\mathrm{H}$ & 3.9895963 & 3.2634364 & 4.9602831 \\
$\mathrm{H}$ & 4.2837539 & 1.4999246 & 4.8072284 \\
& 5.1079740 & 2.6481431 & 3.6976305 \\
\hline
\end{tabular}

\section{TS-EF_Phenol}

$\mathrm{E}(\mathrm{BP} 86 / \mathrm{def} 2-\mathrm{SV}(\mathrm{P}))=-1485.429910183$ C $-0.4861543 \quad-1.2583996 \quad 2.5015231$ $\begin{array}{llll}\text { C } & -0.9536252 & -1.5848969 & 3.9243714\end{array}$

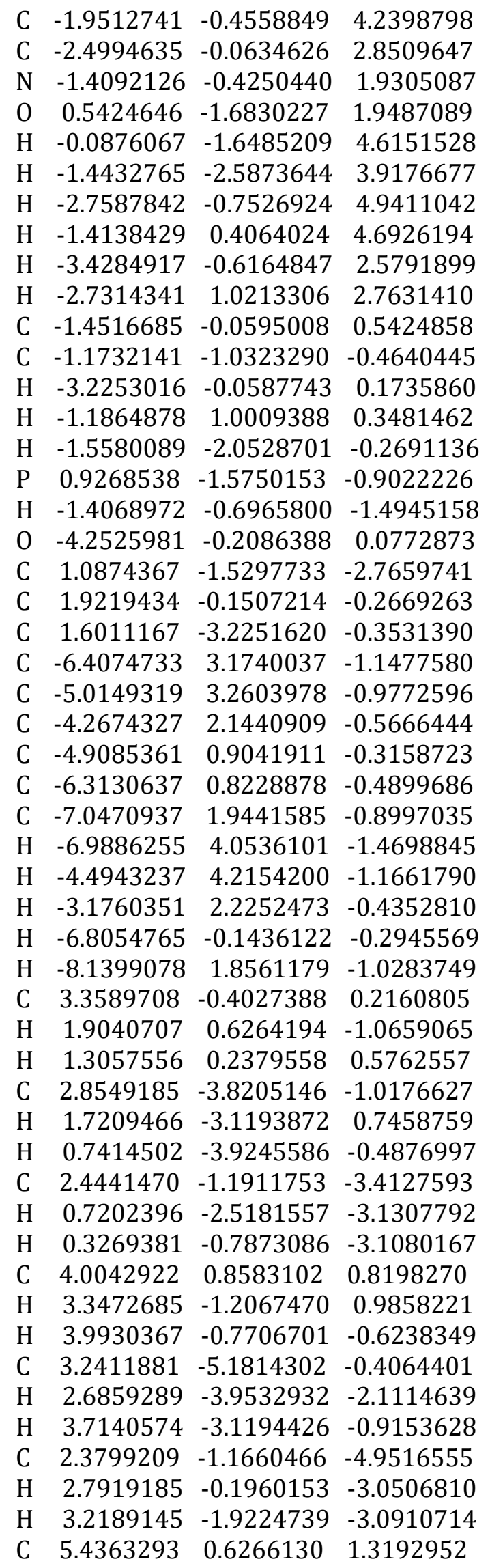




$\begin{array}{cccc}\mathrm{H} & 4.0013257 & 1.6744742 & 0.0588200 \\ \mathrm{H} & 3.3683358 & 1.2220444 & 1.6605219 \\ \mathrm{C} & 4.4858860 & -5.8059419 & -1.0502946 \\ \mathrm{H} & 3.4085661 & -5.0529771 & 0.6885451 \\ \mathrm{H} & 2.3784941 & -5.8825213 & -0.5013842 \\ \mathrm{C} & 3.7189991 & -0.8083501 & -5.6095809 \\ \mathrm{H} & 2.0366346 & -2.1616399 & -5.3198842 \\ \mathrm{H} & 1.5983690 & -0.4376608 & -5.2732771 \\ \mathrm{H} & 5.8704259 & 1.5545397 & 1.7529728 \\ \mathrm{H} & 5.4675114 & -0.1589989 & 2.1078900 \\ \mathrm{H} & 6.1071678 & 0.2971695 & 0.4933347 \\ \mathrm{H} & 4.7360290 & -6.7844149 & -0.5838727 \\ \mathrm{H} & 4.3352737 & -5.9828729 & -2.1395931 \\ \mathrm{H} & 5.3752267 & -5.1448125 & -0.9394935 \\ \mathrm{H} & 3.6367486 & -0.8004084 & -6.7188622 \\ \mathrm{H} & 4.0707275 & 0.1995877 & -5.2923771 \\ \mathrm{H} & 4.5135165 & -1.5397064 & -5.3373068\end{array}$

\section{TS-EF_PMe3}

\begin{tabular}{lrrc}
\multicolumn{4}{l}{ E(BP86/def2-SV(P)) $=-1111.077775178$} \\
$\mathrm{C}$ & -0.6212732 & -2.5923823 & 1.6574535 \\
$\mathrm{C}$ & -1.3749628 & -3.7579917 & 2.2981959 \\
$\mathrm{C}$ & -2.8089344 & -3.2058512 & 2.4290781 \\
$\mathrm{C}$ & -2.9113809 & -2.2017276 & 1.2648133 \\
$\mathrm{~N}$ & -1.5141887 & -1.7526070 & 1.0917327 \\
$\mathrm{O}$ & 0.6276332 & -2.4422910 & 1.6392854 \\
$\mathrm{H}$ & -0.8964951 & -4.0576646 & 3.2539647 \\
$\mathrm{H}$ & -1.3241954 & -4.6401368 & 1.6159509 \\
$\mathrm{H}$ & -3.5941186 & -3.9889650 & 2.3845723 \\
$\mathrm{H}$ & -2.9206307 & -2.6722872 & 3.3990945 \\
$\mathrm{H}$ & -3.2602266 & -2.6876732 & 0.3224120 \\
$\mathrm{H}$ & -3.5703638 & -1.3195750 & 1.4352403 \\
$\mathrm{C}$ & -1.1987369 & -0.6090812 & 0.2589700 \\
$\mathrm{C}$ & 0.0599954 & -0.7298079 & -0.5455975 \\
$\mathrm{H}$ & -2.2946021 & -0.3862019 & -0.6212555 \\
$\mathrm{H}$ & -1.2616683 & 0.3369201 & 0.8478405 \\
$\mathrm{H}$ & 0.1223911 & -1.7083537 & -1.0738404 \\
$\mathrm{P}$ & 1.7637110 & -0.5328404 & 0.2941974 \\
$\mathrm{H}$ & 0.0785273 & 0.0711604 & -1.3213456 \\
$\mathrm{C}$ & -4.4206303 & 0.4551728 & -0.5742114 \\
$\mathrm{C}$ & -5.4940341 & 0.9719659 & -1.5716979 \\
$\mathrm{C}$ & -4.7186627 & 1.0859122 & -2.8922333 \\
$\mathrm{C}$ & -3.5840717 & 0.0366926 & -2.7116717 \\
$\mathrm{~N}$ & -3.3745254 & -0.0482832 & -1.2765550 \\
$\mathrm{O}$ & -4.5415121 & 0.4858298 & 0.6641881 \\
$\mathrm{H}$ & -5.9470365 & 1.9187014 & -1.2087045 \\
$\mathrm{H}$ & -6.3142344 & 0.2174187 & -1.6277388 \\
$\mathrm{H}$ & -5.3306909 & 0.9081688 & -3.8040802 \\
$\mathrm{H}$ & -4.2724531 & 2.1024354 & -2.9816077 \\
$\mathrm{H}$ & -3.8928107 & -0.9517802 & -3.1469238 \\
$\mathrm{H}$ & -2.6510905 & 0.3328310 & -3.2501833
\end{tabular}

$\begin{array}{lrrr}\mathrm{C} & 2.5673859 & 0.8195858 & -0.6874050 \\ \mathrm{C} & 1.7000902 & 0.1655065 & 1.9830205 \\ \mathrm{C} & 2.9889001 & -1.8954145 & 0.1746480 \\ \mathrm{H} & 1.6616762 & -0.6618995 & 2.7166908 \\ \mathrm{H} & 0.7629777 & 0.7584166 & 2.0711795 \\ \mathrm{H} & 1.9460191 & 1.7403949 & -0.6357980 \\ \mathrm{H} & 2.6487340 & 0.5156743 & -1.7541900 \\ \mathrm{H} & 2.6734143 & -2.7117580 & 0.8523670 \\ \mathrm{H} & 3.0059793 & -2.2661207 & -0.8734898 \\ \mathrm{H} & 3.5844837 & 1.0530325 & -0.3045577 \\ \mathrm{H} & 2.5770669 & 0.8243670 & 2.1590745 \\ \mathrm{H} & 4.0032444 & -1.5235928 & 0.4386171\end{array}$

\section{TS-EF_PPh3}

$\mathrm{E}(\mathrm{BP} 86 / \mathrm{def} 2-\mathrm{SV}(\mathrm{P}))=-1685.874745130$

C $-1.1726485-1.8628681 \quad 1.9218549$

$\begin{array}{llll}\text { C } & -2.2172380 & -2.4706343 & 2.8631252\end{array}$

$\begin{array}{llll}\text { C } & -3.3166558 & -1.3927357 & 2.9085263\end{array}$

$\begin{array}{llll}C & -3.1965806 & -0.7145972 & 1.5312585\end{array}$

$\begin{array}{llll}\mathrm{N} & -1.7669949 & -0.8791676 & 1.2036081\end{array}$

$\begin{array}{llll}0 & 0.0220776 & -2.2262175 & 1.8264357\end{array}$

$\mathrm{H} \quad-1.7586692-2.7182727 \quad 3.8431190$

H $\quad-2.5858618-3.4271566 \quad 2.4212221$

$\begin{array}{llll}\mathrm{H} & -4.3335725 & -1.7975252 & 3.0936611\end{array}$

$\begin{array}{llll}\mathrm{H} & -3.0933700 & -0.6580178 & 3.7138575\end{array}$

$\begin{array}{llll}\mathrm{H} & -3.8127167 & -1.2275191 & 0.7552622\end{array}$

$\begin{array}{llll}\mathrm{H} & -3.4697485 & 0.3631304 & 1.4879192\end{array}$

$\begin{array}{llll}\text { C } & -1.2074946 & -0.1300764 & 0.0974857\end{array}$

$\begin{array}{llll}\text { C } & -0.0155988 & -0.7056418 & -0.5850035\end{array}$

$\begin{array}{llll}\mathrm{H} & -2.1789362 & 0.0266262 & -0.8304012\end{array}$

$\begin{array}{llll}\mathrm{H} & -1.1068787 & 0.9435170 & 0.3733295\end{array}$

$\begin{array}{llll}\mathrm{H} & -0.1325439 & -1.7858442 & -0.8155847\end{array}$

$\begin{array}{llll}\mathrm{P} & 1.7464051 & -0.6162206 & 0.2370557\end{array}$

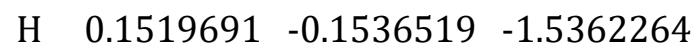

$\begin{array}{llll}\text { C } & -3.8619556 & 1.5622875 & -1.1499878\end{array}$

$\begin{array}{llll}\text { C } & -4.8382811 & 2.0800733 & -2.2437990\end{array}$

$\begin{array}{llll}\text { C } & -4.3188391 & 1.4045600 & -3.5212051\end{array}$

$\begin{array}{llll}\text { C } & -3.6247222 & 0.1252203 & -2.9692569\end{array}$

$\begin{array}{lllll}\mathrm{N} & -3.2122654 & 0.4694937 & -1.6195200\end{array}$

$\begin{array}{lllll}0 & -3.7384759 & 2.0766079 & -0.0212043\end{array}$

$\begin{array}{lllll}\mathrm{H} & -4.8506034 & 3.1903420 & -2.2694631\end{array}$

$\begin{array}{llll}\mathrm{H} & -5.8704040 & 1.7472541 & -1.9805277\end{array}$

$\begin{array}{llll}\mathrm{H} & -5.1035169 & 1.1795814 & -4.2774167\end{array}$

$\begin{array}{llll}\mathrm{H} & -3.5595141 & 2.0561059 & -4.0106634\end{array}$

$\begin{array}{llll}\mathrm{H} & -4.3395038 & -0.7413665 & -2.9769208\end{array}$

$\begin{array}{llll}\mathrm{H} & -2.7580024 & -0.1859509 & -3.6017840\end{array}$

$\begin{array}{llll}\text { C } & 1.7738173 & 1.0048185 & 4.5789679\end{array}$

C $\quad 1.1496663 \quad 1.7349923 \quad 3.5534642$

$\begin{array}{llll}\text { C } & 1.1497034 & 1.2449359 & 2.2366647\end{array}$

$\begin{array}{llll}\text { C } & 1.7737278 & 0.0103017 & 1.9457937\end{array}$

$\begin{array}{llll}\text { C } & 2.3892575 & -0.7268982 & 2.9789712\end{array}$ 


$\begin{array}{lccc}\mathrm{C} & 2.3943186 & -0.2236498 & 4.2885516 \\ \mathrm{H} & 1.7746684 & 1.3935814 & 5.6108724 \\ \mathrm{H} & 0.6552283 & 2.6951375 & 3.7745890 \\ \mathrm{H} & 0.6631956 & 1.8312994 & 1.4414811 \\ \mathrm{H} & 2.8547857 & -1.7000991 & 2.7594645 \\ \mathrm{H} & 2.8796989 & -0.8025832 & 5.0915271 \\ \mathrm{C} & 4.2893119 & -4.4847418 & -0.3195820 \\ \mathrm{C} & 4.8579993 & -3.2203125 & -0.5524068 \\ \mathrm{C} & 4.0959305 & -2.0569281 & -0.3637761 \\ \mathrm{C} & 2.7494128 & -2.1473594 & 0.0676679 \\ \mathrm{C} & 2.1836538 & -3.4220818 & 0.3103644 \\ \mathrm{C} & 2.9559329 & -4.5786074 & 0.1140821 \\ \mathrm{H} & 4.8883179 & -5.3976242 & -0.4743577 \\ \mathrm{H} & 5.9058274 & -3.1346585 & -0.8850177 \\ \mathrm{H} & 4.5552836 & -1.0745946 & -0.5548580 \\ \mathrm{H} & 1.1532421 & -3.4894928 & 0.6927653 \\ \mathrm{H} & 2.5053750 & -5.5662833 & 0.3077803 \\ \mathrm{C} & 4.0473897 & 2.4993579 & -2.3779977 \\ \mathrm{C} & 4.0698321 & 2.6267320 & -0.9791230 \\ \mathrm{C} & 3.3885495 & 1.6997341 & -0.1711716 \\ \mathrm{C} & 2.6722363 & 0.6331942 & -0.7571629 \\ \mathrm{C} & 2.6608667 & 0.5088618 & -2.1679120 \\ \mathrm{C} & 3.3442059 & 1.4354658 & -2.9703368 \\ \mathrm{H} & 4.5795008 & 3.2299764 & -3.0093755 \\ \mathrm{H} & 4.6234997 & 3.4550203 & -0.5068493 \\ \mathrm{H} & 3.4174586 & 1.8093754 & 0.9240875 \\ \mathrm{H} & 2.1275321 & -0.3265645 & -2.6519779 \\ \mathrm{H} & 3.3242114 & 1.3256630 & -4.0671428\end{array}$

\section{TS-HI_cis}

\begin{tabular}{lccc}
\multicolumn{4}{c}{ E(BP86/def2-SV(P) $=-1523.503544972$} \\
C & 1.1583500 & -2.4836156 & 1.5991016 \\
C & 0.7192512 & -3.9487825 & 1.7512777 \\
C & -0.7902935 & -3.8615306 & 2.0374612 \\
C & -1.2013845 & -2.5114662 & 1.4045933 \\
N & 0.0246329 & -1.7222995 & 1.4740235 \\
O & 2.3206630 & -2.0673716 & 1.5587528 \\
H & 1.3142838 & -4.4595862 & 2.5365916 \\
H & 0.9369554 & -4.4686574 & 0.7892035 \\
H & -1.3747090 & -4.7133403 & 1.6307770 \\
H & -0.9672920 & -3.8263522 & 3.1348101 \\
H & -1.5386310 & -2.6293876 & 0.3444103 \\
H & -2.0250253 & -2.0065147 & 1.9569313 \\
C & 0.0757608 & -0.3032704 & 1.1163059 \\
C & 0.3235537 & -0.1052910 & -0.3735037 \\
H & -0.8817701 & 0.1575594 & 1.4632162 \\
H & 0.8672005 & 0.1380749 & 1.7698411 \\
P & 2.5834366 & 1.1441958 & -1.2126300 \\
H & 0.2862280 & -1.0531457 & -0.9415463 \\
C & -0.1123264 & 1.0611224 & -1.1184253 \\
O & 1.2201347 & 1.9679579 & -1.5319725
\end{tabular}

$\begin{array}{llll}\text { C } & 3.6286674 & 2.4279440 & -2.0988789\end{array}$

$\begin{array}{llll}\text { C } & 3.1334700 & 0.9754886 & 0.5441973\end{array}$

$\begin{array}{llll}\text { C } & 2.8316841 & -0.4766482 & -2.0974506\end{array}$

$\begin{array}{llll}\text { C } & -2.9284679 & 3.7981426 & 0.7421832\end{array}$

$\begin{array}{llll}\text { C } & -1.6310127 & 3.6644666 & 1.2672417\end{array}$

$\begin{array}{llll}\text { C } & -0.7036913 & 2.8035275 & 0.6578827\end{array}$

$\begin{array}{llll}\text { C } & -1.0500928 & 2.0542511 & -0.4901277\end{array}$

$\begin{array}{llll}\text { C } & -2.3560840 & 2.2049887 & -1.0096347\end{array}$

C $\quad-3.2868046 \quad 3.0625814 \quad-0.4017576$

$\mathrm{H} \quad-3.6552504 \quad 4.4771278 \quad 1.2188269$

$\mathrm{H} \quad-1.3348092 \quad 4.2421033 \quad 2.1596025$

$\mathrm{H} \quad 0.3172052 \quad 2.7267788 \quad 1.0662664$

H $\quad-2.6452263 \quad 1.6356266-1.9102979$

H $\quad-4.2981775 \quad 3.1645307 \quad-0.8307497$

$\mathrm{H} \quad-0.4548040 \quad 0.8185366 \quad-2.1442393$

H $\quad 2.5522976 \quad 1.7452929 \quad 1.1025721$

H $\quad 2.7450500 \quad-0.0167768 \quad 0.8703003$

C $\quad 4.6285404 \quad 1.0713158 \quad 0.9096151$

C $\quad 4.1380304 \quad-0.7348279-2.8726823$

H $\quad 2.6755097 \quad-1.2528121-1.3183927$

H $\quad 1.9649141-0.5516305-2.7919070$

C $\quad 5.1678856 \quad 2.4600285-2.0882241$

H $\quad 3.2304191 \quad 3.3955558-1.7207372$

$\mathrm{H} \quad 3.2588969 \quad 2.3634737 \quad-3.1487377$

$\begin{array}{llll}\text { C } & 4.8695290 & 0.6273476 & 2.3652711\end{array}$

$\begin{array}{llll}\mathrm{H} & 5.2356043 & 0.4271716 & 0.2319092\end{array}$

H $\quad 5.0056985 \quad 2.1100403 \quad 0.7767442$

C $4.1260718-2.1124065-3.5619873$

$\mathrm{H} \quad 4.2982667 \quad 0.0532687 \quad-3.6435713$

H $\quad 5.0108093 \quad-0.6872022 \quad-2.1817973$

C $\quad 5.7207969 \quad 3.4567767 \quad-3.1250621$

H $\quad 5.5367079 \quad 2.7549210 \quad-1.0823084$

H $\quad 5.6001386 \quad 1.4559297 \quad-2.2894885$

$\begin{array}{llll}\text { C } & 6.3434800 & 0.6983278 & 2.7842776\end{array}$

H $\quad 4.2568624 \quad 1.26651613 .0444698$

H $\quad 4.4829478-0.4092715 \quad 2.4929011$

C $\quad 5.4146069-2.4157060 \quad-4.3375434$

H $3.9560591 \quad-2.9023129-2.7933032$

H $\quad 3.2514428 \quad-2.1662598 \quad-4.2526422$

C $\quad 7.2509158 \quad 3.5671413 \quad-3.1072385$

H $\quad 5.3795969 \quad 3.1530471-4.1431951$

$\mathrm{H} \quad 5.2703550 \quad 4.4611544 \quad-2.9431048$

$\begin{array}{llll}\mathrm{H} & 6.4800647 & 0.3705413 & 3.8387024\end{array}$

$\mathrm{H} \quad 6.9787325 \quad 0.0423956 \quad 2.1462756$

$\mathrm{H} \quad 6.7447230 \quad 1.7343624 \quad 2.7007920$

H $\quad 5.3698714 \quad-3.4161914 \quad-4.8219102$

H $\quad 5.5941613 \quad-1.6629999 \quad-5.1385641$

H $\quad 6.3033923 \quad-2.4078653 \quad-3.6665838$

H $\quad 7.6152351 \quad 4.2898772 \quad-3.8705250$

H $\quad 7.6215471 \quad 3.9118662-2.1152233$

H $\quad 7.7316143 \quad 2.5848657 \quad-3.3186846$ 


\section{TS-HI_trans}

$\mathrm{E}(\mathrm{BP} 86 /$ def2-SV $(\mathrm{P}))=-1523.504008532$

$\begin{array}{llll}\text { C } & -2.5494108 & -0.4882612 & 2.9180048\end{array}$

$\begin{array}{llll}\text { C } & -3.6688927 & -1.4279001 & 3.3936642\end{array}$

$\begin{array}{llll}\text { C } & -4.9627090 & -0.7365512 & 2.9291045\end{array}$

$\begin{array}{llll}\text { C } & -4.5030391 & 0.1180732 & 1.7246654\end{array}$

$\begin{array}{llll}\mathrm{N} & -3.1039020 & 0.3994087 & 2.0292869\end{array}$

$\begin{array}{llll}0 & -1.3602985 & -0.5396023 & 3.2438283\end{array}$

H $\quad-3.5994180 \quad-1.6012741 \quad 4.4876054$

H $\quad-3.5163929-2.41488692 .8978296$

H $\quad-5.7773305-1.4411113 \quad 2.6599120$

H $\quad-5.3467355-0.0699522 \quad 3.7322661$

H $\quad-4.5988126 \quad-0.4369146 \quad 0.7579560$

$\mathrm{H} \quad-5.0801210 \quad 1.0640307 \quad 1.6189113$

C $\quad-2.2830701 \quad 1.3197408 \quad 1.2416171$

$\begin{array}{llll}\text { C } & -1.6748530 & 0.6561247 & 0.0119832\end{array}$

$\mathrm{H} \quad-2.9335297 \quad 2.1909053 \quad 0.9736782$

H $\quad-1.5307035 \quad 1.7232972 \quad 1.9627328$

P $\quad 1.0152256 \quad 0.6218886-0.2203074$

H $\quad-2.0031282 \quad-0.3868679 \quad-0.1441873$

C $\quad-1.3660884 \quad 1.4269842 \quad-1.1647768$

$\begin{array}{lllll}0 & 0.2887464 & 1.6434964 & -1.2567898\end{array}$

C $\quad 2.7001991 \quad 0.9839398 \quad-0.9704966$

$\begin{array}{llll}\text { C } & 1.0251430 & 1.0682422 & 1.5728073\end{array}$

C $\quad 0.7057507 \quad-1.2022518-0.4379134$

$\mathrm{H} \quad 0.7797335 \quad 2.1553253 \quad 1.5887810$

$\begin{array}{llll}\mathrm{H} & 0.1507498 & 0.5394173 & 2.0149181\end{array}$

C $\quad 2.2671632 \quad 0.7867021 \quad 2.4420123$

$\begin{array}{llll}\text { C } & 1.8915054 & -2.1730894 & -0.5978035\end{array}$

$\mathrm{H} \quad 0.0721376-1.4896929 \quad 0.4277387$

H $\quad 0.0501112 \quad-1.2622810 \quad-1.3353712$

$\begin{array}{llll}\text { C } & 4.0391880 & 0.5057777 & -0.3790430\end{array}$

H $2.71137392 .0915126-1.0754294$

H $\quad 2.5913532 \quad 0.6018130 \quad-2.0123398$

C $\quad 1.9540883 \quad 0.9980170 \quad 3.9360225$

$\mathrm{H} \quad 2.6158321 \quad-0.2616726 \quad 2.2914516$

H $3.1160338 \quad 1.4444401 \quad 2.1508637$

C $1.4115746-3.6172773-0.8376959$

H $\quad 2.5391910 \quad-1.8625147 \quad-1.4490284$

H $\quad 2.5319375 \quad-2.1543127 \quad 0.3137447$

$\begin{array}{llll}\text { C } & 5.1978504 & 0.6851965 & -1.3786797\end{array}$

H $\quad 4.2755718 \quad 1.0796175 \quad 0.5429265$

H $3.9933153 \quad-0.5610964 \quad-0.0702992$

$\begin{array}{llll}\text { C } & 3.1525497 & 0.7164491 & 4.8512325\end{array}$

H $\quad 1.6073732 \quad 2.0478813 \quad 4.0870595$

H $1.0929896 \quad 0.3510690 \quad 4.2183906$

C $\quad 2.5606094-4.6222558-0.9909622$

$\mathrm{H} \quad 0.7528557 \quad-3.9272013 \quad 0.0072281$

H $\quad 0.7687134 \quad-3.6401586-1.7490432$

$\begin{array}{llll}\text { C } & 6.5636453 & 0.2843825 & -0.8063028\end{array}$

$\begin{array}{cccc}\mathrm{H} & 4.9861173 & 0.0858055 & -2.2958463 \\ \mathrm{H} & 5.2297570 & 1.7489788 & -1.7138032 \\ \mathrm{H} & 2.8913716 & 0.8795704 & 5.9203876 \\ \mathrm{H} & 3.5015526 & -0.3366490 & 4.7504516 \\ \mathrm{H} & 4.0165986 & 1.3781903 & 4.6125867 \\ \mathrm{H} & 2.1774609 & -5.6522077 & -1.1643218 \\ \mathrm{H} & 3.2179618 & -4.3599700 & -1.8509039 \\ \mathrm{H} & 3.1993951 & -4.6514214 & -0.0791361 \\ \mathrm{H} & 7.3744685 & 0.4209839 & -1.5557303 \\ \mathrm{H} & 6.8254029 & 0.8968296 & 0.0863239 \\ \mathrm{H} & 6.5742832 & -0.7838533 & -0.4912671 \\ \mathrm{C} & -2.5221915 & -0.0063196 & -5.1220762 \\ \mathrm{C} & -3.2673375 & -0.3628766 & -3.9864377 \\ \mathrm{C} & -2.8749021 & 0.0857202 & -2.7127239 \\ \mathrm{C} & -1.7291281 & 0.8934781 & -2.5425829 \\ \mathrm{C} & -0.9912554 & 1.2470464 & -3.6970509 \\ \mathrm{C} & -1.3822918 & 0.8035441 & -4.9704260 \\ \mathrm{H} & -2.8287946 & -0.3555962 & -6.1225423 \\ \mathrm{H} & -4.1661268 & -0.9945349 & -4.0899516 \\ \mathrm{H} & -3.4728983 & -0.1819296 & -1.8267052 \\ \mathrm{H} & -0.0928446 & 1.8711545 & -3.5783982 \\ \mathrm{H} & -0.7892221 & 1.0926098 & -5.8550355 \\ \mathrm{H} & -1.6226530 & 2.5089023 & -1.0889729\end{array}$

\section{TS-S1}

$\mathrm{E}(\mathrm{BP} 86 /$ def2-SV(P) $)=-1178.141634559$

C $\quad 0.8522230 \quad-1.5172990-3.4712332$

$\begin{array}{llll}\text { C } & -0.2628175 & -2.2427933 & -4.2724610\end{array}$

$\begin{array}{llll}\text { C } & 0.2116979 & -2.0513628 & -5.7201148\end{array}$

C $\quad 1.7455465-1.8480667-5.5290076$

N $1.9368725 \quad-1.3083286-4.1904135$

$\begin{array}{lllll}0 & 0.6793834 & -1.2177461 & -2.2321661\end{array}$

H $\quad-1.2698077 \quad-1.8262631 \quad-4.0494198$

H $\quad-0.2825763 \quad-3.3174508$-3.9685479

$\mathrm{H} \quad-0.0452671-2.8901004 \quad-6.4056049$

H $\quad-0.2371089-1.1244119 \quad-6.1451588$

H $\quad 2.2868728 \quad-2.8259439 \quad-5.6454523$

H $\quad 2.1777725 \quad-1.1715840 \quad-6.3057215$

H $\quad 3.3058534 \quad-0.2116585 \quad-2.6429189$

C $\quad 2.8717014 \quad-0.2339261-1.6258524$

$\begin{array}{llll}\text { C } & 2.5386694 & 0.9022234 & -0.9395897\end{array}$

$\begin{array}{llll}\mathrm{P} & 1.3333139 & 0.7828898 & 0.3568148\end{array}$

$\mathrm{H} \quad 2.7874435 \quad-1.2372272-1.1873249$

H $\quad 2.6343329 \quad 1.9007322 \quad-1.4060169$

C $\quad-0.3151348 \quad 1.0062588-0.4376558$

$\begin{array}{llll}\text { C } & 1.4237268 & -0.9062287 & 1.0825427\end{array}$

C $\quad 1.6876846 \quad 2.0889323 \quad 1.6182056$

H $\quad 2.5086264 \quad-1.1541312 \quad 1.1191067$

H $\quad 0.9749978-1.5548537 \quad 0.2928023$

$\begin{array}{llll}\text { C } & 0.7858322 & -1.1367786 & 2.4638583\end{array}$

$\begin{array}{llll}\text { C } & -1.5929400 & 0.5954517 & 0.3143001\end{array}$ 


$\begin{array}{cccc}\mathrm{H} & -0.1775597 & 0.3512727 & -1.3431958 \\ \mathrm{H} & -0.3580962 & 2.0684147 & -0.7748918 \\ \mathrm{H} & 2.5629312 & 1.7205127 & 2.2022900 \\ \mathrm{C} & 0.5494424 & 2.5452727 & 2.5482616 \\ \mathrm{H} & 2.0652391 & 2.9547255 & 1.0255772 \\ \mathrm{C} & 0.8870397 & -2.6097257 & 2.9023605 \\ \mathrm{H} & 1.2887011 & -0.4963506 & 3.2257342 \\ \mathrm{H} & -0.2847835 & -0.8322616 & 2.4567080 \\ \mathrm{C} & -2.8490155 & 0.8245642 & -0.5479567 \\ \mathrm{H} & -1.5343167 & -0.4873189 & 0.5683637 \\ \mathrm{H} & -1.7026459 & 1.1451903 & 1.2776690 \\ \mathrm{C} & 1.0071004 & 3.6253636 & 3.5459309 \\ \mathrm{H} & -0.2914134 & 2.9462708 & 1.9373426 \\ \mathrm{H} & 0.1373891 & 1.6772453 & 3.1111314 \\ \mathrm{C} & 0.2734567 & -2.8733602 & 4.2831038 \\ \mathrm{H} & 0.3869423 & -3.2493406 & 2.1385749 \\ \mathrm{H} & 1.9590533 & -2.9175885 & 2.9022376 \\ \mathrm{C} & -4.1451539 & 0.3796400 & 0.1410939 \\ \mathrm{H} & -2.9178597 & 1.9056775 & -0.8148108 \\ \mathrm{H} & -2.7238852 & 0.2773626 & -1.5102370 \\ \mathrm{C} & -0.1169411 & 4.1091030 & 4.4711039 \\ \mathrm{H} & 1.8497075 & 3.2247757 & 4.1573998 \\ \mathrm{H} & 1.4261775 & 4.4907325 & 2.9803519 \\ \mathrm{H} & 0.3618827 & -3.9455239 & 4.5646035 \\ \mathrm{H} & 0.7795802 & -2.2761766 & 5.0753877 \\ \mathrm{H} & -0.8082850 & -2.6098781 & 4.3043580 \\ \mathrm{H} & -5.0295962 & 0.5606833 & -0.5085780 \\ \mathrm{H} & -4.1240708 & -0.7075956 & 0.3808343 \\ \mathrm{H} & -4.3114996 & 0.9303919 & 1.0951176 \\ \mathrm{H} & 0.2489215 & 4.8857831 & 5.1778011 \\ \mathrm{H} & -0.9580650 & 4.5524130 & 3.8916418 \\ \mathrm{H} & -0.5324745 & 3.2726901 & 5.0774218\end{array}$

\section{TS-S10}

\begin{tabular}{lccc}
\multicolumn{4}{l}{ E(BP86/def2-SV $(\mathrm{P}))=-1046.264607912$} \\
$\mathrm{C}$ & 1.9772520 & 1.1113422 & -3.5673439 \\
$\mathrm{C}$ & 0.8186280 & 1.7810196 & -3.1992722 \\
$\mathrm{C}$ & 0.1186598 & 1.5138904 & -1.9805937 \\
$\mathrm{H}$ & 2.0784423 & 0.1509899 & -2.9853103 \\
$\mathrm{H}$ & 0.5253989 & 2.7110350 & -3.7503483 \\
$\mathrm{C}$ & 2.1136696 & 0.1137740 & -5.9252243 \\
$\mathrm{C}$ & 1.3107647 & -0.8144399 & -6.0004579 \\
$\mathrm{H}$ & 0.5661390 & -1.5955906 & -5.9153646 \\
$\mathrm{H}$ & 2.8727519 & 0.8706619 & -6.0603761 \\
$\mathrm{C}$ & -0.5809277 & 2.4127480 & -1.1636227 \\
$\mathrm{P}$ & -0.7071870 & 4.1315431 & -1.4871371 \\
$\mathrm{C}$ & -1.5851653 & 4.8662347 & -0.0298285 \\
$\mathrm{C}$ & 0.9903060 & 4.8660623 & -1.6435450 \\
$\mathrm{C}$ & -1.7046972 & 4.5228858 & -3.0137325 \\
$\mathrm{H}$ & -1.0410033 & 2.0735414 & -0.2200863 \\
$\mathrm{H}$ & 0.2389821 & 0.4889093 & -1.5742284
\end{tabular}

$\begin{array}{cccc}\mathrm{C} & -1.3619891 & 6.3591886 & 0.2699353 \\ \mathrm{H} & -2.6689457 & 4.6473943 & -0.1712591 \\ \mathrm{C} & 1.1832192 & 6.1718909 & -2.4323568 \\ \mathrm{H} & 1.3594821 & 4.9683551 & -0.5970155 \\ \mathrm{C} & -2.3413163 & 5.9145906 & -3.1767119 \\ \mathrm{H} & -1.0379899 & 4.2831534 & -3.8735454 \\ \mathrm{H} & -1.2533680 & 4.2536536 & 0.8389368 \\ \mathrm{H} & 1.5894729 & 4.0464041 & -2.1037739 \\ \mathrm{H} & -2.4909406 & 3.7337944 & -3.0172231 \\ \mathrm{C} & -2.1400765 & 6.8266034 & 1.5140023 \\ \mathrm{H} & -0.2759424 & 6.5507939 & 0.4291345 \\ \mathrm{H} & -1.6621317 & 6.9812509 & -0.6028876 \\ \mathrm{C} & 2.6620429 & 6.5999937 & -2.4843173 \\ \mathrm{H} & 0.8124361 & 6.0366227 & -3.4745289 \\ \mathrm{H} & 0.5794543 & 6.9960661 & -1.9865392 \\ \mathrm{C} & -3.1150819 & 6.0453379 & -4.5027224 \\ \mathrm{H} & -3.0413082 & 6.1112738 & -2.3316844 \\ \mathrm{H} & -1.5627970 & 6.7089850 & -3.1307567 \\ \mathrm{C} & -1.9149567 & 8.3070399 & 1.8468431 \\ \mathrm{H} & -3.2282863 & 6.6390260 & 1.3562226 \\ \mathrm{H} & -1.8459522 & 6.1969003 & 2.3864206 \\ \mathrm{C} & 2.8909590 & 7.8876418 & -3.2860536 \\ \mathrm{H} & 3.0401523 & 6.7340706 & -1.4433967 \\ \mathrm{H} & 3.2630497 & 5.7704466 & -2.9234066 \\ \mathrm{C} & -3.7809285 & 7.4156150 & -4.6824384 \\ \mathrm{H} & -2.4180240 & 5.8521730 & -5.3513108 \\ \mathrm{H} & -3.8870742 & 5.2422221 & -4.5551638 \\ \mathrm{H} & -2.4926442 & 8.6099706 & 2.7474355 \\ \mathrm{H} & -0.8410200 & 8.5192045 & 2.0505037 \\ \mathrm{H} & -2.2329742 & 8.9656178 & 1.0071612 \\ \mathrm{H} & 3.9673840 & 8.1666595 & -3.3007919 \\ \mathrm{H} & 2.5616843 & 7.7712457 & -4.3433872 \\ \mathrm{H} & 2.3280168 & 8.7450656 & -2.8516061 \\ \mathrm{H} & -4.3272920 & 7.4742235 & -5.6492586 \\ \mathrm{H} & -4.5145277 & 7.6219274 & -3.8702095 \\ \mathrm{H} & -3.0295999 & 8.2374911 & -4.6719782 \\ & & & \end{array}$

\section{TS-S11_cis-C2H3}

$\mathrm{E}(\mathrm{BP} 86 /$ def2-SV $(\mathrm{P}))=-1541.941264078$

C $\quad-1.3549906-2.31319831 .8034812$

$\begin{array}{llll}\text { C } & -1.9166539 & -3.7372574 & 1.9290175\end{array}$

C $\quad-3.3712368$-3.6098433 1.4437914

$\begin{array}{llll}\text { C } & -3.3362129 & -2.3846589 & 0.4992056\end{array}$

$\begin{array}{llll}\mathrm{N} & -2.2098148 & -1.5993418 & 0.9996276\end{array}$

$\begin{array}{llll}0 & -0.3079874 & -1.8877653 & 2.2996313\end{array}$

$\mathrm{H} \quad-1.8032357 \quad-4.1095344 \quad 2.9679834$

H -1.3094708 -4.4075984 1.2762429

$\begin{array}{llll}\mathrm{H} & -3.7581329 & -4.5200926 & 0.9399851\end{array}$

H $\quad-4.0397431 \quad-3.3934885 \quad 2.3053364$

H $\quad-3.1741269-2.6855894 \quad-0.5661694$

$\begin{array}{llll}\mathrm{H} & -4.2734185 & -1.7852775 & 0.5317232\end{array}$ 


\begin{tabular}{|c|c|c|c|}
\hline & 1.20 & ת & \\
\hline & -1.3027844 & 0.0814770 & -0.7182100 \\
\hline & 6025 & 0.2829387 & 05767772 \\
\hline & -1.46 & 366067 & \\
\hline & 0.5003959 & -0.0875458 & 28238 \\
\hline & -1.6697000 & -0.6208758 & -1.5 \\
\hline & -0.8515433 & 2.6953894 & -1. \\
\hline & 1.27 & 1472 & \\
\hline & 0.97 & -1.8 & \\
\hline & 1.240 & 0.80 & $-2.12^{\prime}$ \\
\hline & 1.2608054 & 2.04 & 1.0 \\
\hline & 0.74 & -0.0 & \\
\hline & 2.31 & & \\
\hline & $2.23 \xi$ & -2.3 & -0.1 \\
\hline & 0.0955204 & -2.4 & -0.5 \\
\hline & 1.0523660 & -2.0 & -1.9 \\
\hline & 2.77 & 0.7 & -2.2 \\
\hline & 0.78 & & -3. \\
\hline & 0.81 & & -2.0 \\
\hline & 1.6421712 & 2.35 & 2.54 \\
\hline & 1.97 & 2.5 & 0.3 \\
\hline & 0.26 & 2.4 & 0.8 \\
\hline & 2.53 & -3.8 & -0.4 \\
\hline & 3.12 & $-1.7 \xi$ & -0.4 \\
\hline & 2.09 & -2.2 & 0.9 \\
\hline & 3.28 & 1.5 & -3.4 \\
\hline & 3.21 & & -1.2 \\
\hline & 3.15 & -0.2 & -2.3 \\
\hline & 1.685 & 3.9 & $2.7 \mathrm{C}$ \\
\hline & 0.9 & & 3.2 \\
\hline & 2.6 & 1.9 & 2.7 \\
\hline & 3.770 & -4.4 & 0.2 \\
\hline & 1.6428502 & -4.4 & -0.20 \\
\hline & 2.6652437 & -4.0 & -1.58 \\
\hline & 4.81 & 1.6 & -3.5 \\
\hline & 2.84 & 76 & -4.3 \\
\hline & 2.901 & & -3.351 \\
\hline & 1.950 & 4.1 & 3.852 \\
\hline & 2.43 & 4.4 & 289 \\
\hline & 0.65 & 4.3 & 2.5 \\
\hline & 3.95 & -5.4 & 0.0 \\
\hline & 4.6861962 & -3.8414688 & -0.0279907 \\
\hline & 3.65 & -4.3 & 1.35 \\
\hline & 5.14 & 2.2 & -4.4264934 \\
\hline & 5.286 & 2.0 & -2.6 \\
\hline & 5.2254270 & 0.5808140 & -3.6642400 \\
\hline & -1.7717780 & 1.8811707 & -1.3539815 \\
\hline & -3.12 & 2.1 & -0.7788852 \\
\hline & -4.2191952 & 6902 & -1.6251113 \\
\hline & -3.5335398 & 0.9936403 & -2.8391995 \\
\hline & -2.1263505 & 1.6031185 & -2.8500652 \\
\hline
\end{tabular}

$\begin{array}{cccc}\mathrm{H} & -4.9175327 & 0.9723765 & -1.0940076 \\ \mathrm{H} & -4.8336176 & 2.5518341 & -1.9326869 \\ \mathrm{H} & -3.4872310 & -0.1096804 & -2.6966727 \\ \mathrm{H} & -4.0983107 & 1.1706979 & -3.7793973 \\ \mathrm{H} & -2.1238346 & 2.6014379 & -3.3455565 \\ \mathrm{H} & -1.3746273 & 0.9715527 & -3.3675653 \\ \mathrm{C} & -3.3187529 & 2.9633840 & 0.2808868 \\ \mathrm{C} & -4.5082858 & 3.3367395 & 0.8276435 \\ \mathrm{H} & -4.5211386 & 4.0561216 & 1.6621415 \\ \mathrm{H} & -5.4799858 & 2.9572987 & 0.4670231 \\ \mathrm{H} & -2.3588032 & 3.3662804 & 0.6533346\end{array}$

\section{TS-S11_cis-H}

$\mathrm{E}(\mathrm{BP} 86 /$ def2-SV(P) $)=-1464.592919298$

C $\quad-1.3898510-2.0658002 \quad 2.0040977$

$\begin{array}{llll}\text { C } & -2.1501514 & -3.3811499 & 2.2339400\end{array}$

$\begin{array}{llll}\text { C } & -3.6129308 & -3.0269304 & 1.9136911\end{array}$

$\begin{array}{llll}\text { C } & -3.4939672 & -1.8327775 & 0.9370795\end{array}$

$\begin{array}{llll}\mathrm{N} & -2.2106455 & -1.2303002 & 1.2900517\end{array}$

$\begin{array}{llll}0 & -0.2352879 & -1.8104585 & 2.3614815\end{array}$

H $\quad-1.9765495 \quad-3.7581741 \quad 3.2629976$

H $-1.7374422 \quad-4.1456962 \quad 1.5344213$

H $\quad-4.1952400 \quad-3.8683942 \quad 1.4833205$

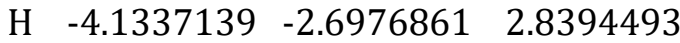

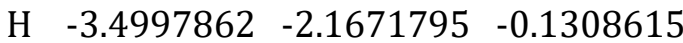

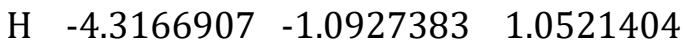

$\begin{array}{llll}\text { C } & -1.8116412 & 0.1031530 & 0.8263861\end{array}$

$\begin{array}{llll}\text { C } & -1.1859131 & 0.2156631 & -0.5636959\end{array}$

$\begin{array}{llll}\mathrm{H} & -2.7279378 & 0.7435549 & 0.7842078\end{array}$

$\begin{array}{llll}\mathrm{H} & -1.1536165 & 0.5342857 & 1.6094038\end{array}$

$\begin{array}{llll}\mathrm{P} & 0.5893092 & -0.0986666 & -0.6358709\end{array}$

$\mathrm{H} \quad-1.6755650 \quad-0.4858796-1.2755266$

$\begin{array}{llll}0 & -0.5955049 & 2.7963484 & -1.0246845\end{array}$

$\begin{array}{llll}\text { C } & 1.4764722 & 0.5030573 & 0.8606146\end{array}$

$\begin{array}{llll}\text { C } & 0.9385324 & -1.9301544 & -0.8088790\end{array}$

$\begin{array}{llll}\text { C } & 1.3187487 & 0.7132857 & -2.1211576\end{array}$

$\begin{array}{llll}\text { C } & 1.5258318 & 2.0305706 & 1.0542565\end{array}$

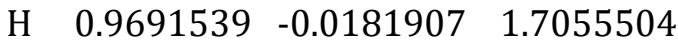

$\begin{array}{llll}\mathrm{H} & 2.5019673 & 0.0732070 & 0.8090775\end{array}$

$\begin{array}{llll}\text { C } & 2.1993342 & -2.4978945 & -0.1347611\end{array}$

H $\quad 0.0416299 \quad-2.4448448-0.4023562$

$\mathrm{H} \quad 0.9496940 \quad-2.1311494 \quad-1.9061716$

$\begin{array}{llll}\text { C } & 2.8442660 & 0.6155293 & -2.2813912\end{array}$

$\begin{array}{llll}\mathrm{H} & 0.8055632 & 0.2688772 & -3.0047200\end{array}$

$\begin{array}{lllll}\mathrm{H} & 0.9508302 & 1.7670167 & -2.0231311\end{array}$

$\begin{array}{llll}\text { C } & 1.9285673 & 2.4144394 & 2.4884862\end{array}$

$\begin{array}{llll}\mathrm{H} & 2.2574121 & 2.4689749 & 0.3361180\end{array}$

$\begin{array}{llll}\mathrm{H} & 0.5515554 & 2.4899367 & 0.7646667\end{array}$

$\begin{array}{llll}\text { C } & 2.3840003 & -4.0014577 & -0.4104206\end{array}$

H $3.1079196 \quad-1.9511464 \quad-0.4788438$

$\begin{array}{llll}\mathrm{H} & 2.1181500 & -2.3380605 & 0.9632562\end{array}$ 


$\begin{array}{cccc}\mathrm{C} & 3.3446582 & 1.3695741 & -3.5278461 \\ \mathrm{H} & 3.3483545 & 1.0363024 & -1.3807220 \\ \mathrm{H} & 3.1677009 & -0.4503978 & -2.3503316 \\ \mathrm{C} & 2.0363403 & 3.9313669 & 2.6924550 \\ \mathrm{H} & 1.1806432 & 1.9944741 & 3.2021091 \\ \mathrm{H} & 2.9005326 & 1.9312747 & 2.7515128 \\ \mathrm{C} & 3.6206999 & -4.5936580 & 0.2781068 \\ \mathrm{H} & 1.4716474 & -4.5460576 & -0.0709215 \\ \mathrm{H} & 2.4505373 & -4.1712433 & -1.5114121 \\ \mathrm{C} & 4.8669201 & 1.3059112 & -3.7061600 \\ \mathrm{H} & 2.8433909 & 0.9517223 & -4.4326424 \\ \mathrm{H} & 3.0154120 & 2.4322413 & -3.4613734 \\ \mathrm{H} & 2.3139972 & 4.1830212 & 3.7403017 \\ \mathrm{H} & 2.8074357 & 4.3763361 & 2.0232371 \\ \mathrm{H} & 1.0710383 & 4.4364675 & 2.4644551 \\ \mathrm{H} & 3.7224851 & -5.6806368 & 0.0644150 \\ \mathrm{H} & 4.5550779 & -4.0951380 & -0.0666200 \\ \mathrm{H} & 3.5648803 & -4.4710989 & 1.3832205 \\ \mathrm{H} & 5.1916932 & 1.8610951 & -4.6137574 \\ \mathrm{H} & 5.3966347 & 1.7515967 & -2.8337720 \\ \mathrm{H} & 5.2219000 & 0.2552049 & -3.8109178 \\ \mathrm{C} & -1.5751959 & 2.0187358 & -1.2820516 \\ \mathrm{~N} & -2.8771539 & 2.3294716 & -0.6550701 \\ \mathrm{C} & -3.9606438 & 2.3677327 & -1.6344649 \\ \mathrm{C} & -3.4717152 & 1.4073719 & -2.7380069 \\ \mathrm{C} & -1.9661675 & 1.6910284 & -2.7680546 \\ \mathrm{H} & -4.9378960 & 2.0507493 & -1.1982592 \\ \mathrm{H} & -4.1120658 & 3.3908613 & -2.0689951 \\ \mathrm{H} & -3.6709678 & 0.3522872 & -2.4388065 \\ \mathrm{H} & -3.9868642 & 1.5801970 & -3.7080933 \\ \mathrm{H} & -1.7560201 & 2.6250945 & -3.3391129 \\ \mathrm{H} & -1.3656833 & 0.8769574 & -3.2244200 \\ \mathrm{H} & -2.7761090 & 3.1699844 & -0.0743244\end{array}$

\section{TS-S11_trans-C2H3}

$\mathrm{E}(\mathrm{BP} 86 / \mathrm{def} 2-\mathrm{SV}(\mathrm{P}))=-1541.935698985$

$\begin{array}{llll}\text { C } & -1.3545739 & -1.8905270 & 2.1106155\end{array}$

$\begin{array}{llll}\text { C } & -1.9162269 & -3.3167138 & 2.2072678\end{array}$

$\begin{array}{llll}\text { C } & -3.3056278 & -3.2191297 & 1.5538857\end{array}$

$\begin{array}{llll}\text { C } & -3.1720371 & -2.0203791 & 0.5840526\end{array}$

N -2.1109928 -1.2116452 1.1812458

$\begin{array}{llll}0 & -0.3836283 & -1.4384995 & 2.7202444\end{array}$

H $-1.9205061 \quad-3.6597942 \quad 3.2622021$

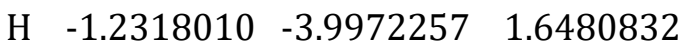

H -3.6239308 -4.1470109 1.0347784

$\mathrm{H} \quad-4.0714825 \quad-2.9878110 \quad 2.3259693$

H $\quad-2.8952396 \quad-2.3484588-0.4485646$

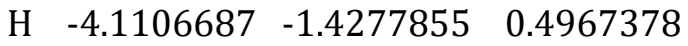

$\begin{array}{llll}\text { C } & -1.8959819 & 0.1945150 & 0.8139993\end{array}$

$\begin{array}{lllll}\text { C } & -1.2390597 & 0.4894222 & -0.5467968\end{array}$

$\begin{array}{llll}\mathrm{H} & -2.9025796 & 0.6639019 & 0.8230178\end{array}$

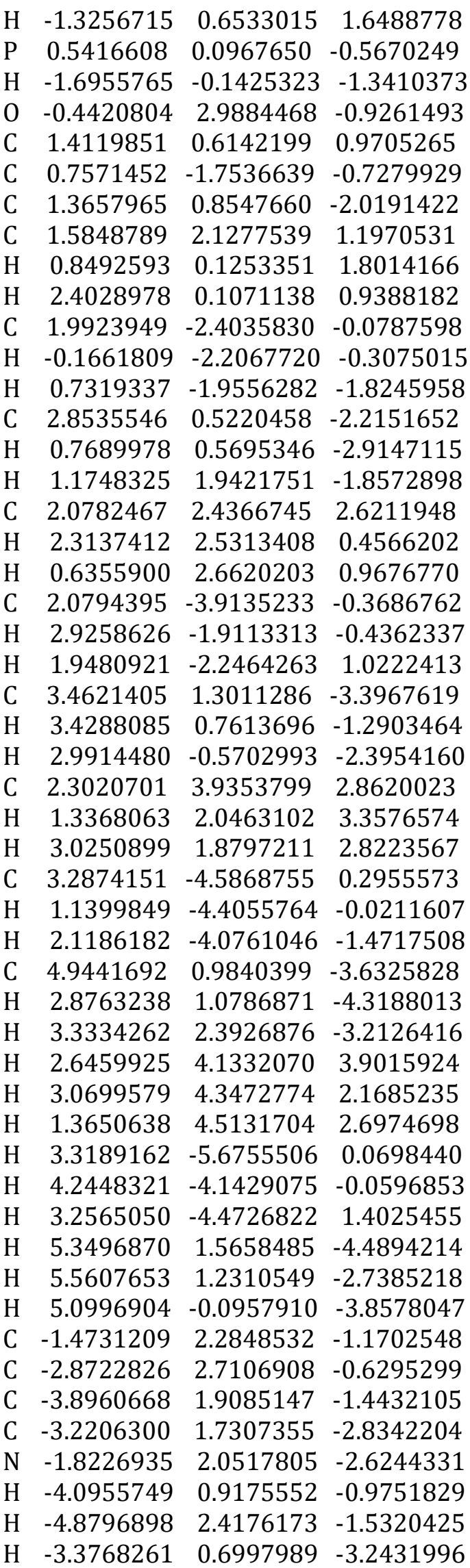

S111 


$\begin{array}{cccc}\mathrm{H} & -3.6449903 & 2.4345594 & -3.5920107 \\ \mathrm{H} & -2.9755633 & 2.6160322 & 0.4713327 \\ \mathrm{H} & -2.9380583 & 3.7969234 & -0.8647940 \\ \mathrm{C} & -1.0231167 & 2.5174124 & -3.6358090 \\ \mathrm{C} & -1.2406381 & 2.4163734 & -4.9787825 \\ \mathrm{H} & -0.5374076 & 2.8925056 & -5.6810838 \\ \mathrm{H} & -2.1107171 & 1.8888814 & -5.4069952 \\ \mathrm{H} & -0.1396981 & 3.0506794 & -3.2388220\end{array}$

\section{TS-S11_trans-H}

\begin{tabular}{lccc}
\multicolumn{4}{l}{ E(BP86/def2-SV(P)) $=-1464.587282257$} \\
C & -1.3279540 & -1.9560292 & 2.2071004 \\
C & -1.8759274 & -3.3867681 & 2.3276030 \\
C & -3.2928322 & -3.2977405 & 1.7350819 \\
C & -3.2071321 & -2.0973783 & 0.7622137 \\
N & -2.1338883 & -1.2792501 & 1.3211915 \\
O & -0.3282235 & -1.5028508 & 2.7686328 \\
H & -1.8320101 & -3.7312440 & 3.3812807 \\
H & -1.2120992 & -4.0619262 & 1.7379111 \\
H & -3.6278639 & -4.2272507 & 1.2291877 \\
H & -4.0259672 & -3.0712326 & 2.5397772 \\
H & -2.9639998 & -2.4221840 & -0.2801597 \\
H & -4.1542005 & -1.5141346 & 0.7088859 \\
C & -1.9239300 & 0.1206408 & 0.9158651 \\
C & -1.2944315 & 0.3792392 & -0.4556089 \\
H & -2.9327498 & 0.5897223 & 0.9298635 \\
H & -1.3414737 & 0.5986983 & 1.7315913 \\
P & 0.4761291 & 0.0302200 & -0.5453798 \\
H & -1.7753421 & -0.2340929 & -1.2459419 \\
O & -0.5701923 & 2.9544610 & -0.8701608 \\
C & 1.3866272 & 0.5716212 & 0.9608068 \\
C & 0.7614706 & -1.8116125 & -0.7237559 \\
C & 1.2157771 & 0.8339273 & -2.0230987 \\
C & 1.5166463 & 2.0912146 & 1.1775777 \\
H & 0.8614213 & 0.0697809 & 1.8085478 \\
H & 2.3912596 & 0.0951546 & 0.9018924 \\
C & 2.0474359 & -2.4178872 & -0.1342671 \\
H & -0.1246751 & -2.2977137 & -0.2608612 \\
H & 0.6905658 & -2.0144014 & -1.8183530 \\
C & 2.7039984 & 0.5583527 & -2.2838876 \\
H & 0.5761662 & 0.5365607 & -2.8835635 \\
H & 0.9880430 & 1.9144214 & -1.8407514 \\
C & 2.0045244 & 2.4251739 & 2.5978580 \\
H & 2.2329196 & 2.5097290 & 0.4328417 \\
H & 0.5520358 & 2.5981923 & 0.9455884 \\
C & 2.1760760 & -3.9237289 & -0.4299121 \\
H & 2.9456311 & -1.8917380 & -0.5318296 \\
H & 2.0479317 & -2.2632867 & 0.9681014 \\
C & 3.2361041 & 1.3617804 & -3.4857880 \\
H & 3.3086374 & 0.8172183 & -1.3831833 \\
H & 2.8771781 & -0.5274909 & -2.4738374 \\
& & &
\end{tabular}

$\begin{array}{cccc}\mathrm{C} & 2.1908950 & 3.9310654 & 2.8255429 \\ \mathrm{H} & 1.2740742 & 2.0229445 & 3.3389259 \\ \mathrm{H} & 2.9653426 & 1.8938729 & 2.8028698 \\ \mathrm{C} & 3.4356473 & -4.5537955 & 0.1788362 \\ \mathrm{H} & 1.2711665 & -4.4490122 & -0.0420812 \\ \mathrm{H} & 2.1722532 & -4.0847879 & -1.5340081 \\ \mathrm{C} & 4.7218592 & 1.1123873 & -3.7744564 \\ \mathrm{H} & 2.6304647 & 1.1094946 & -4.3878085 \\ \mathrm{H} & 3.0643973 & 2.4469130 & -3.2979051 \\ \mathrm{H} & 2.5303870 & 4.1472817 & 3.8630708 \\ \mathrm{H} & 2.9477774 & 4.3559401 & 2.1276193 \\ \mathrm{H} & 1.2394912 & 4.4833524 & 2.6560369 \\ \mathrm{H} & 3.4965875 & -5.6406790 & -0.0500067 \\ \mathrm{H} & 4.3600614 & -4.0753113 & -0.2169554 \\ \mathrm{H} & 3.4494425 & -4.4412391 & 1.2863834 \\ \mathrm{H} & 5.0708057 & 1.7092043 & -4.6459577 \\ \mathrm{H} & 5.3570802 & 1.3900541 & -2.9027841 \\ \mathrm{H} & 4.9186180 & 0.0398913 & -4.0024970 \\ \mathrm{C} & -1.5471717 & 2.2026083 & -1.1824985 \\ \mathrm{C} & -2.9967208 & 2.5721913 & -0.7396182 \\ \mathrm{C} & -3.9098293 & 1.8001036 & -1.6924603 \\ \mathrm{C} & -3.1168298 & 1.8415675 & -3.0252107 \\ \mathrm{~N} & -1.7019492 & 1.8551691 & -2.6326875 \\ \mathrm{H} & -4.0415758 & 0.7470714 & -1.3506153 \\ \mathrm{H} & -4.9279806 & 2.2340822 & -1.8005143 \\ \mathrm{H} & -3.3695123 & 0.9716122 & -3.6792909 \\ \mathrm{H} & -3.3991759 & 2.7612269 & -3.5965966 \\ \mathrm{H} & -3.1997754 & 2.4214942 & 0.3406213 \\ \mathrm{H} & -3.0723880 & 3.6685008 & -0.9273521 \\ \mathrm{H} & -1.1417281 & 2.5343282 & -3.1617620\end{array}$

TS-S12_cis-C2H3

$\mathrm{E}(\mathrm{BP} 86 /$ def2-SV(P)) $=-1541.928842966$

C $\quad-1.8711658 \quad-3.4404078 \quad 1.7474040$

$\begin{array}{llll}\text { C } & -3.0687358 & -4.3937369 & 1.8919160\end{array}$

$\begin{array}{llll}\text { C } & -4.2766571 & -3.4568646 & 2.0682125\end{array}$

$\begin{array}{llll}\text { C } & -3.8274296 & -2.1480717 & 1.3750195\end{array}$

N $-2.3770686 \quad-2.1805304 \quad 1.5096559$

$\begin{array}{llll}0 & -0.6773821 & -3.7364829 & 1.7990509\end{array}$

H $\quad-2.9052942 \quad-5.1053466 \quad 2.7275225$

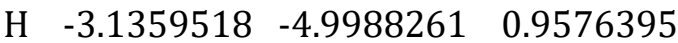

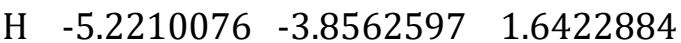

H $\quad-4.4519838$-3.2625978 3.1491277

H $\quad-4.1293415 \quad-2.1234658 \quad 0.2972819$

$\begin{array}{llll}\mathrm{H} & -4.2494280 & -1.2357816 & 1.8535007\end{array}$

$\begin{array}{llll}\text { C } & -1.5034773 & -1.0547686 & 1.1507491\end{array}$

$\begin{array}{llll}\text { C } & -1.3203674 & -0.8331469 & -0.3432483\end{array}$

H $-1.8882072 \quad-0.1589127 \quad 1.6824727$

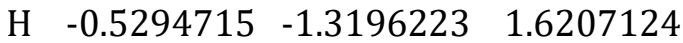

P $\quad 0.5169241 \quad 0.3749608-1.3923006$

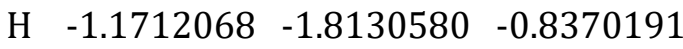




\begin{tabular}{|c|c|c|c|}
\hline & 4 & 4 & -2.0531943 \\
\hline & 1.1860966 & 4084 & \\
\hline & 1.2004760 & -1.3162096 & -1.8384045 \\
\hline & 1.6125338 & 1.4170227 & -2.5 \\
\hline & 1.0180018 & 2.4686173 & 792 \\
\hline & 0.7121950 & 0.3737399 & $1.06^{\prime}$ \\
\hline & 2.2684784 & 0.6923858 & 0.2568424 \\
\hline & 1.6708423 & -2.2134844 & -0.6831644 \\
\hline & 0.3830784 & -1.812 & -2. \\
\hline & 2.0338917 & -1.1630970 & -2.5 \\
\hline & 3.1338459 & 1.4215585 & -2.3259052 \\
\hline & 1.3744303 & 1.0413179 & -3.5675198 \\
\hline & 1.213 & 731 & -2.5 \\
\hline & 1.4586191 & 2.88 & 1.9 \\
\hline & 1.6068346 & 116 & 776 \\
\hline & -0.0428336 & 2.767 & 0.3422135 \\
\hline & 2.0782436 & -3.6229298 & -1.1525375 \\
\hline & 2.53 & -1.73 & -0.1 \\
\hline & 0.87 & -2.31 & \\
\hline & 3.9000569 & 2.151 & 369 \\
\hline & 3.3814812 & 1.90 & -1.3 \\
\hline & 3.5263 & 0.37 & -2.2 \\
\hline & 1.31 & 4.39 & 24 \\
\hline & 0.8 & 2.3 & 452 \\
\hline & 2.5186949 & 2.58 & 178 \\
\hline & 2.4884576 & -4.5378964 & 0.0 \\
\hline & 1.21 & -4.08 & -1.6 \\
\hline & 2.9 & -3.54 & -1.8 \\
\hline & 5.4181305 & 2.18 & -3.2 \\
\hline & 3.6751554 & 1.6 & -4.4 \\
\hline & 3.5082353 & 3.1926307 & 2049 \\
\hline & 1.63 & 4.6 & 3.2 \\
\hline & 1.93 & 4.98 & 03 \\
\hline & 0.2607169 & 4.72 & 762 \\
\hline & 2.7656350 & -5.5531279 & 7550 \\
\hline & 286 & 4089 & 51 \\
\hline & 1.6 & -4.643 & 24 \\
\hline & 5.9370088 & 2.7210305 & -4.0545402 \\
\hline & 5.6784413 & 2.7133498 & -2.2776571 \\
\hline & 5.8455726 & 1.1629957 & -3.1663436 \\
\hline & -2.1743831 & 0.00 & -1.1 \\
\hline & -3.0435408 & 1.0051122 & -0.7179076 \\
\hline & -4.0897300 & 1.3483742 & -1.6968036 \\
\hline & -3.6637061 & 0.6064052 & -2.9751341 \\
\hline & -2.8608544 & -0.5762359 & -2.4 \\
\hline & -5.0886902 & 1.0148583 & -1.3243764 \\
\hline & -4.1336048 & 2.4530367 & -1.8269667 \\
\hline & -4.5284360 & 0.3065046 & -3.6036350 \\
\hline & -2.9904 & 1.2523202 & -3.576222 \\
\hline & -2.1288682 & -1.0049637 & -3.131457 \\
\hline & -3.5387836 & -1.398929 & 0 \\
\hline
\end{tabular}

$\begin{array}{llll}\mathrm{C} & -2.8126410 & 1.8285653 & 0.3741361 \\ \mathrm{C} & -3.6397415 & 2.7970475 & 0.8445962 \\ \mathrm{H} & -3.3214313 & 3.4047364 & 1.7061168 \\ \mathrm{H} & -4.6316756 & 3.0065882 & 0.4115143 \\ \mathrm{H} & -1.8370297 & 1.6628023 & 0.8543885\end{array}$

\section{TS-S12_cis-H}

$\mathrm{E}(\mathrm{BP} 86 /$ def2-SV(P) $)=-1464.589860300$

$\begin{array}{llll}\text { C } & -0.7523950 & -1.8106203 & 2.6269015\end{array}$

$\begin{array}{llll}\text { C } & -0.8625343 & -3.2726022 & 3.0875958\end{array}$

$\begin{array}{llll}\text { C } & -2.2780574 & -3.6934240 & 2.6579221\end{array}$

$\begin{array}{llll}\text { C } & -2.5807806 & -2.7732712 & 1.4507392\end{array}$

$\begin{array}{llll}\mathrm{N} & -1.7509456 & -1.5991221 & 1.7003193\end{array}$

$\begin{array}{llll}0 & 0.0817964 & -0.9839415 & 2.9941390\end{array}$

H $\quad-0.6616989-3.3532847 \quad 4.1755798$

$\begin{array}{llll}\mathrm{H} & -0.0712812 & -3.8584504 & 2.5639800\end{array}$

$\begin{array}{llll}\mathrm{H} & -2.3693820 & -4.7695093 & 2.4007917\end{array}$

$\mathrm{H} \quad-3.0002845 \quad-3.4816258 \quad 3.4767755$

H $-2.3125023 \quad-3.26175890 .4816909$

$\begin{array}{llll}\mathrm{H} & -3.6543597 & -2.4836218 & 1.3849556\end{array}$

$\begin{array}{llll}\text { C } & -1.9740314 & -0.3218087 & 1.0349468\end{array}$

$\begin{array}{llll}\text { C } & -1.6632213 & -0.3312259 & -0.4665878\end{array}$

$\begin{array}{llll}\mathrm{H} & -3.0437989 & -0.0345774 & 1.2234847\end{array}$

$\begin{array}{llll}\mathrm{H} & -1.3516359 & 0.4064211 & 1.6037885\end{array}$

P $\quad 0.3918034 \quad 0.4588273 \quad-1.4719985$

$\begin{array}{llll}\mathrm{H} & -1.8557195 & -1.3294894 & -0.9099438\end{array}$

$\begin{array}{lllll}0 & -0.7526625 & 1.3987413 & -2.0892932\end{array}$

$\begin{array}{llll}\text { C } & 1.1341412 & 0.7402474 & 0.2148097\end{array}$

$\begin{array}{llll}\text { C } & 0.6396906 & -1.2983875 & -2.0805302\end{array}$

$\begin{array}{llll}\text { C } & 1.7413295 & 1.2998624 & -2.5281080\end{array}$

$\begin{array}{llll}\text { C } & 0.8666940 & 2.1580676 & 0.7509184\end{array}$

$\begin{array}{llll}\mathrm{H} & 0.7549892 & -0.0111713 & 0.9370096\end{array}$

$\begin{array}{llll}\mathrm{H} & 2.2330009 & 0.5817652 & 0.1219749\end{array}$

$\begin{array}{llll}\text { C } & 1.0409859 & -2.3270867 & -1.0146983\end{array}$

$\mathrm{H} \quad-0.3210026-1.5888842 \quad-2.5596900$

$\begin{array}{llll}\mathrm{H} & 1.4033408 & -1.2772146 & -2.8914194\end{array}$

$\begin{array}{llll}\text { C } & 3.2078660 & 0.8649461 & -2.3756090\end{array}$

$\mathrm{H} \quad 1.4053470 \quad 1.1454618-3.5794640$

$\begin{array}{llll}\mathrm{H} & 1.6461702 & 2.3942849 & -2.3483934\end{array}$

C $\quad 1.4730839 \quad 2.3781018 \quad 2.1485238$

H $\quad 1.2636653 \quad 2.9231217 \quad 0.0431010$

$\begin{array}{llll}\mathrm{H} & -0.2326595 & 2.3389904 & 0.7948749\end{array}$

C $\quad 1.1172649 \quad-3.7619936-1.5685282$

H $\quad 2.0306913 \quad-2.0525772 \quad-0.5807180$

H $\quad 0.3119723 \quad-2.2876198 \quad-0.1730834$

C $\quad 4.1343194 \quad 1.4832596 \quad-3.4404604$

H $3.5930768 \quad 1.1484566-1.3679742$

$\begin{array}{llll}\mathrm{H} & 3.2993277 & -0.2469738 & -2.4303638\end{array}$

$\begin{array}{llll}\text { C } & 1.1790698 & 3.7685489 & 2.7261667\end{array}$

$\begin{array}{llll}\mathrm{H} & 1.0880851 & 1.5880132 & 2.8325291\end{array}$

$\begin{array}{llll}\mathrm{H} & 2.5766788 & 2.2237803 & 2.0926506\end{array}$ 


$\begin{array}{lccc}\mathrm{C} & 1.5795161 & -4.7936203 & -0.5310709 \\ \mathrm{H} & 0.1165786 & -4.0532352 & -1.9676275 \\ \mathrm{H} & 1.8092372 & -3.7832950 & -2.4436176 \\ \mathrm{C} & 5.6069130 & 1.0823848 & -3.2810846 \\ \mathrm{H} & 3.7732216 & 1.1869241 & -4.4537335 \\ \mathrm{H} & 4.0423421 & 2.5944433 & -3.4007650 \\ \mathrm{H} & 1.6354753 & 3.8943462 & 3.7331393 \\ \mathrm{H} & 1.5788588 & 4.5775755 & 2.0725669 \\ \mathrm{H} & 0.0833824 & 3.9398336 & 2.8342823 \\ \mathrm{H} & 1.6112556 & -5.8193468 & -0.9610209 \\ \mathrm{H} & 2.5988519 & -4.5566428 & -0.1506597 \\ \mathrm{H} & 0.8965037 & -4.8191634 & 0.3486415 \\ \mathrm{H} & 6.2439561 & 1.5447095 & -4.0677382 \\ \mathrm{H} & 6.0098762 & 1.4018645 & -2.2929515 \\ \mathrm{H} & 5.7369673 & -0.0218733 & -3.3506825 \\ \mathrm{C} & -2.3051598 & 0.7125900 & -1.2603093 \\ \mathrm{~N} & -2.9147396 & 1.8016701 & -0.6721315 \\ \mathrm{C} & -3.5651505 & 2.6927472 & -1.6405538 \\ \mathrm{C} & -3.4936115 & 1.8992099 & -2.9739897 \\ \mathrm{C} & -3.1000150 & 0.4720688 & -2.5396712 \\ \mathrm{H} & -4.6134058 & 2.9047080 & -1.3236626 \\ \mathrm{H} & -3.0346157 & 3.6697156 & -1.7184537 \\ \mathrm{H} & -4.4472637 & 1.9374775 & -3.5413358 \\ \mathrm{H} & -2.6874887 & 2.3156877 & -3.6109404 \\ \mathrm{H} & -2.5142163 & -0.0767927 & -3.3025080 \\ \mathrm{H} & -3.9962844 & -0.1403698 & -2.2812844 \\ \mathrm{H} & -2.5010222 & 2.2091275 & 0.1695571\end{array}$

\section{TS-S12_trans-C2H3}

\begin{tabular}{cccc}
\multicolumn{4}{c}{ E(BP86/def2-SV(P) $=-1541.930735321$} \\
C & -0.9385149 & -1.7356205 & 2.4764291 \\
C & -1.0694549 & -3.2185808 & 2.8579799 \\
C & -2.4341810 & -3.6321449 & 2.2821235 \\
C & -2.6357114 & -2.6616311 & 1.0935763 \\
N & -1.8473857 & -1.4918271 & 1.4690360 \\
O & -0.1554186 & -0.9186787 & 2.9591050 \\
H & -0.9675008 & -3.3471218 & 3.9550652 \\
H & -0.2259447 & -3.7721922 & 2.3832613 \\
H & -2.4883628 & -4.6963909 & 1.9711004 \\
H & -3.2310392 & -3.4627014 & 3.0392447 \\
H & -2.2726168 & -3.1054520 & 0.1338612 \\
H & -3.7021010 & -2.3787615 & 0.9402637 \\
C & -2.0432942 & -0.1845954 & 0.8517486 \\
C & -1.6126807 & -0.1258824 & -0.6177461 \\
H & -3.1316083 & 0.0630972 & 0.9851225 \\
H & -1.4842399 & 0.5318039 & 1.4943858 \\
P & 0.6142864 & 0.7078840 & -1.3685582 \\
H & -1.7792793 & -1.0982860 & -1.1191516 \\
O & -0.4832940 & 1.7275748 & -1.9483815 \\
C & 1.2234289 & 0.7819511 & 0.3851754 \\
C & 0.8502455 & -0.9936595 & -2.1171334
\end{tabular}

\begin{tabular}{|c|c|c|c|}
\hline & 81 & & \\
\hline & & & \\
\hline & & 39 & \\
\hline & 212 & & \\
\hline & & 246 & \\
\hline & 3391 & 1774 & -2 \\
\hline & & & -2 \\
\hline & & & \\
\hline & $1.7 \xi$ & & -3.3 \\
\hline & 1.922 & 3852 & -2. \\
\hline & & & \\
\hline & & & \\
\hline & & & \\
\hline & 70 & -3.5 & -1. \\
\hline & 267 & -1. & -0 \\
\hline & 0.2 & -2 & -0 \\
\hline & 4.4 & & \\
\hline & 3.7 & & -0 . \\
\hline & 3.5 & & \\
\hline & 1.5 & & \\
\hline & & & \\
\hline & & & \\
\hline & & -4.6 & -0 . \\
\hline & 21 & -3 & -2 \\
\hline & & & -2 \\
\hline & 5.9 & & \\
\hline & & & 319 \\
\hline & & & -2.8 \\
\hline & 1.9 & & 4. \\
\hline & & & \\
\hline & 0.4 & & \\
\hline & & & -1 . \\
\hline & 2.4 & -4. & 347 \\
\hline & & & -0 . \\
\hline & & & \\
\hline & & & 146 \\
\hline & & & 887 \\
\hline & -2.08 & & 240 \\
\hline & -2.6 & & 903 \\
\hline & -2.9 & & 971 \\
\hline & -3.2 & & -3.0 \\
\hline & -2.7 & $0 . \varepsilon$ & 898 \\
\hline & -3.7 & & -1 . \\
\hline & & & -2.2 \\
\hline H & -4.3 & & -3.2 \\
\hline$\pi$ & -2.80 & 6819 & 0954 \\
\hline & -3.6 & & -0.2 \\
\hline & -1.9759181 & & -0.0 \\
\hline 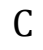 & -2.8 & -0.2 & -3. \\
\hline 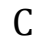 & -3.3737831 & -0.2986166 & -4.6619899 \\
\hline & -3.4158074 & -1.2725526 & -5.174531 \\
\hline
\end{tabular}


H $\quad-3.7605944 \quad 0.5796863 \quad-5.2040881$

H $\quad-2.4906711 \quad-1.1473044 \quad-2.9170727$

\section{TS-S12_trans-H}

$\mathrm{E}(\mathrm{BP} 86 /$ def2-SV(P)) $=-1464.587323890$

$\begin{array}{llll}C & -0.7845813 & -1.6746750 & 2.7363722\end{array}$

$\begin{array}{llll}\text { C } & -0.7707868 & -3.1326737 & 3.2225848\end{array}$

C $-2.1119228 \quad-3.7015109 \quad 2.7300084$

C $-2.4377658-2.8396758 \quad 1.4862046$

$\begin{array}{llll}\mathrm{N} & -1.7370415 & -1.5853782 & 1.7431524\end{array}$

$\begin{array}{llll}0 & -0.0674833 & -0.7593827 & 3.1369577\end{array}$

$\mathrm{H} \quad-0.6219880 \quad-3.1734844 \quad 4.3210777$

$\mathrm{H} \quad 0.1020576 \quad-3.6443510 \quad 2.7538530$

$\mathrm{H} \quad-2.0822705 \quad-4.7860904 \quad 2.4949216$

H $\quad-2.8950893 \quad-3.5474790 \quad 3.5045355$

H $\quad-2.0755085 \quad-3.3217173 \quad 0.5448118$

H $\quad-3.5294032 \quad-2.6560355 \quad 1.3602810$

C $-2.0717689-0.3470667 \quad 1.0476120$

$\begin{array}{llll}\text { C } & -1.7484707 & -0.3688701 & -0.4512175\end{array}$

H $\quad-3.1654379 \quad-0.1681024 \quad 1.2363026$

$\begin{array}{llll}\mathrm{H} & -1.5283539 & 0.4556500 & 1.5937357\end{array}$

P $\quad 0.3157151 \quad 0.4932686-1.4100393$

H $\quad-1.9439372 \quad-1.3764920-0.8776296$

$\begin{array}{lllll}0 & -0.8338348 & 1.4480023 & -1.9874916\end{array}$

$\begin{array}{llll}\text { C } & 1.0619419 & 0.7241291 & 0.2801005\end{array}$

$\begin{array}{llll}\text { C } & 0.5795020 & -1.2476924 & -2.0681542\end{array}$

C $\quad 1.6573779 \quad 1.3668331 \quad-2.4480574$

C $\quad 0.8885117 \quad 2.1531303 \quad 0.8255650$

$\mathrm{H} \quad 0.6365674 \quad-0.0020104 \quad 1.0005117$

H $\quad 2.1473793 \quad 0.4921924 \quad 0.1815648$

C $\quad 0.9787606 \quad-2.3004723-1.0255376$

H $\quad-0.3768998 \quad-1.5309317 \quad-2.5603707$

H $\quad 1.3471526 \quad-1.2030525 \quad-2.8744854$

$\begin{array}{llll}\text { C } & 3.1289251 & 0.9515119 & -2.2912347\end{array}$

H $\quad 1.3285259 \quad 1.2181292 \quad-3.5027315$

$\mathrm{H} \quad 1.5448700 \quad 2.4574739-2.2580519$

$\begin{array}{llll}\text { C } & 1.5500614 & 2.3327600 & 2.2041938\end{array}$

$\mathrm{H} \quad 1.3069415 \quad 2.8978784 \quad 0.1085285$

$\mathrm{H} \quad-0.1973065 \quad 2.3905300 \quad 0.9067623$

C $\quad 1.0268451-3.7278241-1.6010625$

H $1.9783786-2.0465253-0.6017731$

H $\quad 0.2618135 \quad-2.2628217 \quad-0.1733630$

C $4.0502449 \quad 1.5829249 \quad-3.3528285$

H $\quad 3.5050191 \quad 1.2411683 \quad-1.2819982$

$\mathrm{H} \quad 3.2362301-0.1591532 \quad-2.3452770$

$\begin{array}{llll}\text { C } & 1.3520668 & 3.7359842 & 2.7920548\end{array}$

$\begin{array}{llll}\mathrm{H} & 1.1393860 & 1.5643447 & 2.8971117\end{array}$

H $\quad 2.6414297 \quad 2.1173361 \quad 2.1142022$

C $\quad 1.4820679-4.7814232-0.5826560$

H $\quad 0.0172730 \quad-3.9973360 \quad-1.9932329$

H $\quad 1.7092955 \quad-3.7475931 \quad-2.4836324$

$\begin{array}{lrrr}\mathrm{C} & 5.5286092 & 1.2073974 & -3.1857191 \\ \mathrm{H} & 3.6989432 & 1.2787406 & -4.3672754 \\ \mathrm{H} & 3.9393549 & 2.6924262 & -3.3156317 \\ \mathrm{H} & 1.8449520 & 3.8327357 & 3.7850188 \\ \mathrm{H} & 1.7767704 & 4.5235042 & 2.1277892 \\ \mathrm{H} & 0.2714457 & 3.9672382 & 2.9327447 \\ \mathrm{H} & 1.4953457 & -5.8010689 & -1.0277425 \\ \mathrm{H} & 2.5079433 & -4.5651507 & -0.2074165 \\ \mathrm{H} & 0.8053742 & -4.8099680 & 0.3018226 \\ \mathrm{H} & 6.1613116 & 1.6788694 & -3.9704403 \\ \mathrm{H} & 5.9213643 & 1.5359717 & -2.1965067 \\ \mathrm{H} & 5.6778407 & 0.1053666 & -3.2522654 \\ \mathrm{C} & -2.3929849 & 0.6618528 & -1.2578836 \\ \mathrm{C} & -3.0212023 & 1.9435444 & -0.7272102 \\ \mathrm{C} & -3.4829578 & 2.6664449 & -2.0142171 \\ \mathrm{C} & -3.6498223 & 1.5318663 & -3.0669204 \\ \mathrm{~N} & -3.1462539 & 0.3435763 & -2.3715465 \\ \mathrm{H} & -4.4185589 & 3.2451150 & -1.8659955 \\ \mathrm{H} & -2.6916261 & 3.3679119 & -2.3452251 \\ \mathrm{H} & -4.7101448 & 1.3773207 & -3.3734399 \\ \mathrm{H} & -3.0577762 & 1.7503354 & -3.9863930 \\ \mathrm{H} & -3.8885682 & 1.6685653 & -0.0823208 \\ \mathrm{H} & -2.3171949 & 2.5416728 & -0.1176428 \\ \mathrm{H} & -2.8697546 & -0.4706949 & -2.9268640\end{array}$

\section{TS-S13}

$\mathrm{E}(\mathrm{BP} 86 /$ def2-SV(P)) $=-968.9375169005$

$\begin{array}{llll}\text { C } & 2.5968884 & -0.6338122 & 2.6138301\end{array}$

$\begin{array}{llll}\text { C } & 2.1549627 & -1.8283760 & 3.0608911\end{array}$

$\begin{array}{llll}\mathrm{P} & 2.9767860 & -3.0341738 & 4.0699768\end{array}$

H $1.1026909-2.0629625 \quad 2.7304491$

C $\quad 1.9711412 \quad-3.2607159 \quad 5.6071599$

$\begin{array}{llll}\text { C } & 3.0329104 & -4.6168604 & 3.1128605\end{array}$

$\begin{array}{llll}\text { C } & 4.6776928 & -2.4578987 & 4.4886354\end{array}$

$\begin{array}{llll}\text { C } & 0.0697097 & -0.3679867 & 1.3659756\end{array}$

C $-1.0723755-0.3512462 \quad 0.8645770$

$\mathrm{H} \quad 3.6047316 \quad-0.2212290 \quad 2.8293474$

$\mathrm{H} \quad-2.0629180-0.3171914 \quad 0.4188608$

$\mathrm{H} \quad 1.6631753 \quad-0.1873879 \quad 1.9672462$

C $\quad 2.6164857 \quad-3.9258257 \quad 6.8363490$

H $1.6285996-2.2306886 \quad 5.8581515$

H $\quad 1.0564841 \quad-3.8102158 \quad 5.2833571$

C $\quad 5.7206222 \quad-3.5051582 \quad 4.9217378$

H $\quad 5.0220555-1.92714553 .5715586$

$\mathrm{H} \quad 4.5502014 \quad-1.6699449 \quad 5.2662398$

C $\quad 3.2037194 \quad-5.9515408 \quad 3.8599615$

H $\quad 2.0759385 \quad-4.6142278 \quad 2.5409825$

H $\quad 3.8337966 \quad-4.4720850 \quad 2.3509777$

C $\quad 1.6277170 \quad-4.0505144 \quad 8.0110976$

H $\quad 3.0062291 \quad-4.9358219 \quad 6.5768620$

H $3.4959406 \quad-3.3259151 \quad 7.1664332$ 


$\begin{array}{llll}\mathrm{C} & 7.0845003 & -2.8642741 & 5.2400376 \\ \mathrm{H} & 5.3631933 & -4.0678926 & 5.8135547 \\ \mathrm{H} & 5.8546047 & -4.2567015 & 4.1100133 \\ \mathrm{C} & 3.2381444 & -7.1524242 & 2.8955936 \\ \mathrm{H} & 4.1360831 & -5.9438163 & 4.4688221 \\ \mathrm{H} & 2.3617740 & -6.0882959 & 4.5769538 \\ \mathrm{C} & 2.2533011 & -4.6816700 & 9.2613487 \\ \mathrm{H} & 1.2276734 & -3.0398920 & 8.2595435 \\ \mathrm{H} & 0.7485053 & -4.6540718 & 7.6850593 \\ \mathrm{C} & 8.1508746 & -3.8881966 & 5.6491731 \\ \mathrm{H} & 7.4348136 & -2.2918783 & 4.3494666 \\ \mathrm{H} & 6.9536154 & -2.1125660 & 6.0534180 \\ \mathrm{C} & 3.3786504 & -8.4998140 & 3.6150701 \\ \mathrm{H} & 2.3097172 & -7.1504766 & 2.2782734 \\ \mathrm{H} & 4.0813208 & -7.0198842 & 2.1777944 \\ \mathrm{H} & 1.5114208 & -4.7581745 & 10.0858881 \\ \mathrm{H} & 2.6310711 & -5.7084807 & 9.0540809 \\ \mathrm{H} & 3.1101238 & -4.0779407 & 9.6372244 \\ \mathrm{H} & 9.1196373 & -3.3907828 & 5.8730472 \\ \mathrm{H} & 7.8457286 & -4.4526973 & 6.5591736 \\ \mathrm{H} & 8.3348966 & -4.6300809 & 4.8395017 \\ \mathrm{H} & 3.4022533 & -9.3415060 & 2.8890649 \\ \mathrm{H} & 4.3166613 & -8.5468102 & 4.2134531 \\ \mathrm{H} & 2.5274503 & -8.6811486 & 4.3096260\end{array}$

\section{TS-S13_NVP}

\begin{tabular}{lccc}
\multicolumn{4}{c}{ E(BP86/def2-SV(P) $=-968.9375169005$} \\
C & 2.5968884 & -0.6338122 & 2.6138301 \\
C & 2.1549627 & -1.8283760 & 3.0608911 \\
P & 2.9767860 & -3.0341738 & 4.0699768 \\
H & 1.1026909 & -2.0629625 & 2.7304491 \\
C & 1.9711412 & -3.2607159 & 5.6071599 \\
C & 3.0329104 & -4.6168604 & 3.1128605 \\
C & 4.6776928 & -2.4578987 & 4.4886354 \\
C & 0.0697097 & -0.3679867 & 1.3659756 \\
C & -1.0723755 & -0.3512462 & 0.8645770 \\
H & 3.6047316 & -0.2212290 & 2.8293474 \\
H & -2.0629180 & -0.3171914 & 0.4188608 \\
H & 1.6631753 & -0.1873879 & 1.9672462 \\
C & 2.6164857 & -3.9258257 & 6.8363490 \\
H & 1.6285996 & -2.2306886 & 5.8581515 \\
H & 1.0564841 & -3.8102158 & 5.2833571 \\
C & 5.7206222 & -3.5051582 & 4.9217378 \\
H & 5.0220555 & -1.9271455 & 3.5715586 \\
H & 4.5502014 & -1.6699449 & 5.2662398 \\
C & 3.2037194 & -5.9515408 & 3.8599615 \\
H & 2.0759385 & -4.6142278 & 2.5409825 \\
H & 3.8337966 & -4.4720850 & 2.3509777 \\
C & 1.6277170 & -4.0505144 & 8.0110976 \\
H & 3.0062291 & -4.9358219 & 6.5768620 \\
H & 3.4959406 & -3.3259151 & 7.1664332
\end{tabular}

$\begin{array}{cccc}\mathrm{C} & 7.0845003 & -2.8642741 & 5.2400376 \\ \mathrm{H} & 5.3631933 & -4.0678926 & 5.8135547 \\ \mathrm{H} & 5.8546047 & -4.2567015 & 4.1100133 \\ \mathrm{C} & 3.2381444 & -7.1524242 & 2.8955936 \\ \mathrm{H} & 4.1360831 & -5.9438163 & 4.4688221 \\ \mathrm{H} & 2.3617740 & -6.0882959 & 4.5769538 \\ \mathrm{C} & 2.2533011 & -4.6816700 & 9.2613487 \\ \mathrm{H} & 1.2276734 & -3.0398920 & 8.2595435 \\ \mathrm{H} & 0.7485053 & -4.6540718 & 7.6850593 \\ \mathrm{C} & 8.1508746 & -3.8881966 & 5.6491731 \\ \mathrm{H} & 7.4348136 & -2.2918783 & 4.3494666 \\ \mathrm{H} & 6.9536154 & -2.1125660 & 6.0534180 \\ \mathrm{C} & 3.3786504 & -8.4998140 & 3.6150701 \\ \mathrm{H} & 2.3097172 & -7.1504766 & 2.2782734 \\ \mathrm{H} & 4.0813208 & -7.0198842 & 2.1777944 \\ \mathrm{H} & 1.5114208 & -4.7581745 & 10.0858881 \\ \mathrm{H} & 2.6310711 & -5.7084807 & 9.0540809 \\ \mathrm{H} & 3.1101238 & -4.0779407 & 9.6372244 \\ \mathrm{H} & 9.1196373 & -3.3907828 & 5.8730472 \\ \mathrm{H} & 7.8457286 & -4.4526973 & 6.5591736 \\ \mathrm{H} & 8.3348966 & -4.6300809 & 4.8395017 \\ \mathrm{H} & 3.4022533 & -9.3415060 & 2.8890649 \\ \mathrm{H} & 4.3166613 & -8.5468102 & 4.2134531 \\ \mathrm{H} & 2.5274503 & -8.6811486 & 4.3096260\end{array}$

\section{TS-S14}

\begin{tabular}{cccc}
\multicolumn{4}{c}{ E(BP86/def2-SV(P) $=-968.9652173807$} \\
C & -2.6490352 & 0.9192223 & -3.3788992 \\
C & -2.1575374 & 1.3030062 & -2.1848733 \\
P & -1.0332165 & 0.3539392 & -1.1787395 \\
H & -2.3346205 & 2.3971817 & -1.5155215 \\
C & -1.9361970 & 0.1367717 & 0.4133519 \\
C & -0.5342295 & -1.2848265 & -1.8888284 \\
C & 0.4162597 & 1.4710361 & -0.9561185 \\
C & -2.2325195 & 3.5419629 & -0.4332517 \\
C & -1.9098618 & 4.2044988 & 0.5739181 \\
H & -2.4196392 & -0.0426317 & -3.8882635 \\
H & -1.6787090 & 4.8389069 & 1.4255232 \\
H & -3.3409692 & 1.5888473 & -3.9295339 \\
C & -1.3657711 & -0.8141570 & 1.4783927 \\
H & -2.0499997 & 1.1877417 & 0.7831005 \\
H & -2.9583337 & -0.1863998 & 0.1089628 \\
C & 1.3314073 & 1.2889030 & 0.2665401 \\
H & 0.9879822 & 1.4242485 & -1.9124732 \\
H & -0.0750807 & 2.4763481 & -0.9030456 \\
C & 0.7578699 & -1.9430823 & -1.3695298 \\
H & -1.4102756 & -1.9598300 & -1.7480184 \\
H & -0.4529607 & -1.1103030 & -2.9871364 \\
C & -2.2314877 & -0.8328095 & 2.7523071 \\
H & -1.3013825 & -1.8504565 & 1.0704285 \\
H & -0.3269959 & -0.5196927 & 1.7485548
\end{tabular} 


$\begin{array}{cccc}\mathrm{C} & 2.5029017 & 2.2882659 & 0.2485130 \\ \mathrm{H} & 0.7330240 & 1.4583020 & 1.1901487 \\ \mathrm{H} & 1.7335183 & 0.2513779 & 0.3277793 \\ \mathrm{C} & 1.0392299 & -3.2975077 & -2.0460275 \\ \mathrm{H} & 1.6201928 & -1.2609505 & -1.5493044 \\ \mathrm{H} & 0.7009044 & -2.0881254 & -0.2669536 \\ \mathrm{C} & -1.6997018 & -1.7854722 & 3.8305131 \\ \mathrm{H} & -2.2970943 & 0.2020272 & 3.1606113 \\ \mathrm{H} & -3.2744922 & -1.1183827 & 2.4795957 \\ \mathrm{C} & 3.4060826 & 2.1814747 & 1.4837411 \\ \mathrm{H} & 3.1086554 & 2.1270655 & -0.6746153 \\ \mathrm{H} & 2.0910761 & 3.3206511 & 0.1706012 \\ \mathrm{C} & 2.3347759 & -3.9588291 & -1.5585538 \\ \mathrm{H} & 0.1775544 & -3.9823150 & -1.8647732 \\ \mathrm{H} & 1.0851404 & -3.1513513 & -3.1509709 \\ \mathrm{H} & -2.3504059 & -1.7766143 & 4.7323623 \\ \mathrm{H} & -1.6536529 & -2.8353291 & 3.4610520 \\ \mathrm{H} & -0.6735633 & -1.4997177 & 4.1554827 \\ \mathrm{H} & 4.2392014 & 2.9169173 & 1.4385053 \\ \mathrm{H} & 2.8363237 & 2.3788997 & 2.4197321 \\ \mathrm{H} & 3.8583303 & 1.1673163 & 1.5738622 \\ \mathrm{H} & 2.5024675 & -4.9364269 & -2.0613813 \\ \mathrm{H} & 3.2214744 & -3.3183047 & -1.7666731 \\ \mathrm{H} & 2.3074606 & -4.1462963 & -0.4612937\end{array}$

\section{TS-S14_NVP}

\begin{tabular}{lccc}
\multicolumn{4}{c}{ E(BP86/def2-SV(P) $=-968.9652173807$} \\
C & -2.6490352 & 0.9192223 & -3.3788992 \\
C & -2.1575374 & 1.3030062 & -2.1848733 \\
P & -1.0332165 & 0.3539392 & -1.1787395 \\
H & -2.3346205 & 2.3971817 & -1.5155215 \\
C & -1.9361970 & 0.1367717 & 0.4133519 \\
C & -0.5342295 & -1.2848265 & -1.8888284 \\
C & 0.4162597 & 1.4710361 & -0.9561185 \\
C & -2.2325195 & 3.5419629 & -0.4332517 \\
C & -1.9098618 & 4.2044988 & 0.5739181 \\
H & -2.4196392 & -0.0426317 & -3.8882635 \\
H & -1.6787090 & 4.8389069 & 1.4255232 \\
H & -3.3409692 & 1.5888473 & -3.9295339 \\
C & -1.3657711 & -0.8141570 & 1.4783927 \\
H & -2.0499997 & 1.1877417 & 0.7831005 \\
H & -2.9583337 & -0.1863998 & 0.1089628 \\
C & 1.3314073 & 1.2889030 & 0.2665401 \\
H & 0.9879822 & 1.4242485 & -1.9124732 \\
H & -0.0750807 & 2.4763481 & -0.9030456 \\
C & 0.7578699 & -1.9430823 & -1.3695298 \\
H & -1.4102756 & -1.9598300 & -1.7480184 \\
H & -0.4529607 & -1.1103030 & -2.9871364 \\
C & -2.2314877 & -0.8328095 & 2.7523071 \\
H & -1.3013825 & -1.8504565 & 1.0704285 \\
H & -0.3269959 & -0.5196927 & 1.7485548
\end{tabular}

$\begin{array}{cccc}\mathrm{C} & 2.5029017 & 2.2882659 & 0.2485130 \\ \mathrm{H} & 0.7330240 & 1.4583020 & 1.1901487 \\ \mathrm{H} & 1.7335183 & 0.2513779 & 0.3277793 \\ \mathrm{C} & 1.0392299 & -3.2975077 & -2.0460275 \\ \mathrm{H} & 1.6201928 & -1.2609505 & -1.5493044 \\ \mathrm{H} & 0.7009044 & -2.0881254 & -0.2669536 \\ \mathrm{C} & -1.6997018 & -1.7854722 & 3.8305131 \\ \mathrm{H} & -2.2970943 & 0.2020272 & 3.1606113 \\ \mathrm{H} & -3.2744922 & -1.1183827 & 2.4795957 \\ \mathrm{C} & 3.4060826 & 2.1814747 & 1.4837411 \\ \mathrm{H} & 3.1086554 & 2.1270655 & -0.6746153 \\ \mathrm{H} & 2.0910761 & 3.3206511 & 0.1706012 \\ \mathrm{C} & 2.3347759 & -3.9588291 & -1.5585538 \\ \mathrm{H} & 0.1775544 & -3.9823150 & -1.8647732 \\ \mathrm{H} & 1.0851404 & -3.1513513 & -3.1509709 \\ \mathrm{H} & -2.3504059 & -1.7766143 & 4.7323623 \\ \mathrm{H} & -1.6536529 & -2.8353291 & 3.4610520 \\ \mathrm{H} & -0.6735633 & -1.4997177 & 4.1554827 \\ \mathrm{H} & 4.2392014 & 2.9169173 & 1.4385053 \\ \mathrm{H} & 2.8363237 & 2.3788997 & 2.4197321 \\ \mathrm{H} & 3.8583303 & 1.1673163 & 1.5738622 \\ \mathrm{H} & 2.5024675 & -4.9364269 & -2.0613813 \\ \mathrm{H} & 3.2214744 & -3.3183047 & -1.7666731 \\ \mathrm{H} & 2.3074606 & -4.1462963 & -0.4612937\end{array}$

\section{TS-S15}

$\mathrm{E}(\mathrm{BP} 86 /$ def2-SV(P)) $=-1178.117873959$

C $\quad-1.3833677 \quad 2.0034777 \quad-3.3218493$

$\begin{array}{llll}\text { C } & -1.8179712 & 1.8779203 & -2.0439447\end{array}$

$\begin{array}{lllll}\mathrm{P} & -0.8079685 & 0.8588286 & -0.9482295\end{array}$

$\begin{array}{llll}\text { C } & -1.7054797 & 0.5330017 & 0.6359705\end{array}$

$\begin{array}{llll}\text { C } & -0.5611415 & -0.7850250 & -1.7947747\end{array}$

$\begin{array}{lllll}\text { C } & 0.8642588 & 1.6011460 & -0.6387000\end{array}$

$\begin{array}{llll}\mathrm{H} & -0.4851946 & 1.5203194 & -3.7763457\end{array}$

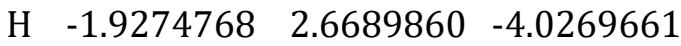

$\begin{array}{llll}\text { C } & -1.3266456 & -0.6987096 & 1.4743735\end{array}$

$\begin{array}{llll}\mathrm{H} & -1.6161949 & 1.4870684 & 1.1995587\end{array}$

$\begin{array}{llll}\mathrm{H} & -2.7729961 & 0.4681169 & 0.3263931\end{array}$

$\begin{array}{llll}\text { C } & 1.6585740 & 1.1697097 & 0.6052369\end{array}$

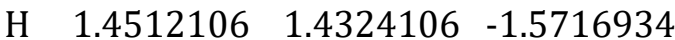

$\begin{array}{lllll}\mathrm{H} & 0.6138440 & 2.6835312 & -0.5791102\end{array}$

$\begin{array}{llll}\text { C } & 0.6016192 & -1.6949080 & -1.3602348\end{array}$

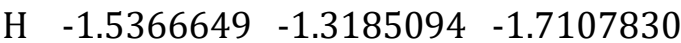

$\begin{array}{llll}\mathrm{H} & -0.4535546 & -0.5352643 & -2.8748346\end{array}$

$\begin{array}{llll}\text { C } & -2.1603261 & -0.7872383 & 2.7668742\end{array}$

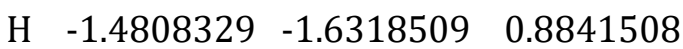

$\begin{array}{llll}\mathrm{H} & -0.2464336 & -0.6739354 & 1.7436751\end{array}$

C $\quad 2.9962883 \quad 1.9243755 \quad 0.7239812$

H $\quad 1.0506847 \quad 1.3736126 \quad 1.5161621$

$\begin{array}{llll}\mathrm{H} & 1.8590416 & 0.0737199 & 0.5954002\end{array}$

$\begin{array}{llll}\text { C } & 0.6521865 & -3.0061365 & -2.1669867\end{array}$ 


$\begin{array}{cccc}\mathrm{H} & 1.5670206 & -1.1538533 & -1.4919329 \\ \mathrm{H} & 0.5290940 & -1.9380093 & -0.2765625 \\ \mathrm{C} & -1.8141175 & -2.0066649 & 3.6308033 \\ \mathrm{H} & -2.0130975 & 0.1469064 & 3.3574709 \\ \mathrm{H} & -3.2433964 & -0.8101527 & 2.5012390 \\ \mathrm{C} & 3.7981253 & 1.5439867 & 1.9754493 \\ \mathrm{H} & 3.6096421 & 1.7281354 & -0.1872704 \\ \mathrm{H} & 2.7931911 & 3.0204389 & 0.7252706 \\ \mathrm{C} & 1.8206481 & -3.9181162 & -1.7710327 \\ \mathrm{H} & -0.3109496 & -3.5533986 & -2.0351287 \\ \mathrm{H} & 0.7169604 & -2.7638113 & -3.2539827 \\ \mathrm{H} & -2.4338231 & -2.0378148 & 4.5540216 \\ \mathrm{H} & -1.9867316 & -2.9588167 & 3.0792314 \\ \mathrm{H} & -0.7456825 & -1.9918806 & 3.9449329 \\ \mathrm{H} & 4.7559161 & 2.1071704 & 2.0296059 \\ \mathrm{H} & 3.2266588 & 1.7650617 & 2.9053095 \\ \mathrm{H} & 4.0465473 & 0.4581282 & 1.9859786 \\ \mathrm{H} & 1.8252322 & -4.8546785 & -2.3711425 \\ \mathrm{H} & 2.8010122 & -3.4139871 & -1.9289463 \\ \mathrm{H} & 1.7638129 & -4.2081399 & -0.6972933 \\ \mathrm{O} & -1.4504701 & 3.3937487 & -0.0848824 \\ \mathrm{C} & -2.4407480 & 3.4438295 & -0.8861056 \\ \mathrm{C} & -3.9091859 & 3.1127188 & -0.4570771 \\ \mathrm{C} & -4.8105625 & 3.9981951 & -1.3490347 \\ \mathrm{C} & -3.8683958 & 4.4578234 & -2.4861273 \\ \mathrm{~N} & -2.5867605 & 4.5205205 & -1.8036238 \\ \mathrm{H} & -4.0096894 & 3.3541337 & 0.6238032 \\ \mathrm{H} & -4.1222664 & 2.0343944 & -0.5923198 \\ \mathrm{H} & -5.7072545 & 3.4704569 & -1.7386096 \\ \mathrm{H} & -5.1635682 & 4.8872672 & -0.7829146 \\ \mathrm{H} & -3.8865545 & 3.7168284 & -3.3285151 \\ \mathrm{H} & -4.1517655 & 5.4503423 & -2.9029714 \\ \mathrm{H} & -1.7498005 & 4.7552903 & -2.3463665\end{array}$

\section{TS-S15_NVP}

\begin{tabular}{lccc}
\multicolumn{4}{c}{ E(BP86/def2-SV(P)) $=-1255.467394771$} \\
$\mathrm{C}$ & -1.9571178 & 1.6430356 & -3.3100130 \\
$\mathrm{C}$ & -1.8198676 & 1.8046143 & -1.9786247 \\
$\mathrm{P}$ & -0.8457116 & 0.6726985 & -1.0051701 \\
$\mathrm{C}$ & -1.7953388 & 0.3675751 & 0.5477348 \\
$\mathrm{C}$ & -0.4928708 & -0.9599146 & -1.8315436 \\
$\mathrm{C}$ & 0.7240533 & 1.5677343 & -0.6339070 \\
$\mathrm{H}$ & -1.5635149 & 0.7921232 & -3.9149986 \\
$\mathrm{H}$ & -2.4971116 & 2.4067646 & -3.9084918 \\
$\mathrm{C}$ & -1.3659840 & -0.7786925 & 1.4773614 \\
$\mathrm{H}$ & -1.7751004 & 1.3646417 & 1.0482804 \\
$\mathrm{H}$ & -2.8453718 & 0.2152484 & 0.2072518 \\
$\mathrm{C}$ & 1.5771683 & 1.1439040 & 0.5735384 \\
$\mathrm{H}$ & 1.3113152 & 1.5544233 & -1.5816332 \\
$\mathrm{H}$ & 0.3329852 & 2.6042943 & -0.4771226 \\
$\mathrm{C}$ & 0.7257002 & -1.7835315 & -1.3753804
\end{tabular}

$\begin{array}{cccc}\mathrm{H} & -1.4312182 & -1.5557116 & -1.7440942 \\ \mathrm{H} & -0.3878642 & -0.7168327 & -2.9149718 \\ \mathrm{C} & -2.2462009 & -0.8580510 & 2.7389798 \\ \mathrm{H} & -1.4232289 & -1.7525181 & 0.9367417 \\ \mathrm{H} & -0.3030480 & -0.6550110 & 1.7840399 \\ \mathrm{C} & 2.8511041 & 2.0012356 & 0.6965913 \\ \mathrm{H} & 0.9758890 & 1.2630694 & 1.5034317 \\ \mathrm{H} & 1.8662064 & 0.0694181 & 0.5163993 \\ \mathrm{C} & 0.8758128 & -3.0968887 & -2.1660663 \\ \mathrm{H} & 1.6521194 & -1.1774792 & -1.5015020 \\ \mathrm{H} & 0.6526489 & -2.0173056 & -0.2891030 \\ \mathrm{C} & -1.8441049 & -1.9934035 & 3.6888514 \\ \mathrm{H} & -2.1970667 & 0.1176702 & 3.2760649 \\ \mathrm{H} & -3.3116025 & -0.9830209 & 2.4332569 \\ \mathrm{C} & 3.7037803 & 1.6465179 & 1.9214991 \\ \mathrm{H} & 3.4608497 & 1.8854100 & -0.2306407 \\ \mathrm{H} & 2.5594427 & 3.0759823 & 0.7385276 \\ \mathrm{C} & 2.0972599 & -3.9231217 & -1.7425765 \\ \mathrm{H} & -0.0502167 & -3.7064023 & -2.0414710 \\ \mathrm{H} & 0.9410975 & -2.8617697 & -3.2545880 \\ \mathrm{H} & -2.4983257 & -2.0190206 & 4.5879802 \\ \mathrm{H} & -1.9194838 & -2.9870756 & 3.1913194 \\ \mathrm{H} & -0.7944867 & -1.8750818 & 4.0417334 \\ \mathrm{H} & 4.6156066 & 2.2811987 & 1.9767623 \\ \mathrm{H} & 3.1353331 & 1.7946214 & 2.8674927 \\ \mathrm{H} & 4.0361574 & 0.5836156 & 1.8935232 \\ \mathrm{H} & 2.1738505 & -4.8630253 & -2.3322329 \\ \mathrm{H} & 3.0434183 & -3.3553730 & -1.8922637 \\ \mathrm{H} & 2.0436933 & -4.2055525 & -0.6667040 \\ \mathrm{O} & -1.4318152 & 3.5273839 & 0.0564383 \\ \mathrm{C} & -2.3083697 & 3.4846619 & -0.8512801 \\ \mathrm{C} & -3.8200297 & 3.2160394 & -0.5448979 \\ \mathrm{C} & -4.5945654 & 4.3398859 & -1.2626569 \\ \mathrm{C} & -3.6588666 & 4.7822558 & -2.4089242 \\ \mathrm{~N} & -2.3328796 & 4.5055435 & -1.8925326 \\ \mathrm{H} & -3.9541876 & 3.2372223 & 0.5572358 \\ \mathrm{H} & -4.1290293 & 2.2162649 & -0.9137921 \\ \mathrm{H} & -5.5923587 & 4.0224903 & -1.6346376 \\ \mathrm{H} & -4.7537650 & 5.1970046 & -0.5722218 \\ \mathrm{H} & -3.8696631 & 4.2272480 & -3.3594698 \\ \mathrm{H} & -3.7569561 & 5.8674644 & -2.6410913 \\ \mathrm{C} & -1.1870892 & 5.1166927 & -2.3441943 \\ \mathrm{C} & -1.0653273 & 5.9806613 & -3.3877812 \\ \mathrm{H} & -0.0833792 & 6.4277057 & -3.6112202 \\ \mathrm{H} & -1.9148510 & 6.2698821 & -4.0300238 \\ \mathrm{H} & -0.3119228 & 4.8447805 & -1.7267130 \\ & & & \end{array}$

\section{TS-S16}

$\mathrm{E}(\mathrm{BP} 86 / \mathrm{def} 2-\mathrm{SV}(\mathrm{P}))=-1178.118091990$ C $\quad-4.05128790 .6496971 \quad-1.5621518$ $\begin{array}{llll}\text { C } & -3.0402659 & 1.4997509 & -1.2922745\end{array}$ 


\begin{tabular}{|c|c|c|c|}
\hline & 9027850 & & \\
\hline & -4.0520101 & 961 & 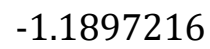 \\
\hline & 714 & 08 & \\
\hline & 1 & & \\
\hline & 908 & & \\
\hline & 528 & 4.11 & \\
\hline & -2.8 & & \\
\hline & -2.7 & & \\
\hline & -2.84 & 214 & -3.1 \\
\hline & 051 & 52 & -0.0 \\
\hline & -4.5 & & \\
\hline & -3.5 & & \\
\hline & -1.8 & & \\
\hline & -3.5 & 4.8 & -4 \\
\hline & -1.7 & 4.8 & -3. \\
\hline & -1.8 & 1.4 & 1.3 \\
\hline & -0.5 & & \\
\hline & 0.82 & 2.1 & \\
\hline & -2.11 & -0.0 & \\
\hline & -2.7 & 2.0 & 1.2 \\
\hline & -1.1 & & \\
\hline & 0.7 & -0.6 & \\
\hline & -1.4 & -0.5 & -1 . \\
\hline & -0.73 & 0.4 & $-2.3^{3}$ \\
\hline & 1.4 & 1.2 & \\
\hline & 1.5 & & \\
\hline & 0.6 & 3.1 & \\
\hline & -2.8 & -0.1 & \\
\hline & -2.73 & -0.4 & 57 \\
\hline & $-1.1^{\prime}$ & -0.6 & \\
\hline & 0.8 & -1.7 & -2.2 \\
\hline & 1.6 & & -1.3 \\
\hline & 0.87 & -1.1 & -0.1 \\
\hline & 2.8 & 1.8 & \\
\hline & 08 & & 74 \\
\hline & & 0.2 & 1.2 \\
\hline & -3.16 & -1.6 & 3.4198992 \\
\hline & -2.23 & 0.3 & 3651 \\
\hline & $-3.7 c$ & 0.4 & 3.0 \\
\hline & 2.16 & -2.5 & -2.2 \\
\hline I & -0.0 & -2.4 & -2.1703931 \\
\hline & 0.75 & $-1.2 \varepsilon$ & -3.2788619 \\
\hline & 3.4 & 1.04 & 3.1 \\
\hline & & & \\
\hline & 2.6971557 & 2.90 & 2.2976839 \\
\hline & -3.7040978 & -1.68 & 4.3933801 \\
\hline & -3.81 & -2.10 & 2.6 \\
\hline & -2.24 & -2.22 & 3.5070294 \\
\hline & 2.20 & $-3.32 \varepsilon$ & -3.0036638 \\
\hline & 3.05 & -1.8899551 & -2.3435550 \\
\hline & 2.2815848 & -3.0653555 & -1.228967 \\
\hline
\end{tabular}

$\begin{array}{rrrr}\mathrm{H} & 4.4711964 & 1.4862674 & 3.4069358 \\ \mathrm{H} & 2.8470499 & 1.0226696 & 4.0173410 \\ \mathrm{H} & 3.6781331 & -0.0092417 & 2.8070588 \\ \mathrm{H} & -2.5497071 & 2.4212826 & -3.7839188\end{array}$

\section{TS-S16_NVP}

$\mathrm{E}(\mathrm{BP} 86 / \mathrm{def} 2-\mathrm{SV}(\mathrm{P}))=-1255.461333248$

C $\quad-4.1640391 \quad 0.6894348 \quad-1.4903262$

$\begin{array}{lllll}\text { C } & -3.0966466 & 1.4949107 & -1.3306329\end{array}$

$\begin{array}{llll}\mathrm{P} & -0.9156471 & 1.6708547 & -0.3083197\end{array}$

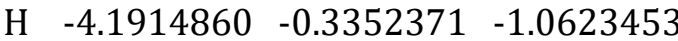

$\begin{array}{llll}\mathrm{H} & -5.0863952 & 0.9775474 & -2.0483501\end{array}$

$\begin{array}{lllll}0 & -1.0875437 & 3.0477096 & -1.0827852\end{array}$

$\begin{array}{llll}\text { C } & -2.9425750 & 2.8317128 & -1.8321534\end{array}$

$\begin{array}{llll}\text { C } & -3.5031213 & 4.0768263 & -1.1683555\end{array}$

$\begin{array}{llll}\text { C } & -2.8873020 & 5.2182366 & -1.9989229\end{array}$

$\begin{array}{lllll}\text { C } & -2.7185007 & 4.5999673 & -3.4036057\end{array}$

$\begin{array}{llll}\mathrm{N} & -2.7599719 & 3.1491459 & -3.1654050\end{array}$

H $\quad-3.2630845 \quad 4.1268880-0.0896048$

H $\quad-4.6151693 \quad 4.0400689-1.2737895$

$\mathrm{H} \quad-3.5100853 \quad 6.1369357 \quad-2.0147378$

$\mathrm{H} \quad-1.8923041 \quad 5.4672192 \quad-1.5785908$

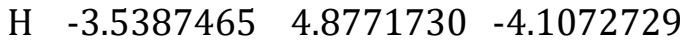

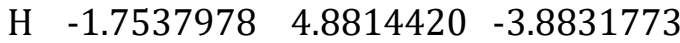

$\begin{array}{llll}\text { C } & -2.5120619 & 2.2279967 & -4.1756801\end{array}$

$\begin{array}{lllll}\text { C } & -2.2467261 & 2.5245661 & -5.4710393\end{array}$

H $-2.08432891 .7049596 \quad-6.1886950$

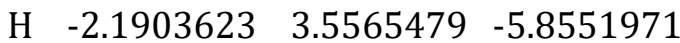

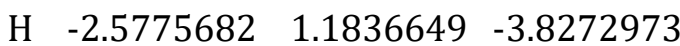

$\begin{array}{llll}\text { C } & -1.8261878 & 1.4098425 & 1.3085012\end{array}$

$\begin{array}{llll}\text { C } & -0.5902312 & 0.1051257 & -1.3000864\end{array}$

$\begin{array}{llll}\text { C } & 0.8038201 & 2.1176937 & 0.3714295\end{array}$

$\begin{array}{llll}\text { C } & -2.0933655 & -0.0553626 & 1.6830553\end{array}$

$\begin{array}{llll}\mathrm{H} & -2.7865489 & 1.9582332 & 1.2193214\end{array}$

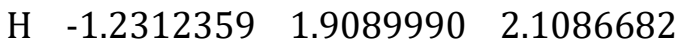

$\begin{array}{llll}\text { C } & 0.7294619 & -0.6783429 & -1.1829055\end{array}$

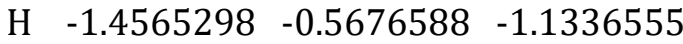

$\begin{array}{llll}\mathrm{H} & -0.7027850 & 0.4813476 & -2.3421243\end{array}$

$\begin{array}{llll}\text { C } & 1.4608403 & 1.3024686 & 1.4984700\end{array}$

$\begin{array}{llll}\mathrm{H} & 1.4804828 & 2.1800883 & -0.5113417\end{array}$

$\begin{array}{lllll}\mathrm{H} & 0.6702585 & 3.1700866 & 0.7092498\end{array}$

$\begin{array}{llll}\text { C } & -2.8260345 & -0.2043262 & 3.0291338\end{array}$

H $\quad-2.7044841 \quad-0.5300557 \quad 0.8825572$

$\begin{array}{llll}\mathrm{H} & -1.1331129 & -0.6220735 & 1.7271063\end{array}$

$\begin{array}{llll}\text { C } & 0.8337858 & -1.7831147 & -2.2520845\end{array}$

$\begin{array}{lllll}\mathrm{H} & 1.6041285 & 0.0030442 & -1.2840309\end{array}$

H $\quad 0.8101839-1.1488050-0.1764807$

$\begin{array}{llll}\text { C } & 2.8160339 & 1.8894589 & 1.9390195\end{array}$

$\begin{array}{llll}\mathrm{H} & 0.7898326 & 1.2650397 & 2.3888034\end{array}$

H $1.6156201 \quad 0.2444713 \quad 1.1913915$

$\begin{array}{llll}\text { C } & -3.1053670 & -1.6639814 & 3.4109399\end{array}$ 


$\begin{array}{cccc}\mathrm{H} & -2.2237452 & 0.2828472 & 3.8321992 \\ \mathrm{H} & -3.7865890 & 0.3607535 & 2.9826768 \\ \mathrm{C} & 2.1258475 & -2.6050914 & -2.1555259 \\ \mathrm{H} & -0.0479004 & -2.4613426 & -2.1658537 \\ \mathrm{H} & 0.7604436 & -1.3187135 & -3.2638794 \\ \mathrm{C} & 3.4815494 & 1.1067868 & 3.0788613 \\ \mathrm{H} & 3.5017480 & 1.9242205 & 1.0595663 \\ \mathrm{H} & 2.6693840 & 2.9511033 & 2.2492738 \\ \mathrm{H} & -3.6383488 & -1.7349351 & 4.3850104 \\ \mathrm{H} & -3.7368352 & -2.1691716 & 2.6452347 \\ \mathrm{H} & -2.1612042 & -2.2478603 & 3.5020733 \\ \mathrm{H} & 2.1680948 & -3.3903342 & -2.9426722 \\ \mathrm{H} & 3.0264893 & -1.9609272 & -2.2755046 \\ \mathrm{H} & 2.2103688 & -3.1147325 & -1.1687351 \\ \mathrm{H} & 4.4561685 & 1.5582178 & 3.3697389 \\ \mathrm{H} & 2.8373032 & 1.0863181 & 3.9874286 \\ \mathrm{H} & 3.6760231 & 0.0497118 & 2.7861542\end{array}$

\section{TS-S2}

\begin{tabular}{cccc}
\multicolumn{4}{c}{ E(BP86/def2-SV(P)) $=-1178.141439584$} \\
H & -0.8614550 & 2.7834585 & -1.9927427 \\
C & -0.1421200 & 2.3774461 & -2.7265924 \\
C & 1.1991794 & 2.1391519 & -2.3479128 \\
P & 1.3213557 & 0.9536581 & -0.9454157 \\
H & -0.3615479 & 2.5874915 & -3.7889540 \\
H & 1.8308695 & 1.7090514 & -3.1551514 \\
N & 2.2717389 & 3.4992311 & -1.9319789 \\
C & 2.1406855 & 4.1222536 & -0.7369016 \\
C & 2.4943366 & 5.6144207 & -0.8924777 \\
C & 2.3216876 & 5.8472308 & -2.4037396 \\
C & 2.5507223 & 4.4355848 & -3.0131840 \\
O & 1.7893392 & 3.5710222 & 0.3296692 \\
H & 1.8410084 & 6.2389401 & -0.2462758 \\
H & 3.5438104 & 5.7772857 & -0.5512581 \\
H & 1.2790186 & 6.1737916 & -2.6148565 \\
H & 3.0053997 & 6.6146316 & -2.8262058 \\
H & 3.5969070 & 4.3123078 & -3.3889617 \\
H & 1.8610184 & 4.2372984 & -3.8689458 \\
C & -0.0454687 & 1.2112838 & 0.2660154 \\
C & 1.0489083 & -0.6769614 & -1.7915367 \\
C & 3.0029116 & 0.8960156 & -0.1666804 \\
C & 0.1779068 & 0.6466339 & 1.6788696 \\
H & -0.1526497 & 2.3142156 & 0.3208428 \\
H & -0.9614231 & 0.7923156 & -0.2056669 \\
C & 0.5389483 & -1.8672032 & -0.9615388 \\
H & 0.3134707 & -0.4172264 & -2.5878757 \\
H & 2.0077484 & -0.9335730 & -2.3005276 \\
C & 3.4887947 & -0.4080910 & 0.4871051 \\
H & 3.6828502 & 1.1922946 & -0.9993662 \\
H & 3.0066437 & 1.7523268 & 0.5416092 \\
C & -1.0120692 & 0.9449427 & 2.6094948
\end{tabular}

$\begin{array}{cccc}\mathrm{H} & 0.3459634 & -0.4547903 & 1.6460256 \\ \mathrm{H} & 1.0970841 & 1.1004286 & 2.1129892 \\ \mathrm{C} & 0.3412026 & -3.1310681 & -1.8196126 \\ \mathrm{H} & 1.2403493 & -2.1004859 & -0.1286338 \\ \mathrm{H} & -0.4328092 & -1.6010351 & -0.4856696 \\ \mathrm{C} & 4.9084759 & -0.2638183 & 1.0679138 \\ \mathrm{H} & 2.7949746 & -0.7142405 & 1.3030900 \\ \mathrm{H} & 3.4874414 & -1.2409647 & -0.2538574 \\ \mathrm{C} & -0.8133350 & 0.4123017 & 4.0342797 \\ \mathrm{H} & -1.1780048 & 2.0467922 & 2.6399679 \\ \mathrm{H} & -1.9399414 & 0.5069010 & 2.1714750 \\ \mathrm{C} & -0.1972884 & -4.3266007 & -1.0233163 \\ \mathrm{H} & -0.3531661 & -2.8950803 & -2.6596349 \\ \mathrm{H} & 1.3123532 & -3.4052803 & -2.2954623 \\ \mathrm{C} & 5.4277314 & -1.5469831 & 1.7291268 \\ \mathrm{H} & 5.6048721 & 0.0476979 & 0.2539836 \\ \mathrm{H} & 4.9142103 & 0.5702123 & 1.8077126 \\ \mathrm{H} & -1.6878080 & 0.6481360 & 4.6800408 \\ \mathrm{H} & -0.6800859 & -0.6936262 & 4.0411831 \\ \mathrm{H} & 0.0869119 & 0.8607623 & 4.5121872 \\ \mathrm{H} & -0.3251247 & -5.2206788 & -1.6725194 \\ \mathrm{H} & 0.4930235 & -4.6085848 & -0.1960763 \\ \mathrm{H} & -1.1877977 & -4.0984182 & -0.5684451 \\ \mathrm{H} & 6.4529243 & -1.4058950 & 2.1369779 \\ \mathrm{H} & 4.7724776 & -1.8632556 & 2.5723584 \\ \mathrm{H} & 5.4690394 & -2.3911557 & 1.0036568\end{array}$

\section{TS-S3}

\begin{tabular}{lccc}
\multicolumn{4}{c}{ E(BP86/def2-SV(P) $)=-1178.080080325$} \\
C & 0.0616292 & -0.7993226 & 1.2051610 \\
C & 0.1251356 & -2.2552215 & 1.6802002 \\
C & -1.3479371 & -2.7042159 & 1.6704822 \\
C & -1.9922786 & -1.7958798 & 0.5969521 \\
N & -1.1712080 & -0.5856572 & 0.6364405 \\
O & 0.9719920 & 0.0412159 & 1.2366248 \\
H & 0.6248210 & -2.3248044 & 2.6692664 \\
H & 0.7467253 & -2.8409370 & 0.9614295 \\
H & -1.4888793 & -3.7836947 & 1.4506698 \\
H & -1.8126829 & -2.4988383 & 2.6600395 \\
H & -1.9685307 & -2.2670777 & -0.4193241 \\
H & -3.0561882 & -1.5518059 & 0.8183183 \\
C & -1.5207485 & 0.6541072 & -0.0023900 \\
C & -0.7655635 & 0.9892481 & -1.2802020 \\
H & -0.7420762 & 0.3457703 & -2.2066613 \\
H & -0.1875067 & 1.0592346 & -0.1172073 \\
P & -0.6154983 & 2.6794499 & -1.7053925 \\
C & 0.4402906 & 2.7921051 & -3.2285559 \\
C & -2.2500256 & 3.4773789 & -2.1491631 \\
C & 0.1290899 & 3.6007180 & -0.2798269 \\
C & 1.2593196 & 4.0819169 & -3.4186614 \\
H & 1.1186461 & 1.9116520 & -3.1638442
\end{tabular} 


$\begin{array}{cccc}\mathrm{H} & -0.2245450 & 2.6198228 & -4.1061637 \\ \mathrm{C} & -2.2932225 & 4.7963029 & -2.9404010 \\ \mathrm{H} & -2.7782894 & 2.6753031 & -2.7169556 \\ \mathrm{H} & -2.7993274 & 3.5693036 & -1.1825452 \\ \mathrm{C} & -0.1895599 & 5.0874180 & -0.0640089 \\ \mathrm{H} & -0.2165603 & 2.9957671 & 0.5938607 \\ \mathrm{H} & 1.2268981 & 3.4324284 & -0.3560743 \\ \mathrm{H} & -2.6033405 & 0.8800029 & 0.1075006 \\ \mathrm{C} & 2.1081746 & 4.0562787 & -4.7030885 \\ \mathrm{H} & 0.5858753 & 4.9689292 & -3.4451461 \\ \mathrm{H} & 1.9329118 & 4.2305382 & -2.5436068 \\ \mathrm{C} & -3.7312390 & 5.2816391 & -3.2053701 \\ \mathrm{H} & -1.7358785 & 5.5939274 & -2.3997157 \\ \mathrm{H} & -1.7743792 & 4.6633027 & -3.9183475 \\ \mathrm{C} & 0.4724826 & 5.6403343 & 1.2123235 \\ \mathrm{H} & 0.1464904 & 5.6936739 & -0.9376032 \\ \mathrm{H} & -1.2922026 & 5.2319606 & 0.0122086 \\ \mathrm{C} & 2.9474990 & 5.3252344 & -4.9000193 \\ \mathrm{H} & 2.7769289 & 3.1640390 & -4.6796054 \\ \mathrm{H} & 1.4369017 & 3.9106504 & -5.5822564 \\ \mathrm{C} & -3.7943626 & 6.5830106 & -4.0155462 \\ \mathrm{H} & -4.2944458 & 4.4798918 & -3.7386409 \\ \mathrm{H} & -4.2537445 & 5.4218368 & -2.2299559 \\ \mathrm{C} & 0.1651597 & 7.1222158 & 1.4631126 \\ \mathrm{H} & 0.1392860 & 5.0323832 & 2.0853702 \\ \mathrm{H} & 1.5758240 & 5.4922823 & 1.1442008 \\ \mathrm{H} & 3.5469874 & 5.2726169 & -5.8353656 \\ \mathrm{H} & 2.3045174 & 6.2323282 & -4.9641217 \\ \mathrm{H} & 3.6570549 & 5.4776407 & -4.0554125 \\ \mathrm{H} & -4.8456891 & 6.9040265 & -4.1856355 \\ \mathrm{H} & -3.2709413 & 7.4141740 & -3.4906291 \\ \mathrm{H} & -3.3148204 & 6.4642547 & -5.0137877 \\ \mathrm{H} & 0.6596438 & 7.4866736 & 2.3904399 \\ \mathrm{H} & 0.5196488 & 7.7601531 & 0.6215392 \\ \mathrm{H} & -0.9289621 & 7.2969136 & 1.5765911\end{array}$

\section{TS-S3_PMe3}

\begin{tabular}{lccc}
\multicolumn{4}{c}{$\mathrm{E}(\mathrm{BP} 86 / \mathrm{def} 2-\mathrm{SV}(\mathrm{P}))=-824.5630488503$} \\
$\mathrm{C}$ & 0.7835864 & -2.0898769 & 0.1372965 \\
$\mathrm{C}$ & 0.7771724 & -3.5738796 & -0.2390558 \\
$\mathrm{C}$ & -0.6898863 & -3.9928802 & -0.0266022 \\
$\mathrm{C}$ & -1.4742168 & -2.6751260 & -0.2307319 \\
$\mathrm{~N}$ & -0.5142118 & -1.6433331 & 0.1618156 \\
$\mathrm{O}$ & 1.7739937 & -1.3614568 & 0.3136059 \\
$\mathrm{H}$ & 1.5132594 & -4.1383253 & 0.3712374 \\
$\mathrm{H}$ & 1.0932615 & -3.6775538 & -1.3046650 \\
$\mathrm{H}$ & -1.0316169 & -4.7978247 & -0.7110253 \\
$\mathrm{H}$ & -0.8328087 & -4.3544456 & 1.0156004 \\
$\mathrm{H}$ & -1.7984007 & -2.5395583 & -1.2943483 \\
$\mathrm{H}$ & -2.3902591 & -2.6143425 & 0.3997523 \\
$\mathrm{C}$ & -0.8454656 & -0.2627777 & 0.3915547
\end{tabular}

$\begin{array}{crcc}\mathrm{C} & -0.3846962 & 0.7318905 & -0.6568664 \\ \mathrm{H} & -0.7011948 & 0.7491236 & -1.7422028 \\ \mathrm{H} & 0.4782412 & 0.1432271 & 0.1210345 \\ \mathrm{P} & -0.0101385 & 2.3305312 & -0.0782016 \\ \mathrm{C} & 0.9291963 & 3.2769894 & -1.3484593 \\ \mathrm{C} & -1.4399425 & 3.4446121 & 0.3142381 \\ \mathrm{C} & 0.9668401 & 2.2818224 & 1.4758198 \\ \mathrm{H} & 1.0498389 & 4.3427737 & -1.0584336 \\ \mathrm{H} & 1.9259737 & 2.8070539 & -1.4852553 \\ \mathrm{H} & 0.3797458 & 3.2254407 & -2.3130619 \\ \mathrm{H} & -1.1061805 & 4.4405050 & 0.6836737 \\ \mathrm{H} & -2.0573510 & 3.5803595 & -0.6004843 \\ \mathrm{H} & -2.0673909 & 2.9554927 & 1.0904688 \\ \mathrm{H} & 1.4121908 & 3.2840697 & 1.6596757 \\ \mathrm{H} & 0.3187763 & 1.9970085 & 2.3281970 \\ \mathrm{H} & 1.7654742 & 1.5156188 & 1.3759017 \\ \mathrm{H} & -1.8202329 & -0.1543600 & 0.9132989\end{array}$

\section{TS-S3_PPh3}

$\mathrm{E}(\mathrm{BP} 86 /$ def2-SV(P) $)=-1399.372112305$

$\begin{array}{llll}\text { C } & -0.1854185 & -1.3515013 & 1.0844425\end{array}$

$\begin{array}{llll}\text { C } & -0.3901443 & -2.8414066 & 1.3675514\end{array}$

$\begin{array}{llll}\text { C } & -1.9213873 & -3.0096334 & 1.3465469\end{array}$

$\begin{array}{llll}\text { C } & -2.4026015 & -1.8735012 & 0.4138220\end{array}$

$\begin{array}{llll}\mathrm{N} & -1.3605447 & -0.8518269 & 0.5577156\end{array}$

$\begin{array}{lllll}0 & 0.8493233 & -0.6881048 & 1.2267771\end{array}$

H $\quad 0.0934726 \quad-3.1267194 \quad 2.3249107$

H $\quad 0.1087191 \quad-3.4331114 \quad 0.5635208$

H $\quad-2.2579410 \quad-4.00889690 .9993553$

$\begin{array}{llll}\mathrm{H} & -2.3324594 & -2.8529834 & 2.3679484\end{array}$

H $\quad-2.4876779-2.2067497 \quad-0.6508063$

H $\quad-3.3934473 \quad-1.4608950 \quad 0.7087725$

$\begin{array}{llll}\text { C } & -1.4677577 & 0.4823520 & 0.0878317\end{array}$

$\begin{array}{llll}\text { C } & -0.5575939 & 0.8776295 & -1.0674305\end{array}$

$\mathrm{H} \quad-0.3996404 \quad 0.1862824 \quad-1.9299737$

$\begin{array}{llll}\mathrm{H} & -0.0960576 & 0.7969289 & 0.1191847\end{array}$

P $\quad-0.4718138 \quad 2.5555884 \quad-1.5240505$

H $\quad-2.4240490 \quad 0.9957869 \quad 0.2964853$

$\begin{array}{llll}\text { C } & 2.8653937 & 3.1157260 & -4.7058474\end{array}$

C $1.8838993 \quad 4.1053479 \quad-4.5340071$

$\begin{array}{llll}\text { C } & 0.8452327 & 3.9209769 & -3.6053519\end{array}$

C $\quad 0.7759435 \quad 2.7306961 \quad-2.8409596$

C $\quad 1.7868764 \quad 1.7521846-2.9943120$

C $\quad 2.8126418 \quad 1.9389871-3.9305295$

H $\quad 3.6809610 \quad 3.2640129-5.4328583$

H $\quad 1.9253830 \quad 5.0353753 \quad-5.1254216$

H $\quad 0.0817502 \quad 4.7063993 \quad-3.4803357$

$\mathrm{H} \quad 1.7732704 \quad 0.8509149 \quad-2.3600879$

H $\quad 3.5870033 \quad 1.1629641-4.0496159$

C $\quad-4.6992161 \quad 3.7744234 \quad-3.0716123$

C $\quad-4.3938820 \quad 3.8398464 \quad-1.7011009$ 


$\begin{array}{cccc}\mathrm{C} & -3.0922491 & 3.5651506 & -1.2503316 \\ \mathrm{C} & -2.0687825 & 3.2266992 & -2.1704847 \\ \mathrm{C} & -2.3943492 & 3.1335745 & -3.5453283 \\ \mathrm{C} & -3.6936070 & 3.4174557 & -3.9910336 \\ \mathrm{H} & -5.7210312 & 3.9924823 & -3.4238667 \\ \mathrm{H} & -5.1765585 & 4.1096009 & -0.9720688 \\ \mathrm{H} & -2.8636268 & 3.6249882 & -0.1727800 \\ \mathrm{H} & -1.6229549 & 2.8325062 & -4.2735331 \\ \mathrm{H} & -3.9248990 & 3.3582439 & -5.0679205 \\ \mathrm{C} & 0.9769604 & 5.3767886 & 1.8901601 \\ \mathrm{C} & 0.2069472 & 5.8847399 & 0.8328116 \\ \mathrm{C} & -0.2973436 & 5.0176450 & -0.1534798 \\ \mathrm{C} & -0.0362790 & 3.6296511 & -0.0872352 \\ \mathrm{C} & 0.7667436 & 3.1274133 & 0.9644938 \\ \mathrm{C} & 1.2547321 & 3.9961951 & 1.9510025 \\ \mathrm{H} & 1.3693295 & 6.0550809 & 2.6661127 \\ \mathrm{H} & -0.0060661 & 6.9650910 & 0.7692540 \\ \mathrm{H} & -0.9090664 & 5.4265367 & -0.9746968 \\ \mathrm{H} & 1.0135237 & 2.0539547 & 1.0266335 \\ \mathrm{H} & 1.8602392 & 3.5884097 & 2.7772269\end{array}$

\section{TS-S4}

\begin{tabular}{lccc}
\multicolumn{4}{c}{ E(BP86/def2-SV(P) $=-1198.991628938$} \\
O & -1.5255809 & 1.5191063 & 0.5830598 \\
C & -1.7073823 & 2.4278002 & -1.8516816 \\
C & -0.6826473 & 1.8285662 & -2.5135567 \\
H & -2.7486645 & 2.2501020 & -2.1746287 \\
H & -1.5738118 & 3.1317376 & -1.0215285 \\
P & 1.0119155 & 1.9252707 & -1.9464424 \\
C & 1.9813832 & 2.8341754 & -3.2361761 \\
C & 1.0805922 & 2.7920450 & -0.3378539 \\
H & -0.8672704 & 1.1203034 & -3.3418612 \\
C & 1.6504023 & 0.1992199 & -1.8142653 \\
C & 3.5040248 & 2.9478751 & -3.0446225 \\
H & 1.7410831 & 2.3341801 & -4.2031327 \\
H & 1.5131533 & 3.8438585 & -3.2896295 \\
C & 1.0284572 & 4.3314988 & -0.3943173 \\
H & 0.1833274 & 2.3793231 & 0.2206848 \\
H & 2.0017572 & 2.4479894 & 0.1816079 \\
C & 2.9073134 & -0.0254116 & -0.9541173 \\
H & 0.7977162 & -0.3913802 & -1.4031248 \\
H & 1.8126478 & -0.1391824 & -2.8653565 \\
C & 4.1631674 & 3.8445704 & -4.1091703 \\
H & 3.7327988 & 3.3524738 & -2.0313039 \\
H & 3.9653517 & 1.9353264 & -3.0856991 \\
C & 0.9319185 & 4.9382048 & 1.0180039 \\
H & 1.9289979 & 4.7412534 & -0.9103617 \\
H & 0.1493230 & 4.6662343 & -0.9918849 \\
C & 3.3934002 & -1.4839614 & -1.0348473 \\
H & 3.7342027 & 0.6579782 & -1.2569092 \\
H & 2.6561059 & 0.2086428 & 0.1047715
\end{tabular}

$\begin{array}{cccc}\mathrm{C} & 5.6849705 & 3.9532663 & -3.9495996 \\ \mathrm{H} & 3.9195651 & 3.4470788 & -5.1225426 \\ \mathrm{H} & 3.7071776 & 4.8613832 & -4.0627044 \\ \mathrm{C} & 0.8596957 & 6.4701601 & 1.0142491 \\ \mathrm{H} & 0.0370853 & 4.5136473 & 1.5277152 \\ \mathrm{H} & 1.8116853 & 4.6046203 & 1.6171737 \\ \mathrm{C} & 4.6051613 & -1.7621051 & -0.1368685 \\ \mathrm{H} & 2.5487539 & -2.1511021 & -0.7472581 \\ \mathrm{H} & 3.6457875 & -1.7307517 & -2.0937847 \\ \mathrm{H} & 6.1259509 & 4.6093840 & -4.7316357 \\ \mathrm{H} & 5.9604150 & 4.3804204 & -2.9588968 \\ \mathrm{H} & 6.1748280 & 2.9568454 & -4.0323727 \\ \mathrm{H} & 0.7911738 & 6.8718290 & 2.0490445 \\ \mathrm{H} & 1.7598736 & 6.9220551 & 0.5381790 \\ \mathrm{H} & -0.0326268 & 6.8333197 & 0.4556213 \\ \mathrm{H} & 4.9323919 & -2.8218602 & -0.2203953 \\ \mathrm{H} & 5.4744538 & -1.1199850 & -0.4081002 \\ \mathrm{H} & 4.3657074 & -1.5695393 & 0.9330622 \\ \mathrm{C} & -0.1647764 & -2.4517392 & 0.5563987 \\ \mathrm{C} & 0.3783061 & -1.5027136 & 1.4492497 \\ \mathrm{C} & -0.0666160 & -0.1739449 & 1.4549907 \\ \mathrm{C} & -1.1219039 & 0.2910850 & 0.5792692 \\ \mathrm{C} & -1.6576582 & -0.7125492 & -0.3144833 \\ \mathrm{C} & -1.1934257 & -2.0351167 & -0.3156773 \\ \mathrm{H} & 0.1828876 & -3.4982275 & 0.5620241 \\ \mathrm{H} & 1.1636407 & -1.8129389 & 2.1635101 \\ \mathrm{H} & 0.3464779 & 0.5509731 & 2.1791500 \\ \mathrm{H} & -2.4764567 & -0.4065657 & -0.9881272 \\ \mathrm{H} & -1.6486065 & -2.7660240 & -1.0095463\end{array}$

\section{TS-S5}

$\mathrm{E}(\mathrm{BP} 86 / \mathrm{def} 2-\mathrm{SV}(\mathrm{P}))=-1992.588413940$

$\begin{array}{cccc}\mathrm{C} & -2.1559576 & 3.7770902 & 0.5196321 \\ \mathrm{C} & -2.1573455 & 2.6181435 & -0.2840915 \\ \mathrm{P} & -0.7026451 & 1.6883700 & -0.6379407 \\ \mathrm{C} & 0.2092956 & 1.3930642 & 0.9319733 \\ \mathrm{C} & -1.3329073 & 0.1018152 & -1.3705436 \\ \mathrm{C} & 0.4218989 & 2.5113451 & -1.8511488 \\ \mathrm{C} & 1.6708061 & 0.9138905 & 0.8957263 \\ \mathrm{H} & 0.1936881 & 2.4039145 & 1.4280053 \\ \mathrm{H} & -0.4391417 & 0.7113023 & 1.5302585 \\ \mathrm{C} & -0.4383847 & -1.1446621 & -1.2581888 \\ \mathrm{H} & -2.3048586 & -0.0867581 & -0.8604182 \\ \mathrm{H} & -1.5718892 & 0.3202261 & -2.4375121 \\ \mathrm{C} & 1.4814345 & 1.6683701 & -2.5840626 \\ \mathrm{H} & -0.2696334 & 2.9733118 & -2.5926534 \\ \mathrm{H} & 0.8921784 & 3.3593542 & -1.2619300 \\ \mathrm{P} & -2.1340372 & 5.8164475 & -0.6295937 \\ \mathrm{H} & -3.0904729 & 2.2274252 & -0.7280869 \\ \mathrm{C} & 2.2253902 & 0.7236673 & 2.3202059 \\ \mathrm{H} & 1.7866246 & -0.0373080 & 0.3253490\end{array}$




\begin{tabular}{|c|c|c|c|}
\hline & 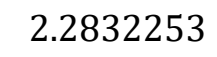 & . & \\
\hline & 301705 & -2.3872548 & -1.9 \\
\hline & & 00 & \\
\hline & 31 & 775 & \\
\hline & 042 & & \\
\hline & 2.1476457 & 1.1581140 & 32601 \\
\hline & 431 & 0.8602189 & 929 \\
\hline & 558 & 26 & \\
\hline & $2.0^{\prime}$ & & \\
\hline & 1.6207837 & -0.05 & \\
\hline & -0.21 & -3.6 & -1.7 \\
\hline & -2.0 & -2.5 & -1 \\
\hline & -1.2 & -2.1 & -2 \\
\hline & 3.4071064 & 1.71 & -4.28 \\
\hline & 1.68 & & 790 \\
\hline & 2.8346 & 3.3 & -2.9 \\
\hline & $4.0^{\prime}$ & & \\
\hline & $3.8 \varepsilon$ & -0.6 & \\
\hline & 4.34 & & \\
\hline & -0.70 & -4.5 & 9921 \\
\hline & 0.7 & -3.5 & -2. \\
\hline & -0.0 & -3.9 & -0 . \\
\hline & 4.0 & 2.3 & -4.9 \\
\hline & & & -3.5 \\
\hline & 2.94 & 0.9 & -4.9 \\
\hline & -0.7 & & -0 . \\
\hline & -2.17 & & \\
\hline & -1.24 & & \\
\hline & -3.11 & 4.0 & 1.0 \\
\hline & -3.74 & & -0.2 \\
\hline & -0.4 & & -1. \\
\hline & -0.8 & & \\
\hline & & 6.3 & -0.3 \\
\hline & -2.984 & 6.13 & 3874 \\
\hline & -1.10 & 5.2 & -2. \\
\hline & -2.5 & & -2. \\
\hline & -3.987 & 8.13 & -0.5 \\
\hline & -4.5445776 & 6.01 & -0.6455501 \\
\hline & -3.84 & & 0.8 \\
\hline & 0.72 & 8.9 & -1.0 \\
\hline & -0.28 & 7.6 & -2.4 \\
\hline & -1.3801231 & 8.7338821 & -1.5816344 \\
\hline & -2.9452058 & 5.519 & 3018 \\
\hline & -4.04 & 6.20 & -3.1 \\
\hline & -2.60 & 7.18 & -3.4 \\
\hline & -5.3732361 & 8.6437686 & -0.1451858 \\
\hline & -3.2026687 & 8.7726879 & -0.1183923 \\
\hline & -3.88 & 8.27 & -1.6 \\
\hline & 1.016 & 10.065 & -2.1470512 \\
\hline & 0.5341290 & 9.4762768 & -0.1059452 \\
\hline & 1.6228901 & 8.3480263 & -0.9476394 \\
\hline
\end{tabular}

$\begin{array}{cccc}\mathrm{C} & -3.7599050 & 6.3111193 & -5.8818590 \\ \mathrm{H} & -1.8841805 & 5.4480339 & -5.1873545 \\ \mathrm{H} & -3.3205518 & 4.4700821 & -4.8018244 \\ \mathrm{C} & -5.6217171 & 10.1182682 & -0.4895001 \\ \mathrm{H} & -6.1624321 & 8.0115507 & -0.6170290 \\ \mathrm{H} & -5.4813809 & 8.4933190 & 0.9550301 \\ \mathrm{H} & 1.8860567 & 10.6950275 & -1.8547160 \\ \mathrm{H} & 1.2509554 & 9.6103178 & -3.1362070 \\ \mathrm{H} & 0.1446823 & 10.7446161 & -2.2910889 \\ \mathrm{H} & -3.7086041 & 5.8402337 & -6.8884413 \\ \mathrm{H} & -4.8341127 & 6.3690853 & -5.5932248 \\ \mathrm{H} & -3.3846956 & 7.3549861 & -5.9807423 \\ \mathrm{H} & -6.6302025 & 10.4487997 & -0.1560088 \\ \mathrm{H} & -4.8726905 & 10.7809353 & 0.0003799 \\ \mathrm{H} & -5.5562002 & 10.2956367 & -1.5870216 \\ \mathrm{C} & 1.6021959 & 4.7047271 & 1.3411184 \\ \mathrm{C} & 2.8443122 & 5.2654200 & 2.0916870 \\ \mathrm{C} & 3.9135555 & 5.2781658 & 0.9936317 \\ \mathrm{C} & 3.0431673 & 5.4026929 & -0.2897712 \\ \mathrm{~N} & 1.7494985 & 4.7986030 & 0.0112889 \\ \mathrm{O} & 0.5984473 & 4.2558387 & 1.9721270 \\ \mathrm{H} & 3.0882188 & 4.6531206 & 2.9862733 \\ \mathrm{H} & 2.6047667 & 6.2929009 & 2.4577730 \\ \mathrm{H} & 4.6747161 & 6.0847479 & 1.0911605 \\ \mathrm{H} & 4.4582685 & 4.3056836 & 0.9830521 \\ \mathrm{H} & 2.9316301 & 6.4874590 & -0.5706937 \\ \mathrm{H} & 3.5281141 & 4.9186268 & -1.1732471\end{array}$

\section{TS-S5_BF4}

\begin{tabular}{lrrr}
\multicolumn{4}{c}{$\mathrm{E}(\mathrm{BP} 86 / \mathrm{def} 2-\mathrm{SV}(\mathrm{P}))=-2130.950759663$} \\
$\mathrm{~F}$ & -2.0937735 & 3.1366629 & 0.0944608 \\
$\mathrm{C}$ & -0.9719387 & 3.6861832 & -3.1521806 \\
$\mathrm{C}$ & 0.2694587 & 3.2577850 & -2.6742285 \\
$\mathrm{P}$ & 0.5534593 & 1.9316987 & -1.5387125 \\
$\mathrm{C}$ & 1.0144464 & 2.5385607 & 0.1445083 \\
$\mathrm{C}$ & -0.9380431 & 0.8567719 & -1.4705739 \\
$\mathrm{C}$ & 1.9960770 & 0.9934323 & -2.2424651 \\
$\mathrm{C}$ & 1.7517434 & 1.6077459 & 1.1234150 \\
$\mathrm{H}$ & 1.6137493 & 3.4585644 & -0.0451608 \\
$\mathrm{H}$ & 0.0412641 & 2.8678954 & 0.5701907 \\
$\mathrm{C}$ & -1.0960088 & -0.0697097 & -0.2513430 \\
$\mathrm{H}$ & -1.7834686 & 1.5772285 & -1.4584993 \\
$\mathrm{H}$ & -0.9665918 & 0.2979825 & -2.4347443 \\
$\mathrm{C}$ & 2.2249985 & -0.4560180 & -1.7778249 \\
$\mathrm{H}$ & 1.8411201 & 1.0219497 & -3.3448289 \\
$\mathrm{H}$ & 2.8961450 & 1.6197148 & -2.0369395 \\
$\mathrm{P}$ & -2.0198310 & 5.5912734 & -1.9564089 \\
$\mathrm{H}$ & 1.1883644 & 3.7899778 & -2.9833953 \\
$\mathrm{C}$ & 1.8622877 & 2.2451483 & 2.5214336 \\
$\mathrm{H}$ & 1.2109869 & 0.6404392 & 1.2181392 \\
$\mathrm{H}$ & 2.7714355 & 1.3698511 & 0.7379342
\end{tabular}




\begin{tabular}{|c|c|c|c|}
\hline & 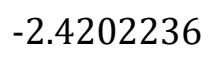 & 346 & \\
\hline & 6709 & 0944 & \\
\hline & & 74 & \\
\hline & 07 & 84 & \\
\hline & 209 & -0.4 & \\
\hline & 537 & -1.071 & -2 \\
\hline & & & \\
\hline & 27 & & \\
\hline & 0.83 & & 282 \\
\hline & -2.63 & -1.73 & 353 \\
\hline & -3.2 & -0.1 & -0 \\
\hline & -2.4 & -1.4 & \\
\hline & 3.6 & -2.5 & \\
\hline & 3.3 & -1.0 & 67 \\
\hline & 4.3 & -0.4 & 730 \\
\hline & 2.6 & 1.8 & \\
\hline & 2.0 & & \\
\hline & 3.6 & 1.1 & \\
\hline & -3.59 & $-2.2 \varepsilon$ & \\
\hline & -1.8 & -2.4 & 09 \\
\hline & -2.6 & -1.1 & \\
\hline & 4.6 & -2.9 & \\
\hline & 3.8 & -2.62 & \\
\hline & 2.8 & $-3.1 c$ & 75 \\
\hline & -2.4 & 3.1 & \\
\hline & -2.0 & & \\
\hline & -3.8 & 2.9 & \\
\hline & -1.6 & 2.16 & \\
\hline & -0.8 & 5.9 & -0 . \\
\hline & -2.0 & 7.0 & \\
\hline & -1.8 & 3.0 & 66 \\
\hline & -0.9 & 4.2 & 5426 \\
\hline & -3.72 & 5.47 & 72830 \\
\hline & -0.5 & 7.3 & 7883 \\
\hline & -1.1 & & 809 \\
\hline & & 5.5 & 164 \\
\hline & -2.77 & 8.33 & -2.7932686 \\
\hline & -0.94 & 7.26 & 33602 \\
\hline & -2.4 & 6.63 & 52116 \\
\hline & -4.0 & & \\
\hline & -4.44 & 5.629 & 21082 \\
\hline & -3.81 & 4.40 & 3397 \\
\hline & 0.42 & 7.42 & \\
\hline & -0.2 & 2 & 18 \\
\hline I & -1.55 & 7.81 & 0.2526369 \\
\hline & -2.6727548 & 9.401 & -3.8957918 \\
\hline & -3.8 & 8.09 & 2362 \\
\hline & -2.39 & 8.75 & -1.8364370 \\
\hline & $-5.4 c$ & 6.02 & 0.5437954 \\
\hline & -3.347 & 6.1711940 & 0.8004458 \\
\hline & -4.0369609 & 7.4325657 & -0.276594 \\
\hline
\end{tabular}

$\begin{array}{cccl}\mathrm{C} & 0.6526541 & 8.8332887 & 1.6260878 \\ \mathrm{H} & 0.0547336 & 6.7490726 & 1.8736043 \\ \mathrm{H} & 1.3930518 & 6.9938182 & 0.7233200 \\ \mathrm{C} & -3.4374422 & 10.6906501 & -3.5653238 \\ \mathrm{H} & -1.5973731 & 9.6413450 & -4.0735859 \\ \mathrm{H} & -3.0522144 & 8.9777367 & -4.8557846 \\ \mathrm{C} & -5.8787009 & 6.8865375 & 1.7499204 \\ \mathrm{H} & -6.2502524 & 6.1427085 & -0.2657610 \\ \mathrm{H} & -5.5023461 & 4.9478196 & 0.8380057 \\ \mathrm{H} & 1.3824512 & 8.8223198 & 2.4658901 \\ \mathrm{H} & 1.0486107 & 9.5236402 & 0.8461927 \\ \mathrm{H} & -0.2939328 & 9.2745823 & 2.0127260 \\ \mathrm{H} & -3.3401336 & 11.4417692 & -4.3802313 \\ \mathrm{H} & -4.5234319 & 10.4918248 & -3.4197195 \\ \mathrm{H} & -3.0568710 & 11.1590701 & -2.6294458 \\ \mathrm{H} & -6.8881665 & 6.6170462 & 2.1329492 \\ \mathrm{H} & -5.1565491 & 6.7510463 & 2.5860154 \\ \mathrm{H} & -5.8957636 & 7.9702569 & 1.4900505\end{array}$

\section{TS-S5_Phenol}

$\mathrm{E}(\mathrm{BP} 86 /$ def2-SV(P)) $=-2013.431622450$

$\begin{array}{llll}C & -2.6026391 & 3.3230871 & 0.3056208\end{array}$

$\begin{array}{llll}\text { C } & -2.4938874 & 2.2953521 & -0.6358888\end{array}$

$\begin{array}{llll}\mathrm{P} & -1.0165807 & 1.4047660 & -1.0289231\end{array}$

$\begin{array}{llll}\text { C } & 0.2082455 & 1.6924128 & 0.3049338\end{array}$

C $\quad-1.5767729-0.3661140 \quad-1.1567329$

$\begin{array}{llll}\text { C } & -0.2828014 & 1.9303665 & -2.6387806\end{array}$

$\begin{array}{llll}\text { C } & 1.6307539 & 1.1258824 & 0.1378145\end{array}$

H $\quad 0.2865745 \quad 2.8146265 \quad 0.2876995$

$\begin{array}{llll}\mathrm{H} & -0.2659916 & 1.3504627 & 1.2536000\end{array}$

C $\quad-0.5420976-1.5002118-1.0488167$

H $\quad-2.3463991-0.4761139-0.3598541$

$\begin{array}{llll}\mathrm{H} & -2.1243409 & -0.4293128 & -2.1273497\end{array}$

C $\quad 0.6786198 \quad 0.9855727 \quad-3.3807911$

H $\quad-1.1552930 \quad 2.1786162 \quad-3.2859032$

$\begin{array}{llll}\mathrm{H} & 0.2036581 & 2.9044162 & -2.3473398\end{array}$

$\begin{array}{llll}\text { P } & -2.4205245 & 5.6207149 & -0.5645219\end{array}$

$\mathrm{H} \quad-3.3801647 \quad 1.9893144 \quad-1.2224180$

$\begin{array}{llll}\text { C } & 2.4991947 & 1.4086215 & 1.3776499\end{array}$

H $\quad 1.6194224 \quad 0.0292255 \quad-0.0561457$

$\begin{array}{llll}\mathrm{H} & 2.1076231 & 1.6090779 & -0.7433941\end{array}$

C $-1.1764004-2.8865952-1.2673161$

H $\quad 0.2800035 \quad-1.3537635 \quad-1.7839727$

H $\quad-0.0675648-1.4738567 \quad-0.0416220$

C $\quad 1.2879067 \quad 1.6548493 \quad-4.6274122$

$\begin{array}{lllll}\mathrm{H} & 1.5054508 & 0.6634479 & -2.7087468\end{array}$

$\begin{array}{lllll}\mathrm{H} & 0.1460189 & 0.0555751 & -3.6922198\end{array}$

C $3.93880890 .9011218 \quad 1.2290586$

$\begin{array}{llll}\mathrm{H} & 2.5104441 & 2.5071403 & 1.5606053\end{array}$

$\begin{array}{llll}\mathrm{H} & 2.0246899 & 0.9415345 & 2.2738026\end{array}$

$\begin{array}{llll}\text { C } & -0.1742347 & -4.0394770 & -1.1258663\end{array}$ 


\begin{tabular}{|c|c|c|c|}
\hline & -2 . & -3 & \\
\hline & -1.6 & 39600 & -2 \\
\hline & 922 & 0.7361845 & -5 \\
\hline & 0.4640046 & 851 & \\
\hline & 492 & 93 & \\
\hline & 093 & 1.1128613 & \\
\hline & 3.9701046 & -0.1989960 & 1.05 \\
\hline & 4.45 & 1.3960856 & 0.37 \\
\hline & 576 & -5.0 & \\
\hline & 0.6 & -3.9 & $-1 . \varepsilon$ \\
\hline & 0.28 & -4.0598570 & -0.1 \\
\hline & 798 & 1.2526368 & -6.25 \\
\hline & $3.0 \mathrm{c}$ & 0.4 & -4.7 \\
\hline & 1.7 & -0.1 & -5. \\
\hline & -1.15 & 6.76 & 0.17 \\
\hline & -2.00 & 5.33 & -2.3 \\
\hline & $-1.7 \varepsilon$ & 3.49 & 1.0 \\
\hline & -3.6 & 17 & \\
\hline & -4.0 & 6.4 & -0.4 \\
\hline & -0.5 & 7.9 & -0.6 \\
\hline & -1.5 & 7.12 & \\
\hline & -0.3 & 6.0 & 0. \\
\hline & -2.3 & 6.3 & -3 \\
\hline & -0.9 & 09 & -2.2 \\
\hline & -2.4 & 987 & -2.5 \\
\hline & -4.2 & 7.97 & -0.72 \\
\hline & -4.7 & 5.8 & -1 \\
\hline & -4.4 & 6.2 & \\
\hline & 0.46 & 8.74 & 0.1 \\
\hline & -0.1 & 7.5 & -1.5 \\
\hline & -1.4 & 8.61 & 99674 \\
\hline & -1.9 & 5.92 & -4.8 \\
\hline & -3.4 & 6.5 & -3.4 \\
\hline & -1.9 & 7.3525708 & -3.18 \\
\hline & -5.6 & 8.4722459 & -0.5992472 \\
\hline & -3. & 8.5 & 0.0 \\
\hline & -3.8 & 81 & -1. \\
\hline & & 9.88 & -0.70 \\
\hline & 0.00 & 9.1510239 & 1.0500055 \\
\hline & 82 & 8.05 & 2683 \\
\hline & -2.2 & 6.93 & -5.9 \\
\hline & -0.8 & 5.7641085 & -4.81 \\
\hline & -2.38 & 4.9389857 & -5.0641492 \\
\hline & -5.82 & 9.9829972 & -0.8263577 \\
\hline & -6.3 & 7.9222767 & -1.32 \\
\hline & $-6.0^{\prime}$ & 8.2069313 & 0.4105967 \\
\hline & 287 & 10.4552663 & -0.1163312 \\
\hline & 1.57 & 9.4994617 & -1.6144387 \\
\hline & & 10.6 & -1.0 \\
\hline & -1.9474366 & 6.5931873 & -6.923701 \\
\hline & -3.3906160 & 7.1006750 & \\
\hline
\end{tabular}

$\begin{array}{cccc}\mathrm{H} & -1.8178566 & 7.9297580 & -5.7325084 \\ \mathrm{H} & -6.8799413 & 10.3098533 & -0.7313551 \\ \mathrm{H} & -5.2228380 & 10.5630114 & -0.0871934 \\ \mathrm{H} & -5.4687381 & 10.2757040 & -1.8415346 \\ \mathrm{O} & 0.6498096 & 4.3780858 & -0.9887277 \\ \mathrm{C} & 4.4793340 & 6.1075768 & -1.1381701 \\ \mathrm{C} & 3.8654137 & 5.8175974 & 0.0962754 \\ \mathrm{C} & 2.5856432 & 5.2449847 & 0.1538885 \\ \mathrm{C} & 1.8391288 & 4.9184044 & -1.0346451 \\ \mathrm{C} & 2.4932013 & 5.2386280 & -2.2779757 \\ \mathrm{C} & 3.7719891 & 5.8111739 & -2.3210528 \\ \mathrm{H} & 5.4854066 & 6.5570708 & -1.1780011 \\ \mathrm{H} & 4.3974258 & 6.0449238 & 1.0384685 \\ \mathrm{H} & 2.1165274 & 5.0294085 & 1.1312382 \\ \mathrm{H} & 1.9491069 & 5.0213716 & -3.2153542 \\ \mathrm{H} & 4.2301120 & 6.0349408 & -3.3021607\end{array}$

\section{TS-S6_cis}

$\mathrm{E}(\mathrm{BP} 86 /$ def2-SV(P) $)=-363.0964728956$

$\begin{array}{llll}\text { C } & -1.8244269 & 0.0373816 & -0.7300839\end{array}$

$\begin{array}{llll}\text { C } & -0.6405977 & 0.4713783 & -0.8004763\end{array}$

$\mathrm{H} \quad-2.4761540 \quad-0.5439661 \quad-0.0689029$

H $\quad 0.1334583 \quad 1.0178636 \quad-1.3447580$

$\begin{array}{llll}\mathrm{N} & 0.9041815 & 0.0613692 & 0.6457713\end{array}$

$\begin{array}{llll}\text { C } & 2.0770329 & -0.0889423 & -0.0064503\end{array}$

$\begin{array}{llll}\text { C } & 2.7698730 & -1.4278045 & 0.4407581\end{array}$

$\begin{array}{llll}\text { C } & 1.5978898 & -2.2169693 & 1.0378737\end{array}$

$\begin{array}{llll}\text { C } & 0.6331604 & -1.0733874 & 1.4879628\end{array}$

$\begin{array}{llll}\mathrm{H} & 1.1061090 & -2.8202337 & 0.2395828\end{array}$

$\begin{array}{llll}\mathrm{H} & 1.8742217 & -2.9155183 & 1.8640884\end{array}$

H $\quad 0.7993246 \quad-0.8369322 \quad 2.5785481$

$\begin{array}{llll}\mathrm{H} & -0.4444748 & -1.3761983 & 1.4007137\end{array}$

H $3.5558498 \quad-1.1993027 \quad 1.2027709$

$\begin{array}{lllll}0 & 2.5745168 & 0.6838069 & -0.8529318\end{array}$

H $3.2804356 \quad-1.9142448 \quad-0.4205665$

\section{TS-S6_trans}

\begin{tabular}{lrrr}
\multicolumn{4}{c}{$\mathrm{E}(\mathrm{BP} 86 / \mathrm{def} 2-\mathrm{SV}(\mathrm{P}))=-363.0892426722$} \\
$\mathrm{C}$ & -1.6083824 & -0.1744264 & -0.7631828 \\
$\mathrm{C}$ & -0.5263253 & 0.4665704 & -0.8297936 \\
$\mathrm{H}$ & -2.5787847 & -0.3491370 & -1.2310443 \\
$\mathrm{H}$ & 0.2168875 & 1.1442188 & -1.2599501 \\
$\mathrm{~N}$ & 0.8582783 & 0.0531965 & 0.6292071 \\
$\mathrm{C}$ & 2.0884716 & -0.0612212 & 0.0764609 \\
$\mathrm{C}$ & 2.7252697 & -1.4367055 & 0.4846048 \\
$\mathrm{C}$ & 1.4934512 & -2.2496002 & 0.9093905 \\
$\mathrm{C}$ & 0.4978794 & -1.1338195 & 1.3533457 \\
$\mathrm{H}$ & 1.0736278 & -2.7834782 & 0.0250109 \\
$\mathrm{H}$ & 1.6892902 & -3.0132231 & 1.7002870 \\
$\mathrm{H}$ & 0.5537163 & -0.9777242 & 2.4671230 \\
$\mathrm{H}$ & -0.5712928 & -1.3872831 & 1.1035347
\end{tabular} 
$\begin{array}{cccc}\mathrm{H} & 3.4376068 & -1.2780146 & 1.3321643 \\ \mathrm{O} & 2.6584394 & 0.7669754 & -0.6637781 \\ \mathrm{H} & 3.3126669 & -1.8644280 & -0.3597798\end{array}$

\section{TS-S7}

$\mathrm{E}(\mathrm{BP} 86 /$ def2-SV(P) $)=-1255.452569993$

$\begin{array}{llll}\text { C } & 0.3624461 & 0.7372239 & 3.0379923\end{array}$

$\begin{array}{llll}\text { C } & 1.7953895 & 0.6842177 & 3.5784089\end{array}$

C $\quad 1.7394249 \quad-0.4112540 \quad 4.6531964$

C $\quad 0.2761499 \quad-0.3717422 \quad 5.1712736$

$\begin{array}{llll}\mathrm{N} & -0.4590526 & 0.3285686 & 4.1312330\end{array}$

$\begin{array}{llll}\mathrm{H} & 2.0181567 & 1.6865571 & 4.0201310\end{array}$

H $\quad 2.5312711 \quad 0.4958437 \quad 2.7720377$

H $\quad 1.9343962 \quad-1.3943769 \quad 4.1783566$

H $\quad 2.4725278-0.2636085 \quad 5.4744168$

$\mathrm{H} \quad-0.1402158 \quad-1.3952469 \quad 5.3285497$

$\begin{array}{llll}\mathrm{H} & 0.1764640 & 0.1764068 & 6.1401714\end{array}$

C $\quad-1.8251693 \quad 0.4945243 \quad 4.1304730$

C $\quad-2.6960253 \quad 0.0652113 \quad 5.0822786$

$\mathrm{H} \quad-3.7707616 \quad 0.2827907 \quad 4.9744690$

H $-2.3746112-0.4908301 \quad 5.9793625$

$\mathrm{H} \quad-2.1619826 \quad 1.0547298 \quad 3.2395119$

C $\quad 0.3737787 \quad-1.0312451 \quad 1.7261130$

$\begin{array}{llll}0 & -0.0809911 & 1.4568900 & 2.1187653\end{array}$

C $\quad 1.1916221-1.1209406 \quad 0.6408169$

P $\quad 0.5632824 \quad-0.7525269-1.0008130$

H $\quad 2.3033848 \quad-1.1220911 \quad 0.7073724$

H $\quad-0.7237402-0.9980016 \quad 1.5203125$

C $1.6978154-1.3951368$-2.3225243

C $-1.1182583-1.5004724-1.1889979$

C $\quad 0.4325926 \quad 1.0878460 \quad-1.1138323$

C $\quad 1.6759058-0.7469082-3.7183094$

H $\quad 2.7155773 \quad-1.2942265 \quad-1.8761026$

H $\quad 1.5064574 \quad-2.4916495 \quad-2.3852818$

C $\quad-1.6569182-1.7198060 \quad-2.6137271$

H $\quad-1.0585547 \quad-2.4658112 \quad-0.6370117$

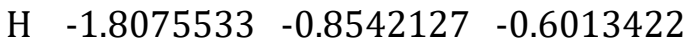

C $-0.5517556 \quad 1.7290738-2.1068671$

$\mathrm{H} \quad 0.1834806 \quad 1.3711855-0.0581436$

$\mathrm{H} \quad 1.4727120 \quad 1.4518028 \quad-1.2889898$

C $\quad 2.7047050 \quad-1.3760586-4.6764237$

H $\quad 0.6601821 \quad-0.8296207 \quad-4.1667437$

$\mathrm{H} \quad 1.8858509 \quad 0.3434673 \quad-3.6252612$

C $\quad-3.0681631-2.3371282-2.6101086$

H $-1.6816528-0.7571135-3.1729881$

$\mathrm{H} \quad-0.9711209-2.3926270 \quad-3.1790829$

C $\quad-0.5455059 \quad 3.2663615 \quad-2.0078534$

$\mathrm{H} \quad-0.3187204 \quad 1.4281634 \quad-3.1547099$

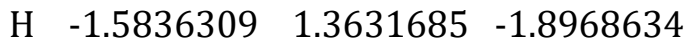

C $\quad 2.7104992-0.7302768-6.0679801$

H $\quad 3.7209032 \quad-1.2986900 \quad-4.2228506$
H $\quad 2.4962207 \quad-2.4678475 \quad-4.7708856$

C $-3.6232723-2.5865522-4.0182144$

H $\quad-3.0456371 \quad-3.2950298 \quad-2.0394128$

$\mathrm{H} \quad-3.7581412-1.6649591 \quad-2.0482970$

C $\quad-1.5470145 \quad 3.9389014 \quad-2.9553818$

$\mathrm{H} \quad-0.7631808 \quad 3.5595657 \quad-0.9552120$

$\mathrm{H} \quad 0.4831221 \quad 3.6411159 \quad-2.2223690$

H $\quad 3.4621817 \quad-1.2096608-6.7328343$

$\mathrm{H} \quad 1.7173421 \quad-0.8228774 \quad-6.5630175$

$\mathrm{H} \quad 2.9561043 \quad 0.3543575 \quad-6.0115586$

H $\quad-4.6422362 \quad-3.0301006 \quad-3.9770199$

H $\quad-3.6930196 \quad-1.6405419 \quad-4.6016207$

H $\quad-2.9759701 \quad-3.2873405-4.5928556$

$\mathrm{H} \quad-1.5133047 \quad 5.0464172 \quad-2.8582248$

$\mathrm{H} \quad-1.3341458 \quad 3.6890824-4.0199800$

H $\quad-2.5907406 \quad 3.6166388-2.7382507$

\section{TS-S8}

$\mathrm{E}(\mathrm{BP} 86 /$ def2-SV(P)) $=-1255.457623399$

C $\quad-2.5589216 \quad 1.4530400 \quad-1.8403148$

C $\quad-4.0090343 \quad 1.8744763-1.9255970$

C $\quad-3.9024966 \quad 3.2256797 \quad-2.6748019$

C $\quad-2.7009139 \quad 3.0299837 \quad-3.6281702$

N $\quad-1.9357458 \quad 1.9331369-3.0330085$

H $\quad-4.4792642 \quad 1.9569869-0.9237975$

H $\quad-4.6195726 \quad 1.1536163-2.5261152$

H $\quad-4.8281085 \quad 3.5059193 \quad-3.2226159$

$\mathrm{H} \quad-3.6799197 \quad 4.0367981-1.9473920$

$\mathrm{H} \quad-3.0103016 \quad 2.7459506 \quad-4.6645199$

H $\quad-2.0814293 \quad 3.9546364 \quad-3.7188839$

C $\quad-0.8450945 \quad 1.3995754 \quad-3.6662489$

$\begin{array}{lllll}\text { C } & -0.1977414 & 1.8938749 & -4.7642628\end{array}$

$\mathrm{H} \quad 0.6365414 \quad 1.3235671 \quad-5.2023224$

$\mathrm{H} \quad-0.4821698 \quad 2.8407699 \quad-5.2518241$

H $\quad-0.5054738 \quad 0.4522209 \quad-3.2145618$

C $\quad-1.9994225 \quad 0.8008398 \quad-0.7784994$

$\begin{array}{lllll}0 & -0.4115674 & 1.1903604 & -0.6606348\end{array}$

$\begin{array}{llll}\text { C } & -2.4360673 & -0.0975998 & 0.1854411\end{array}$

P $\quad 0.6436120 \quad 0.0604002 \quad-0.2640303$

$\mathrm{H} \quad-2.2833723 \quad 0.1102904 \quad 1.2649087$

H $\quad-3.2667817 \quad-0.7823438 \quad-0.0625748$

C $\quad 0.7284004 \quad-0.1799800 \quad 1.5617026$

C $\quad 0.4440162 \quad-1.5661142-1.1235133$

$\begin{array}{llll}\text { C } & 2.2196978 & 0.8399185 & -0.8358741\end{array}$

$\mathrm{H} \quad 1.9326535 \quad 1.3510289-1.7830030$

H $\quad 2.4249153 \quad 1.6557935-0.1046018$

C $\quad 3.4714597 \quad-0.0304761-1.0522939$

H $\quad-0.1682245-0.7869092 \quad 1.8150885$

H $\quad 0.5350583 \quad 0.8374385 \quad 1.9733913$

$\begin{array}{llll}\text { C } & 2.0091541 & -0.7830076 & 2.1661492\end{array}$

$\begin{array}{llll}\text { C } & -0.1981787 & -2.7197911 & -0.3336398\end{array}$ 


$\begin{array}{cccc}\text { H } & 1.4520511 & -1.8638972 & -1.4927454 \\ \mathrm{H} & -0.1714849 & -1.3315311 & -2.0219395 \\ \mathrm{C} & 4.7053979 & 0.8107880 & -1.4292105 \\ \mathrm{H} & 3.7024044 & -0.6289807 & -0.1417638 \\ \mathrm{H} & 3.2801775 & -0.7669756 & -1.8667062 \\ \mathrm{C} & 1.8935471 & -0.9870080 & 3.6885679 \\ \mathrm{H} & 2.2407318 & -1.7624207 & 1.6856081 \\ \mathrm{H} & 2.8785709 & -0.1186550 & 1.9557354 \\ \mathrm{C} & -0.4000077 & -3.9749638 & -1.2004207 \\ \mathrm{H} & 0.4379957 & -2.9813965 & 0.5444248 \\ \mathrm{H} & -1.1774083 & -2.3600174 & 0.0594311 \\ \mathrm{C} & 5.9553860 & -0.0357603 & -1.7010893 \\ \mathrm{H} & 4.4649259 & 1.4260211 & -2.3274620 \\ \mathrm{H} & 4.9156602 & 1.5379916 & -0.6098537 \\ \mathrm{C} & 3.1638347 & -1.5700331 & 4.3205466 \\ \mathrm{H} & 1.6490600 & -0.0102852 & 4.1682771 \\ \mathrm{H} & 1.0266192 & -1.6553672 & 3.9006525 \\ \mathrm{C} & -1.0241066 & -5.1430780 & -0.4256364 \\ \mathrm{H} & -1.0503545 & -3.7142065 & -2.0680526 \\ \mathrm{H} & 0.5772926 & -4.2949288 & -1.6351882 \\ \mathrm{H} & 6.2407143 & -0.6391983 & -0.8095333 \\ \mathrm{H} & 5.7912436 & -0.7432518 & -2.5452338 \\ \mathrm{H} & 3.4148144 & -2.5644490 & 3.8865087 \\ \mathrm{H} & 4.0414695 & -0.9035894 & 4.1597506 \\ \mathrm{H} & -0.3793719 & -5.4562126 & 0.4270561 \\ \mathrm{H} & -2.0177534 & -4.8646283 & -0.0083831 \\ \mathrm{H} & 6.8260340 & 0.6037366 & -1.9654436 \\ \mathrm{H} & 3.0429547 & -1.7049468 & 5.4178391 \\ \mathrm{H} & -1.1696047 & -6.0310369 & -1.0796154\end{array}$

$\begin{array}{cccc}\mathrm{H} & -0.9595794 & -1.4323831 & 0.2371641 \\ \mathrm{C} & 3.2943057 & -0.3568559 & 1.5413253 \\ \mathrm{H} & 1.6800420 & 1.0724094 & 1.2647641 \\ \mathrm{H} & 1.1577170 & -0.6312635 & 1.2304288 \\ \mathrm{C} & -1.9364985 & 2.9200835 & 0.9106440 \\ \mathrm{H} & -2.0075000 & 0.9101645 & 0.0903858 \\ \mathrm{H} & -0.6493028 & 1.1905777 & 1.2093915 \\ \mathrm{C} & -1.7348665 & -4.1079693 & 0.0363964 \\ \mathrm{H} & -2.6783400 & -2.5950553 & -1.2161278 \\ \mathrm{H} & -1.3793037 & -3.4999790 & -2.0270158 \\ \mathrm{C} & 3.2456083 & -0.3655977 & 3.0746559 \\ \mathrm{H} & 3.5799035 & -1.3690893 & 1.1698453 \\ \mathrm{H} & 4.1005704 & 0.3322101 & 1.1955273 \\ \mathrm{C} & -2.8701294 & 2.6434191 & 2.0960870 \\ \mathrm{H} & -1.1660228 & 3.6702059 & 1.2076567 \\ \mathrm{H} & -2.5129282 & 3.3876525 & 0.0788083 \\ \mathrm{H} & -2.4591274 & -4.9088230 & -0.2303234 \\ \mathrm{H} & -0.7442800 & -4.5917675 & 0.1947739 \\ \mathrm{H} & -2.0561571 & -3.6770038 & 1.0119129 \\ \mathrm{H} & 4.2225502 & -0.6727083 & 3.5084987 \\ \mathrm{H} & 3.0013135 & 0.6426898 & 3.4794374 \\ \mathrm{H} & 2.4735164 & -1.0734940 & 3.4526814 \\ \mathrm{H} & -3.3593465 & 3.5768209 & 2.4518076 \\ \mathrm{H} & -3.6769838 & 1.9278041 & 1.8185769 \\ \mathrm{H} & -2.3164536 & 2.2055110 & 2.9577160 \\ \mathrm{C} & -1.9757180 & 1.6899658 & -4.3338213 \\ \mathrm{C} & -1.9871945 & 2.9335622 & -4.3896309 \\ \mathrm{H} & -2.4438552 & 0.7108951 & -4.3410823 \\ \mathrm{H} & -1.5758781 & 3.9386133 & -4.4282458\end{array}$

\section{TS-S9}

\begin{tabular}{lccc}
\multicolumn{4}{c}{$\mathrm{E}(\mathrm{BP} 86 / \mathrm{def} 2-\mathrm{SV}(\mathrm{P}))=-968.9416021873$} \\
$\mathrm{C}$ & 0.2533295 & 0.8236332 & -4.3018723 \\
$\mathrm{C}$ & 1.1187053 & 1.0782637 & -3.2776248 \\
$\mathrm{P}$ & 0.5613449 & 0.5583448 & -1.6612294 \\
$\mathrm{H}$ & 0.6155622 & 1.2342751 & -5.2769637 \\
$\mathrm{H}$ & 2.0877413 & 1.6424390 & -3.2582454 \\
$\mathrm{C}$ & -0.3289600 & 1.9546237 & -0.8269053 \\
$\mathrm{C}$ & -0.6221277 & -0.8431343 & -1.8491954 \\
$\mathrm{C}$ & 2.0391393 & 0.0803090 & -0.6439970 \\
$\mathrm{H}$ & -1.6216314 & -0.3797069 & -2.0056134 \\
$\mathrm{C}$ & -0.6727461 & -1.9000950 & -0.7334998 \\
$\mathrm{H}$ & -0.3491460 & -1.2728814 & -2.8409640 \\
$\mathrm{H}$ & 2.3613600 & -0.9107709 & -1.0399014 \\
$\mathrm{C}$ & 1.9618048 & 0.0610338 & 0.8917123 \\
$\mathrm{H}$ & 2.8257506 & 0.7996581 & -0.9749669 \\
$\mathrm{H}$ & -0.9322207 & 2.3843029 & -1.6615129 \\
$\mathrm{C}$ & -1.2324301 & 1.6558955 & 0.3825229 \\
$\mathrm{H}$ & 0.4473425 & 2.7152108 & -0.5756313 \\
$\mathrm{C}$ & -1.6652602 & -3.0330623 & -1.0557103 \\
$\mathrm{H}$ & 0.3389801 & -2.3424338 & -0.5789398
\end{tabular}

